

\title{
DISCLAIMER
}

This report was prepared as an account of work sponsored by an agency of the United States Government. Neither the United States Government nor any agency thereof, nor Battelle Memorial Institute, nor any of their employees, makes any warranty, express or implied, or assumes any legal liability or responsibility for the accuracy, completeness, or usefulness of any information, apparatus, product, or process disclosed, or represents that its use would not infringe privately owned rights. Reference herein to any specific commercial product, process, or service by trade name, trademark, manufacturer, or otherwise does not necessarily constitute or imply its endorsement, recommendation, or favoring by the United States Government or any agency thereof, or Battelle Memorial Institute. The views and opinions of authors expressed herein do not necessarily state or reflect those of the United States Government or any agency thereof.

\author{
PACIFIC NORTHWEST NATIONAL LABORATORY \\ operated by \\ BATTELLE \\ for the \\ UNITED STATES DEPARTMENT OF ENERGY \\ under Contract DE-AC05-76RL01830
}

Printed in the United States of America
Available to DOE and DOE contractors from the Office of Scientific and Technical Information,
P.O. Box 62, Oak Ridge, TN 37831-0062;
ph: (865) 576-8401
fax: (865) 576-5728
email: reports@adonis.osti.gov

\begin{abstract}
Available to the public from the National Technical Information Service, U.S. Department of Commerce, 5285 Port Royal Rd., Springfield, VA 22161 ph: (800) 553-6847 fax: (703) 605-6900

email: orders@ntis.fedworld.gov

online ordering: http://www.ntis.gov/ordering.htm
\end{abstract}

This document was printed on recycled paper. 


\section{Laboratory Directed Research and Development Annual Report}

Fiscal Year 2010

March 2011

Prepared for

the U.S. Department of Energy

under Contract DE-AC05-76RL01830

Pacific Northwest National Laboratory

Richland, Washington 99352 


\section{Acknowledgments}

Pacific Northwest National Laboratory's LDRD Program office is grateful for the support received throughout the year, particularly while preparing this Annual Report. Special appreciation is given to the Laboratory leadership who shape PNNL's strategic initiatives and whose vision and understanding of the Nation's technological needs help guide and refine the content of PNNL's LDRD portfolio. In addition, we are especially appreciative of the many research staff who perform the research and provide their annual reports, and to the technical and administrative management of PNNL's directorates within which R\&D is pursued.

LDRD Program Office:

Pamela Hughes, Program Manager

Sarah Timmons, Administrative Specialist

Jennifer Eischens, Administrator

Pamela Hartsock, Technical Editor

Debora Schulz, Document Production

Larry Seid, IT Analyst

Stacy Schulze, IT Analyst

Meryl Birn, IT Developer 


\section{Contents}

Acknowledgments.

Laboratory Director's Message.

Mission Overview

Introduction

PNNL's LDRD Management Process.

\section{Advanced Sensors and Instrumentation}

Advanced Environmental Sampling Technology for Safeguards and Proliferation Detection ....................................... 3

Comparison of Assays Utilizing Existing Biological Reagents Specific for Explosives .............................................. 5

Development of a Dual-Sided, Temperature-Controlled, Continuous-Flow Environmental Chamber.............................. 6

Distance-of-Flight Mass Spectrometry for Rapid, Portable Actinide Analysis ............................................................ 8

In Situ High-Pressure X-Ray Diffraction Investigation of Caprock Mineral Reactions With Water Solvated in

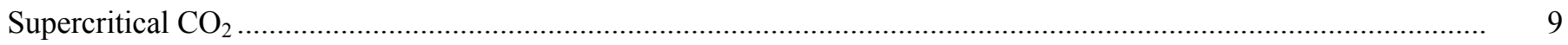

Multi-Modality Sensing Platform for Smart Detection of Explosive Traces ............................................................ 11

Rapid, Sensitive and Selective Explosives Detection Using Tunable Chemical Ionization Drift Mass Spectrometry ........ 12

Standoff Detection of Trace Explosives on Vehicles Using a Cooled Dispersive Longwave Infrared Spectrometer.......... 14

Standoff Hyperspectral Imaging of Explosives Residues Using Broadly Tunable External Quantum Cascade

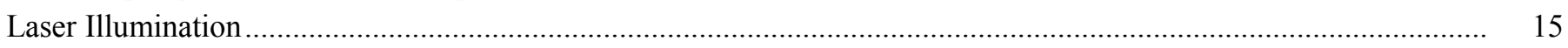

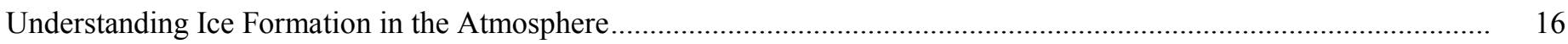

\section{Biological Sciences}

Application of a Systems Biology Approach to Understanding Protein Function .................................................... 21

Combining Proteomic Technologies to Create a Platform for Spatiotemporal Enzyme Activity Profiling ...................... 23

Community Diversity and Functional Redundancy of Cellulytic Microbial Communities in Soil Aggregates.................. 24

Designed Affinity Reagents with Extreme Stability and Selectivity ........................................................................ 25

Distinguishing Yersinia pestis from Natural Host and Laboratory Culture ............................................................... 26

Exploration of Pan-Omics for Biological Research .......................................................................................... 27

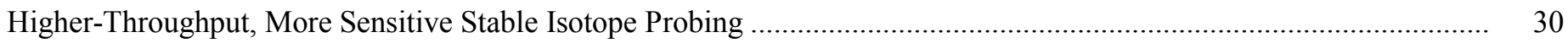

Identification of Functional Proteins Relevant to Bioenergy and Disease Pathology by Multiplexed

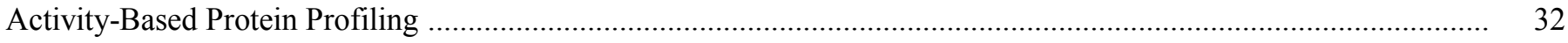

MicroCT Development for Multimodal Imaging in Systems Biology ..................................................................... 33

Micro-Fluidic Models for Studying Microbial Communities - Integration of Micro-Fluidic Model Experimentation,

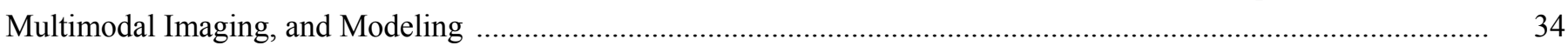

Microscale Spectroscopic Analyses of Cellulose Degradation and Uptake by a Microbial Community........................... 35

Mining the Data from Research on Dogs Exposed to Internally-deposited Radionuclides............................................ 36

Next Generation Software for Automated Structural Identification of Metabolites....................................................... 37 
Proteomics Measurements of Functional Redundancy and Stability Testing of Cellulose Degrading Anaerobic

Microbial Communities Within Engineered Bioreactors.

Synthetic Biology Approach for Hydrocarbon Production in Microbial Photoautotrophs...

\section{Chemistry}

A Real-Time Optical Spectroscopy Platform for Investigating Molecular Mineral Transformations for $\mathrm{CO}_{2}$ Storage .......

Biochemistry of Glycan Signatures: A Novel Approach to Sensor and Vaccine Development.

$\mathrm{CO}_{2}$ Separation Scale-Up

Computational Studies of the Transport and Thermodynamic Characteristics of a Variety of Gases in Ionic Liquids ....... 50

Detection and Characterization of Uranium Hexafluoride Reaction Products in the Environment ................................ 52

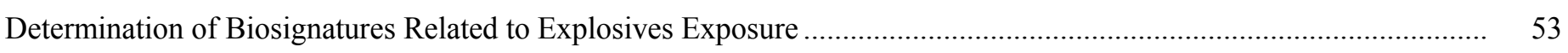

Development of Inorganic Water Oxidation Electrocatalysts .................................................................................... 54

Development of Preparative Mass Spectrometry for the Creation of Novel Catalyst Materials .................................... 55

Development of Techniques for Determining Medium-Range Order on the Surfaces of Catalysts Using Solid-State

NMR Spectroscopy and Computational Chemistry ....................................................................................... 56

Enhanced Ion Detection Mechanisms for Ion Mobility Spectrometry …............................................................ 57

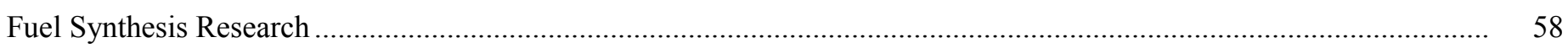

Fundamentals of Carbonate Formation: Interactions of $\mathrm{CO}_{2}$ with Supported Metal Oxide Clusters ................................... 60

High Precision Isotope Forensics via Multi-Collector Multi-Collector Inductively Coupled Plasma

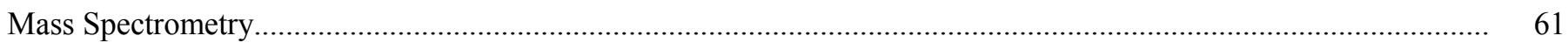

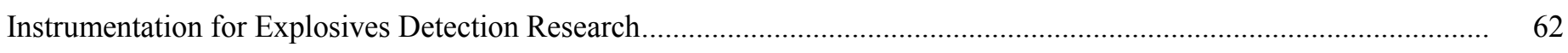

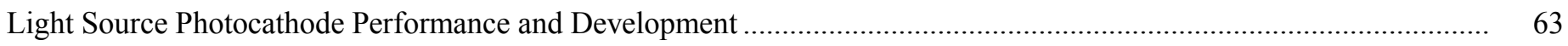

Methodology and Tool Development for Rapid Assessments for $\mathrm{CO}_{2}$ Capture Technologies ...................................... 64

Molecular Structure and Interaction at Aqueous, Non-Aqueous Liquid Interfaces and Catalytic Solid Surfaces .............. 66

Multiscale Investigation of $\mathrm{CO}_{2}$ Behavior in Subsurface Under Extreme Conditions .................................................. 67

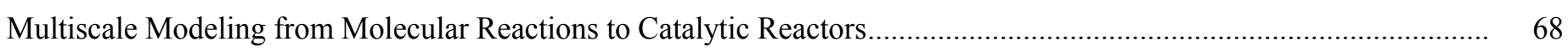

Non-Metal Activation of Hydrogen for Energy Storage in Chemical Bonds ...........................................................

Oxygen Optode for Chemical Imaging in Microfluidic Microbial Models ................................................................. 71

Proof-of-Principle Demonstration of Fluorescence Labeling of Cellulose and Mcroscopic Fluoresence Imaging of

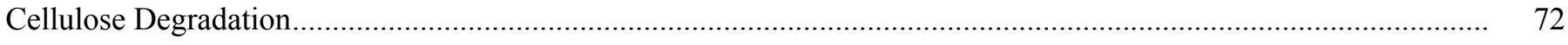

Speciation and Distribution of f-Elements from Enhanced Separations and Safeguards .............................................

Transformational Materials for Advanced Stationary Electricity Storage....................................................................

Transition and Transport of Surface Residue from Non-Volatile Explosives to Gas Phase Ions for Sensitive and

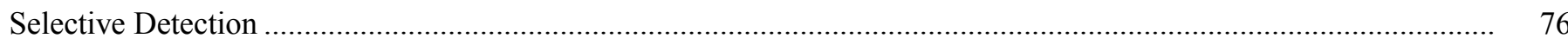

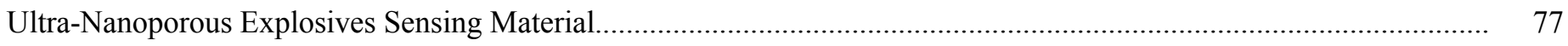

\section{Earth and Space Sciences}

Advanced Scalability for STOMP: Subsurface Simulation and Characterization at Extreme Resolution

Computational Framework for Diagnostics, Validation and Intercomparison of Numerical Simulators for

Geologic Sequestration

Data Assimilation Tools for $\mathrm{CO}_{2}$ Reservoir Model Development 
Developing Ice Nucleation Parameterizations for Large-Scale Models.....

Development and Evaluation of an Externally-Mixed Sectional Aerosol Model

Development of Climate Modeling and Integrated Modeling at Regional Scales Framework and

Functional Specifications.

Development of Prototype Integrated Earth System and Environmental System Models ............................................ 89

Development of Regional-Global Climate Assessment Model ........................................................................... 91

Ideas for Evaluation of Deep Convection Parameterizations With Scanning Radar Data ........................................... 92

Improving the Characterization of Aerosols as Forcing Agents in the Climate System ............................................. 93

In Situ Imaging of Mineral-Supercritical $\mathrm{CO}_{2}$ Reactions with Atomic Force Microscopy ........................................... 94

Integrated Regional Earth System Model (iRESM) Prototype Regional Testbed Specification and Selection .................. 95

Micromodel Pore-Scale Studies of Caprock-Sealing Efficiency and Trapping Mechanisms Related to

$\mathrm{CO}_{2}$ Sequestration

Predicting Climate Change Impacts on Hydropower and Riverine Ecosystems ........................................................ 99

Predicting the Feasibility of Geologic Co-Sequestration of $\mathrm{CO}_{2}, \mathrm{SO}_{\mathrm{x}}$ and $\mathrm{NO}_{\mathrm{x}}$ Under a Broad Range of Conditions........ 100

Transfer and Evaluation of the Community Atmosphere Model Parameterization Suite to Weather Research

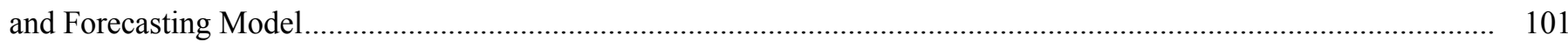

Understanding the Sources and Consequences of Uncertainties ....................................................................... 102

\section{Energy Supply and Use}

Alpha Voltaics

Analytical Framework for Assessing the Economics of Reliable Fuel Services and Supply

Behavioral and Social Modeling to Reduce Energy Use, Climate Change, and Power Grid Vulnerability.......

Centralized Control vs. Decentralized Control: Implications of Demand Response and Distributed Resources on

Power System Security

Development of a State Prediction Methodology Powered by Phasor Measurement Unit Data to Improve Operational

Reliability and Efficiency

Development of a Universal Energy and Infrastructure Systems Framework.......

Development of Rechargeable Li/air Batteries

Development of Regional Agriculture-Land Use Models

Electrolyte Development for Next Generation of Lithium Ion Batteries.

Geological Sequestration Software Suite: Numerical Model Development.

Infrastructure Compatible Fuels and Chemicals from Biomass

Innovative Processes for Integrated Emissions Management..

Marine Biomass and its Conversion to Liquid Transportation Fuels ...

Methodology Development for Optimal Energy Storage Sizing and Placement for Local and Regional Planners...

Novel Carbon Capture Materials.

Operation and Process Optimization of Gasification and Carbon Capture Test Facility 
Simulation of Future Electricity System Operations 132

Tools for Evaluation of Net-Zero Community Concept and Integration of Buildings, Renewables and the Grid.

\section{Engineering and Manufacturing Processes}

Demonstration of On-Line Monitoring and Physics Based Prognostics .

Development of a Ballistic Electron Microfabricated Cathode.

Process Modeling of Chemically Complex Solid-Liquid Suspensions

\section{Materials Science and Technology}

Advanced Cathodes for Sodium-Beta Batteries and Renewable Energy Applications .

Advanced Materials for Capturing Lanthanides and Transition Metals from Fission Products.

Advanced Nuclear Magnetic Resonance Characterization of Energy Storage Materials.....

Advanced Sorptive and Signature Indicating Materials for Ultra-Trace Proliferation Detection.

Application of Imperfection Modeling to Accelerated Fuel Clad Qualification and Characterization

Biotemplated Synthesis of Encoded Bimetallic Nanoparticles

Development and Understanding of Nanostructured Materials for Advanced Energy Storage .....

Energy Absorptive Foams: Space-Age Polymers for Down-to-Earth Applications

Friction Stir Welding of Creep-Resistant Oxide Dispersion Strengthened Alloys.

In Situ Nuclear Magnetic Resonance Investigations of Trapping Mechanisms in $\mathrm{CO}_{2}$ Storage

Ion Beam-Nanoparticle Interactions for Radiation Detection

Leak Rate Measurements for Prototypic Pressurized Water Reactor Primary Water Stress Corrosion Cracks.

Material Interface Optimization in Extremely Thin Adsorber Photovoltaics.

Materials and Methods for Low Cost Photovoltaic Manufacturing

Multicomponent Assembly to Achieve Charge Separation and Transport for Energy Conversion.

Multiphysics Capability Development and Application to Magnesium Alloys

Multiscale Charge and Ion Transport Simulations for Nanostructured Electrodes .

Nanoscale Tantalum Oxide Electrocatalysts for Polymer Electrolyte Membrane Fuel Cells

Simultaneous Charge Transport in Laterally Confined One-Dimensional Systems.

Surface Damage and Environment-Induced Cracking Precursors in Light Water Reactor Components.

\section{Mathematics and Computing Sciences}

A Predictive Defense Model for the Smart Grid

A Scalable Fault Tolerance Infrastructure and Algorithms with Programming Models and Scientific Applications. 
Data Decomposition/Optimizations and Dynamic Load Balancing Mechanisms for Extreme Scale Computing in the

Global Arrays Toolkit

Deception for the Defense of Cyber Systems.

Development of Exascale Algorithms for Molecular Modeling

Development of Gaming Technology for Cognitive Enhancement in Predictive Analytics .....

Enabling Hypothesis Driven Research and Discovery in Extreme Data....

Exploring Architectures Suitable for Scientific Applications at Exascale Levels.

Extending Global Arrays Programming Model on Hybrid Architectures

Extreme Scaling for List Based Pattern Comparisons (LiBaPaC).

Geological Sequestration Software Suite Core Architecture and Simulation Framework

Information Integration for Forecasting Dynamic Organizational Behaviors

Integrating Power and Performance Modeling for Exascale Systems

Knowledge Encapsulation Framework

Machine Learning String Tools for Operational and Network Security.

Managing Complexity of High-Volume Predictive and Adaptive Network Operations.

Multiscale Models for Microbial Communities.

Precision Information Fusion Environments

Scalable Performance Diagnostics and Feedback for Massively Parallel Computers

The Analysis and Discovery of Influencing Factors in Social Media

Ultrascalable Solvers for Subsurface Simulation .

Visualizing Uncertainty in Conceptual and Numerical Models for Geological Sequestration

Vulcan: Unexpressed Communication

\section{Nuclear Science and Engineering}

Adaptation of Existing Probabilistic Risk Assessments to Support Reactor Aging Management

Isotopic Ratio Fluence Monitors for Canadian Deuterium Uranium (CANDU) and Pebble Bed Modular

\section{Physics}

Advanced Computing Architectures for Smart Sensors and Sensor Analytics 
First Operation of a Novel, High Mass Detector as a Weakly Interacting Massive Particle (WIMP) Dark

Matter Detector

Multimodal X-Ray Imaging with a Grating-Based Interferometer ......

Spectroscopic X-ray Computed Tomography for Improved Explosives Detection

Appendix A - Fiscal Year 2010 Refereed Publications

A.1

Appendix B - Fiscal Year 2010 Non-Refereed Publications

B. 1

Appendix C - Index of Investigators

C. 1 


\section{Laboratory Director's Message}

PNNL scientists and engineers are making important scientific discoveries and delivering valuable technical solutions critical to our Nation's future. They work across a broad range of activities with wide-ranging impact on our enduring missions of science, energy, environment, and security. Within these areas, we are distinguishing ourselves through world-leading science and technology aimed at our four institutional leadership positions:

- Design and scalable synthesis of materials and chemicals

- Coupling climate and energy systems for sustainability

- Efficient and secure electricity management from generation to end use

- Signature discovery and exploitation for threat detection and reduction.

Our discretionary investments, especially LDRD, are key to advancing these leadership positions and delivering on our mission outcomes. These investments help us to build our

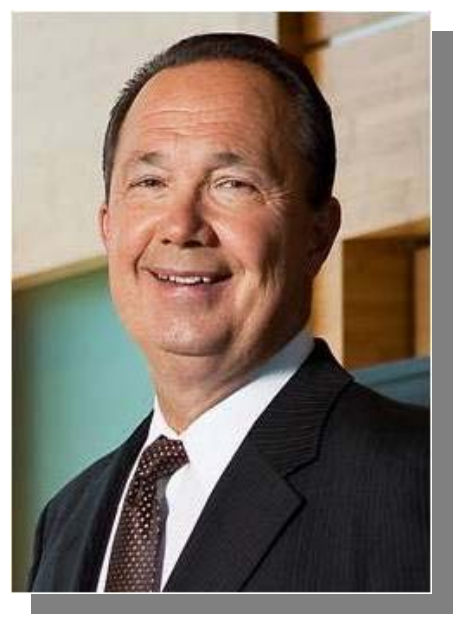
S\&T capabilities and to capitalize on PNNL's talents - all while tackling current and projected problems facing our nation.

We have seen first-hand the benefits of leveraging the discoveries from these investments, which are described in this document. We carefully conduct our LDRD program in compliance with the objectives and guidelines outlined in the DOE Order. And we use rigorous internal and external peer review to maintain the scientific value and soundness of the program and the research projects it enables.

It is with great pride in our researchers' accomplishments that I present PNNL's Fiscal Year 2010 Laboratory Directed Research and Development Annual Report.

\section{Heesere}




\section{Mission Overview}

The Pacific Northwest National Laboratory (PNNL) is a U.S. Department of Energy (DOE) Office of Science (SC) laboratory located in Richland, WA with an enduring mission to advance molecular and environmental sciences and engineering in support of DOE and national needs.

Our vision is to be recognized worldwide and valued nationally for leadership in accelerating scientific discovery and innovation, and deploying solutions to national challenges in energy, national security, and the environment. To do this, we provide distinctive, world-leading science and technology in:

- Design and scalable synthesis of materials and chemicals

- Coupling climate and energy systems for sustainability

- Efficient and secure electricity management from generation to end use

- Signature discovery and exploitation for threat detection and reduction.

PNNL leadership also extends to operating the Environmental Molecular Sciences Laboratory (EMSL), a national scientific user facility providing integrated experimental and computational resources for discovery and technological innovation.

Established in 1965 with 2200 employees and facilities supporting Hanford Site operations, PNNL focused on expanding nuclear fuel cycle research, developing advanced reactor designs and materials, fabricating and testing novel reactor fuels, and monitoring and protecting human health and the environment. PNNL has since become a leading multidisciplinary national laboratory with a long-standing reputation for advancing scientific frontiers through world-class research and development.

PNNL is operated by Battelle Memorial Institute, a private, non-profit, science and technology enterprise that explores emerging areas of science, develops and commercializes technology, and manages laboratories. PNNL currently has approximately 4900 staff members, and total cost for FY 2010 was $\$ 880$ million. In addition to SC, principal PNNL customers include DOE's Offices of Energy Efficiency and Renewable Energy, Environmental Management, Fossil Energy, Electricity Delivery and Energy Reliability, and the National Nuclear Security Administration; U.S. Department of Homeland Security; U.S. Department of Defense; U.S. Nuclear Regulatory Commission; and the National Institutes of Health.

\section{Laboratory at a Glance}

Location: Richland, Washington

Type: Multiprogram Laboratory

Contract Operator: Battelle Memorial Institute

Responsible Site Office: Pacific Northwest Site Office

Website: http://www.pnl.gov/

\section{Physical Assets:}

- 350 acres, 250 acres Battelle, and 101 buildings

- 2,271,000 gsf of buildings

- Replacement Plant Value: $\$ 428,481,552$

- Deferred Maintenance: $\$ \$ 7,966,000$

- Asset Condition Index: .981

- Mission Critical: .983 (Excellent)

- Mission Dependent: .967 (Good)

- Asset Utilization Index: .886 (Adequate)

\section{Human Capital}

- $\quad 4103$ Full-Time Equivalent Employees (FTEs)

- 200 Postdoctoral Researchers

- 417 Undergraduate and Graduate Students

- 1913 Facility Users and Visiting Scientists

FY 2010 Funding by Source (Cost Data in $\$ M$ )

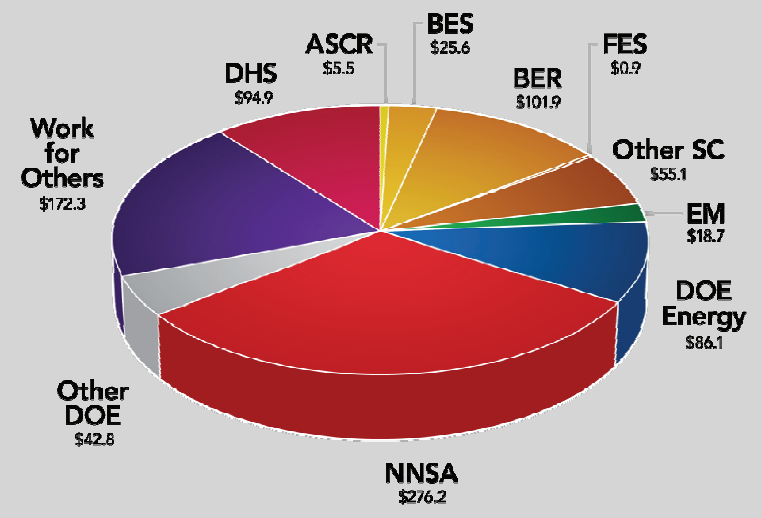

FY 2010 Total Laboratory Operating Costs:

$\$ 880 M$

FY 2010 Total DOE/National Nuclear Security

Administration (NNSA) Costs:

$\$ 612.8 M$

FY 2010 WFO (non-DOE/non-U.S Department

of Homeland Security [DHS]) Costs:

$\$ 172.3 \mathrm{M}$

FY 2010 WFO \% of Total Laboratory Operating Costs:

FY 2010 Total DHS Costs:

$19 \%$

$\$ 94.9 \mathrm{M}$

Recovery Act Obligated from DOE Sources in FY 2010: $\quad \$ 85 \mathrm{M}$ Recovery Act Costed from DOE Sources in FY 2010:

$\$ 96 \mathrm{M}$ 


\section{Introduction}

DOE Order 413.2B sets forth DOE's policy and guidelines for Laboratory Directed Research and Development (LDRD) at its multiprogram laboratories. DOE Order 413.2B requires that each laboratory submit an annual report about its LDRD activities to the Cognizant Secretarial Officer. This document is the PNNL LDRD annual report for fiscal year (FY) 2010.

\section{Program Overview}

A national laboratory must establish and maintain an environment in which creativity and innovation are encouraged and supported in order to fulfill its missions and remain viable in the long term. As such, multiprogram laboratories are given discretion to allocate a percentage of their operating budgets to support research and development projects that align the Laboratory's and DOE's missions and support the missions of other federal agencies, including DHS, DOD, NIH, and others.

DOE Order 413.2B establishes the Department of Energy's policy and guidelines for LDRD at its multiprogram laboratories. LDRD is "research and development work of a creative and innovative nature, which is selected by the director of a laboratory, or his/her designee, for the purpose of maintaining the scientific and technological vitality of the laboratory and to respond to scientific and technological opportunities." It is the mechanism through which PNNL can support the formulation of new theories, hypotheses, and approaches; build new scientific capability; and identify and develop potential applications. The LDRD program is the principal mechanism for renewing capabilities within PNNL and bringing forward novel ideas that will become the next generation of science and technology. LDRD strengthens the Laboratory's fundamental research component, builds capability in support of our applied research and development programs, and translates scientific discoveries into technology applications. Specific objectives are to:

- Foster an environment that encourages creativity and innovation

- Fund novel ideas that have scientific/technical merit but that cannot be funded promptly through programmatic channels

- Investigate new ideas and concepts to the proof-of-principle stage

- Enhance PNNL's core capabilities, which are broad, enduring technical competencies upon which PNNL's reputation is based.

The projects supported by LDRD funding all have demonstrable ties to DOE missions. In addition, many of the LDRD projects are relevant to the missions of other federal agencies that sponsor work at the Laboratory. The program plays a key role in attracting the best and brightest scientific staff needed to serve the highest priority DOE mission objectives. The flexibility provided by the LDRD program allows us to make rapid decisions about projects that address emerging scientific challenges so that PNNL remains a modern research facility well into the 21 st century.

Individual project reports comprise the bulk of this LDRD report. The Laboratory focuses its LDRD research on scientific assets that often address more than one scientific discipline. Though multidisciplinary, each project in this report appears under one of the following primary research categories

Advanced Sensors and Instrumentation

$\checkmark$ Biological Sciences

$>$ Chemistry

- Earth and Space Sciences

Energy Supply and Use

- Engineering and Manufacturing Processes 
Materials Science and Technology

- Mathematics and Computing Sciences

- Nuclear Science and Engineering

Physics

Each project report contains a project relevance statement, an introduction, a project description, and the results and accomplishments during the life of the project. Reports on projects continuing into FY 2011 should be regarded as progress reports; reports on projects completed in FY 2010 should be regarded as final summaries of the projects as a whole. This report also contains three appendices: Appendix A contains refereed publications (by author), Appendix B contains non refereed publications and presentations (by author), and Appendix C contains a list of the principal investigators indexed to the document page numbers on which their report summaries may be found. In addition, a supplement (limited distribution, bound separately) includes new projects funded in FY 2011. 


\section{PNNL's LDRD Management Process}

The major sequential steps governing the Laboratory's LDRD management process are summarized in the table below with more detailed descriptions following.

\begin{tabular}{|c|c|}
\hline Month & Summary Schedule \\
\hline October-February & Update of the Laboratory Agenda. \\
\hline October-March & Deep dive (i.e., proposed new initiatives) activities are conducted. \\
\hline April & Presentation of deep dives and mid-year evaluation of initiatives that began in FY11 conducted. \\
\hline May & $\begin{array}{l}\text { Annual Laboratory business planning guidance is prepared and issued. } \\
\text { The Laboratory Director and Executive Committee approve initial funding for the LDRD accounts } \\
\text { for the next fiscal year. }\end{array}$ \\
\hline June & $\begin{array}{l}\text { Fourth-year project extensions (beyond the normal three-year time limit) are requested by initiative } \\
\text { managers or directorate LDRD representatives to the LDRD Program Manager. } \\
\text { Initiative managers and directorate LDRD representatives solicit LDRD proposals from research } \\
\text { staff for next fiscal year. }\end{array}$ \\
\hline $\begin{array}{l}\text { Late June-early } \\
\text { August }\end{array}$ & $\begin{array}{l}\text { Initiative advisory committee reviews are held. Internal management reviews of mission seed and } \\
\text { Institutional Investment projects held. }\end{array}$ \\
\hline Late July & $\begin{array}{l}\text { The LDRD program prepares and submits an annual LDRD program plan to DOE-SC. The LDRD } \\
\text { program plan includes requests for a fourth-year extension of projects, and a list of potentially } \\
\text { continuing LDRD projects into the next fiscal year. }\end{array}$ \\
\hline August & $\begin{array}{l}\text { Final advisory committee reports delivered to the DDST. These become the basis for guidance and } \\
\text { funding considerations for continuing and new initiatives. }\end{array}$ \\
\hline September & $\begin{array}{l}\text { Principal investigators submit updated and new LDRD project plans to the LDRD Program Office for } \\
\text { review of compliance with DOE requirements. An "electronic prep and risk" form is completed online } \\
\text { by the principal investigator and signed by the appropriate product line manager. }\end{array}$ \\
\hline $\begin{array}{l}\text { September-October } \\
\text { (and throughout the } \\
\text { year) }\end{array}$ & $\begin{array}{l}\text { The LDRD Program Manager forwards new proposals to the DOE-Pacific Northwest Site Office } \\
\text { (PNSO) for review and approval. PNSO signs the project plan, and the LDRD Program Office releases } \\
\text { funding so that work may begin. }\end{array}$ \\
\hline
\end{tabular}

\section{Submission of PNNL's Annual LDRD Program Plan}

DOE Order 413.2B requires that each multiprogram national laboratory submit an annual plan to the DOE-SC for approval of its LDRD program activities and funding levels for the next fiscal year. The LDRD program manager is responsible for the development of this program plan, which includes information about the Laboratory's current-year program goals, the broad scientific and technical areas planned for support, the maximum requested funding level, and requests for fourth-year funding of specific projects. In FY 2010, DOE approved PNNL's LDRD program at \$39 million. Actual LDRD expenditures amounted to $\$ 35.7$ million, resulting in $4.1 \%$ of the Laboratory's operating funds. 


\section{Proposal Submittal and Signature Process}

In FY 2010, 158 projects were funded through PNNL's LDRD program. The LDRD program office manages the process for proposal submissions and obtains the necessary approvals so that work can begin. This process is illustrated in the figure and includes the following major sequential steps:

For newly proposed projects, concept papers (or pre-proposals) are submitted for consideration for funding. A management review is conducted to determine if the project supports the major missions of DOE and capabilities of the Laboratory.

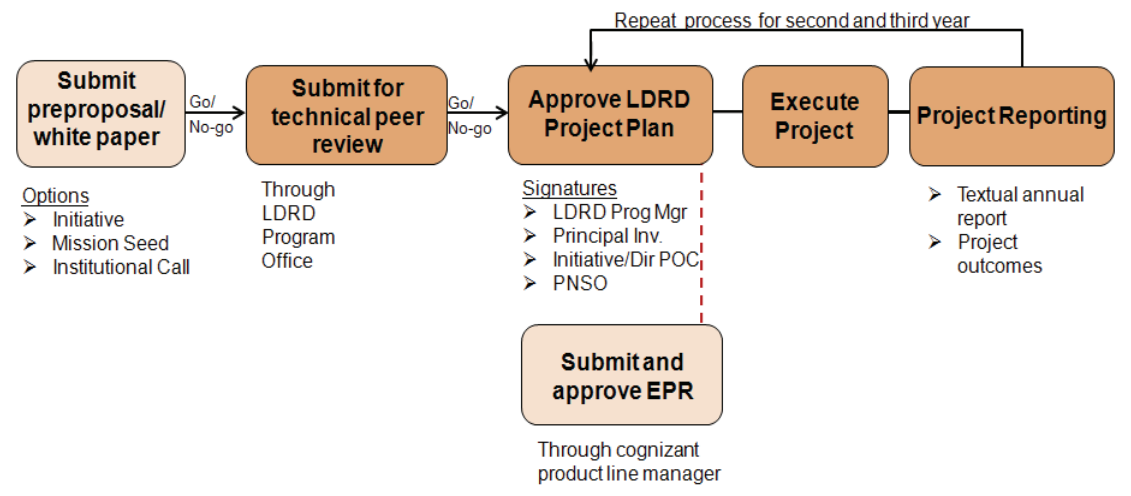
peer review.

- A final project plan is completed based on

LDRD Process Workflow feedback from the peer review. After the project plan has been submitted to the LDRD program office, the project is routed for signature. When approved, principal investigators are notified that their research project can begin.

- For projects continuing from a previous fiscal year, project plans are updated and submitted to the LDRD program office.

- Project work commences throughout the fiscal year. Technical progress and spending are monitored by the LDRD program office throughout the fiscal year.

- At the end of the fiscal year, each project reports on technical progress and completes project outcomes to the LDRD program office.

Details of the composition of projects and the peer review process are provided below.

\section{Peer Review of LDRD Proposals}

PNNL uses technical peer review to evaluate the quality, relevance, and performance of our scientific research. The Laboratory has a formal LDRD peer review process that ensures the technical integrity of our work, enhances our stature within the scientific community, and ensures that our research meets our customers' needs.

Overall, the Laboratory uses management review, internal technical reviewers, and external advisory committees in the annual review of LDRD projects. Each project is evaluated for mission relevance, scientific quality, and progress toward objectives. Midyear reviews and annual summaries of project performance are analyzed. Project costs and spending rates are tracked in the Laboratory's financial system. Annual reports of technical progress are obtained from the principal investigators for annual reporting. Project performance outcomes are obtained electronically from each principal investigator.

The structure of PNNL's LDRD program and subsequent peer review of proposals consists of three separate components as described below. 
Laboratory-Level Strategic Projects. Laboratory-level strategic projects are directed at developing capability in support of the one of PNNL's 13 science and technology investment areas (called S\&T initiatives). These projects are generally multiyear, multidisciplinary efforts. In FY 2010, PNNL's LDRD program funded 98 Laboratory strategic projects totaling \$25.7 million and representing 62\% of the total number of LDRD projects for the fiscal year. Projects in this category span PNNL's core capability areas in chemical and molecular sciences, chemical engineering, systems biology, climate and subsurface science, applied materials science, applied nuclear science and technology, advanced computer science, visualization and data, and systems engineering and integration.

Peer review of Laboratory-level strategic projects. In May, each initiative calls for pre-proposals that are reviewed by the initiative leadership (internal to PNNL), with a subset selected for potential funding. For projects that are selected for potential funding, an LDRD proposal is developed. The proposed new projects are reviewed by two or more internal PNNL technical experts for scientific and technical quality. The criteria used for rating the technical quality of project plans include intellectual merit of the proposed activity and broad impacts of the proposed activities.

In addition to the internal technical peer review of each project, the Laboratory has an advisory committee for each of its initiatives. External experts review both newly proposed and continuing Laboratory strategic LDRD projects. Advisory committees review technical focus areas for alignment with DOE missions and needs and planning and execution. Each advisory committee forwards its final report and recommendations to the Executive Committee through the DDST. The Executive Committee reviews the recommendations, sets guidance, and allocates funding and other resources to the initiatives according to the strategic goals and priorities of the Laboratory. The initiative leadership and principal investigators are then notified of the funding decisions.

Mission Seed Projects. Mission-level seed projects, funded for one or two years, support near-term opportunities and mission needs of one or more of the three research directorates at the Laboratory. Ideas are submitted by individual researchers or small multidisciplinary teams. Projects are reviewed internally by subject matter experts, and at midyear, by management to assess progress against milestones. In FY 2010, 42 mission-level projects were funded, totaling $\$ 7.5$ million, and representing $27 \%$ of the total number of LDRD projects for the fiscal year. Research topics under Science included panomics and biological research, computing architectures, multiscale modeling of molecular reactions to catalytic reactors, and light source photocathode development and performance, to name a few. Research under our energy portfolio included topics such as battery technology, integrated emissions management, HVAC systems, and electric grid research. Environmental research included predicting climate change impacts on hydropower and riverine ecosystems, and process modeling of chemically complex solid-liquid suspensions. Lastly, we explored areas within national security, including projects related to smart sensors and analytics, advanced radiation transport methods, alpha voltaics, cyber warfare assessment, thermally stable chemical markers, and analyzing factors in social media for national security applications to name a few.

Peer review of mission-level projects. Each research directorate at the Laboratory conducts a review and evaluation process for proposed projects that address the directorate's mission objectives. The Associate Laboratory Director for each directorate appoints an internal committee to evaluate and recommend projects for funding. These internal review committees are also responsible for monitoring project progress and for overseeing the appropriate technical progress.

The LDRD program office also conducts a periodic review (usually every 2 years) of the mission-level processes. In addition, the directorate review committees review selected LDRD projects as a part of the directorate's strategy. 
Institutional Investment Projects. PNNL's institutional investments fund projects that are proposed independently by individual researchers and focus on mentoring young science and engineering staff with considerable potential for career distinction. Projects are reviewed annually and at midyear. Eighteen projects were funded in FY 2010, totaling $\$ 2.6$ million and representing 11\% of the total number of LDRD projects for the year. Representative projects in this category include research on advanced materials for capturing lanthanides and transition metals from fission products, inorganic water oxidation electrocatalysts, detector as a weakly interacting massive particle (WIMP) dark matter detector, multiplexed activity-based protein profiling, ion beam-nanoparticle interactions for radiation detection, combining proteomic technologies to create a platform for spatiotemporal enzyme activity profiling, and interactions of carbon dioxide with supported metal oxide clusters to name a few.

Peer review of Institutional Investments. The review process for for projects that fall under institutional investments is similar to that for the mission-level projects in that the reviewers are internal to the Laboratory. These projects undergo midyear and annual reviews by the Laboratory Fellow peers.

\section{Compliance Review and Program Assurance}

The LDRD Office and DOE-PNSO review and approve projects for compliance with DOE Order 413.2B. The oversight and reporting for the LDRD program is the responsibility of the LDRD Program Manager reporting to the DDST. As a part of the Laboratory's process for assuring compliance with DOE Order 413.2B, the Laboratory has developed an electronic system that houses all the project-related information. The system has been designed to manage the life cycle of projects from the technical review of a conceptual idea to the project outcomes that resulted from the funded research. 


\section{Self-Assessment of PNNL's LDRD Program}

During FY 2010, the Laboratory supported a total of 158 LDRD projects (a decrease in the total number of projects from FY 2009). The average project size was $\$ 226.4 \mathrm{~K}$, representing an increase from FY 2009. Projects varied in size ranging from 8 receiving more than $\$ 400 \mathrm{~K}$ and 42 projects receiving $\$ 100 \mathrm{~K}$ or less. The table below shows the number of projects and the associated levels of funding.

\begin{tabular}{|l|l|}
\hline 19 & $\geq \$ 400 K$ \\
\hline 24 & $\$ 250 K-\$ 399 K$ \\
\hline 30 & $\$ 249 K-\$ 200 K$ \\
\hline 61 & $\$ 199 K-\$ 101 K$ \\
\hline 24 & $\leq \$ 100 K$ \\
\hline
\end{tabular}

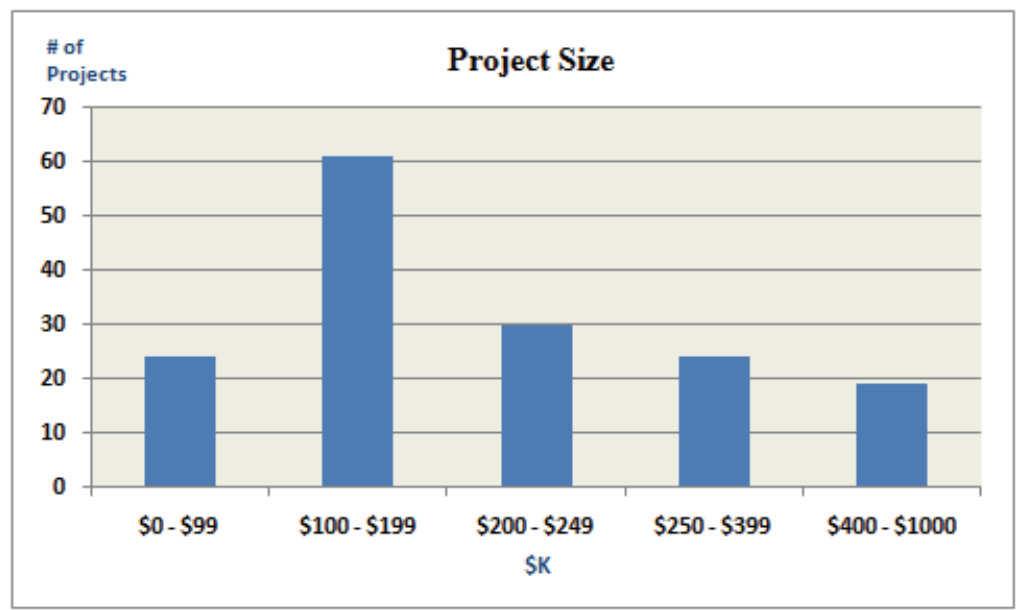

\section{PNNL's Portfolio of Science and Technology Initiatives}

Many of the Laboratory's best scientific ideas are developed through the LDRD program. PNNL's major initiatives during FY 2010 and the associated outcomes for each are described below.

Aerosol Climate. We are funding research that will address critical science questions related to the effects of aerosols on cloud properties and the formation, aging, and transport of aerosols. These advancements will enable more effective and accurate modeling of aerosol effects on climate and aerosol-cloud interactions. This understanding will help transform our ability to predict climate change.

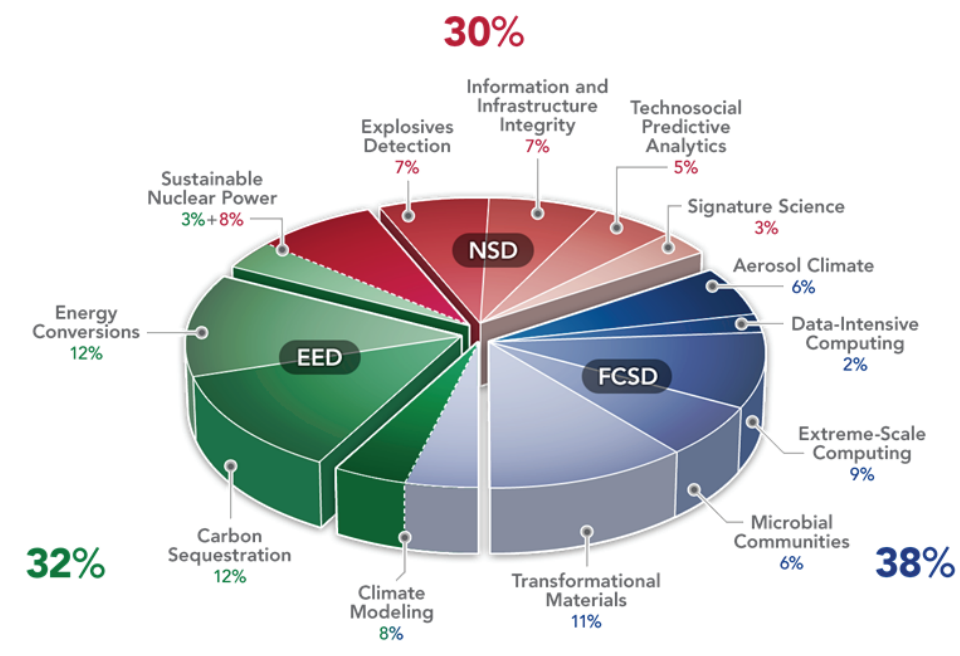

Carbon Sequestration. We are developing a computational/experimental system that enables carbon sequestration by providing science-based models/data to assess the performance of carbon reservoirs. Through this initiative, we will advance the state of science of reservoir simulation through coupling hydrologic-geochemical-geomechanical processes at relevant temporal/spatial scales, mechanistic descriptions and parameters for the interaction of $\mathrm{CO}_{2}$ with geologic media, and validation and identification of science and technology gaps in the models.

Energy Conversions. We are developing and applying new gasification and gas-separation technologies and hydrocarbon synthesis and conversion processes that enable the economically and environmentally viable use of hydrocarbons as energy sources. This will enable PNNL to make rapid progress toward increasing energy independence and significantly reducing U.S. dependence on foreign oil. 
Explosives Detection. We are building the science and technology base and providing the proof-of-principle experiments that enable the development of systems capable of detecting a range of explosives in a dynamic range of missions and operational scenarios. These accomplishments will accelerate progress toward helping to prevent and counter acts of terrorism.

Extreme-Scale Computing. We are developing methods, algorithms, and software to enable the use of next-generation, extreme-scale, massively parallel computer systems for addressing computational grand challenges in the mission critical areas of science, energy, environment, and national security through more effective and accurate modeling and analytics capabilities.

Information Infrastructure Integrity. We are developing new and fundamentally different approaches, methods, and tools for cyber security and information integrity based on the integration of predictive modeling, adaptive response, real-time threat analytics, and a foundation of trustworthy engineering.

Integrated Regional Earth System Model. We are enhancing our understanding of climate change impacts at scales meaningful to decision makers by creating a new, fully integrated modeling framework that delivers insights for managing risks relevant to climate mitigation and adaptation.

Microbial Communities. We are developing technologies and formulating new theoretical frameworks for a predictive understanding of microbial communities, leading to ecosystem stewardship related to energy, environmental science, and global change.

Sustainable Nuclear Power. We are developing the scientific understanding, strategies, and key technologies required for accelerated nuclear fuel qualification, transuranic recycling, and improvements in the proliferation resistance of nuclear power that will enable the development and implementation of sustainable, proliferation-resistant nuclear power generation in the United States and worldwide.

Technosocial Predictive Analytics. We are defining, developing, and evaluating new, predictive analytic methods to combine social network and physical infrastructure modeling. This will enable PNNL to address difficult questions associated with nuclear proliferation, terrorism, and the impacts of energy use and global climate change on national and global security.

Transformational Materials. We are developing integrated synthesis, characterization, and modeling capabilities to create innovative materials that drive revolutionary advances in energy conversion and storage, advanced separations, and signal transduction. This will accelerate progress focused on sustainable energy, environmental stewardship, and homeland/national security.

Mature FY 2010 initiatives that are ending in FY 2010 include:

Data-Intensive Computing. We are developing innovative hardware and software architectures teamed with novel analytical algorithms to address the computational challenges associated with the complex, dynamic, and distributed nature of high-throughput real-time streaming data, advancing scientific discovery and insight, situational awareness and response, and decision support and control. Advancements in these areas accelerate progress toward biological and environmental research, global and homeland security, and power grid operations.

Initiatives expected to start in FY 2011 include the following:

Chemical Imaging. We are developing a new multimodal integration framework that will enable the scientist-driven analysis and integration of experimental results across the spectrum of chemical imaging technologies, facilitating accelerated knowledge creation and exploitation. 
Future Power Grid. Through this initiative, we are expanding the limits of current power grid networking technology to support real-time data flow at very large scales with high security. Deliver real-time, high fidelity tools for grid modeling and simulation, and state-of-the-art advances to visual analytics to convert large volumes of data into actionable information.

Signature Discovery. We are developing an analytical framework and a methodology that are generally applicable to the discovery and validation of new threat signatures across a wide range of domains, and to the analysis of complex composite signatures from heterogeneous data.

\section{Recent Accomplishments}

Over the years, LDRD projects have realized major scientific and technical breakthroughs that have been reported widely in the scientific community. Summarized below are metric data for the Laboratory's LDRD projects in FY 2010.

\section{Awards}

In FY 2010, the Laboratory received five R\&D 100 Awards, one of which derived from LDRD research as described below.

Ion Mobility spectrometer on a Microchip. Scientists at PNNL have developed an Ion Mobility Spectrometer on a Microchip; a miniaturized device that accelerates the speed of analyses using ion mobility separations by over 100 times, enabling capabilities for rapid and confident monitoring of a broad range of chemicals at very low concentrations. Capitalizing on the leading-edge nanofabrication techniques of partner Owlstone Nanotech and the latest developments in Field Asymmetric waveform Ion Mobility Spectrometry (FAIMS) at PNNL, the dime-sized device employs a scalable multichannel architecture for high-speed separations of ions in the gas phase. It can easily be integrated with an

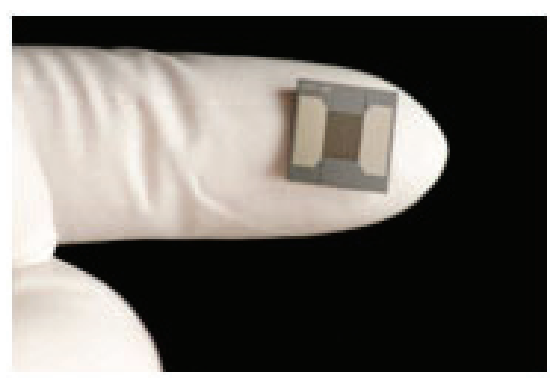
air sampler, ionization source, and detector to yield a powerful gas analyzer and a chemical monitor in a portable, self-contained unit. Current primary applications for this technology include explosive and chemical warfare agent detection and, when integrated with mass spectrometry, complex proteomic and metabolomic sample analysis.

\section{Publications}

Publication in the open scientific literature is an indicator of scientific productivity and quality as well as external recognition. The distribution of both refereed and non-refereed publications from projects funded under the LDRD program is shown over time. The overall number of publications increased from

\begin{tabular}{|l|r|r|r|}
\hline \multicolumn{4}{|c|}{ Publication Count } \\
\hline \multicolumn{1}{|c|}{ Referee Type } & \multicolumn{1}{|c|}{ FY08 } & \multicolumn{1}{c|}{ FY09 } & \multicolumn{1}{c|}{ FY10 } \\
\hline Refereed publications & 108 & 163 & 161 \\
\hline Non-refereed publications & 127 & 177 & 221 \\
\hline
\end{tabular}
FY 2009 to FY 2010. 


\section{Pacific Northwest National Laboratory FY 2010 LDRD Annual Report}

\section{Staff Count by Year}

The number of new hires, postdocs, and students is an indicator that the Laboratory is expanding its staff resource capabilities. In FY 2010, PNNL saw an increase in the total number of new hires, postdocs, and students.

\begin{tabular}{|l|r|r|r|r|r|}
\hline \multicolumn{7}{|c|}{ Staff Count } \\
\hline \multicolumn{1}{|c|}{ Staff Type } & 2006 & 2007 & 2008 & 2009 & 2010 \\
\hline New Hire & 34 & 22 & 29 & 57 & 51 \\
\hline Post Doc & 42 & 44 & 50 & 46 & 48 \\
\hline Student & 57 & 42 & 51 & 53 & 64 \\
\hline Grand Total & 133 & 108 & 130 & 156 & 163 \\
\hline
\end{tabular}

\section{Intellectual Property}

These metrics indicate progress, innovation, and leading-edge development in science and technology. The protection of intellectual property provides the opportunity for technology developed at the Laboratory to be used by DOE. In FY 2010, the Laboratory received 5 patents and 55 invention disclosures.

\begin{tabular}{|c|c|c|c|c|c|c|}
\hline \multicolumn{6}{|c|}{ IP Count } & \multirow{2}{*}{ Total } \\
\hline IP Type & 2006 & 2007 & 2008 & 2009 & 2010 & \\
\hline Patent & 6 & 8 & 10 & 12 & 5 & 41 \\
\hline Disclosures & 42 & 44 & 58 & 52 & 55 & 251 \\
\hline Grand Total & 48 & 52 & 68 & 64 & 60 & 292 \\
\hline
\end{tabular}




\section{PNNL Laboratory Fellows-2010}

In FY 2010, eight researchers earned the title of Laboratory Fellow, the designation of which represents one of the highest levels of scientific and technical achievement and recognition at PNNL. These researchers have led LDRD projects in recent years.

Dr. Daniel DuBois provides key leadership in the Laboratory's Center for Molecular Electrocatalysis, one of DOE's newly established Energy Frontier Research Centers. Dr. DuBois is an internationally recognized expert in metal inorganic catalysts for building and breaking hydrogen bonds using processes similar to those found in nature. His research interests include the catalytic interconversion of fuels and electricity, synthetic organometallic and inorganic chemistry, and thermodynamic studies relevant to catalysis. His research team developed new classes of electrocatalysts for $\mathrm{CO}_{2}$ reduction, hydrogen oxidation, and hydrogen production. In some cases, the activity of these catalysts approach or exceed that

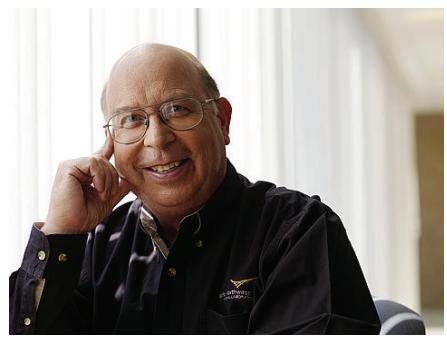
of natural enzymes. New electrochemical methods for $\mathrm{CO}_{2}$ separation and recovery have also been developed. Dr. DuBois' research also involves accurate measurements of thermodynamic properties of transition-metal hydrides, which are of broad utility in catalysis. His studies of proton relays in electrocatalytic reactions involving proton and electron-transfer steps have demonstrated the importance of these relays for both proton transfer and substrate activation.

Dr. Ian Gorton is the Associate Division Director for the Computational Sciences and Mathematics Division, leading the Data Intensive Scientific Computing Group. His research work spans numerous projects in environmental management, systems biology, and carbon sequestration, where he and his team are developing new software systems to manage and analyze vast amounts of data. Dr. Gorton was awarded Research Fellowships with Australian Telecomm (1990) and British Telecomm (1994) and was given the PNNL Lab Director's Award for Exceptional Engineering Achievements in 2009. He has served as Adjunct Professor at the University of Sydney and Conjoint Professor at the University of New South Wales. Dr. Gorton is regularly invited to review papers from leading journals, and he serves on several program committees for leading international conferences.

Dr. Anthony Janetos is the Director of the Joint Global Change Research Institute, a joint venture between PNNL and the University of Maryland. His research interests focus on the interaction of human decisions on land use and energy and the consequences for ecological and atmospheric processes. He is best known for his leading role in several scientific assessments of impacts of climate change on U.S. regions and natural resources, dating from the early 1990s to the present. With many collaborators, Dr. Janetos has written and spoken widely about the need to understand the scientific, environmental, economic, and policy linkages among major global environmental issues, and the need to keep basic human needs in the forefront of the thinking of the environmental science and policy communities. As a member of many national and international study teams, he expands his advocacy for strengthening linkages between science, the environment, the economy, and human choices.
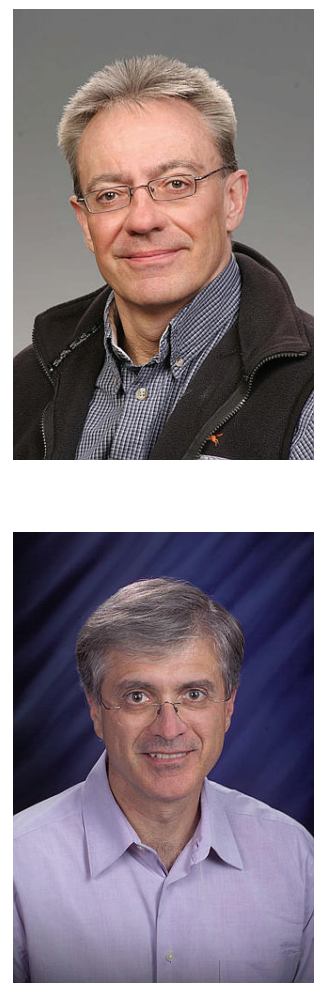
Dr. Kevin Rosso internationally regarded for his work to address creatively the long-standing problems in geochemistry and mineral surface chemistry. His research has enabled breakthroughs in understanding complex molecular and interfacial properties of minerals. A Fellow of the Mineralogical Society of America, Kevin is widely published in top 10 journals for his work in elucidating the relationships between the atomic and electronic structure of natural materials and their reactivity and physical properties. Dr. Rosso has received several accolades, including the Mineralogical Society of America Award recipient for 2004; Mineralogical Society of America Life Fellow; Presidential Early Career Scientist and Engineer Award nominee; and Virginia Tech College of Science 2008-2009 Outstanding Recent Alumnus Award recipient. He has served as Associate Editor for both American Mineralogist (2004-2006) and Geochimica et Cosmochimica Acta (2008-present) and is a frequent invited lecturer at universities and at international symposia.

Mr. Douglas C. Elliott's research has been largely directed toward development of fuels and chemicals from biomass and waste. His experience includes handling and processing of coals, biomass, and wastes primarily in high-pressure batch and continuous-flow processing reactor systems. Mr. Elliott's research has resulted in 84 publications in peer-reviewed journals, books, and scientific and engineering conferences. His recognized international leadership significantly advances PNNL's leading program to development refinery-ready alternative fuels. Mr. Elliott is a Battelle Distinguished Inventor and was recipient of two R\&D100 awards, an Award for Excellence in Technology Transfer from the Federal Laboratory Consortium and a Presidential Green Chemistry Award.

Dr. Gary Yang is an ASM International Fellow who is recognized for his outstanding work in advanced materials and electrochemical energy conversion and storage. Dr. Yang's research centers on the development and studies of energy materials, and electrochemical energy storage and conversion technologies where the materials are applied. In particular, he is recognized by colleagues and clients nationally and internationally for his efforts that have led to major advances in the field of advanced energy materials, solid oxide fuel cells (SOFC) and electrochemical energy storage (EES) technologies or batteries, particularly for renewable integration and smart grid applications. He is widely credited for his pioneering work on conductive protection layers and mechanistic understanding of the degradation of metallic bi-polar plates in high temperature electrochemical devices. In the past few years, he has led efforts in developing novel materials and
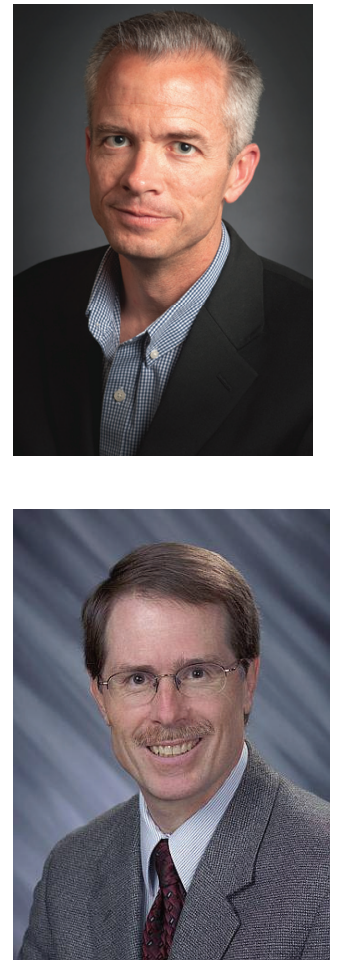
varied battery technologies for renewable integration and electrical grid applications. His work in conducting and directing highly respected, timely research have played key roles in establishing PNNL as a leader in SOFC development and in the emerging field of EES development for the stationary applications. 
Dr. Adolfy Hoisie is PNNL's Director of the Center for Advanced Architectures. Prior to PNNL, he was at Los Alamos National Laboratory, where he served in a variety of scientific and leadership positions, including Director of the Center for Advanced Architectures and Usable Supercomputing and Leader of the Computer Science for High-Performance Computing (HPC) Group, which included the Performance and Architecture Lab. He is an internationally recognized expert in performance analysis and engineering of large-scale parallel computing systems and applications, and system architecture. He is a past winner of the Gordon Bell Award and has won several other awards for technical and teaching excellence. Dr. Hoisie has served extensively in the HPC community in a variety of capacities, such as conference organizer (e.g., Technical Program Chair at SC'08), editorial board member, committees, panels, and advisory boards. He has published extensively in peer-reviewed literature, including 3 books, and has been lead on numerous federal grants (NNSA, ASCR, DARPA, NSF, and others).

Dr. Darren Kerbyson serves as Chief Scientist for Extreme Scale Computing and specializes in modeling and analysis of how well software performs. He is an internationally recognized computer scientist with more than 25 years of experience developing and analyzing high performance computing systems and applications. Dr. Kerbyson's work is focused on developing methods and tools to understand the performance of systems and the applications that run on them. In particular, his work on performance modeling was instrumental in the design and analysis of several large-scale projects, including DOE's Advanced Simulation and Computing program and DARPA's High Productivity Computing Systems. With the push for exascale computing systems, issues are becoming more challenging, with power consumption and its trade-offs with performance, and resilience adding to the complexity of analysis. With this in mind, his current work focuses on the exploration of the performance and power of future systems. 


\section{Advanced Sensors and Instrumentation}




\title{
Advanced Environmental Sampling Technology for Safeguards and Proliferation Detection
}

\author{
Normal C. Anheier Jr., Shane M. Peper, Bruce A. Bushaw, David C. Gerlach, M. Lizabeth Alexander
}

The overall goal of this project is to produce improved sample collection and analysis technologies for detecting proliferation signatures and to verify facility declarations in making determinations about the absence of undeclared nuclear activity.

$\mathbf{E}$ nvironmental sampling is a critical verification tool for the implementation of safeguards and for detecting proliferation activities. Under formal agreements, samples are normally taken by inspectors who are granted access to a nuclear site. Proliferation detection and verification of peaceful nuclear activities are critical national security mission activities, underpinned by sophisticated technology that requires continuous capability improvements as the existing threats grow and new threats emerge. The expected outcomes of this research are to develop and evaluate new environmental sample collection technologies to improve collection efficiency, significantly reduce the time and cost associated with subsequent laboratory analysis, and demonstrate the laser ablation, absorbance ratio spectrometry (LAARS) approach can be used in a purpose built configuration to perform unattended near real-time monitoring of large enrichment facilities.

Our project approach is in developing advanced sample collection, sample analysis, and automated, unattended monitoring techniques. The project consists of three main tasks. First, we will develop improved sample collection methods and technologies for nuclear facility inspectors. Analyte-specific conductive films will be developed that can directly be deposited onto secondary ion mass spectrometry (SIMS) substrates, with the goal of significantly reducing the cost and time associated with laboratory analyses. Next, we will develop and evaluate a high-resolution isotopic fingerprinting system for rapid screening of minor uranium isotopes $(234,235$, and 236$)$ at ultratrace levels. It is expected that minor isotopes can be directly quantified at femtogram levels and with abundance sensitivity below that achievable with conventional mass spectrometry. The unique selectivity provided by high-resolution laser technique is expected to reduce or remove complex sample preparation chemistry substantially, resulting in a faster analysis time. Finally, we will evaluate laser ablation, absorbance ratio spectrometry for highly enriched uranium detection within uranium enrichment plants. An automated, unattended environmental aerosol sample collection approach will be developed and combined with the uranium isotope ratio analysis system to detect enrichment facility misuse in a timely manner.

Continuing from FY 2009, progress on the first project task included evaluating additional new materials for increasing the collection efficiencies of key analytes of interest that are relevant to safeguards and proliferation detection. A polymer synthesis laboratory capability was set up, and several batches of a new methacrylic copolymer were synthesized and evaluated for their ability to adhere particulates. Additionally, several commercially available conductive polymers were evaluated using SIMS to determine which one(s) would be ideal for direct sample analysis (based on degree of conductivity and strength of the analytical signal, processed by the SIMS instrument). It was determined that polyethylenedioxothiophenepoly(styrene) sulfonate (PEDOT-PSS) performed optimally. Initial experiments were directed at combining thin films of the conductive polymer and the adsorptive polymer to evaluate whether the SIMS data quality was preserved without the use of additional sample preparation methods (i.e., sputter coating of metallic conductors such as gold, platinum, and palladium).

General characteristics of the new methacrylic copolymer were presented at the International Union of Pure and Applied Chemistry's World Polymer Congress in Glasgow, Scotland. A fluorescence microspectroscopy system was assembled and configured for performing the uranium analyses. This

capability allows for the direct sample interrogation and the determination of key chemical signatures associated with uranium enrichment. Under our third task, we evaluated the feasibility to adapt the PNNL-developed LAARS into a uranium enrichment facility safeguards technology. During FY 2010, initial isotope analysis began on certified uranium standards. Significant

Histogram of cumulative LAARS U-235 enrichment distributions for natural and low enriched uranium.
LAARS improvements have 
reduced the distribution peak widths by more than 4 times and the relative standard deviation by over 6 times. The deviation of 0.15 percent on certified 2.423 percent enriched uranium was demonstrated. $\mathrm{A} \mathrm{UF}_{6} \mathrm{DA}_{\text {assay concept was conceived }}$ and a feasibility study completed. A feasibility of LAARS division arrested assay on LEU was conducted using four replicate measurements of the 2.4 percent certified standard. A total of 1000 laser enrichment measurements were made on each $1 \mu$ gram sample. The relative standard deviation and the relative error from the certified enrichment value were found to be 1.5 percent, and planned future improvements are expected to reduce this by a factor of 3 times.

We made significant technical progress on this project as reported through conference papers and presentations at the $51^{\text {st }}$ Annual Institute of Nuclear Materials Management meeting in Baltimore, MD; the Pacific Northwest International Conference on Global Nuclear Security - The Decade Ahead conference in Portland, OR; and the International Atomic
Energy Agency Workshop on Advanced Safeguards Technology for the Future Nuclear Fuel Cycle, Tokyo, Japan.

In FY 2011, we will develop methods to improve and modify an aerosol sample collector to accommodate SIMS planchets coated with thin films of conductive polymers. Anthropogenic and naturally occurring particulate uranium samples will be characterized using Raman and fluorescence microspectroscopies in order to elucidate chemical enrichment signatures. Spectral libraries of various anthropogenic and naturally occurring uranium-bearing materials will be developed to facilitate rapid particle classification and rationale selection of particles of interest. The now-extended second task will establish a laboratory capability and permits required to begin LAARS measurements on uranium aerosols. Prepared enriched uranium aerosol samples (both homogeneous and trace heterogeneous) will be analyzed by LAARS to determine the uranium assay performance. 


\title{
Comparison of Assays Utilizing Existing Biological Reagents Specific for Explosives
}

\author{
Marvin G. Warner, Keith D. Miller, Jay W. Grate
}

The objective of this project was to determine the effectiveness and performance of a focused set of biological and biologically inspired reagents specific for explosives using simple optical and competitive binding assay techniques. Our purpose was to determine which reagents perform best (i.e., the lowest limit of detection and most stable signal response) and generate meaningful data regarding the limit of detection of the optical assays used in our laboratory.

he detection of biological materials using antibody-
based immunoassays is a mainstay for biological
science research, biomedical research, clinical diagnostics, biodetection, and food safety. Although generally robust, immunoassays still face significant challenges in obtaining reagents that yield specific, sensitive assays for the detection of small molecules (e.g., explosives) and from issues such as nonspecific binding, signal interference (e.g., from scattered excitation light using fluorescent reporters), and the sample matrix effects in homogeneous and heterogeneous immunoassay formats. This leads to a need to develop and benchmark optical and competitive binding assays that use protein reagents (usually antibodies) that bind explosives tightly with a high degree of selectivity.

Antibodies and antibody fragments have been demonstrated in the detection of small (fewer than $1000 \mathrm{Da}$ ) molecules. For example, it has been demonstrated that synthetic antibody fragments could bind trinitrotoluene (TNT) selectively without displaying appreciable cross-reactivity with a variety of dinitrotoluene (DNT) analogs, thereby reinforcing the potential for high selectivity of these types of antibody-based reagents. Recent assays reported in the literature have shown a fair amount of success and promise for the detection of explosive molecules using protein-based reagents. However, more work must be done to determine the viability of these types of reagents for successful field-based detection of explosives using common assay formats.

The work carried out under this research attempted to determine the efficacy of the commercially available antibodies (many of which have been used in assays reported in the recent literature) and compare them to the in-house prepared antibodies from PNNL. Our antibodies have the unique advantage that they can be selected to have a higher degree of stability under harsh detection conditions, higher specificity, and overall higher affinity than those currently commercially available. Moreover, we sought to demonstrate that the antibodies produced at PNNL have overall lower limits of detection of TNT and RDX. In addition, we aimed to develop selection and assay methods that would allow us to choose our reagents based on their measured stability and performance under the typically harsh (by biological standards) conditions under which successful reagents must function.

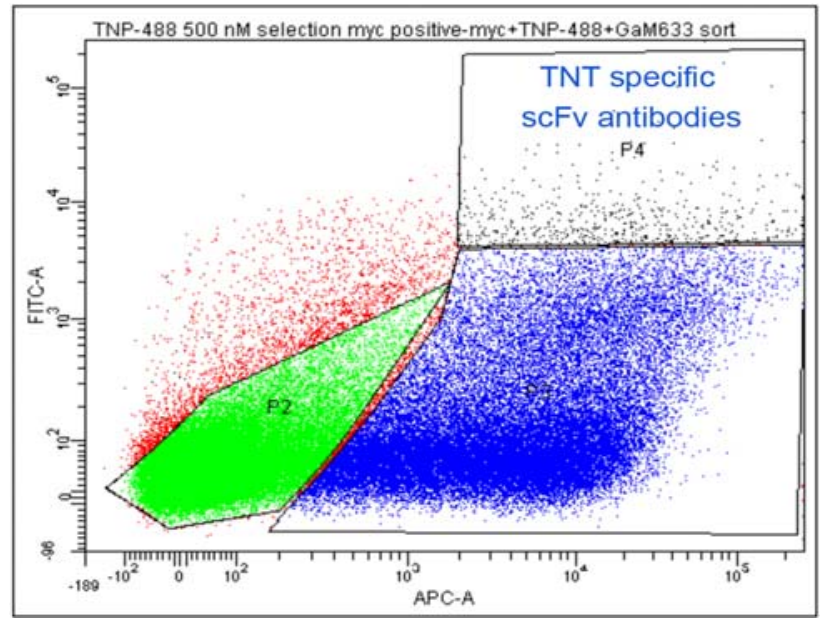

A flow cytometry plot used for the selection and verification of singlechain antibodies produced at PNNL that bind with high affinity and specificity to TNT.

During FY 2010, assays were designed to use the various antibody reagents available from commercial sources. In-house antibodies produced at PNNL were expressed and purified, allowing us to modify the selection conditions to alter and tune reactivity and stability of the protein reagent. We focused on developing simple optical and competitive binding assays that are routinely used in the laboratory. These involved variations of the standard ELISA techniques commonly used for explosives detection using antibody reagents. For competitive binding assays, we used methods and techniques that readily available in commercial kits and have been refined in our laboratory.

We were also able to complete the production of in-house single chain antibodies specific for TNT. We generated small peptide sequences that have a high degree of specificity and affinity for TNT and incorporated these into a secondary protein scaffold that imparts a high degree of thermal and chemical stability to the peptide sequences. These stabilized protein fragments were expressed as soluble proteins and used in the assays discussed above. These reagents have been studied primarily to provide a small (only a few $\mathrm{kDa}$ in size) and stable alternative to the larger antibody based reagents. Due to the limited availability of commercial immunoassay reagents for explosives detection, we also investigated a number of non-immunoassay based methods for the detection of explosives. These kits were used to benchmark the other reagents in our laboratory. 


\title{
Development of a Dual-Sided, Temperature-Controlled, Continuous-Flow Environmental Chamber
}

\author{
John E. Shilling, Chen Song, Naruki Hiranuma
}

As noted by the Intergovernmental Panel on Climate Change, the effect of airborne particles (aerosols) on climate remains a great source of uncertainty for climate models. Laboratory experiments investigating the life-cycle of organic atmospheric particles, which are a large fraction of all atmospheric particles, are the focus of this project.

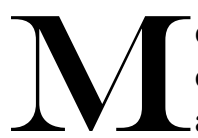

easurements show that organic aerosol particles comprise 20 to 90 percent of the total atmospheric aerosol burden. A significant portion of this organic mass (63 to 95 percent) is secondary organic aerosol (SOA), which forms in the atmosphere from the oxidation of gas-phase precursors. However, state-of-the-art models currently underestimate measured atmospheric SOA concentrations by a factor of 8 to 100 . The inability of models to reproduce measured SOA levels accurately illustrates an incomplete understanding of SOA formation and has important consequences for reducing the uncertainties associated with the effect of aerosol on global climate change. Therefore, insights into SOA formation and transformations, radiative properties, and the ability of SOA particles to form liquid water and ice clouds will directly improve predictions of global climate change.

Currently, most chemistry and transport models calculate SOA concentrations using the equilibrium partitioning theory coupled with experimentally determined SOA yield parameterizations. These laboratory based parameterizations are determined using environmental (smog) chambers, which simulate aerosol formation under controlled and well defined conditions. However, the parameterizations currently used by the models are based on experiments conducted with volatile organic compound concentrations, oxidant concentrations, and organic aerosol loadings far above those representative of the ambient atmosphere. Recent experiments have shown that these parameterizations cannot be accurately extrapolated to atmospheric conditions. In addition, many properties of the SOA such as density, chemical composition, and cloud condensation nucleus activity are central to modeling efforts and dependent on reaction conditions. Therefore, accurate representations of the SOA in models require data from laboratory experiments conducted under atmospherically relevant conditions.

Laboratory studies of SOA formation and subsequent processing have been carried out in environmental chambers for several years. However, due to the traditional experimental design, these studies were conducted using high concentrations of reactants that are not representative of the real atmosphere.
It is becoming clear that data collected under these conditions cannot be accurately extrapolated to atmospheric conditions and are therefore of limited use to modelers. In an effort to reduce uncertainties of previous irregular models, this project contributed to constructing a new environmental chamber to study SOA formation, evolution (aging), and the ability of SOA particles to act as ice nuclei and cloud condensation nucleus. The continuous-flow design has several advantages over traditional chamber designs that allow experiments to be conducted under atmospheric conditions. Initial experiments conducted in the chamber will investigate the link between anthropogenic and biogenic emissions in forming SOA, the yield and chemical composition of SOA produced from biogenic and anthropogenic volatile organic compounds under atmospherically relevant conditions, and the ice nuclei ability of SOA coated mineral particles.

One early outcome of this project is the development of the dual-sided, temperature-controlled, continuous-flow chamber capability at PNNL. This new capability is now being used to conduct laboratory-based research targeted at reducing the uncertainty associated with representing the SOA formation and processing in models. Data collected in these studies will be incorporated into climate models developed by PNNL to determine the effect of aerosol particles on climate change. A specific advantage of the dual-sided chamber is that it consists of a configuration that will increase the overall experiment duty cycle and reduce equipment down time. The chamber represents a new and unique capability for PNNL, but it also retains the ability to operate in the traditional mode. Operation of the chamber in continuous-flow mode suppresses the loss of semi volatile species to the chamber walls, enabling lower, more representative concentrations of volatile organic precursors to be studied.

FY 2009 represented the first year of this project, during which two papers describing the chemical composition and cloud condensation nucleus activity of SOA particles were completed and published. The chamber components and instruments were procured and used in ongoing experiments. A smaller, more limited flow-through chamber was constructed so that scientific progress could be made concurrent with development of the state-of-the-art chamber facility. We completed testing and validation of this secondary chamber and begun employing it for experiments probing possible synergistic interactions between organic compounds emitted naturally from plants and as a byproduct of human activities. At the end of last year, we conducted several experiments to measure the SOA yield from the dark ozonlysis of $\alpha$-pinene 
under atmospherically relevant conditions. The results agreed well with literature data obtained under similar conditions, validating the chamber design and performance.

The $\alpha$-pinene ozonolysis experiments conducted last year served as a baseline for experiments investigating the mixing of anthropogenic and biogenic SOA precursors conducted during FY 2010. During these new experiments, we measured the ozonolysis SOA yield from pure cyclohexene, a model anthropogenic VOC, and from mixtures of cyclohexene and $\alpha$-pinene. Using the yield data obtained for the pure compounds, we predict the results of the mixture experiments assuming two conditions: $\alpha$-pinene and cyclohexene SOA are fully miscible, and $\alpha$-pinene and cyclohexene form separate phases. Comparisons of the predicted results with the experimental data show that SOA from these species are fully and mutually soluble in each other. Thus, the particle mass produced from co-condensation of these SOA species is significantly greater than the sum of yield expected to be produced from the individual species.

Late in FY 2010, a new, dualsided, continuous-flow chamber was constructed. We are currently conducting experiments to investigate the role of water vapor in SOA formation, collaborating with other LDRD research on the role of aerosol in cloud particle activation, and collaborating the University of California, San Diego to investigate the hydrolysis of organic nitrates in SOA particles.

The chamber represents a new experimental capability at PNNL, enabling researchers to conduct laboratory studies of
SOA formation and processing. This technology is an improvement over the current batch mode chamber in several key areas. First, implementation of the continuous-flow mode has several advantages over batch mode operation (vida infra).

These advantages include the ability to conduct experiments under more atmospherically realistic conditions, reduction in the influence of wall artifacts, decoupling of aging time from
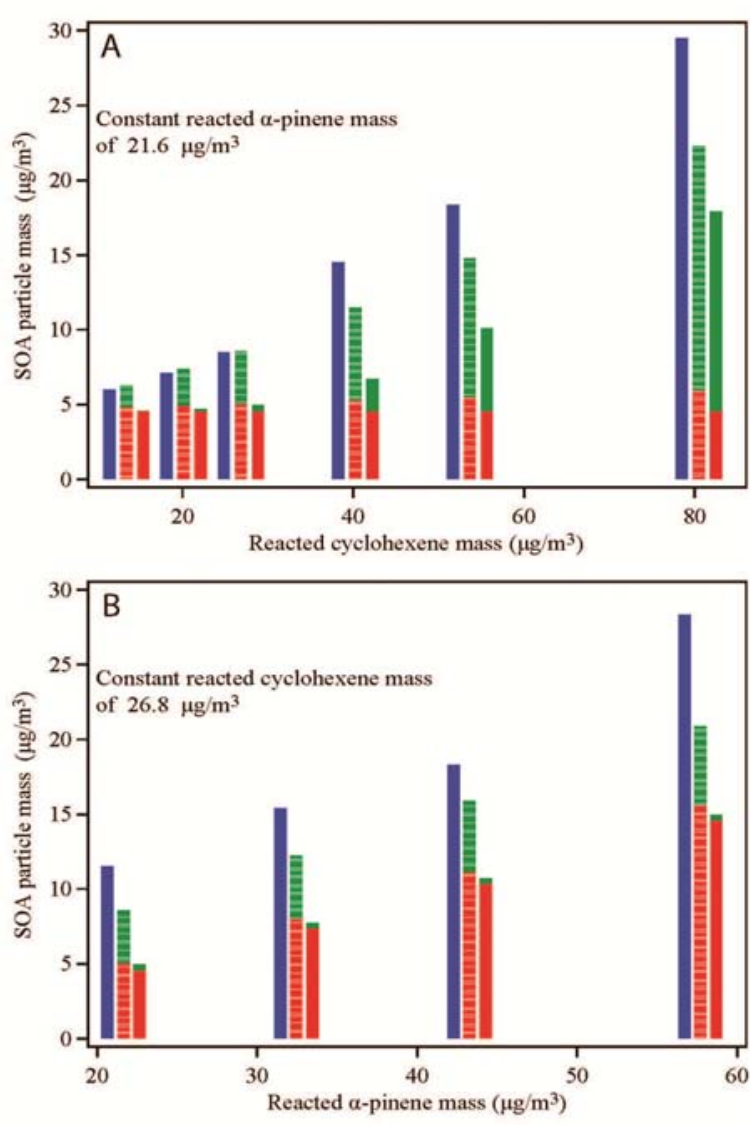

Experiments were conducted with constant reacted $(A)$ a-pinene and variable cyclobexene mass and (B) constant reacted cyclobexene and variable a-pinene mass. Comparison of the measured SOA particle mass (left-hand side solid bars) formed during the dark. ozonolysis of mixtures of a-pinene and cyclohexene to model result assuming a separate (right-hand side solid bars) or fully mixed (dashed bars) particle phase. particle loading, and the ability to serve as a stable particle source for instrumentation or techniques with slow sampling rates. Second, temperature control will be added to the chamber, a critical capability in SOA research. Third, the ability to conduct photoxidation $(\mathrm{OH}$ initiated oxidation) experiments will be added to the chamber. The current facility is capable of utilizing only ozone as the oxidant, severely limiting the scope of possible experiments. Finally, an improved chamber manifold will be designed and implemented.

In FY 2011, we will investigate the yield, chemical, and cloud condensation nucleus activation properties of SOA formed from photooxidation of mixtures of $\alpha$-pinene and 1,3,5 trimethyl benzene. A University of California, San Diego graduate student will visit PNNL in November to conduct experiments investigating the hydrolysis of organic nitrate species in the new chamber facility. We will inject mineral dust particles coated with SOA layers of variable thickness into the chamber to be transferred to the ice nucleation chamber under the Asset Condition Index. This chamber measures the ability of particles to act as nuclei for ice particle formation (cold-cloud nucleation). 


\section{Distance-of-Flight Mass Spectrometry for Rapid, Portable Actinide Analysis}

David W. Koppenaal, Anthony J. Carado, Charles J. Barinaga, Steven J. Ray,

Christie G. Enke, Gary M. Hieftje (Indiana University)

A new approach to chemical analysis using distance of flight mass spectrometry is proposed, which can provide characterization of nonproliferation samples and materials in a more cost-effective manner.

$\mathbf{R}$ esearchers at PNNL took a new approach to mass spectrometry (MS) using distance of flight (DOF) to affect isotope separation and detection. The concept is simple and based on the premise that ions will separate according to mass/charge with transit in a field-free region. Spatially separated detectors or an array of detectors can be located along this path. At any given time, the separated ion packets can subsequently be pulsed to this detector array for observation and detection; the ions are literally sorted by the distance traveled. This is in contrast to the more prevalent practice of time of flight (TOF), whereby ions are detected by arrival time at a fixed (and single) detector.

The DOF mass spectrometer components consist of only an ion source, ion optical elements (a field-free region in which separation by mass-to-charge occurs), and an array detector with associated electronics and controls. Because there are no magnets, radio frequency fields, or sophisticated gating circuitry, the instrumentation is amenable to simple, small packaging (laptop size for a small mass range) along with portable operation. The DOF instrument concept can be employed to collect physically separated isotopes (on an analytical or preparative scale) from complex mixtures. Isotope collection can be useful for isotope preconcentration and subsequent matrix-free analysis. A principal application of this technique is envisioned for field and remote detection analysis of actinide isotopes for safeguards and/or treaty monitoring purposes.

Overall, two primary outcomes were demonstrated in this project. The first was the development of DOF-MS prototype instruments, and the second was the feasibility demonstration of the DOF-MS concept that has been only hypothesized and addressed theoretically to this point. During the first year of this project, modeling and design work was completed for a prototype DOF-MS, and first experimental results were obtained that demonstrated that the new approach was successful. The two prototype instruments constructed and tested initially for this effort were further refined and optimized during the second year. Glow-discharge ion sources were used in the initial and more recent experiments and demonstrations. Ions extracted from this source are shaped (horizontally narrowed and vertically stretched) for pulsing, using constant momentum acceleration into the field-free reflectron region.
Experimental work focused on instrument design and construction as well as proof-of-concept experiments. Much of the effort centered on the efficient operation and characterization of the glow discharge ion source, the ion optics assembly, and the ion mirror performance as well as the multichannel plate, phosphor-type detector. Model metal and oxide samples were examined, with seminal DOF-MS demonstration results for a brass sample obtained during the previous year of the research. Improved DOF-MS performance was achieved during FY 2010 with both improved sensitivity and greatly improved resolution.

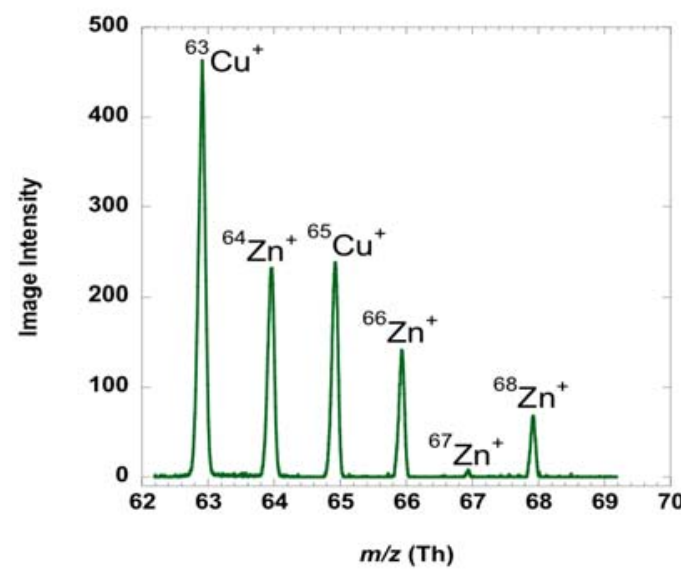

Spectrum of brass sample showing copper and zinc isotopes at high resolution (approximately $500 \mathrm{R}$ ).

Despite the improved resolution, fundamental limitations in the multichannel plate phosphor detector suggested that a shift in the detection scheme would be advantageous. In an effort to improve spatial resolution, the multichannel plate/phosphor detection was replaced with a direct charge detection detector technology based on Faraday arrays and multiplexer electronics (developed under separate funding). Recently, initial spectra were obtained using this new detector. Additional insights and proof-of-principle were obtained for simultaneous DOF-TOF MS capability; the potential for on-demand, selectable higher resolution capability; and the achievement of radical improvements in chromatographic detection speed and power.

A publication and several invited presentations on the promise and potential of DOF-MS have also been realized as a result of this work. 


\title{
In Situ High-Pressure X-Ray Diffraction Investigation of Caprock Mineral Reactions With Water Solvated in Supercritical $\mathbf{C O}_{2}$
}

\author{
H. Todd Schaef, Paul F. Martin, A.T. Owen
}

The objective of this project is to develop a high-pressure x-ray diffraction capability to advance our understanding of reaction processes and kinetics at the fluid-solid interface between water-solvated in supercritical $\mathrm{CO}_{2}$ and minerals with special emphasis on layer silicates.

D eep saline reservoirs with confining layers (caprocks) are considered primary targets for carbon storage by most countries. Post-injected $\mathrm{CO}_{2}$ will reside largely as a water rich supercritical buoyant fluid, constrained from vertical migration by a very lowpermeability caprock seal. This process allows time for slow trapping mechanisms (e.g., $\mathrm{CO}_{2}$ dissolution in pore water, precipitation of carbonate minerals) to sequester $\mathrm{CO}_{2}$ from the system. Despite this well-accepted conceptual model, most of the research on flow, transport, and chemical reaction processes has remained in aqueousdominated geologic systems, which will not represent the vast majority of the rock volume where supercritical $\mathrm{CO}_{2}\left(\mathrm{scCO}_{2}\right)$ is stored. Moreover, recent work at PNNL indicates chemical reaction processes between the water solvated in $\mathrm{scCO}_{2}$ phase and geologic media are significant and often faster than observed in the aqueous dissolved $\mathrm{CO}_{2}$.

This project is developing and conducting proof-of-concept testing using high-pressure micro-focused $\mathrm{x}$-ray diffraction capabilities needed to investigate fundamental reaction processes occurring in mineral systems exposed to water solvated in $\mathrm{scCO}_{2}$. Geologic systems dominated by clays are of inherent interest due to their predominance in shale cap rocks, siliclastic reservoir rocks, and as weathering products in flood basalts. Geochemical processes associated with cap rock systems in contact with $\mathrm{scCO}_{2}$ are complex and range from simple desiccation to structural transformations and secondary mineral formation. The paired an in situ probe with $\mathrm{x}$-rays to examine transformation reactions of minerals and their kinetics when exposed to water bearing $\mathrm{scCO}_{2}$ fluids under pressure, temperature, and fluid composition conditions relevant to geologic sequestration of $\mathrm{CO}_{2}$.

Designing a reactor compatible with x-rays and acid gas mixtures requires special materials and unique design criteria. Additionally, the design should account for beam configuration, geometries at low angles, and $\mathrm{x}$-ray attenuation by dense gas mixtures. After several months of testing, a design for an $\mathrm{x}$-ray transparent high-pressure reactor consisting of three primary components was fabricated and assembled. The reactor base is stainless steel and contains two pressure ports. Constructed from beryllium, inserts are designed to maximize the mineral surface area and allow for minerals to be preferentially oriented toward the x-ray beam, a technique often used to exploit the intensity of 001 basal reflections characteristic of layer silicates. This design also accommodates powdered or whole rock samples as well as a corundum standard panel. Providing a cover is a thin walled ( $1 \mathrm{~mm}$ ) beryllium cap that screws into the steel base. With a total volume of $1 \mathrm{~cm}^{3}$, the reactor is configured for both transmission and traditional reflective $\mathrm{x}$-ray diffraction analysis and has pressure and temperature ratings of approximately 200 bar and $200^{\circ} \mathrm{C}$, respectively.

In situ high-pressure x-ray diffraction testing began with the mineral brucite $\left[\mathrm{Mg}(\mathrm{OH})_{2}\right]$, which was selected because this mineral is a structural analogue to layer silicates, and reactions with aqueous dissolved $\mathrm{CO}_{2}$ are rapid and typically result in the formation of nesquehonite, a hydrated magnesium carbonate. We used a finely focused computer controlled $\mathrm{x}$-ray beam to examine brucite stability in the presence of $\mathrm{scCO}_{2}$ containing varying percentage amounts of water $(0,13,25$,

$50,100,135$, and higher) at 82 bar and $50^{\circ} \mathrm{C}$ for extended periods of time.

Under these experimental conditions, brucite remained stable when in contact with anhydrous $\mathrm{scCO}_{2}$ for the test duration (72 hours); no secondary reaction products were detected in the post-reacted sample. When exposed to wet $\mathrm{scCO}_{2}$, brucite reacted and nesquehonite $\left[\mathrm{MgCO}_{3} \cdot 3 \mathrm{H}_{2} \mathrm{O}\right]$ formed. Experiments conducted close to water saturation of $\mathrm{scCO}_{2}$ (56 to 135 percent) showed the conversion of brucite into nesquehonite ranged between $60 \mathrm{wt} \%$ and $70 \mathrm{wt} \%$ in less than $24 \mathrm{hrs}$. Water concentrations well above the saturation for $\mathrm{scCO}_{2}$ drove the reaction to completion in the same period. In contrast, limiting water content to well below saturation ( $>25$ percent) reduced the reactivity of $\mathrm{scCO}_{2}$ and subsequently produced less carbonation. These types of experiments indicate only trace amounts of water in $\mathrm{scCO}_{2}$ are 
needed to create a highly reactive fluid capable of transforming minerals. This type of capability illustrates the need to understand reaction processes in $\mathrm{scCO}_{2}$ containing water so that reservoir simulation tools can accurately account for the potential impacts in a variety of geologic reservoirs.

Planned activities for FY 2011 will focus on measuring mineral reactivity in the presence of wet $\mathrm{scCO}_{2}$ and quantifying rates of carbonation. This project will test serpentine minerals (antigorite and lizardite) for continued development of higher pressure reactors. Additionally, clay minerals such as montmorillonites, which are used extensively in forming seals around monitoring wells, will be examined in situ at conditions relevant to carbon sequestration. Understanding the stability of clays in contact with $\mathrm{scCO}_{2}$ containing water will better allow modeling of long-term risks associated with $\mathrm{CO}_{2}$ geologic sequestration. 


\section{Multi-Modality Sensing Platform for Smart Detection of Explosive Traces}

Liang (Frank) Wang

We are developing a sensing platform with multiple transductions in concert, for early detection of powder or vapor-phase explosive molecules and discrimination of target analytes at extremely low concentrations. This project will ultimately lead to a selective, sensitive, portable, cost-effective sensor device for remote explosive recognition that has high impact on national security in preventing potential terrorist attacks.

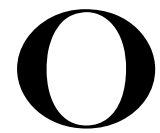
rganic field-effect transistors (OFET) or chemiresistors have been widely used as chemical sensors with signals retrieved from the changes in the bulk or channel conductance upon exposure to an analyte. These sensors have gained increasing interest due to their portable size, widely accessible sensing area, ease of fabrication, availability of modifying active molecule or polymer structure for detection of specific targets, and the simplicity of sensing signals for real-time readout and data process. The response of these sensors only accounts for a single physical property, such as the amount of charge or dipole moment carried by the analyte and thereby limits their distinguishing ability.

We aim to provide a new architecture for chemical sensors built on the transistor, targeting early and real-time detection of explosives with improved selectivity and sensitivity. Our project focuses on the light-emitting OFET, where luminescent organic semiconductors serve as channel material and light emission occurs in the channel from recombining electrons and holes injected from the source/drain electrodes. When a light-emitting OFET channel is exposed to an explosive target, corresponding changes in electrical and optical outputs feed the reading module by multi-modal sensing signals. This approach retains the advantage of OFET- or chemiresistorbased sensors, providing greater resolving power by generating a unique combinational fingerprint for each analyte, leading to smart sensors with improved selectivity and sensitivity.

In FY 2009, the sensing system was completed with analyte vapor delivery in precise control and capability for simultaneous measurement of both electrical and optical signals. The device was exposed to the explosive target with a heating tape in optional operation for facilitating the sublimation of analyte and supplying enough analyte flux. Also, an OFET was fabricated and characterized, which demonstrated gatemodulated hole transport through the channel with mobility of $9 \times 10^{-4} \mathrm{~cm}^{2} / \mathrm{V} \cdot \mathrm{s}$, whereas no electron transport was observed in these devices.

During FY 2010, significant technical progress was made, and the project goal was reached. First, state-of-the-art blue light emission was achieved from a field-effect transistor with phosphorescent emitter blended in the channel material using a novel design of device structure. Next, a green light-emitting field-effect transistor in the similar device structure was fabricated, and both electrical current and light emission successfully exhibited sensing responses upon exposure to the model analyte 2,3-Dimethyl-2,3-dinitrobutane. This prototypical device is the world's first demonstration of light-emitting, field-effect transistor as chemical sensor.

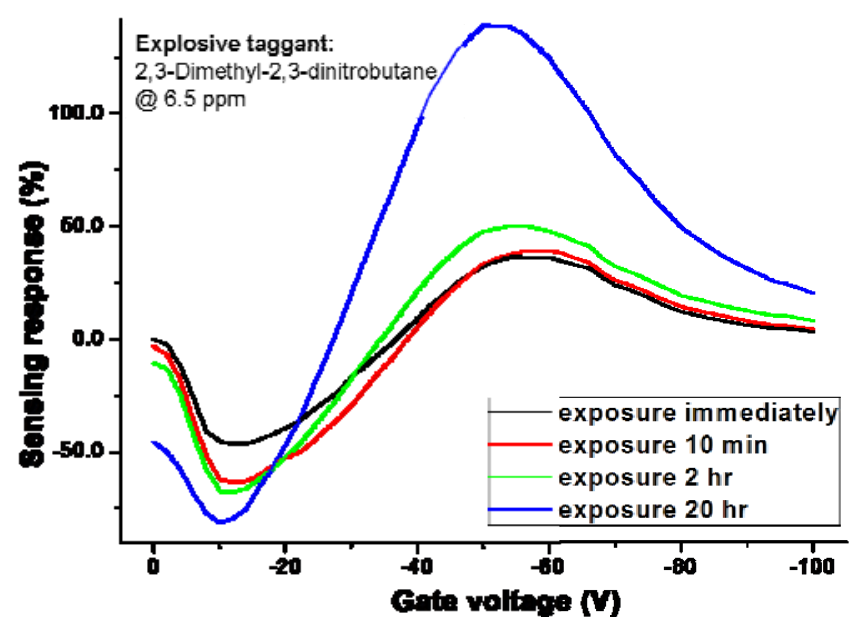

The gate voltage-dependent sensing response of the channel current in a green light-emitting field-effect transistor upon exposure to an explosive taggant 2,3-Dimethyl-2,3-dinitrobutane at a concentration of 6.5 ppm.

The sensing chamber was kept at vacuum to provide a clean environment before introducing analyte vapor whose flux could be finely adjusted. The optimal operating gate voltage for maximal sensing response (\%) is at $\mathrm{Vg}=-54 \mathrm{~V}$. A 6.5 percent immediate increase of channel current and a 200 percent immediate increase of light emission were observed as sensing response upon exposure to the analyte at $6.5 \mathrm{ppm}$ concentration. It is projected that with optimizing the device structure for improved light output, this sensor device could detect the explosive analyte at ppb level of concentrations. The sensor device's reversibility can be improved by heating at moderate (under $100^{\circ} \mathrm{C}$ ) temperatures or a short-time counter operation. Incorporating organic semiconductor materials that stably emit light in air are expected to improve the device's operational reliability.

By examining various physical properties (such as spectral and temporal analysis), this project investigated the interaction between target analytes and transduction elements, including channel materials and interfaces in a light-emitting OFET. These studies will enable the fundamental understanding of the sensing mechanism and pave the way to a selective, sensitive, portable, and cost-effective sensor for explosive detection to prevent potential terrorist attacks. 


\title{
Rapid, Sensitive and Selective Explosives Detection Using Tunable Chemical Ionization Drift Mass Spectrometry
}

\author{
M. Lizabeth Alexander, Brian H. Clowers
}

We worked to modify existing field-portable proton transfer reaction mass spectrometry instrumentation with the addition of new ionization. This non-radioactive source promises to extend the high sensitivity and rapid time response of chemical ionization draft mass spectrometry to the real-time detection of explosives and related compounds critical to national security.

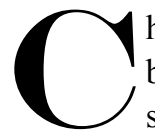

hemical ionization (CI) mass spectrometry has been widely demonstrated as a highly sensitive and selective laboratory technique for the detection of target compounds. Proton transfer reaction mass spectrometry (PTR-MS) uses $\mathrm{H}_{3} \mathrm{O}^{+}$as a CI agent for the specific detection of volatile organic compounds (VOCs) in air at concentrations of tens of parts per trillion with a time response of a few seconds. While proton transfer reactions in the gas phase enable the detection of gaseous species beyond simple hydrocarbons, the high levels of sensitivity observed for VOCs are not reproduced when analysis is directed towards more electronegative gas phase species such as those found in explosives.

Traditionally, ion mobility spectrometry (IMS) has been used to rapidly detect explosive and other warfare agents in a wide range of environments. However, the low-duty cycle (approximately 1 percent) of IMS has inherently restricted its lower limits of detection. Another frequent limitation is frequency of false positive signals. Nonetheless, this technology remains the primary workhorse for remote detection of explosive agents, with much of its success attributed to the variability of ion source conditions chosen for a specific application. As with the use of gas phase protons in PTR-MS, specific CI pathways may be accessed in IMS by tailoring incoming source conditions and reaction kinetics to favor the formation of specific analyte ions.
The objective of this project has been to modify and use exiting laboratory-based proton transfer reaction mass spectrometer (PTR-MS) instrumentation and concepts to demonstrate effective detection of vapor phase compounds relevant to homeland security, evaluate the limitations of standard PTR-MS, a positive ion technique, for the detection of electrochemical compounds, and to identify explosives for which PTR-MS may be used and those for which negative ion $\mathrm{CI}$ is a superior approach. The anticipated outcome of this project is knowledge of the fundamental ion-molecule chemistry and instrumentation required to implement its application to the ultra-trace detection levels of explosives and related vapors.

We successfully demonstrated the concept of a new type of ion-source mass spectrometer interface that will enable a detailed study of necessary ion-molecule science and ultimately the required levels of specificity and trace levels detection of vapor compounds required for a new generation of explosives monitoring equipment. Started in mid-2009, this project focused on a redesign of the new high sensitivity PTR-MS in the Environmental Molecular Sciences Laboratory to allow routine

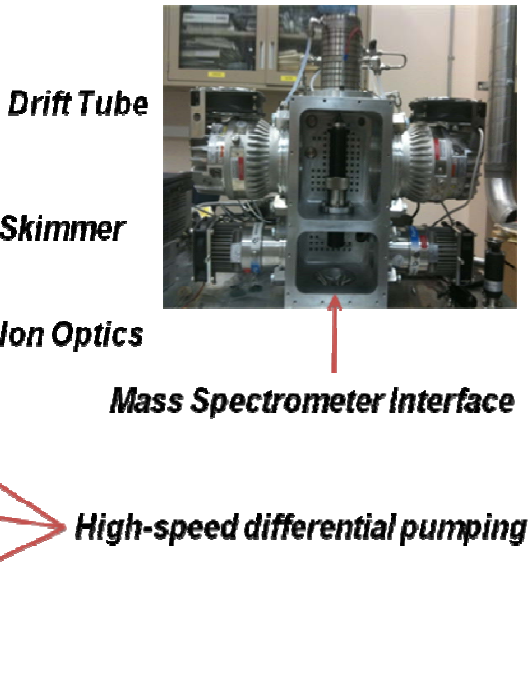

The new CIMS high-sensitivity source and interface showing pumping scheme and ion optics. The projected sensitivity for compounds suitable by PTR-MS is 200 ppq. Sensitivity for negative ion CIMS will be determined in FY 2011. operation for either positive or negative CIMS to develop a dedicated instrument for this and future related projects. A SIMION model was constructed to form ion trajectories and establish optimal potentials for both positive and negative CIMS.

In FY 2010, the SIMION model was expanded to include the variability in pressure through ionization and extraction regions. Developments for the year included: 1) the design and construction of a new CIMS platform with high sensitivity interface, 2) evaluated performance of new CIMS instrument, 3 ) adapted distributed plasma ionization source (DPIS) to PTR-MS/CIMS for negative ions, and 4) evaluated the utility of PTR-MS for 
explosives detection. Work on the new CIMS platform was completed for operation in positive ion mode to allow direct comparison of performance of the new source and interface with existing PTR-MS instruments. Based on the signal-tonoise of results obtained using analog detection of samples at 100 parts per trillion, a detection limit of 200 parts per quadrillion is projected when pulse-counting techniques are implemented. The PTR-MS spectrum of Di-methyl Di-nitro Butane, a tagging compound in plastic explosives that is a chemical analog of nitrate-type explosives, showed considerable fragmentation with very low signal on the parent peak, which would yield definitive identification. This result confirms the need for negative ion CIMS for electronegative compounds such as nitrate explosives.
By the end of the year, the DPIS was adapted to the new CIMS platform and confirmed to operate at atmospheric pressure. Ion generation was not quantified or mass analyzed. Additional work includes establishing the parameters under which negative ions from the DPIS may be produced at reduced pressure (e.g., under 10 Torr); demonstrating operation of PTR-MS and sensitivity in negative ion CI mode using a suitable explosive simulant compound; and initiating the investigation of the ionization and reaction kinetics and mechanisms for negative mode chemical ion drift mass spectrometry for compounds relevant to explosives detection. 


\title{
Standoff Detection of Trace Explosives on Vehicles Using a Cooled Dispersive Longwave Infrared Spectrometer
}

\author{
Thomas A. Blake, James F. Kelly
}

The aim of this work is to demonstrate significantly improved standoff detection of important chemical residues on exposed surfaces that can be applied to a range of practical security and environmental applications.

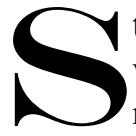
tand-off inspection of suspect containers and vehicles may allow timely detection of explosive residues on exposed surfaces (first or second generation fingerprints, contact with bulk material, and windblown particulates) while working at a safe distance. Active methods such as laser-induced breakdown spectroscopy are potentially feasible but generally require laser power levels that raise eye safety issues. Previous PNNL experiments conducted using a commercial imaging Fourier transform infrared (FTIR) spectrometer showed that it is possible to detect and identify explosive stains with areal dosages of about $15 \mu \mathrm{g} / \mathrm{cm}^{2}$ or greater on bare metal at $50 \mathrm{~m}$ using passive, longwave infrared (LWIR) detection methods. To date, however, efforts to detect explosive residues on painted surfaces such as a car door have proven problematic. Attempts to use an active infrared source with the spectrometer have not demonstrated adequate residue detection because of the instrument's limited dynamic range outside of its black body calibration range.

Improvements in residue detection limits and standoff operating range are needed. We will collaborate with Lawrence Livermore National Laboratory (LLNL) to use their imaging spectrometer to perform proof-of-concept experiments to determine if a LWIR device with superior sensitivity and dynamic range (compared with commercial FTIR imagers) can be used to detect passively explosive residues on a variety of surfaces with areal dosages. Active detection concepts will also be tried using a broadband infrared lamp. In addition to our experimental work, we also developed statistical algorithms for processing data from the hyperspectral data cubes produce by a spectral imaging device. Based on principal component analysis and generalized least squares methods, these algorithms have successfully located regions of interest in the spectral images (anomaly detection) and have identified chemical compounds in the scene (target detection). Recorded laboratory reflection spectra of explosives on various surfaces are used for the least squares analysis. Our results will possibly determine if LWIR imaging is a viable for detecting trace explosives on vehicles (or other surfaces).

This project commenced by preparing a set of metal plates with explosive stains: one set of plates was bare metal (steel), one had a black car finish, and another had a white car finish. Each set of plates had stains of $0.1,0.5,1,10$, and $100 \mu \mathrm{g}$ $\mathrm{TNT} / \mathrm{cm}^{2}$ and $0.1,0.5,1,10$, and $100 \mu \mathrm{g} \mathrm{RDX} / \mathrm{cm}^{2}$, for a total of 30 plates. The stains were $15 \mathrm{~cm}$ in diameter and were made by air brushing the appropriate concentration solutions onto the plates using a circular stencil. The plates were prepared at PNNL and shipped to LLNL, where imaging experiments were performed during a full week. Preliminary statistical analysis of the images from the cooled dispersive instrument showed that we can detect and identify 10 and $100 \mu \mathrm{g} \mathrm{RDX} / \mathrm{cm}^{2}$ on the bare metal plates at 30,60 , and $90 \mathrm{~m}$. We were able to also see $100 \mu \mathrm{gDX} / \mathrm{cm}^{2}$ on the white painted plate. Analysis work is ongoing for the other images in the large (approximately $200 \mathrm{~GB}$ ) dataset.

One difficulty encountered during data collection was instability in the temperature control unit of the focal plane array detector of the imager. This produced considerable noise spikes in the images during the first day of scanning. On the second (final) day of data collection, some changes were made in how the detector array was cooled and this seemed to have improved the detectivity of the instrument somewhat, though it was still not operating optimally. Our analysis shows that the instrument is not performing any better than the FTIR spectrometer in terms of noise equivalent spectral radiance. Despite this, we detected chemicals on the painted plates, an activity that we were unable to do with the commercial system. There is still much to do and understand about the physics of both the instrumentation and data collected.

The results of this work will help us determine the feasibility of detecting and identifying explosive residues less than $10 \mu \mathrm{g} / \mathrm{cm}^{2}$ applied by wet and dry contact on painted surfaces such as a car door. We also anticipate improvements in measurement equipment design, such as proper optical matching of the spectrometer to the spot of interest via an appropriate telescope that will increase residue detection limits by greatly improving the measurement signal-to-noise ratio. Though the experiment was performed passively, understanding the background clutter, how this type of spectrometer responds to it, and how our extant algorithms handle it are vital, as this information will provide the basis for more sensitive, practical instrument designs. Based on our results, we will determine a technical path forward for passive LWIR imaging of explosives. 


\title{
Standoff Hyperspectral Imaging of Explosives Residues Using Broadly Tunable External Quantum Cascade Laser Illumination
}

\author{
Bruce E. Bernacki, Mark C. Phillips
}

Standoff or non-contact detection of explosives residue is essential to meet homeland security threats and to assure military force protection. Active hyperspectral imaging will provide rapid, unambiguous spectral identification of suspicious substances combined with image contextual clues for rapid identification and classification of explosive residues without the need for sample preparation or physical contact.

$\mathbf{R}$ apid identification of explosive threats is an ongoing need for homeland security and force protection. Prior research showed that it is feasible to detect approximately $100 \mu \mathrm{g}$ quantities of explosives on surfaces using ratio images of explosives residue illuminated by infrared lasers tuned to on- and off-absorption features of the explosives. By using a continuously tunable mid-infrared illumination source such as an external cavity quantum cascade laser (ECQCL), one can probe the detailed absorption features of unknown samples that lie within the tuning range of the laser. An image hypercube is formed by recording an image at each tuning step of the ECQCL. The resulting images can then be exploited using hyperspectral image analysis methods developed by the remote sensing community for spectroscopic material identification. The goal of this project is to demonstrate and study hyperspectral imaging of explosives residues with ECQCL illumination. Standoff reflection and transmission microscopy setups were investigated to understand the issues related to this detection scheme, including coherent illumination artifacts, small particle spectroscopy, and limits of detection.

In the project's first year, we constructed the experimental setup and obtained reflection data at near-field and modest stand-off distances. The basic system consists of the ECQCL, LabVIEW control software (used to synchronize the laser scan and the image capture to form the image hypercube), control computer, and microbolometer camera. The illumination source is a custom-built ECQCL operating in a quasi-continuous wave mode with a $100 \mathrm{kHz}$ modulation rate and swept across a tuning range from $950-1095 \mathrm{~cm}^{-1}(9.13-10.53 \mu \mathrm{m})$ in $2 \mathrm{sec}$. The resulting hyperspectral image has 100 wavelength bands, which is adequate to identify the broad spectral features characteristic of explosives and typical solid compounds which could act as interferents.

In FY 2010, we constructed a hyperspectral imaging microscopy apparatus to investigate detection of small individual particles of explosives. The transmission-mode microscope had a spatial resolution of $6 \mu \mathrm{m}$, limited by diffraction at the infrared laser wavelengths used. Small particles of RDX, Tetryl, PETN, and TNT were imaged and distinguished based on differences in their infrared absorption spectra. Individual particles with masses as low as $5 \mathrm{ng}$ were imaged, and the limit of detection for this technique was estimated to be less than $1 \mathrm{ng}$. (For reference, a single $1 \mathrm{ng}$ particle in the field of view of the microscope represents an areal concentration of $30 \mathrm{ng} / \mathrm{cm}^{2}$.) The detection of small particles was found to be limited not by laser or detector noise, nor by the absorption strength of the explosives particles. Instead, the limits were

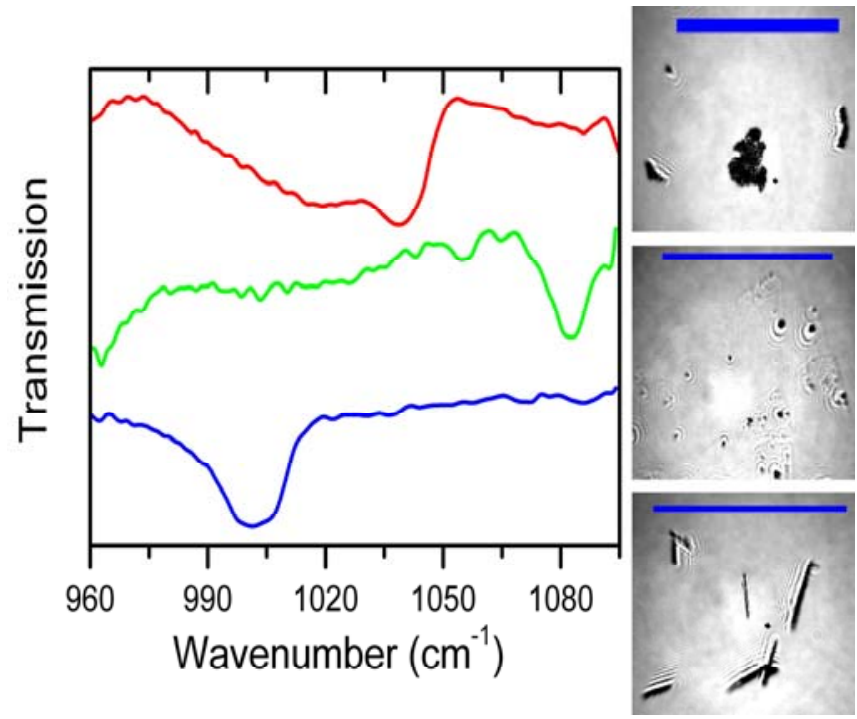

Infrared transmission spectra of $\mathrm{RDX}$ (top), Tetryl (middle), and PETN (bottom) allow identification of explosives in hyperspectral images. Images acquired using the byperspectral microscope show individual explosives particles (scale bars indicate a length of $1 \mathrm{~mm}$ in each image). set primarily by coherent illumination artifacts such as interference and diffraction. In addition, the spectroscopy of small particles with dimensions near the wavelength of the illumination light was complicated due to the interplay between reflection, transmission, scattering, and diffraction.

The study of microscopic particles has increased our understanding of the factors limiting the performance of hyperspectral imaging with ECQCL illumination. The results will be used during FY 2011 to estimate the performance of a system optimized for explosives

detection at larger standoff distances. The microscopy technique itself could have additional applications for non-contact, non-destructive particle identification in forensics or analytical laboratory use, for example. 


\title{
Understanding Ice Formation in the Atmosphere
}

\author{
Daniel J. Criczo, Gouribar R. Kulkarmi, Mikhail S. Pekour
}

According to the most recent Intergovernmental Panel on Climate Change report, the single most uncertain aspect of climate change is the interaction of small atmospheric particles and clouds. While there are several mechanisms by which clouds form, the formation of ice crystals is the most uncertain and is the specific focus of this project.

C arbon dioxide is understood to be the most important of the greenhouse gases in terms of impact on climate change because of both its anthropogenic origin and large radiative forcing. What is not clearly communicated is that the climatic impact of carbon dioxide is resolved, though both its abundance and impact are understood with higher certainty. Other climatic factors are not as well realized, and it is these unknowns that determine our current inability to predict future climate accurately. The most important of these are small particles known as aerosols. By themselves, aerosol particles can affect the earth's climatic balance by absorbing or scattering solar and terrestrial radiation, which is a process known as the "direct effect." It has been understood for several decades that small atmospheric particles can initiate the formation of clouds by creating liquid water droplets or ice crystals. Clouds also play a role in global climate either by trapping terrestrial radiation or reflecting solar radiation back into space. The sign of this forcing - net warming or cooling - is dependent on the altitude, location, persistence, and the thickness of the clouds. Because aerosols affect these specific properties, this is known as the particles" "indirect effect" on climate.

The formation of liquid water clouds has been extensively studied. The indirect effect is typically sub-divided into explicit ways in which aerosols affect clouds, and the first and second indirect effects are generally considered to be formed purely due to warm clouds. The impacts of pure ice as well as clouds that have both liquid and ice (known as "mixed-phase" clouds) are not well known because they are typically at increased altitude or high latitude location, which has made the field study of ice and mixed-phase clouds much more challenging. Likewise, laboratory studies of ice and mixed phase clouds are more difficult due to a requirement of high precisions measurements at low temperatures and water vapor levels. The climatic impact due to ice crystals is the "glaciations effect" portion of the indirect effect and, for the aforementioned reasons, it too remains ill-defined.

It is significant that there are important reasons to study atmospheric ice nucleation beyond climatic uncertainty. For example, ice nucleation is a critical element of the global water cycle. Indeed, the majority of terrestrial precipitation is believed to be initiated by ice nucleation. With this in mind, the foremost objective of this project is to understand which aerosol particles initiate atmospheric ice formation so that we are better able to reduce the uncertainties of climate change and more thoroughly understand the global water cycle. Among the foci for our studies are the following determinations: 1) the exact conditions (relative humidity and temperature) required for ice formation, 2) the effect that anthropogenic activities (specific components such as combustion byproducts as well as secondary surface coatings of sulfate and organics) have on ice formation, and 3) the interrelationship of droplet and ice formation (i.e., which aerosol particles form drops and which form ice).

To address the above issues, we designed and constructed novel instruments that we employed in the laboratory and field. The first is a compact ice chamber that can mimic the formation of ice clouds. This is of extreme importance because it means that researchers need not travel to locations where atmospheric ice formation takes place, nor must they go during the precise moment that the formations are occurring. Instead, the chamber allows researchers to make artificial ice clouds in the laboratory from specific chosen particles, or there is the option to travel to locations where ice clouds form without having to conduct experimentation only at those times when nucleation happens to take place. Examples include laboratory experiments on aerosols that mimic combustion products such as soot and field studies at high altitude sites or from aircraft. 
The compact ice chamber was designed during FY 2008 and completed in FY 2009, during which time it underwent laboratory testing. The initial deployment of the chamber took place during January 2010 at the Desert Research Institute's Storm Peak Laboratory, located in Colorado's Rocky Mountains. This site offers access to particles that form ice clouds without the expense and constraints of a research aircraft, making it an ideal location. This research resulted in the participation in DOE's “StormVEx” study at Storm Peak Laboratory.

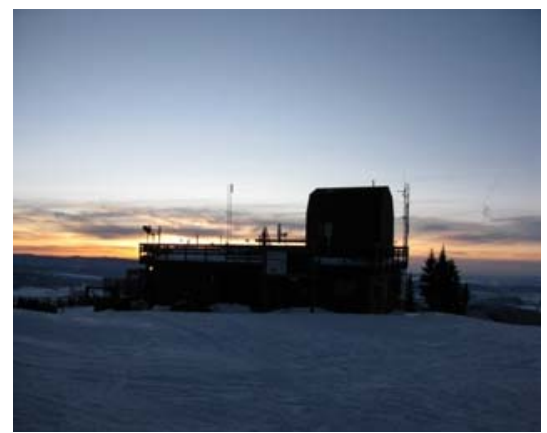

Located at over 10,000 feet above seal level, Storm Peak Laboratory allowed testing of instrument under conditions similar to those encountered on aircraft.
The second novel instrument system is known as a counterflow virtual impactor, which separates ice crystals or droplets from unactivated aerosol particles. In order to build this instrument, we developed an extensive computational fluid dynamic modeling

capability that has for the first time been compared to laboratory experiments as part of this project. This is significant because in the past technology such as this was physically constructed and experimentally tested at considerable material and time cost. Using our modeling capability, we built an instrument of known performance and validated the results by comparison to physical data. With this validation, we could design and test new instruments before physical construction or experimentation. This unique research forms the basis of a currently submitted peer-reviewed publication to Aerosol Science and Technology. The new impactor was coupled with the compact ice chamber for the field deployment, which allowed the separation and analysis of those particles that nucleate ice as a function of relative humidity and temperatures using techniques such as singleparticle mass spectrometry and electron microscopy.

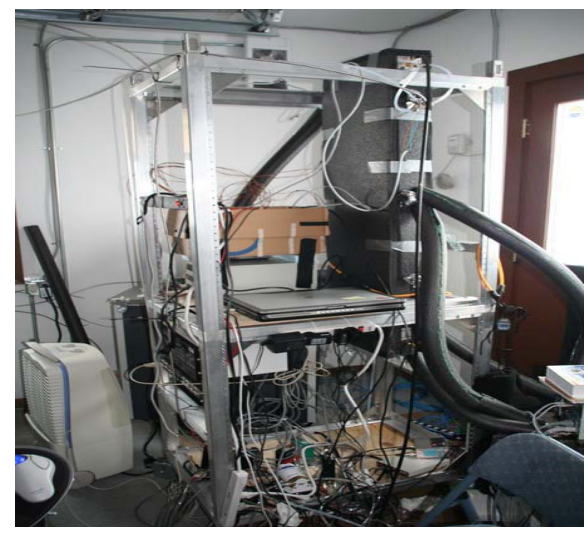

The compact ice chamber during a field study.
We also thoroughly tested the new impactor and described its abilities in a second submitted peer-reviewed publication to Aerosol Science and Technology.

The third novel instrument we developed is analogous to our compact ice chamber. We built an adapted counterflow virtual impactor for use with a commercial chamber available at PNNL so that the composition and size of particles that form droplets can be determined. This extends the capability of a commercial instrument in a way that has never before been done. Through the course of this research, we collaborated with the University of Frankfurt.

FY 2010 has also seen the use of the compact ice chamber in laboratory studies. One of the outcomes of our Storm Peak field study was the observation that combustion generated particles were not observed to effectively form ice clouds. This is significant because previous publications indicated they could be, and this has significant climatic implications. We verified the results internally and with the University of Washington. This study has shown that combustion generated aerosol, both newly generated and after atmospheric aging, does not effectively form ice clouds. This research forms the basis of an peer-reviewed publication to the Journal of Geophysical Research (in preparation).

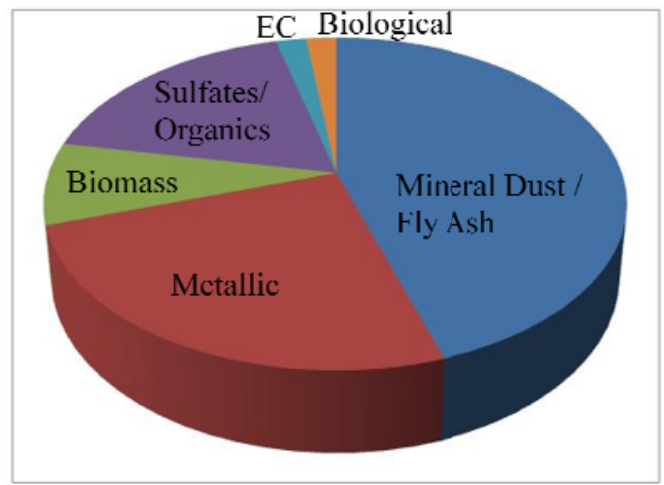

The chemical composition of ice-forming aerosol using the compact ice chamber, counterflow virtual impactor, and mass spectrometer.

A second laboratory study using a Humidified Tandem Differential Mobility Analyzer coupled with a mass spectrometer was at the University of California at San Diego. Currently, we are in the process of analyzing data to determine if a scientific publication can result from these studies.

In addition to instrument development and laboratory activities, we worked on pre-existing data sets during FY 2010. Resulting from our studies and research, a total of four peer-reviewed journal articles on drop formation and ice nucleation were published, two more are currently under review, and two others are in preparation. 


\section{Biological Sciences}




\title{
Application of a Systems Biology Approach to Understanding Protein Function
}

\author{
Karin D. Rodland, Karla D. Thrall, Charles A. Timchalk, Cheryl L. Baird, Garry W. Buchko, \\ Richard E. Jacob, Feng Yang, Bryan E. Linggi, Richard C. Zangar
}

This project uses a new approach of systems biology to understand protein function using a variety of model systems. In addition to furthering PNNL's capabilities in systems biology, we will be exploring applications that are relevant to human health.

$\mathbf{T}$ he genomics revolution of the twentieth century provided a "parts list" for living organisms ranging from microbes of bioremediation to humans. Yet, we still do not comprehend fully how these parts work together to define organism function. We have a vision that includes international recognition by providing the scientific leadership and technology needed to achieve a predictive understanding of multicellular biological systems from microbial communities to humans (i.e., microbes to man). Our motivation is the increasing need for a quantitative understanding of how cells interact with each other and their environment. We will push the state-of-the-science in biology from a descriptive to a predictive science that will allow us to predict, manipulate, and potentially design multicellular systems and bioinspired solutions for bioenergy, contaminant fate and transport, carbon sequestration and global climate change, and human health.

PNNL has already developed novel proteomic technologies. What is lacking is a predictive understanding of complex systems applied to increasingly complex problem sets and the development and refinement of those capabilities to deal with more complex biological problems. The objective of this project is to facilitate the application of capabilities in mass spectrometry, magnetic resonance spectroscopy, systems biology, and bioinformatics to selected problems that represent high impact challenges in predictive understanding. The common theme of these subprojects is the application of proteomic and materials science capabilities to understand the relationship between protein structure, post-translational modifications, and functional capability in complex cellular systems.

During FY 2009, we refined the ability to link protein structure with function, which required more research to explain molecular theory and explore sophisticated computational methods for antiferromagnetic model compounds. We succeeded in defining the conditions for sustained release of immunoglobulin $\mathrm{G}$ from functionalized mesoporous silica particles under physiological conditions, establishing the proof-of-principle for using functionalized mesoporous silica particles in cancer therapy. Further, we validated a system- theoretic model for integrated cell signaling, where it captures all of our experimental observations. These results were used to refine the mechanistic model for oscillations and to validate predictions with targeted experiments.

During FY 2010, we made good progress on the following tasks.

Determine the effects of novel compounds on the decorporation of radionuclides. Relevant to developing radiological/nuclear threat medical countermeasures, the overall goal was to develop an oral product to mitigate the effects of internal contamination with strontium-90. Results obtained using rodents demonstrated that oral administration of alginate results in a significant increase in radioactive strontium elimination and a corresponding decrease in radioactive strontium burden in the bone compared to untreated control animals.

Develop novel imaging technologies. The goal was to develop needed preliminary magnetic resonance proton spectroscopy data to demonstrate a capability to measure in vivo changes in brain chemistry in live neonatal rat pups to enable assessment of subtle changes in regional brain chemistry resulting from low-dose exposure to environmental neurotoxicants. This novel approach has potentially broad implications for evaluation of subtle neurodevelopmental effects; hence, it will be a particularly strong research tool to understand low dose neurotoxicity associated with chemical, drug, and radiological exposures.

Modify the yeast surface display system to utilize singlechain antibody fragments (scFab) as novel affinity reagents. We commenced investigation on formulating affinity reagents based on scFab antibody framework. Our preliminary studies suggest that this antibody format is functional when displayed on the surface of yeast. We created a naïve scFab library for the production of highly stable affinity reagents that retain activity when produced as soluble molecules (not tethered to the yeast surface).

Investigate protein-DNA interactions using a novel labeling approach combined with nuclear magnetic resonance. Our major focus was to explore the use of two different approaches to prepare ${ }^{13} \mathrm{C}$ - and ${ }^{15} \mathrm{~N}$-labelled oligonucleotides. Initial experiments did not result in yields sufficient to attempt a large-scale synthesis. Research will continue into FY 2011. To date, two manuscripts were completed. 
Develop a multi-modal imaging capability for complex biological systems. We worked to develop and implement new non-invasive imaging capabilities that would enable the ability to predict human health risks from inhalation exposures encountered at DOE facilities; characterize defect formation and fracture propagation in materials science applications; and facilitate the investigation of basic properties of rocks, minerals, and fluid dynamics in geosciences and pedometrics. The critical ability to visualize intricate structures (such as pulmonary tissue) at high resolution (i.e., tens of microns) was missing until acquisition of a new micro-CT imager with non-LDRD funds. Under this task, we successfully constructed a new mouse MRI coil that enhanced our imaging sensitivity by about four times over other coils; learned and refined an extremely challenging technique for giving tail-vein contrast agent injections to mice during image acquisition (required for the proposed imaging approach); and developed a unique imaging pulse sequence specifically for monitoring disease.

Develop tools for studying protein glycosylation as a response to metabolic stress. The specific goal was to uncover the mechanism linking altered glycosylation to Alzheimer's disease (AD). These studies will help elucidate the molecular mechanism of neurodegeneration in $\mathrm{AD}$ and, based on this knowledge, help develop strategies to prevent and treat AD. The work will be presented at the Post Translational Modifications Special Symposium hosted by the American Society for Biochemistry and Molecular Biology.

Integration of high throughput transcriptome and proteome data to understand the regulation of autocrine pathways. This task will address the mechanisms by which information from multiple extracellular ligands (growth factors or other extracellular signaling molecules) are integrated into the signal transduction network of cells to produce specific

cellular responses. The central hypothesis is that the primary signal integration takes place at the level of the adaptors that couple receptor activation to downstream signaling pathways. Secondary integration occurs at gene transcription, which depends on the relative strength of multiple primary signaling pathways. Data suggest that many ligands activate the ERK pathway, which appears to control the expression of a core set of target genes that affect cell responses. However, it is also possible that ERK only modulates the expression of ligandspecific genes.

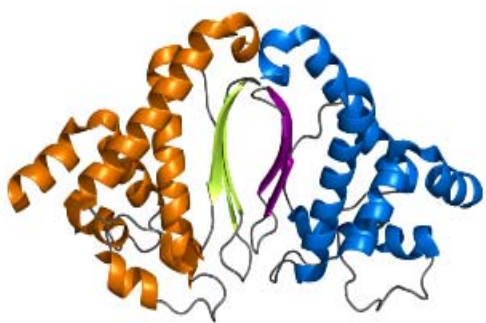

Discriminating between these two ideas is one of the primary objectives of the proposed work.

Characterization of reactive oxygen species as a response to environmental stress. Cytochrome P450 (P450) 3A4 (CYP3A4) is the most abundant $\mathrm{P} 450$ protein in human liver and intestine and is highly inducible by a variety of drugs and other compounds. In this task, low levels of CYP3A4 were expressed in $\mathrm{HepG} 2$ cells, a human hepatocarcinoma line, and effects on intracellular levels of reactive oxygen species (ROS) and on the secretion of a variety of growth factors that are important in extracellular communication were examined. We demonstrated that CYP3A4 expression increases levels of ROS in viable cells. Our results suggested that increased ROS production in the endoplasmic reticulum alters gene expression of secreted proteins that have key roles in paracrine and autocrine signaling.

The following specific tasks will be performed during FY 2011: 1) develop an activity-based protein profiling capability for mitochondrial function, a key to understanding biological processes related to energy conversion and storage, 2) predict the effects of novel nanomaterials on biological systems by developing computational models to describe the fundamental interactions between proteins and nanomaterials, and 3) develop and test bio-nanomaterials for delivery of proteins and lipids for bioengineering of complex systems. 


\section{Combining Proteomic Technologies to Create a Platform for Spatiotemporal Enzyme Activity Profiling}

Susan D. Wiedner, Aaron T. Wright, Ellen A. Panisko

- We are developing spatiotemporal enzyme activity profiling (STEAP) that will combine activity based protein profiling (ABPP) with organelle proteomics. The STEAP technology will provide a platform for assessing and comparing the functional activity of biological systems under various states and conditions.

A BPP and organellar proteomics are techniques used in the field of proteomics to decrease the complexity of the natural cellular proteome, which often contains thousands of proteins. Activity-based protein profiling isolates the active portion of the proteome by tagging functionally active enzymes with chemical activity based probes. In contrast, organellar proteomics reduces complexity by focusing on the proteome of subcellular compartments. Combining ABPP and organellar proteomics into STEAP will provide information about the functionally active proteome on the organelle level. This information will help elucidate on a cellular level fundamental biological processes such as protein translocation, signaling, and cellular response to environmental stress.

The biological system of interest is the lung disease invasive aspergillosis caused by the pathogenic filamentous fungus Aspergillus fumigatus. Invasive aspergillosis is a deadly, costly infection of the lung often found in immunocompromised patients. To date, there is no definitive diagnostic test for invasive aspergillosis except postmortem autopsy, yet early detection and diagnosis is critical. Further, a better understanding of the virulence factors of A. fumigatus and how host enzymatic function responds to infection would increase the potential to find ways to combat this disease.

The goal of this project is to develop and use STEAP to investigate host response to infection with A. fumigatus on a subcellular level and to find enzymatic biomarkers for infection. We will combine the proteomic technologies of ABPP and organelle proteomics. Individually, these two technologies study different "fractions" of the proteome and in combination would provide spatiotemporal information of the functionally active proteome. The new STEAP platform will be used in to compare biologically relevant proteomes such as diseased systems.

During FY 2010, our plan was to investigate the active proteome of $A$. fumigatus with ABPS using liquid chromatography-mass spectrometry (LC-MS) analysis. Known and novel activity based probes that target phosphatases, serine hydrolases, and glutamine synthetase enzymes will be synthesized and evaluated for ABPP. Sample preparation,
LC-MS analysis, and bioinformatics data analysis will be used to identify the tagged enzymes. The active proteome of A. fumigatus will be compared with two closely related non-pathogenic filamentous fungi species: A. clavatus and Neosartorya fisheri.

During the last year, we synthesized a new protein tyrosine phosphatases (PTP) probe in four synthetic steps from commercially available material. The activity based probe contains an alkyne moiety that after derivatization allows for isolation and detection of the protein. For detection, the probe labeled enzyme is attached to a fluorescent indicator via a copper catalyzed [3+2] cycloaddition. The tagged proteins can then be visualized using 1D-SDS PAGE. With this technique, initial studies showed that enzymes in a mouse liver microsome proteome were tagged. To confirm that the probe is specific for PTP enzymes, ABPP samples of mouse liver microsome were prepared and submitted for high resolution mass spectrometry analysis, and the labeled proteins will be identified using SEQUEST-based spectral counting. The data for this experiment is still pending.

Also during FY 2010, A. fumigatus was cultured and grown to produce fungal biomass for ABPP labeling studies. For analysis of the proteome of $A$. fumigatus, the biomass was lysed and homogenized using a bead beating blender to disrupt the fungal cell wall. Once lysed, the standard biological separations and mass spectrometry group sample preparation protocol was used to prepare samples for LC-MS analysis. The data collected from the analysis will be used to generate an accurate mass and time (AMT) tag database for the proteome. The AMT tag database will be used for to identify probe labeled proteins in subsequent ABPP labeling studies. A. fumigatus biomass was processed and fractionated for ABPP labeling. Global and light membrane fraction were labeled with multiple probes to target PTP, P450s, serine hydrolases, and glycoside hydrolases. Initial analysis by 1D SDS-PAGE showed that the probes have tagged enzymes within these proteomes.

Studies in FY 2011 on labeling fractions of A. fumigatus will continue. Studies will focus on sample preparation of the proteome to optimize the labeling studies. Once the sample preparation is optimized, the proteome will be labeled with the small library of activity based probes and analyzed in order to identify the probe labeled peptides. Subsequent cell culture or lung doping studies will test the limits of probe detection for the presence of $A$. fumigatus in a complex proteome. Ultimately, this method will be used to detect A. fumigatus in an infection model. 


\title{
Community Diversity and Functional Redundancy of Cellulytic Microbial Communities in Soil Aggregates
}

\author{
Vanessa L. Bailey, Lee Ann McCue
}

An understanding of the role of natural microbial communities in the global carbon cycle is fundamental to our ability to mitigate the effects of greenhouse gases. To gain this knowledge, we must bridge the current knowledge gap between the community structure (who's there?) and the functional potential (what are they doing?) of microbial communities.

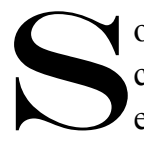
oils are highly complex systems in terms of their composition, dynamics, and heterogeneity, with an estimated $10^{9}$ bacteria and up to $1 \mathrm{~km}$ of fungal hyphae in $1 \mathrm{~g}$ of soil. Aggregates are the building blocks of soils and highly stable individual micro-aggregates (less than $250 \mu \mathrm{m}$ diameter) bind together to form more dynamic (i.e., shorter-lived), larger (up to and greater than $2 \mathrm{~mm}$ diameter) aggregates. These individual and compound aggregates form different habitat types and are likely to feature different microbial community structures and functions. Additionally, the smaller, stable micro-aggregates are the location of the most protected, stable carbon in the soil. As these different types of soil aggregates are what comprise the fabric of an intact soil, it is clear that the fine-scale aggregates exert a strong influence on the behavior of the whole soil.

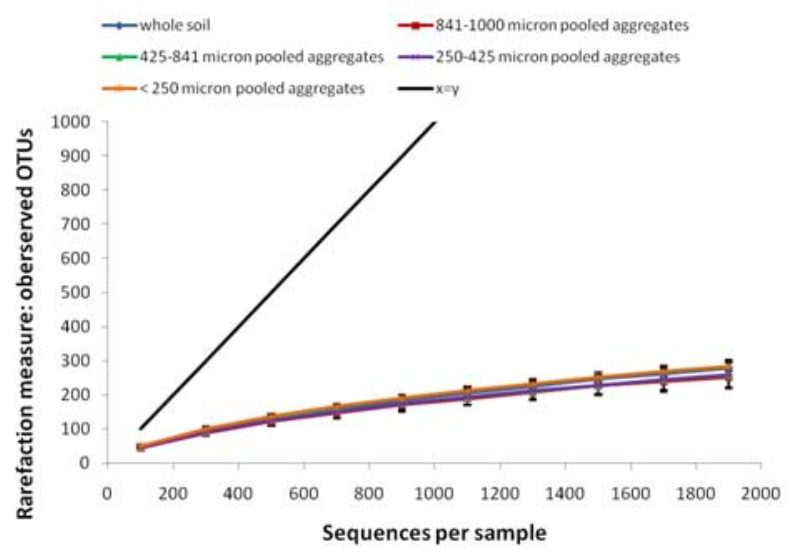

Rarefaction on observed operational taxonomic units plateaus quickly at the individual soil aggregate scale. Species richness varies widely among individual aggregates, though it does not appear to correlate with aggregate size.

With recent advances in DNA sequencing technologies and microscale enzyme assays, it is now possible to study the diversity of soil microbial communities, activities of community members, functional redundancy of the community as a whole, and resiliency to perturbation at the fine scale of soil aggregates, nominally sorted into four size classes (less than $250 \mu \mathrm{m}, 250$ to $425 \mu \mathrm{m}, 425$ to $841 \mu \mathrm{m}$, and 841 to $1000 \mu \mathrm{m}$ ). The soil carbon cycle is complex and diverse; therefore, we focused on the metabolism of cellulose, the most abundant natural biopolymer on the planet. Enzyme assays have been developed for bulk soils, allowing the study of cellulosederived carbon entry into the soil through microbial cellulases action. Examination of soil at fine scales (such as individual aggregates) requires developing highly sensitive fluorescencebased enzyme assays to reveal the functional capability of the community and the resiliency of the community to perturbation.

In FY 2009, DNA pyrosequencing data for the 16S ribosomal DNA from approximately 200 soil aggregate samples were obtained from the University of Colorado. We analyzed these data in FY 2010, examining the microbial community structure to assess community diversity and variability in soil aggregates of similar or different size classes and in whole soil. The rarefaction curves on these data plateaus quickly, demonstrating that the species richness of an aggregate has been sampled with only a few hundred sequences. Analysis of the $\beta$-diversity of the aggregates shows that the number and identity of the species in the aggregates does not exhibit significant differences.

Microscale enzyme assays to measure cellulase activity of individual aggregates were carried out in FY 2010. Classical $\beta$-glucosidase assays were scaled down to reactions in a few microliters with fluorescent detection of the reaction products. These microscaled assays showed that per-volume enzyme activities in the smallest aggregates varied far more than the per-volume biomass of those aggregates, suggesting that either the smaller aggregates were favoring microbial communities that were more responsive to substrate additions or that potentially active extracellular enzymes were protected within the aggregates. The microbial biomass measurements from within these aggregates were also not tightly correlated to the activity of other enzymes (lipase, $\beta-\mathrm{N}$-acetylglucosaminidase). Soil aggregate community structure was also surveyed in FY 2010 through deep coverage sequencing of the phylogenetically informative ribosomal DNA regions. Current DNA pyrosequencing technology using barcoded primers provides measures of microbial diversity and abundance of community members.

During FY 2011, we will sort assayed aggregates according to their enzymatic activities and sequence the most and least active to learn if there are significant differences in community structures of these functionally different aggregates. We will also combine DNA sequencing technology with stable isotope probing to identify the active cellulytic species within the soil aggregate community. 


\title{
Designed Affinity Reagents with Extreme Stability and Selectivity
}

\author{
Cheryl L. Baird, Curt B. Boschek, Garry W. Buchko, Thereza A. Soares, Tjerk P. Straatsma
}

This project is aimed at developing a new platform for the generation of affinity reagents that possess a high degree of stability with engineered specificity for a wide range of molecular targets. Moreover, the reagents developed using this platform will be completely novel reagents with stabilities not observed in natural proteins.

I $\mathrm{n}$ the last decade, new classes of molecular recognition proteins (MRP) based on small, robust scaffolds have emerged as an alternative to immunoglobulin-based MRPs. These novel scaffolds could be used as diagnostic reagents, basic research tools, or therapeutics and may offer potential advantages in production and application over conventional immunoglobulin-based MRPs. We have been developing one such scaffold, Top7, as an MRP for diagnostic, basic research applications and field-based sensors. This small, synthetic protein is thermodynamically stable and can be easily engineered and produced in microbial systems. The stability characteristics of Top7-based scaffolds are especially desirable for field-based biosensors, where variations in temperature and complex environmental samples render standard antibody-based affinity reagents inactive. Our goal is to create a platform for high-throughput affinity reagent production using the Top7 protein as an invariant scaffold.

Our approach to engineering binding specificity into Top7 involves the creation and mining of Top7-variant libraries containing molecular recognition loops inserted at defined regions within Top7. In FY 2009, we demonstrated that insertion of a 12-residue molecular recognition loop (CB1) confers specific binding of Top7 to the CB1 target (CD4) without affecting the scaffold's stability. Because multiple molecular recognition loops improve the specificity and affinity of MRPs, we identified 4 additional loops in Top7 that are not involved in the protein's secondary and tertiary structures and should accommodate insertions without compromising the scaffold's stability. We developed variants of Top7 with the CB1 element inserted into the loop regions and determined whether these sites were available for target binding without affecting the stability of Top7.

The circular dichroism spectra of the Top7-CB1 variants are the same as that of native Top7, suggesting that insertion into these loops does not alter the core Top7 scaffold. The denaturation midpoints of the Top7-CB1 variants in guanidine hydrochloride are also similar to that of native Top7 (5-6M). All four Top7 variants were all able to specifically detect CD4 by ELISA. These experiments suggest that insertion into any one of these loops does not affect the stability of Top7.
A portion of this work was featured as the May 2009 cover story in the journal Protein Engineering Design and Selection.

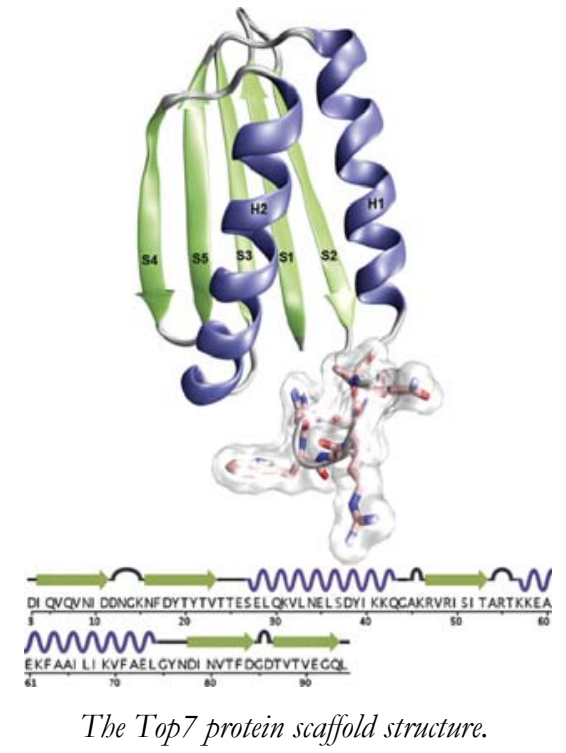

In FY 2010, we continued to study the nature of the Top7 scaffold's tolerance to extremes of $\mathrm{pH}$ and chemical denturants using molecular dynamics simulations to investigate the structural changes of Top7 and the Top7-CB1 engineered at high temperature $(400 \mathrm{~K})$ and low $\mathrm{pH}(2)$. This work was published in the Journal of Molecular Graphics and Modeling. In addition, we constructed libraries of naïve human complementarity determining region insertions into the 4 regions of Top 7 that we previously identified as suitable for modification. These libraries will provide the foundation for an ultra-stable MRP that can be rapidly engineered in vitro.

Our work to date provides a foundation for future development of rapid and efficient methods for generating Top7-based affinity reagents. We will continue this effort by demonstrating the methods we developed by generating Top7-based affinity reagents to a panel of disparate protein targets. We also plan to identify additional applications and sensors which would benefit from the high-stability of Top7 affinity reagents. For instance, antibody-based affinity chromatography is underutilized, particularly in industrial applications, because antibodies are frequently too expensive to be practical and cannot withstand highly reducing/denaturing environments, high ionic strength, or the $\mathrm{pH}$ extremes needed for column regeneration. The goal of studying Top7 as an affinity reagent scaffold is to have a method for rapidly developing an affinity reagent to any clinically or biologically useful target not limited in its application. 


\title{
Distinguishing Yersinia pestis from Natural Host and Laboratory Culture
}

\author{
Helen W. Kreuzer, Douglas C. Duckworth, David S. Wunschel
}

The goal of this project is to produce the organism that causes plague, Yersinia pestis, in a natural host (the rat) in laboratory cultures and compare chemical and biochemical signatures of the various samples to develop means of distinguishing the two cases.

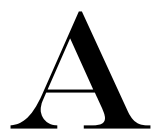

research topic of interest to the homeland security, intelligence, and law enforcement communities is distinguishing natural (e.g., death of an infected animal) from the release of laboratory-produced organisms. Chemical and physical analysis of the material can likely distinguish the two possibilities if genetic analysis cannot provide a conclusive answer. Like other disease agents, $Y$. pestis occurs in nature in the United States and elsewhere. If its presence is detected, it is important to determine whether the detected organisms are naturally occurring or were deliberately released.

This project will apply existing methods for chemical and elemental analysis to virulent $Y$. pestis pathogen cultured in a natural host. Specifically, we will measure stable isotope ratios of hydrogen, carbon, nitrogen, oxygen, and strontium isotope measurements to each sample set. The carbohydrate and lipid makeup of a specific cellular structure, lipopolysaccharide, will be targeted for analysis. The outcome is to determine the potential markers of laboratory culture versus growth in a natural host. A key technical challenge will be to develop methods for separating the bacteria from infected animal tissue for analysis. Because $Y$. pestis causes serious disease in humans, it must be propagated and harvested in a Biosafety Level 3 (BSL3) facility, which is not available at PNNL. One of the goals of this project, therefore, is to establish collaboration with a BSL3 facility for the purpose of sample production. After the samples are produced and inactivated, they will be shipped to PNNL for chemical and biochemical characterization.
E. coli-Rabbit Blood Mixture

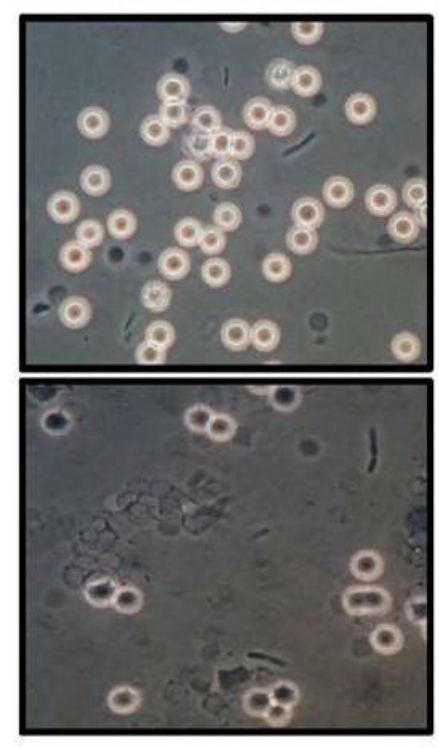

The left panels show a mixture of bacteria (E. coli; small dark rods) and rabbit block cells (bright spheres). The right-hand side panels show the successful separation of the bacterial cells from the blood cells by fluorescenceactivated cell sorting.

FACS separated $E$. coli cells

During FY 2010, we established collaboration with the Battelle BSL3 laboratory in West Jefferson, Ohio and are working with a scientist there to finalize a plan for the rat infections and obtain the necessary approvals. Before conducting the experiment in the BSL3 lab, we wanted to perform a trial run to make sure that all of our procedures work as expected. We will perform that trial experiment using an attenuated (avirulent) strain of $Y$. pestis, which does not cause the disease in humans but will in rodents if a sufficient number is introduced into them intravenously. This trial run can occur at PNNL, and we have worked with the Institutional Animal Care and Use Committee to get a protocol approved and use vivarium staff to equip a laboratory properly for the experiment.

Also during FY 2010, we developed two different methods for separating bacterial cells from animal tissue through the use of blood as a test tissue. One method is based on centrifugation, and the other uses fluorescence-activated cell sorting. These methods are both sufficiently novel that they will be described in a scientific publication that is currently in preparation.

For FY 2011, we will conduct the trial infections at PNNL, harvest infected tissue from the infected rodents, inactivate the organisms, and separate the bacteria from the tissue. Various chemical signatures of the bacteria will be characterized and

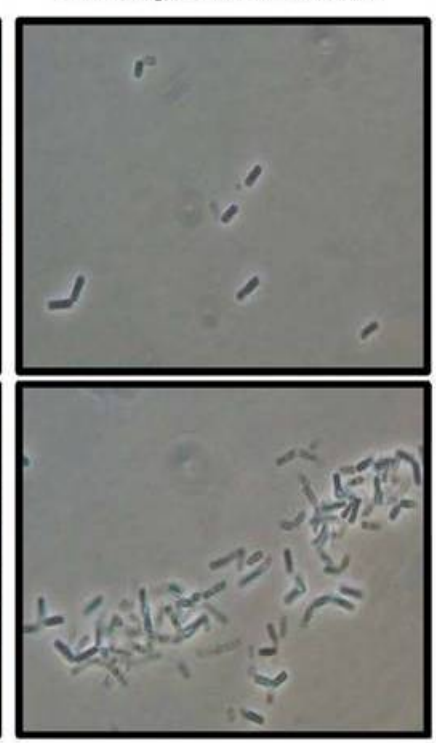

compared with the signatures of the same species of bacteria produced in laboratory culture. The same approach will be replicated in the BSL3 facility in Ohio, with the sterilized infected tissue sent back to PNNL for analysis. The results of our work should describe approaches for potentially distinguishing organisms that were produced via natural infections versus those produced in laboratory culture. Our aim is to expand PNNL's capability in microbial forensics through characterizing and providing proof-of-concept data on $Y$. pestis samples produced in both the natural host and laboratory culture. 


\section{Exploration of Pan-Omics for Biological Research}

Richard D. Smith

This project will result in multiplexed high throughput sample processing analysis capabilities of multiple subproteomes and submetabolomes. Initial demonstrations of capabilities will extend the areas of program growth across multiple biological research areas such as carbon sequestration, bioenergy, environmental processes, biological events in response to radiation, and health related biomarker development.

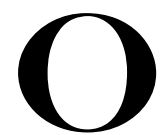
ur "Next Generation Measurement Platform" capabilities will enable the integration of high throughput and comprehensive proteomic and metabolomic quantitative profiling of biological systems. This research will allow effective use of proteomics and metabolomics platforms and will support effective in-depth coverage of many different post-translational protein modifications or other subproteomes and submetabolomes in a high throughput manner. To achieve this objective, our initial emphases are to develop automated multiplexed enrichment technologies for effective fractionation of the global proteome and metabolome into subproteomes and submetabolomes based on their modifications and chemistry, respectively. The initial set of targeted modifications will include phosphorylation, $O$ and $N$ linked glycosylation, ubiquitinylation, and proteolytic processing by profiling $\mathrm{N}$ and $\mathrm{C}$ terminal peptides. Many modifications represent important cell signaling and functional mechanisms.

In FY 2009, we improved a column-based phosphopeptide enrichment protocol to allow batch mode analysis, which will enable future high throughput automation multiplexed with other enrichment technologies. We developed a new click-chemistry protocol for labeling and capturing $O$-GlcNAcylated peptides novel photocleavable reagents synthesized in-house. These reagents were evaluated using model peptides, with the ultimate goal to quantify using an amine specific fluorescent assay.

Informatics advances in FY 2009 involved ion mobility drift time prediction and a new informatics tool that supports the management and manipulation of complex pan-omics data. We developed a support vector regression based model

that accurately predicts peptide cross sections directly from peptide amino acid sequence. When tested on a manually curated high confidence database of experimentally measured cross sections, our prediction method yields areas with $\mathrm{R} 2=90$ percent for doubly charged and 85 percent for triply charged peptides. We developed the Multi-Dataset Analysis and Rollup Tool (MDART) software to allow users to apply a workflow defining data transformation steps to process data stored in standalone SQLite databases populated from the mass and time tag system. MDART workflows are stored as XML files and are designed to be re-used to consistently apply data processing steps to appropriate input data. MDART also includes a built-in workflow editor that provides a mechanism to develop and test queries.

Our progress in FY 2010 is highlighted within the areas of universal sample processing and glycomics pipeline development as well as new, expanded informatics and computational analysis tools. These advancements have enabled effective in-depth coverage of numerous post-translational protein modifications or other sub-proteomes and sub-metabolomes in a high throughput manner.

Development of a Universal Sample Processing Methodology. In part, our efforts have been devoted to developing a multiplexed sample processing for downstream proteomics and metabolomics analyses. Specifically, we enabled methods for "universal" processing of samples, such that the same sample can be used to generate material for proteomics and metabolomics analyses. In preliminary experiments, we performed chloroform/methanol $(2: 1, \mathrm{v} / \mathrm{v})$ extractions of gongylidia from fungal gardens of the leaf cutter ant Atta

Acromyrmex echinatior (Acro)

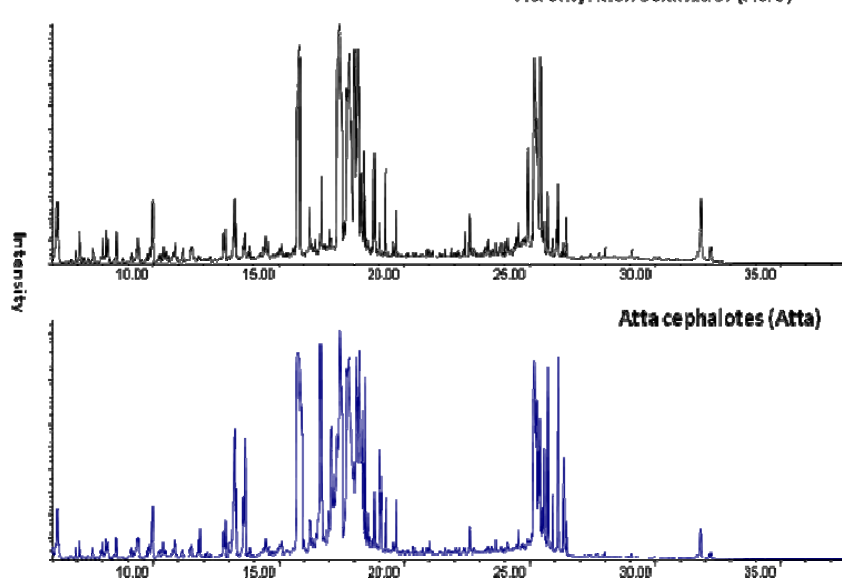

GC-MS total ion chromatograms showing water-soluble metabolites detected in fungal gardens cultivated by either Acromyrmex echinatior or Atta cephalotes. colombica in order to partition the samples into a water-soluble metabolite fraction, a lipidsoluble metabolite fraction, and a precipitated protein fraction. The fungus-growing ant-microbe symbiosis is a paradigmatic example of organic complexity generated through symbiotic association which, over the last decade, has become a model system for studying symbiosis. The ants depend on fungi that they culture for food. In exchange, the ants provide the fungus with substrate for growth, protection from competitors, and dispersal to new colonies. 
Because the fungal gardens are frequently infected with specialized and potentially virulent parasitic microfungi in the genus Escovopsis, the ants engage in a mutualistic association with filamentous Actinobacteria (genus Pseudonocardia), which produce antibiotics with potent inhibitory properties against Escovopsis. Initial gas chromatography-mass spectrometry (MS) analyses of the water-soluble metabolites indicate that this is a very rich fraction: 62 metabolites were structurally identified in high confidence among a total of 143 detected compounds. Importantly, high coverage of amino acids was obtained, and, somewhat unexpectedly, a large number of cellobiose metabolites were identified. Similarly, liquid chromatography (LC)-MS analyses of digested gongylidia proteins revealed reasonably high coverage of the proteome. These results are significant, as the fungal gongylidia samples are typically limited in amount.

Glycomics Pipeline Development. Developing a glycomics sample pipeline requires advances in chemical processing, LC-MS data collection and glyco-informatics software to annotate the data. Human serum samples were used as a source for glycans because of serum's applicability to human disease detection and the diversity of glycans present. The sample preparation protocol includes global enzymatic release of glycans from serum and subsequent purification to a point where the sample is compatible with the mass spectrometer. High performance mass spectrometers are required for analysis (resolution above 40,000 and mass accuracy under 5 ppm) and stable LC platforms are used for separating the complex samples. Glycan data analysis is challenging because glycan structures branch and have several types of linkages between monosaccharide building blocks. A new set of tools and mass libraries are being developed in $\mathrm{CH}$ to annotate the glycans detected in an LC-MS dataset.

Recent developments in this pipeline involve the LC-MS data collection and data analysis portions of the pipeline.

Hypercarb graphitized carbon columns (30 cm length) were packed in house and used to separate glycans in $60 \mathrm{~min}$ per sample. Four columns were packed and implemented in the next-generation high performance LC-nanoelectrospray ionisation (ESI) cart. The next generation high performance LC cart cycles samples through the columns, which themselves cycle through loading, desalting, ESI-MS analysis, and reconditioning states. This four-column system allows

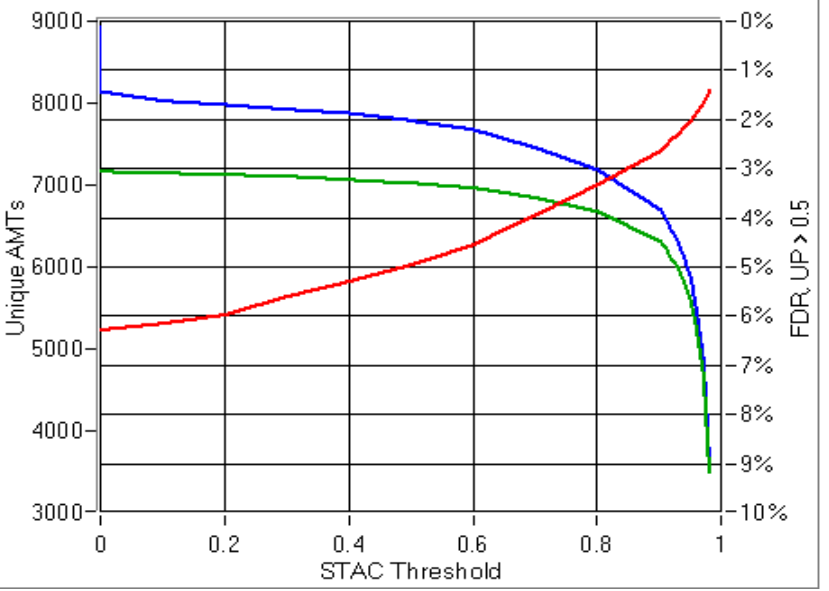

The plotted distribution of STAC scores (top line $=$ matches, middle $=U P$ filtered matches, bottom uptrend=FDR) for a quality control dataset from an LTQ-Orbitrap mass spectrometer. Lowering the STAC threshold increases the FDR slightly but allows for more peptide identifications. for continuous data collection and high-throughput processing. Human serum glycans were analyzed using the carbon columns and over 83 glycan compositions were detected. The columns also demonstrated a unique ability to separate glycan structural isomers and anomers. The glycan data was manually annotated using retrosynthetic glycan libraries and biological filter selection criteria.

Data preprocessing methods have been developed to aid in the automatic annotation of glycans. Synthetic glycan LC-MS spectra are used to model the system and evaluate the feature finding, deisotoping, and annotation algorithms. A new feature finding model is used to sum related spectra together to increase the signal-to-noise ratio. Results from this model or the existing in-house DeconTools platform will be supplied to glycan annotation functions that identify glycan masses in the dataset.

Computational and Informatics. We have integrated a new peptide identification scoring tool, the MS generating function, into the Pan-omics Research Information Storage and Management computational infrastructure, allowing us to compute a search tool agnostic score for peptide identifications from tandem MS data. MS-GF computes the probability that the theoretical ideal match for a MS/MS spectrum will have a higher score than the peptide match determined by the peptide ID software actually used. Results from SEQUEST, X!Tandem, and Inspect are all re-scored with MS-GF. These values are imported into the mass and time tag system when assembling AMT tag databases.

The data management system now generates quality assessment plots for each new dataset captured into the system. These plots include a two-dimensional ( $\mathrm{m} / \mathrm{z}$ vs. time) image and intensity vs. time chromatograms. These plots provide researchers with a high-level overview of the distribution of features detected in the LC-MS(/MS) datasets. Operators use this information to assure that instruments are working properly and to flag datasets that exhibit problems

(e.g., unusual distribution along the elution time axis, a low number of detected species, or a non-uniform intensity distribution).

Overall, new capabilities were developed to aid in the analysis and management of pan-omics data. These advances have been incorporated into our analysis pipeline to accommodate aggregation analysis. We were previously limited to the analysis of a single dataset at a time, but we now have the ability to automate new tools that require aggregation of datasets. The 
intial aggregation tool analyzes phosphopeptides identified across a series of related datasets to compute a phosphorylation site localization score based on the observed ions in the fragmention spectra. An additional advance to the informatics pipeline is the newly integrated capability to perform analyses using the output of a different analysis tool. This allows us to run just a single part of an analysis script using different parameters, allowing for comparison of the results as a function of analysis parameters. Finally, we developed a standard storage format and associated schema (SQLite) to accommodate the large amounts of data that are exchanged between collaborators and researchers. Each analysis tool in our pipeline will eventually be able to read and write in this standard SQLite format. 


\section{Higher-Throughput, More Sensitive Stable Isotope Probing}

Helen W. Kreuzer, M. Lizabeth Alexander, Brian H. Clowers, James F. Kelly, Jon K. Magnuson, James J. Moran, Robert L. Sams

The goal of this project is to develop more sensitive, higher-throughput stable isotope probing technology and apply it to a cellulose-degrading community as a first demonstration.

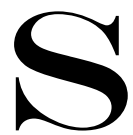
table isotope probing, an experimental approach that allows identification of microorganisms within a community that consume a particular substrate, has been a breakthrough technology that permits a cultureindependent association of function with phylogenetic groups. We propose to develop improved methods for stable isotope probing to enable both higher sample throughput and greater sensitivity. These improved methods would enable outcomes such as:

- The identification of key processes controlling biogeochemical cycles that sustain all life and build the knowledge base required to predict the role of microbial communities in mitigation and adaptation to global change and subsurface contamination.

- The development of new approaches to environmentally sustainable bioenergy systems and other industrial processes.

At present, the sensitivity of stable isotope probing is limited by the methodology, which selects stable isotopically labeled nucleic acids by buoyant density centrifugation. In order for a labeled molecule to separate from its unlabeled brethren, it must incorporate a significant percentage (e.g., 20\%) of the labeled atoms, which can obscure the detection of early incorporation events in a community. Our approach is to isolate ribosomal (r)RNA from a community exposed to a ${ }^{13} \mathrm{C}$-labeled substrate of interest, subject it to a sequence (phylogeny)-based separation, and measure the ${ }^{13} \mathrm{C}$ content of sub-populations of the rRNA directly. Direct measurement of the rRNA isotopic content will provide greatly enhanced sensitivity (less than $0.1 \%$ enrichment will be detectable), permitting the association of phylogenetic groups of micro-organisms with the earliest events in the breakdown of cellulose and enabling finer time-resolution analysis of the process. Further, our approach should greatly decrease the time required for analysis. Although we have selected cellulose degradation as the experimental process to dissect, the improved technology could be applied to any microbial community and process.

In late FY 2009 and FY 2010, we focused on developing new technology for $\mathrm{C}$ isotope ratios to decrease sample size requirements, important for the analysis of microbial communities. We developed the most sensitive laser ablationcombustion-isotope ratio mass spectrometry method to be described in the literature, reducing the sample size required for analysis. Posters about this technology presented at two scientific meetings garnered significant attention. A manuscript describing this technology has been submitted for publication in the scientific literature.

We developed and conducted initial experiments with capillary waveguide spectroscopy and showed that our system could measure isotopic content of $25-30$ pmol CO 2 , offering the potential for analyzing the $\mathrm{C}$ from a few microbial cells. In addition, we initiated development of capillary electrophoresis separation methods for rRNA and construction of a moving wire interface for introducing the electrophoresis eluant into an isotope ratio mass spectrometer (IRMS).

Progress on specific tasks included the following:

Moving wire interface. This task began in FY 2010 with major components of the system built. Continued work is planned for FY 2011.

Laser ablation-IRMS. During FYs 2009 and 2010, we successfully constructed and tested laser ablation-combustionIRMS. Measurement accuracy and precision were similar to standard techniques, and the sample size requirement was reduced (as noted above) nearly 1000 -fold, from about $50 \mu \mathrm{g}$ of $\mathrm{C}$ to $65 \mathrm{ng}$. Because of its high precision, the laser ablation sampling method can be used to distinguish metabolic pathways for lightly isotopically labeled carbon compounds and possibly natural abundance samples, making it superior to other single-cell isotope measurement techniques such as Raman spectroscopy. A manuscript describing this technology has been submitted to Rapid Communications in Mass Spectrometry.

Capillary waveguide spectroscopy. Significant progress was made, as we developed a capillary waveguide system to measure isotope ratios of $\mathrm{CO}_{2}$ with orders-of-magnitude greater sensitivity than has been previously reported.

A manuscript detailing the results is in preparation. This instrument could measure isotope ratios in about 30 pmol of $\mathrm{CO}_{2}$ with precision of about 1 permil, ample for detecting low levels of enrichment. In FY 2010, the system was integrated with the laser ablation unit and combustion reactor, and measurements were made on ablated, combusted solid samples. These preliminary measurements are very promising, as they were made on only $25 \mathrm{pmol} \mathrm{CO}_{2}$, suggesting that it might be possible to ablate a few microbial cells at a time and measure their $\mathrm{C}$ isotopic content. 
In FY 2011, we will complete the development of capillary electrophoretic methods for sequence-based separation of rRNA. As a test of our system, we will create mixtures of RNA from different microbes that have different $\mathrm{C}$ isotopic content, separate them by capillary electrophoresis, and collect fractions. The isotopic content of the fractions will be measured either by laser ablation-IRMS, by moving wire interface-IRMS, or both. These results will represent a significant advance in isotopic analysis of microbial communities and will be described in a publication in the scientific literature. 


\title{
Identification of Functional Proteins Relevant to Bioenergy and Disease Pathology by Multiplexed Activity-Based Protein Profiling
}

\author{
Aaron T. Wright, Jon K. Magnuson, Ellen A. Panisko, Sergey Stolyar
}

This project will develop a novel chemical proteomics technology, a multiplexed activity-based protein for rapidly profiling a myriad of functional enzyme activities from complex biological samples. The application of this method will improve understanding of disease pathogenesis and reveal new sources for bioenergy generation.

$\mathbf{T}$ o isolate, enrich, and characterize functional proteins in complex proteomes, activity-based protein profiling has emerged as a technology in the evolution of post-genomic proteomics. The goal of this capability is to apply chemical activity-based probes that monitor the functional activity of enzymes within the proteomes in which they are naturally expressed. To date, activity-based protein profiling has employed a strategy that uses a single probe per analysis to identify a single enzyme family and has depended on semi-quantitative protein identification. There is a critical need to transform the technology into multiplexed activity-based protein profiling: a high-resolution, high-sensitivity, next-generation mass spectrometry (MS) platform that will characterize and quantify functional enzymes from multiple enzyme families in their native proteome in a single experiment.

Our research will develop a quantitative multiplexed chemical proteomics technology for rapidly profiling functional enzymes from diverse and complex biological systems. We anticipate that this work will contribute to a better understanding of Mycobacterium tuberculosis and to an isolation of disease markers of invasive aspergillosis. Additionally, we will realize enzymes important to biofuels synthesis and lignocellulose degradation in microalgae and extremophilic fungi, respectively.

Our FY 2010 activities focused on integrating organic chemistry and MS-based proteomics to develop multiplexed activity-based protein profiling technology and to employ the technology in human health relevant research. The development of our chemical proteomics technology required four key elements: synthesis of chemical activity-based probes to target specific enzyme families, probe labeling of biological samples, sample preparation for analysis by MS, and data analysis. We used synthetic organic chemistry to prepare a chemical enzyme probe suite to target the following enzyme families: terpene synthases, cytochrome P450s, serine hydrolases, phosphatases, and glycoside hydrolases. Each probe was developed with a chemical handle, permitting the attachment of either a fluorescent tag or an enrichment moiety to the enzyme-probe covalent complex. Upon probe completion, biological sample labeling was optimized. Concentration studies were also performed to determine optimal enzyme labeling conditions by adding a probe to a sample followed by attachment of a fluorescent group and subsequent gel electrophoresis analysis to identify labeling.

The third key element was sample preparation for MS analysis. Following probe labeling, a biotin enrichment moiety was attached to the enzyme-probe complexes using copper catalysis. The excess synthetic materials added to the biological sample for labeling were removed, followed by enrichment of only probe-labeled enzymes, digestion of enzyme samples into their peptide forms, and cleanup of samples to remove salts and detergents incompatible with MS measurements. A key challenge was isolation of the specific protein peptide to which the probe adhered. This required the optimization of an acidic acetonitrile stripping wash while enriching probe-labeled samples, which proved suboptimal for MS measurements. After much effort, a method was determined to clean samples for optimal MS analysis. The final element of the chemical proteomics technology was data analysis. We worked with informatics experts to evaluate probe labeling data. In FY 2011, we will continue to optimize data analysis efforts specifically for isolation of probe-labeled peptides.

Using the multiplexed approach, we profiled serine protease and glycoside hydrolase enzymes in three strains of $M$. tuberculos is differing in virulence and drug resistance. We isolated 38 proteins using the serine hydrolase chemical probe, including identifying enzyme activities found only in single M. tuberculosis strains. This work will continue in FY 2011 with the labeling of glycoside hydrolases and cytochrome P450s. In other health applications, we will culture the fungal pathogen Aspergillus fumigatus and make preliminary labeling measurements with probes for serine hydrolases, phosphatases, and cytochrome P450s.

After establishing the multiplexed activity-based protein profiling technology and performing preliminary biological research in FY 2010, we will complete syntheses of kinase and cysteine protease probes in FY 2011. These are key enzymes in both our health and bioenergy research. Primary efforts will be completing work with $M$. tuberculosis, which should result in high-level publications, and increasing our effort with A. fumigatus to identify biomarkers of lung infection. We will also work to identify novel cellulose degrading enzymes and accessory enzymes in extremophilic fungi. These studies will demonstrate the utility and innovation of our novel chemical proteomics technology while leading to important health and bioenergy findings. 


\section{MicroCT Development for Multimodal Imaging in Systems Biology}

Kevin R. Minard, Richard E. Jacob

This project is focused on augmenting PNNL's multimodal imaging capabilities with a new, high-resolution $\mathrm{x}$-ray computed tomography system.

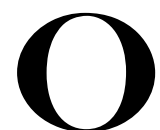
ngoing projects at PNNL that model airflow and particulate deposition require information about local pulmonary function throughout the breathing cycle. Existing capabilities are used to image small animals to detect how inhaled pollutants and/or drugs ultimately affect human health. Data include local tissue compliance (change in volume per change in pressure), tissue strain, conducting airway displacement, and dynamics of lobar boundaries; however, this information cannot be obtained with methods currently available at PNNL. Therefore, the goal of this work was to develop novel imaging capabilities that would enable the acquisition of these critical data.

Our project proposed to develop and implement new, non-invasive imaging capabilities. Though we lack the ability to visualize intricate structures (such as pulmonary tissue at high resolution [tens of microns]), this research will fill the gap by developing novel micro-CT imaging techniques. This system provides detailed three-dimensional images with isotropic spatial resolution as high as 25 microns, accommodates fairly large samples (diameters up to $8 \mathrm{~cm}$ ), and is computercontrolled for versatile data acquisitions.

To demonstrate the new microCT utility for systems biology, images were acquired to quantify lung mechanics in live animals. Generally, this supports ongoing laboratory efforts to develop three-dimensional computer models of inhaled airflow that will ultimately aid in the prediction of human health risks that might be associated with exposure to airborne materials encountered at DOE facilities, in the environment, or through threats posed by weapons and terrorist activities. Collected data generally describe local pulmonary geometry and function throughout the breathing cycle and provide a quantitative basis for improving model predictions through realistic descriptions of local tissue compliance (change in volume per change in pressure), tissue strain, conducting airway displacement, and the dynamics of lobar boundaries. By combining this new technology with existing imaging capabilities, PNNL now has a unique set of imaging tools for solving a wide variety of problems, ranging from materials science to geosciences and pedometrics.

Work began with the successful installation and testing of the x-ray micro-CT imaging system (GE eXplore CT120). A one-of-a-kind small animal ventilator (CWE Inc. model SAR-830/AP with customization) was also procured for controlling the complex breathing maneuvers needed for functional pulmonary imaging. This ventilator allows for breath hold at arbitrary points in the breathing cycle so that image data can be acquired without motional blurring. To facilitate use, a single computer controls the ventilator and a small animal monitoring unit (SA Instruments, Model 1025T), and all are housed on a cart for portability between different imaging systems. In addition, we designed and constructed a unique animal cradle that is compatible with magnetic resonance imaging, CT, and magnetic particle imaging (MPI). Generally, this will facilitate a true multi-modal imaging approach to data collection to support ongoing and future projects.

The result of this research will be to form an integrated, state-of-the-art, multi-modal imaging capability that combines MRI, MPI, and $\mu \mathrm{CT}$. Initially, results will be used to advance computational models of pulmonary airflow dynamics and particulate deposition. Once validated in laboratory animals, these models will provide a quantitative basis for predicting the impact of environmental exposure in human populations.

Overall, the successful integration and implementation of technologies from this project represent a major accomplishment and the establishment of a new capability at PNNL.

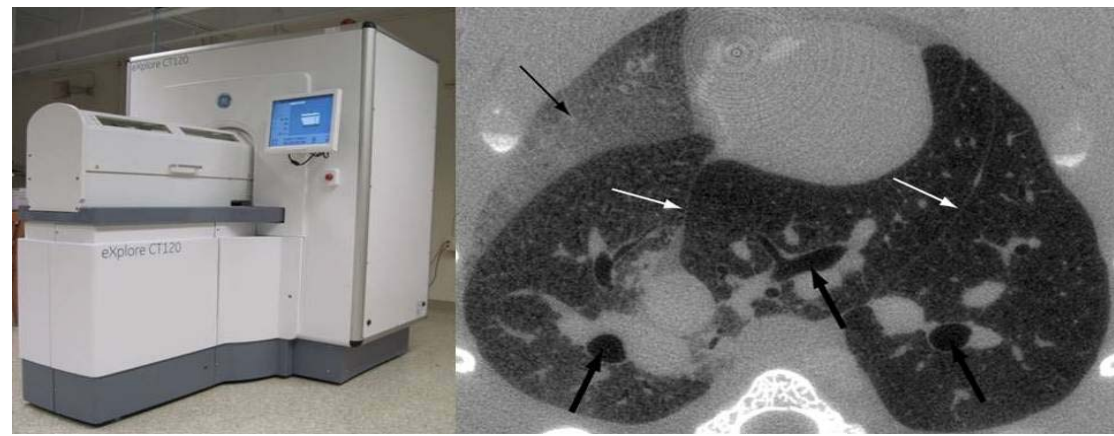

Left: New microCT at PNNL. Right: Typical image of rat lungs. Highlighted are major airways (bottom, heavy black arrows), lobar boundaries (white arrows), and fluid-filled pulmonary tissue (upper, think black arrow). Image resolution is $50 \mu \mathrm{m}$ 


\title{
Micro-Fluidic Models for Studying Microbial Communities-Integration of Micro-Fluidic Model Experimentation, Multimodal Imaging, and Modeling
}

\author{
Michael J. Wilkins, Jay W. Grate, Changyong Zhang, Haluk Resat, Galya Orr, Vanessa L. Bailey
}

An understanding of pore-scale microbial processes affecting rates of cellulose degradation in the subsurface is fundamental to our ability to predict and model aspects of global carbon cycle. To gain this understanding, we must develop a range of technologies that allow us to interrogate microbial communities degrading cellulose in a range of porous media types.

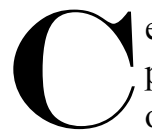

ellulose is the primary structural component of plants and the most common organic compound on Earth. The global carbon cycle is an interplay between carbon reservoirs in the atmosphere, oceans, subsurface, plants and soil, with microbial communities mediating many of the transformations between reservoirs. Within subsurface environments, cellulose degradation occurs within pore spaces where a range of advective and diffusive biogeochemical processes produces local microenvironments. These different environmental conditions can affect both the distribution of microbial communities within the subsurface and subsequent rates of cellulose degradation. Microfluidic models are small cells consisting of a series of vertical columns that act as grains, resulting in specific pore sizes and pore throats. Therefore, these microfluidic models are effective proxies for subsurface environments. In addition, they are engineered to enable the application of multiple imagingbased technologies to interrogate processes occurring at this pore scale in a non-destructive manner.

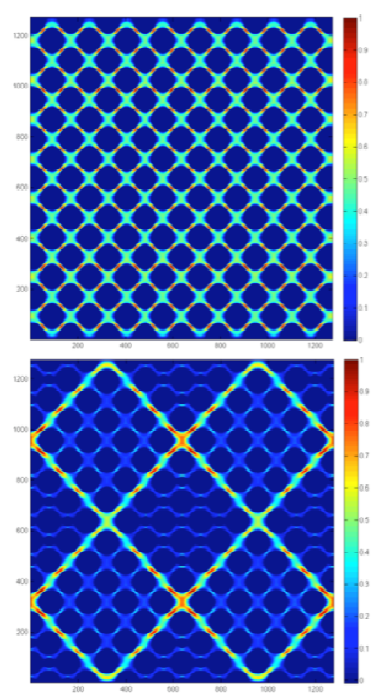

Microfluidic model pore designs illustrating the development of advection-dominated and diffusion-dominated regions. species-specific signatures in single cells. Our general efforts in this project are aimed at developing

- micro-fluidic models for studying the transformation of insoluble polymeric carbon by microbial communities

- multimodal nondestructive imaging of consumption of substrates and production of cells

- micro-fluidic models, multimodal imaging, and individual-based modeling of microbial community processes to improve mechanistic understanding of how complex environmental systems behave

- an improved understanding of how differences in habitat diversity and the spatial coupling of electron acceptor and (initially) insoluble electron donor affects rates of cellulose degradation and microbial community composition.

In FY 2010, our work focused

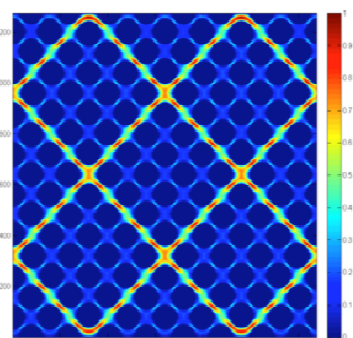
on micromodel design for subsequent experiments. A range of micromodel designs that vary in relative advection and diffusion processes have been modeled, demonstrating the ability to generate zones of variable flux and diffusion within the models. These micromodels have since been manufactured using the polymer PDMS. Work also progressed using a range of cellulose-degrading bacterial species to calculate batch kinetic parameters associated with cellulose degradation in pure culture (cell growth rate, cellulose degradation rate). These parameters are being generated for a specific modeling component in FY 2011.
This project will integrate the technologies arising from a number of other projects. Microcrystalline cellulose will be deposited within microfluidic models, and the subsequent microbial degradation imaged using novel fluorescence-based techniques. In addition, oxygen abundance and the development of microaerophilic zones within the pore structure will be imaged using novel oxygen-sensor probes. This imaging of microbial cells within the microfluidic model will incorporate the advances made by a project that aims to use the Raman detection of ${ }^{13} \mathrm{C}$ incorporation in single cells grown on cellulose and of

Research planned for FY 2011 will include the successful deposition of bioavailable cellulose within the manufactured microfluidic models and the introduction of bacterial species that are able to grow within these environments. These advances will allow an improved mechanistic understanding of how differences in habitat diversity affect rates of cellulose degradation and microbial community composition and will provide additional data for modeling efforts. We will work toward an integration of multimodal imaging (oxygen, cellulose, and Raman). 


\section{Microscale Spectroscopic Analyses of Cellulose Degradation and Uptake by a Microbial Community}

Vanessa L. Bailey, Nancy J. Hess

The acquisition and incorporation of cellulose-derived carbon into individual microorganisms and communities has not been directly observed in a measurable fashion. Complementary work at microscales will provide a foundation for understanding the acquisition and uptake of substrates by microorganisms using extracellular cellulytic enzymes in complex spatially organized systems.

$\mathbf{M}$ icrobial communities are difficult to study. They are a complex network of cells that interact to mediate a wide range of metabolic processes. They are often treated as "black boxes" in the environment where a substrate goes into the community and various products come out. However, there is little knowledge about how these transformations occur in a native, undefined community, whether there is a pattern or organization to the entry and assimilation of substrate or how it is respired. Despite the few species that unilaterally degrade cellulose completely, single cellulytic species will be used for simplicity to determine whether Raman spectroscopic techniques can sensitively and reproducibly track cellulose metabolism through microbial biomolecules at a scale equivalent to or more sensitively than reported elsewhere. Microenvironments resulting from overlapping gradients in physical and/or chemical properties often control the location of a given microbial activity, and we need to be able to map these locations spatially in fine detail.

We used surface-enhanced Raman spectroscopy (SERS) to develop spectra for single, live Escherichia coli suspended in minimal media with silver nanoparticles. The Raman signature observed was consistent with data in the literature for aromatic amino acids and nucleic acid bases previously observed for E. coli, including the identification of tryptophan, guanine, and adenine. The Raman signature appears to change as a function of time of exposure to silver nanoparticles. We collected data for other microbial species (Xylanimonas cellulosilytica and Streptomyces griseus) grown on the same media in order to discern whether the species can be differentiated by Raman spectroscopy. All species were grown using 12C-and $13 \mathrm{C}$-glucose to detect signatures of carbon uptake.

In FY 2009, we optimized spectral acquisition parameters and applied signature processing algorithms to enhance sensitivity. In the interim optimization of Raman, measurements focused on the fabrication of transmitted light capability to facilitate visualization of microbial cells in aqueous media and optimization of detector binning parameters. Building on this activity, the FY 2010 focus was to optimize Raman capability and to study individual microorganisms using E. coli as the model organism. The following details this year's project milestones.

Discrimination of different microbial species using SERS. SERS techniques distinguished between different microbial species. The magnitude of the signal varies on the proximity of the target cell to silver nanoparticles. SERS allows for rapid data collection $(1 \mathrm{sec})$, advantageous when studying live, moving cells. Normal Raman techniques (non-SERS) require greater analysis times $(1000 \mathrm{sec})$; however, we collected spectra for fixed cells that suggest SERS may not be required to differentiate species if cells are fixed through colonization to cellulose. SERS focuses on cell surface features, yet it discriminated between two different gram-positive bacteria. A species such as $S$. griseus presents particular challenges, as it has multiple growth forms with variations of Raman spectra during intervals between cell preparation and analysis. We are transitioning to other

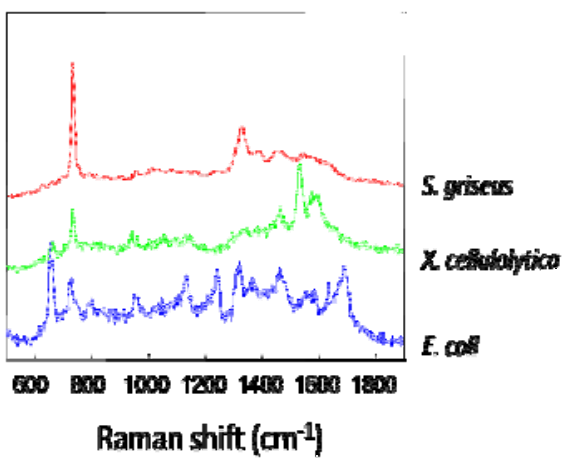

Raman spectra for three model microbial species E. coli, X. cellulosilytica, and S. griseus suspended in minimal media with silver nanoparticles. nonsporulating cellulytic microbial species for future studies. 13C uptake by different microbial species. SERS revealed 13C-glucose acquisition by microorganis $\mathrm{ms}$ in situ.
We anticipate that the use of $13 \mathrm{C}$-cellulose will allow us to develop a greater understanding of carbon use by cellulosedegrading microorganisms and potentially observe the production and secretion of cellulytic enzymes.

In FY 2011, we will continue to develop a library of spectra for our model cellulose-degrading organisms and test whether their spectra change as a result of changes in the primary growth media. The goal will be to use Raman spectroscopy to examine non-destructively microbial species colonizing a microfluidic chip (developed and run elsewhere). We anticipate that the spectroscopy will identify particular species colonizing target niches within the microfluidic platform and determine which (if any) species dominate colonization of the different niches. 


\section{Mining the Data from Research on Dogs Exposed to Internally-deposited Radionuclides}

William F. Morgan

In light of concerns of human exposures during cleanup of radiation-contaminated areas (e.g., the Hanford Site), the increasing use of radiation in medicine and industry, and the potential for dirty bombs and nuclear terrorism, we propose to analyze these studies to understand the potential health effects of radiation exposure in a large animal model system.

$\mathbf{L}$ ife span studies of beagle dogs were started in the early 1950 s to examine the effect of internally deposited radionuclides on a large animal. The studies were funded by the U.S. Atomic Energy Commission and later by the U.S. Department of Energy. The experiments, costing in excess of \$200 million, involved over 8000 dogs and lasted until 1993. The beagle was chosen because it has many positive characteristics, including an easy-going demeanor, optimum size, appropriate life span (mean of approximately 14 years), and physiological and anatomical similarities to humans. Additionally, this breed naturally exhibits similar cancers at the same frequency as those of humans. The aims of this project and research are 1) to relate the observed health effects (diseases and primary causes of death) to radionuclide, route of exposure, initial dose-rate, average lifetime dose-rate, and the total accumulated radiation dose at death and 2) determine the contribution of total dose, dose-rate, and dose distribution in the body on the induction of early acute effects, life shortening, and other health risks associated with radiation exposure, including cancer.

During FY 2010, we determined that it was feasible to mine the above data types for information on the effects of total dose, dose rate, route of incorporation, and specific isotope. The goal was to interrogate these data using new technologies in molecular biology available at PNNL and subsequently apply modern statistical methodologies for data analysis. To this end, we completed the analysis of dogs exposed to ${ }^{137}$ Cesium at Argonne National Laboratory and the Inhalation Toxicology Research Institute. The investigation involved determining the route of exposure (inhaled or ingested), dose, dose-rate, life span, and primary cause of death. This information also became the topic for a graduate student's Master of Science thesis.

In addition to our preliminary findings, we have almost completed our analysis of dogs exposed to ${ }^{60}$ Cobalt at Argonne, the University of California at Davis, and Colorado State University by determining the route of exposure, dose, dose-rate, life span, and primary cause of death. We also made significant progress toward the pedigree analysis of irradiated dogs in order to investigate potential genetic susceptibility to radiation health effects. We identified the primary cause of death and determined whether there is a genetic component to radiation-induced disease. We also invested considerable time and effort toward accomplishing this goal and, with the aid of PNNL investigators using programs that analyze high-throughput proteomic data, we devised protocols for determining pedigree analysis. Currently, we are studying potential genetic susceptibility in the dog population.

As we delved more into the dog studies, it became clear that there were differences in experimental protocols between the various institutions. To benefit all related research, we held a conference in Richland, Washington that involved a number of surviving investigators involved in "dog studies." This symposium revealed how valuable these studies are and where the data are stored, and provided an outline of future studies. A summary of this symposium has been submitted for publication to the journal Health Physics.

We plan to continue our research in FY 2011, focused on the following tasks:

- Compare data, some of which is available electronically, with the appropriate controls. We will verify the data quality at random by comparing electronic versions of the data with laboratory notebooks as well as medical and pathology reports. Data that is currently only available in hard copy will be entered into an electronic format so that all information is available to the scientific community as a whole.

- Determine the initial dose at exposure for individual dogs for each specific isotope or exposure condition. From there, we can determine the total exposure dose depending on the isotope half-life (i.e., decay time) and its retention in the body (i.e., how quickly it is excreted). We can then relate this to the location of the isotope in the body (i.e., whether it concentrated in the bones, liver, lungs, etc.) and the specific cause of death. In this way, we can relate dose and dose rate effects to cause of death.

- Initiate studies to build pedigree profiles of the dogs used in order to determine if related dogs died of similar disease. This information will make possible future studies to determine potential genetic susceptibility to radiation-induced cancers. 


\title{
Next Generation Software for Automated Structural Identification of Metabolites
}

\author{
Lars J. Kangas, Thomas O. Metz, Giorgis I. Mezengie, John H. Miller (Washington State University), \\ Bojana Ginovska, Brian T. Schrom
}

The goal of this project is to reduce the time and cost of biomarker discovery by developing a software product to enable the high throughput and accurate identification of metabolites and other small molecules. The successful development of the software will facilitate studies of fundamental biology and the discovery of disease biomarkers.

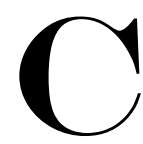
urrently, the structural identification of unknown metabolites detected in liquid chromatographymass spectrometry studies is based on manual interpretation of their tandem mass spectra, which is laborious and typically does not yield a complete identification. The inability to identify endogenous metabolites and other small molecules rapidly and globally has significantly limited the discovery of novel biomarkers of disease and drug toxicity in metabolomics studies. An algorithm that models the fragmentation pathways of molecules in mass spectrometry and generates in silico ion spectra for putative metabolites is required. With a large database of these spectra, unknown metabolites can be identified by comparing the observed spectra against the database.

The software product we propose is anticipated to be of substantial value to laboratories in the medical diagnostic, biotechnology, and pharmaceutical industries, as it will reduce the time and cost of elucidating mechanisms of disease pathogenesis and drug toxicity. In addition, the tool would be indispensible for biofuels research laboratories, as the highthroughput structural elucidation of metabolites could greatly facilitate bioengineering experiments that have as their goal the creation of microbiota that are more efficient in producing hydrogen or ethanol.

In FY 2010, we showed that identifying metabolites accurately, rapidly, and automatically is possible using a software algorithm. An early working version of the algorithm was completed, tested, and trained using spectra from 22 lipid species (metabolites). A set of 45 test lipids were purchased, analyzed, and then identified using the algorithm in conjunction with LIPID MAPS, a database with over 22,000 lipid structures provided by a consortium of lipidomics core laboratories. Of the 45 test lipids, 39 were positively identified, and 5 were identified as the second most likely identifications. The last lipid only made it to the middle of a short list of possible identifications.
The less-than-perfect identifications observed in the test of the algorithm are explained by the type of mass spectrometer used: a linear ion-trap, which does not provide spectral information separating ether and ester lipids (confusion between ether and ester lipids occurred for the 5 lipids identified by the algorithm as the second best match). The worst lipid identification was the result of the algorithm not yet having the ability to predict which molecule fragments are ions and which are neutrals (charged and non-charged). Overall, the test result was positive, solving a very difficult problem.

The algorithm test revealed two specific weaknesses for next year's research. First, it does not yet predict fragment charges, which is a problem that will grow with increasing databases (it is estimated that a complete metabolite database may have between 100,000 and 200,000 entries). Second, it was observed that if the accuracy of masses and structures of molecule fragments improved, identifications would be more accurate, specifically with large databases where the potential number of false positives is higher. This means that rearrangements have to be accounted for properly when molecules fragment in a mass spectrometer.

There are multiple goals and challenges for this project in FY 2011. We aim to:

- Develop a method to predict/calculate charge locations in molecules. The software algorithm generates in silico spectra; knowing charge locations will allow it to generate more accurate spectra, which will enable more accurate metabolite identifications.

- Develop a method to predict/calculate molecular rearrangements. A few known rearrangements described in the literature occur frequently when molecules fragment in mass spectrometers. We will research and implement these rearrangements.

- Incorporate additional mass spectrometers into the algorithm. Currently, the project has developed a model for one type of mass spectrometer, the linear ion-trap. Two other types (orbitrap and triple-q) are considered for implementation. The advantage with these mass spectrometers is that they provide spectra with better mass resolutions and more comprehensive fragment information, respectively, for more accurate identifications of metabolites. 
- Incorporate handling of other adducts in the algorithm. Some metabolites are observed together with an additional small molecule, an adduct, in a mass spectrometer. These adducts have to be accounted for if possible when analyzing spectra. Preliminary research suggests that local charges need to be calculated correctly to predict the affinity between the molecules and the adducts, including the specific site on each molecule that attract the other.
- Test algorithm with other metabolites than lipids, and parallelize software code, which would benefit the speed of the software algorithm. 


\title{
Proteomics Measurements of Functional Redundancy and Stability Testing of Cellulose Degrading Anaerobic Microbial Communities Within Engineered Bioreactors
}

\author{
Stephen J. Callister, Michael J. Wilkins, Brian L. LaMarche, Aaron T. Wright
}

Measuring the redundancy of cellulose degrading enzymes within a microbial community is fundamental to understanding the stability of this process for the development of second-generation biofuels.

$\mathbf{I}$ $\mathrm{n}$ nature, an important service provided by microbial communities is the breakdown of cellulosic biomass. Understanding how microbial communities perform this process improves our knowledge about how biofuels are produced. Unlike a single microorganism, microbial communities work synergistically, often because multiple populations within the community can perform the same process. The number of enzyme catalysts capable of carrying out the same function but produced by different populations in the community is known as functional redundancy. The general belief is that functional redundancy is critical to the stability of important processes in nature, such as cellulose degradation. Given an environmental upset, functional redundancy acts as a "buffer," allowing the community to continue to degrade cellulose.

The role of functional redundancy within a microbial community is fundamental to

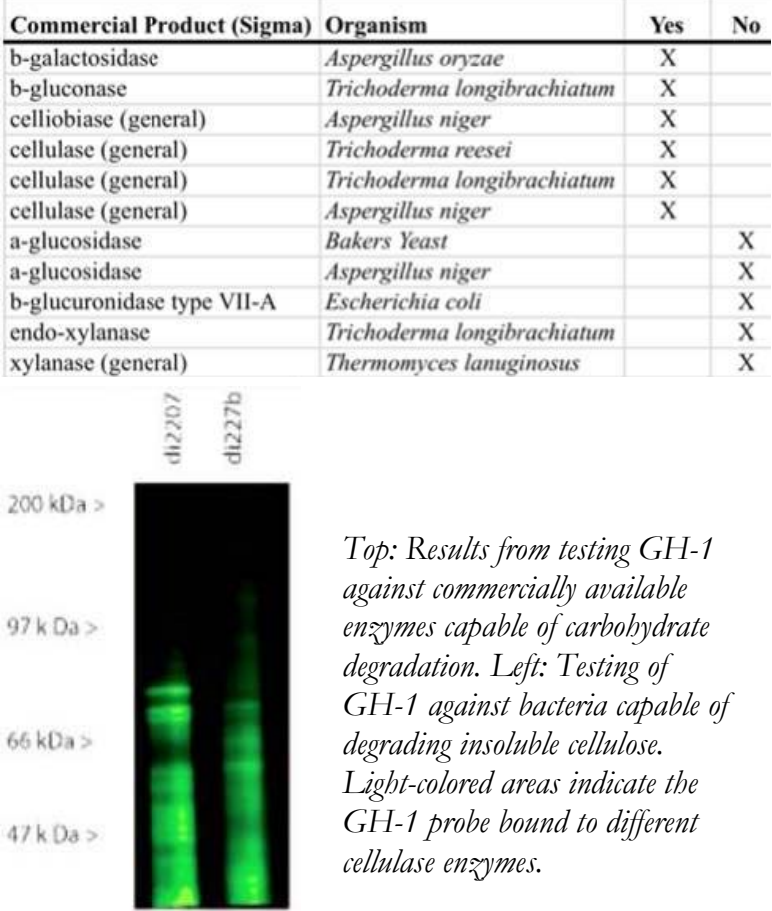
housed in bioreactors capable of degrading cellulose. These measurements enable us to correlate the change in redundancy to cellulose degradation under sudden environmental upsets. Functional redundancy measurements are performed via chemical probes developed to imitate cellulose and irreversibly bind to the enzymes capable of degrading this substance. By placing a probe in a complex mixture of proteins harvested from a microbial community, cellulose-degrading enzymes that bind to the chemical probes are extracted and analyzed using mass spectrometry.

Six chemical probes were designed, with the first probe (GH-1) synthesized. While the first probe design is not original, working out the synthesis steps to make this probe was challenging and represents a breakthrough that will enable the synthesis of the remaining five probes. Initial testing of the first probe against commercially available enzymes shows that it is specific to enzymes capable of breaking $\beta$-glycosidic bonds (that connect sugar [glucose] molecules together in cellulose) and has a high affinity for cellulose, as opposed to other carbohydrates. The probe was used to identify cellulose-degrading enzymes within bacteria recently isolated from a leaf cutter ant dump pile. This research is being conducted in collaboration with the University of Wisconsin.

Research planned for FY 2011 includes synthesizing and testing the remaining cellulase probes against positive controls to confirm their specificity and assess sensitivity. Two anaerobic cellulose-degrading bioreactors will be established in our laboratory: one seeded from a microbial community sample collected from the cow rumen, and the other seeded from a microbial community used to break down manure. The reactors will be monitored for their ability to maintain a steady-state operation. We will also finalize the bioinformatics tools necessary for analyzing functional redundancy measurements, which will allow us to correlate the functional changes in response to environmental upsets.
We are developing methods to measure the amount of functional redundancy associated with microbial communities 


\title{
Synthetic Biology Approach for Hydrocarbon Production in Microbial Photoautotrophs
}

\author{
Alex S. Beliaev, Aaron Wright, Donald Bryant (Penn State University)
}

Through the identification of pathways, genes, and proteins, this project will assess the means to apply genetic and metabolic manipulations to increase hydrocarbon production from sunlight and carbon dioxide. Successful implementation of this research will open new perspectives and provide a platform to design efficient, cost-effective processes for biofuel production.

$\mathbf{U}$ sing solar energy to grow photosynthetic microorganisms is one of the most attractive ways to produce transportation fuels. Among the species of microalgae considered for biofuel production is Botryococcus braunii race B $(B b B)$, a green colonial microalga isolated from a variety of habitats, including freshwater and brackish lakes, reservoirs, ponds, or even ephemeral lakes. This organism has considerable potential for producing lipids and liquid hydrocarbons that can constitute a large fraction of the total biomass. The typical hydrocarbon content of photosynthetically grown $B b B$ cells averages approximately $30 \%$ to $40 \%$ of the dry cell mass. Hydrocarbons extracted from $B b B$ biomass can be readily converted to fuels such as gasoline and diesel by catalytic cracking or thermochemical liquification. While enhancing hydrocarbon production directly in $B$. braunii presents formidable challenges due to low growth rates, undeveloped genetic system, and lack of robust cultivation techniques, availability of genetic and metabolic engineering tools allow for the exploration of other photosynthetic organisms for hydrocarbon (triterpenoid) biosynthesis.

This project uses a synthetic biology approach that will allow reconstructing hydrocarbon biosynthesis in the fastgrowing, genetically manipulable cyanobacterial strain Synechococcus PCC 7002 through optimization of genetic and metabolic controls within the host. Research goals focus on understanding the growth physiology and triterpenoid biosynthesis in $B b B$, isolation and identification of genes involved in isoprenoid metabolism, and conceptual development of synthetic biology approaches required for successful engineering of microbial photoautotrophs (cyanobacteria) for biofuel production. The project also embodies technical objectives, including development of continuous cultivation approaches, biochemical assays, and genetic methods for studying the isoprenoid metabolism that could be adapted for increased hydrocarbon biosynthesis through metabolic engineering. As slow growth and microbial contaminants present major hurdles for the exploration of the biofuel potential of $B b B$, we have focused efforts on identifying optimal cultivation conditions and factors that inhibit growth of the organism.

During the first two years of this project, we optimized the photoautotrophic growth of $B b B$ to where cells grew with doubling times of about 48 hours and displayed an average density of $0.5 \mathrm{~g}$ DW/L biomass. We also optimized mass transfer in continuous cultures of $B b B$ to enhance substrate (carbon dioxide) delivery and byproduct (oxygen) removal. After accumulating copious amounts of extracellular material that encapsulate $B b B$ cells and predominantly consist of secreted hydrocarbons, we learned that gas diffusion inside and out of the cell represents a key factor in controlling organism growth. In addition, we developed methods for biomass collection using differential centrifugation that allow for efficient separation of $B b B$ and bacterial cells. Large samples of hydrocarbon-producing $\mathrm{BbB}$ cells were generated for downstream expression as well as genetic and biochemical studies to identify genes involved in the final reaction of triterpenoid biosynthesis in $B b B$. These experiments are currently underway.

To design and construct synthetic hydrocarbon-producing pathways, we are employing a combination of comparative genomic, genetic, and proteomic approaches. This objective consists of two distinct tasks: assembly of functional isoprenoid pathways for precursor molecule biosynthesis and identification and isolation of $B b B$ genes encoding triterpenoid (i.e., botryococcene and squalene) synthases involved in the final step of hydrocarbon production. In the first task, we used genome sequence and context analyses to identify genes involved in isoprenoid biosynthesis in a wide range of green algae and cyanobacteria. An entire isoprenoid biosyntehsis gene cluster (total of 7 genes) was isolated from a unicellular diazotrophic cyanobacterium (Cyanothece sp. ATCC 51142) and cloned into a subset of expression vectors for functional and biochemical characterization. Enzymatic activity assays are being carried out to validate the predicted physiological function of the putative gene products. Upon successful validation, the entire isoprenoid biosynthetic pathway will be assembled in Synechococcus PCC 7002 and used for metabolic engineering studies.

The second task is conducted in collaboration with another LDRD effort to develop activity-based mass spectrometry approach for studying protein function. The design and synthesis of farnesyl diphosphate analogous probes to test and identify triterpenoid synthases in $B b B$ were initiated during the fiscal year. 
To achieve the metabolic engineering objective, we are working to develop a suitable host system that can be used for the efficient expression of a hydrocarbon-producing pathway. Our organism of choice is Synechococcus PCC 7002, for which a robust genetic system is available and a genome-wide metabolic model is being developed in collaboration with Pennsylvania State University. The metabolic reconstruction of pathways leading to production of farnesyl-PP, a key precursor for hydrocarbon biosynthesis, has been accomplished in 7002 .
Our approach for engineering the hydrocarbon-producing strain will be based on increasing the carbon flux through the isoprenoid biosynthesis (non-mevalonate) pathway and directing it toward the production of $\mathrm{C}_{30}$ triterpenoids using squalene/botryococcene synthases. The bottleneck for the pathway will be identified using metabolomics and mutant strains. The successful implementation of our tasks will open new perspectives for designing efficient, cost-effective processes for biofuel production. Additionally, this research will provide a novel platform for developing consolidated bio-processing methods, leading to production of carbonneutral energy at reduced economic and energetic costs. 


\section{Chemistry}




\title{
A Real-Time Optical Spectroscopy Platform for Investigating Molecular Mineral Transformations for $\mathrm{CO}_{2}$ Storage
}

\author{
Zheming Wang, John S. Loring, Christopher J. Thompson, Alan G. Joly
}

In this project, we are developing and demonstrating an in situ optical spectroscopy platform for investigating the molecular mineral transformation processes involved in carbon capture and sequestration (CCS).

$\mathbf{T}$ he combustion of fossil fuels such as oil and coal has led to a rapid increase of $\mathrm{CO}_{2}$ emission into the earth's atmosphere, which greatly contributes to global warming and causes adverse climatic and environmental changes. With global energy demand projected to increase by 57 percent by 2030, large-scale green house gas emissions will continue and even accelerate. Carbon capture and sequestration in oceans and deep geological formations such as underground saline formations, unused oil wells, and mining seams has become one of the most promising strategies to store the freshly generated $\mathrm{CO}_{2}$. Because of the expected high temperature and pressure present in deep geological "vaults," $\mathrm{CO}_{2}$ is expected to exist as a supercritical fluid. The stored supercritical (sc) $\mathrm{CO}_{2}$ is predicted to remain for hundreds of years as long as the caprock remains intact. For the duration of storage, $\mathrm{scCO}_{2}$ will interact with saline solutions and electrolytes in the liquid phase and is expected to facilitate significant mineral transformation. The nature and amount of the resulting secondary minerals and the extent and rate of $\mathrm{CO}_{2}$ conversion are influenced by many factors including temperature, pressure, the amount of water in the $\mathrm{scCO}_{2}$, surface hydration, liquid flow rate, mineral composition, $\mathrm{pH}$, phase separation and formation, surface reaction site, chemical composition, and interfacial diffusion.

Unfortunately, little is known about the physical and chemical processes that occur with $\mathrm{scCO}_{2}$ and water at solid-liquid and liquid-liquid interfaces. The available thermodynamic database includes mostly aqueous phase phenomena with a limited amount of organic solvent data. Moreover, the scientific community lacks the necessary experimental infrastructure to address these complex issues, at either a process level or molecular level. To address these deficiencies, we will develop an integrated supercritical fluid-optical spectroscopy system, including ultravioletvisible absorption spectroscopy, laser-induced time-resolved fluorescence spectroscopy, Fourier transform infrared spectrometry (FTIR), Raman, and nonlinear optical spectroscopy (second harmonic generation and sum frequency generation). The developed system will be capable of performing in situ optical spectroscopy measurements of mineral dehydration, dissolution, formation, sorption and desorption, and characterization of the solid-liquid and liquid-liquid interfacial processes at temperature and pressure conditions relevant to CCS.

For project progress thus far, we have developed two prototype supercritical fluid manipulation systems capable of optical spectroscopy measurements. The first is a dedicated system for performing UV-visible absorption and transmission mode FTIR measurements. The second system is a mobile platform that can be easily integrated with FTIR, Raman, fluorescence, SFG/SHG and microscopy. Both platforms can operate from room temperature to $75^{\circ} \mathrm{C}$, pressure up to $274 \mathrm{bar}$, and with features such as water vessel inline/offline, $\mathrm{scCO}_{2}$ online dehydration, thermostatted reaction/observation cell, $\mathrm{scCO}_{2}$ circulation as well as thermostatted $\mathrm{scCO}_{2}$ circulation lines. We have also developed separate reaction vessels (also serve as sample observations cells): one is designed for attenuated total reflection FTIR measurement and the other a prototype of reflective model optical spectroscopic measurement.

Using the developed $\mathrm{scCO}_{2}$-IR spectroscopic system, we investigated $\mathrm{H}_{2} \mathrm{O} / \mathrm{D}_{2} \mathrm{O}$ dissolution and speciation in $\mathrm{scCO}_{2}$. The results showed that $\mathrm{H}_{2} \mathrm{O}$ and $\mathrm{D}_{2} \mathrm{O}$ have unique infrared spectral features over a broad spectral range in $\mathrm{scCO}_{2}$ and the spectral intensity is proportional to the concentration of $\mathrm{H}_{2} \mathrm{O} / \mathrm{D}_{2} \mathrm{O}$, allowing in situ $\mathrm{H}_{2} \mathrm{O} / \mathrm{D}_{2} \mathrm{O}$ quantification in $\mathrm{scCO}_{2}$. The dissolution kinetics depend on $\left[\mathrm{H}_{2} \mathrm{O} / \mathrm{D}_{2} \mathrm{O}\right]$ in $\mathrm{scCO}_{2}$. The measured solubility of water is consistent with those reported in the literature. There were clear spectral difference between monomeric dissolved water and the bulk water. Detailed analysis of the $\mathrm{H}_{2} \mathrm{O} / \mathrm{D}_{2} \mathrm{O}$ spectra will help reveal mechanism of water interaction with $\mathrm{scCO}_{2}$.

Methods were designed and tested for making minerals (forsterite) thin films for infrared spectroscopic measurements in $\mathrm{scCO}_{2}$, including pressed powder flakes of ground $\mathrm{Mg}_{2} \mathrm{SiO}_{4}$ using a hand press and mostly opaque slurry film prepared using ground $\mathrm{Mg}_{2} \mathrm{SiO}_{4}$ and $\mathrm{D}_{2} \mathrm{O}$. The slurry was applied directly to $\mathrm{ZnSe}$ cell window and dried under $\mathrm{N}_{2}$. Spectral analysis of the thin films prepared showed spectra similar to published spectra of $\mathrm{Mg}_{2} \mathrm{SiO}_{4}$, demonstrating the appropriateness of the techniques.

We employed in situ mid-infrared spectroscopy to follow the reaction of forsterite for $24 \mathrm{hr}$ with wet $\mathrm{scCO}_{2}$ at $50^{\circ} \mathrm{C}$ and180 atm, using water concentrations corresponding with $0,55,95$, and 136 percent saturation. Results show a dramatic dependence of reactivity on water concentration and the presence of liquid water on the forsterite particles. Exposure to neat $\mathrm{scCO}_{2}$ showed no detectable carbonation reaction. At 
55 and 95 percent water saturation, a liquid-like thin water film was detected on the forsterite particles; less than 1 percent of the forsterite transformed, mostly within the first $3 \mathrm{hrs}$ of exposure to the fluid. At 136 percent, where an (excess) liquid water film approximately several nanometers thick was intentionally condensed on the forsterite, the carbonation reaction proceeded continuously for $24 \mathrm{hrs}$ with approximately 10 to 15 percent transformation. Our collective results suggest constitutive links between water concentration, water film formation, reaction rate and extent, and reaction products in wet $\mathrm{scCO}_{2}$. Preliminary results have indicated that the technique and procedures we developed for forsterite research can be also applied to study the reaction of other metal silicates, such lizardite and antigorite, in $\mathrm{scCO}_{2}$
In FY 2011, we plan to complete the development of the new $\mathrm{scCO}_{2}$ attenuated total reflectance FTIR system and use this technique for the investigation of interfacial reactions on forsterite, lizardite, and antigorite exposed to $\mathrm{scCO}_{2}$. We will also complete the design/modification of a reaction cell capable of in situ study of molecular processes using non-linear optical spectroscopy. Further, we shall conduct research to compare the reactivity of forsterite, lizardite and antigorite at different P-T levels and water concentrations in $\mathrm{scCO}_{2}$. 


\title{
Biochemistry of Glycan Signatures: A Novel Approach to Sensor and Vaccine Development
}

\author{
James A. Campbell, Catherine E. Petersen, George T. Bonheyo, Luke C. Short
}

This research aims to develop sensors that can distinguish real biological threats from environmental background and the development of antibiotics and vaccines to combat bacterial diseases.

$\mathbf{F}$ or many years, sensor research has focused on the nucleic acid and protein signatures of biological warfare-relevant species, which are well characterized and easy to work with in the laboratory. However, nucleic acids and proteins are subject to rapid degradation and chemical transformation in the environment. They are susceptible to chemical, thermal, or other physical degradation in production facilities; often require extensive sample preparation and cleanup procedures; and are exposed to direct manipulation and masking through genetic engineering. In contrast, carbohydrate signatures (glycolipids and glycoproteins) have been underutilized and are likely to provide advantages of enhanced discriminatory properties and greater stability and concentration in collected samples.

We propose a novel chemical differential separation technique to isolate and identify bacterial carbohydrate (glycan) signatures that discriminate Brucella ovis from closely related species. We will employ a novel glycomic differential display to isolate and identify glycan signatures that discriminate closely related starins of $B$. ovis from each other and congener species. Development of this separation technique and identification of signatures will be used as an improved target for detecting live biological warfare agents or residual traces of such agents indicative of recent or historical production. The identification and structural determination of unique glycans will be performed using mass spectrometry techniques, including MS/MS and accurate mass measurements. Finally, the stability of the identified signatures will be evaluated. The unique glycans could be used as signatures to identify target species, and the isolated glycans could potentially develop highly specific antibodies or new vaccines.

To date, we have successfully developed a process that rapidly cleaves glycans from the membrane glycoproteins associated with Gram-negative bacteria and allows for subsequent analysis by mass spectrometry. Using a single glycoprotein standard, we have verified, both with protein assays and ion trap spectrometer that the protocol in place is working. This developed protocol reduces sample preparation time from three days for the typical enzymatic workflow down to between 3 and 4 hours.
The final samples for this project were prepared at the Marine Sciences Laboratory at Sequim, Washington. This sample set will allow us to demonstrate that our process works on complex biological samples and will also allow us to compare the mass spectrometry results of the near neighbor microorganisms. This process will enable us to examine further the differences in the spectra with high resolution spectrometry in order to obtain structural information on significant and unique glycan features. The set will contain membrane material from Brucella ovis, B. neotomae, and Escherichia coli.

B. ovis and E. coli were cultured and processed to recover the total membrane material. ${ }^{1}$ We originally planned to work with only the outer membrane material from Gram-negative species, but we will be starting work with total (inner and outer) membrane material for these experiments. The protocol was specifically developed for the separation of glycoproteins from the outer membrane fraction because the success of the sucrose gradient method for separation of the inner and outer membranes was based on an assumption that the Brucella species would behave similarly to Bacteroides spp. Instead, method development will need to occur to find the best gradient for the Brucella with which we plan to work.

In addition, we have applied a permethylation procedure to derivatize carbohydrates. This allows for more fragmentation in collisionally induced dissociation MS/MS analyses. This provides more informative product ions for structural characterization and is extremely important for the study of carbohydrate isomers. The three spectra show peaks at $\mathrm{m} / \mathrm{z} 697$ which represents the $(\mathrm{M}+\mathrm{Na})$ ion. MS/MS spectra of $\mathrm{m} / \mathrm{z} 697$ show ions at $\mathrm{m} / \mathrm{z} 479$ and 445 . MS 3 spectra of $\mathrm{m} / \mathrm{z} 445$ show ions at 329 and 259. In short, differences are becoming evident in the spectra.

Future studies will include the use of chips packed with graphitized carbon and other chips with N-glycan cleaving enzymes. ${ }^{2}$ This will provide increased savings on consumables and will be less labor-intensive.

\footnotetext{
${ }^{1}$ This material was confirmed dead prior to shipment and will be re-certified as such at the Richland, Washington campus and downgraded to a BSL1 category before any subsequent sample processing takes place. Our proposed protocols for the BSL1 work including the glycan cleavage process and the instrumental analysis have been reviewed and approved. We are still waiting for the other strain of Brucella (B. neotomae) to arrive.

${ }^{2}$ We are presently collaborating with Agilent to use prototype glycan chips.
} 


\title{
$\mathrm{CO}_{2}$ Separation Scale-Up
}

\author{
Dale A. King, Kriston P. Brooks, Feng (Richard) Zheng
}

The objectives of this project are to develop a flexible, modular, and mobile $\mathrm{CO}_{2}$ capture material test capability and to evaluate the performance of novel PNNL capture materials against the goal of 20 percent reduction in energy consumption for flue gas $\mathrm{CO}_{2}$ capture relative to conventional baseline materials.

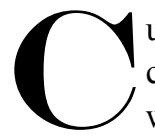
urrently available technical approaches for $\mathrm{CO}_{2}$ capture include pressure swing adsorption (typically with liquid or methanol based solvents) and capture of $\mathrm{CO}_{2}$ by liquid adsorbers such as monoethanolamine (MEA). These technologies for $\mathrm{CO}_{2}$ capture operate at room temperature or below, are costly, and require substantial swings in temperature and pressure. The high cost results from two key factors: the low mass fluxes in the separation units and the high energy consumption during regeneration of the separation agent. For non-pressure swing adsorption approaches, the cost of the separation agent (notably MEA), its operating life and selectivity, and the complexity of the MEA process are also important factors in making any process economically attractive. New technologies that can enable separate or simultaneous economic removal of $\mathrm{CO}_{2}, \mathrm{H}_{2} \mathrm{~S}$ (and $\mathrm{COS}$ ), and $\mathrm{Hg}$ over a range of temperatures, from room temperature to temperatures compatible with the shift reactors, can improve the economic viability of coal-based electricity and fuels in the United States.

A primary drawback applicable for the ability to develop new, cost-effective means of $\mathrm{CO}_{2}$ capture quickly from fossil fuel sources is the limited capabilities for testing materials under a relevant operating regime, including representative impacts on material attrition and performance over life. Developing capabilities for the most promising $\mathrm{CO}_{2}$ capture technologies and testing them on a larger scale in a $\mathrm{CO}_{2}$

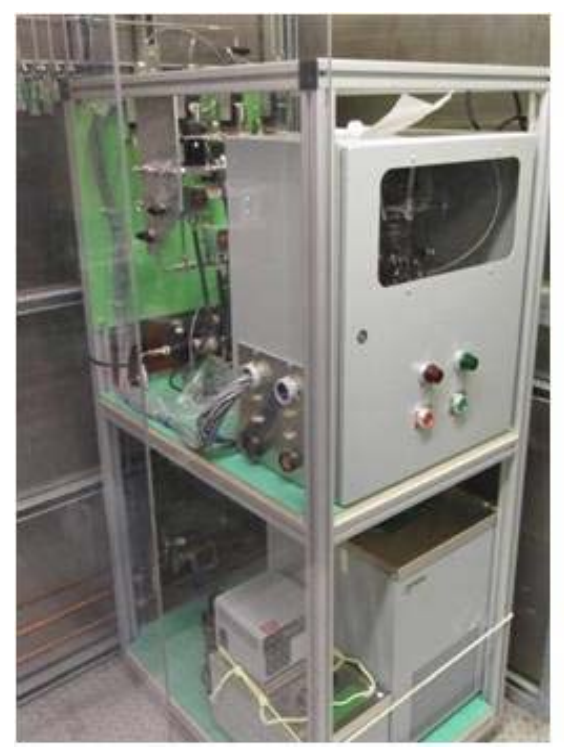

Left: Gas supply and conditioning cart; right: wetted wall column test cart. solid phase adsorption. The liquid phase processes can be distinguished by the method of regeneration - thermal or pressure swing versus electrochemical. For post-combustion capture, which is currently the highest priority, MEA can be used as a baseline technology against which the liquid phase LDRD $\mathrm{CO}_{2}$ capture projects will be compared. We envision that a continuous process should have the ability to be demonstrated that has at least a 20 percent energy savings relative to the MEA process and operating the system so that at least 90 percent $\mathrm{CO}_{2}$ is captured throughout the duration of the absorption test. For the case of solid adsorbents, the performance baseline standard is zeolite $13 \mathrm{X}$, which as a 20 percent energy saving for the novel adsorbent processes relative to this standard and 90 percent $\mathrm{CO}_{2}$ uptake over the duration of the adsorption cycle.

The $\mathrm{CO}_{2}$ capture test capability developed under this project in 2010 consists of three primary test systems: a wetted wall column, a continuous-scale solid sorbent test cart, and a continuous-scale liquid solvent test cart. All three capabilities are applied in the context of a $\mathrm{CO}_{2}$ capture material screening methodology to evaluate the viability of material candidates using progressively more detailed performance data and more representative operating conditions. This set of test hardware and methods brings a unique capability for testing materials in larger quantities and at increased level as material development progresses toward engineering-scale testing.

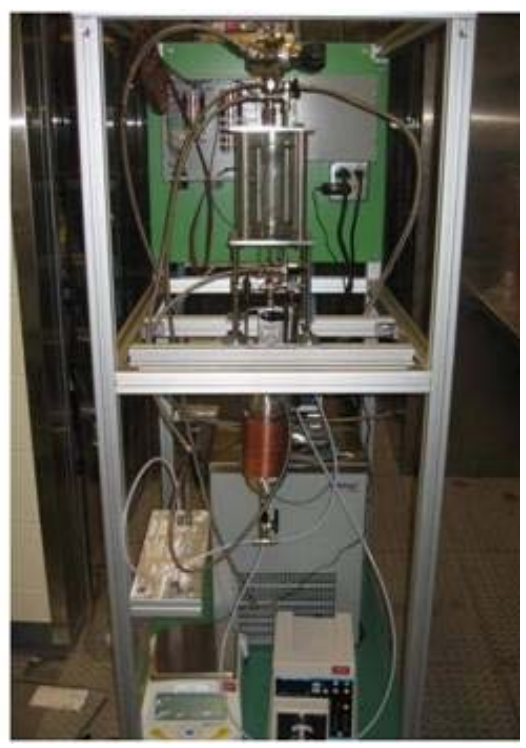

Early in the development and selection of liquid solvents, more fundamental material parameters such as reaction kinetics and $\mathrm{CO}_{2}$ loading capacities are evaluated using the wetted wall column. Similar methods (developed separately) are applied to solid sorbents for fundamental material characterization. Following initial screening, and once higher quantities of materials are available, the continuous-scale test carts enable characterization under industrially relevant operating conditions and through large numbers of loading/regeneration cycles.
There are several different $\mathrm{CO}_{2}$ capture technologies under development that are primarily differentiated by liquid or 
During testing on the wetted wall column, solvent flows downward along the circumference of a vertical metal column with CO2-containing gas flowing upward around the column. This apparatus enables a very well-controlled interface area between the solvent and the gas. By monitoring gas composition changes after flowing past the wetted column perimeter, relationships describing sorption kinetics can be developed. Following design, construction, and startup of the wetted wall cart in FY 2010, it was used to characterize the performance of a proprietary enzyme/ammonia capture system.

The continuous-scale solid test cart consists of two reactors that are filled with a material candidate in a packed-bed configuration. Inlet gas composition simulates a coal flue gas through the use of a set of bottled gases, mass flow controllers, and a gas saturator. A mass spectrometer is used to sample the composition of the reactor outlet continuously to enable characterization of loading profile and breakthrough characteristics. Reactor beds can be regenerated using thermal swing or pressure swing approaches, or a combination of the two. The two-bed approach allows us to explore configurations expected to be necessary to achieve the anticipated $\mathrm{CO}_{2}$ capture and purity levels requirements. In addition to gas compositions, bed temperature profile and pressure drop are monitored through the loading and regeneration steps. After completing the solid cart in FY 2010, it was used to characterize performance of zeolite $13 \mathrm{X}$ as a baseline data set.

For liquid solvents, the continuous-scale solvent test cart includes both contactor and stripping columns. Liquid sorbent candidates are routed through the contactor to absorb $\mathrm{CO}_{2}$ from a simulated flue gas. Solvent can be continuously loaded by circulating through the contactor or can be routed through a heat recuperator and then through the $\mathrm{CO}_{2}$ stripping column, as would be done in a full-scale process. Trim heaters and coolers are incorporated at the inlets of both columns for precise control over operating conditions. The stripping column has a reboiler for desorption of $\mathrm{CO}_{2}$ from the solvent as well as a condenser at the top of the stripping column to remove solvent from the $\mathrm{CO}_{2}$ stream prior to exhaust. As with the solid cart, performance of the material and process are monitored via mass spectrometer analysis of the product gas streams from both columns. The solvent cart is in the final stages of assembly at the end of FY 2010.
Work is continuing into FY 2011 and will include activities to refine the test systems further, develop a combustion gas platform, continue test method development, continue performance testing of candidate materials, and conduct off-site testing of material candidates on real flue gas.

Test system refinement will include limited retrofit as necessary to extend the capability of the test carts to the higher pressure requirements of pre-combustion (syngas) testing, and address any operational issues identified in preliminary post-combustion testing in FY 2010. In parallel, the combustion gas platform development task will provide a capability for reliable production and availability of post-combustion flue gas and any cleaning required to satisfy capture material test requirements. Flue gas sources include a $200,000 \mathrm{btu} / \mathrm{hr}$ flue gas-producing pulverized coal combustion unit and an integrated flue gas sampling facility located at a commercial pulverized coal power plant. The pulverized coal combustion unit is located at PNNL, and the gas sampling facility is envisioned to pull from the flue gas stack at the pulverized coal plant near Boardman, Oregon.

To establish the test carts as a flexible and capable test facility for assessing the $\mathrm{CO}_{2}$ capture performance of a wide variety of materials, a comprehensive test program will be executed. A set of test methods will be developed from experience and data collected over the course of this testing effort. Work will include experimental uncertainty analyses and development of test sequences and limits to minimize variability. After developing a set of test methods and procedures in the laboratory using a mixture of bottled gases as simulated flue gas, the test carts will be coupled with the bituminous coal combustor developed under this project and capture material performance will be compared to earlier simulated flue gas results.

After characterizing $\mathrm{CO}_{2}$ capture material performance using real combustion flue gas on site at PNNL, the test hardware will be transported and tested offsite at one or more flue gas sources. A key element of this effort will be collaborative test method development with industrial and DOE partners at the respective test sites. 


\title{
Computational Studies of the Transport and Thermodynamic Characteristics of a Variety of Gases in Ionic Liquids
}

\author{
Liem X. Dang
}

This research will help us better understand gas sorption and transport in existing room temperature ionic liquids (RTIL) and compare gas sorption with experiments. This understanding will build toward a long-range goal of proposing novel RTIL combinations and testing their gas sorption properties and dynamics computationally without the need to synthesize them.

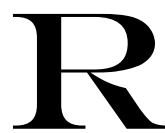
TILs have great potential to serve as new solvents for a variety of processes due to their unique properties and the ability to customize properties by changing their substituents. One of their most useful properties is the ability to absorb certain gases such as $\mathrm{CO}_{2}$ and $\mathrm{SO}_{2}$ preferentially while having near zero vapor pressures and very high stabilities. This makes RTILs good candidates for physical solvents for $\mathrm{CO}_{2}$ and $\mathrm{SO}_{2}$ capture from flue gas streams. Current $\mathrm{CO}_{2}$ capture technology often relies on passing the flue gas through alkanolamine based aqueous solutions, which react with $\mathrm{CO}_{2}$ to create ammonium carbamate. Afterward, the solvent can be recycled; however, this process requires high energy, has problems with loss of solvent through evaporation and degradation, and can cause corrosion. On the other hand, physical absorption has the benefit of not requiring high energy input, as pressure can serve as the mechanism for absorption and desorption. Because coal gasifiers operate at fairly high $\mathrm{CO}_{2}$ partial pressure, this creates conditions favorable for their physical solvation. Some desirable properties for a physical solvent include low-vapor pressures, high selectivity, low viscosity, and noncorrosive behavior. Common RTILs hold many of these properties, including a low volatility and high stability. While RTILs have fairly high viscosities near room temperature, many gasifiers operate at fairly high temperatures (above 450K), in which RTIL viscosities can be fairly low.

With the aforementioned promising properties, RTILs have been investigated extensively as a neat liquid, and their gas sorption properties have been investigated both computationally and experimentally. One interesting factor with RTILs is their interfacial properties with gases and supercritical fluids. Experimental and computational investigations have identified that RTILs generally have a layered surface environment, but how this influences gas sorption and selectivity is not known. For instance, any mechanism for gas sorption into RTILs relies on its transfer across an RTIL interface, and if one wants to optimize this, its mechanism needs to be understood. Further, if the RTIL

surface is found to exhibit unique influences on gas sorption and selectivity, this may be exploited to further improve these by increasing the available surface area.

Computational methods benefit from the ability to extract molecular level information from simulations that can be related to thermodynamic or dynamic properties. One important factor for computational investigations is the utilization of accurate molecular models. For RTILs (especially the investigation of interfaces), the inclusion of many-body interactions in the molecular models has been found to improve agreement significantly between computational predictions and experi-

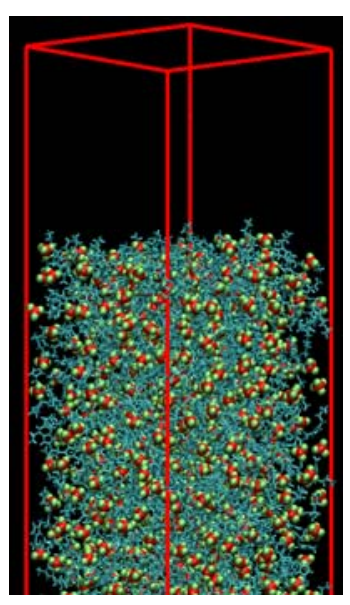

Snapshot of the BMIMBF4 air/ liquid structure at $350 \mathrm{~K}$. ments. To understand RTILs interfacial structure and how it influences gas sorption and selectivity, we performed molecular dynamics simulations with many-body interactions in FY 2010 to determine the mechanism for $\mathrm{CO}_{2}$ and $\mathrm{SO}_{2}$ solvation in butyl-methylimidazolium borate (BMIMBF4). New polarizable force fields were developed for BMIM, BF4, and $\mathrm{CO}_{2}$ and gave good agreement with experiment for the BMIMBF4 liquid density and reasonable agreement with the experimental BMIMBF4 heat of vaporization. Comparisons with the experimental surface tension and $\mathrm{x}$-ray reflectivity at the air-BMIMBF4 interface also showed good agreement. The structure of BMIMBF showed significant layering at lower temperatures with BMIM having enhanced density at the air-BMIMBF4 interface, and BF4 having enhanced density a few angstroms toward the bulk, followed by an oscillation between enhanced BMIM and BF4 densities. This layering would be expected to influence gas sorption significantly.

To understand the results better, we calculated the potential of mean force (PMF) for the transfer of $\mathrm{CO}_{2}$ and $\mathrm{SO}_{2}$ across the air-BMIMBF4 interface. The most prevalent BMIMBF4 functional group is BMIM alkyl. Longer alkyl chains in RTILs have been found to enhance $\mathrm{CO}_{2}$ solubility, so this is somewhat expected. However, the interfacial region will have a different structure than in the bulk, as these dangling alkyl groups will have a lower density. 
Another interesting feature for the solvation of both species is that their free energy clearly oscillates up and down as it crosses the interface. This behavior is not observed for solvation in water for many gas species such as the hydroxyl radical but is clearly present here.

Our results clearly show that the nanostructured ordering in the RTIL creates pockets of enhanced $\mathrm{CO}_{2}$ and $\mathrm{SO}_{2}$ solubility. This effect is less for $\mathrm{SO}_{2}$ than for $\mathrm{CO}_{2}$, likely due to the higher temperature in which $\mathrm{SO}_{2}$ is simulated or from the different interactions between BMIMBF4 and $\mathrm{SO}_{2}$, as $\mathrm{SO}_{2}$ has a more negative free energy of solvation. However, the free energy is expected to be more positive at higher values, so this is consistent with our results. To check our model, we used free energy perturbation to calculate the free energy of solvation at experimental conditions for $\mathrm{SO}_{2}$ and got a value of $-3.00 \pm 0.5 \mathrm{kcal} / \mathrm{mol}$, which is in excellent agreement.
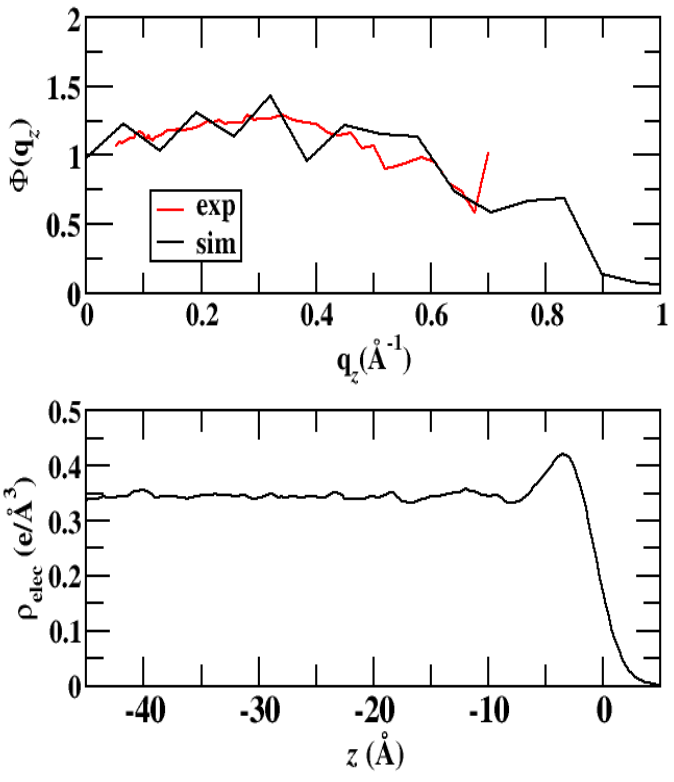

Electron density as a function of position (bottom), and structure factor as a function of frequency (top).
Our PMF results show the importance of the nanostructured interfacial structure on gas solvation.

For FY 2011, we plan on expanding this new understanding to comprehend better how higher gas concentrations, different RTILs, interfaces (such as water-RTIL), and different pressure conditions affects this unique nanostructured solvation behavior. Special efforts will focus on the development of interaction potentials between the solutes $\left(\mathrm{CO}_{2}, \mathrm{SO}_{2}, \mathrm{O}_{2}\right.$, and so forth) and RTIL systems. We will employ a variety of techniques to optimize our molecular models, such as electronic structure methods, ab initio molecular dynamics, and experimental comparisons with our simulated data. One additional, important aspect that will be included in the molecular models will be polarizability, which has been found to be of great importance for the understanding of interfacial properties. 


\section{Detection and Characterization of Uranium Hexafluoride Reaction Products in the Environment}

Steven C. Smith, Leah M. Arrigo, Bret D. Cannon, Herman M. Cho, Paul L. Gassman

This project will attempt to develop new technologies for the detection and characterization of uranium hexafluoride $\left(\mathrm{UF}_{6}\right)$ and its reaction products in the environment.

$\mathbf{T}$ he need for new technologies to detect and characterize $\mathrm{UF}_{6}$ and its reaction products in the environment has been identified and has nuclear forensic applications. This project will develop bulk chemical extraction, nuclear magnetic resonance (NMR), and fluorescence spectroscopy techniques to improve the fundamental understanding of the chemical behavior of uranium oxyfluorides and their reaction products when associated with environmental media. Completed and ongoing projects at PNNL have investigated the chemical behavior of $\mathrm{UF}_{6}$ and its reaction products in the environment and significant, new information has been learned. This project seeks to advance the current understanding of the environmental chemistry of these materials by exploiting the unique characteristics of this family of compounds to develop new methods for their detection and characterization.

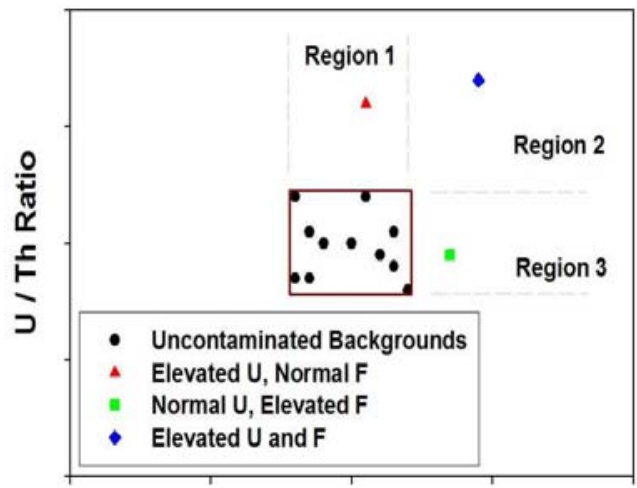

F / Cl Ratio

Conceptual data from soil extractions demonstrate the bypothetical variation of uranium/ thorium and fluorine/ chlorine ratios in uncontaminated and contaminated soil.
Specifically, bulk chemical extraction of soils, NMR spectroscopy of solid and liquid materials, and cryogenic laser-induced fluorescent spectroscopy (CLIFS) techniques will be applied and evaluated. Bulk extractions will focus on the co-extraction of uranium, thorium, chlorine, and fluorine. NMR spectroscopy will characterize the fluorine-uranium chemical association using a new custom-built probe. The two issues addressed by CLIFS are line width-narrowing in aqueous solvents at cryogenic temperatures and exploration of the sensitivity of the cryogenic spectra to changes of the uranyl complex in nonaqueous solvents. A primary outcome of this project will focus on the techniques that leverage advanced analytical methods. Results obtained in FY 2010 show significant progress toward achieving the objective.

Research completed during FY 2010 has been primarily directed at establishing the capability to detect the presence of uranium-fluorine compounds in simple sample types using advanced analytical techniques. The bulk extraction component of the project developed preliminary data indicating the technical approach may yield a promising new detection technique. It is anticipated that careful analysis of soil extracts for uranium, thorium, chlorine, and fluorine may provide a promising new method that does not require the isolation and detection of uranium-fluorine bearing compounds. However, at this time, the accurate determination of fluoride in the extract remains problematic.

An alternative analytical technique for fluoride quantification has been identified and will be explored in FY 2011. Further, the project will investigate the recovery of uranium and

fluorine from three distinctly different soils previously spiked with a uranium-fluorine compound. The NMR spectroscopy component of the project constructed a customdesigned, low-background, variable temperature probe for analysis of solid and liquid materials containing low concentrations of the analyte $\left({ }^{19} \mathrm{~F}\right)$. The new probe was used to demonstrate the capability to quantify the ${ }^{19} \mathrm{~F}$ in solution at 42 parts per billion concentration with a 12-hr scan using a $300 \mathrm{MHz}$ NMR instrument with the possibility of an even lower limit of quantification. Moreover, the detection by NMR of multiple uranium-fluorine chemical species in laboratory synthesized uranium-fluorine solutions has been demonstrated, which indicates the capability to detect and distinguish multiple uranium-fluorine species. Data obtained using CLIFS of uranium in aqueous and nonaqueous solutions show that this technique can be used to detect uranyl species at parts per billion concentrations while probing the local chemical environment of the uranium. Combined with the dramatic suppression of both Raman and broad bandwidth background by gating the detector, these results illustrate the ability to distinguish various uranium-fluorine species from other uranium-bearing chemical forms.

This project has two parallel, interrelated objectives for FY 2011: evaluate the potential to isolate and detect uranium-fluorine compounds from relevant sample types, and evaluate the relative persistence of the uranium-fluorine compounds. These goals will be achieved by the execution of focused laboratory experiments. If successful, the new techniques will provide powerful new analytical methods. 


\title{
Determination of Biosignatures Related to Explosives Exposure
}

\author{
James A. Campbell, Charles A. Timchalk, Catherine E. Petersen, Andrea L. Hjerpe, Luke C. Short
}

In this project, we will identify unique biosignatures associated with exposure to explosives and subsequent use of biosignatures for forensic applications.

B omb-makers are potentially exposed to significant amounts of explosive and other related materials. These compounds are subject to biotransformation. The possibility exists that unique biosignatures may be produced and deposited in biological fluids such as saliva, urine, or blood, which can be used for forensic and investigative purposes. This project provides a proof-of-concept study to determine the fate of pentaerythritol tetranitrate (PETN) and ammonium nitrate (AN), determine possible metabolites and protein modifications as indicators of past exposure, and the best matrix for monitoring. Data to be gathered includes pharmacokinetics, fate, and transport resulting in biosignatures within the body, and identifying the matrix that would be the most ideal for biomonitoring prior to the development of specific assays.

The basis for this project was developed from our studies in organophosphate pesticides. To date, we have analyzed saliva of rats exposed to chlorpyrifos (CPF), an organophosphate pesticide for its major metabolite, trichloropyridinol (TCP). The concentration of TCP in saliva was determined to be approximately two orders less than observed in blood. In this particular case, TCP is a biosignature indicating exposure to $\mathrm{CPF}$. These results potentially provide the foundation for using non-invasive matrices as biological fluids for measuring exposure to explosives, chemical warfare agents, biological agents, and exposure to radiation.

Prior to animal exposure, the protocols for the project were established and approved. As an initial screening study, separate rats were exposed to $50 \mathrm{mg} / \mathrm{kg}$ AN orally (and intravenous [IV]) and $10 \mathrm{mg} / \mathrm{kg}$ PETN orally. There was a solubility problem associated with PETN and IV exposure. Blood was sampled at $0,5,5,15,30$ mins and 1, 2, 3, 6, 12, and $24 \mathrm{hrs}$. Saliva samples were taken after 0 and $3 \mathrm{hrs}$ (sacrificed animal), and urine was sampled at 0, 12, 24, and $48 \mathrm{hrs}$. Sample preparation techniques were developed for PETN and AN in the various biological fluids. The blood samples were washed with acetonitrile and water; urine and saliva samples were diluted and filtered. All samples were filtered and centrifuged prior to analysis. Analytical techniques were developed for the analysis of PETN and AN using high performance liquid chromatography with ultraviolet detection at $214 \mathrm{~nm}$.
Preliminary results for the liquid chromatography/ultraviolet studies show that for blood analysis of AN, the concentration rises after $15 \mathrm{~min}$ of exposure, then falls back to the baseline after $2 \mathrm{hrs}$, then rises to $1400 \mathrm{ppm}$ over $6 \mathrm{hrs}$, and then back to baseline again after $24 \mathrm{hrs}$. There appears to be an unknown peak (possible metabolite) in the blood and urine. For blood and PETN analyses, an unknown peak appears to maximize at $12 \mathrm{hrs}$. The PETN appears to decrease in concentration in the blood $30 \mathrm{~min}$ after exposure. It appears that the residual parent compound could be used to provide an indication of exposure. This result has also been reported recently on studies of RDX, HMX, and tetryl. Urine may be a good matrix for determining the exposure to explosives, and additional studies involving saliva are necessary.

Additionally, select samples were analyzed using an Agilent LC/QTOF mass spectrometer in both the positive and negative ion modes. This instrument has accurate mass capability (determining elemental composition) and MS/MS for additional structural information. The rat blood samples were analyzed using electrospray mass spectrometry. Electrospray positive ion mass spectrometry showed several peaks of higher concentration than the controls. As an example, a metabolite and protein identification library (METLIN) indicated that a component in the $12 \mathrm{hr}$ sample was much higher than in the $0 \mathrm{hr}$ sample. The mass was determined to be $1015(\mathrm{M}+\mathrm{H})$, and the tentative identification was glycerol lipid with an elemental composition of $\mathrm{C}_{67} \mathrm{H}_{114} \mathrm{O}_{6}$. Another example is a peak at $\mathrm{m} / \mathrm{z} 886.4884(\mathrm{M}+\mathrm{H})$. The tentative identification through METLIN was glycerol phosphate with an elemental composition of $\mathrm{C}_{51} \mathrm{H}_{100} \mathrm{NO}_{8} \mathrm{P}$. Another peak at $\mathrm{m} / \mathrm{z} 820.4459$ (present in the $12 \mathrm{hr}$ but not the 0 min sample) was also observed, and the elemental composition was determined to be $\mathrm{C}_{44} \mathrm{H}_{69} \mathrm{NO}_{13}$.

Under negative ion conditions, a peak tentatively identified as glycerol phospholipid at a mass of $949.4306(\mathrm{M}-\mathrm{H})$ with an elemental composition of $\mathrm{C}_{46} \mathrm{H}_{88} \mathrm{~N}_{2} \mathrm{O}_{16} \mathrm{P}_{2}$ was observed. Still another peak observed (in the $12 \mathrm{hr}$ but barely in the 0 min sample) was $\mathrm{m} / \mathrm{z} 769.4458$ with an elemental composition of $\mathrm{C}_{42} \mathrm{H}_{75} \mathrm{O}_{10} \mathrm{P}$ tentatively identified as glycerol phospholipid. These particular compounds are termed "upregulated" as a function of exposure. Other peaks were tentatively identified in both positive and negative ion modes. All of these components need further investigation. 


\title{
Development of Inorganic Water Oxidation Electrocatalysts
}

\author{
Jenny Y. Yang, Daniel L. DuBois
}

This project will develop new classes of homogeneous inorganic water oxidation electrocatalysts that improve upon the efficiency, rate, stability, and cost of known catalysts. With the use of highly modular ligands, this systematic approach toward catalyst discovery is expected to generate new families of effective water oxidation catalysts.

$\mathbf{T}$ he development of a non-fossil fuel energy infrastructure is one of the major challenges facing our country for environmental, economic, and national security reasons. One answer to this challenge is the development of an artificial photosynthetic system. In nature, plants use sunlight to generate chemical fuels by reducing carbon dioxide and oxidizing water to evolve oxygen. A practical synthetic device would require an efficient, fast, stable catalyst capable for oxidizing water under ambient conditions. Despite several decades of research in this area, there are only a few examples of heterogeneous catalysts for water oxidation and most require

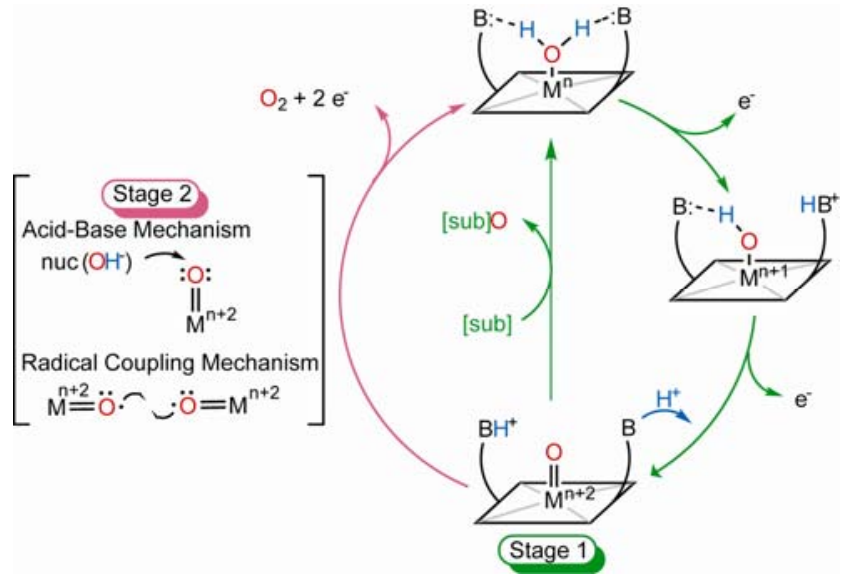

Outline of the catalytic cycle. Stage 1 shows the formation of the metal oxo probed by reaction of an easily oxidized substrate; Stage 2 initiates the oxygen bond formation with the reactive metal oxos.
This project will attack the challenging four-electron process by studying the catalytic cycle in two steps. The first stage will cover the two-electron oxidation of a water molecule bound to a complex to form a metal oxo species, the first intermediate in oxygen bond formation studied spectroscopically and chemically probed by oxygen atom transfer reactions with easily oxidized substrates. The second stage will couple the metal oxo intermediate with another metal oxo or hydroxide to initiate the evolution of oxygen and close the catalytic cycle. The intermediates and chemical steps in our catalytic cycle are consistent with mechanisms proposed for natural and synthetic systems that catalyze this reaction.

Over the past year, we have initiated our studies with a few different ligand classes. Our research with the different systems has provided valuable insights intofuture ligand design and architectures. The first class of ligands studied was a oxidized bidentate phosphine ligand with an amine linker. This ligand can coordinate to a metal and form a six-membered ring; the amine interacts with substrates in the primary coordination sphere when it is in boat conformation. However, addition of less than stoichemetric amounts of this ligand to $\mathrm{Fe}(\mathrm{II})$ and the use of precious metals.

Aside from using expensive metals, current catalysts do not have the efficiency, speed, or stability to function on a practical scale. However, we know from nature that water oxidation can be performed under all of these conditions using cost-effective first row transition metals.

Our approach to catalyst design is to mimic the essential qualities from nature's architecture and incorporate it into synthetic systems. A critical consideration is energy-matching through the catalytic cycle using proton management. We are using thermochemical measurements to assist catalyst design so that we are close to thermodynamic equilibrium at each step of the catalytic cycle, which is essential to maintain high efficiency. Effective proton management is also an important design element to avoiding high or low energy intermediates in the catalytic cycle. Multi-electron and multi-proton chemistry is facilitated in nature by controlling the proton inventory at enzymatic active sites using amino acids. Our synthetic systems will incorporate proton acceptors in the secondary coordination sphere to perform these functions.
$\mathrm{Ni}$ (II) salts resulted in the six-coordinate "tris" complex, which has no open coordination site for substrate. To enforce a coordination geometry that allows for open coordination sites, modified porphyrins were explored. Two meso functionalized porphyrin ligands were synthesized: one with pyridines and the other with ortho-anilines. The ligands can be synthesized on a Gram-scale in one and two steps, respectively. Metallation of these ligands to make the desired metal complexes is currently underway. Another ligand system currently being explored are four-coordinate diamine dipyridine and diamine diphenolic ligands. Modifications to position basic and hydrogen bonding functionalities in these ligand architectures are currently being explored.

For FY 2011, we will have well developed, characterized complexes to initiate catalytic studies. We will incorporate changes to the ligand design, characterize intermediate metal hydroxide or oxo complexes, determine structure-function relationships between ligand functionalities, and initiate studies on oxygen bond formation. 


\title{
Development of Preparative Mass Spectrometry for the Creation of Novel Catalyst Materials
}

\author{
Grant E. Johnson, Julia Laskin
}

This project will develop ion source, mass spectrometry, and soft landing instrumentation to enable size and shape selected deposition of catalytic materials. The reactivity testing, characterization, and computational facilities at PNNL will be employed to establish structure-reactivity relationships and design catalyst materials with unprecedented activity and selectivity.

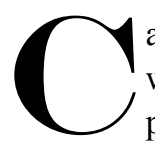
atalysts are used to reduce energy requirements and waste disposal associated with industrial chemical production and to mitigate effects of harmful environmental pollutants. The majority of catalysts consist of either small metal particles immobilized on metal-oxide supports (heterogeneous catalysts) or single metal atoms surrounded by ligands in solution (homogeneous catalysts). The reduction synthesis techniques generally employed for the production of heterogeneous catalysts do not provide atomically precise control over the size and morphology of the resulting materials. This polydispersity results in decreased catalytic selectivity. By contrast, homogeneous catalysts typically exhibit higher selectivity than heterogeneous materials due to their "single site" nature. However, the separation of homogeneous catalysts from products and remaining reactants in solution presents a challenge that may be overcome through surface immobilization of catalytic complexes.

The objective of this project is to investigate soft and reactive landing of mass-selected ions as a technique for the preparation of monodisperse heterogeneous and immobilized homogeneous catalysts. To achieve this objective, ion source, mass spectrometry, and deposition instrumentation will be developed. A fundamental, molecular-level understanding of factors that influence the creation of catalysts using this approach will also be obtained.

We constructed a new ion deposition apparatus coupled to a commercial time-of-flight secondary ion mass spectrometry (TOF-SIMS)

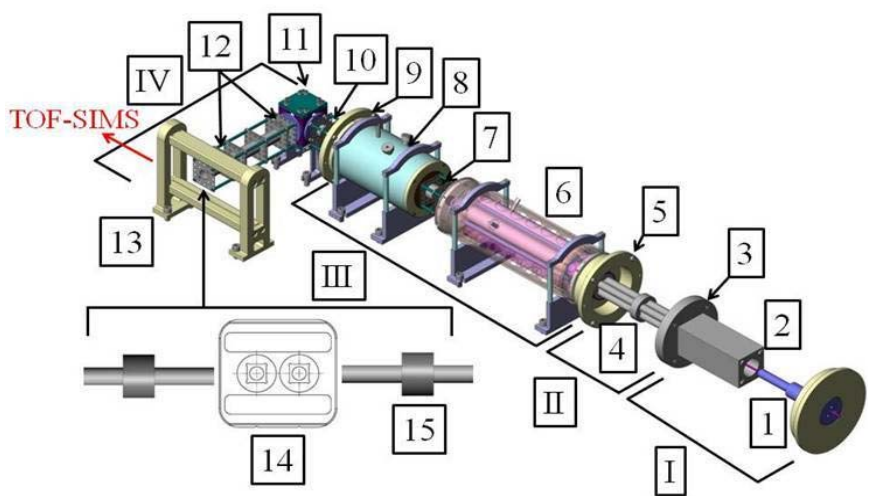

Schematic illustration of the ion deposition instrument: I) ion funnel region $\left(7 \times 10^{-1}\right.$ Torr $)$, II) collision quadrupole region $\left(1 \times 10^{-1}\right.$ Torr $)$, III) mass selection and focusing region $\left(2 \times 10^{-4}\right.$ Torr $\left.), I V\right)$ deposition region $\left(1 \times 10^{-6}\right.$ Torr), 1) heated capillary, 2) electrodynamic ion funnel, 3) first conductance limit, 4) first collision quadrupole, 5) second conductance limit, 6) resolving quadrupole, 7) 2 focusing lenses, 8) second collision quadrupole, 9) third conductance limit, 10) Einzel lens, 11) quadrupole bender, 12) two Einzel lenses, 13) target platform, 14) surface mount, 15) magnetic translators. instrument that enables in-situ analysis of surfaces prepared by soft and reactive landing of mass-selected ions. The TOF-SIMS analysis technique has been validated by comparing results obtained previously by Fourier transform ion cyclotron resonance (FT-ICR-SIMS). TOF-SIMS exhibits less fragmentation, greater dynamic range, and higher sensitivity than FT-ICR-SIMS, allowing catalytic intermediates with low abundance to be detected easily. These results have been published in the journal Analytical Chemistry.

Ruthenium bipyridine complexes, which are used as chromophores for water oxidation catalysis and catalysts for the water-gas shift reaction, were soft and reactively landed onto a variety of different self-assembled monolayer surfaces. It was found that carboxylic acid-terminated surfaces bind the complex strongly, and the extent of reactive landing can be greatly enhanced by removing one bipyridine ligand in the gas-phase through collision induced dissociation. The immobilized complexes showed behavior consistent with catalytic activity when exposed to $\mathrm{O}_{2}$ and $\mathrm{C}_{2} \mathrm{H}_{4}$. These results have been submitted for publication to Chemistry - A European Journal.

As olefin epoxidation catalysts, metal salen complexes were deposited along with proton donor complexes onto fluorinated monolayer surfaces to examine whether it is possible to achieve proton mediated reactivity in a monolayer. Proton mediated loss of oxygen from $\mathrm{VO}(\text { salen })^{+}$to form $\mathrm{V}(\text { salen })^{+}$was observed by in-situ TOF-SIMS. Moreover, the original $\mathrm{VO}(\text { salen })^{+}$complex was regenerated by exposure to $\mathrm{O}_{2}$. A cyclical pattern of oxidation and reduction was observed providing evidence of catalytic behavior. These results have been submitted for publication in Physical Chemistry Chemical Physics.

Future work will evaluate the effectiveness of electrospray ionization for generating metal and metal-oxide clusters for surface deposition. A laser vaporization cluster source will be constructed and evaluated for generating metal and metal-oxide clusters. 


\title{
Development of Techniques for Determining Medium-Range Order on the Surfaces of Catalysts Using Solid-State NMR Spectroscopy and Computational Chemistry
}

\author{
Karl T. Mueller, Nancy M. Washton, Donald R. Baer
}

This project is aimed at developing and applying novel PNNL capabilities for understanding the surfaces of oxide catalysts, combining a specialized solid-state nuclear magnetic resonance (NMR) experiment using an advanced pulse sequence with computational modeling of NMR parameters.

he design of new catalyst systems can build upon
the molecular-level structure of known catalyst
materials through a description of the reactive sites present on the surface of these materials. The deployment of high-sensitivity NMR capabilities will enable information to be collected regarding electronic and other characteristics that affect catalyst performance. Additional data will be gained by coupling the measurements with quantum chemical calculations of reaction product structures and NMR parameters. Once these are confidently mapped onto structural environments, higher-order analyses (such as the determination of distributions or clustering of catalytic sites) may be undertaken. These integrated capabilities will assist catalysis development and be equally important in other areas such as geochemistry and energy production.

Our research focused on developing and deploying novel NMR spectroscopy tools that enable the molecularlevel understanding needed to develop the above-described efficient catalyst materials. Previous studies have demonstrated the power of combining innovative spectroscopic techniques (including NMR) and computational chemistry methods to study advanced materials at the molecular level. The Environmental Molecular Sciences Laboratory (EMSL) provides worldclass facilities for magnetic resonance and computational sciences. Based on advances in computation and magnetic resonance technology, there is significant opportunity to advance the understanding of catalytic processes and materials. This project is part of a long-range goal to develop an
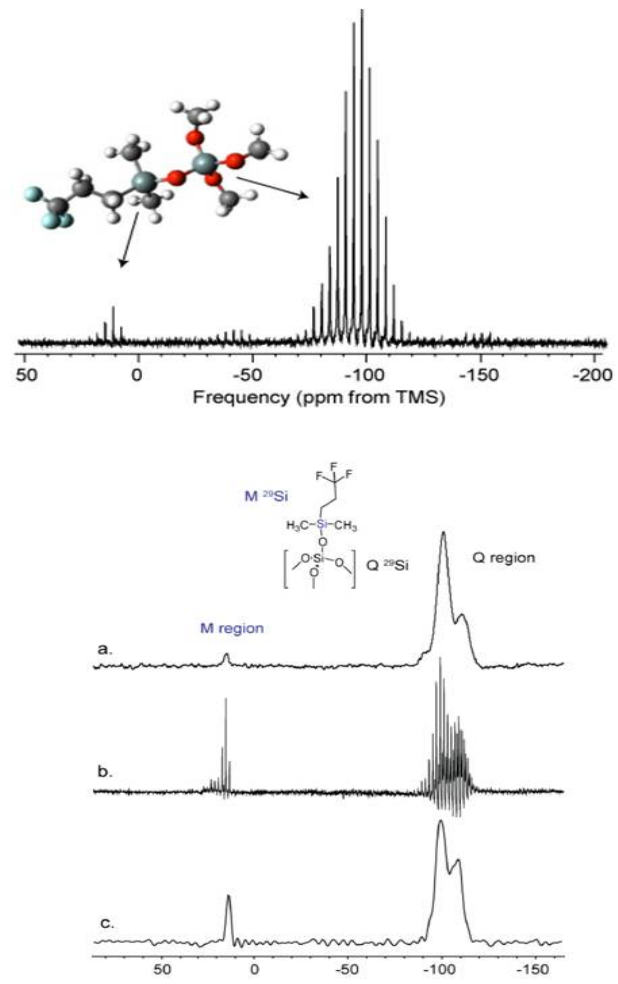

The spikelet-enhanced solid-state NMR spectrum from the silicon-29 nuclei in an aluminosilicate catalyst material contains resonances from silicon in the probe molecule and aluminosilicate surface. The energy minimized model cluster for this system is also shown. understanding of the molecular-level structure of catalyst materials through a description of the reactive sites present on the surfaces of these materials.

During our studies, advanced magnetic resonance pulse sequences were programmed and tested, providing high resolution experimental data with increased sensitivity amenable to modeling based on results from quantum chemical calculations. Experimental data sets were acquired from model systems, and quantum chemical cluster models were constructed describing local binding sites for reactive probe molecules on the surfaces of aluminosilicate catalyst samples. Structures were then energy minimized for the reaction products. As this project concludes, experimental chemical shielding parameters will be calculated from the lowest energy structures and comparisons, with experimental results used to guide the choice of computational methods for further investigations. Ultimately, these coupled experimental and computational data will feed into a statistical model that will describe for the first time the populations of local structures surrounding reactive groups on the catalyst surfaces. Such statistical models will be the focus of a future investigation.

We used state-of-the-art experimental capabilities closely aligned with computational analyses for advanced characterization of catalyst surfaces. These new tools for advanced catalysis studies will be available to collaborators and users at the magnetic resonance facility at EMSL. The approach enabled a significantly improved level of chemical information with impacts ranging from fundamental science to applied applications. Although focused on catalysis studies initially, our methods can apply to other types of materials and biological systems important to DOE, with additional applications in environmental sciences and national security. 


\section{Enhanced Ion Detection Mechanisms for Ion Mobility Spectrometry}

Brian H. Clowers

Despite its resounding success as a field-based instrument for explosives detection, ion mobility spectrometry relies on very few core principles centered in ion generation and gating as well as signal detection. In an effort to leverage recent developments in these core technologies, this project aims to explore the use of alternative ion detection mechanisms to produce higher levels of security through field-based measurements.

I $\mathrm{n}$ addition to being mechanically robust, ion mobility spectrometry is a rapid gas phase separation technique that provides a high degree of selectivity and sensitivity for a range of gas phase analytes. Consequently, it is widely used throughout the world as a field-based method for the detection of narcotics, chemical warfare agents, and explosives. However, the technology used in commercial systems has remained largely static since its introduction. Over the last 30 years, a number of technical developments have been realized with respect to signal detection but have yet to be examined thoroughly in conjunction with ion mobility spectrometry. In order to bolster the absolute detection limits and maximize the utility as an explosives detector, we will explore the use of two general approaches to enhanced signal detection. The first focus area is directed toward the absolute noise reduction in signal recording mechanisms, and the second seeks to explore the practicality of signal amplification at pressures that approach ambient conditions.

During FY 2009, our primary task was to develop the infrastructure necessary to conduct low current measurements with a specific focus on explosives detection. This included the design and design and construction of a research-grade atmospheric pressure ion mobility system that supported electronics and data acquisition software. These project goals were successfully completed in FY 2010 and represent a unique capability at PNNL. Though specifically designed for explosives detection, the apparatus, electronics, and software controls are a core foundation for future ion mobility spectrometry projects.

In tandem with the instrument construction in FY 2009, initial examination of alternative ion detection mechanisms was conducted. Originally, the focus on absolute noise reduction and signal amplification was to be accomplished using capacitive transimpedance amplifiers and electron cascading mechanisms. However, a previous institutional investment in another approach to direct ion signal integration was identified as a superior alternative to the capacitive transimpedance amplifier. Compared with existing ambient condition ion signal recording mechanisms, this approach directly measures the ion signal and mitigates complications that typically arise during signal transduction. Continuing in FY 2010, the evaluation of the new approach demonstrated a moderate yet significant gain over existing technologies with a two- to three-fold improvement in signal-to-noise ratio. Because these studies were conducted using only a single input channel, it is anticipated that this gain may be further improved upon integration with an arrayed detector.

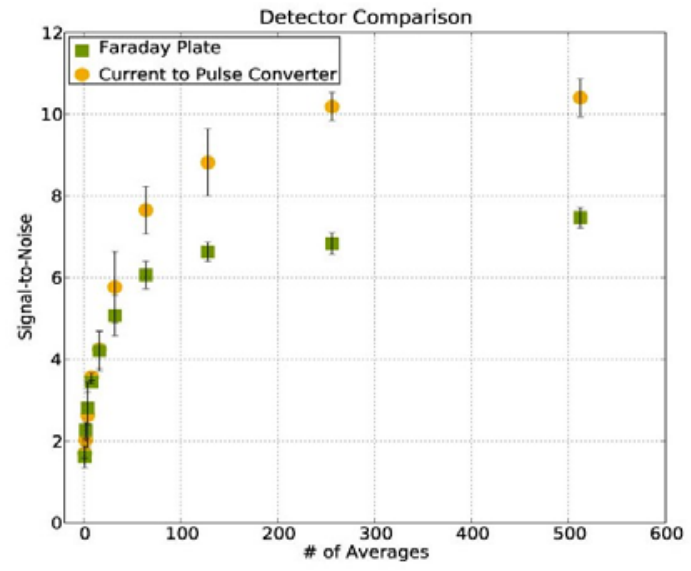

Signal-to-noise ratio gains of the newly developed technology compared with the conventional approach.

The pursuit of ion mobility spectrometry detector technology that directly circumvents the need for external high speed current measuring devices has focused on identifying the high pressure conditions necessary to induce electron cascading. While these conditions can be easily demonstrated within the laboratory, the primary challenge lies in the ability to quench the cascading event so that subsequent measurements can be performed in rapid succession. In order to meet this challenge, we have identified a unique micro-fabricated detector designed specifically to address these concerns.

Most notably, this project led to the successful development of a research-grade ion mobility spectrometer and a new mechanism of ion detection that can be directly integrated into any existing system. Additionally, this effort has established a foundation for the future exploration of electron cascading mechanisms for enhanced ion detection. A more comprehensive understanding of operating conditions required for new detector technologies will allow a more complete assessment regarding the potential for field based applications. While explosives are the primary target for this new class of detectors, the application of these technologies extends to other areas requiring ion detection. 


\section{Fuel Synthesis Research}

David L. King, Daniel R. Palo, Robert A. Dagle, Jair A. Lizarazo-Adarme, Vanessa M. Lebarbier

This project will demonstrate the efficacy of combining the two catalyst functions into a single catalyst bed with good efficiency and yield for the direct conversion of syngas to gasoline.

$\mathbf{T}$ he production of liquid, infrastructure-compatible transportation fuels remains a high priority of the government and DOE as a means to reduce United States dependence on foreign oil. For fuel sources such as coal or biomass, there are two general alternatives for the production of liquid fuels: an indirect method, in which the fuel source is gasified to produce syngas, which is then converted to fuel and direct methods, including liquefaction (for coal) and pyrolysis oil upgrading (for biomass), followed by additional refining as required. This project is based on the indirect method for fuel production.

We have pursued two general indirect approaches: the

Fischer-Tropsch catalysis, which produces hydrocarbons directly with a broad distribution of products, requiring subsequent upgrading, and methanol-to-gasoline (MTG), and requires synthesis of methanol and is then converted to infrastructurecompatible gasoline without further processing. The MTG process requires two (or even three) separate unit operations. It was developed by Mobil Oil Corporation in the 1970s and 1980s and run at a substantial scale in New Zealand but was later shut down due to unattractive economics. Several factors contributed to the unfavorable economics, including a dropping in the cost of oil. However, the operation of a process requiring two or more unit operations contributed to the unfavorable costs.

The technical requirement for two-unit operations was that methanol synthesis needed to occur at temperatures below that required for the subsequent conversion of methanol to gasoline. Recently, through other work carried out at PNNL, an alternative to the existing methanol synthesis catalyst was identified that might be able to operate effectively at higher temperatures than the conventional catalyst. With a catalyst that was stable at the temperature required for the subsequent MTG step, it would potentially be possible to consider combining the two steps into a single step. This would allow development of a process to produce gasoline from syngas that would only require a single unit operation, thereby reducing overall costs and increasing viability of the technology.

Single-Step Syngas-to-Gasoline. The overall focus of this work in FY 2010 was proof-of-concept demonstration for single-step syngas-to-gasoline using high-temperature methanol synthesis catalyst and commercial MTG zeolite catalyst in a single catalyst bed. Figures of merit
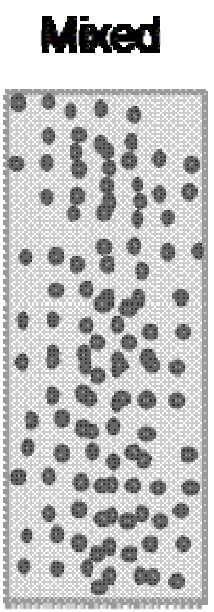

Illustration of the two catalyst bed configurations investigated for single-step syngas to gasoline: sequential bed (left) and mixed bed (right). included degree of conversion of syngas to product, quality of the liquid fuel product, and catalyst stability.

The single-step conversion testing was carried out in two different modes: a sequential bed configuration and a mixed bed configuration (shown schematically). A comparison of the product distributions obtained from the two configurations in terms of product yield per carbon number as a function of temperature was performed both sequentially and mixed bed. The differences in product selectivity are not pronounced, although it appears that there are more $\mathrm{C}_{11}$ and $\mathrm{C}_{12}{ }^{+}$products with the mixed bed. It was noted (not shown) that overall conversion of syngas was higher in the mixed bed, which is expected because methanol synthesis is equilibrium limited. As methanol is converted to gasoline, the syngas conversion to methanol can again occur free of equilibrium limitations. On the other hand, it was found that the catalyst deactivation was more rapid in the case of the mixed bed compared with the sequential bed configuration. The reason for this is not clear at this point, and further work is required.

In addition, there is one other consideration for this single step approach that must be mentioned - the envisioned process operates at elevated pressure to increase the syngas conversion to methanol. The MTG process typically operates at atmospheric pressure, and operation at higher pressures results in a shift of the gasoline range product to include heavier aromatics, from $\mathrm{C}_{6}-\mathrm{C}_{8}$ aromatics at atmospheric pressure to $\mathrm{C}_{10}$ and $\mathrm{C}_{11}$ aromatic products (primarily) at elevated pressures. It is to be determined whether a gasoline product with a higher molecular weight is acceptable from an infrastructure fuel point of view. 
PdZnAl Methanol Synthesis $\mathrm{T}=375 \mathrm{C}, \mathrm{P}=975 \mathrm{psig}$, GHSV=10,000 $\mathrm{hr}^{-1}$, Feed: $\mathrm{H2} / \mathrm{CO}=2, \mathrm{CO}_{2} / \mathrm{CO}=0.13$

\begin{tabular}{|c|c|c|c|c|c|c|}
\hline Run ID & MS-18 & MS-13 & MS-19 & MS-13 & MS-12 ${ }^{\mathrm{a}}$ & MS-14 \\
\hline \multicolumn{7}{|l|}{ Catalyst } \\
\hline $\mathrm{Pd}(\mathrm{wt} \%)$ & 5 & 9 & 20 & 9 & 9 & 9 \\
\hline $\mathrm{Pd} / \mathrm{Zn}$ & 0.25 & 0.25 & 0.25 & 0.25 & 0.38 & 0.75 \\
\hline \multicolumn{7}{|l|}{ Performance } \\
\hline $\mathrm{X}_{\mathrm{CO}}(\%)$ & 36.4 & 37.2 & 45.6 & 37.2 & 25.3 & 33.5 \\
\hline $\mathrm{X}_{\mathrm{C}}(\%)$ & 26.4 & 22.5 & 25.6 & 22.5 & 15.8 & 18.4 \\
\hline $\mathrm{S}_{\mathrm{CO} 2}(\%)$ & 49 & 54.5 & 64.9 & 54.5 & 51.3 & 62.7 \\
\hline $\mathrm{S}_{\mathrm{CH} 4}(\%)$ & 8.9 & 15.7 & 19.8 & 15.7 & 33.9 & 23.9 \\
\hline $\mathrm{S}_{\text {Ethylene }}(\%)$ & 3.2 & 2.1 & 6.6 & 2.1 & 1.2 & $0^{b}$ \\
\hline $\mathrm{S}_{\mathrm{MeOH}}(\%)$ & 3.2 & 5.5 & 1.3 & 5.5 & 3.8 & 2 \\
\hline $\mathrm{S}_{\mathrm{DME}}(\%)$ & 36.5 & 22.5 & 7.9 & 22.5 & 10.6 & 12.2 \\
\hline $\mathrm{Y}_{\mathrm{MeOH}+\mathrm{DME}}(\%)$ & 14.5 & 10.4 & 4.2 & 10.4 & 3.6 & 4.8 \\
\hline
\end{tabular}

Parametric study of $\mathrm{Pd}-\mathrm{Zn} / \mathrm{Al2O} 3$ catalyst for methanol/ dimethyl ether synthesis: effect of $P d$ loading and $P d / Z n$ ratio.

PNNL Methanol Catalyst Development. The catalyst selected for high temperature methanol synthesis was actually developed at PNNL for methanol decomposition or reforming (as a means to produce hydrogen for fuel cell operation).

Because this catalyst operated effectively at elevated temperatures (greater than $300^{\circ} \mathrm{C}$ ), it was reasoned that this same catalyst could carry out methanol synthesis from syngas as well. This catalyst is based on a palladium-zinc $(\mathrm{Pd} / \mathrm{Zn})$ alloy, which is stabilized on alumina. There is flexibility in the design of the catalyst, in terms of both the amount of palladium present and the $\mathrm{Pd} / \mathrm{Zn}$ ratio. The table shows results of a parametric study in which the $\mathrm{Pd}$ concentration (loading) and $\mathrm{Pd} / \mathrm{Zn}$ ratio were varied.

The figure of merit is the combined yield of methanol and dimethyl ether. It can be seen that the catalyst containing the lowest amount of $\mathrm{Pd}$ ( 5 percent) and lowest $\mathrm{Pd} / \mathrm{Zn}$ ratio (0.25) showed the best performance. This is a positive result from a catalyst cost perspective. It is likely that these conditions favor selective production of the alloy: higher levels of $\mathrm{Pd}$ or higher $\mathrm{Pd} / \mathrm{Zn}$ ratios may favor creation of catalyst sites in which the $\mathrm{Pd}$ is a free metal, unalloyed with $\mathrm{Zn}$. It has been shown that unalloyed $\mathrm{Pd}$ is not a good methanol/dimethyl ether synthesis catalyst. This catalyst was compared to the commercial copper-zinc-alumina methanol synthesis catalyst in terms of stability at the desired temperature of operation $\left(375^{\circ} \mathrm{C}\right)$. At this temperature, the $\mathrm{PdZnAl}$ catalyst was found to provide both greater $\mathrm{CO}$ conversion and substantially greater stability than the copper-based traditional catalyst. This is an expected and necessary result to allow us to continue with combining the methanol synthesis catalyst with the MTG catalyst.

Technoeconomic Analysis (TEA). A technoeconomic model was developed for syngas to gasoline using coal as the feedstock. The TEA indicated significant cost savings over the traditional MTG process,

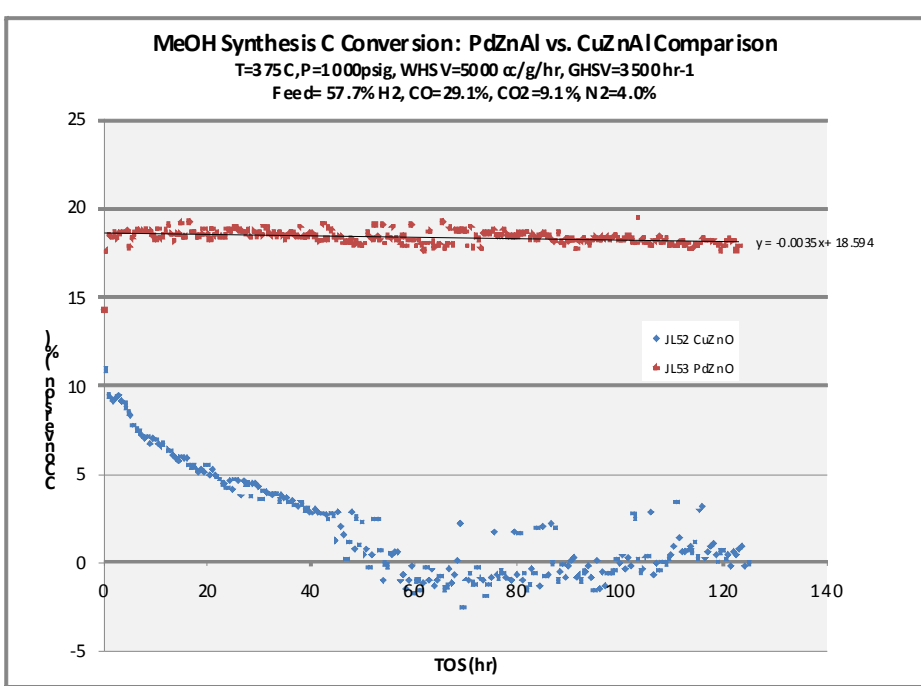

Comparison of catalyst stability for $\mathrm{PdZnAl}$ and $\mathrm{CuZnAl}$ operating at $375^{\circ} \mathrm{C}$, 1000 psig, and WHSV $=5000 \mathrm{hr}^{-1}$. The Pd-based catalyst shows very good stability over the course of the 120-hr test, while the Cu-based catalyst rapidly deactivates, losing virtually all activity after less than $50 \mathrm{br}$. based on several important assumptions about syngas conversion, recycling, and product selectivity. Specifically, the model predicted 23 percent savings in the rack price of gasoline produced by the single-step method, savings based mainly on capital savings and augmented by improved production of both gasoline and electricity for export.

The LDRD funding ended in FY 2010 and has been transferred to programmatic funding. 


\title{
Fundamentals of Carbonate Formation: Interactions of $\mathbf{C O}_{2}$ with Supported Metal Oxide Clusters
}

\author{
Xiao Lin, Zdenek Dohnálek, Bruce D. Kay
}

The goal of this project is to obtain a detailed, integrated understanding on how to control catalytic reaction of $\mathrm{CO}_{2}$ (and relevant molecules) on different oxide catalysts.

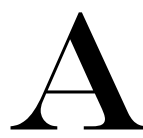
$\mathrm{s}$ industries are relying on fossil fuels for energy and the global warming problem mounts, there is an immediate need for improving the fundamental understanding of $\mathrm{CO}_{2}$ activation, which is instrumental for carbon sequestration and $\mathrm{CO}_{2}$ conversion to fuels. Studying aspects of $\mathrm{CO}_{2}$ adsorption and reactions leading to intermediate species such as carbonates $\left(\mathrm{CO}_{3}{ }^{2-}\right)$ on characterized surfaces and structures is one of the best ways to obtain an understanding of $\mathrm{CO}_{2}$ chemistry. We propose to study the fundamental aspects of $\mathrm{CO}_{2}$ adsorption and reactions leading to $\mathrm{CO}_{3}{ }^{2-}$ formation on well-characterized surfaces and supported nanoclusters of model oxides. Established methodologies will be used to prepare model surfaces of oxide single crystals [e.g., $\mathrm{TiO}_{2}(110)$ ] and epitaxial films [e.g., $\mathrm{MgO}(100), \mathrm{Ni}(100)$, $\left.\mathrm{CeO}_{2}(111)\right]$. Novel methods pioneered in our laboratory will be used to create well-defined oxide nanoclusters ranging from their fundamental molecular building blocks to larger nanoclusters of varying sizes. The effect of cluster size and structure on the reactivity of such well-defined nanoclusters toward $\mathrm{CO}$ and $\mathrm{CO}_{2}$ will be explored.

As the first step of this project, we focused on the interaction of relevant molecules with metal oxides surfaces. In the past decades, titanium dioxide $\left(\mathrm{TiO}_{2}\right)$ has attracted widespread attention due to its unique properties in heterogeneous catalysis and photocatalysis and the potential application in air purification, wastewater treatment, self-cleaning glass, and solar cells. As a part of catalytic carbon cycle, the interaction of alcohols with $\mathrm{TiO}_{2}$ are studied to ascertain the nature of reactive sites (like vacancies and other defects on oxide surfaces) and are expected as a model system to investigate the oxidation of organic contaminants.

Recently, our research team investigated reactivity of organic molecules with $\mathrm{TiO}_{2}(110)$ surface via ensemble-average surface-sensitive techniques and atomically resolved scanning tunneling microscopy (STM). Methanol, 2-butanol, and octanol
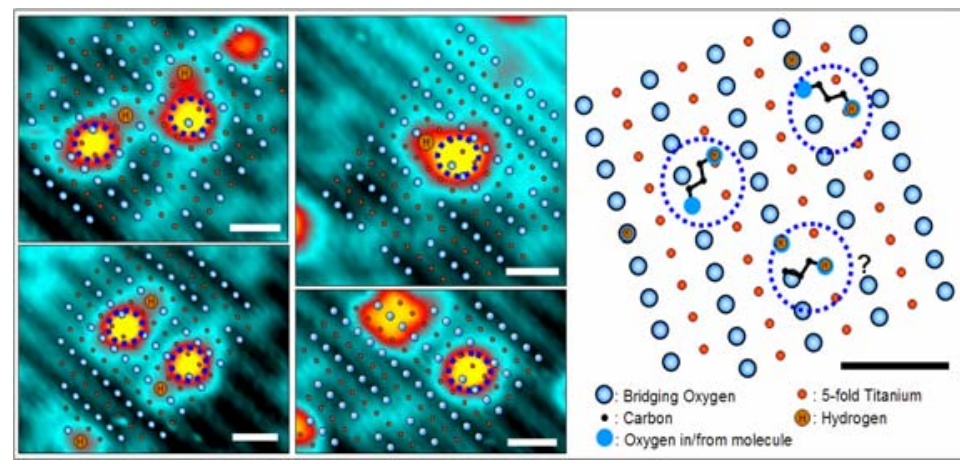

Propane-1,3-diol molecules prefer to adsorb on oxygen vacancies (scale bar: $1 \mathrm{~nm}$ ). were found to adsorb at bridge-bonded oxygen vacancy $\left(\mathrm{V}_{\mathrm{O}}\right)$ sites preferentially on $\mathrm{TiO}_{2}(110)$ and dissociate via O-H bond scission to form paired alkoxy and hydroxyl species. Longchain molecules (octanol) were found to rotate on the surface around the anchoring $\mathrm{C}-\mathrm{O}$ bond. At elevated temperatures (greater than or equal to $400 \mathrm{~K}$ ), mobile $\mathrm{V}_{\mathrm{O}}$ were shown to assist the diffusion of alkoxy group along the bridging oxygen $\left(\mathrm{O}_{\mathrm{b}}\right)$ rows. Dehydration of 2-propoxy groups, dissociated from 2-propanol, will lead to propene formation at $570 \mathrm{~K}$. Our current studies focus on the interactions of diols that contain two hydroxyl groups with $\mathrm{TiO}_{2}(110)$.

In the past months since this project started in April 2010, we studied the adsorption, dissociation, rotation, diffusion, and oxidation of propane-1,3-diol on partially reduced rutile $\mathrm{TiO}_{2}(110)$ surfaces. STM images of $\mathrm{TiO}_{2}(110)$ surfaces obtained before and after in-situ doses of propane-1,3-diol molecules at room temperature show that similar to alcohols, the propane-1,3-diol preferentially binds in $\mathrm{V}_{\mathrm{O}}$ s via one $\mathrm{O}-\mathrm{H}$ bond scission. STM observes the hydrogen species released during the dissociation, and 3-hydroxypropoxide is found centered between the titanium and bridging oxygen rows. Additionally, the 3-hydroxypropoxide rotated from one side of $\mathrm{O}_{\mathrm{b}}$ row to the other at room and elevated temperatures. The strong interaction of 3-hydroxypropoxide with Ti ions was found to reduce the rotational rate strongly. At a higher temperature, not only swing but also diffusion and further dissociation of the 3-hydroxypropoxide species were observed. From STM images, we found that dissociation of 3-hydroxypropoxide leads to new species that center on $\mathrm{O}_{\mathrm{b}}$ rows. Other techniques (e.g., temperature programmed desorption) will be employed to determine the identity of the final gas phase products.

For FY 2011, we will focus on another relevant molecule: ethane-1,2-diol. It has similar properties with propane-1,3-diol but a shorter carbon chain, which should allow us compare their carbon chain length dependent behavior. Following these studies, we will concentrate on the activation and reactivity of $\mathrm{CO}_{2}$ at the $\mathrm{TiO}_{2}$, which is the ultimate goal of this research project. 


\title{
High Precision Isotope Forensics via Multi-Collector Inductively Coupled Plasma Mass Spectrometry
}

\author{
Douglas C. Duckworth, Garret L. Hart, Gary A. Gill, Gregory C. Eiden, \\ James P. McKinley, Benjamin E. Naes, Janet M. Cloutier
}

This project will strengthen PNNL's contribution to national security through a recognized excellence in ultra-trace isotopic analysis capabilities and extending them into new areas of high-precision isotopic analysis using multi-collector inductively coupled plasma mass spectrometry (MC-ICP-MS), specifically nuclear material characterization and material analysis using radiogenic isotope systems.

$\mathbf{T}$ he scientific basis and application of nuclear isotope ratio measurements is well-known within PNNL. The application of radiogenic isotopes in geochemical (and more recently forensic) studies is also well documented and is a complementary technique to stable light isotope analysis for forensic applications. Radiogenic isotopes such as $\mathrm{Sr}, \mathrm{Nd}$, and $\mathrm{Pb}$ are known to vary substantially in nature based on primordial parent-element crustal abundances of the locale and therefore retain a regional signature preserved in mineral laden dust and metal ores. The goal of this project is to evaluate the efficacy of special nuclear material analyses via electrothermal vaporization (ETV) and the use of radiogenic isotope ratio information for provenance determination of soil and metals discrimination. A novel MC-ICP-MS will be evaluated for high precision isotopic measurements, and high efficiency sample introduction techniques will be developed. The ability to perform high precision isotopic measurements on small samples benefits nuclear nonproliferation applications and provenance determination for a broad range of materials.

For FY 2010, three tasks were addressed and are detailed below.

Discrimination of $\mathrm{Cu}$ samples based on $\mathrm{Sr}$ isotope ratios. The analytical method for the isolation and concentration of $\mathrm{Sr}$ from $\mathrm{Cu}$ for isotopic determinations was the focus. A method is being developed that uses a chelating adsorption resin specific for isolating $\mathrm{Sr}$ in a strong acid medium, and initial tests were conducted to verify recovery and suitability. High precision isotopic analyses will determine the feasibility of using $\mathrm{Sr}$ to characterize these samples. The technical approach required developing the $\mathrm{Sr}$ (then $\mathrm{Pb}$ ) separation methods to ensure low processing blanks, evaluating recoveries, and MC-ICP-MS methods. Column yield tests were greater than 95 percent for $\mathrm{Sr}$ in a $\mathrm{Cu}$ matrix. Results from trial $\mathrm{Cu}$ samples show they have low $\mathrm{Sr}$ content, which presents analytical challenges, particularly with isobaric interferences from $\mathrm{Kr}$ in the plasma gas.

Soil provenance classification. Soil contains complex components having regionally specific physical and chemical characteristics. Soil is transferable and is therefore a possible evidentiary material related to criminal activity. The complexity of soil components suggests a composite signature approach to improve discrimination and classification. Mineral inputs are multiple in soils and tend to be heterogeneous. Our aim is to correlate radiogenic isotopes $(\mathrm{Sr}, \mathrm{Nd}, \mathrm{Pb})$ and bulk element distributions in soils. The approach is soils from regions with large predicted isotope ratio variations, which will allow development of a contiguous sample set for mineral type distribution measurement, elemental analysis, and isotope abundance determination to form a composite soil signature.

Uranium analysis. The unique features of state-of-the-art MC-ICP-MS are being evaluated for uranium. The detector array allows simultaneous measurements of all uranium isotopes and an enhanced sampling interface, allowing very high sample utilization efficiency (SUE) for a dry plasma source. A miniaturized electrothermal vaporization device was shown to improve SUE by more than ten-fold over standard solution nebulization systems. Comparisons continue with the goal of over 2 percent SUE for very low uranium concentrations. The goal is performance comparable to the gold-standard thermal ionization mass spectrometer but with much higher sample throughput.

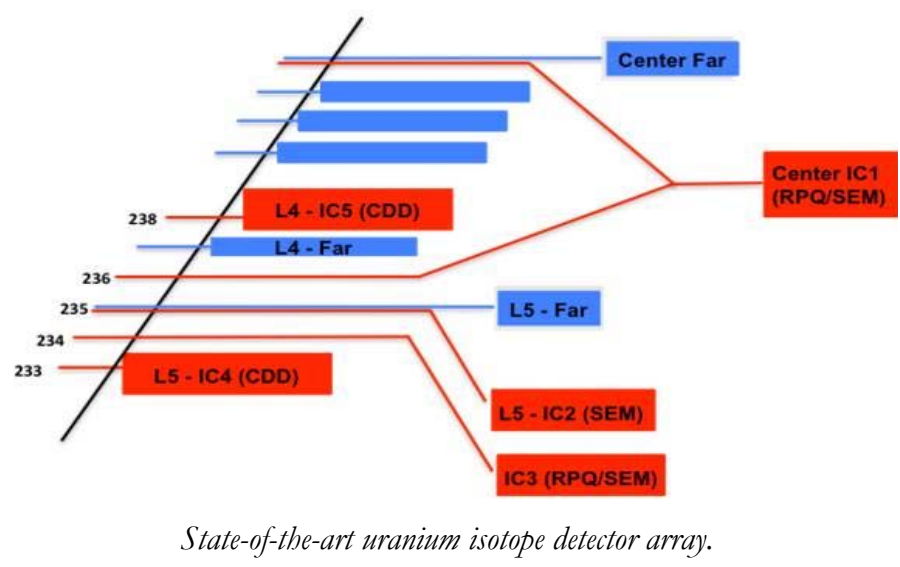

Our path forward in FY 2011 with $\mathrm{Cu}$ samples is to minimize further concentratation of the Sr recovery (approximately 10 times) and focus on $\mathrm{Pb}$ isotopics. We will complete an ASPEX analysis, microchemistry, and elemental surveys for MC-ICP-MS analysis of regional soils. For uranium analysis, we will work on designing and building a small-volume ETV to perform an extensive study. 


\title{
Instrumentation for Explosives Detection Research
}

\author{
Jay W. Grate, Norman C. Anheier Jr., Robert G. Ewing
}

Development of devices and instrumentation for explosives detection requires test and evaluation using samples of explosives molecules. This project formulated a new approach to the challenge of generating pulses of explosives vapors to enable research in explosives preconcentration, sensing, and detecting.

A dvanced sensors and instrumentation systems for trace explosives detection are required in environmental monitoring as well as national and homeland security. The detection of explosives is critical to security applications ranging from defense and homeland security applications to the protection of DOE infrastructure and assets. Detection of explosives vapors in the gas phase carried out using sensors or instruments is feasible; however, improvements are desirable. The development of advanced sensors and instrumentation requires a means to test and evaluate candidate devices and technology against explosives vapor samples. Explosives vapor generation systems in the literature generate vapors from neat solids, from materials adsorbed on inert solid supports, or from solutions containing explosives molecules. Most systems are custom-built arrangements in the investigators' laboratories to support their research.

Generation and delivery of explosives vapors is challenging for several reasons. Most explosive materials have very low vapor pressures. Hence, getting vapor molecules from a source explosive material into the gas phase in sufficient quantities and at sufficient rates is not trivial. Once generated, explosives vapor molecules tend to leave the gas phase by adsorption on tubing walls or other surfaces. Therefore, getting explosives vapor molecules from one place in the generator, transporting them to the outlet of the generator and finally to the device to be tested is complicated by losses to internal surfaces. Schemes are required to provide heating of surfaces as well as tubing, and where possible, sheath flows to keep the molecules away from surfaces. Finally, the explosives materials themselves are hazardous. For systems generating a continuous flow of vapor molecules, calibration is required, and the waste stream must be managed.

In this project, we developed a pulsed method of explosives vapor generation using a solution of the explosive in an organic solvent as the source. The solution is injected onto an inert metal material in a porous foam format, depositing a known mass of explosives compound while the solvent evaporates and is swept away. The porous metal foam is contained in a metal tube with integrated heating wires and thermocouple. A control system in hardware and software provides a method

to heat the metal tube at preprogrammed rates. The explosives molecules are transferred from the metal foam to the gas phase by a programmed thermal desorption process upon heating the tube. Using heated transfer lines, the explosives molecules are transferred forward to a test apparatus.

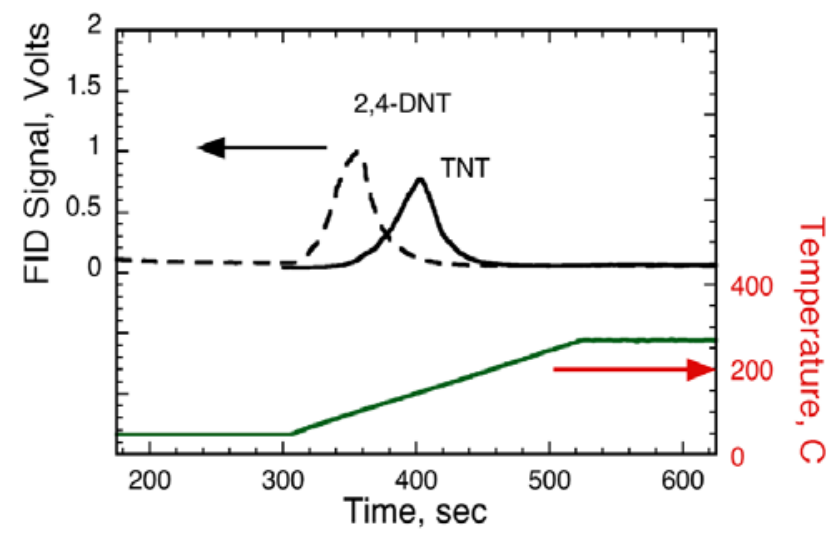

Programmed thermal desorption of 2,4-DNT and TNT from the metal foam core in the explosives generation devices as detected with a flame ionization detector. TNT has a higher desorption temperature than 2,4-DNT.

The above transfer method was demonstrated using a flame ionization detector (FID) downstream from the explosives pulse generator. The FID detected the solvent vapor passing through the system upon injection of the explosive-containing solution onto the metal foam. Additionally, the FID detected the explosives vapor upon programmed thermal desorption.

In further work, we designed and set up a platform that implements this method and will support the research into the development of preconcentrators, sensors, and instruments for explosives detection. The platform has an open architecture with two temperature-controlled ovens and controllers for up to four heating tapes. In addition, a valve switching method has been implemented so that the output from the thermal desorption process can be directed to either of two platform outputs. One output is a waste line to discard the solvent, while the other output goes to the device or sensor under test to which the explosive vapor will be delivered. In this way, the device under test need not be exposed to the solvent vapor from the injection. This platform is also equipped with both an FID and a thermionic ionization detector for use as needed in the research. The open architecture and multiple heating control options provide a versatile platform that can be configured in various ways to support explosives detection research. 


\title{
Light Source Photocathode Performance and Development
}

\author{
Wayne P. Hess, Theva Thevuthasan, Scott A. Chambers
}

This project will develop new research capabilities for making and testing existing photocathode materials and designs. We will develop methods for measuring photophysical properties including work function, photon energy dependent electron yield (bunch intensity), and emittance (electron angular distribution).

A ccelerator photocathode technology must be advanced to meet the needs of fourth-generation light sources. The ultimate output characteristics and cost of next-generation ultraviolet or x-ray sources, whether based on free electron laser (FEL) or storage ring designs, is heavily dependent upon photocathode brightness and emittance characteristics. Novel photocathode designs could potentially reduce light source construction costs enormously — by a factor of two or more - by significantly simplifying downstream accelerator or FEL design. The international light source community recognizes the need for a more scientific approach to new photocathode development and is in the initial stages of addressing this issue; however, no consensus exists within the user community as to the mechanisms of photocathode damage. Materials science, solid-state photochemistry, and surface chemistry and analysis all can make immediate, timely contributions to this essential activity.

This project will develop new capabilities for making and testing photocathode materials and enhancing photocathode designs to improve current and future light sources in a collaborative effort. Initial goals are to increase photocathode longevity by understanding and modeling photocathode failure mechanisms. We will conduct post-mortem analysis of used (expired) photocathodes from the Jefferson Laboratory and other facilities to determine how photocathode emission yield degrades under operating photo-injector conditions. Because different types of photocathodes are in use at various light sources, it will be important to analyze expired photocathodes from various facilities. For this testing, we will interrogate various photocathode types with $\mathrm{x}$-ray photoelectron spectroscopy (XPS), two-photon photoemission (TPPE), and angle resolved photoemission spectroscopy (ARPES). The make candidate photocathodes require the design and construction of a materials growth chamber that is vacuum-coupled to an existing photoelectron spectrometer.

In FY 2010, the post-mortem analysis of expired photocathodes is underway. Initial results from helium ion microscopy, Rutherford back scattering spectrometry, and time-of-flight secondary ion mass spectrometry (TOF-SIMS) have been obtained. The evolution of surface morphology of a
GaAs based photocathode from initial conditioning to use expiration is shown below.

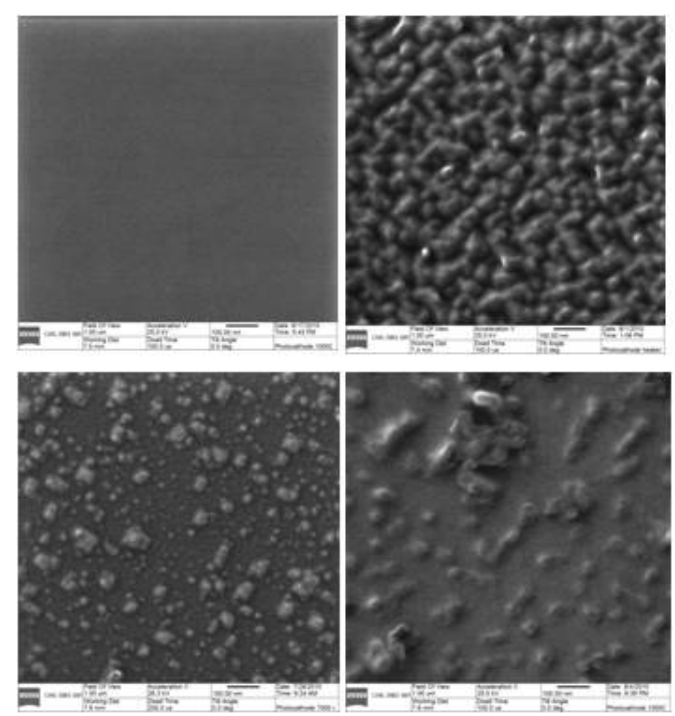

The panels are $1 \mu \mathrm{m}$ field of view helium ion microscope images of photocathode surfaces as the GaAs wafer is progressively conditioned and undergoes operation. Upper left: smooth surface of an unused wafer; upper right: first conditioning step of heating the wafer and adding a $C s$ monolayer, which results in surface roughening; lower panels: changes to surface morphology after the wafer has generated 1000 and 7000 Cs emission, respectively.

We have also begun experiments to determine the Cs surface distribution and surface and bulk impurity concentrations of photocathode samples using TOF-SIMS. Because the damage mechanism is thought to be caused by proton back-bombardment in operation, we have looked for near surface damage and hydrogen implantation in the samples by RBS. Subsequent post-mortem analysis by atom probe tomography is planned.

For FY 2011, capabilities and tools will be developed to understand the angular distribution of the emitted electrons, along with their yield as a function of laser pulse duration and wavelength. With the initial design planning complete for a multi-source thin film deposition chamber, the ultimate design will allow for in-situ growth of coated photocathodes for interrogation by XPS, TPPE, and ARPES. We will modify a photoelectron spectrometer to characterize photocathode properties, including work function, photon energy dependent electron yield (bunch intensity), and emittance (electron angular distribution).These modifications will incorporate a purpose-built photocathode growth chamber to the vacuum load lock of the photoelectron spectrometer. 


\title{
Methodology and Tool Development for Rapid Assessments for $\mathrm{CO}_{2}$ Capture Technologies
}

\author{
Charles J. Freeman, Mark D. Bearden, Greg A. Whyatt, Corinne Valkenburg, \\ Feng (Richard) Zheng, Peter C. Rieke, Paul H. Humble
}

This project is developing an engineering methodology for evaluating novel $\mathrm{CO}_{2}$ capture technologies, which requires a blend of thermodynamics, chemical process engineering, dynamic flow sheet modeling, and economic forecasting. The resulting platform will allow for the assessment of proposed technologies ranging from individual sorbent materials to significant process reconfigurations.

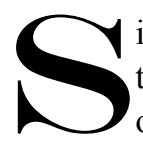
ignificant activity in the United States and around the world centers around understanding the efficacy of capturing and sequestering $\mathrm{CO}_{2}$ from coal-fired power plants for reducing atmospheric greenhouse gas emissions. Commercial $\mathrm{CO}_{2}$ capture technologies exist but at much smaller scales than what will be required for global $\mathrm{CO}_{2}$ reduction targets. Further, the existing capture technologies have forecasted power plant output efficiency reductions of nearly 30 percent, which makes justifying their implementation difficult if not impossible. A large number of energy efficient $\mathrm{CO}_{2}$ capture technologies have been proposed to address the implementation barrier. Technology leaders in the area of gas separations, including groups within DOE, are now each struggling for a rigorous determination of the most promising direction to reach the desired goal. The challenge is forecasting the technology impacts on a power-plant scale, which cannot be achieved with small-scale experimentation alone.
Commercialization of any new carbon capture system for retrofitting power plants will not occur for several years. This delay is based on three significant needs: to prove technology viability at a large scale, to prove $\mathrm{CO}_{2}$ sequestration technology, and for policy implementation to make utilization of these systems economically viable. During this period, numerous research and developmental efforts are ongoing to advance coal-fired power plant retrofit $\mathrm{CO}_{2}$ capture technologies. Current research is focused on retrofitting existing pulverized coal power plants to capture of at least 90 percent of $\mathrm{CO}_{2}$ emissions while increasing the cost of electricity by no more than 20 percent. Many concepts have been proposed for achieving these goals, including advanced aqueous-based solvents, conventional and advanced solid sorbents, oxy-combustion, enriched air combustion, chemical looping, enzyme-enhanced capture systems, cryogenic separation, polymeric and ceramic membranes, ionic liquids, and electrically switchable materials.

The objective of this project is to develop a structured methodology that will allow for efficient assessments of currently proposed and future carbon capture technologies. Our initial focus was on sorbents (solids and liquids), but work was conducted on several other gas separation technologies as well. This included the evaluation of a number of current technologies funded by the DOE to determine overall efficacy.

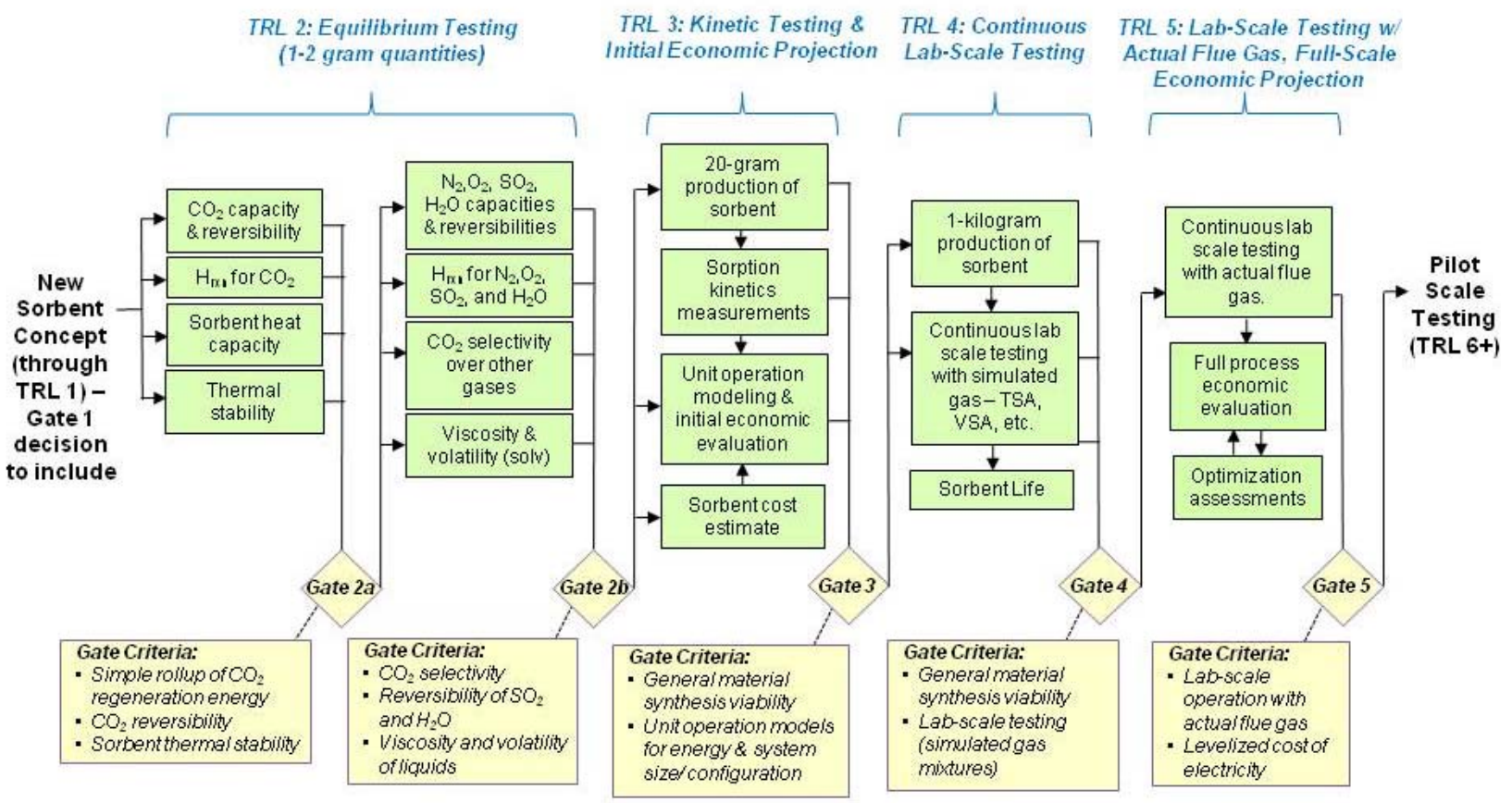

Stage gate process for evaluating and progressing sorbent (solid and liquid) development. 
Key milestones for the sorbent evaluation portion of the project are to: 1) deliver a solid, solvent screening methodology linking laboratory measurements and numerical modeling, 2) validate solid and solvent models using zeolite $13 \mathrm{X}$ and MEA, respectively, and 3) support assessments of PNNL solid and solvents through Gate 3. Summaries of the progress made toward these milestones are provided in the following sections.

The stage gate process shown was developed in the current project for screening both solid and liquid sorbents at PNNL. The stage gates correspond to key decision/evaluation points in the development of sorbent materials. As such, the gates are aligned with the technology readiness level (TRL) definitions currently used by DOE. Gates $2 \mathrm{a}$ and $2 \mathrm{~b}$ correspond to equilibrium testing associated with sorbents, Gate 3 corresponds to kinetic testing, and Gates 4 and 5 correspond to laboratory-scale demonstration/testing with scaled-up amounts of the given sorbent.

At the bottom of each gate, the associated criteria are shown. A given sorbent would need to be assessed against the identified criteria to determine whether advancement to the next gate/TRL is appropriate. A combination of experimental and modeling is required to provide a detailed assessment of each gate criteria (outlined elsewhere). The identified experimental needs were determined based on modeling input requirements in both ADSIM (for solid sorbents) and ASPEN Plus (solvents). The specific analytical techniques were determined by expert assessments of in-house PNNL capability and reported techniques in the open literature. PNNL's involvement with the University of Texas, Austin allowed for specific guidance in solvent testing and modeling. A unique kinetics model was also developed in Comsolv for assessing ammonia-water solvents with carbonic anhydrase. Screening models were developed in each of these platforms to allow for each of the gate evaluations. These screening models were validated against zeolite $13 \mathrm{X}$ (for solid sorbents) and MEA (for solvents) as relative references for advanced materials.

Several gate assessments of the PNNL sorbents were made during in FY 2010. Gate 2a consists of a simple rollup of $\mathrm{CO}_{2}$ regeneration energy, $\mathrm{CO}_{2}$ reversibility, and sorbent thermal stability. Specific data are used to predict the minimum energy required for the $\mathrm{CO}_{2}$ separation for a Gate $2 \mathrm{a}$ regeneration energy calculation. The regeneration energy is compared to that of a MEA base case for solvents, 13X for solids, and a 35 percent increase in the levelized cost of electricity. Hidden data are fit to the temperature-dependent Langmuir isotherm equation. Though there can be some discrepancies between the equation fit and the data, these issues do not typically have a significant impact on regeneration energy calculations. Including the heat of adsorption calculated from the temperature dependence, the equation is used to calculate the amount of $\mathrm{CO}_{2}$ processed during an adsorptionregeneration cycle and the regeneration energy called for in Gate 2a.

The amount of heat needed to regenerate PNNL's silicalite adsorbent was calculated as a function of regeneration temperature. Adsorption temperatures of 25 and $35^{\circ} \mathrm{C}$ were used. Our results showed that the Silicalite did not appear to perform as well as $13 \mathrm{X}$ or MEA as a $\mathrm{CO}_{2}$ sorbent. However, the expected benefits from a kinetics perspective allowed for a decision to pass to Gate 3 testing.

The Gate 2a assessment performed for chitosan included a 10 percent $\mathrm{CO}_{2}$ uptake observed with the first moisture-conditioned sample and 4 percent $\mathrm{CO}_{2}$ uptake for the second condition. None of the higher temperature isotherms produced any significant $\mathrm{CO}_{2}$ uptake or quantifiable isotherm shape. This means that heat of adsorption estimates could not be determined. Further, the results indicated that any realistic operating temperature ranges will compromise the activity of the proposed material. This outcome was also consistent with the earlier "dry" chitosan measurements. Based on this information, the technology was not passed through Gate 2a in the established stage gate process.

Many of the additionally planned gate analyses were not feasible in FY 2010 because of delays in gathering the adequate experimental data for PNNL sorbents and solvents. Nevertheless, the stage-gate evaluation approach has been fully developed to accommodate those gate analyses as data become available. The unexpected time availability during FY 2010 allowed for us to begin work on non sorbent-based technology evaluations that were originally planned for FY 2011. Some of the key activities performed included the following:

- Full plant solid sorbent assessment.

- Initial assessment of cryogenic flue gas cleaning.

- Novel compression physical sorbent concept developed.

- Integrated plant assessment completed for catalyzed potassium carbonate.

- Assessments of multiple carbon capture technologies.

Several of the assessments of current DOE-funded technologies serve as the key foundation for developing the rapid screening process for non-solvent technologies. This will be the key focus our FY 2011 activities. 


\section{Molecular Structure and Interaction at Aqueous, Non-Aqueous Liquid Interfaces and Catalytic Solid Surfaces}

Hongfei Wang

Using cutting-edge instrumentation, this project will lead to new discoveries in surface science and have broad applications on characterization of the structure and interactions at various molecular interfaces that are critical but elusive in many scientific and engineering fields.

$\mathbf{T}$ he interface selectivity and submonolayer sensitivity of the second order nonlinear optical spectroscopy make it a unique tool to study the structure and interaction of molecules at various surfaces and interfaces relevant to energy as well as environmental and biological processes. In the second order optical method, two photons with the same frequency (second harmonic generation) or different frequencies (sum-frequency generation) interact simultaneously with the same set of atoms or molecules to generate a new photon at the sum of the frequencies. The symmetry requirement for these processes forbids the processes from the bulk liquid or amorphous solid with centrosymmetry but allows them to be generated from the surface or interfaces that always have asymmetry. The new photon generated is also background free from the large number of incoming photons, so it allows sensitive detection such as photon counting for submonolayer level vibrational and electronic spectral measurement.

For the past two decades, scientists have been using this tool to understand and characterize the details of the structure and interactions at various molecular surfaces and interfaces. PNNL played leading roles in developing the experimental methodology and theoretical treatment to push forward the

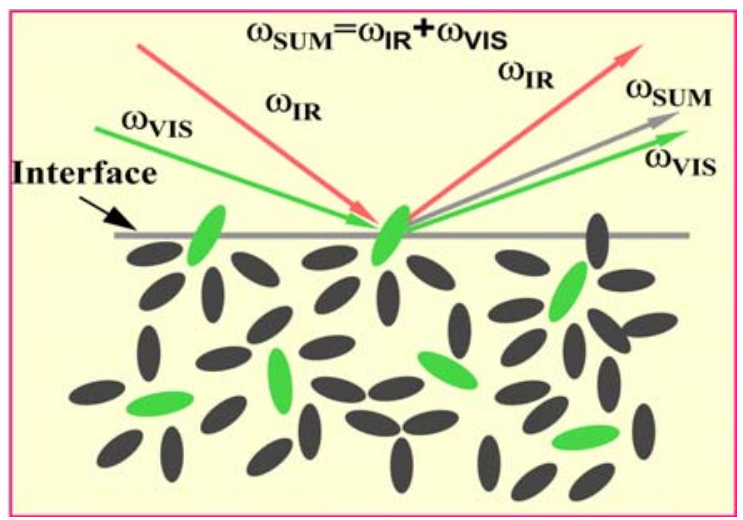

Sum-frequency is selectively generated from the molecules at the interface and is spatially and spectrally separated from the incoming laser beams, allowing background-free photon counting for submonolayer sensitivity measurement of the vibrational or electronic spectra of interfacial molecules. advancement in this field from the simplest air/liquid interface to more complex liquid/solid, liquid/liquid, liquid/membrane, and nanoparticle interfaces. This has been a growing field and is ready for exploring applications related to molecular and biological surfaces and interfaces.

This project aims to employ the recent development of ultrafast laser technology to push the surface nonlinear spectroscopy and dynamics unprecedentedly to higher spectral resolution, time resolution, and detection sensitivity in order to solve problems with complex molecular surfaces and interfaces. It is a unique capability that will positively impact in understanding molecular surface science, especially to complex surfaces that ubiquitously exist in energy, environmental, and biological systems that cannot be accessed or studied in situ and at ambient or extreme conditions using other x-ray, electron, or ion probe tools and instrumentations.

This project commenced early in the 2010 calendar year to support cutting-edge experimental and theoretical studies applicable to various aqueous, non-aqueous liquid interfaces and catalytic solid surfaces. We developed and employed the unique surface nonlinear spectroscopy and dynamics capabilities using state-of-the-art ultrafast lasers together with the quantitative methodology in nonlinear spectroscopy and dynamics to investigate important interfacial problems in the energy, environmental, and biological heterogeneous and homogeneous chemical processes. The key milestone to successful completion of this project rests on the successful design, construction, and testing of the complete laser system; the nonlinear optical experimental setup; and the various optical cells to be used for various surface measurements. All design and construction have been underway and will be setup and tested by the end of 2010 .

Other project progress has included construction of the ultrafast laser instrumentation, anticipated for completion in late 2010. This instrument will be the first system in the world to provide better than $1 \mathrm{~cm}^{-1}$ spectral resolution (ultimate resolution is $0.2 \mathrm{~cm}^{-1}$, compared with $10 \mathrm{~cm}^{-1}$ in other laboratories) with $35 \mathrm{fs}$ ultrafast time resolution (typically $100 \mathrm{fs}$ ). The specific infrared-visible, visible-visible sum frequency generation spectroscopy and ultrafast time resolved dynamics instrumentation using the above laser system have been designed and are under construction. For FY 2011, the design and construction of the liquid flow cell for liquid/solid interface and gas reaction cell for catalytic gas/solid interface will be completed and delivered for testing. 


\title{
Multiscale Investigation of $\mathrm{CO}_{2}$ Behavior in Subsurface Under Extreme Conditions
}

\author{
Alexandre M. Tartakovsky, Bruce J. Palmer, Uditha C. Bandara
}

This project addresses the fundamental challenge of understanding the properties and processes associated with complex and heterogeneous subsurface mineral assemblages comprising porous rock formations and the equally complex fluids that may reside in and flow through those formations. Specifically, we focus on understanding the geochemical interactions of the caprock with ambient water and supercritical and dissolved $\mathrm{CO}_{2}$.

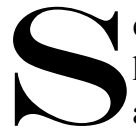
equestration of $\mathrm{CO}_{2}$ in deep geologic reservoirs has proven to be a viable way for stabilizing global atmospheric concentrations of greenhouse gases, and providing the time needed to transition from principally fossil fuel energy sources to renewable alternatives. Despite considerable research effort, little is known about chemical and physical interactions of $\mathrm{CO}_{2}$ with water and mineral phases. At the same time, existing field scale models of $\mathrm{CO}_{2}$ sequestration rely heavily on constitutive relationships that describe interactions of different solid and liquid phases in subsurface environments. Because these relationships are not grounded in first principles, the accuracy of the field-scale models is difficult to estimate a priori. A comprehensive study of the complex coupling between sorption kinetics and $\mathrm{CO}_{2}$ /water composition, temperature, and pressure is difficult to conduct with laboratory experiments due to the extreme pressure and temperature conditions relevant to $\mathrm{CO}_{2}$ sequestration. Pore-scale models proved to be a valuable tool for studying reactive transport, the effect of complex pore morphology on entrapment and dissolution of non-aqueous phases and constitutive relationships governing multiphase flow in porous media.

Given these challenges, we are developing a multiscale, multiphase flow and transport model to simulate $\mathrm{CO}_{2}$ behavior (flow and precipitation) in the caprock at the pore and subpore scales. This model will combine kinetic Monte Carlo (KMC) model of surface reactions with smoothed particle hydrodynamics (SPH) model of pore-scale flow and reactive transport. KMC models alone cannot cover all the length and time scales necessary and relevant to understanding the fundamental processes involved in $\mathrm{CO}_{2}$ sequestration. The hybrid model will bridge the molecular and pore-scale models of the $\mathrm{CO}_{2}$ behavior in subsurface. In addition, the hybrid model will serve as a basis for upscaling $\mathrm{CO}_{2}$ behavior from fundamental scales to scales of practical importance.

To date, we have implemented an efficient atomistic scale approach, namely the KMC method, which can be used for modeling the long time evolution of a particular atomistic system. We applied the $\mathrm{KMC}$ method to model $\mathrm{CO}_{2} /$ water mixtures in contact with calcite surfaces. The model can be used to describe the kinetics of calcite precipitation/dissolution as a function of temperature, $\mathrm{pH}$, and $\mathrm{CO}_{2} /$ water composition. In particular, the model can account for the interplay between the effects of the decrease in $\mathrm{pH}$ and the decrease in calcite solubility as the $\mathrm{CO}_{2}$ content increases. We also developed a hybrid KMC diffusion equation method that couples the standard KMC model with a numerical solution of diffusion equation. This significantly extends the range of application of the KMC method by adding the bulk fluid diffusion.

Also in FYs 2009 and 2010, we developed a pore-scale, smoothed particle dynamics model that simulates the dissolution of liquid $\mathrm{CO}_{2}$ in water and the precipitation/dissolution of calcite. The model is implemented as a part of a general purpose high performance SPH code. The scalability of the code was tested on PNNL's Chinook supercomputer. A good scaling performance was observed for up to 1000 CPUs. A Darcy scale fully Lagrangian particle model for flow and transport was developed and used to simulate gravity driven unstable flow of fluids with different densities. This model will be used to investigate the downward migration of dissolved $\mathrm{CO}_{2}$. The preliminary numerical simulations of micro-fluidic, two-fluid experiments were performed. We developed a novel dimension reduction method for large ODE systems that can significantly accelerate pore-scale simulations regardless of the nature of a numerical solver. The method relies on a computational closure, which is achieved via short bursts of a pore-scale model conducted in small portions of the computational domain, of averaged evolution balance equations. The dimension reduction model was used to simulate flow and transport with mixing controlled reactions and mineral precipitation. The good agreement with microfluidic experiments confirms the accuracy and computational efficiency of the dimension reduction model. Two papers summarizing this research were published in peer-reviewed journals, and a paper describing the model reduction method is in preparation.

Throughout FY 2011, we will continue developments of the multiscale models. The goal is to develop a code able to simulate flow on a laboratory scale and/or scale of a representative elementary volume. Such code can also be used to guide and interpret laboratory experiments and to create a database that can be used in advanced reservoir simulators. Generally, we aim for the project to result in unique capabilities for understanding complex geochemical interactions of heterogeneous subsurface environment with fluid mixtures. 


\title{
Multiscale Modeling from Molecular Reactions to Catalytic Reactors
}

\author{
Donghai Mei, Guang Lin, Alexandre M. Tartakovsky, Roger J. Rousseau, Michel Dupuis
}

This project aims to develop a new modeling capability for reactor-scale catalytic processes informed by lower-scale simulations of chemical transformations on catalyst particles and molecular-scale characterization of reactive processes at interfaces.

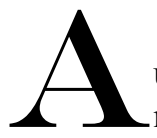
formidable challenge in catalysis research is understanding the mechanisms and dynamics of molecular transformations over catalyst materials under operating reaction conditions. Molecular-level knowledge about catalytic reactions can enable one to design catalysts with improved efficiency (selectivity, yield, and cost) of targeted products. However, operating conditions in reactors cause inhomogeneous temperature and pressure gradients experienced by the catalyst particles, which dramatically complicates the macroscopic prediction of global kinetics and reaction yields.

We are developing a new module that describes heterogeneous catalytic processes from the quantum chemistry level up to reactor level under practical operating reaction and flow conditions. The most challenging task in this unique computational module is linking the various theories and models at different time and length scales seamlessly and dynamically. We attempted this linkage by modeling a nonuniform flow phase using both kinetic Monte Carlo (KMC) and stochastic partial differential equation (SPDE) methods. The power of the new capabilities is highlighted for specific catalytic systems by elucidating and predicting the catalytic transformations under practical reaction and flow conditions of a reactor.

In FY 2009, we developed the KMC simulation module coupling with three-dimensional grids describing the flow phase above the catalysts surface. In order to test the new hybrid module, a benchmark catalytic system was created, with carbon monoxide oxidation kinetics over rutile ruthenium oxide surface at varying temperature and gas phase composition conditions. This microscale model reaction information then was passed to a macroscale model in a form of a mass flux boundary condition.

We expanded the KMC model for FY 2010 with a second order grid-based finite-difference Crank-Nicolson continuum that describes the heat and mass transport in the stagnant reactive gas phase. The new, integrated computational framework consists of a surface phase domain (where catalytic surface reactions occur) and a gas-phase boundary layer domain imposed on the catalyst surface (where the temperature and pressure gradients exist). The temperature and pressure gradients in the gas-phase boundary layer were the consequence of thermal and molecular diffusions of reactants and products under reaction conditions. At each time step, heat and mass fluxes between two domains were calculated simultaneously until the steady-state reaction condition was reached, during which the activity, surface coverages of reaction intermediates, and temperature and pressure gradient profiles in the gas-phase boundary layer are statistically constant.

To demonstrate the idea that proper consideration of heat and mass transfer in the reaction environment is essential to accurate measurement of the intrinsic reaction kinetics, we investigated the kinetics of $\mathrm{CO}$ oxidation over the $\mathrm{RuO}_{2}(110)$ catalysts using the newly developed hybrid model. By varying the catalyst thickness, the pronounced temperature and pressure gradients are formed in the gas-phase boundary layer. Our simulation results indicate that the temperature and pressure gradients caused by the heat and mass transfer could dramatically affect the observed intrinsic reaction kinetics under presumed nominal operating reaction conditions. This work has been submitted to the peer-reviewed journal Catalysis Today.

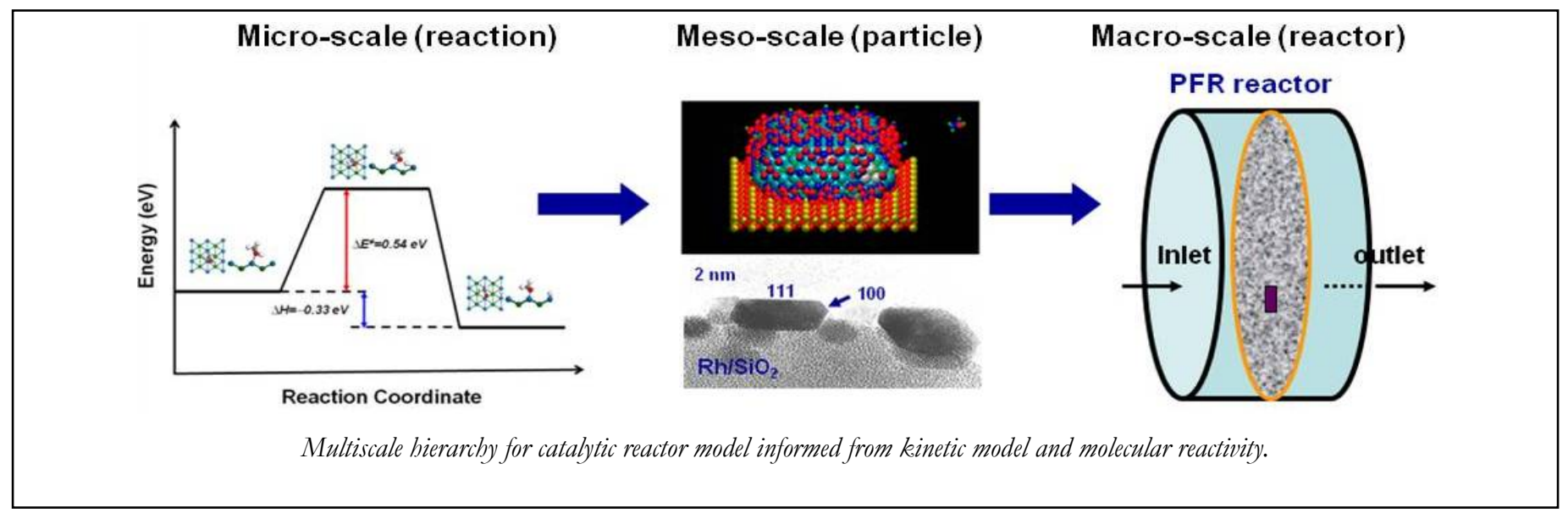


Also in FY 2010, we developed a novel theoretical and numerical model that combines particle simulations and finite-difference partial differential equation (PDE) models applied for reaction diffusion systems at low concentration. Unlike most of existing stochastic and deterministic finite-difference models, instead of deriving from macroscopic PDE or SPDE, our new model is formulated in terms of discrete rather than continuous probability distributions. At high concentration limit, our diffusion model becomes equivalent to the diffusion SPDE. The simulation results of reaction-diffusion systems have shown that our discrete stochastic approach can capture phenomena previously only observed in particle simulations but with much greater computational efficiency. This work was published in the peer-reviewed journal Computer Physics Communications. Currently, we are preparing two more papers and will present an in-depth analysis of this new numerical methodology and how to incorporate proper stochastic boundary conditions and chemical kinetics.
During FY 2011, we will expand the three-dimensional SPDE with a complex (rather than simple, as in the KMC model) multi-component model reaction and couple a three-dimensional (increased from two) KMC with a three-dimensional (increased from one) SPDE under flow condition. Our focus will be on the generalization of the newly developed multiscale module. In particular, we will extend the model to include a more complex reaction system such as methanol synthesis from $\mathrm{CO}_{2}$ hydrogenation on a copper catalyst. To achieve this goal, two major tasks will be performed. First, the hybrid model will be effectively parallelized. A parallelized KMC simulations coupled with reactive flow boundary conditions will be implemented. Second, we will develop the new sub-domain boundary conditions used in the time progression of the simulation. Two different communication techniques between the sub-domains will be tested. This would allow us to select freely sub-domain dimensions (e.g., length, width) and integration time steps, because sub-domains would be integrated separately and coupled only through boundary conditions. 


\title{
Non-Metal Activation of Hydrogen for Energy Storage in Chemical Bonds
}

\author{
Donald M. Camaioni, Abbijeet J. Karkamkar, Kshitij K. Parab, S. Thomas Autrey
}

Activation of small molecules such as hydrogen, carbon dioxide, methane, and nitrogen is critical for development of renewable fuels. This project aims develop mechanistic understanding of the activation of hydrogen by non-metal Lewis pairs to be used in catalytic activation of small molecules.

W hile activation of hydrogen using transition metal catalysts is widely practiced, hydrogen activation with non-metals is less common and little understood. This lack of understanding hinders development of new and inexpensive catalysts for energy storage applications. To solve this problem, we are working to characterize Lewis acid-Lewis base pair properties in solution and solid-phase environments relevant to hydrogen activation using a suite of experimental methods (nuclear magnetic resonance spectroscopy, calorimetry, and x-ray analyses) in combination with computational electronic structure methods (density functional theory and molecular orbital). This information will be used to develop simple predictive relationships. In addition, we are scouting for potential uses of this chemistry as a means to store energy in fuels and chemicals.

During project initiation in FY 2010, we obtained results in three thrust areas: theoretical modeling of structure and reaction paths; synthesis of inter- and intramolecular Lewis acid-Lewis base pairs; and reactivity and thermochemistry in solution, condensed phases, and at interfaces. Our computational studies were aimed at understanding the pathways of prototypical reaction of hydrogen with ammonia and a Lewis acid. Our analysis pointed to advantages of bifunctional systems in which Lewis acid and Lewis base are present in the same molecule to overcome energy and entropy penalties of forming a termolecular reactive complex. We screened several acid-base pairs, computing structures reaction paths and energetics. From this, we selected promising systems to target for synthesis and experimental study. This work is now in progress.

Simultaneously, we are interested in obtaining the thermodynamic parameters for the processes involved in the hydrogen activation by frustrated Lewis pairs using calorimetry. Differential scanning calorimetry experiments in the solid state suggest an enthalpy of dehydrogenation dependent on the Lewis base involved and are endothermic in nature. We are currently developing methodologies to measure these parameters in the solution phase using select solvents capable of solvating both adduct and ion pair. In situ nuclear magnetic resonance experiments are currently underway to determine some of the thermodynamic parameters in solution. Our initial experiments suggest that this is also dependent on the Lewis base involved. An in situ experiment between 2,6-lutidine and tris(penta-fluorophenyl)borane was carried out at room temperature under a pressure of $100 \mathrm{psi}$ of hydrogen. The formation of the ion-pair and disappearance of the Lewis pair was monitored by nuclear magnetic resonance. The reaction went to near completion in $12 \mathrm{hrs}$.

We are currently studying the effect of temperature on rate of ion-pair formation. In contrast, the reaction between tetramethyl piperidine and tris(pentafluorophenyl)borane was very rapid, completed in under $5 \mathrm{~min}$. Based on our initial studies, we assume that we will be able to use these techniques to correlate both the structure and reactivity of these frustrated Lewis pairs. Efforts toward incorporating Lewis pairs on the surface have been developed using grafting of boronic acid functionality on the mesoporous silica surface. Grafting of boron functionality was achieved in loading densities as high as $7 \mathrm{mmol} / \mathrm{g}$ of silica. Formation of the Lewis pair was observed by solid-state nuclear magnetic resonance as a new signal present at a chemical shift consistent with the expected species. We are in the process of measuring hydrogen uptake and further optimizing the system. We also performed preliminary experiments in understanding role of borates in formation of frustrated Lewis pairs. This study is aimed at understanding reactivity of hydrogen toward these Lewis pairs and carbon dioxide.

In the next year, we will continue the above thrusts and begin a thrust on hydrogenation of unsaturated compounds using Lewis pairs with the purpose of elucidate requirements for catalytic hydrogenation $\mathrm{H}_{2}$ by Lewis acid-Lewis base pairs. We will also be elucidating requirements for the transfer of hydrogen from ion pairs to unsaturated molecules.

Linked Lewis pair of 2-pyridyldifluoroborane undergoing reaction with molecular bydrogen The structures and bond lengths for reactant, transition state, and product are shown. 


\title{
Oxygen Optode for Chemical Imaging in Microfluidic Microbial Models
}

\author{
Jay W. Grate, Marvin G. Warner, Norman C. Anheier Jr.
}

Microorganisms that play crucial roles in nutrient, geochemical, and energy cycles on the earth do not act alone; they perform as members of microbial communities. This project is developing analytical methods based on fluorescent imaging (optodes) to observe oxygen concentrations within micromodels containing microorganisms.

A new method of studying the spatial heterogeneity within and models of microenvironments focuses on planar optodes capable of chemical imaging in two dimensions. The optodes consist of thin films that contain chemically sensitive fluorescent dyes. Combined with advances in optical imaging techniques and digital instrumentation, planar optodes extend the previous work in fluorescent point sensors and are compatible with recent advances in developing microfluidic flow cells as models of heterogeneous environments

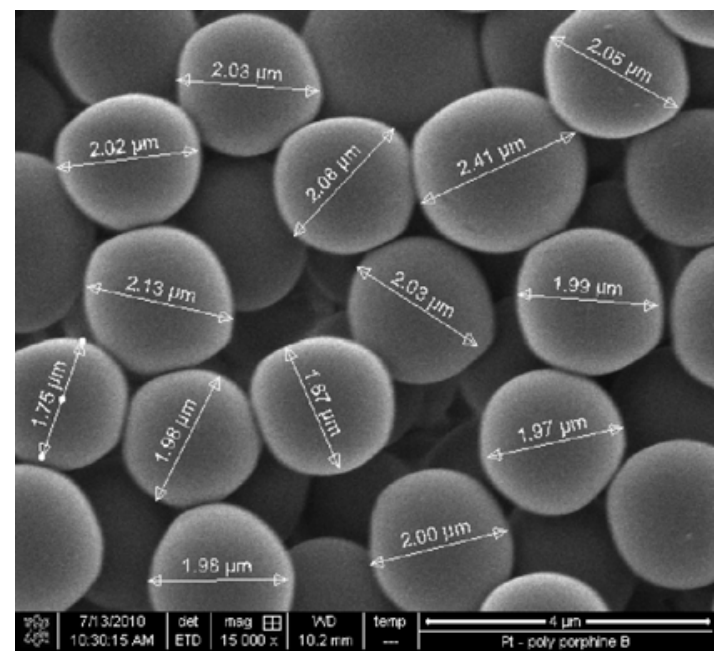

As seen by scanning electron microscopy, polymer microspheres contain oxygen-sensitive fluorophore molecules.

at the submillimeter scale.

This project is both developing planar optodes for sensing in two dimensions to incorporate into microfluidic flow cell model systems and creating the capability to implement multi-parameter imaging platforms for microbial community research. As oxygen is a key parameter in many microbial systems, we will demonstrate for the first time two-dimensional oxygen imaging using a ratio of insensitive and sensitive luminescent peaks from the same dye molecule to simplify the measurement and implementation in microfluidic micromodels. Further, we are investigating the combination of peak ratio measurements and fluorescent lifetime measurements in order to improve the stability of planar optode measurements and advance the precision with which small concentration changes or gradients can be detected.
Key parameters identified for chemical mapping include oxygen, carbon dioxide, and $\mathrm{pH}$. Each of these materials can be sensed optically using fluorescence measurements, where the luminescence (or a ratio of luminescent features) is dependent on the parameter of interest. Devices to detect these analytes were originally developed in FY 2009 as single-point sensors by applying a polymer film containing a chemically sensitive fluorescent dye(s) to the end of an optical fiber. Fluorescent sensors for gases and electrolytes were developed as single particle or bead sensors and are designed to be reversible, thus responding to changes in the sensed parameter.

In developing the measurement capability in FY 2010, we investigated a number of fluorescent sensing materials and material formats. For example, the synthesis of a dithiolene complex for ratiometric oxygen sensing was investigated but judged unlikely to succeed. We tested a commercial sol-gel film containing an oxygen-sensitive ruthenium complex, but film uniformity proved to be unsuitable for imaging applications. Conversely, bead-based methods for lifetime oxygen imaging were much more promising. Nanospheres containing a platinum complex proved to be oxygen-sensitive, and microspheres with an oxygen-sensitive platinum complex were synthesized in our laboratories. Nanosphere- and microsphere-based methods will enable point sensing within the micromodels at specific locations of interest.

In addition to the fluorescent films, this capability required several items of apparatus. We chose planar microfluidic flow cells with inputs for oxygen containing water and oxygen-free water and the means of creating oxygen gradients. To deliver these solutions to the flow cell, we obtained fluidic equipment with a gas-mixing apparatus to generate dilute oxygen concentrations in the gas phase to equilibrate aqueous solutions to desired oxygen concentrations. We also designed and constructed a laser scanning imaging station for optical measurements, enabling the examination of fluorescent films in three distinct imaging modes - intensity, ratiometric, and lifetime. Oxygen sensing is based on the reduced lifetime and intensity of the fluorescence in the presence of oxygen. Using ratiometric or lifetime imaging techniques, one can compensate for processes that might otherwise cause signal drift and inaccuracies.

Work during FY 2011 for this project will focus on the incorporation of oxygen-sensing films and particles into micromodels for microbial research. These methods will enable imaging oxygen gradients that are created fluidically or by microbial activity within the micromodel environment. Intially, we will demonstrate the oxygen-sensing methods in simple flow cells, then in pore network micromodels, and finally in micromodels containing microbes of interest. 


\title{
Proof-of-Principle Demonstration of Fluorescence Labeling of Cellulose and Mcroscopic Fluoresence Imaging of Cellulose Degradation
}

\author{
Fred J. Brockman, Jay W. Grate, Marvin G. Warner, Dehong Hu, Galya Orr, Keith D. Miller, Yongsoon Shin
}

We are developing a method for time-course imaging of the enzymatic removal of fluorescently labeled cellulose from a surface at the micron scale with extremely high analytical precision.

$\mathbf{M}$ icrofluidic models that incorporate key physical, chemical, and microbiological properties of a specific environment can be used to improve the mechanistic understanding of how complex environmental systems behave. A key feature of micro-models is that they are engineered to enable the application of multiple imaging-based technologies to interrogate multiple processes (e.g., cellulose degradation and oxygen consumption) non-destructively over space and time. Existing methods for studying cellulose degradation address the batch scale (versus the sub-millimeter scale) and monitor production of soluble cellulose metabolites (versus loss from the surface). Monitoring of soluble cellulose metabolites does not provide for mass balance and does not provide the parameters needed for mathematical modeling of the initial (and rate limiting) reactions in cellulose degradation. Fluorescent labeling of molecules such as substrates and enzymes is commonplace for studying nanoscale mechanisms such as cellulose synthesis and binding of enzymes to cellulose.

The capability to measure the destruction and removal of insoluble cellulose from a surface at the micron scale with extremely high analytical
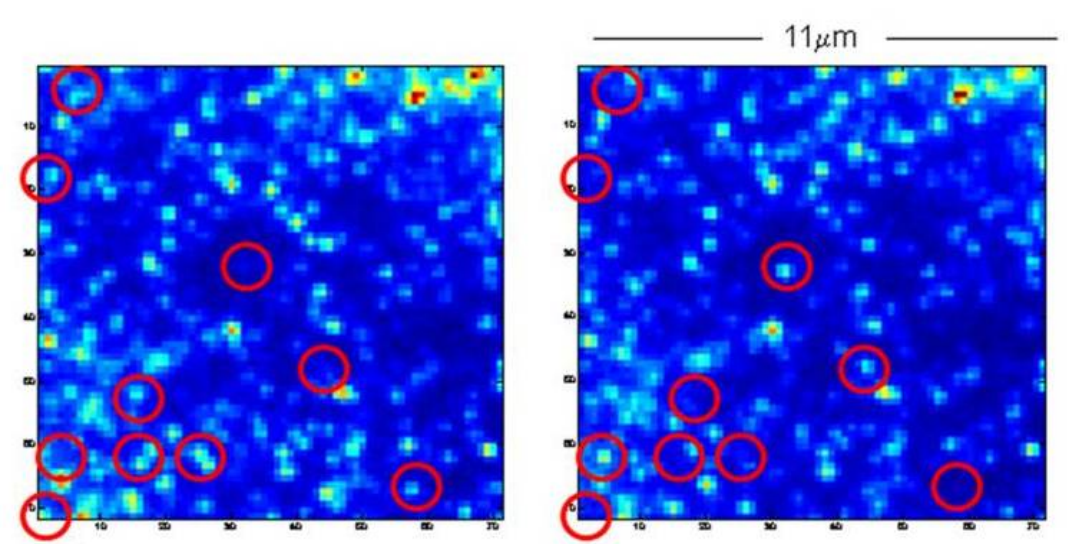

Imaging of individual dye molecules in a discontinuous film of labeled cellulose nanocrystals. To locate all blinking dye molecules, software analyzes an ensemble of several 100-ms exposures. Two such exposures are shown, with dyes shown in only one image circled. As cellulose is degraded, dyes are released from the surface to solution and flow away. Single-molecule imaging is done with total internal reflection fluorescence, which interrogates approximately $200 \mathrm{~nm}$ into the sample.

microbial processes with insoluble cellulose as the carbon and energy source. In short, we expect to develop and demonstrate for the first time proof-of-principle for imaging enzymatic removal of fluorescently labeled cellulose from a surface at the micron scale with extremely high analytical precision.

This research approach involved testing of various combinations of two cellulose starting materials (microcrystalline and nanocrystalline), two fluorescent materials (Alexa dyes and FastLime protein), three labeling methods (two covalent and one molecular interaction), and two imaging modalities (single-molecule TIRF and confocal). Photobleaching was minimized by using blinking dyes and single-molecule imaging (low laser powers and short exposures). All three of the labeling methods were successful. Pre-modification of dye so that it reacted with (unmodified) cellulose provided the best imaging results; this method labeled approximately 1.2 percent of the sugar residues in the cellulose nanocrystals. Nanocrystals prepared with this approach were drop casted onto glass, and individual dye molecules were imaged. Additionally, all of the blinking dye

molecules are identified with an ensemble of exposures. Labeled cellulose films were exposed to a mixture of cellulytic enzymes, and enzymatic removal of cellulose from the surface was followed by imaging loss of dye molecules over time. Cellulosedegrading microbes were shown to grow on suspended cellulose nanocrystals.

The proof-ofcurrently exist. To meet this need, this project will combine fluorescent labeling of cellulose with imaging of the enzymatic removal of fluorescently labeled cellulose from a surface at the micron scale with extremely high analytical precision. We are using micro-models to study the sub-millimeter scale principle was completed at the end of FY 2010. This method will be further refined in a separate LDRD project devoted to conducting microbial cellulose degradation studies in microfluidic models. 


\title{
Speciation and Distribution of f-Elements from Enhanced Separations and Safeguards
}

\author{
Amanda J. Casella, Tatiana G. Levitskaia, Samuel A. Bryan, James M. Peterson
}

Extensive knowledge of real-time solution properties and composition are a necessity for any spent nuclear fuel reprocessing method. This work focuses on the coordination chemistry and interfacial transport behavior of lanthanides and actinides in various reprocessing schemes and the advancement of online spectroscopic techniques that can provide real-time monitoring of solution properties.

$\mathbf{U}$ nderstanding the coordination chemistry and interfacial transport behavior of lanthanides and actinides in complex heterogeneous systems is required to design, characterize, and predictably model an f-metal separation system successfully. Currently, the chemical state of f-metals in liquid-liquid separation systems is focused on the acidic aqueous phase, but there is unfamiliarity with the chemical behavior of f-metal ions in corresponding organic media. With a chemical similarity between lanthanides and actinides, successful separation requires precise knowledge of the coordination and transport behavior in the complicated separation system and constitutes a challenging technical framework for the proposed investigation. Once acquired, this information can be translated to different separation processes.

In this work, TALSPEAK (separation of americium and curium from rare earths) serves as a primary platform for understanding phenomena such as donor-acceptor interactions, chemical selectivity, and solute release during liquid-liquid extraction, leading to enhanced capabilities to monitor, predict, and the model solvent extraction process. TALSPEAK extraction chemistry of f-metals introduces kinetic constraints that may prevent the system from reaching local thermodynamic equilibrium in counter-current separation equipment such as centrifugal contactors, making it difficult to project batch contact data for flowsheet modeling. Using the centrifugal contactor system equipped with spectroscopic monitoring equipment enables investigating chemistry and speciation of f-elements under operational flow conditions.

We investigated speciation and distribution behavior in PUREX- and TALSPEAK-like spent nuclear fuel reprocessing schemes. Testing involved measuring the distribution behavior of extraction system constituents between aqueous and organic phases and speciation phenomena such as reduction/oxidation changes and interaction effects in the organic phase. The liquid-liquid distribution of several components in the equilibrium batch extraction experiments and in subequilibrium flow extraction using centrifugal contactors was compared. In the centrifugal contactor system, the raffinate outlet line was instrumented with optical probes, allowing for continuous Raman and vis/NIR spectroscopic monitoring. Centrifugal contactor testing identified locations and types of instrumentation necessary to enhance online monitoring capabilities. Additional spectroscopic monitoring at the feed and loaded solvent lines were added to the non-radioactive contactor bank, along with continuous data acquisition of temperature, flow rate, density, and conductivity.

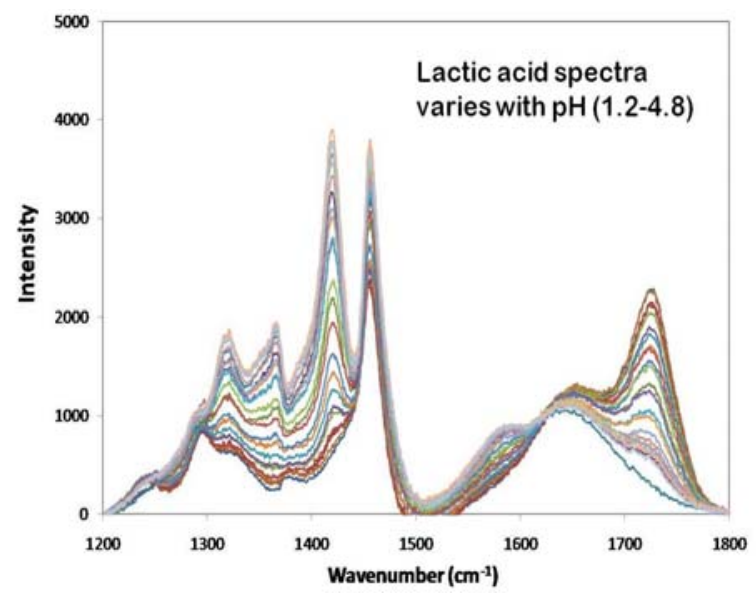

Spectroscopic methods provide a pathway for online $\mathrm{pH}$ measurements in aggressive chemical/ radiation environments.

Free acid/pH online monitoring is of critical importance, as processes such as aggregation, the formation of polynuclear species, coordination to specific receptors, and hydration/ solvation that influence extraction efficiency and selectivity are $\mathrm{pH}$ dependent and cannot be evaluated properly without monitoring the aqueous acid concentration. The potential for online monitoring of strong acid concentration and weak acid buffer $\mathrm{pH}$ using Raman, Fourier transform infrared, and vis/NIR spectroscopic measurements has been investigated, as classic potentiometric $\mathrm{pH}$ measurements are not amiable for online measurements from frequent calibration/maintenance requirements and poor long-term stability in aggressive chemical and radiation environments. Spectral databases were complied for strong and weak acid solutions with effects of chemical composition, temperature, acid concentration, and ionic strength. These spectroscopic measurements were used to generate training data sets and to develop partial least square chemometric models for the quantification of analytes of interest in spent fuel reprocessing streams.

In FY 2011, we will continue validating spectroscopic modifications and implementing the radioactive contactor bank. Chemometric models will expand as needed to incorporate flow parameters and validated using the centrifugal contactor extraction system. 


\title{
Transformational Materials for Advanced Stationary Electricity Storage
}

\author{
Zhenguo. (Gary) Yang, Yulain Chao, Gordon L. Graff, Jun Liu
}

This project intends to discover new materials and synthetic approaches that can lead to advanced electrochemical storage devices or technologies that are suitable for renewable integration and smart grid applications.

A

long with the current trend of monetization, environmental concerns about using fossil fuels and their resources constraints have spurred great interest in generating electrical energy from renewable sources such as wind and the sun. However, the variable and stochastic nature of renewable sources makes solar and wind power difficult to manage and use, especially at high levels of penetration. An effective approach to managing the intermittent nature of renewable energy and enabling its delivery is through electrical energy storage. This capability can be a critical element for improving the reliability, stability, and efficiency of the future electrical grid that is also expected to support plug-in electrical vehicles and enable real-time, two-way communication and the balancing of supply and demand. Although much attention has been given to energy storage for transportation needs, there has been a lack of understanding and public awareness of the critical challenges in the storage for renewable resources and the future grid. Significant breakthroughs are needed to reduce the cost and improve the performance and reliability of technologies for these applications.

To strengthen PNNL's qualification in the critically important field of energy storage, we will study and develop transformational materials for stationary electricity storage applications. By drawing on PNNL's synthesis and self-assembly capabilities, combined with our extensive solid oxide fuel cell and catalyst expertise, we can develop novel nano-structures and architectures (and eventually new chemistries and catalysts) for electrodes and electrolytes used in advanced stationary battery applications. We expect to develop novel nanostructured materials and composites for next-generation batteries while obtaining the fundamental scientific knowledge necessary for the successful development of advanced materials and advanced electricity storage systems.

Covering the range of storage options, electrical energy storage can be realized either by direct storage in electrical charges or conversion of electrical energy into other forms of energy, among which may include chemical, potential, and kinetic. Direct storage technologies such as supercapacitors and superconductive magnetic electrical storage are highly efficient (close to 100 percent) but have low-energy densities and discharge all stored energy in only seconds or sub seconds. Similarly, flywheels that store electricity via conversion into kinetic energy are capable of high power but not much energy.
Thus, these technologies are mainly used for power management instead of energy storage. The electrical storage in potential energy such as pump hydro and compressed air storage may be appropriate for the gigawatt level of storage but unfortunately have geological and social/ecological limitations and require large initial capital investment.

Our project is emphasizing technologies of great potential to store a large electrical energy, while flexible for varied stationary markets. These technologies include flow batteries, sodium batteries, and even the unique lithium ion batteries. With our previous success efforts in developing sodium beta alumina electrolyte batteries and long cycle life/low-cost lithium ion batteries, we have for the past year concentrated on sodium ion batteries that operate at room temperature. Compared to lithium ion, the sodium ion batteries use lowcost sodium instead of lithium in lithium ion that is concerned about resource availability and cost. The challenge is to discover electrochemically active materials that area capable of easy intercalation and deintercalation of sodium ion at 70 percent larger than the lithium ion.

Initial efforts in FY 2009 were focused on developing a novel oxide membrane of a satisfied sodium ion conductivity at temperatures below $280^{\circ} \mathrm{C}$ and cost-effective, optimized positive electrodes for next-generation sodium beta batteries based on planar designs. For lithium ion community storage, our emphasis was on advanced electrodes made from $\mathrm{TiO}_{2}$ base anodes and $\mathrm{LiFePO}_{4}$ cathodes, and we assembled and tested the anode and cathode for over 700 cycles with negligible degradation. Our emphasis on flow batteries was the study of all vanadium sulfate solution electrolyte to obtain insights into the aqueous electrolytes and gaining knowledge about optimization and modification of the chemicals. In particular, we carried out a nuclear magnetic resonance study on the all vanadium sulfuric acid base electrolyte at varied concentrations and temperatures. The work helped to determine transport properties of active species in the electrolytes and mechanistically understand the charge transfer process. Our research yielded the publication of eight articles in scientific journals.

We also studied sodium batteries, particularly the one with chemistry that allows operation at room temperatures. The use of low-cost, abundant sodium instead of lithium potentially leads to a storage technology that meets the cost targets for both transportation and stationary applications. However, the sodium ion battery faces a great challenge in finding suitable materials for both cathode and anode, as the radius of sodium ion is approximately 70 percent larger than that of lithium ion, making difficult its insertion and deinsertion through solid 
materials. Thus, a major objective of this project has been to search suitable electrode materials or structures that can allow facile sodium ion insertion/deinsertion, yielding a satisfactory electrochemical performance.

During FY 2010, we successfully synthesized single crystalline $\mathrm{Na}_{4} \mathrm{Mn}_{9} \mathrm{O}_{18}$ via by a polymer-pyrolysis method using polyacrylates of $\mathrm{Na}$ and $\mathrm{Mn}$ as precursor compounds. These precursor compounds were prepared by solution polymerization of $\mathrm{NaNO}_{3}$ ( $\geq 99.0$ percent, Sigma-Aldrich), $\mathrm{Mn}\left(\mathrm{NO}_{3}\right)_{2}$ (hydrate, 98 percent, Aldrich) and acrylate acid (99 percent, Aldrich) that was followed by heat treatment. The process was optimized to make $\mathrm{Na}_{4} \mathrm{Mn}_{9} \mathrm{O}_{18}$ nanowires of a high crystallinity and homogeneous structure. This unique structure was found stable structure and offers a shortened diffusion path to sodium ion during interecalation/ deintercalation or charge/discharge process.
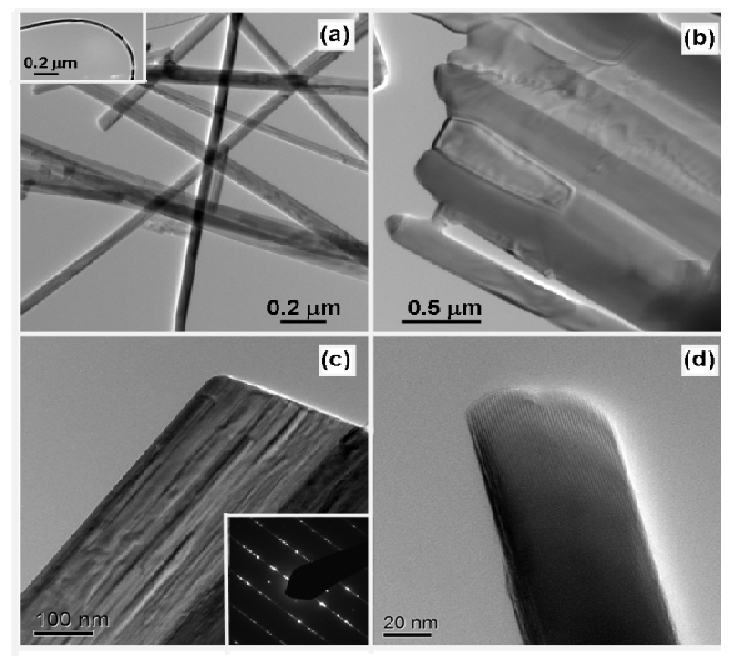

TEM images of $\mathrm{Na}_{4} \mathrm{Mn}_{9} \mathrm{O}_{18}$ nanowires calcined at $750^{\circ} \mathrm{C}$ :

a) dispersing nanowires (inset is a single nanowire),

b) incorporated nanowires, c) expanded image of a single nanowire (inset of $S A D P$ of $c$ ), d) bigh-resolution nanowires.

The synthesized materials were then made into electrodes by mixing $80 \mathrm{wt} \% \mathrm{Na}^{2} \mathrm{Mn} 9 \mathrm{O}_{18}$ powder, $10 \mathrm{wt} \%$ SP-type carbon black and $10 \mathrm{wt} \%$ polyvinylidene difluoride dissolved in N-methyl-2-pyrrolidone to form a slurry and then coating on $\mathrm{Al}$ foil. Electrochemical tests of the electrode materials were performed on coin cells that were made from the prepared $\mathrm{Na}_{4} \mathrm{Mn}_{9} \mathrm{O}_{18}$ cathode and sodium metal as counter and reference electrode, along with $1 \mathrm{M} \mathrm{NaClO}_{4}$ dissolved in a mixture of ethylene carbonate and dimethyl carbonate (1:2 by wt) as the electrolyte. A microporous membrane (Celgard 2400) was employed as a separator. Electrochemical investigation on the assembled coin-cells indicated a capacity $128 \mathrm{~mA} \mathrm{~h} \mathrm{g-1}$ and retention of 82 percent of the capacity after 500 cycles at $0.1 \mathrm{C}$. The graphs below show the charge/discharge profiles and capacity of the assembled cells as a function of cycling.
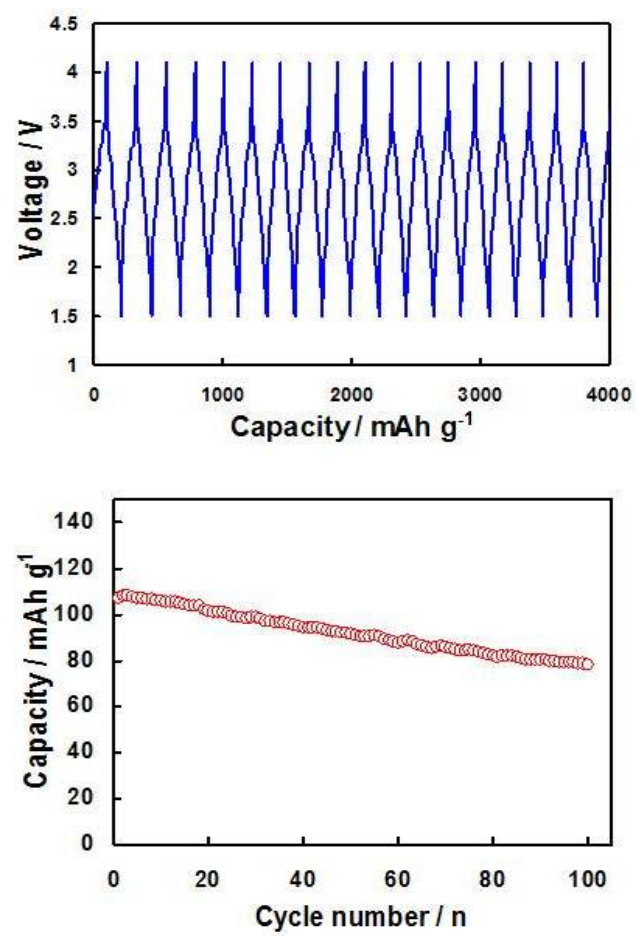

The charge-discharge performance of the $\mathrm{Na}_{4} \mathrm{Mn}_{9} \mathrm{O}_{18} /$ pyrolyzed carbon sodium-ion battery in potential range of $1.5 \sim 4.1 \mathrm{~V}$ (vs. $\left.\mathrm{Na} / \mathrm{Na}^{+}\right)$at a constant current of $50 \mathrm{mAg}^{-1}(\sim 0.5 \mathrm{C})$.

To our best knowledge, such good electrochemical performance from the $\mathrm{Na}_{4} \mathrm{Mn}_{9} \mathrm{O}_{18}$ nanowires has not reported in literature for the sodium ion rechargeable battery. The synthesized nanostructured sodium compounds appeared promising electrode materials to make sodium ion batteries that can be viable alternatives to the traditional lithium ion batteries that have been increasingly concerns over the lithium resources and the incurred high cost. 


\title{
Transition and Transport of Surface Residue from Non-Volatile Explosives to Gas Phase Ions for Sensitive and Selective Detection
}

\author{
Robert G. Ewing, Melanie J. Waltman, David A. Atkinson
}

A significant challenge associated with explosives detection by ion mobility spectrometry (IMS) is the transfer of less volatile explosive samples from the sampling media into the instrument as a vapor without significant background contaminants from surroundings or the material itself. Development of these areas will dramatically advance the efficacy of IMS for explosives detection via enhanced sensitivity with concurrent increase in selectivity.

$\mathbf{T}$ he conversion of explosives from a surface residue into the gas phase is currently accomplished by thermally heating the sample swipe to transform the explosive into a vapor. There are several obstacles associated with this approach. Increasing the vapor pressure through heat with a fixed heating inlet does not treat all samples alike. A compromise must be made between compounds with a variety of vapor pressures. In order to desorb less volatile components, more heat can be applied, but at the cost of increasing the background matrix of other materials, including thermal degradation of the swab. Higher temperatures can also cause thermal degradation of the sample, causing a reduction in sensitivity. Surface ionization may be a solution to this particular problem. Sources such as DESI and DART have been described at length. However, the problem with these two sources is that they only provide surface ionization at a small point and not over an area. One goal of this effort is to investigate the possibility of sample desorption and ionization occurring efficiently over a larger surface area.

A challenge associated with ion mobility spectrometry is selectivity, a process that has been improved by others with the addition of a gas chromatograph (GC) to pre-separate the analytes prior to entering the IMS. Although increased selectivity has been achieved with the addition of a GC, it comes at a significant cost in analysis time, requiring several minutes. For security screening scenarios, this amount of time is prohibitive. Although the IMS performs well as a stand-alone instrument, some separation of the analyte from background interferents will enhance the selectivity, thus increasing the detection probability while reducing false alarms. Because

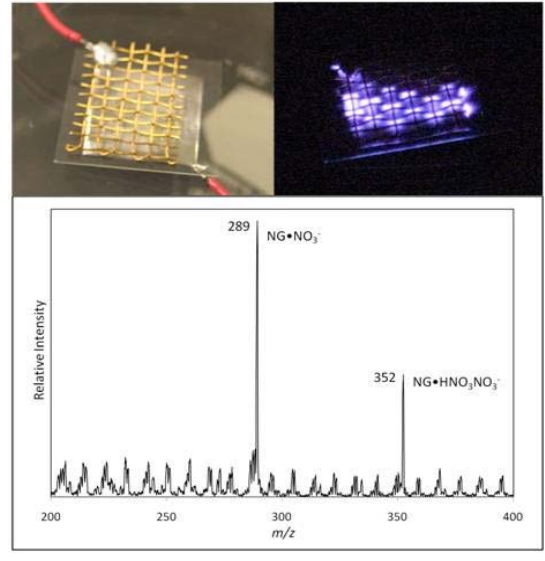

An example of a broad area plasma that occurs between the metal screen and glass slide, with subsequent mass spectrum of nitroglycerine showing clusters with the reactant ion peaks of $\mathrm{NO}_{3}^{-}$and $\mathrm{HNO}_{3} \mathrm{NO}_{3}$. the ionization at atmospheric pressure is based upon competition for available charge, reduction in background interferents will increase sensitivity. The long analysis time is the main reason that GC-IMS is not widely accepted; thus, fast pre-separation prior to IMS analysis could be the answer. Another goal of this research is to investigate potential advantages of fast, partial pre-separation combined with IMS for improved selectivity in explosives detection.

Surface desorption and ionization was achieved by assembling an ionization source consisting of a copper sheet, a thin glass slide and a metal screen. A RF voltage was placed between the copper sheet and stainless steel screen, resulting in plasma occurring at the surface of the slide and screen. This ionization source was placed in front of a mass spectrometer, which was used to identify ions and compare relative peak intensities with an atmospheric pressure ionization interface. Chemical samples of explosives in a solvent were deposited onto the screen, and the solvent was allowed to dry before the source was turned on and a mass spectrum collected. RDX, PETN, TNG, and NG were evaluated. Both TNT and NG provided observable peaks in the mass spectrum. TNT exhibited a peak at m/z 197 (TNT-NO). NG was observed from peaks at $\mathrm{m} / \mathrm{z} 289$ and 352 resulting from clusters of $\mathrm{NG}$ with the reactant ions $\mathrm{NO}_{3}{ }^{-}$and $\left(\mathrm{HNO}_{3}\right) \mathrm{NO}_{3}{ }^{-}$. Even though TNT and NG appeared in the spectra there intensities were lower than expected. Further, no response was observed for RDX and PETN, likely due to low vapor pressures of these compounds. The location of the plasma and deposition of the analyte may also play a role and need further investigation.

For improved selectivity, rapid gas chromatography was used prior to the IMS. Original IMS scans would require approximately $15 \mathrm{sec}$ for the complete analysis time. With the added separation, complete scans were analyzed in under 30 seconds. Mixtures of TNT with NG and TNT with RDX were evaluated and resolution values of approximately 0.5 were obtained. This provided IMS spectra of pure compounds at the front and end of the two peaks, with a mixture of the two compounds occurring during a brief period in the middle. This enables less complex chemical ionization and more detailed analytical information. 


\title{
Ultra-Nanoporous Explosives Sensing Material
}

\author{
Jay W. Grate, Praveen K. Thallapally, Scott D. Harvey
}

Sampling and preconcentration represent significant issues in explosives detection. Selective concentration of target explosives from the air allows collection of fractions that simplify field portable analysis techniques and allows larger samples to be analyzed successfully, leading to lower detection limits. These capabilities are critical for locating hidden explosives. We propose to investigate metal organic frameworks (MOFs) as the sorptive material of a new generation of devices for this purpose.

A dvanced detectors are required in national security, homeland security, and environmental monitoring. In particular, the detection of explosives is critical to security applications ranging from defense and homeland security applications to the protection of DOE infrastructure and assets. Sampling and preconcentration represent significant issues in chemical detection in general and explosives detection in particular. Research performed on nanostructured sorptive materials for preconcentration and sensing is essential for creating advanced sensor systems needed to meet requirements for greatly enhanced chemical sensitivity and lower detection limits.

In this project, we have begun to investigate MOFs as a new material for use in adsorptive preconcentrating devices for explosives. Such devices could also be used as collectors in monitoring applications and in trace detection instrumentation. In the latter case, the use of a preconcentrator could reduce detection limits, improve selectivity, and/or allow other instrumentation to be used as the detector. Additionally, nanostructured sorptive materials with high affinity or selectivity for explosives molecules may be useful on sensor devices.
MOFs represent a new type of nanoporous material consisting of metal centers linked by organic bridging ligands. These components readily self-assemble into materials with an exceptionally open framework structure, where all the porosity is accessible. The materials have high thermal stability; to be sure, the structure and the selectivity of MOFs can be tailored by the selection of metal ion and organic bridging ligand. Compared with conventional sorbents, MOFs have more surface area, less dead volume, and more rapid diffusion within the material. These unique characteristics are highly favorable for the development of sorptive materials for preconcentration and sensing. Nevertheless, the affinity and selectivity of MOF materials for explosives molecules are either not known or little understood. Accordingly, this project has begun collecting basic research information on the sorptive characteristics of MOF materials as they relate to explosives molecules. We are interested in the uptake affinities for explosives compounds, the selectivity among explosive and nonexplosive vapors, and the effects of the metal centers of the MOF structures on these properties.

In our approach, we first synthesize and characterize a select set of MOF materials with different metal ions. These materials have been characterized for key properties using methods such as x-ray diffraction, surface area measurements, and thermogravimetric analysis in order to obtain each item's structure, porosity, and stability. Second, the well characterized materials are investigated for vapor affinity and selectivity using methods such as programmed thermal desorption and inverse gas chromatograpy.

MOF materials were prepared with metal ions, including nickel, magnesium, lanthanides, and copper. The material with copper, benzene tricarboxylate (CuBTC), was further investigated using inverse gas chromatography, which evaluates the interactions of probe vapors with $\mathrm{CuBTC}$ by examining the chromatographic retention times on a stationary phase that contains CuBTC. Retention time data were converted to Kováts retention indices, which were compared with the indices for the same vapors on a similar stationary phase lacking the CuBTC. It was found that $\mathrm{CuBTC}$ showed very strong retention of oxygenated compounds with high selectivity over hydrocarbons. However, the affinities were unexpectedly low for aromatic and nitro compounds. These results are specific for CuBTC, as other MOFs may have different selectivities.
For each compound, the difference between the retention index on the CuBTC-containing stationary phase, minus the retention index on the control material. Positive numbers indicate bigher affinity and selectivity for a given compound. 


\section{Earth and Space Sciences}




\title{
Advanced Scalability for STOMP: Subsurface Simulation and Characterization at Extreme Resolution
}

\author{
Steven B. Yabusaki, Yilin Fang, Bruce J. Palmer, Guang Lin, Anderson L. Ward, Timothy D. Scheibe
}

Advanced computers and computational technology are enabling the development of more reliable predictions of the movement of fluids and contaminants in the subsurface environment. The subsurface simulation capabilities developed under this project will lead to a reduction in risks to human health and the environment and cost-effective solutions for waste cleanup, carbon sequestration, and hydrocarbon recovery.

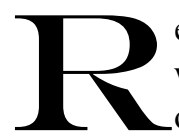
eactive transport simulation codes have become widely used for simulating the movement and fate of contaminants and other dissolved constituents in the subsurface. They are useful not only as engineering tools for site analysis but also, importantly, as fundamental research tools. While major advances have been made in our ability to simulate numerically complex hydrologic, geochemical, and microbiological processes of interest, accurate and detailed characterization of spatial property distributions in the subsurface remains the primary challenge facing reactive transport modeling efforts. The extreme resolution targeted for computation-based research on critical subsurface science issues requires highly scalable simulators that can fully exploit the high performance and large memory of the most advanced computers.

Three broadly defined approaches were used to address the problem of incomplete subsurface characterization: inverse modeling, stochastic simulation, and hydrogeophysical interrogation. This research will draw on all three approaches in an integrated manner to develop and test a general approach to subsurface characterization and modeling with extremely high resolution of the model parameters and processes. PNNL's Subsurface Transport Over Multiple Phases (STOMP) simulator is a state-of-the-art code used for all simulations of subsurface multifluid flow and reactive transport at the Hanford Site. A key component of the project is re-engineering the STOMP simulator to achieve a high degree of scalability on high-performance computational platforms.
In FY 2009, a parallel processing operational mode for variably saturated flow and multicomponent reactive transport, eSTOMP- $\mathrm{H}_{2} \mathrm{O}$, was successfully developed. Scalability was demonstrated across 16,384 processor cores on both the EMSL Chinook and NERSC Franklin clusters. The restructuring of the STOMP code involved separating the physics and chemistry kernels from the data management and grid services and implementing the Global Array Toolkit (GA). GA supports a high-level global shared memory programming style where the programmer is not responsible for process-to-process communication and does not need to know where data resides in the distributed memory. An important benefit of this approach was the rapid and efficient development through significant re-use of the existing source code.

The general restructuring approach from last year was used in FY 2010 to develop and test successfully the nonisothermal, multiphase, multifluid eSTOMP$\mathrm{CO}_{2}$ operational mode for deep geologic $\mathrm{CO}_{2}$ sequestration. With four primary variablesaqueous pressure, supercritical $\mathrm{CO}_{2}$ saturation, temperature, and brine concentration - this was a significantly larger re-engineering effort than eSTOMP- $\mathrm{H}_{2} \mathrm{O}$. Identifying and removing bottlenecks resulted in very good weak scaling up to 8192 processors (134 M degrees of freedom) for a simulation of multiple supercritical $\mathrm{CO}_{2}$ injection wells ( 1 million metric tons per year per well) in a vertically heterogeneous

representation of the Illinois basin.

Application and development of eSTOMP- $\mathrm{H}_{2} \mathrm{O}$ also continued in FY 2010. The successful simulation of a uranium bioremediation field experiment was one of the most complex, coupled-process, field-scale, reactive transport simulations of its kind: acetate biostimulation of two functional microbial groups including biomsass, four terminal electron accepting processes, uranium aqueous and surface complexation, seven minerals, and 102 biogeochemical species under transient three-dimensional, variably saturated flow through physically and chemically heterogeneous porous media. A multilevel 
parallelism simulation framework based on GA processor groups was applied to uncertainty quantification in the Hanford 300 Area aquifer: 317 alternative conceptual model simulations in 23 hours using 4096 processors versus 1 week for a single desktop simulation. A new load balancing scheme based on GA counters was implemented to address spatially variable computational "hot spots" of reaction solver activity. A new project with Professor Warren MacEvoy investigating the use of graphical processing units (GPUs) for reactiondominant simulations successfully developed and tested a differential algebraic equation solver on Nvidia Tesla systems at Mesa State College and PNNL. The conclusion is that this is a viable approach for current and future general purpose GPU architectures.
Our principal objectives for FY 2011 include extending the scalability of eSTOMP to more than 100,000 processor cores, developing a large-scale parameter estimation and uncertainty quantification capability for multiple simultaneous iterations/ realizations using eSTOMP as the computational engine, developing and implementing new eSTOMP chemistry solver options (e.g., including GPU solvers), incorporating energy and multiphase capabilities into eSTOMP- $\mathrm{H}_{2} \mathrm{O}$, and producing manuscripts on computational framework and applications. 


\title{
Computational Framework for Diagnostics, Validation and Intercomparison of Numerical Simulators for Geologic Sequestration
}

\author{
Mark D. White, Kenneth C. Carroll, Chandrinka Sivaramakrishnan, Sumit Purobit
}

The national laboratories have developed and are continually improving a suite of numerical simulators for modeling geologic sequestration of greenhouse gases that collectively represent the state of science and coupled-process modeling. An Internet-based computational framework is being developed that will dynamically document the scientific state and capabilities of simulators for geologic sequestration, providing the nation with a scientific standard for these analytical tools.

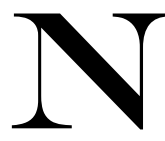
umerical simulation capabilities for modeling sequestration associated with greenhouse gases injected into geologic reservoirs have evolved rapidly during the last decade. At that time, the accepted state of the science was numerical simulators that could address dissolution, structural, and mineralization trapping; isothermal conditions; aqueous-based geochemistry; and decoupled geomechanics for idealized subsurface conceptualizations. The standard for geologic sequestration modeling is continually advancing but includes capabilities for predicting hydraulic trapping, nonisothermal conditions, transitions to subcritical conditions, ground-surface interactions, injection wells, co-sequestration, supercritical $\mathrm{CO}_{2}$-based geochemistry, coupled hydrology-geochemistry-geomechanics, heterogeneous basin-scale domains, and wettability transitions. Numerical simulators for geologic sequestration are founded on mathematical descriptions of physical processes and solution algorithms. Critical components for developing analytic tools are verifying, benchmarking, and validating computer code and implementing mathematical models and numerical solution schemes.

Historically, simulators are verified against analytical solutions, benchmarked against alternative implementations, or validated against laboratory or field experiments. All three checks are generally used during simulator development through extensive application. Publications of code validations against laboratory or field experiments, simulator verification workshops, and code comparison publications are all static snapshots of the state of numerical simulation science for greenhouse gas sequestration. This project is developing a computational framework to make validation and benchmarking a dynamic process where numerical solutions evolve with modeling capability advances and benchmark problem complexity evolves with growth in available field and experimental data. The project will produce a computational framework for diagnosis and intercomparison, a suite of validation problems and solutions, observational data sets for model validation, standardized documentation of code features and characteristics, and systematic accounting of disagreements for validation problems.

Seven years ago, an intercomparison of numerical simulation codes for geologic sequestration of $\mathrm{CO}_{2}$ was conducted. Findings from these studies involving problems of moderate complexity were that the suite of internationally recognized simulation codes was in satisfactory agreement, with some discrepancies being traced to differences in fluid property correlations as well as spatial and temporal discretizations. For numerical simulation to be applied confidently to geologic sequestration, a systematic accounting of these disagreements is needed. We propose three missions for this project: intercomparison of numerical simulators for geologic sequestration, a model validation suite, and integration into the data management and assimilation, numerical model development, computation, and analytics tools of the Geologic Software Simulation Suite $\left(\mathrm{GS}^{3}\right)$. Though the developed software will have broader applicability, this project will be directed at geologic sequestration in deep saline formations.

This project started with three principal foci: assimilating validation problems, acquiring and installing four national laboratory codes (FEHM and PFLOTRAN from Los Alamos, TOUGH from Lawrence Berkeley, and STOMP from PNNL) on the $\mathrm{GS}^{3}$ server, and identifying new scientific research directions for extending the current suite of code comparison problems for geologic sequestration (i.e., multifluid geochemistry, nonwetting fluid entrapment and hysteretic relative permeability, compositional greenhouse gases coupled with nonisothermal effects, and wettability transformations). Each new research direction will require support from the experimental component, In Situ Sequestration Suite (IS ${ }^{3}$ ). We made substantial advances on the experimental data collection and mathematical model development for understanding multifluid geochemistry.

For FY 2011, our aims are to define code diagnostics and intercomparison approaches and develop the user interface for prototyping and demonstration (for computational framework); diagnose a foundational suite of code comparison problems for deep saline formations (in the suite of validation); develop a suite of observation benchmarks from field-scale demonstrations (for observational data sets for model validation); and define a standard documentation format for the core numerical simulators for geologic sequestration and post content for each in the standard format on the $\mathrm{GS}^{3}$ framework/wiki. 


\title{
Data Assimilation Tools for $\mathrm{CO}_{2}$ Reservoir Model Development
}

\author{
Mark L. Rockhold, Christopher J. Murray, E. Charlotte Sullivan, George V. Last, Gary D. Black
}

We aim to develop improved, robust software tools and workflows for data analysis and assimilation tasks associated with the development and parameterization of subsurface reservoir models for $\mathrm{CO}_{2}$ sequestration. Ultimately, our research results will help mitigate adverse effects of excessive $\mathrm{CO}_{2}$ production on climate change by providing tools to facilitate modeling of subsurface injection for geologic sequestration of $\mathrm{CO}_{2}$.

D evelopment and parameterization of conceptual and numerical models of large-scale subsurface reservoirs for $\mathrm{CO}_{2}$ sequestration is a difficult, time-consuming process that requires the assimilation of multiple data types (core, borehole geophysical logs, seismic) representing different properties, processes, and scales of measurement. Development of large-scale reservoir models has generally been the purview of petroleum exploration and production companies. $\mathrm{CO}_{2}$ sequestration involves additional processes such as geochemical reactions and buoyancy-induced plume migration that must be considered in developing reservoir models.

The objective of this research is to build workflow and software tools that support data analysis and assimilation tasks for the development and parameterization of models of subsurface reservoirs for $\mathrm{CO}_{2}$ sequestration. One of several collaborative research efforts, this project interfaces with a software development project that is developing a wiki-based framework for organizing and tracking data and modeling results associated with numerical model simulations. This framework and associated analysis tools are referred to as the Geologic Sequestration Software Suite, GS ${ }^{3}$.

This project is assisting this other project with designing the layout of $\mathrm{GS}^{3}$, providing recommendations on functionality and developing software tools for data analysis and assimilation that will be accessible from the wiki and facilitate building and parameterizing large-scale models of subsurface reservoirs. The $\mathrm{GS}^{3}$ wiki and associated analysis tools are being demonstrated using application examples from real sites with active sequestration projects. A goal of the combined projects is to develop a software framework or platform and associated toolkits that streamline the process of reservoir model development and analysis.

During FY 2009, we interfaced with the software framework group to design the layout and recommend features/capabilities to incorporate into the $\mathrm{GS}^{3}$ wiki. We also developed or linked to existing rock and fluid property databases and created programs and scripts for tasks associated with data analysis and assimilation, some of which are used in the wiki. Project activities in FY 2010 focused primarily on software tool development and implementation in the wiki to support the upcoming release of $\mathrm{GS}^{3}$. Our progress to date on the data assimilation project is detailed below.

- $\mathrm{A} \mathrm{CO}_{2}$ Plume Area Calculation Program was converted into a Perl script now callable from the $\mathrm{GS}^{3}$ wiki. This calculator allows a user to enter information about injection depth, temperature, salinity, porosity, and injected mass of $\mathrm{CO}_{2}$. It then calculates plume area with the results being viewable from the $\mathrm{GS}^{3}$ wiki via Google Maps.

- Log ASCII Standard (LAS) File Plotting and viewing from $\mathrm{GS}^{3}$ wiki. LAS files are a standardized format for geophysical well log data, and programs were developed for reading and plotting LAS files. In conjunction with staff from the $\mathrm{GS}^{3}$ platform project, capabilities were developed for invoking LAS file plotting from the $\mathrm{GS}^{3}$ wiki and viewing well locations on Google Maps.

- A prototype R-based Multivariate Analysis Toolkit has been developed. The toolkit consists of modules for performing different types of statistical analyses: descriptive statistics, correlation, classification, prediction, and geostatistical analysis. Capabilities include principal components analysis, cluster analysis, and neural network modeling. This multivariate analysis toolkit is applicable to analysis of many different types of geologic and geophysical data used in the development and parameterization of numerical models of $\mathrm{CO}_{2}$ sequestration. A user of $\mathrm{GS}^{3}$ will be able to invoke this toolkit from the wiki.

- A prototype Graphical User Interface (GUI) for Model Attributes is being developed in Jython, which includes worksheets for the following categories: general site description; infrastructure and operations; geologic framework; processes and properties; and monitoring, history matching, and model comparisons. The model attributes defined through this GUI pertain primarily to the site conceptual model and the data used in its development, which are also used for parameterization of numerical models. A separate GUI is being developed for Numerical Model Specifications (Williams project). Both of these GUIs will eventually be invokable from the $\mathrm{GS}^{3}$ wiki.

- A Geophysics Toolkit is being implemented in Matlab to perform various tasks related to site characterization and monitoring. This toolkit will include capabilities for 
viewing seismic data (e.g., SEGY files), developing synthetic seismograms, modeling and interpretation of vertical seismic profiles (VSP), identification of reservoir and cap rock intervals, and site monitoring.

- Finally, a prototype Rock Properties Catalog is being populated with data for physical, hydraulic, and mineralogical properties of reservoir formations and cap rocks. This prototype rock properties catalog is currently in spreadsheets, but the development of a data model for a wiki-based repository is under discussion. The spreadsheet data are augmented by URLs to publically accessible external databases.
For FY 2011, our project will continue developing and testing subsurface characterization, data analysis, and data assimilation software tools, which will in turn be integrated into the $\mathrm{GS}^{3}$. We intend to demonstrate the full $\mathrm{GS}^{3}$ software environment and integrated toolkits using data from realistic analogs or actual sequestration sites. We will also continue to submit our results for publication in top-tier, peer-reviewed journals. 


\section{Developing Ice Nucleation Parameterizations for Large-Scale Models}

Xiaohong Liu, Jennifer M. Comstock, Gourihar R. Kulkarni, Daniel J. Cziczo, Jiwen Fan, Mikhail Ovchinnikov, Steven J. Ghan

We propose to develop a new ice nucleation parameterization so that we can quantify the climatic impact of aerosol effects. Such a parameterization will improve representation of aerosol-cloud interactions in cloud-resolved, regional, and global models.

$\mathbf{M}$ ore than half of the global precipitation originates from the ice phase. Because primary ice particles are formed on a tiny fraction $\left(10^{-5}\right)$ of atmospheric particles (called ice nuclei), natural and human activities can potentially impact ice formation in the atmosphere, leading to the alteration of earth's energy budgets and hydrological cycles. The limited state of knowledge of ice formation processes (including both primary and secondary ice formation) limits our ability to forecast precipitation production and to predict climate change. While the influence of aerosols on cloud droplet nucleation has received considerable attention from the climate research community, the effects of aerosol particles on ice formation are currently insufficiently understood and present another level of challenge for both observations and modeling.

To study ice phase cloud microphysics and aerosol effects on climate through ice formation, a parameterization for ice nucleation needs to be implemented in the model. There are two basic categories of ice nucleation parameterizations: empirical (founded on laboratory- or aircraft-based measurements) and classical nucleation theory (CNT).

Empirical parameterizations have appeal because they are easy to implement and computationally efficient for coarse resolution regional/global models that typically use bulk microphysics schemes. The drawback is that empirical schemes often have limited temperature or supersaturation ranges where they can be applied. CNT approaches provide a specific link between aerosol or ice nuclei characteristics and the nucleation process in high spatial resolution models with size-resolved aerosol and cloud microphysics. However, the CNT approach is fraught with uncertainty because there are a number of unconstrained parameters from the lack of observations of specific aerosol or ice nuclei properties.

To address the above parameterization issues, we propose to develop a new ice
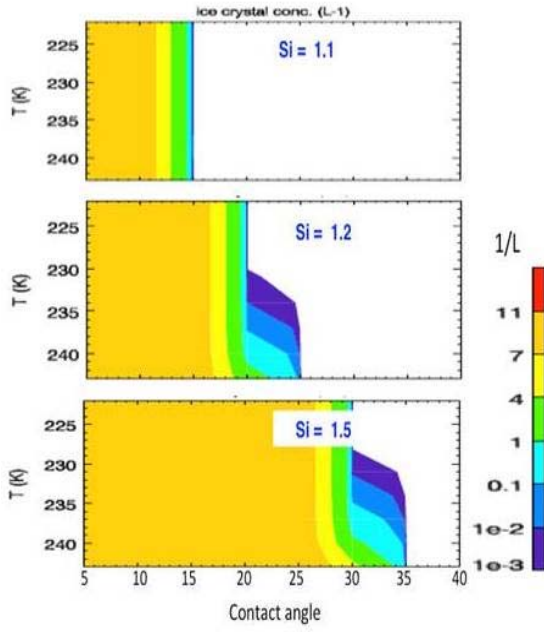

Ice nucleation through deposition mechanism as a function of temperature, ice supersaturation, and contact angle calculated from the classical nucleation theory. As shown, ice number depends critically on ice supersaturation and contact angle. nucleation parameterization that links directly concentration of nucleated ice particles with aerosol properties and air dynamics (updrafts) from first principles so that we can quantify the climatic impact of aerosol effects. We will make use of the most recent data from laboratory measurements, combined with field observations through CNT, to constrain uncertain parameters. The parameterization will be applicable to different temperature regimes (ice clouds and mixed-phase clouds) and will account for different mechanisms (immersion and deposition), depending on aerosol surface properties due to aging in the atmosphere. We expect that this new parameterization will be used in the next generation of regional and global models, which will add the capability to study aerosol effects on mixed-phase and ice clouds.

In the first year of the project, we collected experimental data on ice nucleation published in the literature. The data contain ice nucleation onset conditions (temperature, relative humidity, and aerosol particle size) of mineral dust particles investigated under deposition and immersion nucleation freezing mechanisms. These conditions are used to calculate nucleation rates. Using the tabulated experimental data for deposition mode nucleation onset conditions of mineral dust particles, we computed the contact angle between the ice embryo and ice nucleus using two existing approaches in the literature, both based on the CNT. The benefit of using the CNT approach is that it includes a particular link between the nucleation rate and aerosol properties (i.e., size and composition).

The experimental measurements of nucleation rate, ice saturation ratio, and temperature are the necessary inputs, and the contact angle are computed for each data point. We introduced the probability distribution function (PDF) of contact angle to the CNT to reflect the complexity of particle surface inhomogeneity. Offline testing of CNT will be used to examine the dependence of ice nucleation on temperature, supersaturation, and contact angle.

In FY 2011, we plan to finish the work of PDF of contact angle for all the data collected and apply the CNT parameterization to a case study from ICE-L using the parcel model framework and a cirrus case at the ARM Southern Great Plain site using a cloud-resolving model framework. 


\title{
Development and Evaluation of an Externally-Mixed Sectional Aerosol Model
}

\author{
Rabul A. Zaveri, Richard C. Easter, James C. Barnard; \\ Nicole Riemer, Matt West (University of Illinois at Urbana-Champaign)
}

Atmospheric aerosols (suspended fine particulate matter) are widely recognized as key elements in many environmental issues ranging from global climate change and visibility to adverse effects on human health. To address DOE concerns about the safe production and use of energy, it is necessary to improve our understanding of the origin, fate, and properties of different types of particles commonly found in the atmosphere.

A tmospheric aerosols range from a few nanometers up to a few microns and can be composed of a wide variety of species such as soluble salts, soot, insoluble dust, and organic compounds of anthropogenic and biogenic origins. Primary particles (i.e., emitted) and particles formed via homogeneous nucleation (e.g., sulfuric acid) are initially "externally mixed" (i.e., exist as separate populations). Upon interacting with semi-volatile gases in the atmosphere via condensation and with other particles of similar or different compositions via coagulation, these particles undergo changes in size and chemical composition and gradually become "internally mixed" to varying degrees. This process is called evolution or "aging" of aerosols. The radiative properties, cloud condensation nuclei $(\mathrm{CCN})$ activation super-saturations, and chemical reactivity of fresh and aged aerosol particles can differ significantly.

Accurately tracking the mixing state in conventional aerosol models requires treating a multi-dimensional size distribution, which is computationally prohibitive. Consequently, current aerosols models employ simplified treatments that may

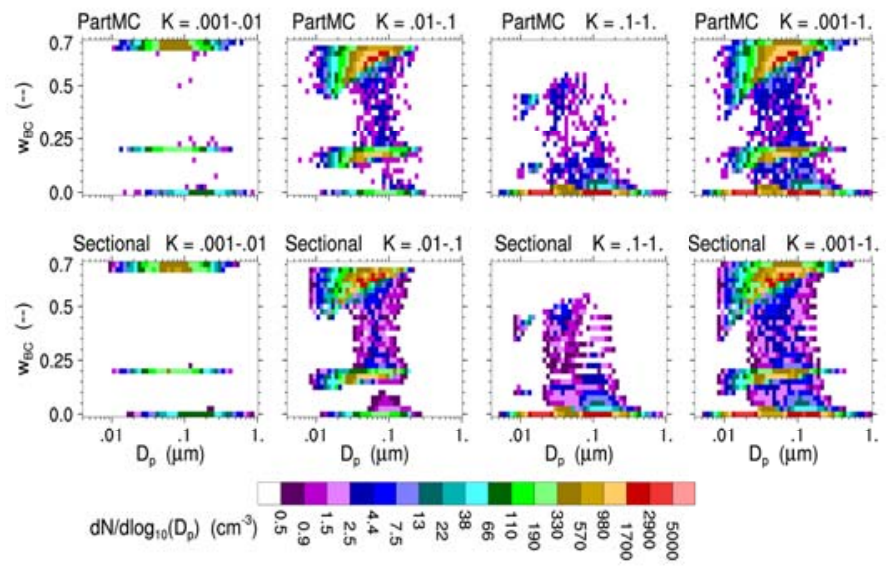

Example of comparison of results from particle-resolved (top row) and mixing state resolving sectional (bottom) versions of MOS AIC. Plots of black carbon mass fraction $\left(W_{B C}\right) v s$. dry particle diameter $\left(D_{p}\right)$ are shown after 2 hours of aging in an urban plume for four ranges of aerosol bygroscopicities. not adequately resolve aerosol mixing state. In this project, we use the newly developed particle-resolved aerosol model PartMC-MOSAIC as a numerical benchmark to guide the development of an accurate and efficient representation of the different particle types and mixing states in MOSAIC-ext to predict the optical and $\mathrm{CCN}$ activation properties reliably.

For FY 2009 and into FY 2010, the PartMC-MOSAIC model was applied to an idealized urban plume case, where pollutant trace gases and particles from diesel and gasoline engine exhaust and meat cooking were emitted for 12 hours into a well-mixed urban air shed containing background aerosols composed of ammonium sulfate and aged organic aerosol. Urban emission profiles were based on Los Angeles emission inventory. Gas-phase photochemical reactions and gas-particle partitioning of various semi-volatile species continued for 36 more hours in this urban air as it was advected from the source region. By resolving more than 100,000 particles in a representative volume in this urban plume, the PartMC-MOSAIC model simulated the evolution of the mixing state of urban soot particles as well as the associated optical and $\mathrm{CCN}$ activation properties without any simplifying assumptions of particle mixing states.

The particle-resolved results were benchmarked to evaluate errors in the predicted optical and $\mathrm{CCN}$ activation properties due to the internal mixture assumption and the choice of bin size resolution commonly used in sectional aerosol models. We developed a 3D sectional version of MOSAIC, which resolved dry size $\left(\mathrm{D}_{\mathrm{p}}\right)$, black carbon mass fraction $\left(\mathrm{W}_{\mathrm{BC}}\right)$, and hygroscopicity (K). The size, black carbon fraction, and hygroscopicity axes were kept flexible in both resolution and uniformity so that an optimal configuration of the 3D sectional framework could be determined by evaluating the performance of different configurations in predicting optical and CCN activation properties against the PartMC-MOSAIC results.

An optimal configuration of 24 size bins and 3 bins for black carbon fraction and hygroscopicity each was determined, comparing well with results for the idealized urban plume case. An empirical parameterization of homogeneous nucleation was implemented in PartMC-MOSAIC to evaluate CCN production from newly formed particle growth. Further development and testing of the new MOSAIC sectional version using field measurements will be performed using external programmatic funding. In FY 2010, we published two articles in peer-reviewed journals and made four presentations (one invited) at various national and international conferences. 


\section{Development of Climate Modeling and Integrated Modeling at Regional Scales Framework and Functional Specifications}

Ian Gorton

We are designing a flexible software framework to integrate existing global models with high resolution regional models of climate, land usage, building energy demand, and power demand and supply.

G lobal models of climate and integrated assessment address large-scale questions to understand the physical, biophysical, and socio-economic consequences of climate and its interactions with human systems (climate and land use or land cover). Global models are ill-suited, however, to address regional-scale questions on impacts, adaptation, and mitigation. This project will result in a flexible software architecture that will allow model components with disparate temporal and spatial resolutions to pass information in a dynamic fashion. The functional specifications for both the operational (software engineering) and systems (socio-economic, climate, energy, ecosystem) components will be defined and implemented.

This project's software integration framework will link the various models involved in the Integrated Regional Earth Systems Modeling (iRESM) program, which consists of the following models: regional climate, regional assessment, building and land use, and energy infrastructure. All of these models work on different spatial and temporal scales, and are not designed to be linked in terms of input data required and output data produced. They also have radically different run time platforms from supercomputers to workstations and are built using multiple software programming languages. Together, these differences make model linking highly complex. In addition, model outputs for runs of several decades can be in the hundreds of megabyte to terabyte range,

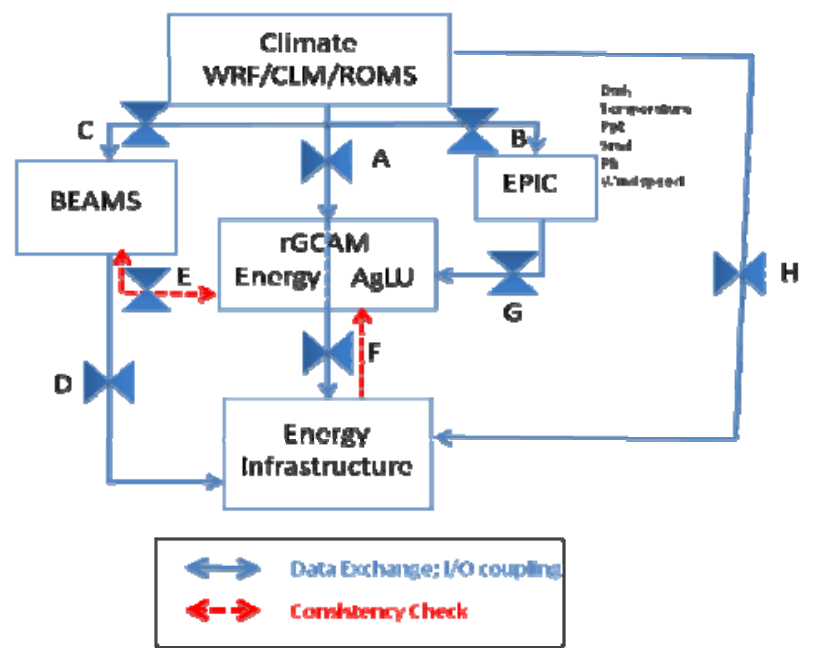

Linking together models in iRESM. making the filtering, transformation, and transfer of data between models difficult.

During FY 2010, we worked extensively with iRESM modeling projects to understand current and planned capabilities and to analyze the scope and complexity of the required integration framework. To drive this analysis, we defined an initial use case to address. Based on this topology of model integration, we worked to define the various data requirements that are identified by the arrows in the diagram. This has allowed us to understand:

- the complexity of data transformation algorithms that need to be created to link the outputs from one model to the inputs of another

- the frequency with which the various models must exchange data as a simulation progresses

- mismatches in the data that one model requires as inputs from another model's output data.

These investigations are driving the design of a prototype integration framework that will demonstrate the feasibility of model integration in a simple, one-way fashion.

In developing the modeling framework and functional specifications for the iRESM model, design is crucial. The framework will be planned so that the capability can address four overarching issues: 1) regional constraints on mitigation and adaptation, 2) effect of changes in regional average or extreme climate states on mitigation and adaptation, 3 ) interactions between management and natural processes that contribute to rapid or nonlinear environmental change, and 4) potential interactions between mitigation and adaptation decisions. We are investigating detailed scientific use cases, software integration, and data issues relevant to the integrated modeling environment. In future years, we will implement the model framework to enable regional modeling scenarios for set up and execution.

In FY 2011, we will complete the prototype based on simple file exchanges and use it demonstrate an initial version of model integration in iRESM. Based on the prototype experiences, we will design the initial integration framework version with mechanisms that can evolve as model input requirements change over its lifetime. The initial version will also abstract the precise software integration approach used to link the various models. This is necessary to evolve the framework to leverage more tightly coupled integration mechanisms that will eventually be needed to execute the models at high spatial and temporal resolutions on supercomputer platforms. 


\section{Development of Prototype Integrated Earth System and Environmental System Models}

Lai-Yung (Ruby) Leung

This research will provide a new modeling capability to represent interactions between the atmosphere, ocean, terrestrial ecosystems, sea ice, and biogeochemical processes that allow us to investigate climate change impacts at the regional scale.

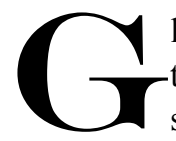
lobal climate models (GCMs) are the primary tools used to predict the evolution of the climate system. Through decades of development, GCMs have demonstrated the useful skill of simulating the global mean temperature response to various natural and anthropogenic forcings of the last century. More recently, global earth system models (ESMs) that integrate multiple climactic processes have emerged to enable the global carbon cycle to be included for projections of global climate change at the century time scale. Further, some progress has been made in demonstrating potential skill in climate predictions at the decadal time scales. Despite these efforts, our ability to understand and predict regional climate change and its impacts on the adaptive capacities and vulnerabilities of environmental and human systems is still limited because current GCMs/ESMs do not represent the myriads of human-earth system interactions that influence global and regional climate change. In addition, important human-earth system interactions that manifest at the local to regional scales cannot be explicitly represented in global models that have limited grid resolutions because of the hydrostatic formulation and computational constraints.

This project aims to develop the prototype models to represent regional earth system processes and the environmental systems. This project includes the development of two major components: the Regional Earth System Model (RESM) and the environmental system models of the Regional Integrated Model (RIM). The RESM will provide downscaled climate simulations to assess climate impacts and adaptation options. For this component, we will couple state-of-the-science community models of the regional atmosphere and aerosol/chemistry, ocean, land, and sea ice through a flux coupler to represent regional earth system processes and their interactions. The RESM will also include representations of land use and water use that fully interact with the natural water and biogeochemical cycles to characterize regional human-earth system interactions that are critical for projecting regional climate change.

For the second component, we will develop a fully integrated environmental system model for RIM to assess local/regional mitigation and adaptation strategies. Motivated by the need to reconcile the ubiquitous redundancy and inconsistency that exists across hydrology and ecosystem models in their representations of water and biogeochemical cycles, we will develop a model to represent hydrology, soil, managed and unmanaged ecosystems, and biogeochemical processes in a single modeling framework. The model will be scalable to the extent supported by data to enable coupling with the integrated human system models to capture human and earth system interactions at a wide range of scales. Additionally, this model will be based on the Community Land Model (CLM) that is also used in the global and regional earth system models. For scalability, we will develop a distributed extension of CLM (DCLM), incorporate more detailed water management in DCLM that takes advantage of the distributed framework, and couple DCLM and a crop model for more detailed representations of the managed ecosystems and land management.

During FY 2010, progress was made in several areas. On development of a prototype RESM, the Weather Research and Forecasting (WRF) model has been coupled with the CLM through a flux coupler. High-resolution CLM input data at 0.05 degree has been developed to support the application of WRF-CLM at high resolution. In addition, initial conditions for CLM and grid mapping data for a western U.S. domain have been developed. A one-month simulation of WRF-CLM produced reasonable results, and the team is refining the methods to generate initial conditions and other high-resolution input data for CLM.

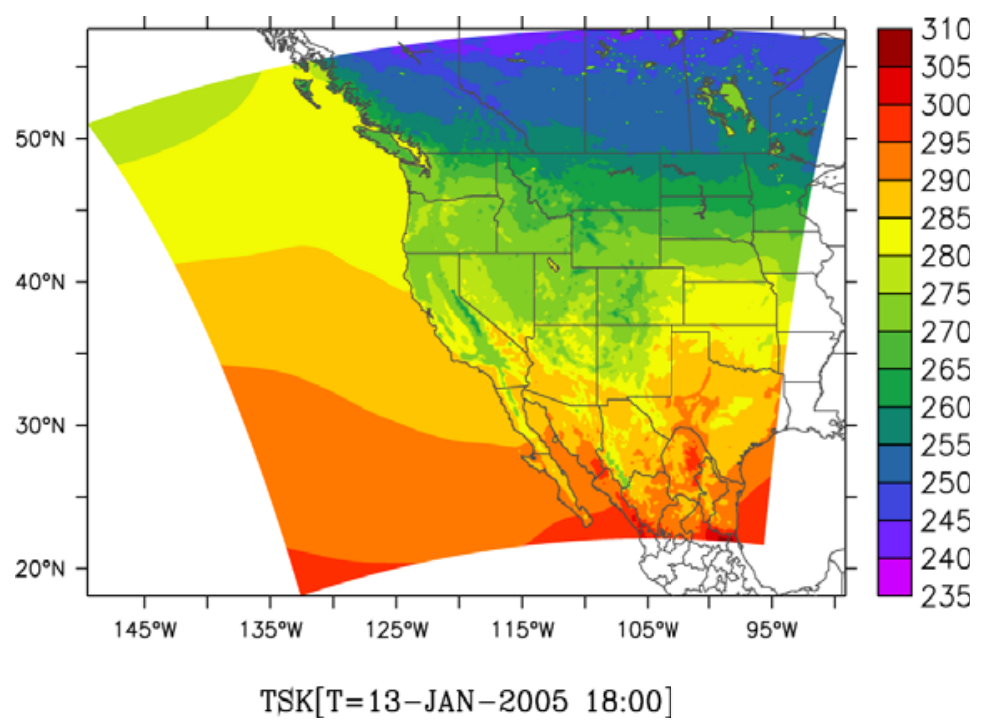

Skin temperature simulated by WRF-CLM coupled through the CCSM flux coupler. WRF-CLM was applied to a regional domain of the western United States at 12-k.m grid resolution. 
On development of environmental models, CLM has been selected to provide a consistent modeling framework with for modeling land surface and carbon cycle processes across the global model (CCSM), regional model (WRF), and spatially explicit models that will be coupled with energy infrastructure and operation models. To enhance the capability of CLM for modeling across scales, the soil hydrology component of CLM has been replaced with that of the widely tested macroscale hydrological unit: the Variable Infiltration Capacity (VIC) model. The merged CLM-VIC has been applied to several flux tower sites and examined against observations and simulations with the original CLM. The comparison suggests that CLM-VIC more realistically captures the seasonal and interannual variability of soil moisture and surface fluxes in sites with bare soil or short vegetation. On the other hand, CLM performs better in sites with deep root ecosystems because it includes a treatment of shallow aquifer. This motivates the need to include a groundwater component to CLM; therefore, we are working on incorporating a more detailed groundwater component to CLM.

In addition to merging CLM and VIC, testing of CLM applied in a semi-distributed mode has begun. In the semi-distributed approach, subbasins rather than raster grids are used as the basic modeling unit. Because subbasin

boundaries follow the natural topography, each subbasin becomes hydrologically independent, so that lateral redistribution of soil moisture between adjacent subbasins is not needed even when the subbasins are delineated at high spatial resolution. The size of the subbasins can vary depending on the land surface heterogeneity. The American River on the east side of the Cascades has been selected for our first demonstration of the semi-distributed approach. Preliminary results show unrealistically high stream flow amounts simulated

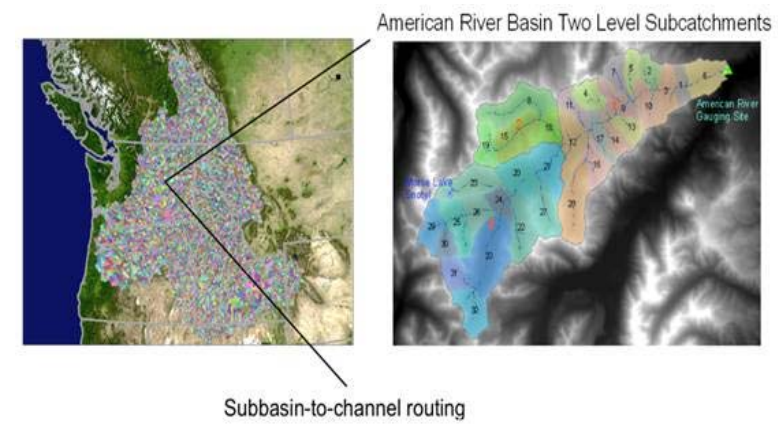

Observed and simulated SWE at Morse Lake SNOTEL

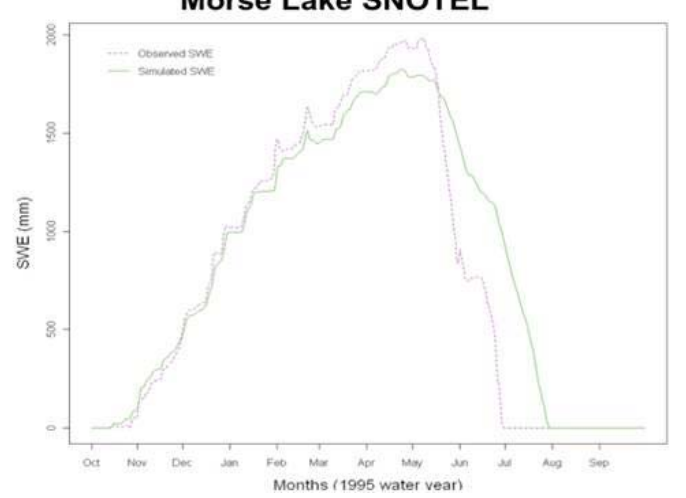

Top: The two level subbasins of the American River Basin used to test CLM in the semi-distributed approach. Bottom: The observed and simulated snow water equivalent (SWE) at the Morse Lake SNOTEL station within the American River Basin. by the model compared to observations. Sensitivity experiments are also being performed to determine the model sensitivity to hydrological parameters and other input data.

During FY 2011, we plan to: 1) merge the WRF-CLM and WRF-Regional Ocean Modeling System, 2) test of RESM with aerosols/chemistry and implement water use within, 3) test a semidistributed approach in CLM, and 4) couple a DCLM and EPIC-agricultural and land use model to provide an integrated framework to represent soil, vegetation, managed and unmanaged ecosystems, biogeochemistry, and water/ land management at the regional scale. We anticipate publishing our findings in the applicable professional journals. 


\title{
Development of Regional-Global Climate Assessment Model
}

\author{
Leon E. Clarke, James A. Edmonds, James A. Dirks, Son H. Kim, Michael J. Scott, Marshall A. Wise
}

This project will result in a new and unique modeling capability to explore climate change and climate change responses. It will result in a regional integrated model that will enable analysis at sub-regional scales (such as states or provinces) in an integrated framework that are explicitly linked to global-scale phenomena.

I ntegrated assessment modeling in the context of climate change is a form of analysis in which all of the relevant human and natural processes are brought into a single computational framework. They combine human systems such as economic, energy, and agricultural into a single platform that contains key natural systems such as climate and the carbon cycle. Although integrated assessment modeling has a long history at the global scale, no regionallevel integrated assessment model or modeling projects exist. As such, this project is part of a larger effort to perform climate change integrated assessment at a regional scale. This research effort will build such a regional-scale integrated assessment model within the context of a global modeling framework.

Our project takes advantage of the fact that PNNL uses the PNNL Global Change Assessment Model (GCAM), an integrated assessment model that combines human systems and natural systems, into a single platform for consideration of climate change and climate change response strategies. We are developing a new model based on GCAM referred to as the Regional-Global Climate Assessment Model (R-GCAM) that can incorporate sub-regional resolution. We are creating a generic capability, and we will implement and test it within one United States sub-region. The development of the generic capability and the testing and implementation in a specific region are taking place simultaneously as interactive processes. The generic capability will require new, innovative coding within the R-GCAM concept, and the testing will require necessary data, along with analysis of model behavior with this new data.

The implementation of this project is based on the completion of two prerequisites, both of which are nearing completion. One critical prerequisite is the completion of a new method of modeling agriculture and land use that is particularly amenable to subregional analysis. The code for this material is complete and has been successfully tested with preliminary data. An effort is underway to produce the necessary final data set and produce an operating version in GCAM. This requires development of consistent data from sources such as FAO and GTAP. These data sources are often inconsistent or limited, so serious data challenges must be overcome to create this first version of a spatially resolved agriculture and land use model.
The second critical prerequisite is the ability to run GCAM at arbitrary time steps. At present, the GCAM model runs on 15 -year time steps. This temporal resolution is based on the long timeframe generally used for integrated assessment analysis. However, in the context of this larger effort to conduct integrated analysis at a regional scale, fine temporal resolution (particularly in the near-term) will be desirable. In addition, the R-GCAM model will need to be linked to a range of other modeling tools (e.g., a detailed land use model, a detailed regional climate model, and a detailed electricity model), all of which operate at temporal scales well below 15 years. The code for this capability has also largely been completed. Preliminary tests indicate that the new code not only works but also provides meaningful, temporal resolution in model behavior. The behavior of the new approach continues to be tested and analyzed, and model revisions are undertaken as appropriate.

Activities that have been completed or are underway through this project include the following. Strategic planning has been initiated to identify methods for implementing a subregional modeling architecture in the R-GCAM. The first draft of a planning document should be complete in several months, with initial coding to follow soon after. A strategic plan for addressing hydrology in the R-GCAM was also completed. Efforts have begun to develop representations of water demands in electricity demands that can be used in R-GCAM. Initial results have been generated within the GCAM framework, which is in the prototype phase. In addition, we have commenced a literature review to explore methods for incorporating uncertainty analysis into the R-GCAM structure. The detailed buildings model (BEAMS) has been modified to allow for changing climate conditions, taking climate-related data and converting it into necessary inputs for both the R-GCAM and BEAMS.

Activities in FY 2011 will focus on three main areas. The first is development of a water-modeling capability within the R-GCAM framework. Water is a critical input to a range of human and natural systems (including agricultural), and changes in supply from a changing climate will be a critical area of climate impact. In addition, changes in energy and agricultural systems over time and potentially as a means to reduce emissions through biomass production will also interact with water. The second area will be in the core subregional U.S. energy system of R-GCAM. Efforts here will focus on development of energy transformation (electricity) sectors and end-use sectors (buildings, industry, and transport). The final area of development will be to take the subregional AgLU capability and build the specific data for the upper Midwest. 


\section{Ideas for Evaluation of Deep Convection Parameterizations With Scanning Radar Data}

Sally A. McFarlane, Julia E. Flahert

The goal of this project is to develop ideas for evaluating model parameterizations of convective clouds with data from the Atmospheric Radiation Measurement (ARM) program tropical measurement sites.

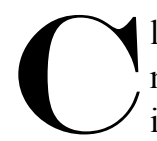
limate change from carbon dioxide emissions may impact aspects of the earth's climate system, including clouds and precipitation. The simulation of future precipitation patterns requires accurate modeling of convective clouds processes. However, creating a good representation of cloud and precipitation processes is one of the most difficult aspects of climate modeling because the relevant processes occur on scales much smaller than a climate model grid-box. Instead of processes explicitly represented at the scales at which they occur, their mean effects over the grid-box are represented by a simplified set of equations governed by a few parameters, known as parameterization. Current parameterizations of tropical convection in climate models have difficulty correctly representing several aspects of cloud and precipitation processes, including the frequency and intensity distributions of precipitation, the diurnal cycle of cloudiness and precipitation, and the transition from shallow to deep convection. The inability to model tropical convection accurately has implications for predicting tropical phenomena such as the El Niño-southern or Madden-Julian oscillations and regional monsoons.

DOE recently invested in a suite of new instruments for the ARM tropical facilities, including scanning cloud and precipitation radars and enhanced microwave radiometers. These new instruments will provide an unprecedented opportunity to evaluate and improve the way climate models connect precipitation and radiative processes. To utilize these
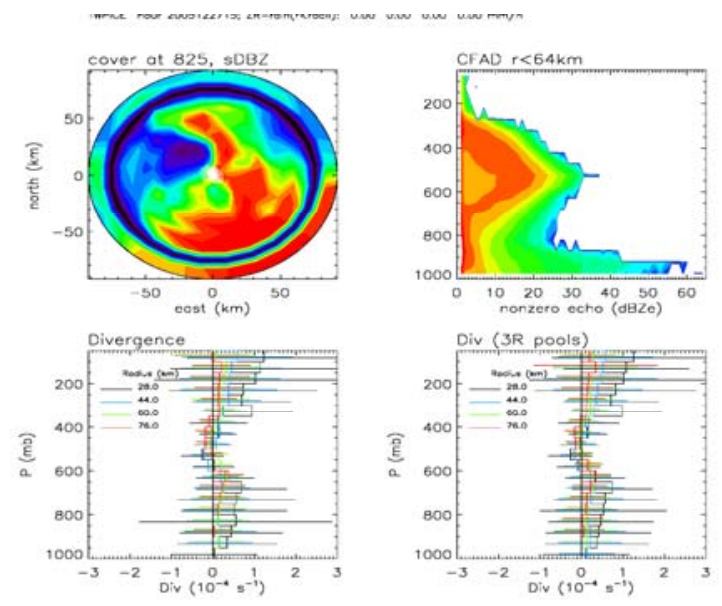

Example output from radar analysis code. new instruments fully, preliminary work on existing datasets will determine the key parameters to examine, understand instrument limitations, and develop methodology and computer codes that can be applied to the new datasets.

This year, we collaborated with an expert in modeling of deep convection, which led to a detailed understanding of the current issues in convective parameterization and innovative ideas for how they could be addressed with ARM measurements. We spent a week at the National Center for Atmospheric Research working with Dr. Richard Neale, co-lead of the Community Atmosphere Model (CAM) working group. We identified several key issues in the new CAM 5.0 model that we can evaluate with ARM measurements: detrainment height of convective anvil, vertical velocity, and multivariate relationships between rainfall and humidity metrics at a range of time scales.

The second accomplishment was to develop and implement code to work with scanning radar datasets. We spent one week at the University of Miami with Dr. Brian Mapes, an expert in tropical deep convection and precipitation radar. We used existing precipitation radar datasets from Darwin, Australia to examine convective storm evolution, the height at which cirrus anvils detrain from convection, and vertical divergence profiles. We wrote code to read, analyze, and plot reflectivity data, including movies of storm evolution and calculation of contoured frequency by altitude diagrams. Additionally, we implemented code developed by Dr. Mapes to calculate divergence from scanning radar data and modified it for use with the Darwin data. We performed a detailed analysis of one storm system to understand the key features observable from radar and to link divergence to anvil evolution. The code can be easily adapted to work with future scanning precipitation radar data at Manus and the ARM mobile facility deployment in the Indian Ocean.

Finally, we performed a statistical analysis between humidity metrics and rainfall using 6 years of measurements from multiple instruments at the ARM site at Nauru (near Australia). Dr. Neale will use this to compare with CAM results. We manually examined data quality reports from sensors to identify problem periods, developed a method for identifying and removing condensation on the precipitation sensor, and wrote code that can be easily modified to work for other datasets, time scales, or locations. Results show that the relationship between column water vapor and rain amount is fairly consistent across time scales from 0.5 to $3 \mathrm{hrs}$; thus, a single parameterization could represent this relationship, even as model grid-box sizes decrease. 


\section{Improving the Characterization of Aerosols as Forcing Agents in the Climate System}

Philip J. Rasch, Vinoj Velu, Dilip Ganguly, Nathaniel Beagley, Hailong Wang

The focus of this project is on understanding how the improvements to representation of aerosol and cloud processes manifest themselves in a fully coupled climate model, and how aerosols influence precipitation and associated dynamical features of monsoons.

V irtually all assessments of climate change have identified aerosols as among the most uncertain of forcing agents and very important to climate.

Aerosols are strongly influenced by anthropogenic activity, and it is important to produce an accurate representation in climate models to understand how mankind is influencing climate. With a long history of producing fundamental research involving aerosols, PNNL has generally focused on a process level understanding but has not attempted to determine the climate response, particularly at the global scale.

This project is designed to strengthen PNNL contributions by understanding responses to climate forcing, examining how the improvements derived using field programs and detailed model frameworks influence the model climate, and altering the projections of climate change at the global scale. The project contributes to answering the following scientific question:

How do changes to the model physical parameterizations manifest themselves in producing "aerosol forcing," cloud feedbacks and cloud forcings, and modifying fundamental circulation features of the climate system like the monsoon circulations?

Our goal is to explore the role of some aspects of aerosols important to climate change in a national tool, the Community Earth System Model (CESM), formerly known as the Community Climate System Model. The project focuses on identifying mechanisms that involve aerosols and climate feedbacks in the CESM and studies the influence of aerosols on monsoon systems, with a goal of a better description how they change the spatial and temporal distribution of precipitation and the climate. We also hope to explore consequences of the improvements to climate feedbacks and monsoon systems.

The CESM has been under development over the last 5 years. In early 2010 , the first satisfactory coupled simulations were demonstrated that are capable of producing information close the historical record of surface temperature changes over the globe. The simulations also produce a reasonable estimate for many climatic features for the last few decades, including distributions of precipitation, El Niño/La Niña-Southern
Oscillation, cloud radiative forcing, aerosol concentrations, and aerosol forcing. The model was officially released in July 2010. Prior to this event, work for this project used unstable development versions; thus, our focus shifted to the stable released model in July, during which time we also hired two new staff members to assist with our work.

The coupled ocean atmosphere climate model was ported to computers, and simulations with this model made for the first time at PNNL. Various model configurations were run for short periods, using: 1) prescribed sea surface temperatures, 2) a simplified Slab Ocean Model using prescribed heat transport and heat capacity, and 3) a full ocean model that allows ocean density, heat storage, and currents to evolve in time. The main goal was to familiarize staff in running the model and interpreting the output. A series of simulations have been designed and started to explore the response of monsoon circulations to changes in aerosol forcing from various geographic regions. An abstract was submitted for presentation at the fall meeting of the American Geophysical Union.

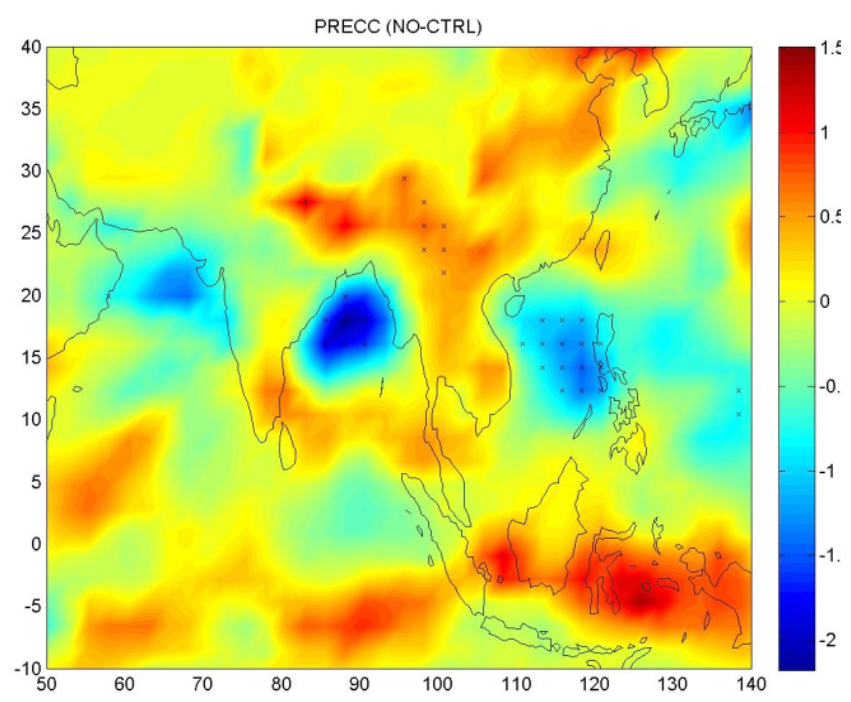

An example of the change in precipitation due to carbonaceous aerosols.

During FY 2011, we will isolate the effect of the direct changes in the radiative heating by aerosols from the indirect effect of aerosols on clouds in monsoon regions. With the response, we will contrast to aerosol emissions from Asia in the vicinity of the Tibetan Plateau, with the response similar to forcing in other regions that do not have that strong orographic feature. Additionally, we plan to produce two journal articles on the results and present them at national and international meetings. 


\title{
In Situ Imaging of Mineral-Supercritical $\mathrm{CO}_{2}$ Reactions with Atomic Force Microscopy
}

\author{
A. Scott Lea, James E. Amonette; Steven R. Higgins (Wright State University)
}

This project seeks to develop and demonstrate a novel, state-of-the art capability to investigate the site-specific reactions that occur at the molecular scale at the interfaces of minerals in conditions relevant to geosequestration in deep, underground aquifers.

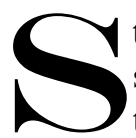

torage of anthropogenic $\mathrm{CO}_{2}$ in deep underground saline aquifers is viewed as a viable method to slow the increase of $\mathrm{CO}_{2}$ in the earth's atmosphere while continuing to allow the use of fossil C-based fuels for energy. In these aquifers, $\mathrm{CO}_{2}$ will exist in a supercritical (sc) state. Consequently, numerous bulk laboratory studies are underway to measure the thermodynamic and kinetic properties of the reactions that would occur for various mineral systems in the presence of hydrous $\mathrm{scCO}_{2}$. At the molecular scale, sitespecific reactions occurring at these mineral fluid interfaces are entirely unknown. These fundamental reactions ultimately sum to produce the interfacial phenomena observed in bulk studies and described macroscopically by equilibrium thermodynamics or kinetic rate laws. An attempt to understand and control the chemistry at these interfaces, which is necessary to assess the practicality of subsurface carbon sequestration, should be based on explicit and quantitative understanding of these fundamental interactions.

To address the above issues, we are designing and building an atomic-force microscope capable of operating at the high pressures and elevated temperatures that are expected to exist in these underground locations. This capability will be the first of its kind to operate in both $\mathrm{CO}_{2}$ and water-dominant $\mathrm{scCO}_{2}$ water phases where dehydration, mineral trapping, and solubility trapping reactions relevant to carbon sequestration occur. We will demonstrate the use of this capability to study the interfacial aspects of silicate dissolution and carbonation chemistry under supercritical conditions.

During FY 2009, we designed and manufactured a high pressure test cell with a Digital Instruments multimode hydrothermal atomic force microscope (AFM) head modified to accommodate the test cell. Connections were made in the cell permit inlet and outlet ports for the $\mathrm{scCO}_{2}$ and the introduction of a K-type thermocouple for the measurement of temperature within the test cell when noise measurements. It was necessary to regulate the temperature to within $0.1{ }^{\circ} \mathrm{C}$ over a period of seconds to minutes to permit observation of atomic scale steps on calcite.

After the successful test cell design, we designed and built an AFM head capable of operating at pressures up to $100 \mathrm{bar}$ (to replace the test cell and interface with the commercial AFM controller and $\mathrm{scCO}_{2}$ delivery system) so that we will have a fully functional high pressure AFM head. The plumbing system was redesigned to incorporate a high pressure $\mathrm{CO}_{2}$ pump to enable flow through the AFM fluid cell and to provide system pressurization without rupture of the Kalrez membrane separating the fluid cell from the scanning and translational system components. Calibration of the piezoelectric scanner was accomplished using calibration grids and $20 \mathrm{~nm}$ step height standards. Under ambient conditions (pressure at 1 bar and temperature at $22^{\circ} \mathrm{C}$ ), the AFM head is capable of imaging mineral surfaces with atomic resolution. All scanning and translational components of the AFM head have been demonstrated to operate as intended for up to 100 bar and up to $50^{\circ} \mathrm{C}$.

Under target conditions of 80 bar, the AFM head imaged single atomic steps on the surface of calcite in a nitrogen environment. This is the first time any AFM demonstrated atomic-scale imaging at these pressures. While this and other images were not taken in $\mathrm{scCO}_{2}$, this system should be capable of imaging mineral surfaces under $\mathrm{scCO}_{2}$. Time-dependent measurements of pressure and temperature at $100 \mathrm{bar}$ and $35^{\circ} \mathrm{C}$ demonstrate that these parameters vary under 0.05 bar $\min ^{-1}$ and less than $0.01^{\circ} \mathrm{C} \min ^{-1}$ which, according to calculations using the Helmholtz equation, is adequate to keep noise level due to density fluctuations below that necessary to see atomic-scale steps. Currently, we are charging our AFM head with $\mathrm{scCO}_{2}$ at 80 bar and $35^{\circ} \mathrm{C}$ and expect to obtain first-ever AFM images of mineral surfaces in situ.

In FY 2011, the kinetics of anorthite and forsterite carbonation, which are involved in "mineral trapping" processes that are expected to occur in some silicate-rich underground storage sites, will be investigated as specimens to facilitate the full development of our system.

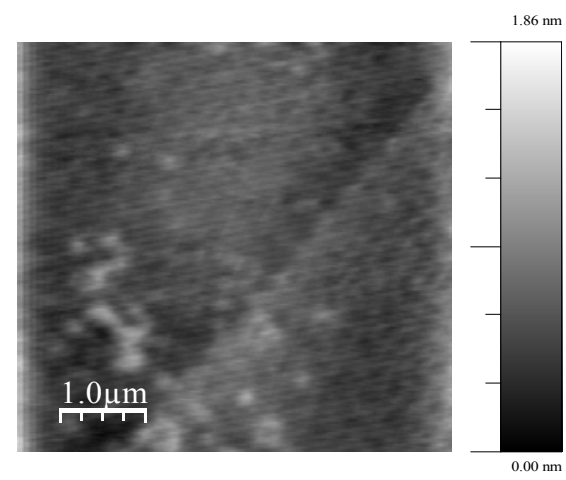

A $5.0 \mu \mathrm{m} \times 5.0 \mu \mathrm{m}$ AFM image of calcite at 83 bar and $22^{\circ} \mathrm{C}$. The single $3.2 \mathrm{~A}$ bigh monatomic step extending from the lower left to the upper right demonstrates the first-time atomic-scale imaging achieved at these pressures. 


\title{
Integrated Regional Earth System Model (iRESM) Prototype Regional Testbed Specification and Selection
}

\author{
Lai-Yung (Ruby) Leung
}

The development and testing of new systems through modeling frameworks and tools that integrate climate with hydrology, land systems, socio-economics, and energy at the regional scale enable the evaluation of regional feedbacks and interactions between energy, hydrology, climate, biogeochemistry, and global implications of socioeconomic processes. This project aims to execute a systematic approach to developing testbeds studies to demonstrate and evaluate the unique capabilities of the integrated regional earth system models (iRESMs).

A $\mathrm{n}$ important component of any modeling activity is evaluation or validation. Establishing a robust evaluation process through careful and systematic specification and the selection of testbeds or pilot studies are of vital importance to ensure that the model evaluation can improve the understanding and quantification of key model biases and uncertainties and to provide insights for model improvements. Four types of scientific approaches have been developed and applied to evaluate and understand model behaviors and sensitivities:

- Hindcast experiments, where models are evaluated on their skill in reproducing the historical conditions and the associated spatial and temporal variability

- Analysis and diagnostics of model simulations, to attribute the sources of model errors

- Sensitivity experiments, to understand the impacts of model parameterizations, model feedback processes, or the effects of external perturbations

- Model intercomparison, to assess model biases and uncertainties under a common set of simulation conditions.

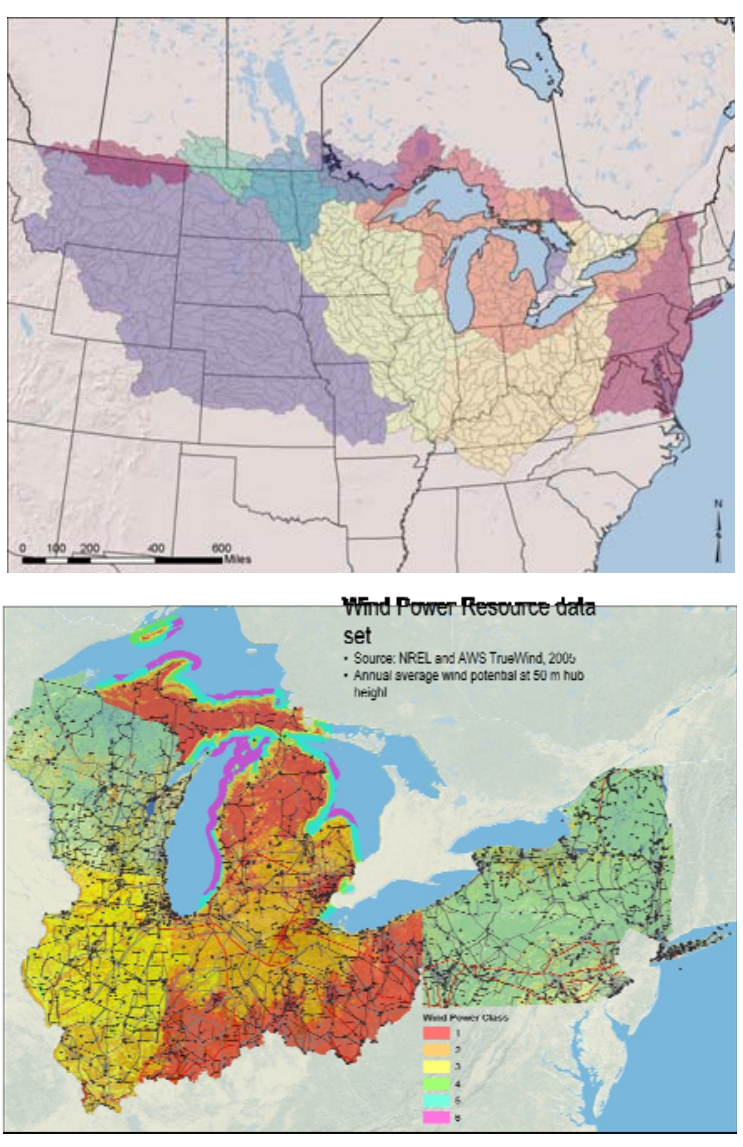

Examples of database developed for the first regional pilot study focusing on the U.S. Midwest. Top: Delineation of subbasins for bydrologic modeling using a semi-distributed approach. Bottom: Energy infrastructure data, including transmission lines, locations of power plants, and wind power resources.
This project will develop and execute a systematic process to select the iRESM regional testbed that will be used to evaluate the iRESM model and its components. This includes developing a focused set of science questions or hypotheses to be addressed by the iRESM, developing the scientific approach for model testing and evaluation, selecting geographic regions and time periods, developing the testbed database, and developing evaluation metrics. The prototype iRESM testbeds will highlight the interactions between the natural systems and energy, water, and land use.

During FY 2010, progress was made in three main areas. On the selection of a region for the first pilot study, the team decided to adopt the region definition used in the U.S. National Assessment, which divides the conterminous states into Northwest, Southwest, Great Plains, Midwest, Northeast, and Southeast, as a first cut. The Midwest emerged as the region of choice for the first pilot study because it represents significant interactions among climate, land use, energy, and water (the Midwest includes the 10 states defined in the North American Carbon Program, plus KY, WV, PA, and NY, which represents interesting contrast in terms of energy use and population, and the sliver of Canadian region that is part of the Great Lakes Drainage).

After the selection of the pilot region, the team worked on developing a database that supports modeling of the pilot region. This included the development of data for energy infrastructure (e.g., wind resource, power transmission lines) as well as data for hydrologic modeling (e.g., delineation of subbasins for the Midwest region).

PNNL then began to map a strategy for model evaluation and uncertainty characterization. Aprototype iRESM model has been defined with mostly one-way coupling among models. The prototype model is being used to 
define numerical experiments that will be performed with uncoupled and coupled models to evaluate the models.

Regardless of the methods used to evaluate models, specification and selection of the testbeds are critical to the subsequent design of the numerical experiments to provide information about model biases and uncertainties. Ultimately, this project will develop and execute a systematic process to design prototype testbeds.

For FY 2011, we have two tasks: further developing the testbed database and identifying evaluation metrics. The database task will focus on the geospatial representation of energy infrastructure and the cost and performance characteristics of existing and future energy supply, energy end-use, and emissions reduction technologies. It is envisioned that this database will become the de facto standard for GIS-based energy technology, infrastructure, and natural resource representations. For the testbeds to provide insights on model biases and uncertainties, a set of metrics must be used to assess and document model skill systematically. Based on the science questions, scientific approach, geographic regions and time periods, and the testbed database, the project team will compile a set of metrics that have been documented in the literature of the relevant fields and assess the strengths and weaknesses of each for the specific testbed objectives. 


\title{
Micromodel Pore-Scale Studies of Caprock-Sealing Efficiency and Trapping Mechanisms Related to $\mathrm{CO}_{2}$ Sequestration
}

\author{
Martinus Oostrom, Jay W. Grate, Changyong Zhang
}

To improve our understanding of subsurface supercritical (sc) $\mathrm{CO}_{2}$ storage and caprock sealing efficiency, pore-scale experimental and numerical studies of processes related to caprock-sealing efficiency and trapping are being conducted. Research at the pore scale is an essential first step to facilitate upscaling of fluid displacement processes to the field injections.

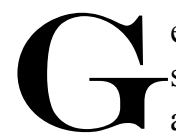
eological storage of $\mathrm{CO}_{2}$ is considered in deep saline aquifers, depleted oil and gas reservoirs, and unminable coal beds. For the first two options, interactions at fluid-fluid and fluid-mineral interfaces (such as capillarity, mass transfer, interfacial tension, and wettability) greatly influence fluid displacement in porous media. Two of the main issues related to any subsurface sequestration project are the potential leakage of $\mathrm{scCO}_{2}$ out of the reservoir into the caprock, followed by transport into the atmosphere and the storage capacity of the reservoir rock. The integrity of the overlying caprock and the storage capacity of $\mathrm{scCO}_{2}$ in deep saline aquifers or reservoirs are affected by interfacial interactions. Both caprock-sealing efficiency and free-phase $\mathrm{scCO}_{2}$ trapping are to a large extent determined by porous medium properties (e.g., pore geometry, grain size and distribution), fluid properties (e.g., density, viscosity, interfacial tension), and porous medium-fluid interactions (e.g., wettability) occurring at the pore-scale. Data related to interfacial interactions are scarce, and knowledge of displacement of brine by $\mathrm{scCO}_{2}$ (main drainage) and vice versa (imbibition) under various wettability conditions is limited.
To improve our understanding of subsurface $\mathrm{scCO}_{2}$ storage, pore-scale experimental and numerical studies of processes related to caprock-sealing efficiency and trapping are needed. The caprock-sealing efficiency is a measure of the capillary pressure at the caprock: reservoir interface that needs to be exceeded before $\mathrm{scCO}_{2}$ can move into the caprock. The major trapping mechanisms include storage of free-phase gas through hydrodynamic and capillary trapping, dissolution in formation brines, and mineral trapping through geochemical reactions. Of these trapping mechanisms at the reservoir scale, hydrodynamic and capillary trapping processes may occur on much smaller timescales than mineral and dissolution trapping.

In this project, we will complete a series of micromodel experiments at supercritical conditions addressing the following scientific challenges:

- identify roles that porous medium properties, fluid properties, and wettability (including contact angle hysteresis) play during hydrodynamic (primary) trapping, when $\mathrm{scCO}_{2}$ displaces brine, and during capillary (secondary) trapping when brine displaces $\mathrm{scCO}_{2}$

- examine the effects of fluid-fluid interfacial tension, pore-size geometry, and wettability on caprock-sealing efficiency

- study and understand the relationships between capillary pressure, fluid saturation, relative permeability during pore-scale displacement processes.
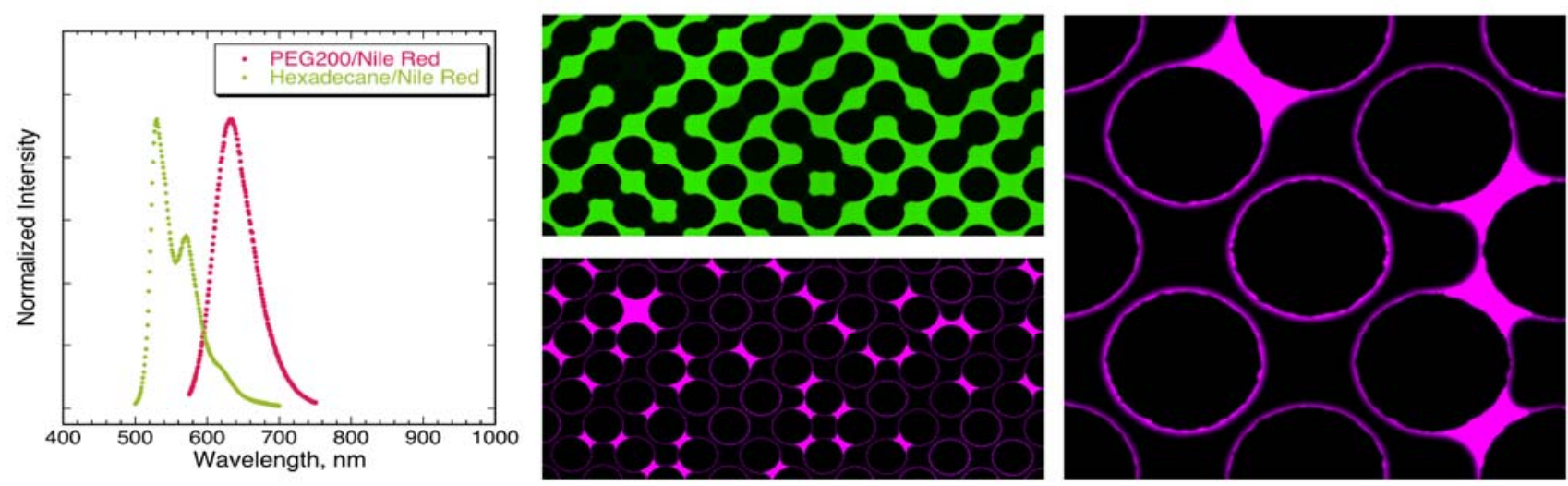

A novel method using solvatochromic dyes has been developed to distinguish between two fluids in a micromodel. This method allows us to quantify brine and $\mathrm{scCO}_{2}$ saturation separately in porous medium systems using a single dye dissolving in both the wetting and nonwetting phases. 
To address these challenges, we will design and construct a unique high-pressure system for micromodel experimentation and a capability to obtain interfacial tension and contact angle data at supercritical conditions. Methods will be developed to modify micromodel wettability and to improve displacement visualization using solvatochromic dyes and potentially planar optodes.

In the first year of this project, we developed a novel method to distinguish between competing fluids at the pore-scale using solvatochromic dye. Nile Red was demonstrated as a single dye whose solvatochromism enables selective visualization of two immiscible liquid fluids in a micromodel containing a homogeneous array of pillars creating a pore network. A descripton of this novel technique was recently accepted by Water Resources Research. The dye dissolves in and partitions between hexadecane as a nonwetting fluid and polyethylene glycol (PEG200) as a hydrophilic fluid that wets the silicon oxide surfaces in the micromodel. Bright field, epifluorescence, confocal fluorescence, and hyperspectral microscopy methods were used to image the micromodel after displacing PEG200 in the model with hexadecane. The use of Nile Red with these imaging methods facilitates visualization of phase identity at specific locations; the interfaces between the two immiscible liquid phases; wetting behavior of the wetting phase within the porous structure; and retention of the wetting phase as thin films around pillars and as bridges across the pore throats.

Also during FY 2010, we demonstrated the ability to investigate $\mathrm{CO}_{2}$-related chemistry issues at the micromodel scale. In this experimental study published in Environmental Science \& Technology, a pore-scale structure etched into silicon wafer was used to study the mechanisms of transverse mixing limited mineral precipitation relevant to geological carbon sequestration. Solutions containing $\mathrm{CaCl}_{2}$ and $\mathrm{Na}_{2} \mathrm{CO}_{3}$ were introduced through two separate inlets, and they mixed along the center of the micromodel, resulting in precipitation of the $\mathrm{CaCO}_{3}$ polymorphs calcite and vaterite. Fluorescent tracer tests conducted both before and after $\mathrm{CaCO}_{3}$ precipitation indicate that pore spaces were completely blocked by $\mathrm{CaCO}_{3}$ precipitates along the transverse mixing zone, resulting in reduced porosity and permeability. The experiments show that mineral precipitation along $\mathrm{CO}_{2}$ plume margins may affect injection and sealing efficiency.

Another important accomplishment was the completion of a series of micromodel displacement experiments to investigate the impacts of viscous and capillary forces on fluid distribution in a silicon-based homogeneous pore network micromodel. Seven wetting-nonwetting fluid pairs with viscosity ratios ranging four orders of magnitude were used. The micromodel was initially saturated with a wetting fluid and then displaced by a nonwetting fluidunder different flow rates, characterized by the capillary number ranging three orders of magnitude. Results showed two distinctive fingering mechanisms associated with viscosity ratio at low capillary number: viscous fingering and capillary fingering. Stable displacement occurred at higher capillary numbers. Results from these experiments also revealed that there is a linear correlation between interfacial area and nonwetting fluid saturation. The displacement experiments have been submitted for publication in Energy \& Fuels.

Finally, a new method was developed and tested to change the wettability of initial water-wet micromodels to oil wettability using treatment with diphenyltetramethyldisilazane. An analysis of treated micromodels indicated a contact angle change from less than 30 degrees to greater than 150 after applying the alteration method. Although extensive quantitative displacement experiments will be conducted in FY 2011, the initial scoping experiments have clearly demonstrated large differences in nonwetting fluid saturation and distribution depending on the porous medium wettability.

In FY 2011, methods to alter wettability will be developed. The goal is to manufacture both models that are uniformly wet and models that have at least two distinct wettability zones such as a water-wet zone representing the reservoir and an intermediate-wet zone representing the caprock. The solvatochromic dye techniques will be expanded to include covalently bound dyes for investigation of wetting films. In addition, the feasibility and rationale of $\mathrm{scCO}_{2}$-optode development will be established. Micromodel experiments will be conducted related to trapping mechanisms and caprock-sealing efficiency. Pore-scale models will be used to design the micromodel experiments and the obtained experimental data will be used to test and verify the numerical model.

The micromodel experiments at $\mathrm{scCO}_{2}$ injection conditions planned for FY 2011 will be conducted with a newly designed and constructed high-pressure cell. The new cell is equipped with high quality syringe pumps for accurate and precise fluid injections at constant pressure or at constant injection rate. Full control of either boundary pressure and injection rate is needed to understand observed phenomena and for modeling purposes. The design of the new pressure cell allows for fluid displacement visualization using epifluorescent microscopy equipped with charge-coupled device camera and/or hyperspectral cameras. 


\title{
Predicting Climate Change Impacts on Hydropower and Riverine Ecosystems
}

\author{
Timothy P. Hanrahan, Marshall C. Richmond, Mark S. Wigmosta
}

The future management of water resources for hydropower production in the Columbia River Basin and around the world requires attention to long-term implications of climate change. The purpose of this project is to develop a better scientific understanding and analytical tools to enhance the predictive capability of climate change effects on hydropower production and riverine ecosystems.

$\mathbf{R}$ ecent assessments of climate change impacts on the aquatic environment have been limited to large, spatial-scale predictions of future hydrologic and temperature regimes and the subsequent general ecological effects on a selected group of organisms. For example, The Washington Climate Change Impact Assessment evaluated future hydrologic and temperature changes across the entire Columbia River Basin, and the subsequent impacts to the aquatic environment were limited in scope to evaluating the habitats of salmon as a group and not individual species. Not only was this assessment on the aquatic environment simplistic in its scope, but also the fundamental methods used for predicting stream temperature changes were based on statistical regression rather than on physics- and processbased models. We require a better predictive capability of climate change effects on hydropower production and riverine ecosystems in order to improve the information available to water management decision makers and the public policy and planning officials responsible for integrating this information into social and economic forums.

Using the Columbia River Basin as an example, decisions on system water management such as timing, frequency, magnitude, and duration of reservoir releases will vary significantly depending on the predicted riverine ecosystem impacts of climate change. Thus, this project will focus on climate change impacts on hydropower production in the Columbia River. Fine-scale projections of precipitation and temperature from regional climate models will be used to predict future hydrological conditions through the application of hydrologic models. The resulting information will be used to model hydrodynamic and water quality conditions in the Columbia River and mainstream reservoirs. Riverine ecosystem changes will be predicted by integrating hydraulic and water quality conditions into predictions of primary and secondary productivity, higher order trophic level changes, and life history dynamics of aquatic organisms through the application of ecosystem models. This approach will foster the quantification of climate change impact on hydropower production and riverine ecosystems in a comprehensive manner and at a scale that is useful for decision-making.

The modeling system is being developed by linking a set of well established models that simulate watershed hydrology, reservoir and river hydrodynamics, water quality, and ecosystem processes. In FY 2010, the models were integrated and applied to a test watershed, the North Fork Clearwater River Basin, including the Dworshak Dam and Reservoir. Historic climate data and predictions of future climate scenarios were compiled for the North Fork Clearwater River Basin in order to provide temperature and precipitation input to simulate watershed hydrology. The Distributed Hydrology Soil Vegetation Model (DHSVM) was set up to model the water cycle dynamics within the study watershed. Daily average streamflow and water temperature were modeled for the existing and future climate conditions. Simulated streamflow and temperature were used as input for modeling the hydrodynamics in Dworshak Reservoir and downstream of Dworshak Dam.

Hydrodynamics were modeled with the one-dimensional Modular Aquatic Simulation System (MASS1), two-dimensional MASS2, and three-dimensional Transient Environmental and Engineering Turbulent Hydrodynamics Simulator (TE THYS). Digital elevation models for river channels and the reservoir were developed and used to construct the computational domains. Project efforts for this task focused on simulation linkages with DHSVM and the ecosystem model. Reservoir/ river water quality and trophic structure were modeled with AQUATOX, which was set up at seven locations in the reservoir and one location downstream from Dworshak Dam. Empirical data for model calibration and validation were acquired for all model locations and incorporated into a database. Hydrodynamic and temperature boundary conditions were acquired from the output of MASS1, MASS2, and $\mathrm{TE}^{2} \mathrm{THYS}$.

In FY 2011, work will continue on model development, linkage, and testing. The modeling system will be run first under current climate conditions and then underaltered climate scenarios to evaluate spatial-temporal impacts of climate change on discharge and water quality, assuming current reservoir operating strategies. A detailed representation of power generation will be developed for the modeling system, and an ensemble approach will be implemented to consider the uncertainty of climate forecasts on tributary inflows and water quality. The MASS2, TE ${ }^{2}$ THYS, and AQUATOX models will be deployed in selected mainstem reaches to evaluate impacts to hydropower production, water quality, and riverine ecosystems. 


\title{
Predicting the Feasibility of Geologic Co-Sequestration of $\mathrm{CO}_{2}, \mathrm{SO}_{\mathrm{x}}$ and $\mathrm{NO}_{\mathrm{x}}$ Under a Broad Range of Conditions
}

\author{
Diana H. Bacon, Mark D. White, Steven B. Yabusaki
}

Using the mineralogy of deep saline systems to co-sequester $\mathrm{NO}_{\mathrm{x}}$ and $\mathrm{SO}_{\mathrm{x}}$ along with $\mathrm{CO}_{2}$ could significantly lower the cost of retrofitting coal-fired power plants for carbon sequestration. A new simulator is being developed that will prove predictions of the impact of geologic co-sequestration of $\mathrm{CO}_{2}, \mathrm{NO}_{\mathrm{x}}$, and $\mathrm{SO}_{\mathrm{x}}$ on target formation and caprock hydraulic properties under a broad range of mineralogical and phase conditions.

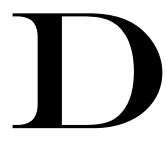
ue to limitations of previous simulators in handling the injection of multi-component and multi-phase mixtures, previous modeling studies of co-injection of $\mathrm{SO}_{2}$ with supercritical $\mathrm{CO}_{2}$ have either simplified hydrogeological systems (batch conditions) or, by injecting $\mathrm{H}_{2} \mathrm{~S}$ and $\mathrm{SO}_{2}$, have already dissolved in an aqueous phase rather than acted as constituents of the nonaqueous phase. This is an extreme case scenario in which there is no limitation on contact of $\mathrm{SO}_{2}$ with the brine. The opposite and equally unrealistic extreme case is a scenario in which $\mathrm{SO}_{2}$ is limited by diffusion through a stationary supercritical $\mathrm{CO}_{2}$ phase. A better approach is to simulate the injection of supercritical/gas mixtures, where each component is in equilibrium between multiple fluid phases. What is lacking is an approach that combines the physics of multicomponent/ multi-phase mixtures with the chemical effects of mineral dissolution and precipitation. Also lacking are modeling studies on the impacts of co-sequestration of $\mathrm{NO}_{\mathrm{x}}$. Although $\mathrm{NO}_{\mathrm{x}}$ may form a smaller percentage of the flue gas, it forms a stronger acid and could have a significant impact on mineral dissolution and precipitation rates.

In order to determine the geologic scenarios where co-equestration is practical, we calculated the carbon sequestration and co-sequestration potential of 150 minerals to use as a guide for future simulations. Injection of supercritical $\mathrm{CO}_{2}$ into geologic formations lowers the $\mathrm{pH}$ of the formation pore fluids. Under these conditions, the dissolution of minerals containing calcium, magnesium, and iron drives the sequestration of $\mathrm{CO}_{2}$ to form carbonate minerals. Co-injection of $\mathrm{SO}_{2}$ may also lower the $\mathrm{pH}$ and drive the precipitation of sulfate, sulfite, or sulfide minerals. Co-injection of $\mathrm{NO}_{\mathrm{x}}$ is problematic because nitrate forms neither insoluble minerals nor is adsorbed significantly. One method for nitrate removal from formation waters is microbial reduction of nitrate to $\mathrm{N}_{2}$ by organic matter.

Geochemical simulations indicate that co-injection of $\mathrm{CO}_{2}$ and $\mathrm{SO}_{2}$ results in $\mathrm{SO}_{2}$ mineral sequestration. The amounts sequestered are greater and more persistent in dolomite and basalt than in glauconitic sandstone. In a predominantly dolomite formation, dissolution of calcite (and to a lesser extent dolomite) will provide $\mathrm{Ca}$ in solution to promote the precipitation of anhydrite, thus removing $\mathrm{SO}_{2}$ from solution. In basalt, dissolution of basaltic glass under acidic conditions provides $\mathrm{Ca}$ and $\mathrm{Fe}$ in solution, which promotes $\mathrm{SO}_{2}$ sequestration as anhydrite and eventually pyrite.

As magnetite in the formation is consumed, pyrite redissolves. In basalt, 86 to 47 percent of the $\mathrm{SO}_{2}$ remains sequestered after 5,000 years. In glauconitic sandstone, $\mathrm{SO}_{2}$ precipitates as alunite, but it eventually redissolves. After 5,000 years, 87 to 0 percent of the $\mathrm{SO}_{2}$ remains sequestered in the glauconitic sandstone. In all cases, co-injection of 1 percent $\mathrm{SO}_{2}$ with $\mathrm{CO}_{2}$ did not appreciably reduce the amount of $\mathrm{CO}_{2}$ sequestered nor did it induce a measureable change in porosity versus injection of $\mathrm{CO}_{2}$ alone.

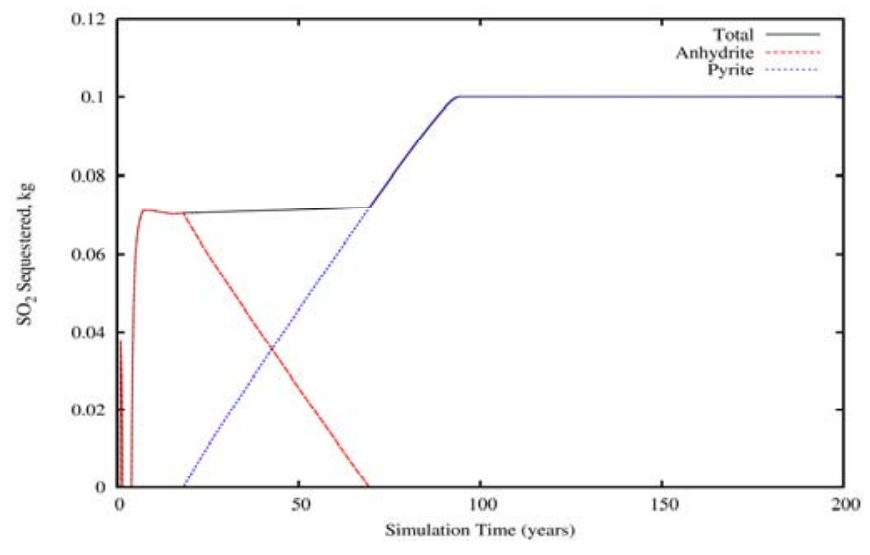

Mineral sequestration of $\mathrm{SO}_{2}$ in basalt when co-injected with $\mathrm{CO}_{2}$.

In FY 2011, we will focus on building numerical simulation capabilities for geologically sequestering a mixture of gases in deep saline, depleted oil, and natural gas reservoirs. We will develop a new three-phase, nonisothermal, multi-component operational mode for the PNNL-developed STOMP simulator. The new operational mode will be founded on the premise that all three phases will have variable compositions and component numbers. Phase and component flexibility will be handled numerically through the implementation of the Peng-Robinson cubic equation of state with a three-phase flash equilibrium model, where phase composition is defined through fugacity equilibria. The new equation of state will be written so that a general number of co-contaminants may be specified and not limited to $\mathrm{NO}_{\mathrm{x}}$ and $\mathrm{SO}_{\mathrm{x}}$. This will allow the simulation of co-sequestration of $\mathrm{Hg}$ and other co-contaminants as well as other flue gases such as $\mathrm{O}_{2}$. 


\title{
Transfer and Evaluation of the Community Atmosphere Model Parameterization Suite to Weather Research and Forecasting Model
}

\author{
Philip J. Rasch, Jerome D. Fast, William I. Gustafson Jr., Baldwinder Singh, Richard C. Easter
}

The goal of this project is to compare the treatments for processes used in global climate models with those commonly used in cloud/mesoscale models and enable a systematic, consistent methodology to evaluate various treatments of physics, chemistry, and feedback processes for both types of models that will help improve both model classes.

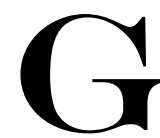
lobal climate models are tools used to represent a comprehensive statement of interactions between complex processes that govern evolution of the earth's climate and to assist science and society in understanding and projecting climate change. Although they aim to be comprehensive, the models are by nature incomplete, imperfect, and require many approximations to make calculation affordable. Regional models and those resolving features down to a single cloud system can utilize fewer approximations and may be conceptually simpler because fewer features occur at the sub-grid scale. They can also use more complex, elaborate representations for processes because they simulate features for shorter time scales over smaller regions. Thus, two communities have evolved that view the world differently and focus on unique methods. As science has evolved, these communities' interests have gradually intersected, but the tools (models) used make it challenging for one community to exploit the strengths of the other.

The aim of this project is to explore the transfer of a suite of physical parameterizations (radiative transfer, convection, turbulent boundary layer, aerosols, and cloud microphysics) from the Community Atmospheric Model (CAM) in the Weather Research and Forecasting (WRF) model and produce an initial evaluation of this suite of parameterizations in WRF. This exercise will provide an opportunity for comparing the parameterizations commonly used in cloud/mesoscale models with those in global climate models and provide a means of quantifying the performance of new process modules before they are incorporated into and used by global climate models. The merger will produce a synergy in modeling activities for weather and climate.
Configured as an aerosol modeling testbed at relatively high spatial resolution, WRF facilitates comparison to field measurements. By adding a suite of physical representations from CAM to WRF, we can help separate sensitivity of solutions to spatial resolution from changes in process representation. This provides a useful tool to aid community climate system model simulations by assessing the performance of aerosol-cloud-radiation interactions, where they can be evaluated at regional scales more appropriate for atmospheric radiation and science field measurements.

During FY 2010, the cumulus, shallow convection, boundary layer, and aerosol schemes from CAM were ported to WRF, although some work remains in finalizing the last two schemes. A methodology was established as to the best way of coupling the codes to WRF that consists of an interface layer so that the original CAM CAM modules are largely untouched. In
Grell 3D Ensemble

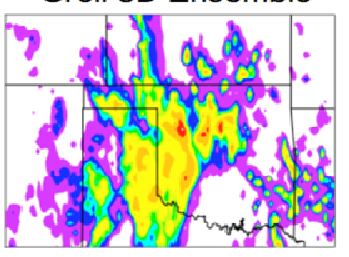

Observed 24-hr accumulated precipitation in June 2007 compared with simulated values using the CAM convection scheme and two existing schemes in $\mathrm{WRF}$.

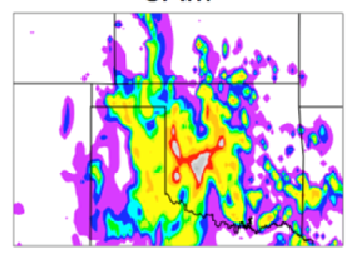
this way, updates in CAM modules can be easily ported to WRF without significant reprogramming. To ensure that our efforts are recognized and embraced by the broader scientific community, we continued discussions and solicited input regarding software engineering strategies and goals with the relevant National Center for Atmospheric Research (NCAR) global and mesoscale communities and gave presentations at the CAM and WRF workshops. Currently, our NCAR collaborators are porting the simplified version of the MOZART chemical mechanism used in CAM into WRF. Tests over the central United States were performed for the convection schemes to ensure that they operated as expected in WRF, with an example shown in the figure.

For FY 2011, we will link the photochemistry and aerosol modules in WRF, port the microphysics and macrophysics scheme into WRF and check that the new code is code compliant, verify the interoperability of CAM physics and existing chemistry options, confirm whether WRF driven by boundary conditions from CAM can reproduce the same biases, conduct a case study, produce a journal article on strategy and case study findings, and present at the CAM and WRF workshops. 


\title{
Understanding the Sources and Consequences of Uncertainties
}

\author{
Richard H. Moss, Lai-Yung (Ruby) Leung, Jennie S. Rice, Paul J. Runci, Michael J. Scott, Stephen D. Unwin
}

This project is characterizing the various sources of overall uncertainty in the integrated Regional Earth System Model (iRESM) framework. Particular attention on the sources using the model is intended to support regional climate change mitigation and adaptation decisions and to use insights to inform iRESM model development and the design of model experiments. Addressing uncertainty rigorously is key to evaluating model results and providing useful information regarding climate change.

$\mathbf{T}$ he iRESM modeling framework aims to capture important feedbacks and interactions among three natural earth (climate, hydrology, and ecosystem) and two human (energy and socioeconomic) systems. These interactions occur within and between individual sub-national geographic regions in the context of national and global changes in these same systems. In addition, iRESM is intended to provide policy-relevant insights into the complex interactions between these five systems in a regional context and to help decision-makers understand the regional consequences of climate change as well as the consequences of policies to mitigate or adapt to such change within regions. To meet the twin requirements for scientific accuracy and policy relevance, the characterization of uncertainty must be accomplished in a computationally feasible, transparent, and practicable manner using a variety of methods.

In FY 2010, the project focused on a review and recommendation of methods for uncertainty characterization (UC), uncertainty quantification (UQ), and uncertainty propagation (UP) for the iRESM framework. This effort was documented in a white paper that included a substantial bibliography. The research spanned the climate domain and other areas of science-based policy and decision-making. After this preliminary researched-based investigation, we worked on a design of the first numerical experiment for the iRESM. The project's most important findings are the investigation and evaluation of alternate methods for uncertainty characterization, quantification, and propagation that will address the need for defensibility and robustness in the policy analyses conducted with iRESM.
We developed several different uncertainty taxonomies in various scientific domains. From the integrated modeling architecture of iRESM, we focused the uncertainty characterization process on the sources of uncertainty in the individual as well as coupled models. Thus far, we have defined the uncertainty taxonomy for iRESM as follows:

- Quantifying the model input parameters accurately. We identified that the aggregate uncertainty associated with the quantification of the input parameters constitutes this first class of uncertainty. This is generally the most accessible to quantification and analysis.

- Skillful modeling. Given the scope of the analysis and the phenomena and factors for which it is intended to account, is the model accurate? The notion of prediction accuracy cannot always be completely disentangled from the vagueness inherent in the model outputs.

- Completed model. This third form of uncertainty is associated with the question, given the intended scope of application, of whether the model or model suite has captured the phenomena and factors necessary to produce accurate predictions. The completeness issue has two components: 1) the phenomena and factors known to be absent from the model but not sufficiently well understood to allow incorporation into the analysis, and 2) the acknowledgement that we may not have thought of everything that could influence outcomes, which is reflected as uncertainty in the validity of our predictions.

- Implementing the model accurately. This form of uncertainty is routed in the question of the veracity of the calculations underlying implementation of a model or model suite. The more complex and extensive a set of models, the greater the likelihood that software errors, hardware faults, and analyst mistakes will occur. Inability to confidently verify all such aspects of a large, interacting set of calculations is the source of this fourth form of uncertainty.

UC methods and their uses. A key result of our research during FY 2010 was clarifying the differences between UC, UQ, and UP and their applications. As shown in the figure, $\mathrm{UC}$ is the most general category 
and includes quantitative and non-quantitative methods. UQ methods are by definition quantitative but are more broad than the set of propagation methods typically associated with it. UP methods include both quantitative and non-quantitative methodologies. Much recent attention in uncertainty research and application focuses primarily on UQ, when in fact a broader range of methods is required to perform meaningful UC.

UC process for iRESM. We recommend that the UC process for iRESM be structured to inform regional decisions so that decision-makers will understand the implications of the uncertainty embodied in the framework's outputs for their decision-making. This means that the decision context necessarily provides the organizing principles for UC. More specifically, we identified the following 10 steps to the UC process:

1. Characterize stakeholder decision support needs in terms of regional mitigation and adaptation decisions and iRESM science questions.

2. Identify the decision criteria for making these decisions and parameterize.

3. Identify the iRESM component models that produce relevant output variables.

4. Identify the relevant input parameters for each component model and upstream model couplings.

5. Identify the sources of uncertainty in each of the models and model couplings from (4) according to the taxonomy.

6. Select a UC method and develop uncertainty bounds (i.e., not probability distributions) for each source of uncertainty.

7. Determine the significance of these uncertainty bounds through sensitivity analysis using the impact on the parameterized decision criteria as the basis for the sensitivity test.
8. Characterize uncertainty in the most important sensitivity drivers (i.e., those that have the largest impact on the decision criteria) according to the UC method selected in (6).

9. Propagate the uncertainty through iRESM using surrogate models as necessary to facilitate UP.

10. Evaluate and interpret the results for the decision context.

Scenario analysis as an approach to $\mathrm{UC}$ is relatively unique to the climate change domain and involves the characterization of a range of alternative futures. Scenario analysis as a UC method incorporates both qualitative and quantitative methods that define the boundary conditions for key variables. Each scenario is defined as a combination of quantitative point estimates for these factors and is intended to portray a consistent possible future. Subsequent analysis of climate impacts is then subject to the boundary conditions defined by each scenario. Research conducted under this project contributed to the development of a new process for integrating socioeconomic, climate, and ecosystems research in more tightly coupled scenarios of emissions, climate change, and related environmental changes such as land cover change. This new scenario process is expected to play a role in both $\mathrm{UC}$ and engagement of decisionmakers in iRESM.

In FY 2011, the project will conduct a series of roundtables with the iRESM modeling team and make a selection of stakeholders identified in a separate LDRD project. The project will also implement, test, and assess the UC methods selected within the iRESM framework. The goal is to determine the validity and usefulness of the UC methods to answer archetypal stakeholder questions. Numerical experiments will be designed for the pilot study to demonstrate the capability of the iRESM models to address mitigation and adaptation issues for the Midwest region of the United States. Feedback from these experiments regarding the usefulness of the UC methods will be incorporated into revised recommendations as necessary and will be recorded in a white paper. 


\section{Energy Supply and Use}




\section{Alpha Voltaics}

David E. Meier, Randall D. Scheele, Anne E. Kozelisky, Jon M. Schwantes, J. David Robertson, Jae W. Kwon (University of Missouri)

This project investigates the feasibility of converting the emissions from alpha emitting radionuclides into usable electrical power using a liquid-based semiconducting matrix. If successful, our research could provide a longer-lived power source at a reduced volume and mass than that of current conventional chemical cell technologies.

C onversion technologies that use radioactive decay as a power source were first conceived in the early 1900s. This knowledge could theoretically provide orders of magnitude more energy density over current conventional chemical cell technologies of similar volumes. Miniaturized power sources that are long-lived, lightweight, and have high energy densities are usable for many different types of applications, including independently powering critical components or systems, anti-tamper protection, and as a power source for persistent sensing. Significant accomplishments were achieved during the 1960s and 1970s with the development of BetaCel batteries for use in cardiac pacemakers. Historically, "soft" (emissions less than $300 \mathrm{keV}$ ) beta emitters were used to mitigate efficiency loss due to radiation-induced lattice defects within solid-state semiconducting matrices. The emissions from an alpha source into a solid-state semiconductor would dislocate atoms and create a non-homogeneous electrical field within the devices effectively trapping electrons, eventually rendering it unusable.

Wide application of radioisotope micro power sources (RIMS) are an ideal solution to the challenge of independently powering miniaturized remote sensors and micro-electromechnical systems. For various reasons, however, wide application of RIMS technology has not occurred. While solid-state betavoltaics have been developed, traditional solid-state semiconductors are vulnerable to self-absorption and radiation-induced lattice defects. The former limits the overall conversion efficiency of the process, while the latter degrades performance over time. Studies have questioned the ability of solid-state alpha voltaics to provide power for an extended period of time due to radiation induced lattice defects.

An alternative method for developing an alpha voltaic involves using a liquid-based semiconductor (or semi-metallic liquid), which is the goal of this project. The principle of the device is to convert the energy associated with alpha decay as efficiently as possible into usable power. The device behaves like a Schottky diode, with the semiconducting material (liquid selenium) acting as a depletion region placed between two dissimilar metals. This project uses a liquid-based semiconducting matrix as a way to mitigate the radiation induced lattice damage through simultaneous annealing. Theoretically, this would also permit the conversion of alpha particles (as well as other large ionizing particles) into usable electrical power.

The devices are produced by our collaborators at the University of Missouri Research Reactor, Dr. Jae W. Kwon and his team, who have demonstrated successful power generation using a beta-emitting isotope $\left({ }^{35} \mathrm{~S}\right)$ as its source material. The University's role in this project is to provide devices that would be tested at PNNL. Information and data would be shared between the two institutions and used to modify the devices for further testing.

In FY 2010, we modified a glove box. Available isotopes for study included Plutonium-238 (due to its availability) and a potential energy density of $550 \mu \mathrm{W} / \mathrm{mg}$ of $\mathrm{PuO}_{2}$ and Polonium-210 with a potential energy density of $141 \mathrm{~mW} / \mathrm{mg}$. After thorough surrogate testing, an alpha-emitting radionuclide was loaded into a device in the late spring and achieved minimal power production. Because the device produced power for a very short time, work is currently being conducted isolating the cause of the short lifetime of the cell.

Further testing in FY 2011 will focus on identifying the root cause of the device failure and correcting it. We will also identify, acquire, and/or produce the ideal alpha emitting radionuclide source material for this device. We plan to determine whether the semiconducting materials and the epoxy holding the device together will degrade in a high radiation field. This will be accomplished at PNNL using the High Exposure Facility using a ${ }^{60} \mathrm{Co}$ source.

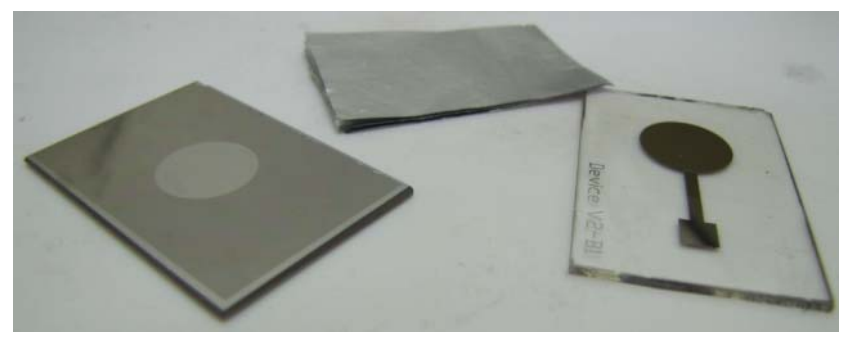

Liquid semiconductor conversion device. The slide to the left is coated in nickel, and the slide to the right has been plated with aluminum. 


\title{
Analytical Framework for Assessing the Economics of Reliable Fuel Services and Supply
}

\author{
Steven M. Short, Mark R. Weimar, Seán J. Kreyling, Zoe N. Gastelum, Julia B. Harvey
}

We developed an analytical construct and software toolkit to perform systematic comparative studies of reliable fuel services and supply options that will further the understanding of the economics and risks associated with potential reliable fuel services and supply offerings.

A long with the anticipated global expansion of nuclear energy, concerns have been raised by the international nonproliferation community about the consequences of additional states acquiring enrichment and reprocessing (ENR) technologies and know-how as part of a nuclear energy program. These concerns, however, are not new. Interest in creating policy measures to control the use of nuclear energy has been present since the 1940s and can be seen in the Atoms for Peace proposal initiated by President Eisenhower in 1953. Efforts to reach an international consensus on how to control and secure nuclear fuel supply were undertaken extensively during the 1970s and 1980s by the International Atomic Energy Agency (IAEA) to no avail. The desire to develop an international consensus has been rekindled by renewed international interest in nuclear power and recent international incidents involving states' development of ENR capabilities outside of the nuclear nonproliferation treaty. As a result, to date, at least 12 proposals offering varying degrees of multinational or multilateral reliable nuclear fuel service (RNFS) assurances have been made to the IAEA by various nations and non-governmental organizations, with additional proposals expected.

PNNL is developing a framework for assessing the economics and nonproliferation merits of alternative RNFS arrangements. The global use of nuclear power is poised to expand substantially over the next few decades to address national desires for energy security, economic parity, and climate change mitigation. However, the expansion of use of nuclear power also increases the potential for further proliferation of sensitive nuclear technologies, namely uranium enrichment and spent fuel reprocessing. Multilateral options for providing RNFS are being proposed to address these conerns. PNNL is proposing the use of a systematic analytical framework (and modeling tool) to consistently assess and compare the economic and nonproliferation benefits of proposed RNFS options.

A cash flow model was developed having modules or components representing each major process in the nuclear fuel cycle using data and approaches predominantly already developed and available in the literature. The analytical framework provides the flexibility to assess the economic impact of alternative fuel supply (front-end) regimes and alternative spent nuclear fuel (SNF) disposition (back-end) regimes. The analytical construct spans the entire breadth of the nuclear fuel cycle because the proposed RNFS options range from providing reliable fuel supply on the front-end to providing fuel-cycle services on the back-end, plus proposals for making fuel suppliers ultimately responsible for SNF management and disposition.

The current version of the model only reflects modules for enrichment, dry storage of SNF, direct disposal, PUREX recycle facilities, and MOX fuel fabrication. Advanced fuel cycle modules are not currently incorporated into the model, although the prototype model has been constructed with the flexibility to incorporate additional modules in the future. The model's analytical framework identifies the necessary fuel type, quantity, characteristics, and cost factors, and quantifies the cash flow analysis, economic analysis, and logic. Currently, the analysis figures-of-merit include 1) total and incremental life cycle cost, cash flow (revenues and expenses), and unit cost $(\$ / \mathrm{kWh})$ based on constant dollar analysis, and discounted dollars, 2) logistical attributes, such as total and annual metric tonnes heavy metal, and fuel-cycle facility requirements (e.g., number, capacity, timing, etc.), and 3) fuel characteristics (e.g., time since discharge, burn-up, etc.). 
During FY 2009, three fuel services scenarios were evaluated to demonstrate the utility of the model and compare the cost of fuel leasing relative to the current nuclear fuel service market. Each of these scenarios was in turn evaluated for three different assumptions regarding back-end fuel management approaches. The results are presented as a cost per unit of electricity produced $(\$ / \mathrm{kWh})$ for nations having one, three, and six power reactor programs. The model results clearly show that for both open- and closed-fuel cycles, fuel leasing is preferred from an economic cost standpoint and that the cost premium or penalty for not using fuel leasing can be substantial.

Our work in FY 2010 included development of a proliferation potential index model, which includes first order effects of technical capability, nonproliferation regime effectiveness, and national motivation influences. Outlines of a comparative proliferation potential analysis and an integrated economic/ nonproliferation value added analysis approach for RNFS option studies were developed. The models appear to have acceptable sophistication to test the comparative value of different proposed arrangements. This model includes a Proliferation Potential Index based on the technical capability, nonproliferation regime effectiveness, and national motivation, yielding an index normalized from 0 to 1 as a first-order approximation of the complex problem of quantifying proliferation potential. This model also includes the development of constructed scales for evaluating asymmetric weapons of mass destruction (presence or absence of chemical, biological, and nuclear weapons in neighboring countries), asymmetric conventional forces, a regional climate of war or peace, and the proliferation potential of neighboring states. Beyond completing the above-mentioned tasks, we made two Institute of Nuclear Materials Management conference presentations regarding our work.

Our initial research focused on the development of an analytical framework and model for assessing the economics of RNFS alternatives. The utility of the framework was demonstrated in an assessment of fuel leasing as an RNFS alternative. While initial efforts were made in FY 2009 to develop a metric for assessing the nonproliferation benefits of RNFS arrangements, this was a primary focus in FY 2010.

The nonproliferation metric of FY 2010 was also integrated with the economic model, completing the prototype integrated model and demonstrating its utility as a tool for consistently and systematically assessing the economic and nonproliferation merits of alternative RNFS arrangements.

An early exercise of an economic analysis framework for evaluation of RNFS arrangements has been applied to full-service, fuel-leasing arrangements.

Calculations showed that for states having a small nuclear fleet, fuel leasing may have a significant cost advantage over indigenous fuel-service arrangements and even over fuelservice arrangements using the current supplier market. However, our analyses also show that the fuel leasing cost advantage decreases with increasing reactor fleet size and will reach a limit for open fuel cycle scenarios. This is apreliminary conclusion since the model is still under development and does not account for the cost of research, development, and deployment and nonperformance risk mitigation, which could be incorporated in future work. Nevertheless, these results are encouraging because they demonstrate that leasing can provide significant economic value to entry-level nuclear power states that would elect to lease fuel under such arrangements. 


\title{
Behavioral and Social Modeling to Reduce Energy Use, Climate Change, and Power Grid Vulnerability
}

\author{
Thomas F. Sanquist, Bin Shui, Heather M. Orr, Steven C. Smith
}

This project is concerned with developing a modeling capability to understand the impact of specific social and behavioral lifestyle actions and patterns on energy usage and greenhouse gas emissions.

$\mathbf{T}$ raditional physical-technical-economic modeling approaches as represented by integrated assessment models can be enhanced by addressing behaviorally specific actions that can be taken in shorter timeframes to reduce energy requirements and greenhouse gas emissions. The modeling can eventually lead to policies and programs that are tractable from an implementation standpoint and can move action on climate change into the arena of specific behavioral changes at the individual, group, and community level.

We will develop a series of social and technological models that predict the energy use and greenhouse gas impacts of various human-based demand reduction interventions. The research outcome is a series of plausible scenarios on social dynamics, energy grids, and national security implications that are intended to provide policymakers with options for implementing socially- and technically-based demand reduction interventions to reduce power grid vulnerability to supply shortfalls. We will enhance a previous modeling system (known as consumer lifestyle analysis) with technical models of electricity supply and demand. Our prototype end-user for the model is an energy policy planner charged with developing federal-level interventions to reduce consumer-based energy consumption and greenhouse gas emissions.

Our project developed a quantitative, multivariate model of lifestyle factors in relation to U.S. residential electricity consumption. We specifically focused on electricity consumption for several reasons: 1) the proliferation of consumer electronics represents a growing source of electricity consumption that is likely reflected in social, cultural, demographic, and behavioral measures, 2) there is increasing interest in "behavioral wedges" to reduce energy usage through efficiency and conservation have clear implications for a range of lifestyles, and 3) the rapid development of "smart grid" technologies for changing consumption patterns through feedback can benefit from better knowledge of the lifestyle factors that influence energy consumption. While our analysis is focused on electricity because of the relative availability of data, the findings are applicable to other sources of energy as well.
There has been an increase in the amount of data available to for this type of analysis in recent Residential Energy Consumption Surveys, including more detailed questions concerning miscellaneous electrical loads and usage. The data can support an enriched approach to segmentation and cluster analysis of residential energy consumption, to complement prior analyses that have been based primarily on household demographic variables. By combining factor analysis to develop composite variables expressing relationships between demographics, technology choices, usage, and preferences, specific lifestyle factors can be defined. Further use of those lifestyle factors as predictors in multiple regressions can indicate their relative importance in predicting energy usage and thus identify prospective targets for efficiency and conservation interventions, such as smart grid-based in-home display technologies.

Work accomplished during the FY 2010 included the development of both a database of energy consumption for the 2005 and 2001 Residential Energy Consumption Survey single-amily households and multiple regression models for 2005 and 2001. To this end, we also performed an extensive exploratory data analysis using factor analytic and multiple regression methods, development of specific lifestyle, and demographic/economic factors for predicting electricity consumption. Additional analysis for this project included future energy scenarios using the PNNL Global Change Assessment Model (GCAM) model to evaluate behavioral interventions related to smart grid implementation. Further, we evaluated validation methodologies of building energy models and the extent to which they account for occupant effects in buildings. Finally, we analyzed the overall growth patterns of energy consumption in the United States in relation to direct and indirect sources of energy.

The most important findings of the work this year are that residential electricity usage can be modeled by five lifestyle factors: usage of air conditioning, laundry appliances, personal computer, and television as well as climate zone. Additional demographic/economic factors include electricity price and income, although in a structural regression model, income adds very little predictive capability. Overall, these factors account for 55 percent of the variance in electricity usage. Application of the 2005 factors score regression weights to 2001 data shows similar predictive capability, suggesting a stable behavioral model. 
Use of the GCAM integrated modeling tool indicates that implementation of smart grid behavioral measures such as information feedback or critical peak pricing will result in either a continuous small percentage offset in electricity usage or a small reduction in peak load over the scenario periods analyzed. Enhancements to the GCAM tool are required to evaluate issues related to adoption rates, geographic effects, lifestyle variables, and measure persistence. Similarly, individual building models have been shown to yield moderately useful predictions about energy performance on average but tend either to over- or under-perform on an individual building basis. Validation and enhancement of the models with enriched behavioral representations is a method for making these models more accurate. 


\title{
Centralized Control vs. Decentralized Control: Implications of Demand Response and Distributed Resources on Power System Security
}

\author{
Shuai Lu, Nader A. Samaan, Marcelo A. Elizondo, Ruisheng Diao, Chunlian Jin, \\ Harold Kirkham, Ebony T. Mayhorn, Yanfeng Zhang
}

The objective of this project is to investigate the potential impacts on power system reliability from applying centralized and decentrailized control approaches for demand response and distributed resources

A dvancements in communications, information, and computer technologies have opened new opportunities to the control of the generation, storage, and load resources in distribution systems. A well designed implementation strategy will help to maximize the benefits that distributed energy resources (DERs) can bring to the power system, including improved reliability, higher operation efficiency, optimal use of renewable generation, and deferred infrastructure investment. The control of DERs, including demand response and distributed resources in response to bulk power system changes, can be categorized into centralized control and decentralized control, depending on where the intelligent controller making the response decision is located. Centralized control sends the command signal from a central location (the utility or load aggregation company) to turn on or off the load and adjust the output of the distributed resources. Decentralized control features autonomous response by each individual load or resource, monitoring signals containing system status information such as price, system frequency or voltage, or their derivatives. The performance and impacts of each specific DER controller design should be thoroughly evaluated before the actual implementation. Capability of modeling the steady-state and dynamic characteristics of the DER will help greatly in the design and operation of ongoing pilot programs and future

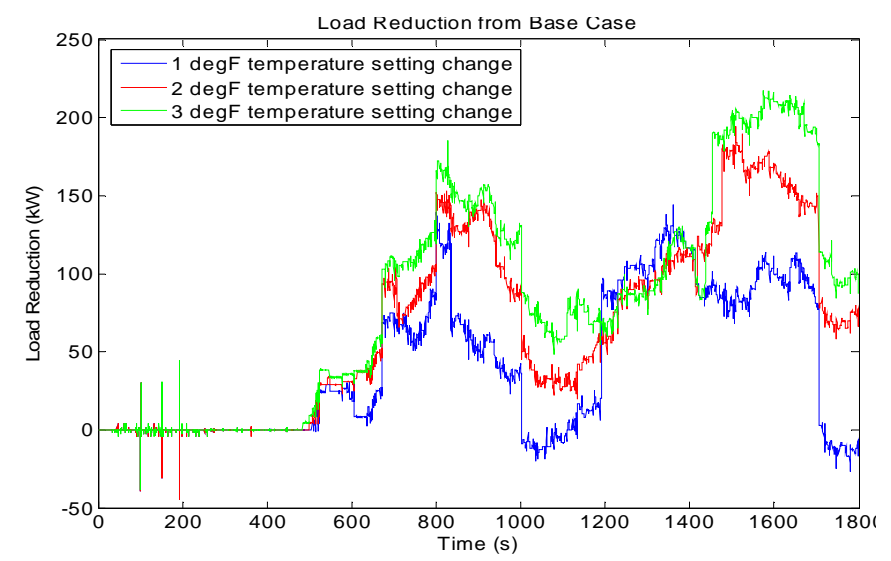

Load reduction as a function of changes in the temperature settings of water heaters and air conditioners. large scale implementations. However, no systematic study has been conducted in this regard.

We will study the performance and effects on power system reliability from the application of these two control approaches for DERs using comprehensive dynamic modeling and simulations. The investigation is an initial attempt to identify potential challenges and guide the design of such technologies. Important outcomes of the project include the study results on the characteristics and impacts of each DER control approach as well as a testing platform and testing cases that can be used to evaluate different DER controller designs for both steady-state and dynamic performances. These outcomes will provide necessary information and tools for the formation of smart grid DER control strategy and will help maximize the benefit of DERs for the power grid.

During FY 2010, we developed a test benchmark system with generic quasi-steady state and dynamic DER models such as rooftop photovoltaic, distributed wind generators, and detailed air-conditioning and water heater models. Basic demand response control strategies were implemented and simulated. Different patterns of interaction among DERs and how traditional load shapes in distribution systems will change with the inclusion of DERs were investigated. The control performance of centralized control and decentralized control in terms of delay time and predictability were demonstrated through simulation results. The research performed in FY 2010 will position PNNL at the leading edge in analyzing smart grid techniques through comprehensive simulations.

In FY 2011, this simulation capability will be used to conduct studies on the impacts of DER controls on power system reliability, for example, simulating the dynamic impact of grid-friendly appliance controller on a transmission system, interactions of DER with transmission system disturbances, and the effect of high penetration of renewable distributed generation on transmission network. We will develop study cases and perform comprehensive dynamic simulations taking into consideration the significant amount of distributed resources expected to be installed in distribution systems in the next decade; analyze simulation results to evaluate the performance of both the decentralized control and centralized approaches, with the purpose of ensuring power system reliability; and evaluate the challenges facing each control approach to be widely used in the future. 


\title{
Development of a State Prediction Methodology Powered by Phasor Measurement Unit Data to Improve Operational Reliability and Efficiency
}

\author{
Ning Zhou, Francis K. Tuffner, Guang Lin, Thomas A. Ferryman
}

The goal of this project is to establish a predictive methodology to provide accurate estimates of power system states for current and near-future time periods. Our objective is to improve the efficiency and reliability of power system operation.

A state estimator is an essential tool for power system operation. From delays in communications (typically between 1 and $5 \mathrm{~min}$ ) and calculations, current state estimators can only provide power system status in the past. Operating a power grid based on past data is acceptable only when a power grid does not change very much or does not deviate significantly from the forecasting mode. However, with the high level penetration of renewable generation (e.g., wind and solar), the North American power grid is going to experience significant levels of variation and uncertainty in power flow. With quick changes and great uncertainty from renewable generation, operations based on past deterministic states can lower the reliability and efficiency of power grid operation.

The proposed state prediction method aims to give current and "look-ahead" view for power system states through a prediction model. Combined with the past states from traditional estimators, the proposed study can provide the whole picture of power system operations, providing guidance and allowing time for power system operators to take proactive actions. Thus, this project is motivated by the challenges of increasing uncertainty and variation to power system operation. We aim to develop a state prediction methodology to facilitate the high penetration of renewable generation by increasing situational awareness for grid operation, and we expect to create a set of algorithms for power system state prediction.
To accommodate the high penetration of renewable generation, we propose to develop a prediction methodology to estimate power system states of current and future time under a statistical framework to improve efficiency and reliability of power grid operation. The two objectives of the investigation are to develop dynamic probabilistic prediction methods to predict power grid variables based on PMU data and to develop an uncertainty quantification method is to estimate the prediction errors.

For the first objective, information was extracted from the phasor measurement unit (PMU) data from 38 sites in the Western Electricity Coordinating Council reliability region of the western United States from May, August, and December 2009. The data were reduced by calculating the median value for each 5-minute time segment. Several prediction methods (including naïve prediction, moving average prediction, two-point regression prediction, and multi-point linear regression prediction) were coded and run on the data. The results of the investigation show that for each prediction interval $(5,10,15,25,50,75$, and $300 \mathrm{~min})$, at least one method "beat" the naïve method. This result successfully demonstrates that the real power on transmission lines can be successfully predicted.

For the second objective, a probabilistic collocation method (PCM) was developed to quantify the uncertainty in state estimation. Compared with the classic Monte Carlo method, the PCM is based on sparse grid points and uses a smaller number of ensembles to quantify the uncertainty. Thus, the PCM can quantify the uncertainties of a large number of power system variables with relatively lower computational cost. The PCM was applied to an Institute of Electrical and Electronics Engineers (IEEE) 14-bus system to quantify the uncertainty of power system state estimation. Comparisons were made with the Monte Carlo method.

Simulation results show that the proposed PCM can achieve same accuracy as the Monte Carlo method with smaller ensemble size and is thus computationally more efficient.

Our project efforts yielded the following outcomes: we presented at MIT's international conference on computational sustainability and have two potential publications/presentations at the IEEE annual conference. We have also created a research quality code and demonstrated an approach using simulation and real-world data. For our research and development oriented task, we anticipate investigating capabilities related to this research. 


\title{
Development of a Universal Energy and Infrastructure Systems Framework
}

\author{
Michael C.W. Kintner-Meyer, Jennie S. Rice, Timothy E. Siple, James A. Dirks, David Anderson,
} Chunlian Jin, Erin L. Hamilton, Jerry D. Tagestad, Nino Zuljevic

The objectives of this modeling framework are to provide planning tools with the necessary detail to explore energy technology and infrastructure investments options under climate change constraints, and provide detailed emissions and land-use information for energy production to other models of the integrated Regional Earth System Model (iRESM) modules to be analyzed in the broader earth systems and global climate context.

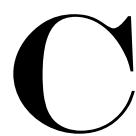
urrent toolsets do not provide an efficient and integrated way to address the technical and economic complexities of siting, expanding, and operating new infrastructure at a sufficient level of detail for investment decisions in the context of climate change and climate policy uncertainties. This project initiates a development process to fill this methodological gap, including developing and testing new systems modeling frameworks and tools that integrate climate with hydrology, land systems, socio-economics, and energy at the regional scale to enable regional feedbacks and interactions between energy, hydrology, climate, biogeochemistry, and global implications of socioeconomic processes. Specifically, we are developing a Universal Energy and Infrastructure Systems Framework (UEISF), consisting of tools and models to analyze the impacts of climate change and the associated mitigation and adaptation strategies on the energy infrastructure. This analytics framework will address the knowledge gap of regional infrastructure planners to design energy infrastructure that will be resilient, affordable, and flexible to sustain new conditions imposed or caused by climate change.

This project will develop a set of models. A power plant siting model considers land-use availability and spatial considerations such as exclusion zones and distances to necessary infrastructures for fuel or the transmission grid. This model will be using highly resolved geographical data sets of land-use, water resource availability, renewable energy resources, transportation infrastructures, and energy infrastructure for fuel input and energy service delivery. Next, a building model represents zonal building stock and their response to population and economic growth and weather dependency, and advanced demand response strategies enabled by smart grid technologies. This model will calculate the electricity consumptions hourly for 1 year. Finally, an operations model simulates the operation of the bulk power electric grid. It considers the economics of individual power plants and characteristics of the bulk power transmission system to perform an economic dispatch of power plants to

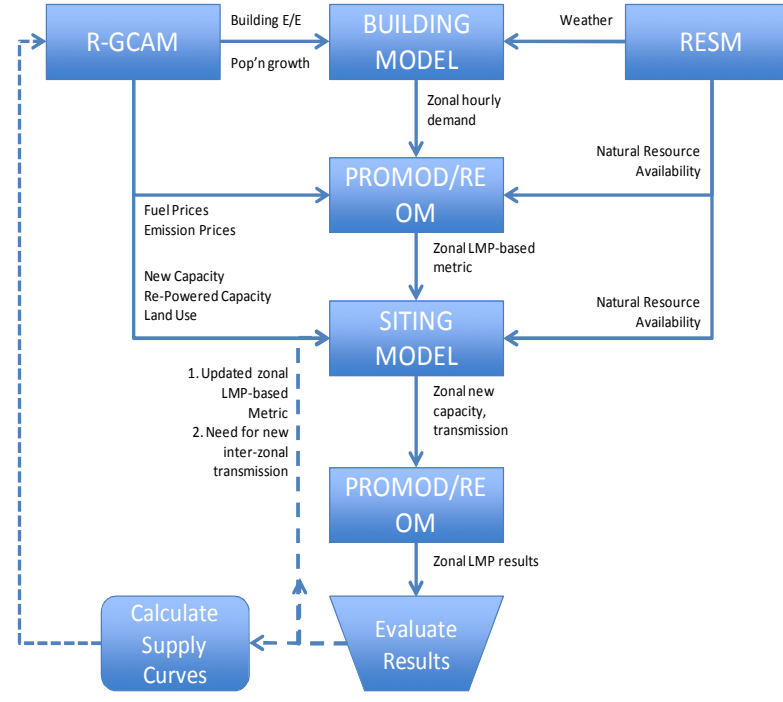

The models and their interactions. meet hourly loads. The model will be linked to a hydrological model to assess whether or not sufficient water resources are available for normal operation of power systems. The model will be responsive to weather induced loads such that severe weather conditions and their full impact on the infrastructure can be analyzed.

During FY10, progress has been in the following areas:

- We developed geographic information databases that would store relevant geographic data sets in a format that can be easily accessed, analyzed, and used for visualization purposes. All data are now stored and available as raster data sets. We developed analytics tools to perform complex, nested queries on geographic datasets. This would allow the user community to apply our models without requiring licenses for GIS analytics tools.

- We tested feature extraction methods to characterize buildings stock for buildings electric load modeling. This effort appeared to be more complex than first anticipated, and we determined that it would be terminated at the end of this fiscal year. 
- We developed a building model. The particular functionality used for the UEISF project is its ability to generate hourly load estimates for the building stock in a particular utility zone. We made significant progress in disaggregating system load profiles to attribute the contribution of the commercial and residential building sectors to the entire system load. We estimated statistically the weights of

20 commercial building types and 2 residential types to represent their contribution to the entire system load.

- We developed a tool to adjust the electric load inputs into our current electricity operations model, and analyzed the impacts of severe weather in the summer on the grid in the Great Lakes

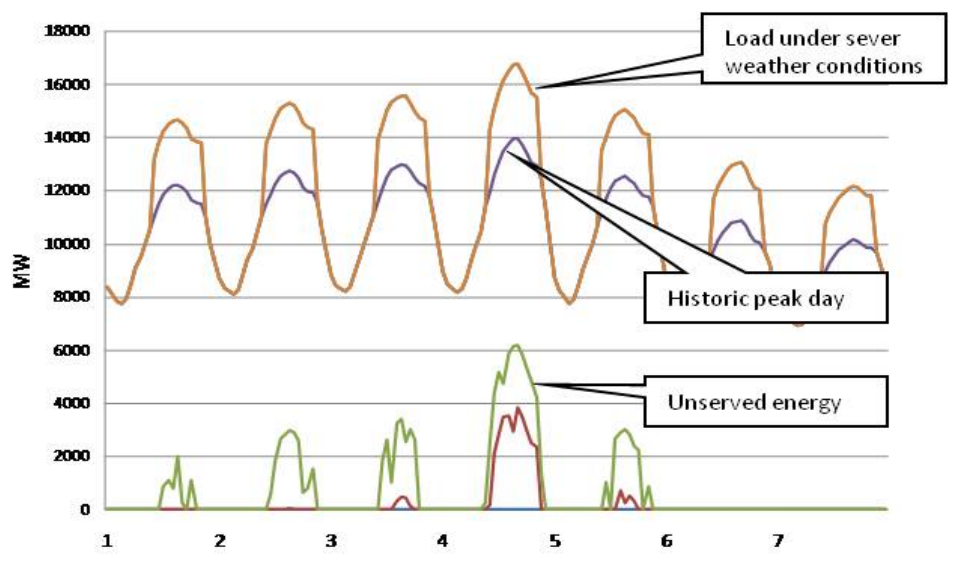

Preliminary results of weather feedback on grid operations. Feedback was simulated as an increase of peak demand by 10\% due to an increase in air conditioning and a reduction of baseload power plants output by $10 \%$ because of cooling water limitations. region to test the sensitivity of the electric grid to severe weather conditions. The preliminary results are suggestive of a high sensitivity of the electric grid with respect to hot and humid conditions over longer periods of time. In addition, these preliminary results indicate selected outages as indicated in the lower curves as unserved energy (i.e., the electric energy that cannot be provided by the grid).

During FY 2011, we will complete an early prototype siting model for one generation technology type and implement and test interactions between this and PROMOD, an existing proprietary production cost model. By the end of the next fiscal year, we also hope to have completed a stand-alone prototype regional generation siting model for multiple generation types. 


\section{Development of Rechargeable Li/air Batteries}

Jiguang (Jason) Zhang, Wu Xu, Jie Xiao, Deyu (David) Wang, Vilayanur V. Viswanathan, Jianzbi Hu, Silas A. Towne

Energy storage devices, especially batteries with good reliability, affordability, and environmental friendliness for plug-in hybrid electrical vehicles (PHEVs) and pure electrical vehicles (EVs), are critical for the United States. While the current lithium (Li) ion battery technology can satisfy the short-term need of EVs, rechargeable Li-air batteries are one of the most promising energy storage technologies for the next generation of EV applications.

I i-air batteries have a much higher specific energy than most available primary and rechargeable batteries, and they are unique in that the cathode active material $\left(\mathrm{O}_{2}\right)$ is not stored in the battery when the battery is assembled. Oxygen from the environment diffuses into the battery during discharge and is catalytically reduced at the surfaces in the air electrode forming either an oxide or peroxide ion that further reacts with cationic species in the electrolyte. Li-air batteries could give a high specific energy of 2,790 Wh/kg due to the extremely high theoretical specific energy of $\mathrm{Li}-\mathrm{O}_{2}$ redox couple. Although several researchers have reported the significant rechargeability of Li-air batteries, there are still many questions and uncertainties about the stability of these batteries. Our main goal is to investigate the rechargeability of these batteries and understand the fundamental mechanism of rechargeable $\mathrm{Li}$-air batteries. New electrolyte, carbon, and catalysts will also be developed to improve battery stability and cycle life.

Progress has been made in several areas in FY 2010. First, we have built four customer designed containers which can be filled with $\mathrm{O}_{2}$ so the rechargeability of $\mathrm{Li}-\mathrm{O}_{2}$ batteries can be tested in a pure oxygen environment. The container can also be connected to a residual mass spectrometer so that the composition of reaction product can be analyzed. The rechargeability of different air electrodes containing pure Ketjen black (KB) carbon, and $\mathrm{KB}$ carbon with vanadium oxide $\left(\mathrm{V}_{2} \mathrm{O}_{5}\right)$ or manganese dioxide $\left(\mathrm{MnO}_{2}\right)$ were first tested in $\mathrm{Li}-\mathrm{O}_{2}$ coin-type batteries containing carbonate-based electrolytes under pure $\mathrm{O}_{2}$ atmosphere. All three air electrodes can give high discharge capacities during the first cycle. However, their cycle lives are still limited to less than 5 cycles. The cycleability of lithium peroxide $\left(\mathrm{Li}_{2} \mathrm{O}_{2}\right)$ in air electrodes with Super $\mathrm{P}(\mathrm{SP})$ conductive carbon and with or without catalysts such as cobalt oxide $\left(\mathrm{Co}_{3} \mathrm{O}_{4}\right)$ and iron oxide $\left(\mathrm{Fe}_{3} \mathrm{O}_{4}\right)$ was also tested. Poor cycle life is found for these electrodes.

The $\mathrm{Li}_{2} \mathrm{O}_{2} / \mathrm{SP}$-based air electrodes with and without catalysts were analyzed by in situ gas chromatography/mass spectroscopy (GC/MS) technique during the charge processes. The $1 \mathrm{M}$
LiTFSI in carbonate-based solvents (EC/PC in 1:1 weight ratio) was used as electrolyte. During the first charge (up to $4.5 \mathrm{~V}$ ), the gases released during the charge process were measured by GC/MS analysis. The amount of $\mathrm{O}_{2}$ release is consistent (within 95 percent) with electrochemical decomposition of $\mathrm{Li}_{2} \mathrm{O}_{2}$ measured by the charge capacity. Only a small amount of $\mathrm{CO}_{2}, \mathrm{CO}$, and moisture were observed during the charge process. This result indicates that $\mathrm{Li}_{2} \mathrm{O}_{2}$ can be decomposed in the given electrolyte at less than $4.5 \mathrm{~V}$.

After the cells were discharged to $2 \mathrm{~V}$ in $\mathrm{O}_{2}$ atmosphere and charged to nearly $4.5 \mathrm{~V}$ again, the composition of the released gases was dominated by $\mathrm{CO}_{2}$. Only a small amount of $\mathrm{O}_{2}$ was found via the in situ GC/MS analysis. Apparently, there is no significant amount of $\mathrm{Li}_{2} \mathrm{O}_{2}$ formed during the process when $\mathrm{Li}$-air batteries were discharged to $2 \mathrm{~V}$ in a carbonate-based electrolyte. To understand further the reaction process in the discharge process of Li-air batteries, the reaction products formed in the air electrodes of the discharged $\mathrm{Li}-\mathrm{O}_{2}$ batteries were analyzed by several techniques, including x-ray diffraction (XRD), Raman spectroscopy, and ${ }^{6} \mathrm{Li}$ nuclear magnetic resonance (NMR) spectroscopy. These investigations indicate that $\mathrm{Li}_{2} \mathrm{CO}_{3}$ and/or lithium alkyl carbonate are the main reaction products during the discharge process of $\mathrm{Li}^{-} \mathrm{O}_{2}$ batteries when they were discharged to $2 \mathrm{~V}$ in a carbonate-based electrolyte. $\mathrm{Li}_{2} \mathrm{O}_{2}$ is not the major product. The latest literature reports suggested that these carbonate compounds were probably produced from decomposition of carbonate solvents in electrolytes. Therefore, $\mathrm{Li}_{-} \mathrm{O}_{2}$ batteries are not stable when discharge to $2 \mathrm{~V}$ in a carbonate-based electrolyte. To quantify our project achievements, we have totaled nine publications on Li-air batteries during FY 2010.

In FY 2011, our efforts will focus on the development of new electrolytes and air electrodes that are stable in both the charged and discharged state. The discharge products of $\mathrm{Li}-\mathrm{O}_{2}$ batteries with different air electrode materials (carbonbased and non carbon-based) in different organic electrolytes (carbonate-based and non carbonate-based) at different discharge cutoff voltages will be investigated. The reaction product will be analyzed by NMR $\left({ }^{6} \mathrm{Li},{ }^{13} \mathrm{C}\right)$, XRD, Raman spectroscopy, and Fourier transform infrared spectrometry. The charge/discharge mechanism will be investigated. Different $\mathrm{O}_{2}$ reduction and evolution catalysts will be tested to improve the battery performance and to reduce the overvoltage during the charge process of the $\mathrm{Li}-\mathrm{O}_{2}$ batteries. The success of this project will enable a practical application of a novel $\mathrm{Li}-\mathrm{O}_{2}$ battery system and significantly improve the energy density of $\mathrm{Li}-\mathrm{O}_{2}$ batteries for $\mathrm{EV}$ applications. 


\title{
Development of Regional Agriculture-Land Use Models
}

\author{
Allison M. Thomson, Roberto C. Izaurralde, Tristram O. West, Marshall A. Wise, \\ Benjamin Bond-Lamberty, Katherine V. Calvin
}

We are developing an advanced capability for simulating climate impacts on agriculture and forestry to allow investigation of adaptation needs and mitigation potential of land management. This will inform questions about food security and greenhouse gas mitigation from regional-scale agriculture of interest to decision-makers.

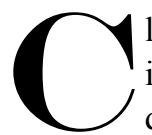
limate change and food security are global issues that are increasingly linked through decision-making that takes place across all scales from on-farm management to international climate negotiations. While still supplying sufficient food to feed the global population, understanding how agricultural systems can respond to climate change through mitigation or adaptation thus requires a multi-sector tool in a global economic framework. Integrated assessment models are one such tool; however, they are typically driven by historical aggregate statistics of production in combination with assumptions of future trends in agricultural productivity; they are not capable of exploring agricultural management practices as adaptation or mitigation strategies. Yet, there are agricultural models capable of detailed biophysical modeling of farm management and climate impacts on crop yield, soil erosion, and carbon and greenhouse gas emissions, although these are applied at point scales incompatible with coarse resolution integrated assessment modeling.

To combine the relative strengths of these modeling systems, we are applying the agricultural model Environmental Policy Integrated Climate (EPIC) in a geographic data framework for regional analyses to provide input to a regional integrated assessment model (Regional Global Change Assessment Model [RGCAM]). The initial phase of our approach focuses on a pilot region of the Midwestern United States. We will apply EPIC across the domain at $56 \mathrm{~m}$ spatial resolution and use the results to inform RGCAM. This will enable integrated assessment to take into account the different crop yield, bioenergy production, soil carbon, greenhouse gas emissions, and economic costs of different management practices for agriculture. Using this information, RGCAM can simulate the

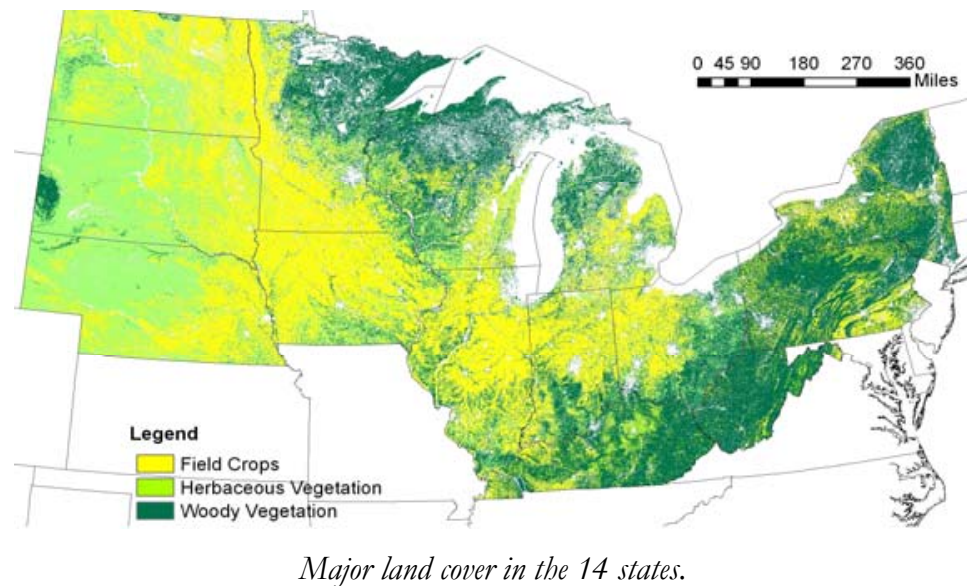

Major land cover in the 14 states. optimum mix of land management to achieve specific climate mitigation or adaptation targets.

In FY 2010, substantial progress was made on several tasks. The agriculture and land use components of the integrated assessment model were re-developed to allow for subregionalization of the land area and to allow multiple production methodologies in the simulations. The model code is complete, and the underlying global data structure is nearly complete. This global data framework will be used as boundary conditions, and the relevant variables replaced with output from the agricultural model for the region of interest. For the EPIC model, a spatially explicit modeling framework was completed. This integrates highly spatially resolved land use, soils, and weather data with the model and has been tested for a region in Michigan. The necessary input data for the 14-state Midwest pilot region has been acquired and processed into the modeling framework, and a draft model run matrix has been produced.

The new integrated assessment model code structure was tested in the simulation of multiple agricultural technologies. Initial results illustrate how the model behaves with two competing options for growing corn: a conventional tillage case and a higher yielding but higher cost management case. The results show that when the second technology is available in a climate mitigation policy, it is selected for over 50 percent of the area used for corn in the Midwest. Further, adopting a higher yield technology allows for greater production on less land. This new capability will be tested further and fully implemented with simulation results from the agricultural model in FY 2011.

The outcome of this project will be an integrative element of the final integrated Regional Earth System Model and will connect human and physical science elements of the model framework through land productivity and management. These improvements and capabilities will enable new, regional simulations of climate impacts on agriculture and forestry, climate mitigation potential of land use options, potential supply of bioenergy, and nitrogen and water-cycle impacts of projected changes in land management. 


\title{
Electrolyte Development for Next Generation of Lithium Ion Batteries
}

\author{
Wu Xu, Jiguang (Jason) Zhang, Phillip K. Koech, Sarah D. Burton, Deyu (David) Wang, Jie Xiao
}

Electric vehicles (EVs) and plug-in hybrid electric vehicles (PHEVs) demand advanced energy storage systems to have higher energy density, longer cycle life and calendar life, higher safety, and lower cost than the state-of-the-art lithium (Li) ion batteries. The development of new electrolytes with high thermal stability and good electrochemical stability will significantly increase the life time (both cycle and calendar) of $\mathrm{Li}$ ion batteries, thus leading to more reliable products.

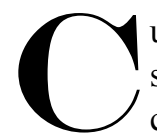
urrent $\mathrm{Li}$ ion batteries have several drawbacks, such as low energy density, high cost, limited cycle life, and some safety concerns, especially in the case of abuses. To increase the cycle life and calendar life of $\mathrm{Li}$ ion batteries, the electrolytes should have high thermal stability and good electrochemical stability. The state-of-theart lithium ion batteries use lithium hexafluorophosphate $\left(\mathrm{LiPF}_{6}\right)$ as the solute in non-aqueous electrolytes based on comprehensive considerations of possible lithium salts. The thermal instability and moisture sensitivity of $\mathrm{LiPF}_{6}$ lead to corrosion of electrode-surface-protection-films, dissolution of cathode active materials, and degradation of electrolyte solvents, which result in reduced life time and safety problems of $\mathrm{Li}$ ion batteries. Therefore, there is an urgent need to design and develop new electrolytes that are thermally and electrochemically stable to improve significantly the cycle life and calendar life of $\mathrm{Li}$ ion batteries required for the large-scale application of EVs and PHEVs.

In FY 2008, the preliminary electrochemical test on effect of salt additives on silicon $(\mathrm{Si})$-based electrodes reveal that the addition of 2 percent $\mathrm{NaPF}_{6}$ in the control electrolyte leads to a lower capacity of $\mathrm{Si}$ based electrodes, while the addition of 2 percent $\mathrm{KPF}_{6}$ improved capacity. During FY 2009, we found that some of the SOA Li ion battery electrolytes were stable at $4.5 \mathrm{~V}$ and even closely stable up to $4.8 \mathrm{~V}$. New electrolytes for high capacity anode materials such as $\mathrm{Si}$ and tin oxide $\left(\mathrm{SnO}_{2}\right)$ and for high energy $\mathrm{Li}$ air primary batteries were also investigated.

In FY 2010, our work was focused on developing new lithium salts and solvents with high thermal stability and good electrochemical stability suitable for long-term, safe operation of Li ion batteries. Several new lithium salts have been designed and synthesized using different starting materials and synthesis routes. We found that lithium bis(carbonato)borate is difficult to synthesize because of the instability of the intermediates at room temperature and the high stress of the four-member ring structures. Lithium bis(sulfato)borate has been successfully synthesized and characterized; however, it decomposes slowly in solvents even in inert atmosphere. Therefore, it is not suitable for $\mathrm{Li}$ ion battery applications.

In a task related to this project effort, we successfully synthesized a new additive, 4-methylene-1,3-dioxolan2-one (or 4-methylene ethylene carbonate [MEC]), for the formation of solid electrolyte interface (SEI) on anode surface. The effects of this additive on cycling performances of $\mathrm{Li}$ ion batteries have been investigated, with comparison of conventional SEI formation additives vinylene carbonate (VC) and vinyl ethylene carbonate (VEC). On the graphite anode, MEC improves battery capacity and has the best battery performance in the MEC content range of approximately 1 to 4 percent by weight in electrolytes. VEC shows the best capacity and rate capability, while $\mathrm{VC}$ isthe poorest among the three additives. MEC shows similar good performance like VEC at low discharge rates. On $\mathrm{LiCoO}_{2}$ cathode, all the three SEI formation additives decrease capacity and rate capability. However, MEC improves battery performance at high discharge rates, and it shows the best performance from among the three additives. Thus, MEC should be a good SEI formation additive over the conventional SEI formation additives to achieve long cycle life of $\mathrm{Li}$ ion batteries.

We have continued to study the electrolytes for $\mathrm{Si}$ anode and have found that the $\mathrm{NaClO}_{4}$ electrolyte can increase Si discharge capacity and capacity retention when compared with the $\mathrm{LiClO}_{4}$ electrolyte, which is opposite to the case of addition of $\mathrm{NaPF}_{6}$ as an additive in $\mathrm{LiPF}_{6}$ electrolyte, while the $\mathrm{KClO}_{4}$ electrolyte can improve the capacity retention of $\mathrm{Si}$ anode. A sulfone-based electrolyte with $5.5 \mathrm{~V}$ stability has been developed for the study of new, high-voltage cathode materials developed in our research laboratory. One technical paper has been submitted for publication in a peer-reviewed journal. Two additional papers based on the FY 2010 work are in preparation.

For FY 2011, we will develop high-voltage electrolyte additives for high voltage (greater than $4.8 \mathrm{~V}$ ) cathodes to improve the energy density of $\mathrm{Li}$ ion batteries and develop flame-retarding additives/solvents that greatly improve the safety of the batteries. It is anticipated that the new additives and organic solvents developed in this project will provide significantly improved reversible energy density, a long cycle life, and safety stability for $\mathrm{Li}$ ion batteries used for EV and PHEV applications. 


\title{
Geological Sequestration Software Suite: Numerical Model Development
}

\author{
Mark D. Williams, Signe K. Wurstner, Paul D. Thorne, Andrew P. Kuprat, Vicky L. Freedman
}

This and other Geological Sequestration Software Suite $\left(\mathrm{GS}^{3}\right)$ projects will develop an integrated software system with a consistent user interface and data structures to provide for comprehensive, rapid development of simulations for greenhouse gas sequestration at specific sites. The ability to develop and run multiscale simulations with multiple conceptual models and operational complexity in high performance computing will facilitate evaluation of the efficacy of the sequestration approach, given the uncertainties of the natural environment.

$\mathbf{F}$ ossil fuels will remain the mainstay of energy production well into the $21^{\text {st }}$ century. The ability and availability of these fuels to provide clean, affordable energy is essential for the prosperity and security of the United States. However, increased concentrations of $\mathrm{CO}_{2}$ due to carbon emissions are expected unless energy systems reduce the carbon emissions to the atmosphere. One way of accomplishing this is through carbon sequestration: the storage of $\mathrm{CO}_{2}$ in geologic formations, including oil and gas reservoirs, unmineable coal seams, and deep saline reservoirs. The primary goal of the DOE's sequestration research is to understand the behavior of $\mathrm{CO}_{2}$ when stored in geologic formations. This information is key to making sure that sequestration will not impair the geologic integrity of an underground formation and that $\mathrm{CO}_{2}$ storage is secure and environmentally acceptable.

Advanced multi-phase flow and transport codes provide the capability to investigate coupled processes occurring at various scales, thereby advancing the science and providing a set of tools for evaluating the feasibility and long-term effects of sequestering carbon dioxide in large-scale, deep geologic reservoirs. However, the process of managing and interpreting raw data, building the conceptual model of the subsurface domain from those data, and transforming/ scaling the conceptual model into the numerical model while maintaining the detail captured in the conceptual model is currently a tremendous challenge even for experienced modelers. The complexity of this task indicates the need for a fully integrated software framework that provides a suite of tools and options to streamline the modeling process and aid in the creation of defensible models that incorporate our most current understanding of the subsurface and the technology.

The focus of this project is to develop algorithms and tools for building multi-scale numerical models from site conceptual models. This application will aid in the translation of conceptual models to a numerical modeling framework and provide the capability to generate multiscale (spatial and temporal) numerical models that support a variety of collaborative geologic sequestration studies. User specifications for numerical models include grids, geology, boundary and initial conditions, source terms, material properties, geochemical reactions, and geomechanics. To preserve the distinction between the conceptual model and its translation to a numerical modeling framework, the application manages data associated with each specification independently. This facilitates 1) building multiscale numerical models from a common conceptual model, 2) building numerical models from multiple conceptual models, 3) building numerical models and input files for different simulators from a common conceptual model, 4) ease in regenerating numerical models in response to revisions of the conceptual model, and 5) revising the numerical model specification during the development process (e.g., grid modifications and resulting reassignment of material property values and distributions).

A key aspect of the model definition software is the ability to define features in the numerical model by specifying them as geometric objects, eliminating the need for the user to specify node/element numbers that often change when the grid is revised. The $\mathrm{GS}^{3}$ platform provides the capability of tracking provenance and dependencies for data files used in the numerical model. Within this framework, metadata is generated to support configuration control for a specific simulation. In addition, the user will be notified when simulation input files would be impacted by changes in the conceptual model. The software will also provide three-dimensional previews of the numerical grid, material property distribution, and node/element selection providing for visualization early in the model development process.

During FY 2010, a design concept was developed and presented to the project team and a prototype numerical model development tool with limited functionality was developed focused on supporting the $\mathrm{CO}_{2}$ injection based operational mode of the Subsurface Transport Over Multiple Phases (STOMP) $-\mathrm{CO}_{2}$ simulator. In the design concept development phase, the mediawiki functions and features were found to be inadequate for development of this tool. The Java programming language was selected because the code is platform independent (Linux, Windows, OS X) and programs can be launched via Java webstart from the $\mathrm{GS}^{3}$ wiki. The team adopted the Netbeans Integrated Development Environment for Java as a development platform because it provides for rapid graphical user interface form prototyping, design, and development. The XML format was selected for storing the model definition with JAXB parsers for reading and writing the files based on a pre-defined XML schema. Also, the XML format is widely supported, and the JAXB 
parsers are automatically generated within the Netbeans IDE. Further, Java3D is being used to provide a preview function with mouse-based zooming and rotation during the model definition process. Portions of the model domain can be selected and properties specified based on simple indexing, geometric objects, and linkage to external files for more complex specification. For supporting complex hydrostratigraphy based on solid earth models (e.g., Earth Vision or Petrel), a work flow was developed for exporting the numerical grid, assigning properties to the grid within Petrel or EarthVision, and then importing the zonation back into the model definition process.

Also in FY 2010, we commenced the development of unstructured grid generation using the existing Los Alamos Grdding Toolbox (LaGrit) code (PNNL and Los Alamos National Laboratory [LANL] versions). The initial focus is on developing templates and workflows for generating three-dimensional unstructured meshes for a layered system with faulting. These geologic surfaces and features are defined in solid earth models and exported for use by LaGriT, which has an extensive library of functions and requires a fairly complex scripting input. However, templates are being developed for non-expert users to produce unstructured meshes for classes of problems without understanding all the intricacies of the LaGriT command structure. This work is being conducted in collaboration with the LaGriT developers at LANL.

Work during FY 2011 will focus on the addition of features to transform the prototype developed in FY 2010 to a more functional tool for numerical model definition and input file generation for the STOMP simulator, the integration of this tool within the $\mathrm{GS}^{3}$ platform including file access with data provenance and metadata, and the development and linkages to LaGriT using templates for creating unstructured meshes for classes of hydrogeologic settings. 


\section{Infrastructure Compatible Fuels and Chemicals from Biomass}

Douglas C. Elliott, Hanwu Lei (Washington State University-Tri-Cities)

The aim of this research is to develop a microwave heating capability to allow torrefaction and fast pyrolysis. With this capability, we can directly compare the results of the more conventional fluidized bed pyrolysis with the microwave heated products.

$\mathbf{F}$ ast pyrolysis of biomass has been developed for production of liquid fuels from biomass and is a near-commercial technology in engineered systems using fluidized-bed reactors. Fast heat-up and cool-down is the essence of fast pyrolysis with temperatures of approximately $500^{\circ} \mathrm{C}$ required with residence times of less than a second. Biomass fast pyrolysis can be achieved by microwave radiation. The key advantage of the microwave pyrolysis process over the conventional furnace heating method is that thermochemical reactions can take place rapidly in large-sized biomass materials such as woody biomass or cornstalks or wheat straw, thanks to the nature of fast internal heating by microwave energy. Therefore, very fine feedstock grinding required by conventional pyrolysis is not necessary for the microwave pyrolysis process, resulting in substantial energy savings.

While the of fluid-bed technology for fast pyrolysis is a current capability at PNNL, an innovative method for fast pyrolysis using microwave heating is proposed under this project. With this capability, we can directly compare the results of the more conventional fluidized bed pyrolysis with the microwave heated products. Our research is expected to lead to an important capability in thermal processing of biomass to liquid fuels and energy products by enabling direct comparisons of novel microwave processing of complex biomass with conventional methods. Additionally, this work will provide direct comparisons of the quality of the energy products/oils produced and the relative economics and energy savings between the novel and conventional processes.

During FY 2010, the microwave-heated reactor system was assembled within the Washington State UniversityTri-Cities laboratories. Comparative tests were made with a representative biomass feedstock, prairie cord grass (PCG), in both the microwave heated reactor and the bench-scale fluidized-bed fast pyrolysis reactor in Chemical Engineering Laboratory. Analysis of the products was made to develop elemental balances around the pyrolysis step in each reactor. The bio-oil quality from each reactor was also determined and compared. A preliminary technoeconomic assessment of the microwave heating system was also attempted to compare to the base case fast pyrolysis model developed for DOE based on fluidized bed pyrolysis technology.
Significant differences in the products from the two systems were identified. As generally practiced, fast pyrolysis produces a high yield (as much as 70 percent) of a single phase bio-oil product containing a significant amount of water. Herbaceous feedstocks are reported to give lower bio-oil yield, and the PNNL test recovered only 60 percent of miscible combined condensates. The microwave assisted reactor operated more like a slow pyrolysis system in that it produced a higher char yield ( 25 percent), and the smaller amount of liquid condensates ( 35 percent) were recovered in two phases. The results of the analyses of these products are shown in the table below.

\begin{tabular}{|l|l|l|l|}
\hline Properties & $\begin{array}{l}\text { Fluid-bed } \\
\text { PNNL }\end{array}$ & $\begin{array}{l}\text { Microwave } \\
\text { water phase } \\
\text { WSU }\end{array}$ & $\begin{array}{l}\text { Microwave } \\
\text { oil phase } \\
\text { WSU }\end{array}$ \\
\hline Carbon wt\% & $51.6-54.5$ & $5.1-20.5$ & $65.0-65.9$ \\
\hline Hydrogen $w \mathrm{t} \%$ & $8.1-8.7$ & $10.5-9.3$ & $7.7-8.6$ \\
\hline Nitrogen $\mathrm{wt} \%$ & $0.5-0.6$ & $0.9-0.3$ & $1.9-2.4$ \\
\hline Oxygen $\mathrm{wt} \%$ & $33.9-39.2$ & $51.1-45.4$ & $24.0-24.4$ \\
\hline Sulfur wt\% & $0.03-0.05$ & $0.005-0.013$ & $0.09-0.30$ \\
\hline Water wt\% & $13.2-15.4$ & $85.9-58.5$ & $0.2-1.6$ \\
\hline $\begin{array}{l}\text { Acid } \mathrm{number} \\
\mathrm{mgKOH} / \mathrm{g}\end{array}$ & $75.6-94.1$ & $47.9-142.5$ & $23.2-131.2$ \\
\hline
\end{tabular}

Composition of bio-oils produced from PCG.

An economic comparison was made for the microwave system and the fluid bed system. The analysis was based on the already completed fast pyrolysis study in fluid-bed systems. When comparing to microwave-assisted pyrolysis, the overall product cost is about twice that from the fluid-bed system. The products are not comparable, and a devaluation of the bio-oil because of its two-phase structure would mean an even less economical process. The value of the aqueous condensate is not known, and if it cannot be remixed in the oil phase, it might actually become a waste disposal cost. The char product might have exceptional value as a soil amendment rather than as a solid fuel and that would improve the overall economics. The large amount of gas product is sufficient to generate the electricity required by the microwave system, but the investment for the generator adds to the capital cost.

These results confirm that microwave-assisted pyrolysis does not produce the same products as fluid-bed fast pyrolysis. The microwave system may still hold some potential as a pre-pyrolysis torrefaction-type processing system to modify biomass and, as a result, produce a modified fast pyrolysis bio-oil. 


\title{
Innovative Processes for Integrated Emissions Management
}

\author{
Michael L. Elliott, Eddie G. Baker, Mark D. Bearden, Charles J. Freeman
}

This project generated new concepts and estimated the cost of electricity (capital and operating costs) for integrated emissions control.

$\mathbf{T}$ he Department of Energy has set the goal for carbon capture and sequestration (CCS) to be: By 2020, have available for commercial deployment, technologies, and best practices for achieving $90 \% \mathrm{CO}_{2}$ capture and $99 \%$ storage permanence for post combustion systems for $<35 \%$ increase in the cost of electricity. The DOE goal is aggressive and cannot be met with incremental improvements to existing technologies. It also cannot be met with improvements to the capital and operating expenditures - both costs must be significantly reduced to meeting this goal.

The significance of finding an economical solution to CCS is momentous. The concept of integrated emissions management has the potential to significantly reduce the cost of carbon capture and sequestration from fossil fuel power plants. Integrated emissions management considers $\mathrm{CO}_{2}$ capture in conjunction with the control of other emissions (e.g., $\mathrm{SO}_{\mathrm{x}} / \mathrm{NO}_{\mathrm{x}} / \mathrm{etc}$ ) rather than as an additional "bolt on" unit operation. Several integrated emissions management concepts have been identified for investigation to determine their efficacy and uniqueness. These concepts integrate $\mathrm{CO}_{2}$ capture using new separation materials and processes and heat integration into the facility to reduce operating costs and increase plant efficiency. New materials are being discovered that will enable new CCS flowsheets to take advantage of their unique characteristics.

Making CCS economical will require an integrated system design. This project performed a high-level screening of identified concepts, and ranked candidates for further investigation and estimate the cost of electricity (capital and operating costs) for the top one or two options.

An initial set of candidate integrated emissions management processes and novel materials were researched. Candidate ideas were summarized into tabular and graphic formats to document the raw ideas. The most promising new processes were further researched using first principles reaction chemistry, energy, and exergy thermodynamic calculations, as well as specialized modeling tools such as Aspen Plus modeling software, ChemCAD modeling of unit operations, and internally developed spreadsheets.

Research results were compared with the energetics and thermodynamics of the current state-of-the-art process for carbon capture. A series of papers summarized the research findings in areas such as feasibility, technical uncertainty, energetics, and economic issues for each of the top candidates.

This project gathered and researched a broad set of ideas for integrated emissions. There were over 80 novel materials and flowsheets recommended, ranging from minor, near-term retrofit ideas that could be accomplished for existing power plants to major stepout ideas that would require significant development. Many of the ideas could result in improved economics for carbon capture and storage. We identified technology gaps for materials development for carbon capture. Specifically, many of the recommended processes would benefit from a single unit operation, using a unique material that can capture acid gases such as $\mathrm{CO}_{2}, \mathrm{SO}_{\mathrm{x}}, \mathrm{NO}_{\mathrm{x}}$, and trace contaminants while not being impacted by water or chlorides.

The research and development done through fundamental calculations and modeling as well as process design also identified unique ideas for providing the energy to drive the gas cleanup system while having minor impacts on the existing power plant infrastructure. Examples include using low quality steam that is normally lost in a power plant to regenerate the acid gas sorbent and combining a natural gas fuel cell system or solar reforming system whose rejected heat would be used to regenerate the $\mathrm{CO}_{2}$ sorbent.

This project ended in FY 2010. Further research is needed, and in FY 2011, a new effort will investigate and develop integrated processes to improve the economics of carbon capture in fossil fuel (coal) power plants. The focus will integrate new processes (groups of unit operations and materials) into existing power plant infrastructures or designs. The goal is to develop a single, integrated system to capture both $\mathrm{CO}_{2}$ and criteria pollutants $\left(\mathrm{NO}_{\mathrm{x}}, \mathrm{SO}_{\mathrm{x}}, \mathrm{Hg}\right)$ using a whole-plant solution rather than incremental (i.e., "bolt on") emissions control units. This offers the advantage of using new capture materials to reduce operating costs while using the existing plant infrastructure to reduce capital expenses. An example would be to use the existing infrastructure of the sulfur $\left(\mathrm{SO}_{\mathrm{x}}\right)$ removal system (FGD) in a power plant and replace the normal sorbent with a novel solvent that would remove $\mathrm{CO}_{2}, \mathrm{NO}_{\mathrm{x}}, \mathrm{SO}_{\mathrm{x}}$ and $\mathrm{Hg}$ without requiring major capital expenses. Additional energy will be required to drive the $\mathrm{CO}_{2}$ removal process because of the thermodynamics involved (for most cases). An example of integrated emissions would be to utilize energy that is currently unusable in a plant (e.g., low quality steam) and therefore not impact the efficiency of the plant and increase operating expenses. 


\title{
Marine Biomass and its Conversion to Liquid Transportation Fuels
}

\author{
Guritno Roesijadi, Michael H. Huesemann, Ronald M. Thom, Douglas C. Elliott
}

This research is intended to demonstrate the capability and provide findings to establish a path to a broader research and development program in marine biomass as feedstock for liquid transportation fuels.

A lthough marine biomass in the form of macroalgae or seaweed is an untapped resource with a biomass potential that rivals that projected for terrestrial biomass, the technical foundations for its propagation and conversion to biofuels, which are considered central to the successful development of the resource, are at early stages of the research and development cycle and require further development. The challenges include the ability to grow macroalgae at the scale and cost needed for an economically viable feedstock and the conversion of the feedstock to liquid transportation fuels.

The feasibility of utilizing marine biomass as feedstock for conversion to liquid transportation fuels has yet to be rigorously tested. Thus, this project focused on the conversion of macroalgae to alcohols using fermentation technology and bio-oils using hydrothermal liquefaction. Bioconversion of macroalgae to ethanol has only recently been demonstrated and, to our knowledge, there are currently no reports on the bioconversion of seaweed to butanol, which has characteristics that are more desirable in an advanced biofuel. There is also little evidence that hydrothermal liquefaction of macroalgae can produce bio-oils for further processing to hydrocarbon products. The test species was Saccharina latissima, a kelp that has a high water content rich in mannitol, laminarin, fucoidan, and alginates, primary carbohydrate constituents, with the initial two representing the predominant fermentable feedstocks.

Experiments in FY 2009 tested the ability of the yeast Pichia angophorae to convert glucose, mannitol, and extracts of S. latissima to ethanol. The results showed that the yeast converts mannitol to ethanol, and this conversion is enhanced by seaweed extracts. During FY 2010, our work focused on fermentation of glucose, mannitol, and seaweed extracts to butanol by bacterium Clostridium acetobutylicum and conversion of whole seaweeds to bio-oils using hydrothermal liquefaction.

Conversion to butanol. The butanol conversion showed that either alone or in combination with glucose, mannitol was readily fermented into organic acids and solvents by C. acetobutylicum. Cultures containing mannitol and glucose exhibited diauxic growth, with glucose being utilized first. A lag period of about one day occurred following glucose depletion before mannitol utilization began, indicating that some time is needed for the induction of the enzymes involved in mannitol uptake and utilization.

Butanol yields in cultures with seaweed extract were comparable to those containing mannitol and slightly less than those containing glucose, indicating that extracts of S. latissima are a suitable feedstock for fermentation to butanol. Cultures containing seaweed extract exhibited triauxic growth associated with sequential utilization of glucose, mannitol, and "total complexed sugars" (i.e., sugars incorporated into polysaccharides). This last fraction most likely consisted of laminaran, which requires specific extracellular saccharolytic enzymes such as laminarase to break down the polysaccharide into fermentable sugars.

Hydrothermal liquefaction. To prepare macroalgae for hydrothermal liquefaction, samples must be preprocessed to a pumpable slurry. A wet mill was used to prepare the appropriate particle size slurry that can be sufficiently stable and yet fluid enough to be pumped with the high-pressure metering pumps. Challenges were presented by the high water content (results in a dilute sample) and the presence of alginates, which causes tissues to gel under preprocessing conditions. Several trials using a bench scale processing system resulted in dry solids-in-water slurry concentrations from 5 to 13 percent.

The results of hydrothermal liquefaction of the preprocessed samples showed that macroalgae could be converted into a bio-oil with an oxygen content of less than 8 percent calculated to a dry basis. The oil yield was low, and much or most of the organic fraction remains dissolved in the water byproduct, which also contains little gas. Oil increased with elevated concentration of slurry feedstock, exceeding 40 percent on a carbon basis with feed concentration at 13 percent dry solids. This is clearly the direction for future research. The mineral matter was for the most part recovered in the aqueous byproduct as well, and only to a limited degree did it precipitate at hydrothermal conditions. Because a large fraction of remaining seaweed dissolved in the aqueous byproduct, energy recovery from that stream was tested using catalytic hydrothermal gasification. We determined that the organic compounds dissolved in the aqueous byproduct could be gasified by catalytic hydrothermal gasification as a means to recover energy value and clean the water.

The results demonstrate that butanol and bio-oil can be produced from macroalgae through fermentation and hydrothermal liquefaction, respectively. Additional research is needed to assess conversion technologies at the efficiencies and scale needed for macroalgae to serve as an economically feasible biofuels feedstock. 


\title{
Methodology Development for Optimal Energy Storage Sizing and Placement for Local and Regional Planners
}

\author{
Michael C.W. Kintner-Meyer, Michael T. Batdorf, Nirupama Prakash Kumar, Michael J. Hoffman, \\ Artyom Sadovsky, Pengwei Du, David P. Chassin
}

This project is developing a methodology that helps determine the optimal sizes and locations of energy storage devices, which minimizes the operation cost of the electric power system while ensuring system reliability at high penetration level of intermittent generation resources.

E nergy storage has been recognized as a critical technological solution that helps accommodating intermittent renewable energy sources and improving power system efficiency. Many utilities explore options for energy storage to be integrated into their infrastructure. However, no tools exist to quantify all of the values that storage may provide. The methodologies for quantifying the value of energy storage are not clear at this point. To fill this methodology gap, new algorithms and methodologies will be developed to quantify the value of storage and to size and place energy storage systems optimally. These new methodologies will be tested and evaluated for the use in future grid planning tools. In addition, the project is designed to develop optimal control and sizing methods to compete energy storage against conventional generation technology for providing energy arbitrage and ancillary services. The methods can be used to estimate the total size of energy storage potential for 13 United States regions.

In FY 2010, we developed the mathematical formulation of an optimal control and sizing problem for a type of energy storage that captures the cost and performance characteristics of energy storage and competing alternative technology options, such as conventional generation capacity and demand response, to minimize the total cost of purchasing new capacity and operating new and existing capacity to meetthe load and ancillary services requirements.

Two distinctly different formulations for the operation of the grid were implemented, and their advantages and disadvantages were analyzed. The first representation applied the commonly used representation of a load duration curve, in which the entire hourly load time series ( 8760 hours of a year) are sorted in a descending order and then usually simplified by a small number of "time slices" (between 3 to 20) with a constant load for a given time slice to approximate the monotonically decreasing load duration curve. The advantage of this representation is its computational efficiency because the size of the time domain is greatly reduced from multiple hours to manageable time frames. The disadvantage of the load duration format is particularly applicable for energy storage applications. The chronological order of time is lost when the time series is sorted to a load duration curve. This greatly reduced the degree of storage controls option and sets some a priori defined cycling (charging/discharging) solutions as optimal outcome.

In the second representation, we optimized the hourly operation and size of the storage over 1 year, which provided a true optimal solution. The optimization in this formulation is free to select cost-optimal size for daily charging/discharging or a larger size for a weekly charging/ discharging (as shown below graphically) - or even a seasonal - cycle. This formulation is unique and has never been performed. It solves simultaneously the optimal sizing of generators assets and energy storage systems as well as optimally dispatches them.

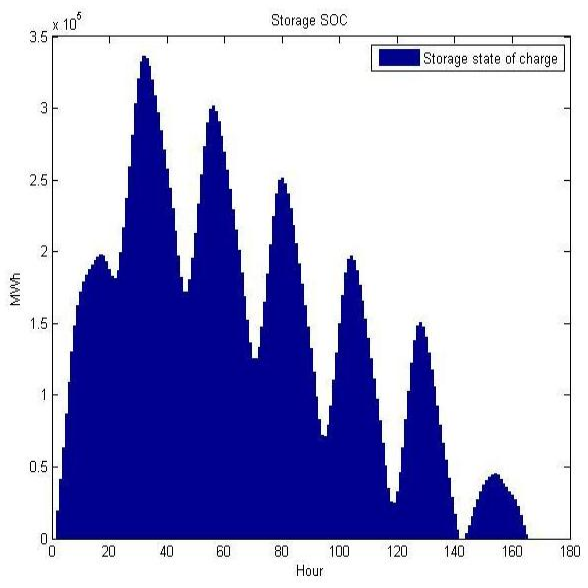

The state of charge for a weekly storage cycle.

In addition, we performing a spectral analysis that estimated the total plausible limits of the country's energy storage needs based on the 13 modified North American Electric Reliability Corporation regions. This research explored novel ways to apply spectral analyses of system loads to a sizing task of energy storage potential for regional grids in the United States. The analysis defined stereotypical cycling behaviors of energy storage over a period of a day, a week, and one season and estimated the total storage potential that could be economically plausible. The results were summarized in an IEEE paper. 


\section{Novel Carbon Capture Materials}

David L. King

This project will develop analytical capabilities to quantify the amount of $\mathrm{CO}_{2}$ taken up and released, along with determining the heat of adsorption of $\mathrm{CO}_{2}$ with solid absorbent materials. It is envisioned that this capability can be applied to both internally developed materials and materials that might be provided by others.

$\mathbf{T}$ he use of fossil fuels, especially from coal-derived sources, has the potential to adversely impact the world climate through generation of greenhouse gas $\mathrm{CO}_{2}$. Major efforts in various laboratories have been devoted to carbon capture and sequestration to mitigate the effect of $\mathrm{CO}_{2}$ generation. The most costly aspect is the actual selective capture and release of $\mathrm{CO}_{2}$ and, for this reason, minimizing the cost of $\mathrm{CO}_{2}$ recovery is a significant driver in technology selection. The overall goal of this project is to develop a portfolio of novel, cost-effective, material-based approaches for the capture of carbon dioxide, primarily from coal-based power plants where $\mathrm{CO}_{2}$ is present in the flue gas. These materials must not only be able to capture $\mathrm{CO}_{2}$ but also release it with a minimal amount of energy expenditure. In order to support development of these materials and to assist in the quantification of performance, an additional task was incorporated under this project.

There are several approaches being investigated for $\mathrm{CO}_{2}$ capture. First, the medium may be either a liquid or a solid. Liquid capture systems are practiced in industry, with the current standard from flue gas uses mono-ethanolamine (MEA) solvent. The advantage of the liquid system is that it is easy to move the solvent from the point where $\mathrm{CO}_{2}$ is absorbed to the point where it is released. However, the energy penalty with $\mathrm{CO}_{2}$ release with MEA is significant, as the system retains water that is not involved in absorption (it is present to retard corrosion) but must be heated when $\mathrm{CO}_{2}$ is released. Non-corrosive solvents with high $\mathrm{CO}_{2}$ capacity and low binding energy are most attractive. Solid systems are much less developed for $\mathrm{CO}_{2}$ capture. Moving solids are more challenging than moving liquids, and it is more difficult to heat a bed of solid material rather than liquid. The primary incentive for using solid absorbents is the potential for lower heat capacity so that the energy required to raise a solid absorbent to regeneration temperature may be less than the corresponding liquid.

In 2010, we focused on developing three systems that had previously been worked on in FY 2009. The first is absorbents referred to as $\mathrm{CO}_{2}$-binding organic liquids $\left(\mathrm{CO}_{2} \mathrm{BOLs}\right)$ originally comprised of two separate units: base and alcohol molecules (two-component system), which do not interact unless $\mathrm{CO}_{2}$ is present. With $\mathrm{CO}_{2}$ presence, a strong, selective interaction develops within the liquid medium. The second is chitosan, matter based on a naturally occurring material in shellfish that can be used as either a solid or (when properly engineered) is soluble in an aqueous medium. The third material is a class of solids called zeolites that, when properly modified, have satisfactory $\mathrm{CO}_{2}$ capacity while avoiding strong water adsorption (a frequent problem with such materials).

$\mathrm{CO}_{2}$-Binding Organic Liquids. At the end of FY 2009, two-component $\mathrm{CO}_{2} \mathrm{BOLs}$ had good $\mathrm{CO}_{2}$ capacity but unacceptably high volatility that would lead to solvent loss during regeneration. As a result, we focused on developing a novel, single-component $\mathrm{CO}_{2} \mathrm{BOLs}$ based on combining base and alcohol functions on a single molecule. These materials were found to perform as well as the first generation materials in terms of $\mathrm{CO}_{2}$ capacity with far lower volatility-greater than 95 percent material recovered after 10 cycles of $\mathrm{CO}_{2}$ absorption and desorption compared to under 40 percent recovered from the first generation.
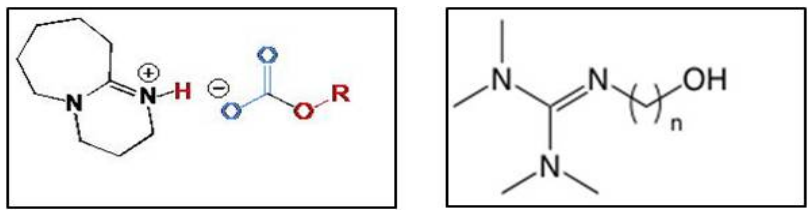

Left: first generation $\mathrm{CO}_{2} \mathrm{BOL}$; right: second generation $\mathrm{CO}_{2} \mathrm{BOL}$.

As a downside, the viscosity of the second generation materials was found to be significantly (and unacceptably) higher when $\mathrm{CO}_{2}$ was absorbed; thus, a good portion of FY 2010 work was devoted to developing strategies for reducing viscosity. Conversely, the presence of water in the flue gas was not a problem with $\mathrm{CO}_{2} \mathrm{BOLS}$. The performance of $\mathrm{CO}_{2} \mathrm{BOLs}$ remained unchanged with the presence of up to $10 \mathrm{~mol} \%$, showing that water has no adverse effect on selectivity toward $\mathrm{CO}_{2}$ or hydrolytic stability of the bases. Further, water was boiled off during regeneration, indicating that $\mathrm{CO}_{2} \mathrm{BOL}$ can be dehydrated during thermal stripping of $\mathrm{CO}_{2}$. This effort will continue into FY 2011, with a focus on developing a single component $\mathrm{CO}_{2} \mathrm{BOL}$ that avoids high viscosity. A viscometer was purchased for these studies.

Chitosan. $\mathrm{CO}_{2}$ interacts strongly with the amino group (-NH2) on chitosan. In its as-produced state, chitosan has a relatively low surface area, and many amino groups are inaccessible (they are in the bulk of the material, not at the surface); as a result, the highest capacity achieved was about $0.35 \mathrm{mmol} \mathrm{CO}_{2} / \mathrm{g}$ sorbent. Freeze-drying and cross-linking of chitosan provided enhanced surface area and $\mathrm{CO}_{2}$ capacity. The best of such "engineered" materials had an increase in 
specific surface area

(approximately 6 times that of the original FLC chitosan), increased water content (about 2 times that of the original FLC chitosan) and showed about 20 times the uptake of $\mathrm{CO}_{2}$ compared with the original FLC chitosan. Preliminary sorption/ desorption cycling experimentation showed that the engineered form regenerated without significant decrease in sorption capacity, with stable chitosan material in operation. These higher performing materials will be characterized for $\mathrm{CO}_{2}$ adsorption capacity and strength as a function of temperature in FY 2011, with no additional synthesis work on solid chitosan.

We have also identified manufacturers who are able to convert chitosan (a polymer) to smaller, water soluble units. Use of a water-soluble chitosan allows operation analogous to the other liquid systems $\left(\mathrm{CO}_{2} \mathrm{BOL}, \mathrm{MEA}\right)$, but because the solvent is water, it is totally compatible with water present in the flue gas. There are no corrosion issues, unlike MEA, and no viscosity issues, unlike $\mathrm{CO}_{2} \mathrm{BOLs}$. Surprisingly, the monomer of chitosan, glucosamine, showed little $\mathrm{CO}_{2}$ uptake. Optimizing the molecular weight of the oligomer to maximize the $\mathrm{CO}_{2}$ uptake and oligomer solubility are the key tasks to be addressed in FY 2011.

Nano ZSM5/Silicalite. A major premise of this effort is that it is necessary to develop solid adsorbents of very small (nanometer) size in order to facilitate rapid kinetics of capture and release of $\mathrm{CO}_{2}$ within the solid. The materials of choice are a particular aluminosilicate zeolite type known as ZSM-5 or silicalite, depending on whether the material contains aluminum atoms in the

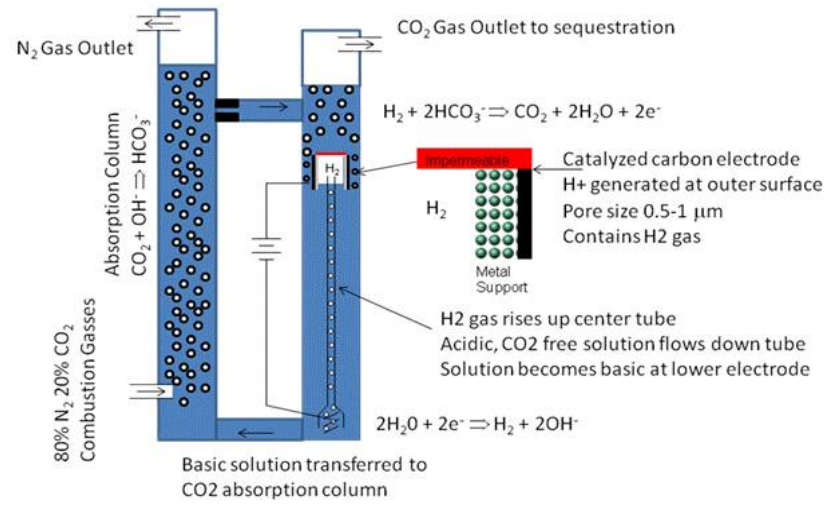

Schematic of the electrochemical $p \mathrm{H}$ swing $\mathrm{CO}_{2}$ capture device. and the structure was confirmed by NMR, TEM, XRD, and SEM analyses. A combinatorial zeolite synthesis methodology was employed to generate a large quantity of zeolite samples for comparative performance. The silicalite material was evaluated for $\mathrm{CO}_{2}$ uptake and demonstrated over $7 \mathrm{wt} \% \mathrm{CO}_{2}$ uptake at $25^{\circ} \mathrm{C}$ and 1 bar pressure. Thermo-analytical characterization of many of these zeolite samples will be completed in FY 2011. No additional synthesis work will be carried out, with only performance testing to continue.
Electrochemically Driven $p H$ Swing. An electrochemical aqueous-based method for capturing $\mathrm{CO}_{2}$ from flue gas streams was developed and tested for proof-of-principle at the laboratory scale. The method used an electrochemical reactor to create a $\mathrm{pH}$ gradient which can be used to capture and release $\mathrm{CO}_{2}$ from solution via the formation of $\mathrm{H}_{2} \mathrm{CO}_{3}$ and $\mathrm{HCO}_{3}{ }^{-}$. Acid can be electrochemically generated from $\mathrm{H}_{2}$ gas to acidify a $\mathrm{HCO}_{3}{ }^{-}$solution and cause release of $\mathrm{CO}_{2}$ gas. Concurrently, acid is consumed through the generation of $\mathrm{H}_{2}$ gas at the cathode. At this location, the solution becomes more basic and able to absorb $\mathrm{CO}_{2}$. The focus of this work was to demonstrate concept feasibility and identify primary technical barriers to achieving targeted efficiency. Our findings are that the design of the acid-generating anode and management of solution flow over this electrode is critical to structure. The all-silica (silicalite) material is most capable of rejecting water but has lower $\mathrm{CO}_{2}$ capacity. Hence, the project has focused on finding a balance between capacity and $\mathrm{H}_{2} \mathrm{O}$ co-adsorption.

A challenge has been to obtain these materials in nanometer size, hence some effort of the project focused on synthesis of such materials. The crystal framework structure of small, uniform ZSM-5 crystals was prepared in a novel flow reactor, setting up and maintaining a $\mathrm{pH}$ gradient between the anode and cathode. An electrode was developed that showed stable behavior. $\mathrm{CO}_{2}$ was generated at nearly 50 percent chemical efficiency. This task will continue in FY 2011, where we will focus on producing a more efficient system.

Thermoanalytical (HIDEN) Testing of Adsorbent Materials. Training of personnel and testing of solid materials for
$\mathrm{CO}_{2}$ and $\mathrm{N}_{2}$ adsorption isotherms was carried out. A number of experimental methods were developed for optimal material pretreatment and data gathering. These data were used to support a stage-gate process wherein we down-selected the best solid adsorbent materials for scale up testing. Nano ZSM5 zeolite was the material down-selected for scale up tests. $\mathrm{CO}_{2} \mathrm{BOLs}$ and $\mathrm{pH}$ switching are liquid phase processes and were therefore not evaluated on this equipment. 


\title{
Operation and Process Optimization of Gasification and Carbon Capture Test Facility
}

\author{
James E. Cabe, Mark D. Bearden, Brian K. Boyd, Douglas J. Reid, Liyu Li
}

This project is focused on the development and operation of reliable fossil-based engineering scale pre-combustion and post-combustion testing platforms. The capabilities of the testing platforms enable the effective research and advancement of proof-of-principle concepts related to the cleaning and capture of gasification-based syngas, and combustion-based flue gas.

$\mathbf{T}$ he benefits of research level gasification technologies for solid feedstocks have not yet beenadequately leveraged due to several drivers. Of the primary issues limiting development is simply the sheer size, lack of operational knowledge as it relates to the durability of materials, and lack of operational flexibility. The pilot-scale facility will be operated with the expressed intent of providing realistic syngas conditions and constituents to the variety of downstream processes under evaluation. Due to size and flexibility, the gasifier may be operated in fuel-rich combustion, oxy-combustion, and pre-combustion modes depending on the needs dictated by operational targets and downstream processes being evaluated.

This test facility project and ensuing research objectives aligns with national goals regarding clean fossil energy and carbon management strategies. The gasification and combustion platforms provide for continuing research into clean coal technologies and carbon capture, offering viable solutions to reducing the traditional emissions associated with the combustion of coal, and providing a demonstrated pathway to the capture of $\mathrm{CO}_{2}$. There are three primary objectives of the project: advancing the gasification platform to produce realistic syngas at atmospheric conditions targeting research objectives for hot syngas clean-up and refractory degradation evaluations; design and commission a fuel flexible coal combustion unit including flue gas conditioning equipment targeting research objectives for $\mathrm{CO}_{2}$ capture using liquid and solid sorbents; and the development of a flue gas conditioning and testing platform at a regional $600 \mathrm{MW}$ sub-bituminous pulverized coal fired power plant targeting research objectives for $\mathrm{CO}_{2}$ capture using liquid and solid sorbents.

The expected outcomes of this work are capabilities to support the safe operation of a gasifier in multiple modes, including fuel-rich combustion, oxy-combustion, and pre-combustion. We are also developing the capabilities to effectively evaluate methods to providing a stable and reliable syngas or flue gas source respective of the fired fuels for downstream research and analysis, and establish the gasifier system as a reliable platform for applied research and proof-of-principle activities. Initial activities will be focused on proof of design operations for dry feed of pulverized coal through an integrated burner. Secondary activities include slurry feed of coal/methanol through positive displacement feed systems for more rigorous control in gasifier operation. Additional outcomes are intended to define and initiate activities to minimize emission/off-gas operational issues such as high solids syngas conditions and elevated temperatures (may exceed current instrumentation limits).

The project team made significant advances in FY 2010, advancing both gasification and combustion platforms. We addressed mechanical constraints in the gasification platform by re-engineering the fuel feed system, and burner nozzle. The modifications resulted in successive increases in both length of run, and overall carbon conversion as calculated. Specifically, we re-designed the fuel feed system to be capable of overcoming backpressure developed in the burner nozzle at the point of combustion, and we were also able to address flame impingement and refractory degradation at the burner nozzle by modifying the location and direction of oxidant (oxygen) feed. As a final measure, we made sure that the appropriate refractory was placed in the gasifier vessel at the time of replacement and increased the residence time, which improved carbon conversion.

To date, we are capable of operating the gasifier unimpeded for a complete fuel cycle, which is represented by gasifying all of the coal in fuel storage without stopping. Refilling the fuel storage currently requires stopping operations, allowing the burner nozzle to cool, and isolating the fuel storage system; therefore, continuing efforts in FY 2011 will include evaluations for expanding the fuel storage system or modifying operations to enable generating syngas for a longer duration than has been demonstrated.

We conducted analytical work on the syngas. The full water-quench gasifier performed as expected. However, our research regarding the warm syngas clean-up was compromised because the syngas exit temperatures were below dew point, and many targeted contaminants were removed in the quench water. Warm coal syngas clean-up research continues using spiked syngas to evaluate the sequential removal of particulate, condensable species, $\mathrm{HCl}$, and ammonia. Objectives evaluated in FY 2010 included deep sulfur removal using $\mathrm{ZnO}$ beds, selective oxidation of $\mathrm{NH}_{3}$ to $\mathrm{N}_{2}$ and $\mathrm{H}_{2} \mathrm{O}$ using $\mathrm{Cu}-\mathrm{Ni}$ oxides, and developing an integrated system for overall warm syngas cleanup demonstration. 
During our evaluations of the gasifier, we concluded that it would not feasible to conduct full combustion tests using the same vessel and configuration. Given these constraints, we pursued the design of combustion specific equipment and configuration for use in FY 2011 carbon capture and integrated emissions research. For our limited fuel firing rate and for added fuel flexibility, we were able to develop a small and mobile cyclone combustion platform. The combustion unit will generate flue gas which we will be conditioned and supplied to downstream research platforms evaluating liquid and solid $\mathrm{CO}_{2}$ sorbents and for continuing integrated emissions capture research. Designed by PNNL, flue gas conditioning equipment supplies an emissions conditioning cart, which was completed in FY 2010. The emissions cart was originally designed to condition and shift syngas from the gasification process; however, we established that by changing to an appropriate catalyst, the emissions cart will be able to scrub flue gas removing water, particulate matter, and sulfur oxides.

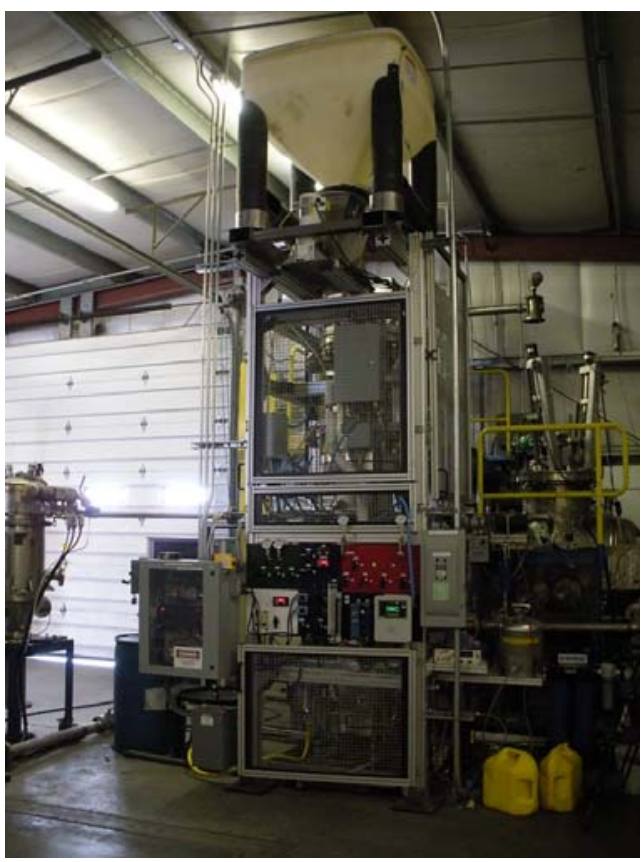

The PNNL gasification and syngas test platform.
In FY 2011, we will commission the cart using common flue gas constituents. Once commissioned, we will put the cart in service scrubbing the flue gas from the combustion unit and providing a slip stream to the $\mathrm{CO}_{2}$ cart systems for sorbent research. We also developed a flue gas testing platform at a conventional pulverized coal power plant. The testing platform enables FY 2011 sorbent research and evaluation using sub-bituminous derived flue gas. In concert with the onsite flue gas testing platform, the offsite testing platform expands our capabilities for sorbent testing and evaluation using actual flue gas from a traditional coal fired power plant. A secondary purpose of the platform is to enable continuous carbon capture and integrated emissions testing during

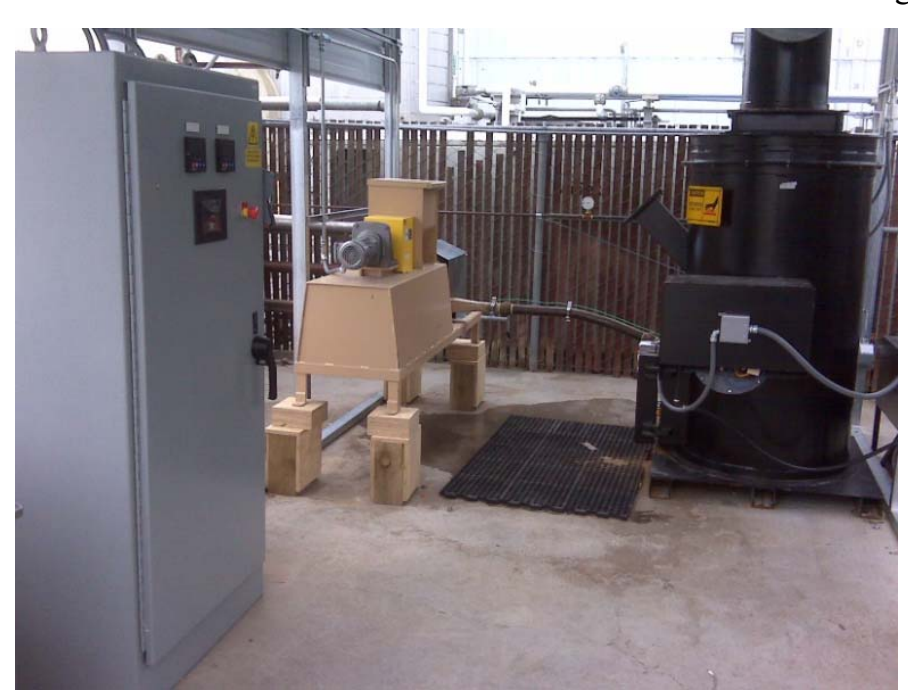

The PNNL solid fuel combustion and carbon capture test platform. the plants actions addressing targeted reductions of $\mathrm{SO}_{\mathrm{x}}$ and $\mathrm{NO}_{\mathrm{x}}$ in the flue gas. 


\title{
Photoelectrochemical Flow Battery
}

\author{
Guan-Guang (Gordon) Xia, Cheng Huang, Birgit Schwenzer
}

We propose to develop a novel photoelectrochemical flow battery (PEB-FB) system for solar energy conversion, storage, and use. Success of this project will demonstrate a new capability to increase our energy options and reduce dependence on oil, thereby reducing vulnerability to disruption and increasing the flexibility of the market to meet U.S. needs.

$\mathbf{T}$ he world demand for energy is expected to more than double by 2050 and more than triple by the end of the century. Although it is the most abundant renewable source in the planet, solar energy and its utilization remains limited (less than 2 percent of total energy consumed worldwide) due to the lack of cost-effective technologies available to compete with other energy sources. Moreover, solar power is variable due to the intermittency and terrestrial variation of the sun shining on the earth, affecting its usage effectiveness and burdening its transmission. A massive scale energy storage system to store converted solar energy would be ideal to balance solar energy harvesting and utilization.

The goal of this project is to develop and demonstrate a novel photoelectrochemical flow

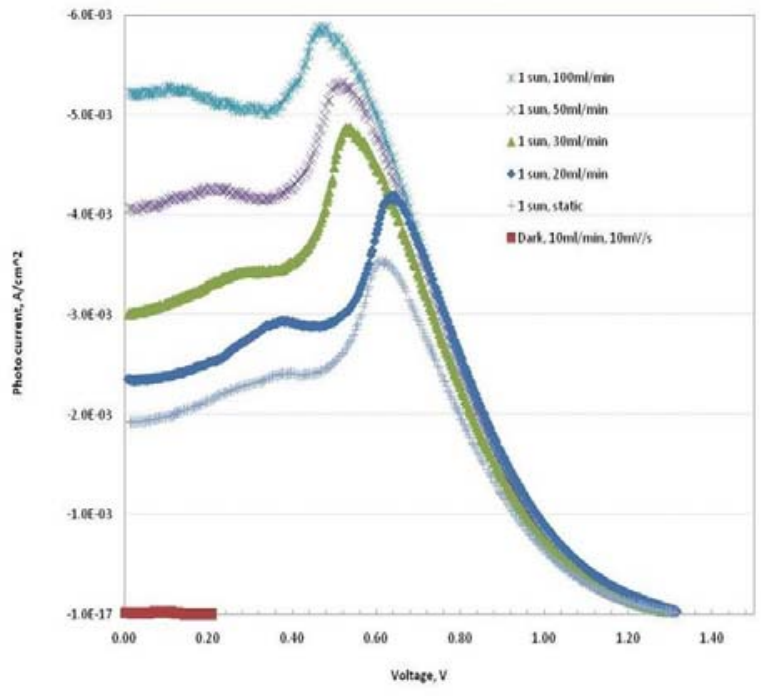

The performance comparison of $G a A s$ as a photoanode in a proposed new mixture solution under static and flow conditions. innovation, and 3) investigating suitable redox electrolytes and solvents with optimal properties for better understanding solid/liquid interaction, charge transfer kinetics, and corrosion mechanism in order to achieve maximum energy conversion efficiency and extended system life.

In FY 2010, our effort was devoted to prove the novel concept by selecting suitable photoelectrodes and redox couple solutions and testing in our conceptual photoelectrochemical flow cells. We screened a variety of semiconductor materials for photoelectrodes, including single crystalline silicone, gallium arsenide (GaAs), cadmium selenide, indium phosphide, and thin films such as tungsten diselenide and cadmium telluride that were fabricated by electroplating and RF magnetron sputtering deposition methods, respectively. Metal oxide semiconductor materials such as $\mathrm{TiO}_{2}$ and $\mathrm{ZnO}$ nanoparticles were also investigated in parallel for dye-sensitized flow cells. Although most semiconductor materials for photoanodes are not stable in aqueous electrolyte solutions under illumination, we learned that they can show reasonable performance and stability under flow conditions. High quality $\mathrm{WSe}_{2}$ thin film may be a good choice for PEC-FB systems.

We discovered a new flow battery system that can be used for developing PEC-FB systems. This new battery has an open circuit of about $1.1 \mathrm{~V}$ fully charged and over 80 percent round-trip efficiency (electrical charge-discharge mode). This system has also demonstrated good stability over 50 cycles, although the ion-conductive membrane and counter electrode need to be optimized. Preliminary results confirmed that it could be charged by solar energy using a photoanode (e.g., GaAs) in this PEC-FB system.

For FY 2011, we will continue to investigate these high performance photoelectrodes, especially photoanodes, based on the new flow battery system. Most activities will be devoted to synthesizing and fabricating highly stable semiconductors and improving their energy conversion efficiency by modifying their compositions and structures. 


\title{
Sensitivity Analysis of Kalman Filter and Its Applications in Power Systems
}

\author{
Zhenyu (Henry) Huang, Pengwei Du
}

This project aims to investigate the sensitivity properties of the Kalman filter to determine power grid observability and model parameter identifiability used to determine optimal sensor placement and model parameter calibration, respectively. The success of the proposed work will significantly improve dynamic state estimation performance and other applications in power systems and other domains.

A s complex engineered and critical national infrastructure, power systems pose fundamental challenges in real-time operations and control because they are highly dynamic, consisting of a large number of interacting elements with severe nonlinearities. Today's tools for real-time grid operations are mostly based on steady-state models with response time in the order of minutes, unable to capture the dynamic nature and too slow to prevent system failures. Tackling this challenge, the extended Kalman filter (EKF) can estimate power grid dynamic states based on a dynamic model and high-speed sensor data. Two key success factors in dynamic state estimation are dynamic model quality and sensor locations. Given the complexity of power grids, the large number of candidate sensor locations and model parameters are not of the same importance in terms of the quality of the estimated dynamic states. Identifying key locations and parameters is an immediate need in the power grid dynamic state estimation application.

To ensure good performance, EKF-based dynamic state estimation needs adequate sensor measurements and a good dynamic system model. This raises technical issues on sensor placement and model parameter identifiability. Both issues point to the need of sensitivity analysis of the EKF formulation. This project focuses on the fundamental underlining mathematics of the Kalman filter, aims to derive its sensitivity formulation, and applies the sensitivity information to assess the significance of parameter uncertainty and evaluate the location value of phasor measurement units (PMUs). The resulting capability of optimal sensor placement and model parameter calibration will lead to significant performance improvement of dynamic state estimation for real-time power grid operation. The derived sensitivity property of the Kalman filter is also applicable to other engineering domains.

In FY 2009, we focused efforts on exploratory studies of the sensitivity formulation of EKF and numeric experiments on sensitivity using power grid models and data. We explored two approaches for formulating Kalman filter sensitivity and performed numeric studies of sensitivities associated with power plant parameter calibration. In FY 2010, we developed a framework to quantify the sensitivity of the EKF performance with respect to different parameters to be calibrated and sensor placement.

Because the Kalman filter is a two-step process (namely prediction and correction), the proposed method will evaluate the filter's sensitivity in an integrated frame. In this context, the effect of all the factors such as system models, process, and measurement noises can be considered. Different from other methods like time-domain simulation and relevant sensitivity studies, this approach has great advantages because it can provide a quantitative measure of the EKF performance without a need of running time-consuming, time-domain simulation. Simulation results tested on a 16-machine, 50-bus system demonstrated that when the PMUs are reduced to 14, the system becomes marginally stable. In other words, some parameters are not observable because of an inadequate number of PMUs. Through these studies, it is easy to determine whether a given sensor placement plan can ensure good performance of the EKF for dynamic state estimation. Two conference papers reporting these methodologies and results were submitted and accepted.

The sensitivities of EKF to parameters can be also evaluated by state variable tracking errors (i.e., estimation biases) and the errors bars on estimated state variables which may be unrealistically small if the uncertainties in the input parameters are not incorporated. The solution to these problems is to augment the state variables. In additional FY 2010 studies, we compared EKF results on a single machine model with noisy data when augmenting the state variables or not. Preliminary results show that EKF is not very sensitive to errors in inertia of machines. An estimation of the state variables is only slightly better when augmenting with the inertia. The EKF can produce improved estimates of the inertia but convergence is slow. EKF is sensitive to errors in transient reactance, but small errors in reactance result in large errors in some state variables when not augmented. When augmented, EKF produces improved estimates of reactance with fast convergence and very good tracking of the state variables. These studies quantify the effect of parameter uncertainties over the performance of the Kalman filter. In this special case, a correct value of transient reactance is very critical to ensure a satisfactory performance of EKF. A paper discussing more details on this method is in preparation.

The application of EKF to power system dynamic state estimation is the first of this kind to dynamically track the status of the power grid in real time. These efforts and 
accomplishment

in FY 2010 have

significantly

enhanced our

fundamental

understanding

of how the

performance of

EKF relates to

parameter

identifiablities

and observability.

Thus, it will

enable us to

implement and

improve the

filter's performance for large, complex power system models.

To this end, an invention disclosure was filed in FY 2010.

In FY 2011, research will continue in application of the Kalman filter for dynamic state estimation and parameter calibration. Because sensors and related communications infrastructure are often monetarily expensive (or the associated estimations are often computationally expensive), one wants the sensor placement to be optimal in the sense that it maximizes observability while minimizing cost. Our emphasis in the upcoming fiscal year will seek to determine the most sensitive locations for sensor placement to estimate dynamic states. We will demonstrate application of the Kalman filter for a future (smart) grid, which is characterized by the presence of smart load and renewable resources like wind and solar power.

Another focus of next year's activities will address the model calibration challenge (complexity of models involved and size of the problem) in order to meet the needs in a smart grid operation environment. While model calibration is deemed to be critical and necessary for power system operation, utilities lack of easy-to-use tools to help them improve model integrity. Also in FY 2011, a software prototype tool will be built with the integrated functions of model calibration and sensor placements. 


\title{
Simulation of Future Electricity System Operations
}

\author{
Harold Kirkham, David P. Chassin
}

The aim of this work was to have a tool that demonstrates, educates, and illustrates the difficulties and intricacies of system operation with deregulated power sources and with cost-dependent loads on a workstation. In particular, it created an interactive program to teach the evolving nature of the power supply business and the role of a market-based approach to power system operation.

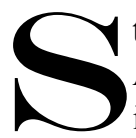
tarting with the Public Utilities Regulatory Policy Act of 1978, deregulation of the electric power industry has had increasing impact on the world, as much of the nation's power system is now operated as a price-driven, renewable-rich market. Yet many phases of the operation remain poorly understood, with some aspects largely untested. For example, there is no control theory that establishes that operation with price-sensitive load as well as price-sensitive generation is bound to be stable. Through this project, we have developed a new software tool that would simulate such an operation, allowing a number of alternatives to be tested realistically. The result is a new, cutting-edge simulation capability that will allow the user to examine future operations scenarios.

In operating the electric power system, control of generation is aimed at precisely meeting demands of the load and doing so for minimum cost. Historically, cost was minimized according to an optimization that included reserve power (in case of faults) but assuming that load was a relatively predictable quantity largely independent of system characteristics such a frequency, voltage, and costs. The traditional utility operations paradigm involves a sequence of processes that includes calculation of power interchange with neighbors (contractual calculation), unit commitment (machines ready when needed) and economic dispatch (true optimization). These aspects of system operation can be simulated and inform the user about the way the system works.

Since deregulation, the customer has been able (at least in theory) to play a role in that operation. For example, a price signal is sent to the customer, who then makes a decision to buy or not. The idea was first proposed about 30 years ago by MIT professor Fred Schweppe, but it has not been until the advent of deregulation and the development of low-cost

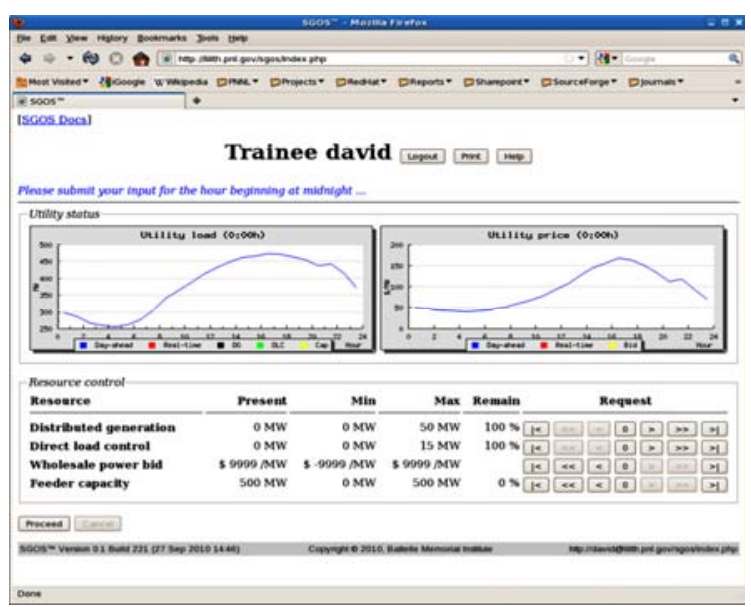

Screen shot showing representative load and price information presented to a participant in the Future Electricity Systems Operation simulator. computing power that such operation has been feasible. The impact of the customer (now known by the term "demand response") can also be part of the simulation.

Another aspect of deregulation has been the increasing use of non-utility generation. Run-of-the-river hydrolic and wind systems were encouraged by this regulation change, and since then renewable energy has been incorporated into generation portfolios by most states. A problem has been (and will continue to be) the unpredictable nature of some renewables. This issue leads to changes in power system operations and may cause increasing amounts of energy storage to be needed. This too is an aspect of system design and operation that can be simulated interactively.

The system prototype has been operating in training mode since September 2010. It is a bulk power system based in the northeast United States with a system capacity ranging from $5000 \mathrm{MW}$ to about 100,000 MW and up to 12 participants. Before a session begins, participants are given the opportunity to "purchase" resources for utility management, including distributed generation, direct load control, and fixed reserve contracts. Once trainees have purchased resources, they are confronted with the operation of their utility on a peak day. For each hour, they must allocate the resources acquired, taking into account the cost of the resources, any resource limitations, and resource benefits. At the end of the day, the trainee's performance is projected for a year of operation and evaluated with respect to customer satisfaction and financial outcomes. Trainees are then scored with respect to other trainees.

This research resulted in a set of tools that allow the user to participate in a market-driven and/or renewable-rich power system operation. A simulated timeframe (day or week) of system operation was modeled with different assumptions about market operation. An important part of the program is economic modeling, with displays showing the price and demand on the simulated system. In addition, the user can explore the impact of different levels of wind power penetration on the system. Alternatively, the effect of altering the price signals of demand response in mitigation of the variability of wind power might be of interest. 


\title{
Tools for Evaluation of Net-Zero Community Concept and Integration of Buildings, Renewables and the Grid
}

\author{
Srinivas Katipamula, James A. Dirks, Nick Fernandez, Will J. Gorrissen, Michael R. Brambley, Manish Dixit
}

Numerous organizations are focused on research and development for net-zero energy homes and commercial buildings. Seeking net-zero energy at the community scale creates the possibility of using renewable generation technologies beyond rooftop photovoltaic, such as solar thermal electricity generation, solar-assisted heating/cooling systems, on-site battery storage, and wind energy. Developing tools and a methodology to evaluate net-zero energy concepts at both building and community scales will allow researchers and policymakers to evaluate the benefits and risks associated with net-zero energy concepts.

B uildings use over 40 percent of total energy and over 70 percent of electricity in the United States. Many organizations are focusing on net-zero energy homes and buildings as a solution to reduce the use of fossil fuels and carbon emissions. Although net-zero energy homes and buildings are feasible with today's technology in some climate locations, significant improvements in efficiency are needed in the renewable generation technologies as well as the buildings and their systems for broader adoption. Seeking net-zero energy at the community scale opens the possibility of using more renewable generation technologies. If the push for net-zero energy homes/community is realized as many organizations hope, it will have significant repercussions to the stability and reliability of the electric grid because the onsite variable generation will introduce volatility to the transmission and distribution load profile, which challenges the transmission and distribution system planning process.

The two specific objectives of the project include: 1) development of a simulation framework for conducting detailed energy and power demand estimations for net-zero energy buildings and high-performance buildings (HPB), and 2) conducting a detailed analysis to highlight the advantages of building net-zero energy communities as opposed to communities with individual net-zero energy homes and buildings. The simulation framework will be used to generate hourly electricity loads for comparing the relative merits a community of net-zero energy buildings and a community that is net-zero energy and for assessing the impact of the high penetration of net-zero energy buildings/communities on the electric grid.

In FY 2009, a simplified economic analysis of comparing conventional net-zero energy approaches to net-zero energy community concepts was conducted. The results from this preliminary analysis showed that even in the United States best case for net-zero energy buildings (Phoenix, AZ), there are more cost-effective approaches to achieving net-zero energy than the conventional suite of technologies (rooftop photovoltaics, with aggressive efficiency measures) used at the building level. By expanding the conceptual boundary for net-zero, a community can take better advantage of economies of scale, as well as having other generation options at its disposal.

In FY 2010, we performed a more detailed economic analysis. To investigate the economics of pursing net-zero energy buildings versus net-zero energy communities, two separate models were developed. The first model, Aggregate Building Simulator (ABS), is a Microsoft Excel-based tool that generates EnergyPlus input files in a semi-automated way. Based on selection of high-level building attributes, the ABS generates EnergyPlus input files, runs each file individually, and aggregates the results among the set of buildings to produce hourly load profile for the entire community. The second model, a net-zero energy community modeling tool developed in MATLAB environment, simulates the hourly flows of electricity in a community. In addition, the model approximates the renewable systems used and estimates the cost of generating electricity based on the capital and recurring costs of the renewable systems and the sale and purchase price of electricity.

Using these two models, a host of parameters that affect the economics of net-zero energy on either the building or community scale (including renewable electricity generation technology, geographic location/climate, level of end-use energy consumption, electricity rate structure, use of thermal energy storage for solar-thermal electric power, use and level of electric energy storage, year of installation, and community size) were investigated. The analysis indicated that suitable net-zero energy community solutions can be 50 to 85 percent less expensive than net-zero energy building solutions. Often the difference between 6 to 12 cents per $\mathrm{kWh}$ and between 30 and 50 cents per $\mathrm{kWh}$ (for community- versus buildingscale net-zero energy), this cost barrier makes net-zero energy buildings less viable on a large scale. At the community scale, wind power is strongly favored in most cities regardless of the other parameters.

Wind power is the most mature of the renewable energy technologies and is not expected to decrease in cost. However, solar photovoltaics and solar-thermal electric technologies are expected to level out in cost as those technologies are refined and up scaled. Eventually, installed and operations and maintenance costs for solar technologies (especially solar-thermal electric) are expected to approach levels that should make them competitive with wind power for net-zero energy ommunities in most locations. At very favorable sites, wind may always remain superior to solar technologies. 


\section{Engineering and Manufacturing Processes}




\title{
Demonstration of On-Line Monitoring and Physics Based Prognostics
}

\author{
Pradeep Ramuballi, Mukul Dixit, Jeffrey W. Griffin, Leonard J. Bond
}

This project will investigate innovative sensing technologies and prognostic methods to support residual life predictions for materials subjected to stressors. The resulting capability will form the basis for advanced diagnostics and prognostics tools needed to support U.S. nuclear power plant "life beyond 60" and provide novel online monitoring tools for incorporation in new advanced nuclear power plants. The methods proposed will contribute to maintaining and potentially improving the safe and economic long-term operation of legacy plants.

A central issue in life extension for the current fleet of light water nuclear power reactors (LWR) is the early detection and monitoring of materials degradation. Related is the ability to estimate remaining useful life (RUL) of components and systems based on condition assessment or degradation information. Material aging and degradation due to stresses and irradiation is a critical element in the failure of components in legacy nuclear power plants, and the detection of early stage damage in materials and assessment of remaining life is important in proactive- or prognostic-based life management of these facilities. Sometimes referred to as "condition-based maintenance," this strategy can potentially improve safety and reduce costs by detecting damage and scheduling appropriate maintenance/mitigation strategies early in the component lifecycle.

For early detection of degradation, novel nondestructive (i.e., without destroying the utility of the specimen) methods suitable for continuous monitoring over extended time periods (months to years) are needed, as are new techniques to data integration. The challenge of predicting remaining life starting from earlier phases of degradation is also largely unsolved and will require new prognostics tools. Research activities in this project are being conducted within this framework. The objectives of this project are to develop online nondestructive monitoring methods to assess the condition of materials used in legacy nuclear power plants and prognostics algorithms that use the measurements to estimate RUL.

The research focus during FY 2010 was on quantifying the degradation state of mechanically fatigued austenitic stainless steel specimens and using this information, together with models of damage accumulation, to estimate the RUL probabilistically. Austenitic steels are commonly used in LWR components and in an LWR operating environment can develop fatigue cracking, particularly near structurally significant locations such as welds. Early stages of fatigue in austenitic steels result in changes in the material microstructure, such as the formation of dislocations, slip bands, and a ferrite phase. These changes result in local variations in electrical conductivity, magnetic permeability, and elastic constants. Sensitive to such changes, electromagnetic nondestructive methods (such as multi-frequency eddy currents and magnetic Barkhausen noise) were therefore selected (during the FY 2009 period of the project) for early material degradation state evaluation.

In order to determine the sensitivity and selectivity of these techniques to early-stage damage, multiple specimens with different levels of damage were prepared in both FYs 2009 and 2010. The measurements of these specimens obtained to date indicate good correlation with early stage damage. A Bayesian prognostic algorithm was also developed for estimating RUL from nondestructive measurements of material degradation. The algorithm was initially evaluated using data from nonlinear acoustics measurements, and results show the feasibility of RUL estimation from early damage measurements.

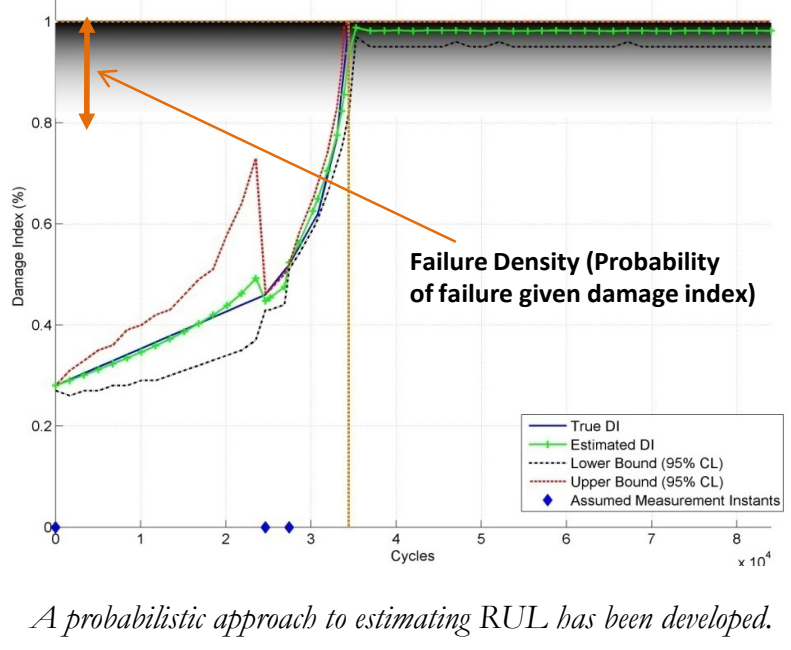

This project has thus far resulted in nine publications and two invited presentations, and is contributing to a draft international report through an International Atomic Energy Agency coordinated research program that is assessing advanced diagnostics and prognostics for nuclear power plants. During FY 2011, the focus of our effort will be on examining other degradation modes, particularly where more than one stressor (such as thermal and mechanical fatigue) is involved. Online monitoring (i.e., during the degradation evolution process) using nondestructive tools developed in this project will also be explored. The diagnostics and prognostics algorithms will be integrated to provide a first demonstration of such technology for nuclear power applications. 


\section{Development of a Ballistic Electron Microfabricated Cathode}

Kyle J. Bunch, Mukul Dixit, Hong (Amy) Qiao

We propose to develop a solid-state cathode based on ballistic electron travel within thin film materials. With a high emission density, this cathode will serve as an enabling technology for many different fields and devices.

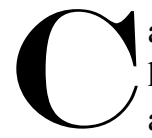
athode technology is used in applications such as high-powered microwave amplifiers, oscillators, and potentially next-generation flat-panel displays. Conventional cathodes rely on thermionic emission of electrons from a material surface but have the disadvantages of short lifetimes, difficulty in miniaturization, and issues with heat dissipation. Research over the last 30 years has focused on replacing thermionic cathodes with field emission tips. This approach has also met with difficulty, including poor emission densities and tip degradation. Our project involves exploiting advances in the processing and deposition of materials, especially diamond and diamond-like materials, in order to create a cathode that directly accelerates electrons within an intrinsic material region and into a vacuum. The potential advantages of this theory include high current densities, long cathode lifetimes, and compatibility with microfabrication processing methods. Such a solid-state cathode would represent an enabling technology leading to novel devices combining vacuum electronics with solid-state electronics.

For our project start in FY 2008, we selected three materials as promising: diamond $\mathrm{n}$-doped silicon, silicon carbide $(\mathrm{SiC})$, and gallium arsenide. These materials have a combination of high breakdown field strength, long electron mean free path (for ballistic transport), and practicality for use with current microfabrication methods. They are also the best suited materials for creation of a solid-state, ballistic-electron cathode. In FY 2009, we focused on simulation, fabrication, and testing. Our testing showed that the ballistic electron cathode concept as a viable means for creating a solid-state cathode.

In FY 2010, we finished testing of devices fabricated consisting of substrates with CVD diamond coating, which were designed for optimal electron emission into vacuum. Two sets of wafers were produced: $0.2 \mu \mathrm{m}$ intrinsic CVD diamond on $\mathrm{n}$-doped silicon and $0.2 \mu \mathrm{m}$ intrinsic $\mathrm{SiC}$ on n-doped SiC. Composed of deposited platinum, the grids were fabricated on the wafer surface. We placed the devices into a vacuum chamber and applied a high voltage for electron collection and current measurement. The grid can vary from $0-50 \mathrm{~V}$, while the high voltage collector electrode is $0-5 \mathrm{kV}$. We measured the current into each element, which reached a threshold of about 20, after which voltage increased exponentially. This result was unexpected, as its I-V curve resembles that of an avalanche breakdown diode. The device showed no emission current at the collection plate, even at high voltages $(5 \mathrm{Kv})$.

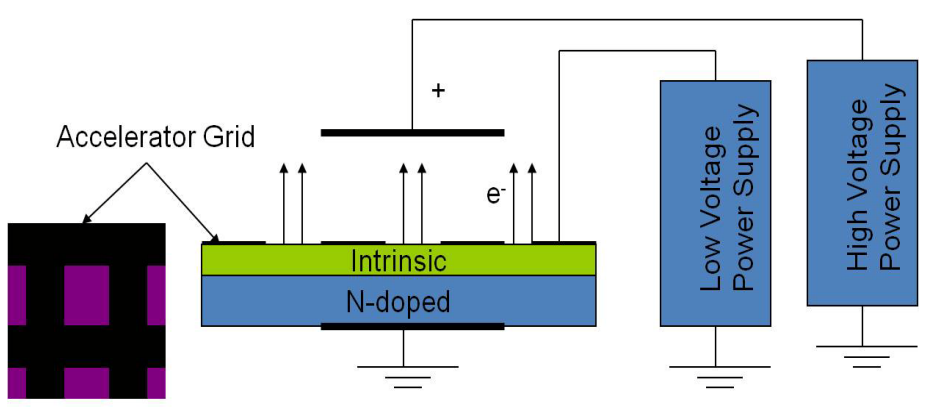

The setup for cathode testing.

Our results yield different possibilities. First, the device is operating in avalanche breakdown mode. Given the diamond film thickness $(0.2 \mu \mathrm{m})$, it is likely that the mean free path of electrons in the diamond is $10-20 \mathrm{~nm}$, as multiple collisions typically occur for operation in avalanche breakdown. With the grid breakdown voltage at 30 , it is probable that the electrons injected into the diamond film are not being accelerated beyond a few volts because no emission current was observed. Another option is that the diamond film is polycrystalline, rather than monocrystalline as expected.

A SiC substrate was chosen because of the high state of fabrication capabilities available for this material. Unfortunately, it appears that the effective lattice constant for $\mathrm{SiC}$ is a poor match with monocrystalline diamond. Because the cathode requires collisionless acceleration up to several electron volts, a monocrystalline material is needed. A $n+$ doped monocrystalline diamond substrate would be the ideal follow-on material to investigate, although an $\mathrm{n}+$ doped $\mathrm{SiC}$ material with an intrinsic layer as the accelerating region could also be promising. Given the behavior of the tested device, it is likely that its use as an avalanche-breakdown device has advantages. Both diamond and $\mathrm{SiC}$ are good conductors of heat, plus, diamond has a high breakdown voltage. High voltage, high power transient suppression devices may be a suitable market niche for such a device.

Fabricating a thin monocrystalline accelerating layer has been more difficult than originally anticipated. Although combining diamond thin films with $\mathrm{SiC}$ has not resulted in the desired ballistic electron behavior, the avalanche breakdown within the film may prove advantageous for creating high-powered transient suppression devices. For the next phase of cathode development, it is necessary to create a monocrystalline electron acceleration layer. Diamond is the preferred material, although it is likely to require a doped diamond substrate on which to grow the intrinsic layer. 


\title{
Process Modeling of Chemically Complex Solid-Liquid Suspensions
}

\author{
David R. Rector, Mark L. Stewart, Andrew R. Felmy
}

The design and construction of successful chemical processing systems depend heavily on fundamental processes developed during bench- and pilot-scale testing. Our objective is to develop a general simulation capability to predict the performance of chemically complex multiphase chemical processes for a wide range of applications.

$\mathbf{M}$ any examples exist in the chemical processing industry, illustrating the difficulty of chemical process scale-up from benchtop or pilot-scale experiments, including high-level waste treatment. Current simulation methods use assumptions and approximations to fit the existing computational methods and resources. These assumptions can result in poor scaling predictions. The objective of this work is to develop and demonstrate a general simulation capability to predict chemical process performance that uses highly parallel computational resources to reduce assumptions and approximations. Physical models are incorporated that are appropriate for specific applications, and the method is being validated using available data. The simulation approach used in ParaFlow is based on the lattice kinetics methods.

Distribution functions containing information about the pressure tensor streams to adjacent lattice

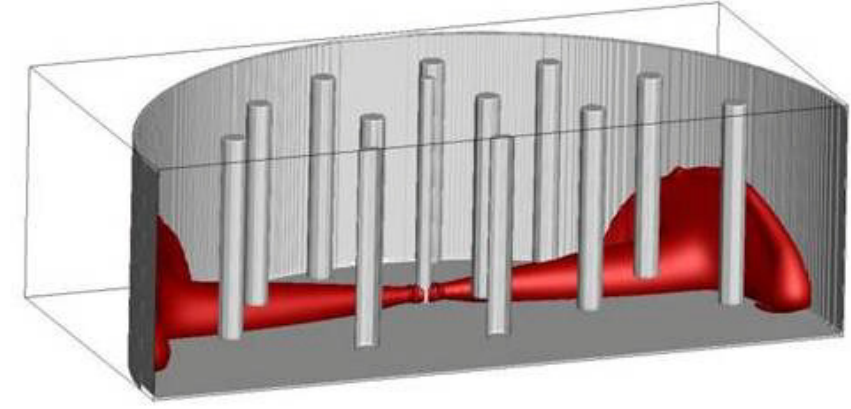

Velocity isosurface of jet pump tank mixing in a Hanford waste storage tank.

turbulent mixing. A series of simulations were performed to determine the flow verses pressure drop behavior of suspension transport pipelines. Comparison of simulation results with experimental data showed very good agreement.

During FYs 2009 and 2010, we developed additional capabilities for ParaFlow. A hierarchal grid structure was incorporated that allows computational nodes to be subdivided in regions where additional detail is desired. The time step was also subdivided to maintain the same Courant number, plus this capability is used to focus computational resources where they are most needed in a simulation. Many environmental and energy flow applications, including dissolution and precipitation, involve chemical reactions; for this reason, a thermodynamically based chemical reaction model was added. The solution method used is a Gibbs free energy minimization scheme

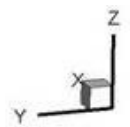

that is especially useful for systems with high ionic concentrations. Application simulations included the flow and heat transfer in a section of a 19-pin LMFBR wire-wrapped fuel, a catalyst bed reactor downstream from a coal gasification and a pulse jet mixing tank.

More specific activities for FY 2010 focused on the application of ParaFlow to a variety of multiphase systems. The first was both the pore-scale and sites. The summation of these quantities results in equations solved for mass, momentum, and scalar transport. The method depends only on local information and scales nearly linearly with the number of computer processors.

In FY 2008, we incorporated solid-liquid multiphase flow models for the sediment and suspended solids. The sediment is modeled using a representation where the phase interface embodies a continuous order parameter profile that moves smoothly through the computational grid. The sediment bed changes through deposition by hindered settling and erosion when the surface shear stress exceeds a specified yield stress. A dynamic lithostatic model was included, where the internal stresses are calculated and the sediment can flow when a yield stress is exceeded. The transport equation for suspended solids includes convection, hindered settling, diffusion, and device-scale simulation of filters used in leaching process to determine fouling and pressure drop behavior. The pore-scale modeling involved discreet particle transport and deposition in a detailed filter material geometry. Next, a series of simulations were performed of sediment resuspension in large waste storage tanks. A rotating jet boundary condition was developed based on rotating grids centered on the rotating jets and a stationary grid for the rest of the tank. Information was passed through an interface between the grids at each time step during the transient. The simulations also included sediment erosion and transport of different particle species throughout the tank. The base numerical algorithm used in ParaFlow, the lattice kinetics method, has been documented in a journal article published in the Journal of Computational Physics. 


\section{Materials Science and Technology}




\title{
Advanced Cathodes for Sodium-Beta Batteries and Renewable Energy Applications
}

\author{
John P. Lemmon, Xiaochuan Lu, Guosheng Li, Gregory W. Coffey, Zhenguo (Gary) Yang
}

Due to environmental concerns, there has been increased interest in using energy generated from renewable resources such as solar and wind. Energy storage systems or batteries are typically needed to enable integration of the renewable energy into the grid. One such technology is sodium-beta alumina batteries (NBBs), which are extremely promising for renewable storage in stationary as well as transportation applications.

$\mathbf{T}$ wo kinds of NBBs (Na-S battery and sodium-metal halide batteries) have been extensively developed since a few decades ago. The Na-S battery offers high energy density, high energy efficiency, and good cycle life. The main problem with the battery is the sulfur/ polysulfide cathode. The cathode materials are highly corrosive and reactive at operation temperatures, making the battery unsafe. A closely related system is sodium-metal halide batteries, which use metal halide as the cathode material plus a molten secondary electrolyte. The cathode and secondary electrolyte are less corrosive compared to polysulfide and thus make the battery safer. One notable disadvantage of the battery is its lower power than the Na-S battery.

Tubular design has been the dominate geometry of NBBs. Planar design, however, has a number of advantages over tubular, such as higher power/energy densities, lower operation temperature, and higher packing density. In this project, sodium-nickel chloride battery with planar design will be used to achieve high performance at reduced temperatures, which will in turn improve battery material durability, allow the use of more cost-effective cell and stack materials, and eventually reduce the overall battery cost.

In FY 2009, we developed and successfully tested halide-based cathodes in single cells. We tested a new approach to make the electrolyte and cells with a variety of materials, which enabled fabrication of variable thicknesses. To evaluate the cathodes and cells, we built a cell test setup that was considered a novel approach and provded effective in sealing alumina electrolyte and metal parts.

Continuing our work in FY 2010, the $\beta "-\mathrm{Al}_{2} \mathrm{O}_{3}$ electrolyte discs for a sodium-nickel chloride battery were fabricated via the vapor phase conversion process. The slurry with $\alpha-\mathrm{Al}_{2} \mathrm{O}_{3} / \mathrm{YSZ}$ powders was tape-casted into a thin sheet. After lamination, the sheet was punched into circular discs, which were fired at $1600^{\circ} \mathrm{C}$ in the air to achieve a full density. The $\alpha-\mathrm{Al}_{2} \mathrm{O}_{3} / \mathrm{YSZ}$ discs were then packed in a $\beta^{\prime \prime}-\mathrm{Al}_{2} \mathrm{O}_{3}$ powders and heat-treated to $1450^{\circ} \mathrm{C}$ for a certain time to complete the conversion process. The disc was glass-sealed to an outer thicker $\alpha-\mathrm{Al}_{2} \mathrm{O}_{3}$ ring. The cell was then moved into a glove box and assembled in a discharged state with starting powders consisting of $\mathrm{Ni}, \mathrm{NaCl}$, and additives. The powders with the amount of $2 \mathrm{~g}$ were thoroughly mixed and granulated. After a drying treatment to eliminate moisture, molten $\mathrm{NaAlCl}_{4}$ was vacuum-infiltrated into the granular at $250^{\circ} \mathrm{C}$. At the anode side, a metal shim was inserted into the anode compartment, and copper wool filled the gap between the electrolyte and shim. Anode and cathode end plates were compressed to the $\alpha-\mathrm{Al}_{2} \mathrm{O}_{3}$ ring with the help of alumina washers. Platinum leads were welded to the electrode end plates as current collector. The assembled cell was placed in a furnace inside the glove box, and cell testing was carried out at $300^{\circ} \mathrm{C}$.

A constant current of $10 \mathrm{~mA}$ was used for initial five charge/ discharge cycles. After cell performance was stabilized, it was further cycled at a higher rate (e.g., C/3). The capacity gradually decreased from 146 to $87 \mathrm{mAh}$ after 20 cycles even with the low current rejuvenation cycles. The capacity loss during cycling was diagnosed to be related to growth of sodium chloride grains within the cathode. During charge, small grains are consumed and they tend to redistribute on the surface of large grains during the following discharge. As a result, the large grains become even larger while the small ones disappear with cycling. This process apparently causes more and more materials unavailable for further charge/discharge (i.e., loss of cell capacity with time).

In a summary, planar sodium-nickel chloride cell using the BASE was successfully constructed and operated for about 20 days without cell failure. The loss in cell capacity during cycling was observed, which was likely related to irreversible $\mathrm{NaCl}$ grain growth during charge/discharge process. More work will be needed to optimize the cathode chemistry so as to minimize the cell capacity loss. 


\title{
Advanced Materials for Capturing Lanthanides and Transition Metals from Fission Products
}

\author{
Glen E. Fryxell, Wilaiwan Chouyyok, Yongsoon Shin; Wassana Yantasee (Oregon Health \& Science University)
}

This project seeks to develop and evaluate advanced materials for the selective capture of specific decay species (lanthanides, transition metals) such as might be encountered in spent nuclear fuel or nuclear waste, from human blood, dialysate, high acid solutions, and natural waters. Successful development of sorbent materials for these species in the challenging matrices will enable not only their collection and separation from nuclear wastes and natural waters but also their monitoring and treatment strategies of the workers at the DOE sites.

F our classes of functional materials (functional mesoporous silica, functional mesoporous carbons, functional activated carbon, and functional magnetic nanoparticles) have been successfully developed at PNNL for the capture of heavy metals, actinides, lanthanides, and transition metals in the aqueous phase. Removal of lanthanides (Ce, $\mathrm{Pr}, \mathrm{Gd})$ and transition metals ( $\mathrm{Ru}, \mathrm{Rh}, \mathrm{Pd}$ ) from challenging matrices (human blood, high acid solutions, dialysate, and natural waters) have not been investigated, thus will be the focus of this work. The physical and chemical properties of the four advanced nanoporous materials (functional silica, mesoporous carbon, and activated carbon) have been evaluated in acid solutions, natural waters, and dialysate. These materials can withstand a wide range of solution $\mathrm{pH}$, and the small pore sizes are ideal for excluding particulates and microbes from clogging the pores or degrading the sorbent and releasing the toxic metal. The goal of this project is to develop and evaluate new sorbent materials for selective capture of lanthanides and transition metals.

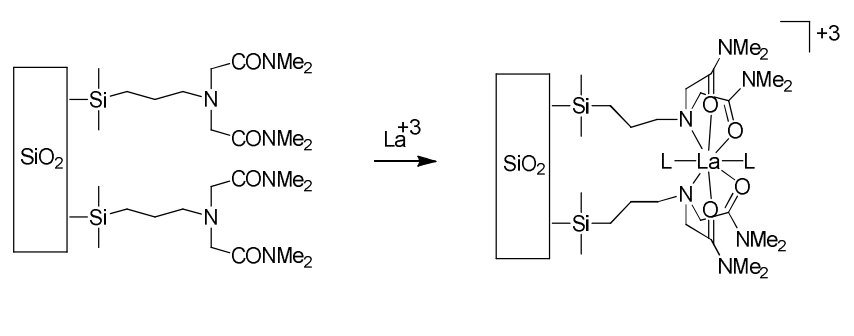

$L=$ unspecified ligand (e.g. water)

ID A-Amide SAMMS chelating a lanthanide cation.

In FYs 2008 and 2009, we tested six and seven (respectively) organically modified mesoporous silica (SAMMS) for capturing lanthanides in nitric and hydrochloric acids, a variety of waters (river, ground, and sea), and dialysate against commercial activated carbon and zirconium phosphate. We also measured the uptake capacity, selectivity, removal rate, stability, and regenerability of the SAMMS materials in relevant matrices. A novel sorbent was designed and built in FY 2009 by decorating an activated carbon framework with thiol groups. During FY 2010, the focus of our work with silica-based sorbents was on neutral chelating polyamides, tailored with the intent of selectively chelating lanthanide cations. Chelating diamides are known to have good affinity for rare earth cations and have been used for solvent extraction processes.

From the previous fiscal years' success with iminodiacetic acid (IDAA) SAMMS, our first target was the diamide analog or iminodiacetamide (IDA-Amide) SAMMS made on MCM-41. Synthesis of the IDA-Amide silane proceeded uneventfully and in quantitative yield. Deposition of this silane onto MCM-41 $\left(880 \mathrm{~m}^{2} / \mathrm{g}, 35 \AA\right.$ pores, and $1.29 \mathrm{cc} / \mathrm{g}$ pore volume) proceeded smoothly to give IDA-Amide SAMMS ( $486 \mathrm{~m}^{2} / \mathrm{g}, 25 \AA$ pores, and $0.77 \mathrm{cc} / \mathrm{g}$ pore volume). Thermogravimetric analysis (TGA) revealed a population density of 0.94 ligands $/ \mathrm{nm}^{2}$. Several attempts were made to synthesize the ED3A-Amide analog (essentially the triamide analog to EDTA), all of which resulted in the formation of an intractable red-brown polymeric resin. Further attempts to synthesize ED3A-Amide SAMMS were abandoned.

Batch contact studies with IDA-Amide SAMMS revealed good affinity for lanthanide cations between $\mathrm{pHs}$ of about 6 and 7, but low affinity under more acidic conditions (and precipitation problems under alkaline conditions). This sorbent was unique, as the kinetics of sorption were rather slow relative to other "flavors" of SAMMS, taking several hours for the system to reach equilibrium and the rate of sorption being somewhat different for each lanthanide cation. Studies revealed that there was no competition from alkaline earth cations (e.g., $\mathrm{Mg}, \mathrm{Ca}$ ), but some observed certain transition metal cations (primarily from $\mathrm{Mn}(\mathrm{II})$ and $\mathrm{Ni}(\mathrm{II})$ ). To determine if this chemistry was due to the amide terminus or was a chelation effect, we performed control experiments using SAMMS terminated with a simple amide (i.e., a nonchelating analog with no basic amine in the ligand). Synthesis and vacuum distillation of the amide terminated silane was carried out uneventfully, and it was subsequently deposited onto MCM-41. TGA revealed a population density of

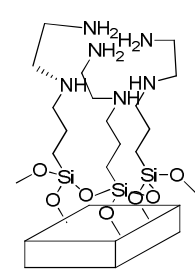

EDA SAMMS

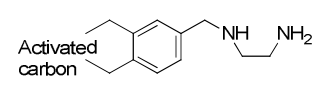

$\mathrm{AC}^{-\mathrm{CH}_{2} \mathrm{EDA}}$

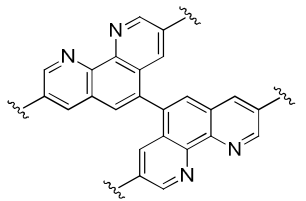

Phen-FMC
Chemical structures of three different nanoporous sorbents built around the chelating diamine motif. 
2.64 ligands $/ \mathrm{nm}^{2}$. Batch contact studies revealed that these Amide SAMMS had a much weaker interaction with the lanthanide cations than did IDA-Amide SAMMS, confirming the importance of chelation for this chemistry (and need for a basic amine).

For transition metal separations, we undertook a comparison of three sorbents (ethylenediamine [EDA] SAMMS, functional mesoporous carbon made with 1,10-phenanthroline [Phen-FMC], and activated carbon-bound EDA) designed around chelating diamines, which are known to be good general purpose transition metal complexants. The AC- $\mathrm{CH}_{2}$-EDA was found to have a specific surface area of $1200 \mathrm{~m}^{2} / \mathrm{g}$, and a functional loading of 1.5 mmole EDA per gram of sorbent. Batch contact experiments showed that both EDA SAMMS and $\mathrm{AC}-\mathrm{CH}_{2}$-EDA were effective $\mathrm{Cu}$ sorbents at near-neutral
$\mathrm{pH}$ (between 6 and 8) in filtered river water $\left(\mathrm{K}_{\mathrm{d}}>10^{6}\right)$ and even in the high ionic strength of filtered sea water $\left(\mathrm{K}_{\mathrm{d}} \sim 10^{5}\right)$. Likewise, Phen-FMC binds late transition metal cations (e.g., $\mathrm{Co}, \mathrm{Ni}, \mathrm{Cu}$ ) very effectively under near neutral conditions $\left(\mathrm{Kd}>10^{5}\right)$. Particularly noteworthy is the observation that Phen-FMC is also effective for binding $\mathrm{Cu}$ (II) under strongly acidic conditions (e.g., $\mathrm{pH}=1$ ), or high ionic strength (e.g., filtered seawater). Phen-FMC sorption kinetics are rapid, with equilibrium being achieved in nearly 10 minutes.

There has been some interest in using EDA and DETA SAMMS for separations of catalyst metals (e.g. Rh) under certain specific conditions. These sorbents might also be applied towards other separations (e.g., Au) as well. For FY 2010, we had six manuscripts appear in peer-reviewed technical literature. 


\section{Advanced Nuclear Magnetic Resonance Characterization of Energy Storage Materials}

Jianzhi Hu, Murugesan Vijayakumar

We propose to develop and apply unique nuclear magnetic resonance (NMR) capabilities to provide a molecular level understanding of the detailed ion transport mechanisms and chemical environments in lithium-ion battery, flow-battery, lithium (Li)-air battery, and other conducting materials related to energy storage.

D eveloping advanced energy storage materials for emerging applications such as plug-in hybrid vehicles or efficient use of renewable energies have been identified as one of the critical enabling technologies to accomplish DOE's strategic goals. Although Li-ion batteries have gained commercial success for mobile electrics applications, they do not meet the requirements for the emerging, large-scale applications in terms of energy/power density, life, safety and cost. Fundamental improvements on the electrochemical storage performance and developing new battery technologies such as flow- and Li-air are demanded for the emerging applications in the sectors of transportation and electricity production. To reach this goal, a molecular-level understanding of the detailed ion transport mechanisms, the chemical status of the ions and the chemical compositions both inside the electrodes and at the electrodeelectrolyte interface is critical for designing next generation of Li-ion, flow-, and Li-air batteries with significantly improved performance.

We propose to apply our unique capabilities in high-field NMR, including the application of a range of low to moderate field NMR combined with computational modeling to study the ion transport mechanisms and the chemical status of the ions inside the electrolytes, electrode and at the interface of electrolyte-electrode on existing and emerging battery materials. For FY 2010, our focus is on carrying out a comprehensive ${ }^{1} \mathrm{H},{ }^{17} \mathrm{O},{ }^{19} \mathrm{~F}$, and ${ }^{51} \mathrm{~V}$ NMR investigation on the redox chemistry associated with all vanadium redox flow battery to support DOE's energy storage mission in the field of stationary battery. The methods and technologies established in this project are applicable to the study of a wide range of other energy storage materials, including other batteries such as proton batteries, supper capacitors, hydrogen storage materials, and proton membranes.

During FY 2009, we carried out NMR spectroscopy, $\mathrm{x}$-ray diffraction, transmission electron microscopy, and density functional theory studies. These studies reveal that the host rutile nanorod holds $\mathrm{Li}$ insertion only up to $0.12 \mathrm{Li} / \mathrm{TiO}_{2}(x=0.12)$ without significant changes in either nanorod morphology or structure. Also using high-field ${ }^{6} \mathrm{Li}$ NMR, we detected a new tetrahedral Li occupation detected, with the three crystallographic octahedral sites in the monoclinic $\mathrm{Li}_{2} \mathrm{TiO}_{3}$ corroborated by molecular dynamic simulations.

For FY 2010, we engaged in two significant tasks, which are described in detail below.

Understanding the poor thermodynamic stability of $\mathrm{V}^{5+}$ electrolyte solution in vanadium redox flow batteries. The $\mathrm{V}^{5+}$ electrolyte solution is studied by variable temperature ${ }^{17} \mathrm{O}$ and ${ }^{51} \mathrm{~V}$ NMR spectroscopy. The vanadium specitation and respective structure of $\mathrm{V}^{5+}$ species in the electrolyte solutions are explored to explain the poor thermal stability of the solution. It was found that $\mathrm{V}^{5+}$ species exist as hydrated penta co-ordinated vanadate ion $\left(\mathrm{VO}_{2}\left(\mathrm{H}_{2} \mathrm{O}\right)_{3}{ }^{1+} \times \mathrm{H}_{2} \mathrm{O}\right)$. This hydrated structure is not stable at elevated temperature and change in to neutral $\mathrm{H}_{3} \mathrm{VO}_{4}$ molecule by deprotonation process. This structural transition from hydrated vanadium cation structure to neutral $\mathrm{H}_{3} \mathrm{VO}_{4}$ molecule is verified by molecular modeling and NMR chemical shift measurements.

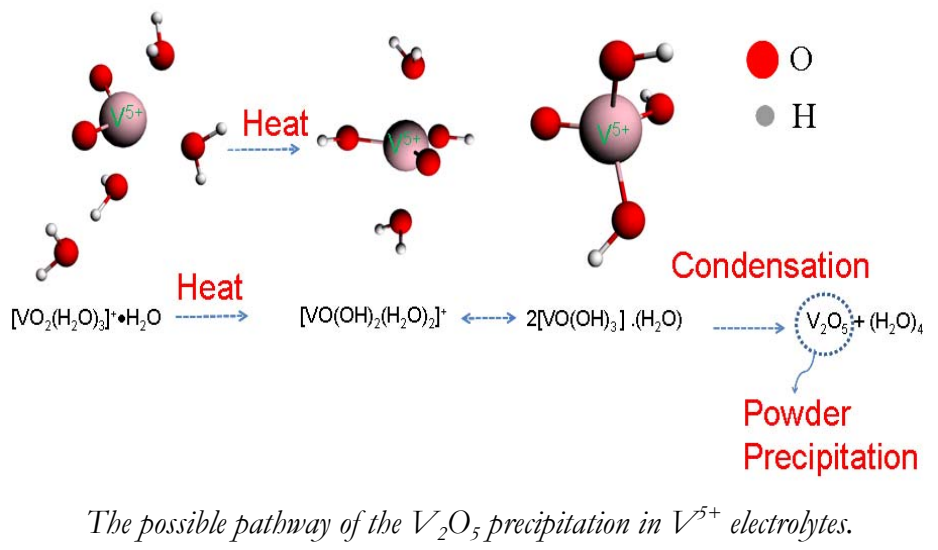

The possible pathway is identified for the $\mathrm{V}_{2} \mathrm{O}_{5}$ precipitation from different vanadium species in $\mathrm{V}^{5+}$ electrolyte solutions.

Spectroscopic Investigations of the Fouling Process on Nafion Membranes in Vanadium Redox Flow Batteries. The Nafion membrane is a critical component in redox flow batteries that are widely investigated for grid scale energy storage. However, there has been limited understanding of the fundamental properties of the membrane materials in the working environment relevant to redox flow batteries. In this work, the Nafion-117 membrane used in a vanadium redox flow battery (VRFB) is analyzed by $\mathrm{x}$-ray photoelectron spectroscopy (XPS), NMR spectroscopy, and UV/Vis spectroscopy. The XPS study reveals the chemical identity 
and environment of vanadium cations accumulated at the surface. On the other hand, the ${ }^{17} \mathrm{O}$ NMR measurement explores the nature of the diffused vanadium cations inside the bulk part of the Nafion and shows the chemical bonding of cations and the host membrane. The ${ }^{19} \mathrm{~F}$ NMR shows the basic Nafion structure is not altered due to the presence of the vanadium cations diffused inside. Based on these spectroscopic studies, the chemical identity and environment of the diffused vanadium cation in the
Nafion membrane is identified. This study reveals important information on the origin of performance degradation of the membrane materials in vanadium redox flow batteries and provides clues on how to improve the chemistry and properties of the energy storage devices.

Our investigations for this project have resulted in two peer-reviewed journal publications and the submission of two new manuscripts, with a third scheduled for prior to the end of FY 2010. 


\title{
Advanced Sorptive and Signature Indicating Materials for Ultra-Trace Proliferation Detection
}

\author{
Jay W. Grate, R. Shane Addleman, Keith D. Miller, Praveen K. Thallapally, Jon M. Schwantes,
} Richard M. Ozanich, Marvin G. Warner

The collecting, enhancing, and detecting signatures of undeclared nuclear activity from extremely dilute environmental samples taken at a distance from nuclear sites are essential technical capabilities addressing the risk of nuclear proliferation.

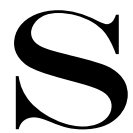
ignatures for nuclear proliferation can occur in the gas phase as gaseous molecules or aerosol particles, in the liquid phase as dissolved ions, or as residues or components of solid samples. This project will develop three different but complementary technologies for concentrating and detecting signatures of activities that could be associated with nuclear weapons proliferation. Under the first task, we target noble gases produced during fission, selecting and synthesizing appropriate metal organic framework (MOF) candidates and characterizing their physical properties that are relevant to noble gas sorption, collection, and separation. The second task is about enhancing signatures of a wide variety of dissolved or suspended nuclear materials, fission products, and related process chemicals in water or other liquids. The third task involves enriched or depleted uranium species generated in the front end of the nuclear fuel cycle to enable the design of uranyl ion binding TCC-cpGFP proteins for engineering sensors capable of uranium detection for national security applications. These tasks and their specific project progress are described below.

Metal Organic Frameworks for Noble Gas Sorption. With regard to the gas phase, the collection of noble gases is an important aspect of monitoring and detection of undeclared nuclear activity. Sorptive materials play a key role in this collection. This project addresses a new type of sorbent, MOFs, with superlative properties compared with current carbons and zeolites for gas capture and separations. We are developing and investigating MOF materials for gas collection and separation of noble gases such as xenon that are produced during nuclear fission in reactors. The development of these materials will lead to more effective collection of noble gases from greater distances in the face of rising backgrounds.

\section{Magnetically Active Sorbents for Proliferation Signature} Collection and Detection. Capture of low concentration signatures from complex matrices and/or large volume samples is challenging. Magnetic nanoparticles offer a novel means to capture and pre-concentrate trace level signatures of interest selectively including radionuclides, heavy metals, and organic compounds. By functionalizing these high-surface area nanoparticles with a variety of proven chemosorbents, specific elements of interest may be targeted and preferentially extracted away from complex matrices at a capacity and selectivity greater than commercial sorbents. Once sorbed, the magnetic character of the nanoparticles allows them to be magnetically manipulated. This provides the ability to trap the functionalized nanoparticles magnetically in one geometry for a sample collection to release and retrap in a specific geometry for signature detection using a variety of measurement platforms.

Protein Based Uranium Detection. This project will significantly enhance the capability for stand-off detection of upstream uranium processing using novel protein-based fluorescent sensors that bind to uranium species. These proteins were designed to fluoresce only in the presence of labile uranyl species and are being functionally tested for high affinity/specificity to demonstrate effective and enhanced fluorescence detection. Design of the sensor material was based on metal binding trimeric coiled coil peptides fused to circularly permuted variants of fluorescent proteins. Our research used the green fluorescent protein (GFP) because its structural and fluorescent properties have been well characterized. However, the results can also be extended to the design of other novel metal binding fluorescent proteins.

In FY 2009 with a post mid-year start, we made significant progress with the first task in synthesizing and characterizing MOF compounds that were originally identified as good candidates for noble gas sorption studies. These include MOF-5, where we prepared and scaled up the synthesis to gram scale and confirmed the structure; $\mathrm{Cu}-\mathrm{MOF}$, where on a small scale, we synthesized this material successfullly and characterized by x-ray diffraction, leaving the scale-up synthesis to begin; lanthanide MOFs, with initial work showing lanthanide-containing MOFs to be more challenging to obtain the proper porous structure; therefore, our work with this material is ongoing. We strategized on methods to prepare MOFs containing nanoparticles that may influence gas sorption.

For the second task, we explored the means to make and manipulate magnetically active sorbents, including two sizes of synthesized magnetic particles, functionalized the nanoparticles with glutathione and acid capture ligands, developed initial flow-through nanoparticle capture columns and capture/release protocols, and compared capture/release of in-house synthesized and commercially available nanoparticles. 
For the third task, all task milestones were met. The majority of time was spent designing codon optimized genes for expression of cpGFP-TCC variants displayed on the surface of yeast, which will be tested for their ability to bind uranium ions. In addition, all of the relevant cpGFP-TCC controls were designed. These constructs are being constructed using a polymerase chain reaction based gene synthesis approach, which will then be incorporated into the yeast surface display vector.

In FY 2010, we investigated a number of MOF candidates and found one that had twice the uptake of xenon compared to MOF-5, the first MOF we investigated for noble gas capture. We characterized the physical properties of the MOF materials to verify structure, in addition to determining gas uptake properties relevant to noble gas collection. We also gained a better understanding of how to make/select, modify, and manipulate magnetic nanoparticles for trace level analyte capture from large volumes. Also, we demonstrated novel functionalization methods that have resulted in unprecedented affinity and selectivity toward radionuclides of interest. Additionally, we designed and constructed novel protein structures for fluorescent sensing of uranium. A high throughput method was used to characterize and select high affinity GFP fusion structures with the desired fluorescence sensing properties.

A highlight in FY 2010 was the publication of part of our research from the project's second task in the journal
ChemSusChem (June 2010). The team describes the synthesis of magnetic nanoparticles and the installation of a variety of heavy-metal chelating ligands onto their surface. A simple, one-step ligand exchange technique allows the incorporation of a ligand with specific affinity towards the toxic analyte of interest. These materials are promising functional heavy sorbents for a diverse set of remediation applications due to their high binding affinity toward a given analyte and their ability to be easily separated from a sample system through the application of a magnetic field.

For FY 2011, we have several goals to reach, including the following:

- Synthesize rationally designed MOF compounds for noble gas adsorption based on the evaluation of results obtained in the first year and a half of the project, and complete characterization and determination of gas adsorption isotherms at several temperatures on these compounds.

- Demonstrate isotoptically labeled sorbing nanoparticles that will function as self-referencing internal standards as well as demonstrate a high level of preconcentration of radiological signature for an aquatic matrix.

- Characterize flow cytometrics of the GFP and its multiple variants and determine if the metal variants can bind uranyl ions. 


\title{
Application of Imperfection Modeling to Accelerated Fuel Clad Qualification and Characterization
}

\author{
Curt A. Lavender, Elizabeth V. Stephens, Stan G. Pitman, Richard W. Davies
}

The novel imperfection modeling approach will shorten qualification time by providing a quantifiable measure of performance of a new clad material rather than typical pass/fail outcomes based on acceptance limits. The technique uses a speckle pattern digital image to measure strain and describe quantitatively the sample quality, which may be used to predict future clad performance without costly, time-consuming in-reactor testing or extensive ex-reactor parametric studies.

$\mathbf{I}$ n-reactor fuel clad performance is sensitive to the numerous quantity and type of manufacturing defects encountered during tube fabrication and end-plug welding. This sensitivity can be dependent on the clad base material and processing method that affects microstructure, texture, surface quality, mechanical properties, and defect population. The challenges of selecting a new material or joining method for cladding are similar to those encountered in most engineering applications. To address this issue, PNNL developed a method for rapid material and process selection. The focus was on rapid qualification of advanced high strength steels and new joining methods for aluminum alloys. Through this process, new steel alloys and aluminum joining methods were implemented into high volume auto manufacturing with predictable performance without the normal 10-year development cycle. The purpose of this project is to apply related techniques to sample geometries and materials of specific interest to the nuclear industry.

This research has two advantages over conventional nuclear fuel manufacturer test methods. First, we used digital image correlation to quantify in real time the strain that develops during internal pressurization, tube expansion, and subsequent localization and fracture of fuel cladding. Second, by using this approach and test samples, a quantitative statistical distribution of fuel clad performance during internal pressurization and subsequent burst will be derived. The advantage of statistical distribution is a probabilistic understanding of material performance under internal pressure conditions that can be used to predict the overall performance of a fuel cladding population, which should yield a more detailed prediction of long-term performance of a given fuel clad material.

In FY 2008, the tube burst and digital imaging system were completed, and tubes with three heat treatments and a variant with ultrasonic test indications were evaluated. The test method was found to be indicative of tubing quality and produced data of interest to the Nuclear Regulatory
Commission and tubing users and manufacturers. Several key outcomes occurred during this first year: 1) uniform strain was validated as a better method to evaluate tubing quality, 2) flawed tubes demonstrated a higher imperfection level, and 3) irrespective of heat treatment, the method predicted the same level of manufactured tubing quality.

During FYs 2009 and 2010, research expanded by investigating $\mathrm{Zr}-4$ end joining methods and applying the modeling approach to tubes with weldments. In addition, oxide dispersion strengthened alloy clad fabrication was initiated, pre-cursory investigations of hydride tubes were performed, and the imperfection modeling development continued. Tube burst testing and digital imaging spectroscopy were used to determine optimum processing parameters for TIG welded end plugs to produce a quality weldment population. Electron beam tube weldments were also evaluated using previously developed processing parameters for nuclear cladding evaluations. The burst test results and uniform strain of the two weldment populations were compared with the results of acceptable $865^{\circ} \mathrm{F}$ annealed $\mathrm{Zr}-4$ tubes.

We observed that the burst test does not discriminate between tubes or the optical strain analysis. For example, similar burst pressures, well above the specification minimum of 11,000 psi, were observed among annealed tubes and tubes with electron beam weldments. However, different uniform strains were observed, showing that the uniform strain is a better indicator of tube variability than burst pressure alone. In addition, we saw that the Weibull analysis demonstrates that fuel cladding material can be treated probabilistically. Although similar burst pressures were observed between the tubes with weldments and acceptable annealed tubes, different slopes were observed in the Weibull probability plot, indicating different probabilistic behaviors. This difference is more pronounced when extending the tail end of Weibull probability plots.

Results and observations from this work indicate that imperfection modeling can be used to predict fuel clad behavior in a probabilistic manner. However, a more robust mechanics of materials analysis and further development of the imperfection modeling approach are needed. Further development of this approach, if successful, may be the basis for a performance-based standard that can have significant influence on the characterization and qualification of cladding materials by providing a better understanding and predicting of the cladding performance and integrity. 


\title{
Biotemplated Synthesis of Encoded Bimetallic Nanoparticles
}

\author{
Yuehe Lin, Yongsoon Shin, Jun Wang, Zhaobui Li
}

This project developed novel approaches to synthesize bi- and trimetallic nanoparticles with controlled architectures inside protein cavities, prepare protein cage-based nanomedicine platforms for drug delivery, and investigate biofunctionalized graphene for biosensing and imaging.

1 lthough protein cages are widely investigated for synthesizing inorganic and metallic nanoparticles, bimetallic nanoparticles with controlled composition and uniform sizes remain a significant challenge. In this project, we focused mainly on bimetallic and trimetallic nanoparticle formation, nanomedicine platform fabrication, and graphene nanosheets investigation for biosensing and bioimaging. These nanomaterial-based platforms can be developed for detecting pathogens or toxins from biological fluids in vitro or in vivo as well as for target therapy. This work will build synthesis capabilities and materials characterization approaches to address mission relevant issues to homeland security.

In FY 2008, we prepared Au-Ag alloys using native apoferritin as a model consisting of a spherical protein shell surrounding an aqueous cavity of about $8 \mathrm{~nm}$. Channels were generated by the multi-subunit construction of the shell. Eight hydrophilic channels of about $4 \AA$ were thought to facilitate passage of metal ions and small molecules of appropriate size into the protein cavity. For FY 2009, we successfully prepared $\mathrm{Au}-\mathrm{Ag}$ and $\mathrm{Cd}_{1-\mathrm{x}} \mathrm{Zn}_{\mathrm{x}} \mathrm{S}$ alloys using an apoferritin cavity. Simultaneously, we explored graphene, a new nanomaterial as a novel nanoscalfold, for biosensing and imaging. FY 2010 highlights are detailed below.

Tempalte Synthesis of Trimetallic Alloy. During FY 2010, we successfully synthesized metal alloys in the apoferritin cavities using ammonia water. Most of metal ions are stable in 5.0-10.0 $\mathrm{mM} \mathrm{NH}_{4} \mathrm{OH}(\mathrm{pH} 8.0-8.5)$ from forming metal amine complexes. Stable metal ion complexes can diffuse into an apoferritin cavity by a diffusion route through $4 \AA$ channels between subunits at $\mathrm{pH}$ 8.0-9.0. An equilibrated sulfide precursor can form metal sulfides in and out of the apoferritin cavity, and bulk metal sulfide in solution can be easily separated by centrifugation. The core formation ratios for all samples are over 80 percent. TEM images showed homogeneous particle size distributions with mean diameters of 5.60-6.20 nm, slightly smaller than an apoferritin cavity $(8.0 \mathrm{~nm})$. X-ray energy dispersive spectroscopy (EDS) was used to analyze the ratio of the alloy products: $\mathrm{CuS}, \mathrm{NiS}$, and $\mathrm{CoS}$ showed band gap energies of 4.42, 4.60, and $3.20 \mathrm{eV}$ in the apoferritin cavities, while (Cu:Ni:Co)S showed $3.35 \mathrm{eV}$, meaning that alloy sulfides were near the lowest limit of value. We proved this phenomenon in alloy sulfides with different molar ratios: (Cd:Zn:Co)S. CdS, $\mathrm{ZnS}$, and $\mathrm{CoS}$ showed band gap energies of 3.38, 3.92, and $3.20 \mathrm{eV}$. However, (Cd:Zn:Co)S showed 3.14-3.30 eV regardless of the composition. We also synthesized alloy sulfides with $\mathrm{Pb}$. In our experiment, $\mathrm{Pb}(\mathrm{II})$ ion was successfully infiltrated into an apoferritin cavity and formed alloy sulfide with $\mathrm{Cd}$ and $\mathrm{Co}$. The quantitative analysis of $\mathrm{Pb}, \mathrm{Cd}$, and $\mathrm{Co}$ was examined by stripping voltammetry.

Constraint of DNA on Functionalized Graphene to Improve its Biostability and Specificity. We found that single-strand DNA adsorbed on graphene surface is effectively protected from enzymatic cleavage by DNase I, an encouraging discovery for biomedical applications involving complex cellular and biofluid samples. Further, constraining a single-stranded DNA probe on graphene improves specificity of response to a target sequence. The unique features of DNA-graphene interactions are promising traits that may be exploited to construct nanobiosensors with facile design, excellent sensitivity, selectivity, and biostability. Considering the low, large-scale cost of producing grapheneona, these findings will promote graphene use in basic research and practical applications.

Aptamer/Graphene Oxide Nanocomplex for In Situ Molecular Probing in Living Cells. We report the illustration of cellular delivering and in situ molecular probing in living cells by using graphene oxide nanosheets (GO-nS) as DNA cargo and sensing platform. With particular interaction between GO and DNA molecules, aptamer/GO-nS nanocomplex was designed and employed to demonstrate the dramatic DNA delivering enzymatic cleavage, protecting and biosensing capabilities of GO-nS in living cells. Results showed that GO-nS could successfully transfer DNA aptamer into living cells, efficiently protect oligonucleotides from enzymatic cleavage during the delivery, and selectively monitor intracellular target in situ. The advantages of graphene oxide will encourage many biological fields such as DNA and protein analyzing, gene and drug delivering, intracellular tracking, and in vivo monitoring.

Sensitive Immunosensor for Cancer Biomarker Based on Signal Amplification Strategy of Graphene Sheets. A novel electrochemical immunosensor for sensitive detection of cancer biomarker $\alpha$-fetoprotein (AFP) uses a graphene sheet sensor platform and functionalized carbon nanospheres labeled with horseradish peroxidase-secondary antibodies. The proposed method could respond to $0.02 \mathrm{ng} \mathrm{mL}-1$ AFP with a linear calibration range from 0.05 to $6 \mathrm{ng} \mathrm{mL}-1$. This amplification strategy is a promising platform for clinical screening of cancer biomarkers and point-of-care diagnostics. 


\section{Development and Understanding of Nanostructured Materials for Advanced Energy Storage}

Jiguang (Jason) Zhang, Daiwon Choi, Wu Xu, Jie Xiao, Xiaolin (Shari) Li, Deyu (David) Wang, Anqiang Pan, Jianguo Yu

Fundamental improvements to electrochemical storage performance are essential for emerging applications, particularly in the transportation and electricity production sectors.

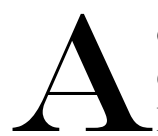
dvanced batteries, particularly the high power/ energy lithium (Li) ion, have been identified by DOE as critical enabling technologies for the development of advanced, fuel-efficient vehicles, including plug-in hybrid (PHEV) and electric(EV). The applications remain limited by several barriers, including reliability, longevity, safety, and cost concerns. Higher energy and power than current Li ion are highly preferred and, as with PHEVs, required for the applications. Given that the performance of electrochemical storage devices intimately depends on the properties of their electrode and electrolyte materials, the emerging applications require transformational materials that can demonstrate a much improved charge transport and electrode kinetics and a satisfied structural and interfacial stability as well as enable high voltage and multi-electrons per redox center for high energy density while being cost-effective.

One group that potentially satisfies the stringent requirement is nanomaterials. However, there has been lack of fundamental knowledge about the role of nanostructure materials in energy storage and scientific understanding on the processing of nanostructures, both concepts of which would help design and develop optimized nanostructures for electrochemical energy storage applications. Accordingly, our project focuses on: 1) a mechanistic understanding of the effects of nanostructures or architectures on electron/ion transport, electrode reactions, and charge storage at the nanoscale and 2) the development of transformational electrochemically active materials via synthetic approaches that enable cost-effective, scale-up production of nanostructures with desirable features for energy storage applications.

In FY 2008, our research focused on understanding the charge transport in nanostructured materials and the initial synthesis and assembly of anode, cathode, and electrolyte/ cathode interface nanomaterials for $\mathrm{Li}$ ion batteries. Along with selective structural and chemical analyses via advanced diagnostic tools (including nuclear magnetic resonance and transmission electron microscopy), electrochemical studies were performed to understand fundamental charge transport in the electrochemically active materials and the effect of processing/nanostructure on charge transport and separation. We also initiated the computer modeling effort to understand and predict the nanosize effects on electron/Li ion transport in the synthesized nanostructures. In FY 2009, silicon-based anode was investigated via electrochemical properties of carbon-coated nanoporous silicon powder, the morphology change of original silicon and carbon-coated silicon before and after electrochemical cycling, and the preparation of high capacity silicon-based anode in the gas-induced three dimensional growth of silicon nanowires.

In FY 2010, the electrochemical active cathode material $\mathrm{LiMnPO}_{4}$ was prepared via a single-step solid-state reaction in molten hydrocarbon. This substance exhibits a unique porous nanoplate shape of approximately $50 \mathrm{~nm}$ in thickness with a highly preferred crystallographic orientation. Oleic acid was used as a surfactant and paraffin acted as a non-polar solvent that facilitated thermodynamically preferred crystal growth without agglomeration. After the optimization of carbon coating through ball milling process, carbon coated $\mathrm{LiMnPO}_{4}$ demonstrated a flat potential at $4.1 \mathrm{~V}$ with specific capacity of up to $168 \mathrm{mAh} / \mathrm{g}$ and good rate capability.

Iron-doped $\mathrm{LiMnPO}_{4}\left(\mathrm{LiMn}_{1-\mathrm{x}} \mathrm{Fe}_{\mathrm{x}} \mathrm{PO}_{4}\right.$ where $\left.\mathrm{x}=0-0.2\right)$ was also synthesized and showed excellent high capacity. The first principle calculations using density function theory (DFT) indicated that de-lithiated $\mathrm{LiMnPO}_{4}$ (the pure phase of $\mathrm{LiMn}_{1-\mathrm{x}} \mathrm{Fe}_{\mathrm{x}} \mathrm{PO}_{4}$ where $\mathrm{x}=0$ ) may not be stable at charged conditions, but the de-lithiated 20 percent iron-doped $\mathrm{LiMn}_{1-\mathrm{x}} \mathrm{Fe}_{\mathrm{x}} \mathrm{PO}_{4}\left(\mathrm{LiMn}_{0.8} \mathrm{Fe}_{0.2} \mathrm{PO}_{4}\right.$ where $\left.\mathrm{x}=0.2\right)$ was stable at elevated temperatures. The DFT calculations found that $\mathrm{MnPO}_{4}$ was stable up to $450 \mathrm{~K}$ which is consistent with experimental value of between 423 and $473 \mathrm{~K}$ reported in the literatures, and $\mathrm{Mn}_{0.8} \mathrm{Fe}_{0.2} \mathrm{PO}_{4}$ could be thermally stable up to about $650 \mathrm{~K}\left(377^{\circ} \mathrm{C}\right)$. It is suggested that $\mathrm{LiMn}_{0.8} \mathrm{Fe}_{0.2} \mathrm{PO}_{4}$ should be a safe cathode material comparing to most other cathode materials.

Nanostructured $\mathrm{Li}_{3} \mathrm{~V}_{2}\left(\mathrm{PO}_{4}\right)_{3} /$ carbon $\left[\mathrm{Li}_{3} \mathrm{~V}_{2}\left(\mathrm{PO}_{4}\right)_{3} / \mathrm{C}\right]$ cathode composite was successfully prepared by incorporating precursor solution into expanded pore structure of highly mesoporous carbon. The particles sizes of the samples were centered at about $20 \mathrm{~nm}$ and well dispersed in the carbon matrix. When cycled in a voltage range of 3-4.3 V, this composite delivered a reversible capacity of $122 \mathrm{mAh} \mathrm{g}^{-1}$ at $1 \mathrm{C}$-rate and maintained a specific discharge capacity of $83 \mathrm{mAh} \mathrm{g}^{-1}$ even at $32 \mathrm{C}$-rate. Therefore, nanostructured $\mathrm{Li}_{3} \mathrm{~V}_{2}\left(\mathrm{PO}_{4}\right)_{3} / \mathrm{C}$ composite has a great potential to be used as cathode material for high power $\mathrm{Li}$ ion batteries. We also 
studied organic cathode materials based on polyacene quinine radical polymers. High reversible capacity of above $200 \mathrm{mAh} / \mathrm{g}$ was obtained. The electrolyte and binder showed significant effects on the performance of the organic cathode materials. The cathode demonstrated increased initial capacity, reduced polarization and improved cyclability in ether-based electrolyte than in carbonate-based electrolyte.

For nanostructured anode materials, we developed a unique approach to prepare silicon $(\mathrm{Si})$ /graphene superstructured nanocomposite anode using self-assembly method.

Micron-sized Si particles with nano-pore structures were first coated with a thin layer of carbon by chemical vapor deposition and then mixed with graphene to form $\mathrm{Si} / \mathrm{C} /$ graphene nanocomposite anode. Such $\mathrm{Si}$ anode showed a reversible capacity of more than $2300 \mathrm{mAh} / \mathrm{g}$ after 35 cycles. Threedimensional macroporous copper sheets as anode current collector and three-dimensional macroporous tin oxide $\left(\mathrm{SnO}_{2}\right) / \mathrm{Cu}$ composite as anode were also investigated in FY 2010. Significantly improved battery performances were obtained compared to using conventional smooth copper sheet and $\mathrm{SnO}_{2} / \mathrm{C}$ composites.

In FY 2010, we had multiple accomplishments for the conclusion of our research. Six scientific papers based on this project have been published in peer-viewed journals. 


\title{
Energy Absorptive Foams: Space-Age Polymers for Down-to-Earth Applications
}

\author{
Aashish Rohatgi, Brad J. Busche, R. Shane Addleman
}

This project will develop a method to fabricate high-performance, light-weight polymer foams for crash-energy absorption resulting in safer and lighter cars and trucks. It is envisioned that the foam developed will also be suitable as a core material in sandwich composite structures for transportation applications.

$\mathbf{F}$ oams are lightweight materials with good energy absorption, thermal, and acoustic insulation properties, making them attractive candidates in the automotive and transportation industry for vehicle weight reduction, improved crash-safety and a quieter, smoother ride. However, in practice, foams (polymer-based) in vehicles are primarily used for cushioning and acoustic insulation rather than for light weighting or crash safety. This limited use of (polymeric) foams in the auto industry is due to their inherent low mechanical properties. Unlike polymeric foams, metallic foams possess the mechanical performance suitable for light weighting or crash safety. However, metallic foams have not penetrated the automotive market significantly due to processing issues: they are fabricated at close to melting point (approximately $660^{\circ} \mathrm{C}$ for aluminum) and in sheet form, making them unsuitable to fill in complex contours of hollow automotive structural members such as the A-pillar. Thus, owing to material and processing issues, the full potential of foams for safer and lighter vehicles remains untapped.

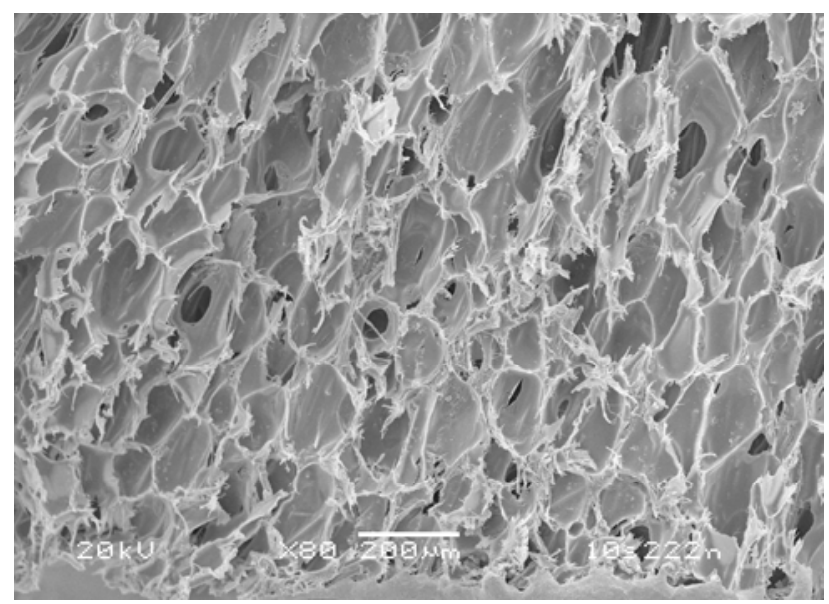

A scanning electron micrograph showing the transverse cross-section of a cylindrical sample of the polymer foam; the axis of the cylinder is along the vertical direction.

The objective of this project is to develop a high performance foam that combines the performance of metallic foams with the ease of processing of polymeric foams. This will enable foams to be used for crash-energy absorption and for structural support, thus helping the auto industry in producing safer and lighter vehicles. At the end of this project, it is anticipated that we would have identified a suitable foamable material and develop a foaming technique such that the resulting foam possesses high strength and high stiffness akin metal foams but is processed at lower temperatures and is moldable into hollow structural members.

In order to meet our project objectives, a literature search was performed initially to identify polymers demonstrating high mechanical performance and to determine foaming techniques suitable for such polymers. A bench-top experimental capability to foam polymers was developed, and process parameters to fabricate the foams were determined. The mechanical performance of the foams was determined and compared to existing metallic foams. Finally, compatibility of the foams with the auto industry's "paint-bake" (approximately $175^{\circ} \mathrm{C}$ for $20 \mathrm{~min}$ ) heat treatment was evaluated.

A new experimental capability to fabricate foams was developed. The liquid crystalline polymer Vectra was chosen as the candidate foam materials due to its potential for high strength (10 times stronger than aluminum on a weight basis) and demonstrated robustness (Vectra-fiber airbags were used by NASA to cushion the Mars landing of Pathfinder and Rover spacecrafts). Cylindrical foam samples (up to 1 in in diameter and height) of Vectra were fabricated at about $300^{\circ} \mathrm{C}$. Microstructural characterization of the foam showed the cells to be a semi-closed and approximately 100 to $200 \mu \mathrm{m}$ in size. The best performing samples possessed a density in the range $0.4-0.6 \mathrm{~g} / \mathrm{cc}$, compressive strength about 10 to $20 \mathrm{MPa}$, Young's modulus about 150 to $250 \mathrm{MPa}$, and densification strain around 50 percent. These mechanical properties resulted in quasi-static energy absorption values between 10 and $20 \mathrm{~J} / \mathrm{g}$. A preliminary finite element model using the actual foam microstructure (obtained from x-ray computed micro-tomography) was also developed to describe the plastic deformation behavior of the foams.

The key project accomplishment was that the polymer foam was fabricated and possessed similar mechanical energy absorption capability as aluminum foams. Further, the foam did not show any loss in mechanical performance after having been twice subjected to a simulated paint-bake heat treatment. Thus, the polymer foam developed in this work is an excellent candidate for implementation in the auto industry for crash energy absorption and structural support. 


\title{
Friction Stir Welding of Creep-Resistant Oxide Dispersion Strengthened Alloys
}

\author{
K. Scott Weil, Glenn J. Grant, Yuri Hovanski, Jens T. Darsell, Curt A. Lavender
}

We are developing a cost-effective method of joining high performance oxide dispersion strengthened steels and demonstrating their high-temperature strength and both creep and corrosion resistance. The work will help to build PNNL's capabilities in high-temperature materials development.

B ecause of their low cost, excellent thermal properties, and suitable corrosion and radiation resistances, ferritic/martensitic steels are key materials in next-generation nuclear plant designs and primary candidates for advanced fast reactor cladding/duct materials. Recently, there has been a push within the nuclear plant design community for higher operating temperatures to improve plant efficiencies. Unfortunately, the utilization of most ferritic/martensitic steels is limited to about $600^{\circ} \mathrm{C}$ due to a significant drop off in tensile and creep strength. It is well known that the addition of insoluble, nanoscale oxide dispersoids to these alloys greatly improves their high temperature mechanical properties and resistance to radiation induced damage mechanisms. These oxide dispersion strengthened (ODS) alloys are important materials for future reactors, but barriers need to be overcome before widespread use is possible. Two critical issues are implementing the technologies into final assemblies and the cost of ODS alloys. Joining or welding of ODS alloy components is especially problematic, as liquid phase methods such as brazing and fusion welding lead to regions that are devoid of dispersoids and the associated strengthening effects, making the joined components susceptible to failure by creep (even though the base ODS alloy is not).

This project addresses the barriers to implementing ODS alloys in two ways. We are evaluating the efficacy of a solid-state joining approach known as friction stir welding (FSW). Because FSW does not involve the formation of a fusion zone, it has the potential to produce joints that will maintain the creep and radiation swelling resistant properties of the original base ODS material. To address the current high cost of producing the ODS alloy, this project is investigating a novel, single-step method of consolidating ODS alloy powder directly into semi-finished product form (such as tubing, rod, and plate).

Friction stir welding. In FSW, two metal work pieces are placed end-to-end, and a spinning tool is brought into contact along the common join-line, simultaneously heating both pieces through a combination of friction and plastic work energy dissipation. As the materials are heated, their flow stresses drop, and the shearing action of the plunging tool causes plastic deformation to take place. As a result, material from each work piece flows into the other, leading to the formation of a solid-state, metallurgical bond across the original join-line. For ODS alloys, our initial goal was to determine whether a sufficient concentration of dispersoids could be maintained across the weld region to ensure that the joined part would exhibit creep strength essentially equivalent to that of the original pre-joined material.

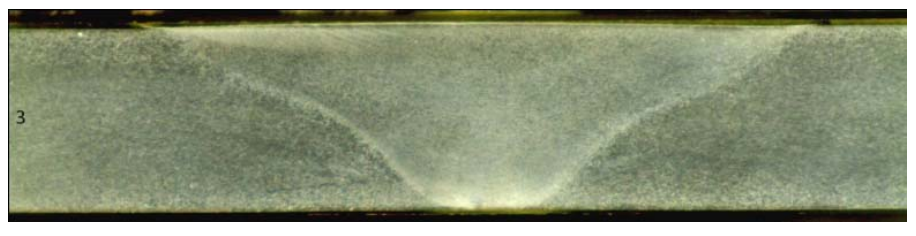

Fully consolidated and defect-free FSWs are possible in this ferritic ODS alloy.

In FYs 2009 and FY 2010, we conducted a series of parametric welding studies on Kanthal APMT, a commercially available dispersion strengthened high chromium containing ferritic stainless steel. An envelope of successful welding conditions (e.g., linear speed, rotational tool speed, rake angle, etc.) was identified and room temperature mechanical properties were measured. Microstructural observations indicate that successful defect free welds can be made in this material. Welds developed in this program are on 0.25 -inch thick material and are the thickest demonstrations of FSW in ferritic ODS materials to date in the open literature. Mechanical testing showed that the weldments displayed roughly the same yield and ultimate strengths as the base material at room temperature. Elevated temperature creep testing is underway,

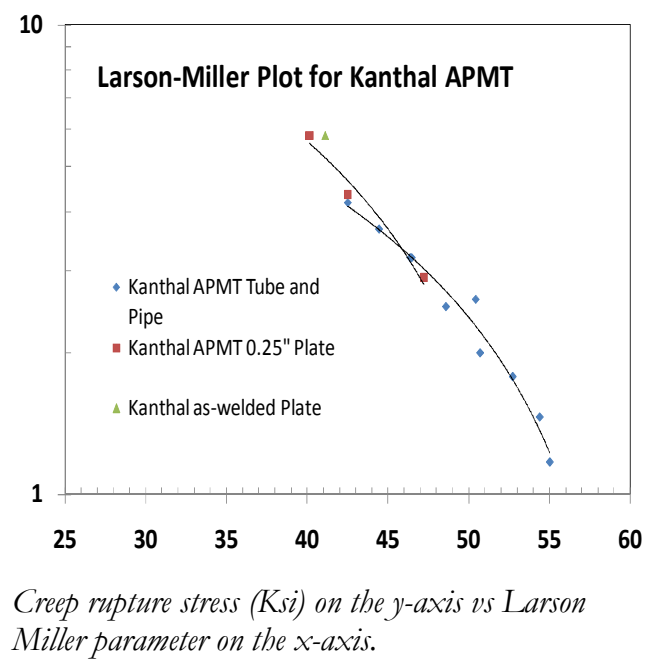


but preliminary testing indicates that FSW weld metal is displaying Larson Miller parameters (a measure of creep performance) that are the same as those of the base metal.

Reducing the cost of ODS materials. As discussed, one means of increasing creep resistance in ferritic alloys is to incorporate a dispersion of fine-scale oxide particles (such as $\mathrm{Y}_{2} \mathrm{O}_{3}$ ). Currently, this is done by mechanically mixing a small amount of the oxide powder (typically less than $1 \mathrm{vol} \%$ ) with a ferritic steel powder in a high energy attrition mill. Batch sizes typically are less than $1 \mathrm{~kg}$, and milling times are generally between 80 and 120 hours to achieve uniform oxide dispersion. Once the final mechanically alloyed powder is formed, it is poured into a steel can that is subsequently welded shut (after evacuating the vessel of any intrinsic atmosphere, such as air). The sealed can is then hot isostatically pressed (HIPed) at temperatures ranging from $1200-1750^{\circ} \mathrm{F}$ and $10-30 \mathrm{ksi}$. After HIPing, the densified material undergoes a series of rolling and annealing steps, followed by mechanical removal of the outer can layer, and subsequent pickling to form a final ODS sheet product. Thus, even though the raw powders for an ODS alloy cost between
\$1-\$2 per pound, the final wrought semi-finished product ends up costing on the order of $\$ 200-\$ 500 / \mathrm{lb}$, often making ODS material too expensive for commercial application. In addition, due to the methods of sheet or tube rolling, these materials tend to display very anisotropic properties and often exhibit low hoop creep strength and poor pressure retention capability at high temperature. Rolling also often leads to the formation of larger scale oxide stringers that can deleteriously impact the mechanical properties of the final product form.

In FY 2011 we will continue investigating direct powderto-product methods of ODS fabrication that will: 1) eliminate many of the steps currently employed in producing these materials, 2) generate material on a continuous (as opposed to batch) basis, and 3) avoid property anisotropy and oxide stringering. We are working with a university collaborator to initially demonstrate direct formation of consolidated ODS buttons measuring approximately 1 inch in diameter by a 0.5-inch height from MA 956 powder. Once demonstrated, we will begin fabricating 0.25 -inch rod and tubing product from both mechanically alloyed powder and mixed stainless steel and yttria powders using the same consolidation process. 


\title{
In Situ Nuclear Magnetic Resonance Investigations of Trapping Mechanisms in $\mathrm{CO}_{2}$ Storage
}

\author{
David W. Hoyt, Jianz̧bi Hu, Romulus V. Turcu, Ja Hun Kwak, Jesse A. Sears,
} Kevin M. Rosso, Andrew R. Felmy

We propose to develop unique in situ nuclear magnetic resonance (NMR) capabilities, including the application of existing developed techniques, to advance the understanding of geochemical processes associated with the precipitation and dissolution of $\mathrm{CO}_{2}$ at molecular level.

$\mathbf{F}$ ossil fuel usage such as coal, a major source of $\mathrm{CO}_{2}$ emissions to the atmosphere, will continue to provide a significant portion of total energy in both industrialized and developing countries. It is estimated that the global emissions of $\mathrm{CO}_{2}$ to the atmosphere would increase from 7.4GtC (Giga tons, or billion tons, of carbon) per year in 1997 to $26 \mathrm{GtC} / \mathrm{yr}$ by 2100 . Increased levels of greenhouse gases such as $\mathrm{CO}_{2}$ are adversely affecting the global environment, as evidenced by recent trends in global warming and dramatic changes in weather patterns. These facts have made it critical to develop technologies to stabilize $\mathrm{CO}_{2}$ in the environment. Global increases in carbon emissions and the rapid decrease in energy securities have resulted in an increased global commitment to develop technology for stabilizing $\mathrm{CO}_{2}$ released into the environment. One proposed method for reducing this greenhouse gas in the atmosphere is to store the $\mathrm{CO}_{2}$ underground. Natural reservoirs of the gas exist, suggesting that geologic carbon sequestration is feasible. However, little is known about the molecular based mechanisms that govern the storage of $\mathrm{CO}_{2}$. This requires an understanding of the geochemical processes associated with $\mathrm{CO}_{2}$ sequestration in complex and heterogeneous subsurface mineral assemblages comprising porous rock formations, and in the equally complex fluids that may reside in and flow through those formations.

We propose to develop and apply unique high-pressure magnetic angle spinning (MAS) NMR capability for in situ characterization of mineral carbonation mechanisms and kinetics at super critical $\mathrm{CO}_{2}$ pressure. This research will advance the understanding of geochemical processes associated with the precipitation and dissolution of $\mathrm{CO}_{2}$ at molecular level. Our studies will unravel the role of water activity for catalyzing mineral transformation to metal carbonates, understand water activity thresholds that enable mineral transformations, discover important chemical mechanisms involved in these transformations (dissolution, ligand-exchange, nucleation, etc.), and discover time scales controlled by mineral transformation rates or by water availability.
In particular, we will study the interfacial structure, kinetics, and mechanisms of common primary and secondary mineral phase dissolution in $\mathrm{CO}_{2}$ /water mixtures and carbonate nucleation and mineralization reactions as a function of composition, temperature, and pressure. We will study the detailed molecular interaction between the $\mathrm{CO}_{2}$ molecules and the surface moieties/functional groups of selected geological media, including mineral interfacial reactions with $\mathrm{CO}_{2}$, or mixed solvent $\mathrm{CO}_{2} /$ water solutions in porous media, and the interaction of $\mathrm{CO}_{2}$ with $\mathrm{H}_{2} \mathrm{O}$ molecules at the relevant geological temperature (from $-20^{\circ} \mathrm{C}$ to $80^{\circ} \mathrm{C}$ ) and pressure (from less than 1.0 to $80 \mathrm{atms}$ ). Our research will provide new insights into the physical/chemical phenomena that underlie the carbon sequestration at the subsurface, in particular the molecular mechanisms of mineral interfacial reactions with $\mathrm{CO}_{2}$, the effects of a varied amounts of water on this reaction, the effects of structural and chemical heterogeneity at the interfaces on the reaction kinetics, and the macroscopic dynamics of $\mathrm{CO}_{2}$ and water at relevant geological temperature and pressure.

In situ High Pressure MAS NMR Capability Development. This capability consists of a special high pressure sample cell, a high pressure sample loading chamber, and a special large sample volume MAS probe that provides low ${ }^{1} \mathrm{H}$ and ${ }^{13} \mathrm{C}$ background. After more than 10 iterations, we have successfully achieved our goal of holding $\mathrm{CO}_{2}$ pressure at supercritical conditions for more than 72 hours at $50^{\circ} \mathrm{C}$, at higher pressures than have previously been reported in the literature. For proof of principle, in situ ${ }^{13} \mathrm{C}$ MAS NMR experiments have been successfully carried out on forsterite $+\mathrm{H}_{2} \mathrm{O}+\mathrm{scCO}_{2}$ at 95 bars and $50^{\circ} \mathrm{C}$ using a MAS speed of $2.1 \mathrm{kHz}$. For the first time, magnesium carbonation products, reactants, and reaction intermediates, have all been observed in a single experiment. A technical manuscript summarizing the initial in situ results is currently in preparation.

Ex situ ${ }^{29}$ Si and ${ }^{13} C$ NMR investigations. The mechanisms of forsterite metal carbonation at supercritical $\mathrm{CO}_{2}$ with water varied from dry to well above saturation, including at saturation, were investigated by a combination of solid state NMR $\left({ }^{13} \mathrm{C}\right.$ SP-, CP-MAS, ${ }^{29} \mathrm{Si}$ SP-, CP-MAS), XRD, TEM and XPS. Run conditions were $80^{\circ} \mathrm{C}$ and 96 atm. Major results are as follows: at high water contents where an aqueous solution was co-present with $\mathrm{H}_{2} \mathrm{O}$ saturated $\mathrm{scCO}_{2}$ phase forsterite was converted into magnesite, and an amorphous $\mathrm{SiO}_{2}$ reaction product that is free from $\mathrm{Mg}$ and dominated by $\mathrm{Q} 4$, and to a less extent by Q3. 
As the amount of added water became limiting, intermediate reaction products could be identified until at zero water no reaction products were observed. The intermediate reaction products identified were a complex mixture of partially hydrated/hydroxylated metal carbonate species and a variety of surface silica species. Formation of these intermediate species requires water via hydrolysis of $\mathrm{Mg}-\mathrm{O}-\mathrm{Si}$ linkages. If insufficient water is available, the reaction does not proceed far enough to form magnesite and $\mathrm{SiO}_{2}$ reaction products. The intermediates were mainly in an amorphous state, forming a thin layer on the surface. Water in excess of this limit yields the crystalline reaction products, a process that in turn is expected to liberate water. Hence, for a given fluid/forsterite ratio there is a water threshold (i.e., the formation of $\mathrm{H}_{2} \mathrm{O}$ film with sufficient thickness somewhere between 2.4 and $17.4 \mathrm{~nm}$ ) above which a significant portion of the water serves in a catalytic role for the carbonation process.

In addition to forsterite investigations, for ex-situ NMR we also initiated comprehensive studies on the metal carbonation mechanisms, in particular on understanding the role of $\mathrm{H}_{2} \mathrm{O}$, of other natural minerals such as antigorite and lizardite. Our ex situ NMR has resulted in one publication in The Journal of Physical Chemistry C, with a second publication submittal pending.

In FY 2011, we will perform a full-scale application of the high pressure MAS probe by increasing the pressure to 80 atms. We will then comparison the magnesite precipitation and forsterite dissolution rates to those found at lower pressures and conditions from the previous years' results. Also, we will study the mechanisms and kinetics of interlayer dehydration for a hydrous clay mineral in $\mathrm{CO}_{2}$-dominant supercritical $\mathrm{CO}_{2} /$ water fluids. If applicable, $\mathrm{CaSiO}_{3}$ (wollastonite) may also be studied as an alternative reactant phase if $\mathrm{Mg}_{2} \mathrm{SiO}_{4}$ (forsterite) fails due to its higher expected reactivity with $\mathrm{scCO}_{2} /$ water. Continuing the ex situ investigations, we will investigate computational modeling of the NMR chemical shifts to establish firmly the unique role of in situ detection. Additionally, we will initiate the task of investigating the effects of sulfide (i.e., contrasting results with acidified water) by adding a controlled amount to the mixture of mineral phase. For this effort, we will make adjustments according to a faster or slower rate of reaction accordingly. 


\title{
Ion Beam-Nanoparticle Interactions for Radiation Detection
}

\author{
Marvin G. Warner, Yanwen Zhang, Jay W. Grate
}

The specific aim of this project was to gain a fundamental understanding of the scintillation properties of semiconductor nanoparticles and to test the hypothesis that their radioluminescence properties correlate with the easily observed photoluminescence properties using innovative ion beam measurement techniques and sample preparation methods. These studies have contributed a scientific basis for the predictive understanding that enables a rational approach to the use of engineered nanomaterials in radiation detection applications.

$\mathbf{R}$ ecently, there has been an explosion of scientific interest in semiconductor materials in nanoparticle form, where quantum effects play a role in their optical and electronic properties. Called quantum dots, these semiconductor nanoparticles are typically made with a semiconductor core, a shell of additional inorganic material of a different composition, and an organic surface layer to stabilize the dot and prevent aggregation. While photoluminescence properties of quantum dots, which depend on parameters such as size, shape, and composition, are relatively well-understood in the open literature, research on the materials' radioluminescence response to ionizing radiation (e.g., ion beams, alpha and beta particles, and gamma rays) is just commencing. Studies have focused primarily on the empirical development of individual quantum dots containing composites to prove that they scintillate. Even more noteworthy is the lack of correlation between well-characterized photoluminescence properties and by extension their composition and structure, with the radioluminescence responses observed upon exposure to ionizing radiation.

If a correlation exists between photo and radioluminescence responses, then the first predictive understanding of the radiation physics of quantum dots will be developed.

Knowing how photoluminescence properties correlate to radioluminescence responses would allow a synthesis of new material for radioluminescence applications a priori to possess specific characteristics. If there is no direct correlation, these

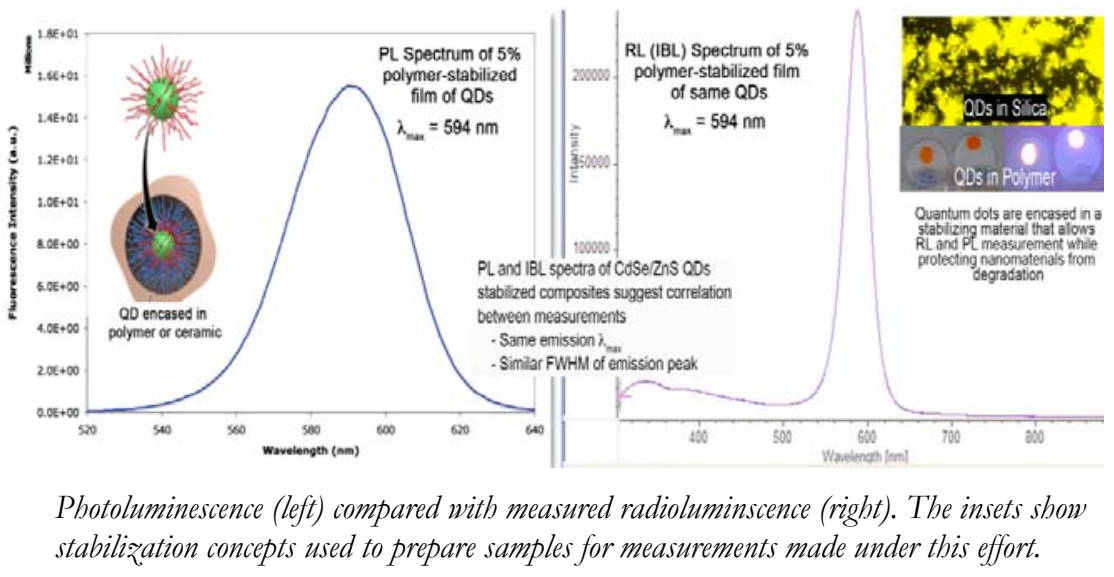

studies will represent some of the first measurements of energetic ion interaction with quantum dots, determine the potential as materials for ionizing radiation detection, and provide insight into chemical composition and structure effects on radioluminescence properties. Moreover, systematic studies will help understand scintillation mechanisms, where photo-luminescence consists of photon excitation and emission processes and radioluminescence includes ionization energy deposition, electron hole production, migration and recombination, and photon emission and absorption properties and qualities.

Under this project, we have sought to understand the correlation of photo and radioluminescence properties in nanostructured materials. Our integrated research program included synthesis, along with characterization and physical measurements, of quantum dots. In FYs 2008 through 2010, we investigated how quantum dot composite composition, shell structure, surface modification, and local environment influence photo and radioluminescence properties, measuring parameters such as fluorescence quantum (light) yield, ion induced luminescence spectra, resolution, and decay time. We investigated a wide range of quantum dot synthesis, including but not limited to $\mathrm{CdSe}, \mathrm{CdSe} / \mathrm{ZnS}, \mathrm{PbS}, \mathrm{PbSe}$, $\mathrm{CdSSe}$, and CdSTe. These materials were produced using previously published methods adapted in our laboratory for these studies.

Once materials were synthesized and purified, stabilized polymer films were produced using methods developed in our laboratory for analysis using the ion beam system. The thin films were characterized using scanning electron microscopy to determine their morphology and quality such that the stabilizing organic ligand on the quantum dot surface served as film plasticizer. This served the dual role of stabilizing the dots against aggregation or decomposition while affording films that were pliable, rugged, and easy to handle from one measurement apparatus to another. Using the films, we measured ion and electron induced luminescence of the quantum dots as a function of loading (by wt \%) into the polymer matrix and for the first time to correlate the observed radiolumin 
escence properties successfully to measured

photoluminescence properties of the same film.

We continued measuring radioluminscence response of specific quantum dots materials to correlate how changes in structure and functionality affect observable properties. Namely, we focused on three parameters: quantum dot size, chemical functionality, and homogeneity of the resulting polymer films formed to stabilize the quantum for photo and radioluminscent measurements. The radiation response of the quantum dots studied were demonstrated to have good correlation to the observed photoluminescence properties independent of the methods used for preparing the composite samples for measurement. 


\title{
Leak Rate Measurements for Prototypic Pressurized Water Reactor Primary Water Stress Corrosion Cracks
}

\author{
Mychailo B. Toloczko, Stephen M. Bruemmer
}

Leak rate measurements of through-wall stress corrosion cracks found in light water nuclear reactor primary cooling pipe systems are needed to establish a sound leak before break criterion for stress corrosion cracks in these piping systems. The goal is to establish a laboratory-based experimental method to reliably measure leak rates in prototypic through-wall stress corrosion cracks.

L eak before break is a criterion to determine what safety measures are needed for light water nuclear reactor primary cooling pipes in the event that a crack forms and grows. Using a combination of experimental measurements, computer modeling, and statistical analysis, it is possible to predict with high accuracy whether a cracked pipe will leak before it catastrophically breaks. In the event that catastrophic failure is predicted to occur before a leak is detected, it is necessary for the utility companies to install massive pipe restraints and shielding at a great cost. DOE and several other governmental regulating bodies are strongly involved in the development and use of this criterion so that it is used accurately and correctly. Thus, there is strong interest both from the government and utility companies in determining whether piping systems will leak before breaking.

Originally developed for fatigue cracks, the leak before break analysis models are being modified to predict leak or break for stress corrosion cracks. Part of this process includes generating accurate assessments of leak rates in through wall stress corrosion cracks. Contemporary methods used for measuring leak rates in fatigue cracks are not suitable for measuring leak rates in stress corrosion cracks. With these bases, this project will develop a unique laboratory-based method to measure reliably and relatively inexpensively leak rates in prototypic through wall stress corrosion cracks. The technique undergoing development makes use of new methods and equipment to measure stress corrosion crack growth rates in materials for piping systems. These experimental techniques will be assessed on a fatigue and stress corrosion cracks in weld and base metal samples.

In FY 2008, we modified the necessary laboratory equipment to allow leak rate measurements and prepared a fatigue-cracked specimen to test the initial equipment modifications. During FY 2009, a suitable technique to modify a test specimen for leak rate measurements was developed, along with our completing baseline modifications to allow leak rate measurements using our state-of-the-art crack growth test systems.
Work for FY 2010 focused on further shakedown testing of the leak rate method and on obtaining specimens with long stress corrosion cracks. The continuation of the shakedown testing from FY 2009 consisted of further leak rate measurements at room temperature and at elevated temperature using fatigue cracked specimens. Good reproducibility was found with further room temperature testing as shown for two specimens that were fatigue cracked and leak rate tested under almost identical conditions. A single elevated temperature test on a fatigue cracked specimen was performed, and it was found that the heat exchanger to cool the high temperature leak water was inadequate to bring the water to room temperature; thus, revised, higher cooling capacity heat exchangers were manufactured. These have been installed and are ready for use. Equipment to perform pressure measurements along the length of the crack was also obtained, but with limited finances in FY 2010, this equipment could not be included in shakedown testing.

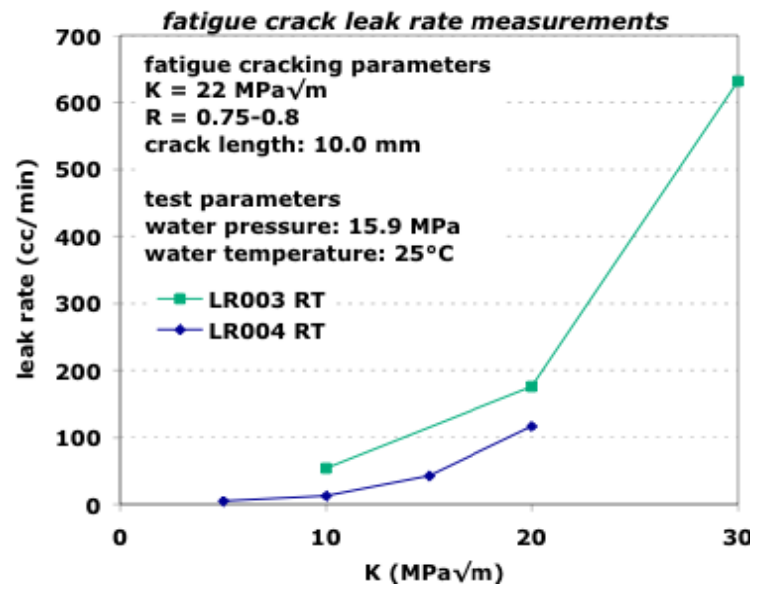

Leak rate as a function of crack opening stress intensity factor for two fatigue-cracked specimens tested at room temperature.

The remaining project effort was directed toward obtaining stress-corrosion-cracked specimens made from alloy 600 base metal and alloy 182 weld metal, both of which are prototypic pressurized water reactor class one piping materials that have been prone to stress corrosion cracking in plants. The intent was to grow the cracks in these specimens using equipment at PNNL, but due to other commitments for the needed equipment, this work had to be subcontracted to provide raw material, machine specimens, and grow a stress corrosion crack into the specimens. Final crack length on both specimens is $6.0 \mathrm{~mm}$. Leak rate testing of these specimens will be performed in FY 2011. 


\title{
Material Interface Optimization in Extremely Thin Adsorber Photovoltaics
}

\author{
Tiffany C. Kaspar, Timotby C. Droubay
}

To meet the world's growing demand for energy, breakthrough technologies such as nanostructured photovoltaic (solar) cells are required. This project investigates the fundamental charge transport properties of relevant photovoltaic materials to aid in material selection and optimization.

$\mathbf{N}$ anostructured solar cell designs using novel materials are necessary to realize a revolution in solar cell efficiency and cost. One such design is the all-solid extremely thin absorber (ETA) solar cell, which consists of a nanostructured $n$-type semiconductor $(\mathrm{ZnO})$ coated with a thin layer of photon absorber material (CdTe) and impregnated with a $p$-type semiconductor $(\mathrm{CuSCN})$. With other solar conversion efficiencies at 2.3 percent for the $\mathrm{ZnO} / \mathrm{CdTe} / \mathrm{CuSCN}$ structure, there is room for significant improvement. To achieve highly efficient charge separation and transport in ETA solar cells, materials must be engineered so that band alignments at heterojunction interfaces favor the desired charge transport (electrons into $n$-type $\mathrm{ZnO}$ and holes into $p$-type $\mathrm{CuSCN}$ ). Despite the importance of resultant alignments to overall photovoltaic efficiency, band alignments in solar cell materials are rarely measured directly. By measuring with x-ray photoelectron spectroscopy (XPS), this study will open new paths to material selection, optimization, and solar cell design.

This project aims to provide a fundamental scientific foundation to understand the charge transport in novel oxide-based solar cells and tailor the transport by doping and/or interface modification of the material systems. These results will provide the basis for improvements in solar cell efficiency, a primary goal in the quest to harness solar power to meet the world's energy demands. We will focus on proposed ETA solar cell designs that consist of a study of the band alignments between $n$-type $\mathrm{ZnO}$ and common inorganic photon absorber, $p$-type semiconductor, and electrode materials in both thin-film form and nanostructured devices.

The thin film heterojunction chosen for the first band alignment measurements in FY 2009 by XPS was $\mathrm{ZnO}$ on the $n$-type transparent top electrode (In, $\mathrm{Sn})_{2} \mathrm{O}_{3}$ (ITO) because these oxides are easy to work with and the heterojunction is used in many technological applications. Pulsed laser deposition was used in FY 2010 to deposit various thicknesses of $\mathrm{ZnO}$

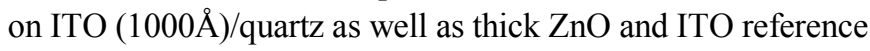
standards. A series of $\mathrm{ZnO}$ films of varying thickness, as well as an analogous series of $n$-type $\mathrm{Al}$-doped $\mathrm{ZnO}$ films, revealed surprising behavior of the electronic band alignments. For pure $\mathrm{ZnO}$ films, substantial band bending resulted in a nearly $0.6 \mathrm{eV}$ barrier for electron injection into ITO, making charge transfer highly unfavorable. This barrier is unexpected because even undoped $\mathrm{ZnO}$ deposited in an oxygen environment is generally weakly $n$-type, not insulating, and thus a favorable band offset with ITO would be predicted. Doping the films with $\mathrm{Al}$, a known $n$-type dopant, eliminated the band bending and instead produced a flat-band condition that was favorable for electron injection into ITO. Further work is necessary to determine whether this effect is due to the increase in carriers in the $\mathrm{ZnO}$ film or the $\mathrm{Al}$ segregation to the interface or diffusion into ITO. In FY 2011, Ga-doped ZnO films will be deposited on ITO to begin to answer these questions.

Despite its wide application in solar cell devices, the electronic structure of the $p$-type inorganic semiconductor $\mathrm{CuSCN}$ has not been explored either theoretically or experimentally. Thus, basic information such as what causes $p$-type behavior and what the role defects play in the conductivity is lacking. To remedy this, we performed density functional theory (DFT) electronic structure calculations of $\beta-\mathrm{CuSCN}$ (hexagonal crystal structure). Results for the band structure of $\mathrm{CuSCN}$ were somewhat unexpected. $\mathrm{CuSCN}$ was predicted to have an indirect bandgap, contrary to literature reports of a direct optical gap. Also, the bottom of the conduction band consisted of cyanide $(C \equiv N)$ antibonding states, which gives $\mathrm{CuSCN}$ a "molecular" character. The defect structure of $\mathrm{CuSCN}$ was explored, and both $\mathrm{Cu}$ and $\mathrm{CN}$ vacancies were found to be stable and generate $p$-type conductivity.

To corroborate DFT calculations, $\mathrm{CuSCN}$ films were deposited and analyzed. Spin-casting deposition of CuSCN onto a heated substrate $\left(80^{\circ} \mathrm{C}\right)$ produced films, which crystallized (at least partially) in the desired $\beta$ phase. Core-level XPS analysis indicated the films had correct $\mathrm{Cu}: \mathrm{S}: \mathrm{C}: \mathrm{N}$ stoichiometry. The valence band of $\mathrm{CuSCN}$ was measured by XPS in very good agreement, which confirmed the accuracy of the calculations. Optical absorption measurements on thin CuSCN (partially crystallized) resulted in a bandgap value of $3.9 \mathrm{eV}$, which matches well with literature. A weak absorption tail below the band edge may indicate an indirect transition as predicted by DFT. These experimental and theoretical results on CuSCN were published in The Journal of Physical Chemistry C.

In FY 2011, the work on ZnO/ITO band offsets will be completed. In addition, work will begin on $\mathrm{ZnO} / \mathrm{CdTe}$ and $\mathrm{CuSCN} / \mathrm{CdTe}$ band alignments. Deposition of $\mathrm{ZnO}$ nanorods by pulsed laser will also be attempted. 


\title{
Materials and Methods for Low Cost Photovoltaic Manufacturing
}

\author{
John P. Lemmon, Cheng Huang, Christopher A. Coyle, Feng Chen
}

Developing new solution-based thin film photovoltaic (PV) manufacturing methods versus the current costly high vacuum processes is significant to decreasing our nation's dependence on petroleum imports while reducing carbon emissions.

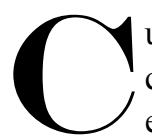
urrently, PV cells based on thin-film copper-indium-gallium-selenide (CIGS) have emerged as one of the leading contenders for thin-film solar technologies. Thin-film solar cells utilizing CIGS as the absorber material have demonstrated over 19 percent efficiency in the small cell configuration, greater than 16 percent efficiency in production sub-modules and 11 percent in large-scale production modules. Despite offering great promise, CIGS and its complicated nature renders the deposition process complex and therefore time consuming and costly. An attractive approach to decreasing production cost has been the development of a non-vacuum, solution-based CIGS (and other low-cost absorbers), which is the focus of this project.

Our approach combines several steps: facile, low-cost methods to synthesize processable CIG nano-metal inks, a non-vacuum deposition of nano CIG inks selenized into uniform CIGS thin films, fabrication and processing steps to form a functional photovoltaic cell, and performance testing and materials evaluation. The CIGS materials and cell fabrication methods will be validated using benchmarked materials with known performance properties and risk will be minimized through the use of a go/no-go timeline. Characterization of materials and devices combined with performance data will be used to derive structure-property relationships and their correlation to performance. The insights will allow for improvements to the device and provide real data as a base to develop mechanistic modeling.

During FY 2010, our first task aimed to develop inks and deposition techniques that produce thin-film alloys of CI or CIG with targeted composition, thickness, and uniformity. Inks with variable composition were synthesized from low cost inorganic salts.

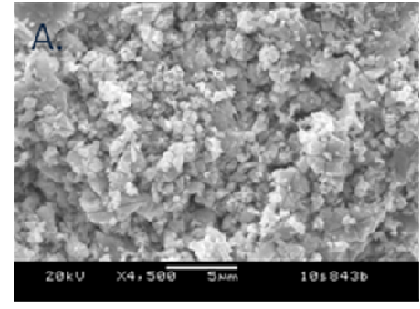

Scanning electron microscopy of thin-film CIGS in A) small grain growth conditions, and B) targeted grain size from design of experiments.

to optimize these CIGS films with the targeted thickness, phase, microstructure, and uniformity.

The selenization process of CIG films has several optimization parameters, including temperature profile, selenium pressure, and atmosphere to create grain size and orientation important to CIGS cell performance. First, we determined the initial conditions using a new capability based on an in situ infrared reactor that allowed for the conversion of CIG to CIGS to be monitored in real time. To expedite the optimization process further, a level IV screening design of experiments was used, and data were analyzed for main effects and binary interactions using an $\mathrm{x}$-ray diffraction peak width and 112 peak shift, lattice parameter fitting, and grain size from SEM as the response factors. Based on the design of experiments analysis, selenization reaction temperature had the greatest single effect on CIGS morphology, while post-anneal time and temperature had significant interactions. Scanning electron microscopy images were created from two CIGS films in the process. The contrast in grain size is profound given that the experimental parameters are not diverse. The next step is a focused design of experiments that contains several replicate samples for improved statistical correlations for detail in process parameter.

Also in FY 2010, an in-situ optical reactor that uses sapphire window for monitoring chemical reactions and processes with a 3 to $5 \mu \mathrm{m}$ thermal imaging camera was developed. The new capability can monitor real temperature changes from free energy differences in chemical reactions or emmistivity changes in reaction surfaces correlating to the formation of new products. It can also determine thermodynamics, kinetics, and end point of reactions or be used for rapid discovery of new materials using a combinatorial, high-throughput screening approach. In addition, a turn-key PV performance measurement system was purchased. The system replaces an inaccurate,

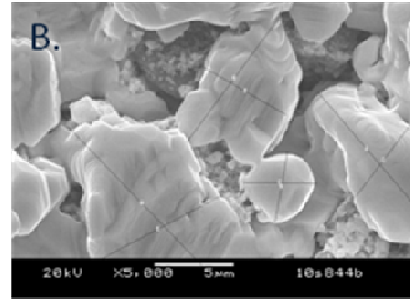
outdated system that yielded highly irreproducible results. The addition of this new capability will allow for publication of performance data.

In FY 2011, the program will move into Phase II, where CIGS microstructure will be further optimized and perfor-
The inks were dispersed on molybdenum (Mo)-coated glass substrates and selenized at $500^{\circ} \mathrm{C}$ in flowing $\mathrm{H}_{2} \mathrm{Se}$ to form single-phase CIGS. In the second task, our objective was mance tested in PV cells to determine the overall efficiencies and impact on cost. 


\section{Multicomponent Assembly to Achieve Charge Separation and Transport for Energy Conversion}

Daniel L. DuBois, Jun Liu

The potential use of biological agents by terrorists is a real threat. The goal of this project was to advance magnetic nanoparticle handling approaches to enable low cost, portable devices for rapid detection of biothreats that could be used to safeguard the homeland and allow rapid response to biological outbreaks and events.

A $\mathrm{n}$ abundant supply of clean and affordable energy is vital to the economic growth, quality of life, and security of the United States. The increasing energy demand will create worldwide competition for the gradually depleting fossil fuel reserves, endangering our quality of life that depends on a supply of energy at a low cost. On the other hand, increasing greenhouse emission is mostly likely linked to global warming. The concentration of carbon dioxide - the key contributor to global climate change in the atmosphere - is at the highest recorded level since records began and will increase further. The need to increase our energy supply while simultaneously reducing carbon dioxide emissions is one of the major challenges facing our global society today.

This research is designed to develop the capability of assembling multicomponent materials composed of designed functional materials (for electron and proton transport and catalysis) that are integrated across a range of scales from the size of individual molecules through nanoscppic and microsopic scales to macrosopic devices. The demonstration of highly active integrated structures will provide an excellent basis for designing more complex multiscale structures, such as membranes, that integrate the functions of light-harvesting and charge separation, proton and electron transport, and catalysis. The focus of this particular project is on the integration of electron transport, proton transport, and catalysis at catalytically active interfaces for $\mathrm{H} 2$ production and $\mathrm{CO}_{2}$ reduction.

The specific aim of this project is to construct organized multiscale materials containing arrays of nanorods, nanotubes, or nanochannels that allow for efficient electron and proton transfer over nanometer dimensions. We wish to discover new approaches for multiscale assembly to achieve efficient energy conversion and improve our understanding of electron and ionic conductivity in such multicomponent systems. Our approach involves a shift in perspective in materials science from one of obtaining a single material with optimal properties to precisely assembled multicomponent materials composed of designed functional materials (for electron and proton transport and catalysis) integrated across a range of scales from the size of individual molecules through nanoscppic and microsopic scales to macrosopic devices. The demonstration of highly active integrated structures will provide an excellent basis for designing more complex multiscale structures such as membranes that integrate the functions of light-harvesting and charge separation, proton and electron transport, and catalysis.

In FYs 2008 and 2009, we examined five different approaches to modifying electrode surfaces with catalysts for $\mathrm{H}_{2}$ production: adsorption, the use of thin layers of ionic liquids containing the catalyst, the use of ion exchange membranes such as Nafion for incorporating the catalyst cation, covalent attachment, and electropolymerization. Of these approaches, two appeared most promising: adsorption and the use of thin layers of ionic liquids. The modification of glassy carbon electrodes by adsorption of $\left[\mathrm{Ni}\left(\mathrm{P}_{2}^{\mathrm{Ph}}{ }_{2} \mathrm{~N}_{2}\right)_{2}(\mathrm{NCMe})\right]\left[\mathrm{BF}_{4}\right]_{2}$ (1, where $\left.\mathrm{R}=\mathrm{C}_{6} \mathrm{H}_{4}\left(\mathrm{CH}_{2}\right)_{5} \mathrm{CH}_{3}\right)$ resulted in electrodes with catalytic activity for $\mathrm{H}_{2}$ production at much higher rates and lower overpotentials than for unmodified electrodes. This led to further studies in this area during FY 2010.

Our initial focus in the area of ionic liquid thin films in FY 2008 was on the use of 1-butyl-3-methylimidazolium bis(trifluoromethylsulfonyl)imide ([BuMeIm] $\left.\left[\mathrm{NTf}_{2}\right]\right)$ as the ionic liquid and encouraging results were obtained in that catalyst 1 retained both its electrochemical activity and its catalytic activity when confined to the electrode surface using a thin film of this ionic liquid. Because of their initial promising performance, two new ionic liquids were studied in 2009: dodecyltrihexylphosphonium bistriflylamide $\left\{\left[\mathrm{C}_{12} \mathrm{H}_{25} \mathrm{P}\left(\mathrm{C}_{6} \mathrm{H}_{13}\right)_{3}\right]\left[\left(\mathrm{CF}_{3} \mathrm{SO}_{3}\right)_{2} \mathrm{~N}\right]\right\}$ and an ionic liquid formed by the protonation of dibutylformamide with $\mathrm{H}\left(\mathrm{CF}_{3} \mathrm{SO}_{3}\right)_{2} \mathrm{~N}$.

The thin films of $\left[\mathrm{C}_{12} \mathrm{H}_{25} \mathrm{P}\left(\mathrm{C}_{6} \mathrm{H}_{13}\right)_{3}\right]\left[\left(\mathrm{CF}_{3} \mathrm{SO}_{3}\right)_{2} \mathrm{~N}\right]$ were more stable than those of $\left([\mathrm{BuMeIm}]\left[\mathrm{NTf}_{2}\right]\right.$ ionic liquid, but incorporation of $\mathbf{1}$ into these films did not result in high catalytic activity. This low activity was attributed to the inability of this ionic liquid to provide an adequate supply of protons (i.e., the acids used were of low solubility in this ionic liquid). The catalytic performance was enhanced by the incorporation of Nafion (a known proton conductor), but the performance was still less than desirable. To overcome this problem, the ionic liquid formed by the protonation of dibutylformamide with $\mathrm{H}\left(\mathrm{CF}_{3} \mathrm{SO}_{3}\right)_{2} \mathrm{~N}$ was studied as a thin film containing catalyst $\mathbf{1}$. A very active catalyst was observed with a turnover number 
of at least 50,000 and turnover frequency of approximately $10 \mathrm{~s}^{-1}$ over a period of $1.5 \mathrm{~h}$. This performance is quite exciting, and studies in this area were pursued in 2010 with an emphasis on extending these results to include catalysts for $\mathrm{CO}_{2}$ reduction.

In FY 2010, we extended our studies of adsorbed $\mathrm{Ni}$ complexes by synthesizing a $\left[\mathrm{Ni}\left(\mathrm{P}^{\mathrm{Ph}}{ }_{2} \mathrm{~N}^{\mathrm{R}}{ }_{2}\right)_{2}(\mathrm{NCMe})\right]\left[\mathrm{BF}_{4}\right]_{2}$ (2, where $\mathrm{R}=\mathrm{C}_{6} \mathrm{H}_{4}\left(\mathrm{CH}_{2}\right) \mathrm{C}_{6} \mathrm{H}_{5}$ ) with benzyl group attached to the para- position of the benzene ring. This complex readily adsorbs to glassy carbon electrodes, and electrodes modified with this complex produce hydrogen, but at a slower rate than films using acidic ionic liquids. For this reason, we returned our attention to ionic liquid thin films.

In an effort to develop modified electrodes that would catalyze the electrochemical reduction of $\mathrm{CO}_{2}$ at high rates and low overpotentials, we prepared a catalyst prepared previously in our group, $\left\{\mathrm{Pd}\left[\mathrm{PhP}\left(\mathrm{CH}_{2} \mathrm{CH}_{2} \mathrm{PCy}_{2}\right)_{2}\right]\right.$ $\left.\left.\left(\mathrm{CH}_{3} \mathrm{CN}\right)\right]\left(\mathrm{BF}_{4}\right)_{2}\right\}$, where $\mathrm{Cy}$ is cyclohexyl. This complex was shown to be very stable in the acidic ionic liquid described above, and electrodes modified with ionic liquid thin films of this catalyst exhibited a significant catalytic current in the presence of acid and $\mathrm{CO}_{2}$. However, the major product was $\mathrm{H}_{2}$ and not the desired product, $\mathrm{CO}$. This observation was attributed to the strongly acidic nature of the ionic liquid, which favored $\mathrm{H}_{2}$ production over $\mathrm{CO}_{2}$ reduction.

In the course of these studies, a number of different methods have been assessed for their potential as a general method for modifying electrode surfaces with highly active molecular electrocatalysts for $\mathrm{H}_{2}$ production and $\mathrm{CO}_{2}$ reduction. As previously projected, the two most promising approaches are adsorption and the use of ionic liquid thin films. For the latter method, including acidic functional groups are necessary for the delivery of the protons required for fuel production as exemplified by the construction of electrodes that are highly active for $\mathrm{H}_{2}$ production. However, our studies of modified electrodes using acidic ionic liquid thin films also indicate that the acidity must be carefully controlled to achieve the desired selectivity. 


\section{Multiphysics Capability Development and Application to Magnesium Alloys}

Erin I. Barker, Ramaswami Devanathan, Dongsheng Li

The primary aim of this project is to develop and demonstrate multiphysics material models relevant to energy technologies, especially in advanced nuclear energy systems and lightweight alloys for transportation. The secondary aim is to enhance a valuable code used for nuclear fuel performance modeling, namely FRAPCON. Development of this needed capability will add value to material modeling efforts, create a much-needed capability, and enhance the scientific stature of PNNL in nuclear energy research.

G iven the essential nature of nuclear energy, it is important to extend the lifetime of existing reactors, and deploy advanced reactor technologies that are safe, reliable, proliferation-resistant and economical. In order to evaluate the safety and performance characteristics of advanced reactors and fuel concepts such as mixed oxide and $\mathrm{ThO}_{2}$-based fuels in place of the conventional $\mathrm{UO}_{2}$ fuel, we need dependable computational tools that are informed by sound fundamental physics. The computer code FRAPCON-3 is an analytical tool that is used to calculate light water reactor fuel rod behavior under a variety of conditions. The code describes the complex behavior of a fuel rod during reactor operation with equations that are based on experimental data and phenomenological models. Gaseous swelling is not well described, and extension to new fuel types is not straightforward. The code is implemented using one-dimension calculations at a series of locations along the length of the fuel rod, thus ignoring any non-symmetric variation or localization in the fuel response. To improve the reliability of the code to predict fuel rod failure, there is a need to consider off-normal operating conditions, incorporate fundamental physics-based data and extend the code to three dimensions. This requires extracting parameters from hierarchical multiscale models starting from the lowest length scales and moving upward toward the microstructure scale and finally incorporating parameters into coarse-grained models or directly into a computer code such as FRAPCON.

Multiscale, multiphysics models are widely applicable to material science problems, such as fuel performance and alloy development. In this regard, there is growing interest in developing lightweight alloys for transportation technologies. DOE has set an automotive target of 50 percent weight reduction relative to steel without loss of performance. While one could replace the body panels of cars with expensive lightweight alloys, the weight savings cannot be justified by the higher cost. The most promising area for improving vehicle efficiency through lightweight alloy substitution is in the engine because of its weight. Lightweight $\mathrm{Mg}$ alloys are quite promising, but their elevated temperature mechanical performance requires rare-earth element addition. Because one has to depend on suppliers in sensitive foreign markets to obtain rare earths, it is desirable to develop magnesium alloy compositions that have the desired elevated temperature characteristics without the use of rare-earth elements. The development of non-rare earth cast Mg alloys will overcome a major scientific barrier to achieve DOE's goal for reducing automobile weight and also reduce U.S. dependence on foreign suppliers. Traditionally, alloy development has followed the expensive, cumbersome heat-and-beat approach. With the development of sophisticated material models and availability of high-performance computing resources, rational development of alloys based on fundamental science is increasingly becoming a reality. One can combine information from multiphysics simulations, data from literature and experimental results to develop rules that can guide alloy selection.

During FY 2010, we conducted a review of FRAPCON to determine the limitations of the code and developed the software design for a new three-dimensional fuel performance code. The design leverages an existing finite element solver package, such as PETSc or Trilinos, wrapped with a multiphysics driver and utilizing a material model library. This will allow us to focus our efforts on developing the material models while utilizing packages developed through other PNNL and DOE projects. We also surveyed the available interatomic potentials for $\mathrm{Mg}$ alloys and have chosen a potential for our simulations. Based on the modified embedded atom method, the potential provides a satisfactory description of point defect properties, thermo-mechanical properties, and $\mathrm{Mg}-\mathrm{Al}$ alloy behavior. At the microstructure scale, we have developed a preliminary algorithm for generating two-dimensional realizations based on $\mathrm{Mg}$ alloy microstructures. A sensitivity study of the material parameters of an elastic-viscoplastic material model describing Mg alloy grains is being conducted using three-dimensional generic microstructures.

In FY 2011, we propose to enhance the software framework with higher dimensional implementations of the material models currently available in FRAPCON. Improved models will also be implemented by incorporating fundamental parameters from lower length scales. For Mg alloys, therodynamic parameters from atomistic simulation will be included in the larger length models. The algorithm for modeling Mg alloy microstructure will be expanded to three-dimensions and combined with the finite element solution framework. 


\section{Multiscale Charge and Ion Transport Simulations for Nanostructured Electrodes}

Kevin M. Rosso

This project will develop and perform first ever simulations of collective charge transport dynamics from the atomic to micron scales for coupled charge and ion transport in polycrystalline metal oxide electrodes. A deep, fundamental understanding of the basis for charge and ion conductivity in select systems will also be developed.

$\mathbf{T}$ itania $\left(\mathrm{TiO}_{2}\right)$ is an attractive alternative for carbon-based anode materials in lithium ion batteries because of its high surface area, chemical stability, and high theoretical capacity. Several polymorphs of $\mathrm{TiO}_{2}$ have been shown to benefit significantly from nanostructuring or from the incorporation of nanoporosity. Increased rate capability, capacity, and tolerance for strain from lithium insertion/extraction have all been reported for $\mathrm{TiO}_{2}$ nanomaterials. Although nanostructuring and nanoporosity offer improved performance because of higher contact areas between electrolyte-electrode and shorter diffusion distances for lithium ions and electrons, there is a lack of fundamental understanding about the effects on charge and ion transport efficiency of nanosizing electrolyte-electrode interfaces and of the interdependence of electrode materials and electrolytes, contact areas, and diffusion distances, thus preventing reliable predictions of performance.

As a result of the issues raised above, a long-term goal of our research is the discovery of design principles for nanostructured metal oxide electrodes that maximize charge and ion diffusivity. The first step in this process is to improve our understanding of charge and ion transport in electrode materials such as the $\mathrm{TiO}_{2}$ polymorphs. In particular, two fundamental questions are identifying the mechanism of charge transport at the nanoscale and determining how defects and grain boundaries influence the elementary and mesoscopic $\mathrm{Li}^{+}$and electron conductivity.

Our FY 2009 activities focused on performing molecular dynamics simulations of the kinetics of coupled electron and lithium ion transport in $\mathrm{Li}_{x} \mathrm{TiO}_{2}$ polymorphs for comparisons with experiments and also to examine fundamental processes that potentially impact anode performance in lithium batteries. We concentrated on the two most stable and most studied $\mathrm{TiO}_{2}$ polymorphs: rutile and anatase. Also, molecular dynamics simulations were performed with a potential shell model to investigate the diffusion of $\mathrm{Li}^{+}$and electron polarons in rutile and anatase. In all, this project met or exceeded its fiscal year milestones. Multiscale simulations with a key staff member of $\mathrm{Li}^{+} /$electron transport in $\mathrm{TiO}_{2}$ nanoparticles led to the publication of one manuscript and submission of another in high quality journals. Density functional theory simulation coupled $\mathrm{Li}^{+} /$electron transport in olivine phosphates and the embedded cluster simulations of $\mathrm{Li}^{+}$in reduced $\mathrm{TiO}_{2}$ both led to the submission and publication, respectively, of one manuscript each to a high-quality journal.

In FY 2010, this project focused on elucidating the mechanism of coupled electron and $\mathrm{Li}^{+}$transport in $\mathrm{TiO}_{2}$ nanoparticles to predict the size, crystal structure and orientation of $\mathrm{TiO}_{2}$ nanoparticles, which would enhance the performance of nanostructured anode materials for Li batteries. We have also continued the investigation of elementary $\mathrm{Li}^{+} /$electron transport in bulk materials and in the vicinity of defects. We targeted the following milestones: 1) publish findings in a high-quality scientific journal, 2) develop novel software for multiscale simulations of one-dimensional charge transport and equilibrium properties of multicomponent systems, 3) study charge transport in $\mathrm{TiO}_{2}$ nanoparticles, and 4) develop quantum mechanical embedded cluster model for simulation of elementary $\mathrm{Li}^{+} /$electron transport in the vicinity of defects and grain boundaries.

The results on the elementary $\mathrm{Li}^{\prime} / \mathrm{e}^{=}$transport in $\mathrm{TiO}_{2}$ polymorphs obtained in $\mathrm{FY} 2009$ were used in FY 2010 to simulate charge transport on the nanoparticle level. In particular, diffusion barriers for $\mathrm{Li}^{+}$and electron polaron elementary transport in bulk materials were used to parametrise the kinetic Monte Carlo and Poisson-NernstPlanck models. Kinetic Monte Carlo simulations were used to study the influence of explicit $\mathrm{Li}^{+} / \mathrm{e}^{-}$coupling, interfacial contact area with the carbon matrix, charge injection flux and grain size on $\mathrm{Li}^{+} / \mathrm{e}^{-}$conductivity in $\mathrm{TiO}_{2}$ nanoparticles. These simulations revealed that charge diffusion rate increases with the increase in injection flux and with the decrease in the grain sizes.

To elucidate the fundamental mechanism for the dramatic increase in $\mathrm{Li}^{+}$conductivity with the decrease in the size of the nanoparticles, we employed the Poisson-Nernst-Planck method. Using this approach, we showed that the competition between $\mathrm{Li}^{+}$and electron accumulation at the nanoparticle boundaries competes with the steady ion and electron fluxes. For nanoparticles smaller that a critical size (equal to $20 \mathrm{~nm}$ for rutile and $5.7 \mathrm{~nm}$ for anatase), the balance is shifted towards the steady charge transport (hence high conductivity), while for larger nanoparticles, charge separation prevails. Size effects are also manifested in the change in the nature of charge transport from dual ionic/electronic for small 
nanoparticles to predominately ionic for lager ones. Simulations revealed that the temperature dependence of $\mathrm{Li}^{+}$conductivity in anatase is very weak, while in rutile the conductivity decreases with temperature in small nanoparticles and increases in large nanoparticles.

We have studied the effect of oxygen vacancies on $\mathrm{Li}^{+}$ diffusion in rutile $\mathrm{TiO}_{2}$ using the embedded cluster approach. We found that the calculated $\mathrm{Li}^{+}$diffusion pathway in vacancy-containing lattice is similar to that found for the ideal lattice. However, vacancies strongly modify the shape of the $\mathrm{Li}^{+}$potential energy surface and increase the diffusion activation energies by the factor of two.
In FY 2011, we will build on these simulations to study more realistic systems, which have defects and grain boundaries. The new simulations will include molecular dynamics simulations of the boundaries between two $\mathrm{TiO}_{2}$ nanoparticles and the boundaries between $\mathrm{TiO}_{2}$ nanoparticles and graphene and continue the work on the kinetic Monte Carlo simulations of grain-scale coupled $\mathrm{Li}^{+} / \mathrm{e}^{-}$transport. The one-dimensional Poisson-Nernst-Planck model will be extended to three dimensions and used to study the mechanism of charge transport across grain boundaries. Elementary charge transport in the boundary region will be studied using the embedded cluster model. 


\title{
Nanoscale Tantalum Oxide Electrocatalysts for Polymer Electrolyte Membrane Fuel Cells
}

\author{
Jin Yong Kim, Yongsoon Shin
}

The objective of this project is to develop economical catalysts for polymer electrolyte membrane (PEM) fuel cells in order to replace the current expensive platinum $(\mathrm{Pt})$-based catalysts.

I $\mathrm{n}$ reports from the National Academy of Sciences, American Physical Society, and DOE, one of two bottlenecks to the success of PEM fuel cells is cost and developing an appropriate means of hydrogen storage. Recent economic studies indicate that fuel cell stack cost can be reduced by decreasing or eliminating the amount of platinum required in the cell electrodes, the largest quantity of which is employed in the cathode where it catalyzes the oxygen reduction reaction. While substantial progress has been made in understanding why platinum is such an effective catalyst for oxygen reduction reaction and in explaining the catalyst degradation mechanisms that currently limit the operational lifetimes of PEM fuel cells, little success has been achieved in identifying promising alternative electrocatalysts.

We aim to investigate the synthesis, microstructure, and properties of a new PEM fuel cell catalyst. We studied carbon-supported nanoscale tantalum oxide materials that show promise as an alternative oxygen reduction reaction electrocatalyst in partial or full replacement of platinum. This project will focus on synthesizing an optimized form of this material via a sol gel approach. Methods will be employed to investigate the microstructure and composition of the catalyst and catalyst support, particularly along the interface where the two materials meet, and catalytic activity will be measured using a previously established half-cell measurement in an effort to coxygen reduction reactionelate interfacial structure and composition to catalytic activity.

During FYs 2008 and 2009, we attempted to develop economical oxygen reduction catalysts for PEM fuel cells by replacing expensive $\mathrm{Pt}$ with tantalum oxide. The basic concept is to enhance the catalytic performance of tantalum oxide by making nanoscale tantalum oxide $\left(\mathrm{Ta}_{2} \mathrm{O}_{5}\right)$ /carbon composites that contain increased triple-phase boundaries working as reaction sites for oxygen reduction. The novel direct synthesis technique was developed, and the composite catalysts were tested using a rotating-disc electrode cell. We found that the size of oxide particles decreased with the amount of oxide, and the electrochemical performance of tantalum oxide was enhanced by smaller particle size and tungsten oxide replacement. The best performance was achieved with $32 \mathrm{~mol} \%$ replacement of tungsten with tantalum. For this tungsten-doped tantalum oxide composite catalyst, the mass-specific current density reached 7.2 percent at $0.6 \mathrm{~V}$ and 35 percent at $0.2 \mathrm{~V}$ vs. normal hydrogen electrode (NHE) compared with Pt at the high rotating speed of $2400 \mathrm{rpm}$, where the diffusion limitation is minimized.

In FY 2010, our goals were to understand the effects of tungsten addition on electrochemical performance, to optimize the oxide content and particle size for the maximum electrochemical performance, and to enhance the performance of the oxide-based catalyst by adding a small amount of Pt. First, to understand why tungsten doping improves electrochemical performance of tantalum oxide-based catalysts (in particular, reducing resistance polarization), the valences of the tantalum and tungsten cations in the $32 \mathrm{~mol} \%$ tungsten-doped catalyst were examined by x-ray photoelectron spectroscopy. Results show that the oxidation states of tungsten and tantalum are +6 and +5 , respectively, the former of which indicates that tungsten cations can generate excess oxygen anions in the tantalum oxide structure. In turn, this excess can enhance surface transport mechanisms such as surface diffusion. Thus, the improvement observed in the catalytic performance of tungsten-doped catalysts appears related to the formation of excess oxygen in the tantalum oxide structure.

To make nanoscale oxide particles even smaller, the content of oxide with respect to the carbon support was decreased up to $5 \mathrm{wt} \%$. Though slightly finer oxide particles were achieved by lowering oxide content in direct synthesis, the reduction current did not increase, because the lowering content also decreases the number of particles; hence, triple-phase boundaries. Below $17 \mathrm{wt} \%$, the reduction current density was not changed significantly.

Finally, a small amount of $\mathrm{Pt}$ addition was attempted to enhance the catalytic performance. With $1 \mathrm{wt} \%$ Pt addition into $15 \mathrm{wt} \%$ tantalum oxide/tungsten oxide catalyst, the electrochemical performance of tantalum oxide-based catalyst increase drastically. Compared to the commercial $10 \mathrm{wt} \%$ Pt catalyst (Pt loading: $0.17 \mathrm{mg} / \mathrm{cm}^{2}$ ), the mass-specific current density at $60^{\circ} \mathrm{C}$ reached 70 percent at $0.6 \mathrm{~V}$ vs. NHE even though the tantalum-oxide based catalyst contained only $0.011 \mathrm{mg} / \mathrm{cm}^{2}$ of $\mathrm{Pt}$ ( 6.2 percent of $\mathrm{Pt}$ compared to the commercial Pt catalyst). In other words, the composite catalyst can generate more than 10 times current per the weight of $\mathrm{Pt}$ at $0.6 \mathrm{~V}$ than the commercial Pt. This oxide-based composite catalyst also reached 70 percent 
of area specific current density at $0.6 \mathrm{~V}$ compared to the commercial Pt catalyst. With $2 X$ loading $\left(0.021 \mathrm{mg} / \mathrm{cm}^{2}\right.$ of $\mathrm{Pt}$, 12.4 percent $\mathrm{Pt}$ loading compared to the commercial $\mathrm{Pt}$ ), it reached almost the same area specific current density as the commercial Pt catalyst. Therefore, the small Pt addition to nanoscale tantalum based composite catalyst can lead to drastic decrease in the Pt loading (only 12 percent needed for nearly identical performance) for PEM cathode catalysts. Transition electron microscopy analysis revealed that the size of Pt particles in the nanoscale oxide-based catalyst is less than $3 \mathrm{~nm}$, while the commercial Pt catalyst contains the agglomerates of 10 20 nm particles. We also expect the better stability of performance with Pt-added oxide-based catalysts because Pt bonds better to oxide than carbon.
In summary, nanoscale tantalum oxide-based catalysts (oxide particle size of approximately $20 \mathrm{~nm}$ ) on carbon support show high ORR onset potential comparable to $\mathrm{Pt}$. The catalyst of $\mathrm{Ta}_{2} \mathrm{O}_{5}$ with $32 \mathrm{~mol} \% \mathrm{WO}_{3}$ showed 7.2 percent of mass-specific current density at $0.6 \mathrm{~V}$ vs. NHE at $60^{\circ} \mathrm{C}$, compared with the $10 \mathrm{wt} \%$ commercial Pt catalyst. With $5 \mathrm{X}$ loading of this oxide-based catalyst, the area specific current density reached 35 percent of the commercial Pt catalyst at $0.6 \mathrm{~V}$. The addition of a small amount of Pt to the nanoscale oxide-based catalyst drastically enhanced catalytic activity. Nearly the same electrochemical performance was achieved with only 12 percent of Pt use compared with the commercial Pt catalyst. Thus, the nanoscale oxide-based catalyst demonstrates the potential as an economical alternative for the reduction catalysts of PEM fuel cells. 


\section{Simultaneous Charge Transport in Laterally Confined One-Dimensional Systems}

Xiao-Dong Zhou

This project investigated the transport of electrons and ions in hydrothermally grown nanometer wires of $\mathrm{MnO}_{2-\mathrm{d}}$ one-dimensional structures. Superior electronic conduction and charge storage capacitance in these nanostructures were observed.

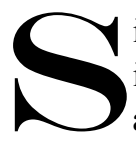
imultaneous electron and ion conduction is of great importance in understanding transport phenomena and for developing various energy conversion and storage systems. Of particular interest is charge transport on the nanometer scale, within which confinement effects may be observed. Electron and ion transport lie at the heart of recent DOE sponsored workshops that addressed the current status and possible future directions of some important research areas: solar energy utilization, solid state lighting, hydrogen fuel, superconductivity, and energy storage.

In this project, crystal and local chemical structures will be evaluated, and transport dynamics will be investigated by in situ nuclear magnetic resonance and impedance spectroscopy with a blocking electrode. The structure transport property relationship will be evaluated with respect to short- and long-range ordering of lithium ions, channel size for ion migration, exchange between lithium and transition metal ions, and magnetic ordering. A transport theory will be developed based on a space charge model and will be closely coupled with experimental studies.

In a conductor with reduced dimensionality, electron motion is free electron like along one-dimensional system axes, such as quantum wires or in planes parallel to the surface in a two-dimensional electron gas structure. One can assume that electron transport is parallel to confining potentials; hence, homogeneous transport properties in reduced dimensionality systems can be discussed in the same context as bulk systems. Further, homogeneous transport properties enable macroscopic phenomenological parameters such as mobility, conductivity, and thermopower to be measured in appropriately designed experiments.

During FY 2008, we employed in situ nuclear magnetic resonance with respect to $\mathrm{ZnO}$ proton characteristics to investigate proton dynamics in $\mathrm{ZnO}$ nanorods. Protons were clearly present in $\mathrm{ZnO}$ at ambient temperature; however, protons remained at elevated temperatures (between 200 and $500^{\circ} \mathrm{C}$ ) only in some highly aligned and uniform nanowires. The transport dynamics of protons associated with defects in $\mathrm{ZnO}$ nanorod lattices were determined from measurements of the temperature dependent spin lattice relaxation time. Because we could differentiate signals associated with proton defect states from the weakly bound surface hydroxyl groups, site specific dynamic information was obtained.

For FY 2009, $\mathrm{MnO}_{2-\delta}$ nanowires were chosen for our studies because they exhibit rich chemistry and crystalline phases. Also, the use and modeling of nanometer-scale $\mathrm{MnO}_{2}$ in the fields of catalysis, supercapacitors, and battery electrodes prompted further exploration to understand anomalous observations of size dependent behavior. In most of these applications, the redox reaction between $\mathrm{Mn}^{4+}$ and $\mathrm{Mn}^{3+}$ contributes to the unique property of $\mathrm{MnO}_{2}$. The $\mathrm{MnO}_{2}$ nanowires were synthesized by oxidation of $\mathrm{MnSO}_{4}$ with ammonium persulfate in a hydrothermal reactor, and precipitates were filtered and washed for further characterization. The nonstoichiometry of nanowires was studied by using a combined differential scanning calorimetry and thermogravimetric analysis.

During FY 2010, charge storage capacity measurements were taken on $\mathrm{MnO}_{2}$ nanostructures with various morphologies. From the non-rectangular shape of the cyclic voltammogram, it is clear that $\mathrm{MnO} 2$ with nanobelt shape (specimen D) exhibited pseudocapacitor behavior, whereas other morphologies (specimens A, C, and D) showed interior pseudocapacitance, thereby lack of sufficient redox process. The specific capacitance of specimen D was approximately $310 \mathrm{~F} / \mathrm{g}$, considerably greater than that reported in literature $(115 \mathrm{~F} / \mathrm{g}$ by Staiti and Lufrano). The capacitance (C) was calculated, following $\mathrm{C}=\mathrm{i} \cdot \Delta \mathrm{t} /(\mathrm{w} \cdot \Delta \mathrm{V})$, where $\mathrm{i}$ and $\Delta \mathrm{t}$ are the applied current and discharge time, respectively, and $\mathrm{w}$ and $\Delta \mathrm{V}$ are the weight of the manganese oxide on the electrode and the potential difference, respectively. 


\title{
Surface Damage and Environment-Induced Cracking Precursors in Light Water Reactor Components
}

\author{
Matthew J. Olszta, Danny J. Edwards, Mychailo B. Toloczko, Bruce W. Arey, Stephen M. Bruemmer
}

Unique insights into the influence of surface preparation and reactor aging will be obtained for the first time, which will define relevant precursor states. The fundamental understanding of near-surface microstructures as a function of key service parameters will lead to improved predictive methodologies for crack initiation and significant advances in the non-destructive detection of crack precursors.

A paramount issue impacting the performance, safety, and life extension of current light water reactors is the environment-induced cracking of structural components. Nuclear regulatory and industry groups worldwide have identified specific concerns within materials degradation management programs. Considerable work is still needed to be able to model long-term performance effectively and to secure the reliability of critical components. The stress data is used to understand stress states and water conditions that the components may have experienced during their lifetime and how crack propagation occurred. The results of this project will enable a better understanding of surface structure and morphology in light water reactor components exposed to practical surface treatments and service environments.

State-of-the-art, multiscale investigations of the restructured surface will provide quantitative insights into the depth, extent, and microcharacteristics of near surface damage layers. Detailed knowledge of deformation microstructures will improve understanding of time dependent corrosion/ oxidation processes and stress corrosion crack initiation pathways. Exposure to light water reactor environments at high temperatures will help elucidate the development of precursor states in restructured near surface morphologies leading to crack initiation and propagation. Using site-specific sample preparation techniques allowed by focused ion beam milling, the entire surface and near surface regions can be prepared and analyzed as one continuous uniformly electron transparent sample. The preparation and analysis will allow for a complete picture of surface structures and precursor crack initiation sites in light water reactor components.

During FY 2009, we developed a transmission electron microscopy sample preparation technique that provides a uniform electron transparent model at least 15 to $20 \mu \mathrm{m}$ into the sample. This was achieved using a unique focused ion beam technique. We also aimed to recreate surface damage conditions observed in industrial components using controlled laboratory experiments. Using a Struers polisher/grinder, we reached a level of polishing that induced little to no damage on the surface but also removed contamination from the cutting process used to make sample coupons. This was considered a satisfactory starting condition for all future grinding experiments.

\section{In FY 2010, we prepared} samples with ground surface microstructures and arranged them in a reverse u-bend geometry under constant loading, and exposed the samples in an autoclave to highly controlled high temperature pressurized water reactor water conditions in for an extended period to induce crack initiation. In FY 2011, we will analyze the exposed surface conditions.

Concurrent research was also performed to understand the various types of grinding that can occur in real-world nuclear reactors. Our research was designed for repeatable grinding conditions in order to study surface initiation,

but with grinding conditions in actual reactor settings performed manually, conditions can vary between operators. We collaborated with Pal Efsing at Ringhals Vattenfall to obtain a sense of what types and how grinding is performed. Samples of alloy 690, which had been studied for crack growth tests and showed unique surface oxidation characteristics, were treated to surface grinding protocols and exposed to high temperature water for $1000 \mathrm{hrs}$. The preliminary results indicate that the unique surface phenomenon is also observed in the surface damage layers, and thus may play an important role in initiation of this highly used reactor metal. The goal during FY 2011 will be to characterize this material fully and publish these results. 


\section{Three-Dimensional Structured Composite Nanomaterials for Energy Storage}

Xiaolin (Shari) Li, John P. Lemmon, Jiguang (Jason) Zhang, Jie Xiao

Next-generation energy storage devices such as high capacity or low-cost batteries are key enabling technologies to accomplish DOE's targets for transportation and stationary power supplies. Developing new three-dimensional structured composite nanomaterials will bring revolution to electrode materials and structures, therefore leading to advanced electrochemical storage devices.

$\mathbf{P}$ romising technologies such as the lithium-sulfur (Li-S) and sodium ion battery suffer from extremely poor stability or extremely poor cycle life due to the instability or the breakdown of the electrode materials during charge/discharge cycles. As the advance of electrochemical storage devices strongly depends on new electrode materials and structures, breakthroughs in electrode materials and structures are urgently needed. Three-dimensional structured composite nanomaterials will integrate different functional components (electroactive material, mechanically stable buffer material, highly conductive material, and capping material) into organized three-dimensional architecture in which the individual components will contribute to the desired properties while maintain the integrity of the overall structures. It is important to develop three-dimensional structured composite nanomaterials for energy storage.

To begin this mid-FY 2010-start project, we synthesized an ordered sandwich structured graphene-sulfur nanocomposite as cathode materials for the Li-S battery. The designed structure has a layer of few-layer graphene sheets and a layer of sulfur nanoparticles integrated into a three-dimensional architecture. Making full utilization of the efficient physical and electrical contact between sulfur and the large surface area, the composite highly conductive grapheme provides a high loading of active materials of around 70 percent, a high tape density of $0.92 \mathrm{~g} \mathrm{~cm}^{-3}$, and a high power Li-S battery with the reversible capacity of $505 \mathrm{mAh} \mathrm{g}^{-1}$ at the current density of $1680 \mathrm{~mA} \mathrm{~g}^{-1}$. Coated with a thin layer of cation exchange Nafion film, the shuttle of polysulfide anion from the GSS nanocomposite was effectively alleviated, leading to a reasonable cycling stability of 75 percent capacity retention over 100 cycles.

Transition metal sulfide based nanocomposites were also investigation as materials for $\mathrm{Li}$ based storage devices. More specifically nanocomposites of molybdenum disulfide $\left(\mathrm{MoS}_{2}\right)$ and poly(ethylene oxide) (PEO) were prepared by an exfoliation/ absorption method that involved the hydrolysis of lithiated $\mathrm{MoS}_{2}$ in an aqueous solution of PEO. The absorption and subsequent interaction of PEO on the colloidal $\mathrm{MoS}_{2}$ formed a nanocomposite which restacked into layered secondary particles.

Both x-ray diffraction and high resolution TEM indicated that highly disordered nanocomposites were produced when the $\mathrm{Li}_{\mathrm{x}}(\mathrm{PEO})_{\mathrm{y}} \mathrm{MoS}_{2}$ stoichiometry was limited to $\mathrm{y}<1$. A significant improvement in capacity and cycling stability was observed for the disordered nanocomposites. After further incorporation of graphene during the exfoliation process, extremely stable cycling performance is achieved in the $\mathrm{MoS}_{2} / \mathrm{PEO} /$ graphene nanocomposite.

For the sodium ion battery, we synthesized single crystalline $\mathrm{Na}_{4} \mathrm{Mn}_{9} \mathrm{O}_{18}$ nanowires by polymer-pyrolysis method using polyacrylates of $\mathrm{Na}$ and $\mathrm{Mn}$ as precursor compounds. The optimized $\mathrm{Na}_{4} \mathrm{Mn}_{9} \mathrm{O}_{18}$ nanowires have high crystallinity and homogeneous nanowire structure, which provide stable structure a)
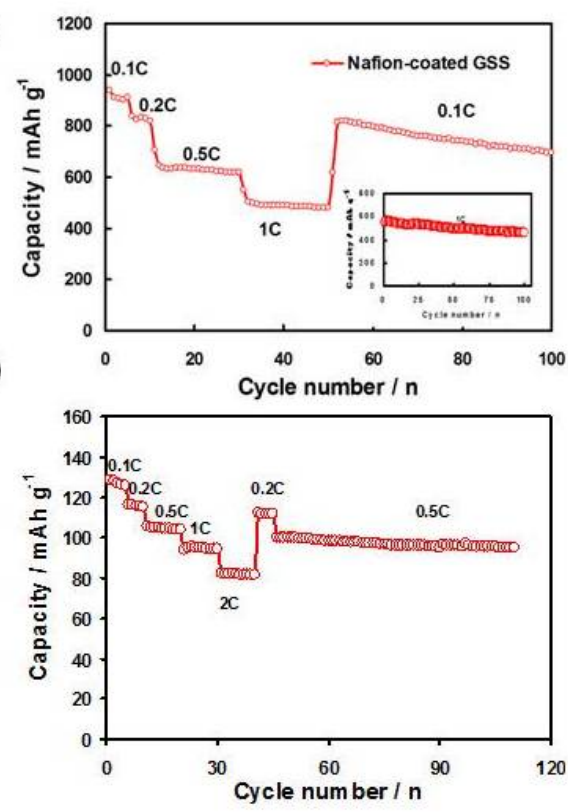

a) Cyclibility of nafion-coated GSS nanocomposite at different charge/ discharge rate. Inset shows cycle life of Nafion-coated GSS nanocomposite at a current density of $1 \mathrm{C}$, and b) cyclibility of $\mathrm{Na}_{4} \mathrm{M} n_{9} \mathrm{O}_{18}$ nanowires at different charge/ discharge. and short diffusion path for sodium ion intercalation/ deintercalation. The $\mathrm{Na}_{4} \mathrm{Mn}_{9} \mathrm{O}_{18}$ nanowires has high capacity of $128 \mathrm{~mA} \mathrm{~h} \mathrm{~g}^{-1}$ at $0.1 \mathrm{C}$, excellent cycleability of 90 percent capacity retention for 200 cycles and promising rate performance.

In FY 2011, we will try to optimize the graphene-sulfur composite cathode to increase cyclibility further. We will try different kinds of conductive carbon frameworks to optimize the electrode structure. We will also test different coating layers to alleviate the capacity fading. On the anode side, we will attempt different methods in order to protect the lithium metal. Finally, we will build a full cell with the best composite cathode and protected lithium anode. The success of this project will enable high capacity and low-cost energy transportation and stationary storage devices. 


\section{Mathematics and Computing Sciences}




\section{A Predictive Defense Model for the Smart Grid}

Ning Lu, Mark D. Hadley

It is critical to modernize our national electricity transmission and distribution grids while maintaining a reliable and secured electricity infrastructure. This research builds innovative and unique capabilities that bridge the critical gaps between data rich and information rich and between smart and secure to make sure that security is built into the smart grid from the beginning.

$\mathbf{T}$ he massive use of low-cost communication and electronics provides an explosion of information that bears different data formats, timestamps, with/without secured information interchange mechanisms. However, the smart grid is at its beginning stage with a yet-to-be designed predictive defense supervisory system that can efficiently process myriads of data to evaluate the status of the system, identify failures, predict threats, and suggest remediations. The innovation behind this research is the detection-in-depth process of information by dynamic filtering, multi-layer triggering, and cross-diagnosis to identify the nature of system events and incidents as security vulnerabilities in personnel, technology, or operation. Then, response can be taken effectively.

As proposed for this project, the prototype predictive defense modeling (PDM) tool will analyze data collected by multiple control and communication networks, characterize the states of the smart grid, recognize possible failure modes, vandalization attempts, and malicious attacks, and respond proactively or provide mitigation recommendations to remove or neutralize the threats. This proposed research and development will make full use of the abundant data collected by smart grid information technology layers while cross-checking to enforce data integrity. The idea behind the detection-indepth is similar to defense-indepth approach. It detects anomalies using several varying filtering methods against information

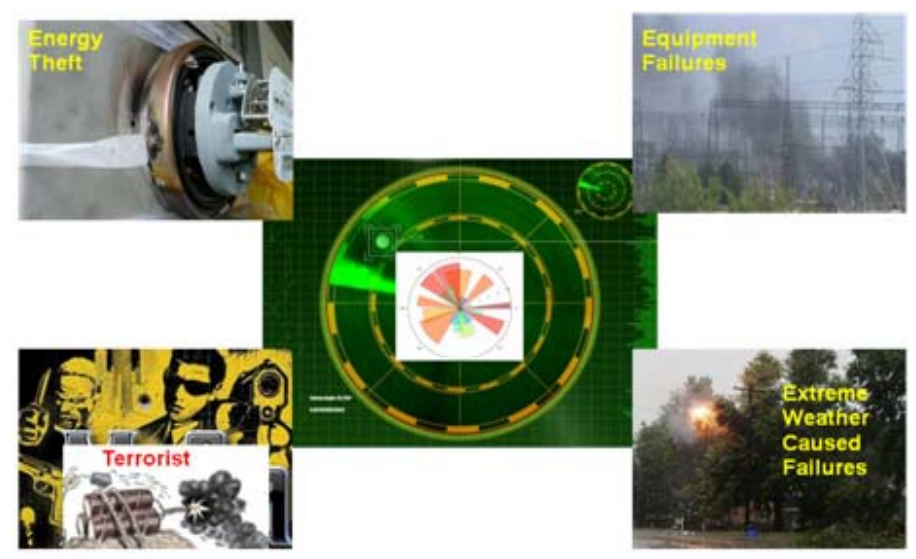

An illustration of the power grid cyber radar. collected from different control and communication networks. This will also provide actionable outputs to aid grid operators in responing to different events. Our goals for this project are to: 1) develop a power grid cyber radar that detects physical and cyber threats, predicts the likelihood of threats, and aids the grid operator to take proactive actions to remove, prevent, or mitigate threats, 2) focus on the PMU, SCADA, IT, and AMI networks, 3) reveal interdependency and correlations between data sets collected by different networks, and 4) create a multilayer reasoning process to rank the causes of an event.

The first phase of the project focused on setting up a smart distribution grid testbed to test or compare designs of an integrated information management system (I2MS). An I2MS extracts and synthesizes information from a wide range of data sources to detect abnormal system behaviors, identify possible causes, assess system status, and provide grid operators with response suggestions. The testbed provides a modeling environment with sufficient data sources for the I2MS design. The testbed includes five information layers anda physical layer; it generates multi-layer chronological data based on actual measurement playbacks or simulated data sets produced by the physical layer. The testbed models random hardware failures, human errors, extreme weather events, and deliberate tampering attempts to allow users to evaluate the performance of different I2MS designs. A centralized decision-maker "detector" examines the individual data sources for abnormalities. Then the detector cross-checks the synthesis of data sources to identify the cause and to determine the appropriate response. The data for all data sources were either generated from experiments or selected from typical data sets through simulations with cross-correlations and interdependency preserved. Initial results of I2MS performance tests showed that the testbed created a close-to-real-world environment that allowed key performance metrics of the I2MS to be evaluated.

A prototype I2MS design. A prototype has been built to demonstrate how I2MS is designed and how it performs. The user interface serves as a gateway to multiple data sources in a smart grid operation environment. Each modeled entity represents an information layer, and the major modeled entities in the prototype are the smart meter network, the supervisory control and data acquisition, the phasor measurement unit and outage management system layer, the utility customer information systems layer, and home energy management system. A centralized decision-maker "detector" examines individual data sources for abnormalities and cross-checks data source synthesis to identify the cause and determine appropriate response. The data were either 
generated from experiments or selected from typical data sets through simulations with cross-correlations and interdependency preserved.

Scenario settings. In an I2MS environment, abnormalities can be simulated. An abnormality can have several possible causes, like bad weather, equipment failure, cyber attack, and energy theft. For example, in energy theft scenario, the smart meter reading saved in "baseline" is manipulated. As a consequence, it can result in several abnormal phenomena (or data corruption):

- energy bill is significantly lower than average

- electricity consumption of neighboring households is higher than normal

- electricity consumption pattern is irregular

- energy consumption deviates from its past patterns

- outage rate of the tampered smart meter is high

- communication package from the tampered smart meter can be easily lost

- no energy discount issued to the customer.

One scenario (equipment failure, cyber attack, or energy theft) can trigger multiple changes in "baseline" data set; on the other hand, a change in "baseline data set" may be tracked back to different causes (or scenarios). This highlights the difficulty of detecting an abnormality using a single database and shows the need for and advantage of the synthesis of multiple data sources.

Test results. An I2MS design gives operators the ability to detect the altered situations and provides mitigation guidance. After scenario setup, corresponding data are examined by "detectors;" the question is whether we can identify the scenario by looking at the tampered data set. Essentially, the reasoning process built into detectors uses logic steps as a descriptive means for determining the interdependence between data and different scenarios and calculating probabilities. The logic process is adaptively changed to reflect different operation conditions (e.g., seasonal variations). One example of detector output can list several scenarios with bars to represent probability. The most likely cause for tampered data is bad weather, while other events (cyber attack or energy theft) are not possible.

In FY 2010, we finished design considerations, physical and information network configurations, data flow generations, detector selection and placement, and testing results of a distribution grid testbed that allows researchers to design and test integrated information management systems. The test system models the interdependency and correlation among different data sets and provides an environment that closely resembles actual data collection systems. It provides us with a less expensive yet effective tool to evaluate the performance (such as hit rate and false alarm rate) of an I2MS in anomaly detection.

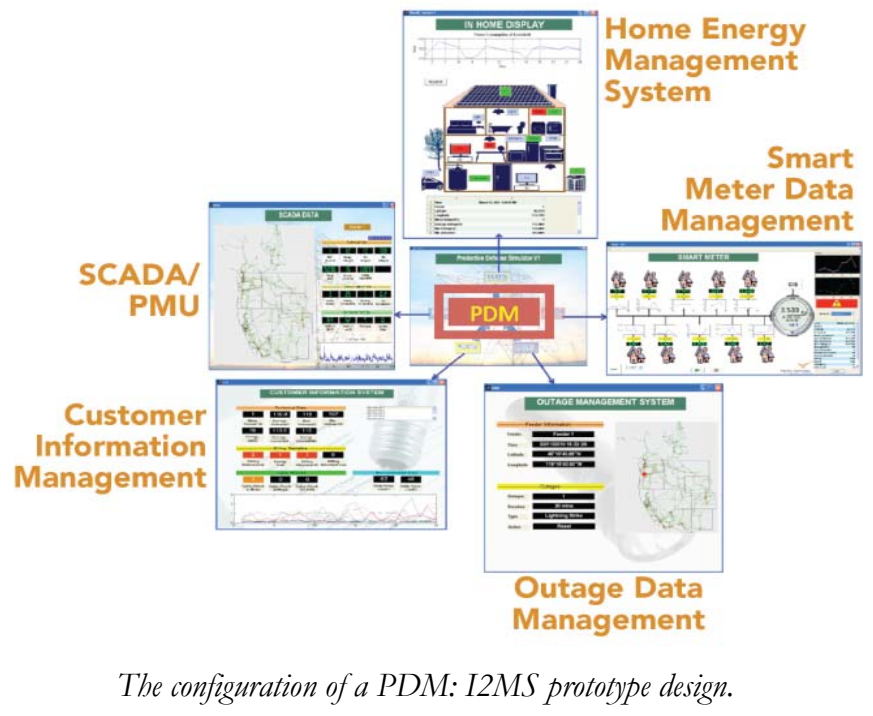

Because I2MSs for different anomaly (behavior) detections require different information flows, the testbed allows its user to add data flows (either real measurements or physical-based models), as long as the correlation and interdependency of data streams are correctly maintained. Uncertainty in the detection process has been accounted for by using a fuzzy membership function. Grid operators are notified of all possible causes (ranked by likelihood) of an anomaly to help them take actions based on information from the smart grid infrastructures.

The investment in smart grid infrastructure is availability of high resolution data collected from more measurement points. To monetize this benefit, information that can facilitate the grid operation, maintenance, and planning needs to be extracted from these data sets and made available to grid operators in an actionable manner. A testbed that can model information flows is therefore important to test and benchmark the performance of information management systems. In this way, our research can focus on developing detection and reasoning algorithms to enforce situation awareness and the proactive response to system anomalies. Specifically in FY 2011, we will transform our PDM tool into a cyber radar that can scan smart grid data to detect anomalies. A cyber radar is a radar scanning data in the cyber space, not simply a cyber attack radar. It detects events or possible events such as equipment failures, abnormal operation (intended and non-intended) and ranks their causes by confidence levels. Based on the smart distribution grid testbed, we will develop a procedure to allow anormalies to be created by a red team. A blue team will focus on detection and reasoning algorithm development. Students can be involved to form red teams to prepare anormalies. Different algorithms can be evaluated for effectiveness to detect those anormalies. Because real data sets are difficult to obtain and rarely sufficient for low probability events, this approach can make the algorithm development cheaper and more robust. 


\title{
A Scalable Fault Tolerance Infrastructure and Algorithms with Programming Models and Scientific Applications
}

\author{
Abhinav Vishnu, Wibe A. de Jong, Hubertus J. J. van Dam, Shuaiwen Song
}

Delivering fault tolerance on exascale scale computing platforms with millions of processing elements and components will enable leading edge scientific applications to fully utilize the performance of these systems to accomplish scientific discovery and innovation that will transform the nation.

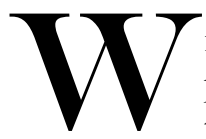

ith the arrival of petascale scale computing platforms and the advent to exascale systems, the high performance computing community is entering an era of combining millions of processing elements and components. Besides achieving peak performance out of these systems, one of the major remaining challenges is how to handle reduced mean time between failure of various system components. Providing fault tolerance to the system software stack is imperative to achieving any realistic goals of sustained peak performance as well as enabling scalable application software to utilize these systems effectively to accomplish leading edge scientific discovery and innovation.

We are developing a fault tolerance management infrastructure layer comprised of various modules like fault detection, propagation, prediction, and prevention for one or more system components. This process will underpin a fault tolerant global arrays toolkit layer and utilize infrastructure to deliver fault tolerant computational chemistry algorithms for high accuracy coupled cluster triples and density functional theory in the high performance scalable computational chemistry software NWChem. Specifically, we will add capabilities to NWChem to handle processor faults and be able to recover from them without using the checkpoint/restart mechanism.

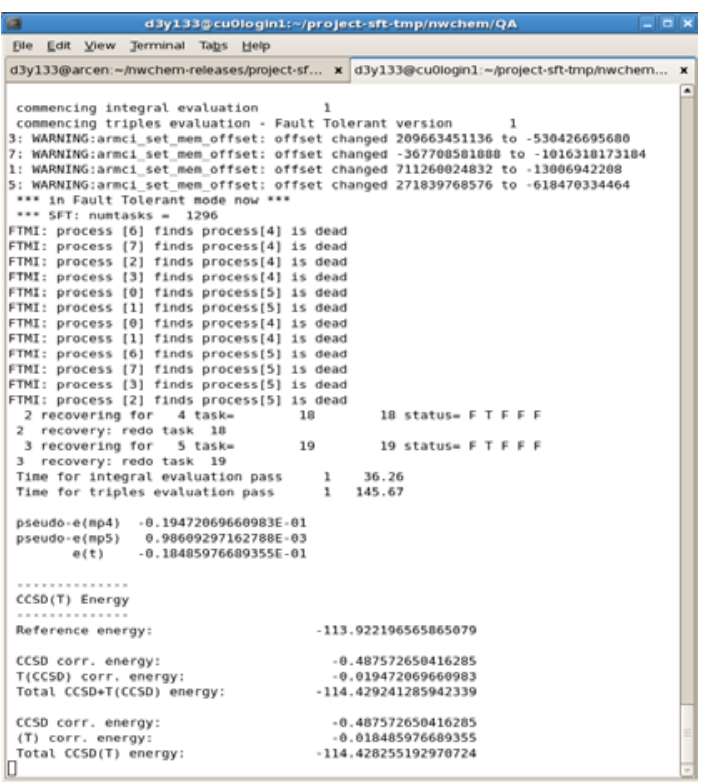

The output of a run on eight processors across four nodes, demonstrating the successful recovery from a single-node failure. detection, resilient communication protocols, a messaging layer for arbitrary data communication, and a fault tolerant process manager in addition to fault resilient, non-data moving collective communication operations. Using global arrays and ARMCI as the research and development vehicle, we began to demonstrate the fault resilient execution of communication benchmarks with a hard node fault. The fault tolerant communication infrastructure is in publication for the 2010 International Conference on High Performance Computing.

Within NWChem, an approach to building fault tolerant applications using (and on top of) global arrays was designed and implemented to provide required fault tolerant infrastructure to the application. With this infrastructure, a fault tolerant version of the coupled cluster with single, double, and perturbative triple excitations (CCSD(T)) was implemented. The $\operatorname{CCSD}(\mathrm{T})$ capability was an initial target because it is computationally very expensive. This capability was successfully demonstrated for a single hardware failure, and a manuscript documenting this approach was submitted to the Journal of Chemical Theory and Computation.

In FY 2011, we will target mechanisms for efficient fault information propagation, providing FTMI for handling simultaneous and consecutive faults, reducing its overhead introduced by introduced redundancies and making the code more robust. We will also develop the fault tolerance management infrastructure for leadership class machines such as BlueWaters and Cray based systems. On the application side, fault tolerance for the iterative CCSD part and the draft functional theory will be developed. Additionally, we will integrate the current infrastructure layer with global arrays to offer it to a larger developer community. This will help with wider recognition for the work and enable making other scientific applications fault tolerant.

In FY 2010, we developed the initial fault tolerance management infrastructure that provides efficient fault 


\title{
A Statistical Framework for Integrated Explosives Detection
}

\author{
Kristin H. Jarman, Kenneth D. Jarman, Nathaniel Beagley, Mark F. Tardiff
}

Next-generation devices for explosives detection and characterization need to be portable, robust, and insensitive to various backgrounds introduced by diverse environments. The current project facilitates this goal by developing integrated data analysis of information from different screening and detection devices. provide enhanced results and how the framework can be used to make sensor system design decisions. We expanded our efforts to accommodate systems with temporally or spatially varying components, such as systems that monitor vehicles as they move or an aerial survey of potentially hidden explosives over a pre-defined area. A hierarchical Bayesian net model was developed for this type of explosives detection problem. Key insights included error rates being generally lower with integrated sensors; characterization of clutter, interferents, or nuisance factors on each sensor as crucial to improving performance; and increasing the number of sensors to reach work alone or in combination with other sensors, these technologies collect, preconcentrate, and detect explosives and analyze results that enable end users to make reliable decisions. For optimal performance, a decision analysis framework is required to take data from multiple disparate sensors and convert the information into meaningful results. The objective of this research is to develop a sensor integration and decision analysis methodology can propagate uncertainty through a multi-sensor detection system and perform Bayesian integrated explosives detection.

The Bayesian framework was applied to two operational scenarios: passenger screening and the passive surveillance of people entering a large event. The first was demonstrated on existing data in FY 2008. In FY 2009, available data were used to demonstrate the potential for the Bayesian framework in the second scenario. The case study used was passive screening of spectators entering a large venue using three different camera technologies (near-infrared, mid-infrared, and mm-wave). As we integrated the three sources, we found that error rates were generally lower than if a single or two data sources were used. Layers of clothing on screened spectators affected performance of infrared (but not the mm-wave) camera, thus lowering the performance of the overall integrated system. This underscores the need for system designers to understand how interferents, nuisances, and background clutter might adversely affect sensor performance and explosives detection integrated systems.

Our FY 2010 objective was to build on the Bayesian integration framework. Our previous years of project work demonstrated how data from various sources are integrated to rapidly diminishing return in performance improvement.

The hierarchical Bayesian net model used the previously developed framework for integrating multisensor data at a single location at a single time within an area of interest, and aggregated threat probabilities over Markov chain concern index. The model is appropriate for monitoring scenarios using sequential testing methods and is in a class of stochastic processes with a long history of theory and application. Data from the guardian angel sensor fusion tests performed by the Department of Defense Joint Improvised Explosive Device (IED) Defeat Organization were used to demonstrate hierarchical sensor data integration with the following conclusions:

- The modeling exercise indicates data (specifically that on which individual sensor false alarm probabilities can be estimated) and feature information from raw data that enable generating benign and threat probability distributions needed to evaluate sensor systems in application using this approach.

- The modeling exercise suggests further designed data collection and system evaluation exercises that better mimic field operations (e.g., monitoring areas for IEDs over time, including areas with no IEDs, as opposed to target characterization studies).

- Existing theory and application to different monitoring scenarios (e.g., nuclear safety and manufacturing systems) enable the straightforward calculation of performance estimates and system design methodology. multiple locations and time into a 


\title{
Adaptive Cyber-Defense Using an Auto-Associative Memory Paradigm (ACAMP)
}

\author{
Ryan E. Hohimer, Frank L. Greitzer, Shawn D. Hampton, Patrick R. Paulson
}

We intend for this project to create advanced methods of pattern recognition in cyber streams, enabling more secure systems. This research is visionary in its employment of a conceptual model informed by a functional mimicry of the neocortex - the ultimate pattern recognition system - that seeks to develop an unprecedented dynamic, adaptive cyber defense system with potential applications to diverse problem domains.

$\mathbf{T}$ he trend in software exploits is changing from operating systems to application software, and the list of potential exploits is rapidly growing along with the application space. Hackers can take advantage of this trend, as it provides a large library of exploits to use to gain access, traverse, manipulate, and pilfer. Understanding and analyzing the changing patterns in the use and sequences of software exploits is crucial. Attack graph calculations are tools used in the fight against hackers. Modeling potential exploits within a network can help defenders to understand potential weakness in their IT systems. If a network configuration exactly matches the requirements needed

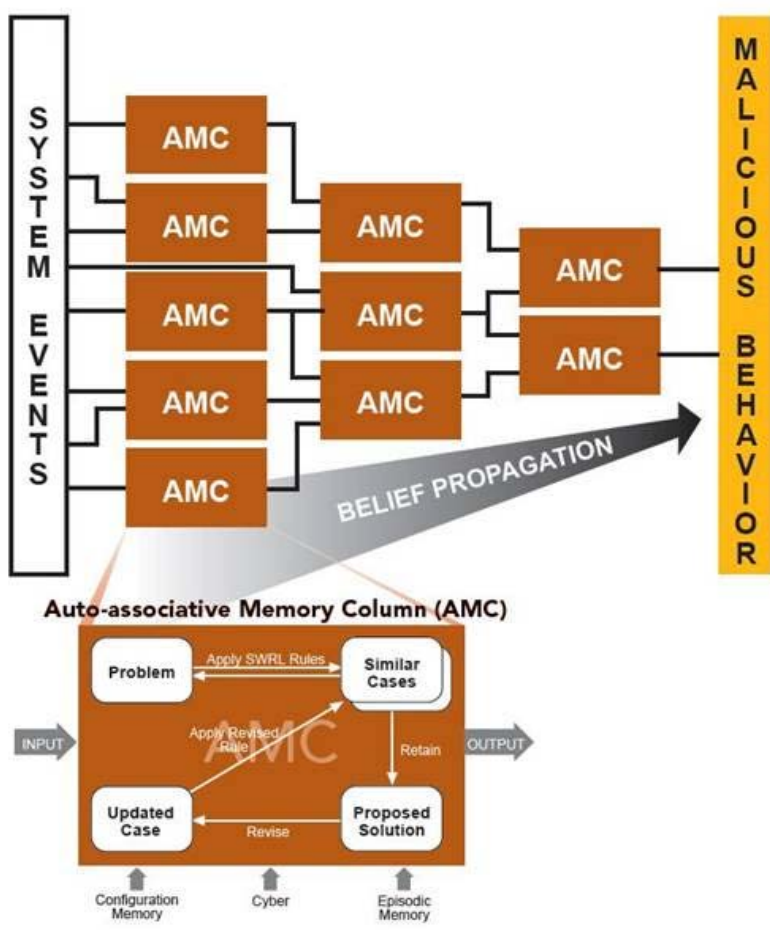

The ACAMP beliefpropagation system is comprised of a bierarchical set of case-based reasoners (e.g., AMCs) that enable adaptation and support learning and generalization. We use rules (such as semantic web rule language) to determine of the input is recognized; if so, it is retained in memory. for a modeled exploit, then attack graphs can determine and highlight the network vulnerabilities to the exploit. If, however, the network vulnerability or new exploit varies from those used to construct the attack graph, the vulnerability becomes undetectable. The more creative (i.e.,unusual or unfamiliar) the exploit, the more likely that existing attack graphs will not be useful. To this end, an adaptive and dynamic understanding of the changing network configuration and patterns of exploit activity is needed.

Inspired by the human sensory system and the brain's ability dynamically and adaptively process sensory input, this research will functionally mimic the neocortex to bring dynamic and adaptive capabilities to the cyber-defense domain. While existing technologies are limited in pattern matching ability to compare inputs with fixed templates, the functionality of the neocortex exhibits a richer, more adaptive capability that "remembers" similar past patterns that have been experienced. The neocortex creates and stores sequences of patterns, recalls these patterns auto-associatively, stores them in an invariant form, and stores them in a hierarchy. With this auto-associative memory paradigm, the neocortex is incredibly good at pattern recognition. Using a software architecture inspired by the functional model of the neocortex, we will provide a new and transformational technology that advances understanding of the context and complexities of external attacks on an information technology system.

Based on an inspirational metaphor, we seek to create significant advances in adaptive pattern recognition processes that yield dynamic, adaptive functions for recognizing and anticipating malicious behaviors such as malware exploits. Following from the functional model of the neocortex, the ACAMP functional requirements are to:

- create and store sequences of patterns to raise the level of analysis beyond individual/isolated data to enable the system to identify behavioral patterns

- recall sequences of patterns auto-associatively to produce dynamic detection capability within a "memoryprediction" framework

- store the sequences of patterns in invariant forms to enable system functions that support adaptation and generalization capabilities

- store the sequences of patterns in a hierarchy to facilitate growth and "learning" functions of the reasoning system. 
This research advances the state of the art in model development and reasoning system design in two key respects: the system design uses an innovative hierarchical network of reasoners, and the reasoner design extends traditional case-based reasoning to yield knowledge-intense case based reasoners (KI-CBR), which are themselves enhanced by modifying the traditional reasoning cycle to include analysis of invariant forms of stored patterns (i.e., patterns previously gained from experience or elicited from domain experts). To demonstrate domain independence, we selected a second application context (insider threat) and initiated knowledge acquisition to implement this application.

Accomplishments and products of our research during FY 2010 include the following developments. We created a conceptual and technical design (specifications) of the system captured in the ACAMP design document, executed an alpha reference implementation, and formulated a prototype demonstration system for selected domain applications. Specifically, we defined a dynamic signature based approach that analyzes malcreant behavior patterns, focusing on semantic content, not just syntax. We have designed and implemented an initial hierarchical structure of reasoners that can interpret sequences of patterns in inputs based on memories of cyber-defense knowledge, episodic knowledge of past experiences, and knowledge of system configuration.

Project plans for FY 2011 are to continue the development of the KI-CBR technology and auto-associative features of the ACAMP model, instantiation of the model and associated ontologies for two application domains and to design and conduct a modest evaluation of the system's performance. 


\title{
Battle Damage Assessment for Cyber Warfare
}

\author{
Richard W. Quadrel, Thomas E. Carroll
}

- The goal of this project is to develop automated capabilities for the (near) real-time assessment of damage inflicted by a computer network attack to an organization's information technology (IT) infrastructure. The damage is reported in terms of high-level concepts such as missions and capabilities, not systems.

$\mathbf{T}$ he use of IT hardware and software has become an integral component of modern enterprises and organizations. Because of this importance, the technology is constantly subjected to threats. Virtually every organization is dependent on information that is accessed, stored, and processed in cyberspace to support quality and timely decision-making. This dependence introduces significant risk to cyber assets that impacts real-world missions and operations. Despite our best efforts at developing cyber defense capabilities, attacks do and will occur, and some will successfully compromise the operation of mission-critical systems. Thus, we need quickly and accurately to identify damage and reports its impact.

Unfortunately, the IT system implementation is often flawed, which makes it vulnerable to disruption and degradation. The implementation of cyber security technology is necessary just to maintain nominal system operation. Cyber security typically focuses on detection and prevention, but little focus is given when defenses fall and systems are compromised. Presently, there are limited capabilities for assessing cyber battle damage to networks and systems. The current state of the practice involves a multitude of security tools and utilities that were never designed to interoperate manual resource-intensive methods and techniques. Contemporary practices are time-consuming and often provide an incomplete assessment of the damage.

With the above challenges in mind, we intend to provide an automated, near real-time assessment of the nature and extent of damage to networks and systems following a cyber attack. We propose a multi-agent system that monitors for damage and robustly transmits notification of it to gain a rapid, comprehensive assessment and evaluation of mission capabilities. Information about capabilities will assist cyber attack victims to assess rapidly the extent of damage following an attack and determine the impact on the organization's missions and capabilities. From this information, leaders can make informed decisions in managing resources, public relations, and other facets of organizational operations.

For FY 2010, we limited our focus of cyber threats to computer network attacks, which are actions that degrade performance or capability (effectively denying service to those resources) or compromise data integrity. A multi-agent system monitors performance and data trustworthiness in evaluating mission capability. Agents are categorized into types, each of which dictates what tasks or duties agents perform. Upon detection of the damage, agents report the information via a virtual overlay network to analysis agents who aggregate the data and perform an assessment. Components of multi-agents systems are typically distributed throughout a network and, because of this quality, the system is better able to tolerate failures and survive cyber attacks.

The monitoring framework is a distributed multi-agent system, which is inherently extensible and scalable due to its decentralized nature. It is more likely to survive cyber attacks by way of redundancy and tolerance to failure that is extrinsic to distributed systems. The framework comprises a hierarchy of agents; each agent is responsible for verifying the functionality of the agents below it. Our efforts focused on the lowest levels of hierarchy: sergeants and virtual sensors that share monitoring resource duties. Sergeants are pre-positioned in the network. Once the battle damage assessment functionality is invoked, the sergeants generated mobile virtual sensors to detect the damage. Using a challenge-response protocol, data are transmitted by virtual sensors back to the sergeants, where information is interpreted. The sergeants then generate reports on resource fitness. We developed virtual sensors that can be used to detect damage to web applications. The sensors collect information on the availability and integrity of world-wide web servers, application response integrity, and content integrity. We have also exercised the agents on a testbed simulating a small network.

The mission risk assessment models allow us to reason about the inter-relationships between networks, systems, and other resources and enable tracing impact of degradation due to dependencies. Instead of providing a numeric risk impact statement (e.g., mission impact is scored a real number in the interval $[0,1]$ ), we report impact in terms of quantifiers: critical, high, medium, low, and none. We developed a framework to support the development of assessment models, including an ontology to describe battle damage assessment. To date, we have modeled a small network of seven systems that executes four mission-critical services.

This project is slated to continue into FY 2011. The objectives for the year include developing schemes for the agents to self-organize, resilient communications between agents, and the processing of live monitoring reports into the mission risk framework. 


\title{
Comparing Performance on Different High Performance Computing Architectures
}

\author{
Mahantesh M. Halappanavar, John T. Feo, John R. Johnson, Daniel G. Chavarria-Miranda
}

The aim of this project is to conduct performance analysis on different architectures for applications that have irregular memory access patterns such as graph algorithms. Performance will be conducted on datasets chosen to be representative of a variety of applications of interest.

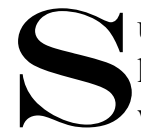
upercomputers are increasingly characterized by hundreds of thousands of compute nodes linked with fast interconnects. The nodes communicate by passing messages. With the introduction of multi-core processors and GPUs, the architecture of individual nodes has increasingly become complex with deep hierarchies for memory caches and communication. Developing scalable implementations for applications with irregular memory access patterns such as graph algorithms on traditional distributed-memory architectures is challenging. The key bottlenecks to performance include data partitioning (load balancing), irregular memory access patterns, low computation-to-communication ratio, and inherently serial nature of algorithms. Moreover, the structure of input directly influences performance.

Special hardware-software co-designed architectures such as the Cray XMT have been shown to address these challenges well. Features of XMT of interest to this project include the large globally addressable memory (1 TeraByte) that alleviates the data partitioning problem, massive multithreading (128 hardware threads per processor) that provides latency tolerance not only for memory stalls but also for synchronization, light-weight synchronization mechanisms (full/empty bit and extended memory semantics) that enable development of fine-grained parallel algorithms, and compiler-driven automatic parallelization that allows rapid software development. While similar studies have been done previously for graph algorithms such as breadth-first search and betweenness-centrality, this project is a controlled scientific study to compare architectures objectively on a combination of algorithms and inputs for which no large-scale study currently exists. In this way, our work is different from past studies in its selection of algorithms, architectures, and test datasets. While being simple to understand, graph coloring and matching are prototypical graph algorithms with numerous applications in science and engineering.

In FY 2010, we developed multithreaded implementations for distance-1 graph coloring and half approximation weighted matching. We conducted extensive experiments on Cray XMT, Sun Niagara2, and Intel Nehalem platforms using a carefully chosen set of synthetic graphs representing a variety of real-world scientific and informatics applications. We characterized input data to provide insight on performance. The three platforms cover a broad spectrum of architectural features, including clock speed $(0.5 \mathrm{GHz}$ for XMT vs. 2.5GHZ for Nehalem), cache hierarchy (cache-less for XMT vs. three levels for Nehalem), and multithreading (128 threads per processor for XMT vs. 2 threads per processor for Nehalem). While XMT and Nehalem are two extremes, Niagara2 provides a middle path by synergizing hardware multi-threading ( 8 threads per processor) with shallow cache hierarchy. We also developed new algorithms for coloring and matching based on dataflow principles to exploit special hardware features on XMT. We also compared performance of these algorithms to our existing implementations and results on IBM BlueGene/P and Cray XT-4. The coloring algorithms are being integrated into a separate project application for hierarchical Bayesian modeling of large, complex data.

In addition to the above-mentioned progress, we demonstrated a near-linear speedup for Erdős- Rényi random graphs and moderately structured Scale-free graphs with power-law degree distribution and small-world characteristics, and moderate speedups for scale-free graphs with difficult structures across all three architectures. We characterized the input graphs and provided insight on performance bottlenecks. To the best of our knowledge, the size and variety of input graphs explored (up to a billion edges), amount of concurrency used (up to 12,800 threads), and variety of architectures considered is first of its kind for graph coloring and matching.

Backed by experimental results, we can draw general conclusions about parallel graph algorithms: 1) multi-threading provides an efficient way to tolerate latency, which can be seen from experiments on the massively multithreaded XMT and Niagara2 platform, where we observe that $T$ threads running on one processor has similar runtime as one thread running on $T$ processors, 2) the negative impact of clock frequency and cache hierarchies can be tolerated with a greater thread concurrency, as can be seen in the better performance obtained on XMT and Niagara relative to Nehalem, 3) parallelism should be exploited at fine grain if supported by light-weight synchronization mechanisms in hardware, and 4) graph structure critically influences performance of parallel graph algorithms. We expect these insights to be helpful in the design of high performance algorithms for irregular problems on the impending hybrid and many-core based architectures. 


\title{
Cyber-Attack Risk Inference Model
}

\author{
Patrick R. Paulson, Jonathan Young, Thomas E. Carroll, Peter A. Neorr, Chitra Sivaraman
}

- This project uses formal risk modeling methodologies to describe cyber assets of an installation in terms of the risk that compromise of those assets will present to the organization. We feel that this approach will improve the ability of administrators of cyber systems to prioritize their time and resources, offering improved security for both cyber systems and associated infrastructure.

D ialogue with the intelligence community indicates that cyber security professionals generally assume that their systems are vulnerable to infiltration or have already been infiltrated to some degree. With limited cyber security resources, the question arises of how those resources might be most effectively allocated to prevent or mitigate the prospective consequences of infiltration. This project will provide methods to prioritize responses available to a system administrator to minimize potential consequences to an organization's mission.

We are creating a model to provide a dynamic risk-informed framework to prioritize the proactive protection of assets and mitigation of attacks already initiated, thus resulting in a basis for the effective use of limited security resources, achieved through development of: 1) the analytic framework in which to characterize modes of cyber attack systematically, relevant protective systems, and potential consequence severities (in terms of cost impact of compromised assets and disruptions to operational continuity), 2) a repository of attack scenarios identified through analysis, elicitations, and literature review, and 3) a means of risk quantification based on probabilistic or more recently explored non-probabilistic methods.

During FY 2009, we evaluated candidate application domains on specific criteria and selected supervisory control and data acquisition. We defined a model framework for calculating consequences and effects of mitigations and a data model for holding application domain specific knowledge. Also, we elicited and constructed a prototype model in the supervisory control and data acquisition domain. In FY 2010, we developed a model exploration tool that allows domain experts to examine the state of a modeled system in terms of potential risk and assets with the greatest influence on risk. When a particular mission is selected, the model displays assets that contribute most heavily to the risk associated with that mission. Lines between assets indicate the strength of influence other assets have to particular asset risks; these links can be followed to determine how CARIM determined which asset contribute to mission risk.
A major accomplishment was creation of a planning facility that allocates resources efficiently to address risk across multiple organizational missions. The planning is carried out in a manner that improves the risk profile across all organizational missions, rather than balancing the risk in one mission against other missions. The planning tools allow a planner to specify the levels of manpower, dollars, and calendar time to determine which actions will produce the best reduction in risk across all organizational missions. Additionally, we instantiated the domain and installation data models and created initial domain models for SCADA, business continuity, cyber security, and emergency power grid response. We also developed several software tools to facilitate model definition and instantiation.

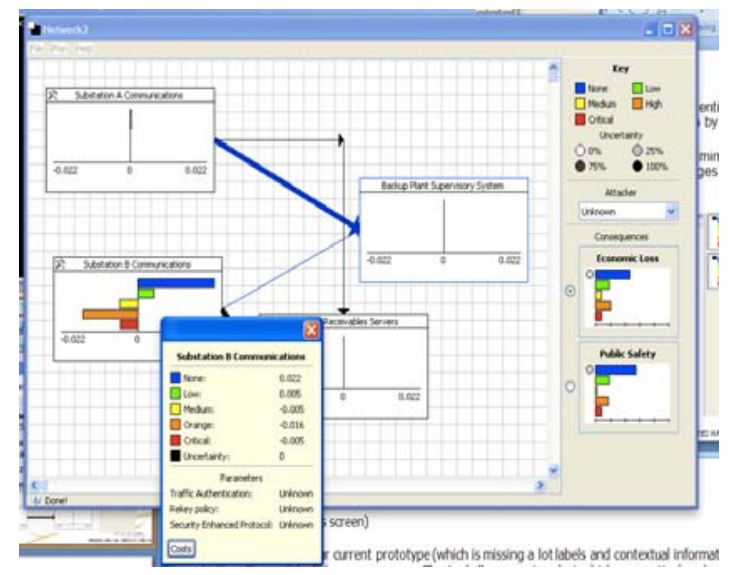

The CARIM model exploration tool, showing interactions between modeled assets and potential risk from multiple perspectives.

One of our goals for 2010 was to determine if our methodology was best suited for configuration or for active monitoring of systems. Our research showed that active deployment of CARIM required little modification, and we developed API specifications to notify a deployment model of detected events to provide dynamic risk assessment.

For FY 2011, a new GUI for the deployment model will be designed that will meet the needs of operators, rather than subject matter experts and developers. An interface to the resource allocation and planning capability will be developed. In addition, the scalabilty of the approach will be examined. While there are few theoretical constraints, we are limited in practice by the COTS Bayesian network software we currently use. We will also make improvements to the user interfaces for domain model and installation model elicitation and deployment model generation and provide domain models as needed for proof-of-concept in additional problem domains. 


\title{
Data Decomposition/Optimizations and Dynamic Load Balancing Mechanisms for Extreme Scale Computing in the Global Arrays Toolkit
}

\author{
Manoj Kumar Krishnan, Bruce J. Palmer
}

The goal of this research is to develop capabilities in the global arrays (GA) toolkit to support flexible data decomposition, communication optimization, and dynamic load balancing approaches for communication intensive molecular simulations and subsurface modeling. To address the extreme-scale computing challenges in GA applications, we will focus on developing flexible data decomposition, dynamic load balancing, and locality and communication optimization capabilities.

D ata decomposition is a highly effective technique used to distribute data among processors. The performance of applications can be significantly improved by selecting an appropriate data distribution. Balancing the computation load amongst the available processors is crucial to effectively exploit and scale applications on extreme scale systems. Different applications exhibit different types of load imbalance, and the appropriate choice of load balancing scheme depends on the computational structure of the applications. In addition, the complexity of extreme-scale systems presents several challenges to largescale scientific applications. In order to achieve scalability and performance in these systems, data decomposition, communication, and dynamic load balancing play a key role for several applications, including molecular simulation and subsurface modeling.

We will focus on providing the following capabilities in GA: flexible data decomposition, dynamic load balancing, and locality and communication optimization. This project will add new capabilities within the GA programming toolkit to address extreme-scale computing challenges in GA applications. We will demonstrate the effectiveness of new capabilities in the context of two important applications areas (molecular simulation and subsurface modeling) of interest to the larger extreme-scale computing community. We expect to publish our research findings in leading high performance computing conferences and journals.

In FY 2010, we developed a limited feature flexible data distribution mechanism for GA toolkit based on Restricted Arrays capability in the GA toolkit (e.g., Hilbert curve and Morton's Z-order). We designed and implemented symmetric multi-processor (SMP)-aware topology distribution for better performance as the current GA model (and most PGAS models) assumes flat hierarchy for data distribution. We also designed and implemented network-topology aware GA for infiniband Linux clusters (e.g., Chinook at PNNL).
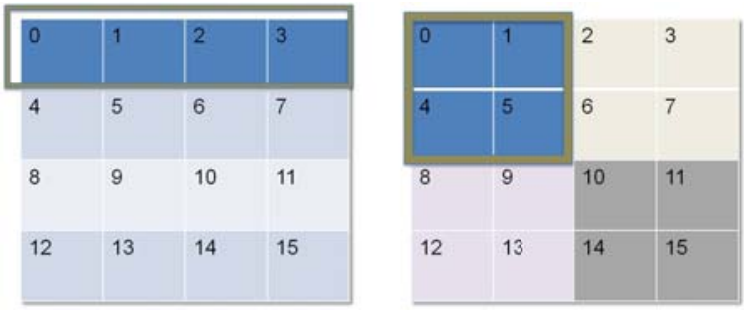

Block-based data decomposition (regular, SMP-aware).

The performance of topology-aware GA is demonstrated using a GA-based ghost cell update kernel on Chinook. We noticed that the topology aware ghost cell update performed 30 percent better than the topology oblivious kernel. We have also designed a GA based scalable FFT algorithm to demonstrate the benefits of various data distribution. Additionally, we have an initial design of scalable task counters to develop fine-grain scalable work stealing schemes for dynamic load balancing. We worked to optimize the nearest neighbor communication and synchronization in order to improve GA scalability in STOMP's ghost cell update algorithm.

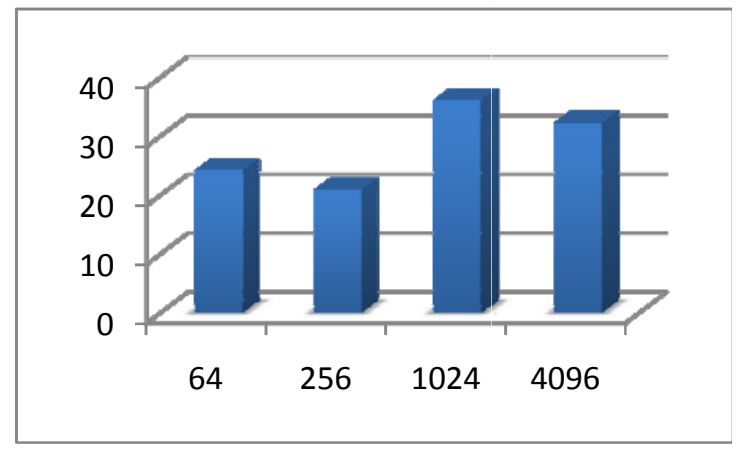

Performance improvement in ghost cell update algorithm using topology-aware distribution (topology aware versus topology oblivious $G A$ ).

For FY 2011, we will extend the current block-based distribution in GA to support block distributions based on data access pattern in applications and topology (intra-node and inter-node). The data access or communication pattern in applications can be classified as regular and irregular. For the regular access pattern, we will develop techniques for topology-aware mapping of data to processors. Based on the data access pattern, we will develop data distribution templates for GA applications. For irregular applications, we will develop dynamic load balancing techniques to deploy topology-aware placement of tasks in an application. 


\section{Deception for the Defense of Cyber Systems}

Thomas E. Carroll

This project investigates deception and its value in cyber defenses. We are modeling deception mechanisms using game theory and other tools from economics in order to draw conclusions about the viability of deception in augmenting system defenses.

D eception has a long history within warfare. The successes in this area have led researchers and practitioners to use it in the defense of cyber systems. Deception creates uncertainty for attackers, as they will respond by expending additional resources and taking disadvantaged courses of action. Defenders will conserve their resources, and the attackers' disadvantaged courses of action will contribute to detection. The study of deception is complex; there is no current unified theory that sufficiently models it. One reason for this is that historically attackers witness a specific deceptive technique only once in their lifetimes; another is that it is difficult to prepare for deception. However, in the context of cyber systems, effective defenses are widely deployed, giving the attackers many opportunities to experiment. This benefits us in that game theory, the study of adversarial interactions, can then be applied.

For this project, we are studying deception for the defense of cyber systems, and we are examining specific deceptive techniques by developing models based on game theoretic foundations. Conclusions are drawn from these models about the value of deception and how to deploy it efficiently. By applying models that combine game theory and cognitive reasoning, we are investigating the benefits of deception in protecting cyber systems. Each deception technique is being examined under various attacker capabilities and with basic learning. The outcome is to demonstrate that deception efficacy depends not only on the attacker's capability but also exposure to the technique.

As an attacker becomes familiar with a specific technique, efficacy should diminish.

We first examined fake honeypots, which are a common practice used when defending a network. A honeypot is a system formulated to detect or deflect unauthorized use and access. Attackers are aware of honeypots and will avoid them when possible. Through experience as well as experimentation, attackers have cataloged

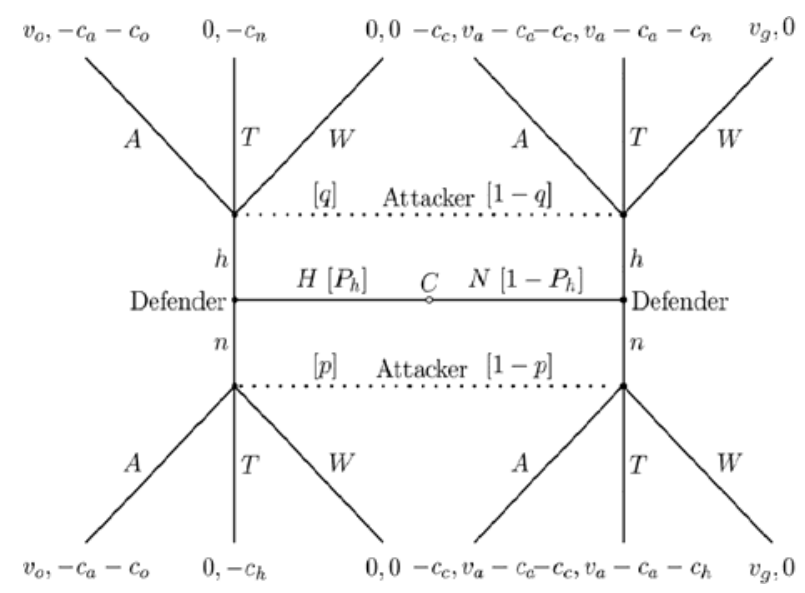

The signaling representation of the fake honeypots game. observances that can be used to distinguish honeypots from normal systems. Fake honeypots take advantage of the attackers' aversion to honeypots. Because the system shares attributes with a honeypot, attackers believe that the system is a honeypot and will avoid them.

We developed a mathematical game that models the interaction between network defenders administrating honeypots and fake honeypots and attackers that are attempting to breach the network. Designed as a signaling game, this dynamic process has the defender and attacker alternate turns. In the first move, the defender integrates honeypots and fake honeypots into the network defenses. The attacker then probes the defenses and determines whether to attempt to breach the systems. If a honeypot is encountered, the attacker loses, as the defender can detect the attacker's presence; if attacking only normal systems, the attacker successfully breaches the network unobserved. Solving the game identifies defender and attacker strategies, and the choice of strategies identifies the game outcome(s). We computed strategies using perfect Bayesian equilibrium, a solution concept in which neither player has anything to gain by changing her strategy unilaterally. From the outcomes, we determined efficient strategies for including honeypots and fake honeypots in the network.

We next examined network shuffle, a concealment technique in which one-to-one mapping between network addresses and computer systems is permutated. Network shuffle shortens the timeframe that information obtained by reconnaissance and network probing is effective. We are modeling the effects of the technique using statistical urn models and computing optimal defender strategies. We have shown that an attacker's odds for success declines little between infrequent and frequent network shuffles. We then accounted for the costs and determined the defender's cost-effective strategies. Because we also devised efficient strategies for attackers, we draw conclusions on techniques to enhance detection.

For FY 2011, we will continue investigating the modeling deception techniques. We will combine our conclusions in a guide to assist network designers and administrators in defending networks. 


\title{
Development of Exascale Algorithms for Molecular Modeling
}

\author{
Karol Kowalski, Eric J. Bylaska, Marat Valiev
}

Our main purpose is to develop new modeling tools that can be effectively used on next generation computer architectures. In particular, we will significantly advance time and spatial scales of multiscale simulation methods and excited state methodologies.

A wide range of molecular processes require highly diversified methodologies to describe systems characterized by different time and spatial scales. In a vast majority of situations, this problem entails a need for having multiscale methods built upon fundamental solvers whose scalability and accuracy define the final performance of combined approaches. Given the dramatic increases in computer technology over the last decade, it is expected that exascale capability will lead to a significant shift in the system size and time scales. The progress in enhancing parallel performance of multiscale methodologies rests upon concomitant, balanced development of all constituent parts of a given multiscale approach. In the proposed research, which aims at the achieving scalability across $10^{6}$ or more processors, this translates into designing novel upscale spatial and time methods, including automated, self consistent algorithms for securing new quality of inter atomic classical potentials (as well as its extensions to excited state simulations) and development of efficient multilevel time solvers in order to push the time limits for molecular simulations.

We propose to develop a fully automated, self consistent, and dynamic method to generate classical potentials based on smaller scale ab initio calculations done asynchronously and in conjunction with a classical molecular dynamics simulation. The proposed scheme has several advantages over conventional methods of generating classical molecular dynamics potentials. By providing a concise definition of the effective molecular dynamics potential, we will remove the ambiguity from choice of the fitting parameters or from the fitting process itself. It also guarantees that within a given functional form, resulting parameters will yield the best approximation to the full partition function of the system. Unlike the case of traditional empirical potentials fitted to a small set of equilibrium experimental data, different structural configurations will be sampled in the course of the calculation, leading to a better transferability of the resulting potentials. There is no restriction on choice of the functional form of potential (including grid or basis expansion), and new analytical forms better suited to model complex materials can also be explored under the proposed scheme.

During FY 2009, we made significant progress with several project tasks. A new coupled cluster excited state, non-iterative methodology based on the reduced iterative core was developed. In addition, a new parallel in time algorithm was implemented and tested on several benchmark systems, and a novel ground state coupled channel formalism was fully integrated with multiscale, multi-physics formalism. Preliminary tests clearly showed the scalability of newly developed ab initio codes across the 6144 excited state coupled cluster methods as well as the 20,000 hybrid density functional theory/molecular modeling approach central processing units. The reported parallel performance was demonstrated in contemporary excited state calculations for fused porphyrin dimer and hybrid density functional theory/molecular modeling approach calculations for 80 atom cell of hematite. Substantial improvements in speed, memory management, and communication pattern were achieved in the first year. Six papers reporting on this research were either accepted or submitted in FY 2009.

For FY 2010, we again made good progress in several key areas of this project, our research, and results. First, we initiated the development of scalable GPGPU-based implementations of the $\operatorname{CCSD}(\mathrm{T})$ and regularized $\operatorname{CCSD}(\mathrm{T})$ approaches. Specifically, the scalability of the CR-EOMCCSD(T) approach was demonstrated across 34,008 cores. All tests were performed on the Franklin Cray XT4 computer at NERSC, and the CR-EOMCCSD(T) and active-space CR-EOMCCSD $(\mathrm{T})$ approaches were shown to be applicable in calculations for excitation energies of large molecular systems (942 basis set functions and 270 correlated electrons). We demonstrated that the errors in calculated excitation energies can be reduced to within $0.1 \mathrm{eV}$ with respect to the experimental data.

For task scheduling during FY 2010, we implemented new algorithms for the iterative CCSD and EOMCCSD methods. We demonstrated that grouping CSD/EOMCCSD procedures into layers collecting independent routines increases size of task pool, reduces the impact of load imbalance, and allows the use of larger tile sizes, which is essential for effective utilization of the GPU-based accelerators. In addition, we enabled the integration of newly developed high-order methods with MS/MP methods, which enabled us to perform calculations for molecular systems in solution or for surface localized states in materials. A new framework for task and data management in MS/MP calculations was implemented and applied to the optimization of large molecular structures. Further, we implemented a new parallel algorithm for hybrid DFT (incomplete butterfly) which reduced communication costs by half. A stand-alone AIMD program was converted to CUDA, and a stand-alone MPI/C++ code was being developed as well as converted to three different platforms. The implementation and testing of task level parallelization 
with global array groups in NWChem is in progress using the python interface. Finally, we implemented a general two-level parareal multiscale model using the task level parallelization python code in NWChem. For this year, seven papers reporting on project progress were either published or submitted.

In FY 2011, we are planning to implement root-level parallelism for the excited state calculations and develop large-tile-size algorithms for $(\mathrm{T})$ methods. We will extend adaptive coarse grained algorithms to multilevel parallel in time simulations and develop multilevel parallel in time algorithms to make practical use of error indicator-driven adaptive multilevel finite element techniques based on a solve-estimate-refine iteration based on local time mesh refinement. In addition, we will demonstrate the performance of the developed codes on chosen scientific problems.
Ultimately, our project will potentially have a long-standing effect on the routine calculation performed on the next generation of computers. We anticipate a significant shift in both system size and accuracy level characterizing exascale molecular simulations. The expected outcomes of this project are computational chemistry algorithms that will be published in the scientific literature, including scalable, excited state approaches for exascale molecular simulations, the efficient interface between ab initio theories and multiscale, multiphysics simulation module, and the exascale parallel in time algorithms (or parareal algorithms) integrated with terascale ab initio molecular dynamics and the terascale molecular dynamics programs. 


\title{
Development of Gaming Technology for Cognitive Enhancement in Predictive Analytics
}

\author{
Roderick M. Riensche, Patrick R. Paulson, Gary R. Danielson, Lyndsey R. Franklin, \\ Louis M. Martucci, Nino Zuljevic
}

This project aims to develop techniques and a systematic framework to enable the creation of a specific type of serious game in which users collaboratively and competitively explore plausible scenario outcomes as part of a predictive modeling process.

\section{s computational ability increases, models also increase in complexity, simulating an ever 1 increasing range of physical and behavioral} phenomena. Ultimately, human users and builders of these models must define complex systems, reason over implications, identify and mitigate risks and opportunities, and formulate and implement plans to capitalize on opportunities. We seek to streamline this entire interface, allowing users to maintain an interactive role in the modeling process, tapping their creativity to facilitate better predictions of real world behavior.

Today, the use of gaming as a mechanism to create stimulating user interaction with computer systems is widely recognized, as evidenced by the wide array of training games. In these training application games, content delivery and interactions are designed to stimulate the user's senses and enhance user experience by augmenting cognitive functions such as memory, attention, processing speed, and cognitive control. These games also present a natural environment for problem solving. In many games, success is achieved by repeatedly solving similar but non identical problems; in others, players solve a specific problem repeatedly by doing so more quickly and efficiently. Previous work has acknowledged this concept, creating serious games for exploring possible decisions rather than focusing on training. These efforts have typically dealt with specific problem domains. We believe that a generalized approach can add value by making game based approaches more accessible in modeling and analysis problems. In our project, we are maintaining the computational modeling and game software separately. While not seeking to create a purely generic game engine that could be plugged into an arbitrary computational model without modification, we are attempting to advance a unique method in that direction by developing a methodology and framework to facilitate easier application of gaming approaches to model outputs.

In our previous two fiscal years of work, we created a formal schema by which we define analytical games as a collection of distinct elements, including underlying computational models, user roles, data elements that connect models to users and to an encompassing simulated environment, and the interfaces by which users may affect the game environment and underlying data elements. We constructed the Analytical Gaming Framework (AGF) software architecture to facilitate construction of games defined using this schema in a fashion that encourages reusability and enables data collection of player actions as well as model and game environment data parameters. In addition, we defined and implemented two proof-of-concept prototype games to exercise and test the AGF by connecting to two different types of models: systems-dynamic and the Bayesian network. Through the construction and evaluation of the IED Game prototype, we devised methods for mapping a Bayesian network to a game environment.

During FY 2010, we continued work on the IED Game. We addressed computational complexities by refining our Bayesian network interface method. In conjunction with this activity, we developed a structured process to elicit subject-matter expert knowledge that we use to control the game-model interface. We defined and constructed a third prototype, the Illicit Trafficking Game, to explore the use of the analytical gaming method as a tool for producers and consumers of predictive models. In this game definition, we emphasized role-playing and focused on player communication rather than a prescribed list of potential actions, encouraging players to devise their own actions.

We included an explicit role for a human Game Master (GM) and added capabilities to the AGF to enable the GM to manipulate the game environment directly and even alter the underlying game definition by defining custom player actions. By allowing players and GM to work constructively, we facilitate a structured brainstorming exercise. By using the AGF to operate this exercise, we make sure that the data from each exercise are recorded to be used for a variety of purposes, including:

- Analysis of a single instance of game play to identify interesting courses of action devised by players

- Analysis of multiple instances of game play to identify common patterns of activity, which in turn may be used to calibrate existing models and to define new agentbased models

- Identification of common player requests for ad hoc activities that can be encapsulated in the formal game definition for future game play iterations. 


\section{Enabling Hypothesis Driven Research and Discovery in Extreme Data}

Kerstin Kleese-Van Dam

The goal of this project is to discover the significant limiting factors preventing today's scientists from making better use of the existing archives by using a wider range of investigative methodologies. This project will explore strategies from the aspect of several different types of sciences to overcome previously identified limiting factors and develop a prototype research environment.

dvances in experimental and computational
technologies have lead to an exponential increase
in scientific data volumes and their complexity. This data has resulted in the creation of a fast growing number of data collections, at times of extreme size. Unlocking the inherent potential in these data collections by enabling scientists to carry out hypothesis-driven research and discovery through effectively synthesizing and probing data as a community and across boundaries will provide extraordinary scientific advantages. However, to achieve the translation of the collected data into exploitable knowledge requires a paradigm shift both in terms of tools and human behavior. The advent of extreme data has led to the development of hypothesis-neutral data mining and social data analysis as a means to interact with the data. A new generation of tools, taking into account the human behavior, could play a pivotal role in enabling the necessary paradigm shift to support hypothesis-driven scientific research and discovery.

In this project, our goal is to discover the significant limiting factors that are preventing today's scientists from making better use of the existing archives by using a wider range of investigative methodologies. We explored strategies from behavioral science, cognitive psychology, business sciences, and information technology that will enable us to overcome the previously identified limiting factors. By the end of this project, we will develop a prototype research environment that will enable greater knowledge from data collections by researchers. We will establish quantitatively and qualitatively the boundaries to exploiting extreme data collections by the communities they serve, and we will develop strategies to overcome these boundaries based on methodologies from the aspect of various difference sciences.

During FY 2010, background research was conducted into the usage patterns of different types of data archives, both through literature research and interactions with staff (this project identified and assessed the current boundaries to the exploitation of extreme data as experienced by the scientific community, observing user groups at Environmental Molecular Sciences Laboratory and the Earth Systems Grid). This helped to form a hypothesis about the limitations and enabling factors for research collaborations around data, the scientist's interactions with others and the data, and the scientist's requirements for future research work with extreme scale data. The behavioral and cognitive aspect of the scientist (software interaction and in their extension data) was also investigated. A rich body of cognitive psychological studies has shown that researchers create mental models of technology used for their work to conceptualize and systemize its behavior. These clear mental models allow for the utilization of the technology for research without necessarily requiring an indepth understanding of its internal structure and function. The level of alignment of the mental model with the actual underlying system will determine how effectively researchers can exploit the capabilities of the technology.

Further studies have shown that researchers will assign and display human emotions toward any technology with which they are interacting; therefore, behavioral and social concepts that govern human-to-human interaction will to some extent also govern the researchers' interaction with technology and digital information. Therefore, any new user environment has to take common practices in the research environment into account required to build the necessary trust in the data, its handling, and analysis, such as trust in the creator. Further research studied successful strategies in software environments to stimulate desired behavior, such as collaboration and data reuse.

By the end of FY 2010, this project established a 100 TB data storage system that supports up to $800 \mathrm{MB} / \mathrm{sec}$ data transfers (to remote systems with matching capability). These results enable implementation and testing in FY 2011. The project lead has been invited to speak at the annual conference of the U.S. Environmental Protection Agency and has contributed to two book chapters on data intensive science expected to be published in early 2011.

Plans for FY 2011 include extending the project team and developing an initial prototype research environment that supports the collaborative, hypothesis-driven research in extreme-scale data. Involvement with other research in chemical imaging and BELLE II should provide this project with access to extreme-scale data repositories and additional user groups. 


\section{Exploring Architectures Suitable for Scientific Applications at Exascale Levels}

Oreste Villa, Andres Marquez

The aim of this project is to provide guidance to PNNL domain scientists on the refactoring and design of algorithms suitable for future exascale platforms. Current and future DOE applications will benefit from the ability to run faster and more accurately on large-scale machines.

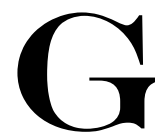
iven recent computer hardware design, it is likely that a future exascale system would contain hybrid processors composed of hundreds of cores and/or special purpose hardware accelerators. This project focuses on the evaluation of novel architectures that, in large-scale system configurations, can potentially achieve performance at exascale levels ( 1 billion billions of floating point operations/sec [FLOPS]). A recent promising trend in computer architecture design is stream processing, an execution paradigm that allows some applications to exploit parallel processing. Such applications can use multiple computational units (such as floating point) without explicitly managing allocation and/or communicating among units. One of the most common forms of stream computing is in graphic processing units (GPUs). Current GPUs are highly parallel multicore, multithreaded accelerators capable of performing general purpose computations. As accelerators, they must be interconnected through a central processing unit (CPU)-based host system. This project addresses the computational challenges involved in molecular simulations and subsurface flow and transport applications at PNNL.

In FY 2009, we investigated the use of GPUs in the context of molecular dynamic simulations, a computationally intensive method used to study the time evolution of a system of atoms using Newton's classical equations of motion. We implemented a functional and complete GPU-enabled molecular dynamic simulator capable of calculating bonded and non-bonded forces between atoms of a periodic system. As reference, we implemented a multithreaded non-bonded force calculation on conventional CPUs to test performance and correctness of the GPU counterpart. On double precision, the GPU code outperformed the CPU base code 15 times with cutoff and 10 times without cutoff.

During FY 2010, we investigated the use of the NVIDIA GPU "Fermi," which is used as base for the NVIDIA TESLA C20 family. This processor is composed of up to 512 cores running at $1.15 \mathrm{GHz}$ and is able to achieve a theoretical peak performance of about 1 TFLOPS in single precision and about 500 GFLOPS in double precision. A cluster of GPUs of the same size as PNNL's Chinook supercomputer (2130 nodes) would be able to achieve a theoretical peak performance of about 10 PFLOPS in single precision and 5 PFLOPS in double precision. As reference, Chinook has a peak performance of about 0.2 PFLOPS. NVIDIA GPUs are programmed using Compute Unified Device Architecture (CUDA) software architecture for issuing and managing computations on GPUs. CUDA treats the GPU as a data-parallel computing device without the need to map computations to the graphics pipeline explicitly.

We investigated the use of "Fermi" GPUs in PNNL NWChem software suite specifically for the CCSD methods. CCSD methods are used to calculate the approximate solution of the Schrödinger equation, and they are successfully used to understand fundamental optical processes in solar cells, photosynthesis, and other optically active materials. We applied our accelerated code to the CCSD calculation of the Green fluorescent protein (GFP) with 284 and 476 basis functions.

We observed the completion times for a portion of the calculation called "triple correction $-\operatorname{CCSD}(\mathrm{T})$ " on a 16 nodes GPU accelerated cluster. Each node is composed of two quad-cores Intel Nehalem $2.66 \mathrm{GHz}$ processors and a Tesla C2050 "Fermi" GPU. On conventional cluster, the $\operatorname{CCSD}(\mathrm{T})$ portion of the calculation is responsible for greater than 60 percent of the total computation time. A single GPU outperforms two quad-cores Intel Nehalem $2.66 \mathrm{GHz}$ processors by a factor of 4 . All the calculations are performed in double precision.

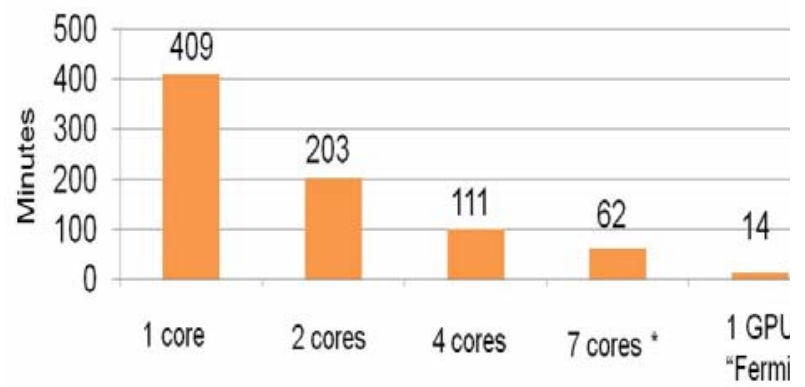

Performance of the CCSD $(T)$ calculation on a 16 nodes cluster.

* 1 core is used by the communication engine.

Our plans for FY 2011 will focus on increase of scalability and in the acceleration of the full CCSD method. In particular, we will focus on the implementation of atom to molecules functions for GPU cluster as part of NWche. Other studies will involve the STOMP applications for which we are currently developing a domain-specific compiler for the reactions that maps the computation on GPGPUs. 


\title{
Extending Global Arrays Programming Model on Hybrid Architectures
}

\author{
Daniel G. Chavarría-Miranda
}

The goal of this project is to extend the functionality of global arrays (GAs) to graphic processing unit (GPU) accelerated clusters. We will develop this capability in GA (including memory management, communication layer, and similar) generically so that it can be ported with limited effort to future hybrid architectures

$\mathbf{T}$ he development of extreme scale computational science capabilities continues to face major challenges in adapting or developing software to use emerging hardware environments effectively. High performance computing systems are characterized by large processor counts and increasing the use of heterogeneous and specialized environments in which GPUs, cell processors, and other hardware are harnessed to accelerate general scientific computing. Recently, GPUs have become a ubiquitous component of modern clusters; they have inspired researchers to accelerate general purpose computation on GPUs due to its increased performance, memory bandwidth, and programmability.

GA operations facilitate an intermediate programming model between message passing and shared memory known as remote memory access (RMA). This model

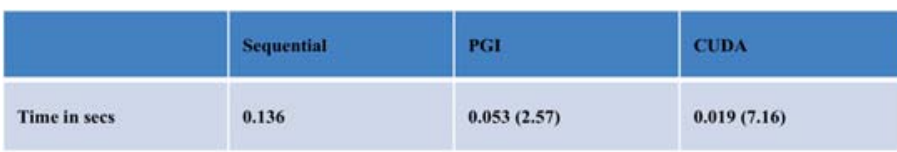

Performance of software written tensor contraction calculation (sequential), the PGI directive-based implementation, and the hand-coded CUDA implementation. Numbers in parenthesis indicate the speedup obtained over the software-based version. The calculation has been done using double precision floating point numbers (all cases). combines some advantages of shared memory, such as direct access to shared/global data and the message passing model, namely control over locality and data distribution. Certain types of shared memory applications can be implemented using this approach. In other cases, memory operations can be used as a high performance alternative to message passing. On many modern platforms, RMA is hardware supported and is the lowest level but often the most efficient communication paradigm available. Traditional RMA communication facilitates data transfers between a buffer of a local processor and another in the remote processor memory.

GPUs are typically programmed using models such as CUDA and OpenCL, which are quite different from the standard $\mathrm{C} / \mathrm{C}++/$ Fortran programming available on general purpose processors. These are special purpose environments targeted toward the fine-grained, multi-threaded, SIMD-style parallelism available on GPUs. Implementing algorithms and porting applications to GPUs can require significant algorithmic and data structure redesign. The techniques and approaches for this are being explored within the context of other LDRD work. The current study focuses on enabling a multi-node, multi-GPU programming model based on RMA operations.

GPUs do not have direct access to the main node memory banks or other attached I/O devices on the node, particularly the high-performance network interface card or other GPUs. A GPU accelerator has direct access only to the memory resources on its board. Access to other resources will have to be mediated by one of the CPU cores, which implies that data must be copied multiple times in order for computation to occur, particularly for execution models in which data is assumed to reside on CPU memory. Thus, the main objective of this work is to enable higher level, more integrated approaches to programming hybrid CPU/GPU clusters, including support for traditional programming languages $(\mathrm{C} / \mathrm{C}++$, Fortran) executing on the GPUs. A key factor is integration of the GPUs' local computation into the high level parallel framework provided by GAs.

Our preliminary work in FY 2010 demonstrated the use of the Portland Group's PGI Accelerator compiler to enable Fortran-level programming of GPUs using a directive-based approach. This model manages data copies fairly

transparently. Integration with GAs has been done transparently because the accelerator programming model can deal with local buffers populated by a GA call directly. Performance of this approach has been good. Our preliminary comparison against software-based implementation and highly optimized, hand-coded CUDA implementations indicate that the performance and productivity tradeoffs are in favor of the PGI directive approach.

We identified some issues with the management of pinned memory pages, which are required for efficient data transfers by both GA and GPU-based programming models. Currently, GA and CUDA do pinned memory management separately and independently. In the future, this approach will need to be unified to optimize the performance of data transfers to and from other nodes, as well as to and from the GPU. We expect that the programming model will enable more productive use of hybrid CPU/GPU clusters, making them more accessible to application scientists accustomed to using GA on CPU-only clusters. We also anticipate that many of the techniques that we discover will be applicable to hybrid systems with other types of accelerators. 


\title{
Extreme Scaling for List Based Pattern Comparisons (LiBaPaC)
}

\author{
Christopher S. Oehmen, Douglas J. Baxter, Kevin A. Glass
}

We aim to develop core software capabilities to enable list-based comparison applications to scale efficiently far beyond the terascale in which they currently operate.

I $\mathrm{n}$ the context of core applications that will effectively use computational resources at the extreme scale (i.e., peta-, exa-, and beyond), there is a growing collection of applications with computational requirements that fall outside the domain of parallelized linear algebra libraries or by optimized differential equation solvers. Some of those fundamental algorithms are methods that compare patterns in a list of queries against patterns in a reference database. This type of list-based pattern comparison ( $\mathrm{LiBaPaC})$ application is common in bioinformatics, in applications such as ScalaBLAST, proteomics such as polygraph, and also in emerging cyber applications. These software applications have already demonstrated highly efficient scaling, in part because of the native ability to partition list-based pattern matching into independent calculations. However, to continue to advance these methods beyond the terascale as the driving problem spaces grow exponentially into the foreseeable future, we must develop new underlying capabilities such as dynamic scheduling, fault resilience and more sophisticated algorithms for interacting with data structures that may not fit in-core.

Making the power of multiprocessor computing available to a community of researchers and analysts will require the development of many novel solutions and techniques to enable novice users to develop rapidly new applications that scale efficiently. MapReduce is an example of a library that enables a particular class of algorithms to be implemented as a scalable application with only a few lines of code. We are abstracting design features from an existing set of scalable applications with relevance to biological sciences into a library in the same spirit as MapReduce. This project will enable future developers of list-based comparison tasks common in bioinformatics and proteomics and other domains to develop novel scalable applications rapidly.

Our library enables robust, efficient scaling for $\mathrm{LiBaPaC}$ applications toward the exascale. This will be achieved via the following specific areas of development:

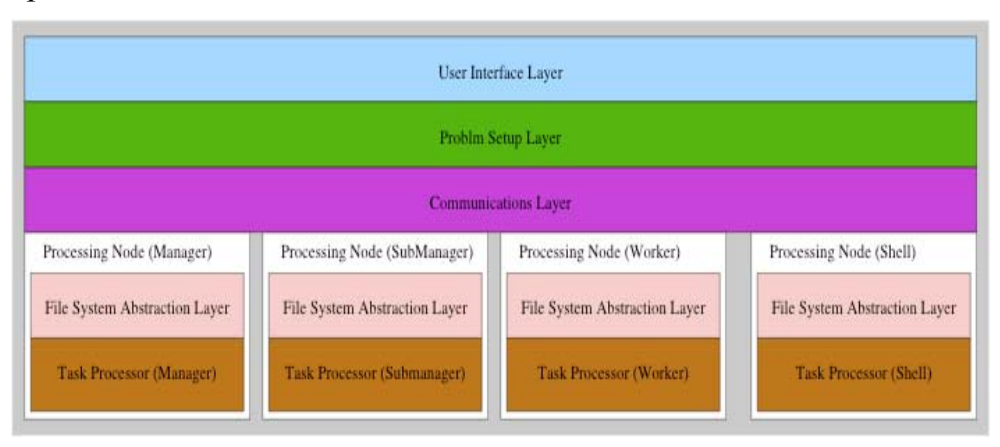

An overview of the $\mathrm{LiBaPaC}$ architecture.
Dynamic Scheduling. Used in the newest version of ScalaBLAST, this framework will be abstracted into a general-purpose scheduling architecture. This will form the basis for efficient scaling of independent tasks specified by the user for different applications and the core of functionality allowing for fault-resilient operation on large-scale systems.

Flexible Memory Management. As dataset sizes increase, they often exceed the available memory on a single processor or core; thus, managed resources include disk and communication resources as well as processor cycles and memory. We will add data set partitioning schemes to facilitate a variety of data storage and movement techniques including asynchronous IO, global arrays, MPI message passing (one-sided and two-sided non-blocking), and memory mapping.

Scalability. For client-server code to scale effectively with up to millions of clients without overloading the server communication capacity, we will implement a hierarchic server model with a server managing sub-servers that manage a limited number of clients or other subservers.

Fault Resilience. Fault resilience will be addressed in stages. The first is creating tracking data for task states and adding logic to reschedule incomplete tasks, which effectively handles failure in a client. The server/sub-server resiliency will be provided by mirroring the server(s) using heartbeat data to do software failover.

In FY 2010, this project completed the basic $\mathrm{LiBaPaC}$ design and developed a prototype of the communication layer independent of the underlying communication fabric on a particular system. This is so that $\mathrm{LiBaPaC}$ can be constructed on conventional message-passing based systems using MPI libraries, or using other communication interfaces available on grid or cloud architectures. A second aspect of the design is that the file system is viewed as an abstraction. This allows users to interact with files without having to know details about the underlying architecture's file system and is essential for operation in cloud and other distributed systems for which true file paths may not be available to the end user. The ScalaBLAST application design has also been completed and will be a use case for implementing the $\mathrm{LiBaPaC}$ library software. 


\section{Geological Sequestration Software Suite Core Architecture and Simulation Framework}

Ian Gorton

Our geological sequestration software suite $\left(\mathrm{GS}^{3}\right)$ is a set of tools integrated through a software framework that supports the modeling process used to evaluate and monitor carbon dioxide sequestration sites. We have worked extensively with geoscientists to design and build an initial version of $\mathrm{GS}^{3}$ for use in sequestration projects.

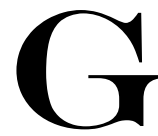
eologic sequestration is a technology that is currently being developed to mitigate global warming through the long-term storage of greenhouse gases. Finding suitable subsurface (underground) sites for sequestration of greenhouse gases requires performing extensive studies that include modeling the site geology and the processes that determine the fate and transport of injected gases. Unfortunately, subsurface modeling is an iterative process that can often take months or even years to complete and is continually revisited as new information is gathered. In addition, work is performed not by a single scientist but by interdisciplinary teams comprised of geologists, hydrologists, engineers, geochemists, and computational scientists who are unlikely to be co-located.

In this project, we are building a framework that incorporates off-the-shelf tools with which many modelers are already familiar; facilitates the creation/integration of new custom tools, utilities, and scripts; and integrates seamlessly with data management capabilities, including provenance capture and user annotation. We are working with carbon dioxide sequestration experts to prototype this framework in the context of two alternative candidate sequestration sites and to identify data and tools needed to characterize and model these sites. Our specific contribution will be in the deployment of data management services, standardized formats, data translations, automated computational job management, and the component integration framework.

Collaboration through data and knowledge sharing is a driving force behind our architecture, as is the goal of providing infrastructure that enables a self-sustaining community. For this reason, we specifically chose wiki technology for the core of our project and $\mathrm{GS}^{3}$. Most wikis provide a rich web application development platform and a mechanism to add plug-in modules that operate on the wiki content. Of particular interest for $\mathrm{GS}^{3}$ are the semantic extensions that enable a range of dynamic views of the content. From project commencement, we have worked extensively with geo-scientists to understand the modeling and simulation process for carbon sequestration. This process involves multiple tasks that follow a loose, highly iterative workflow. Based on elicit requirements, our designed prototype software tool contains a highly flexible and extensible underlying software architecture. Thus, for project initiation, we spent a considerable amount of time gathering requirements from the geo-scientists and proceeded to design and prototype a reusable, extensible software architecture based on satisfying all of those requirements.

During FY 2009, we designed and built a prototype version of $\mathrm{GS}^{3}$ and demonstrated it to various groups involved in carbon sequestration. The software adopts a novel layered architecture that enables it to be highly customizable for generic modeling and simulation environments. Based on our experiences in the validation of our prototype, we found that the $\mathrm{GS}^{3}$ environment is an excellent match to a scientific user base in terms of usability; the auto-generation of useful content leads to a consistent look and feel for the environment with straightforward semantic queries that allow quick site modification in response to user requests; provenance and model versioning provide an appropriate, lightweight solution for keeping track of the complex modeling process; and the ability to build the reference catalog incrementally under user control is well received, with the automated knowledge extraction pipeline being a simple process for users.

For FY 2010, we focused on how modelers use a range of data throughout the modeling process. Data types that are important include:

- site data measured from well logs and experiments that is used to build geological models of the subsurface

- model input data that maps the geological models to a numerical grid so that it can be simulated

- simulator output data that is produced by numerical simulators such as STOMP.

Crucially, assessing the significance of simulated outputs requires detailed assessment of the simulator inputs and how they are derived. As modelers cannot "see" underground, various parameters must estimated and a number of different input models constructed to assess the effects of these estimates. This makes it highly challenging to effectively manage the large volumes of data associated with modeling a given sequestration site.

For this reason, we created $\mathrm{GS}^{3}$ as a core knowledge management system for carbon sequestration. We have created an initial version of $\mathrm{GS}^{3}$ that is based on a number of integrated software components. These components that 
together comprise the $\mathrm{GS}^{3}$ knowledge management system that we have designed and built this year are as follows:

Data storage: $\mathrm{GS}^{3}$ includes a data repository for storing the heterogeneous data files associated with a modeling project.

Versioning: $\mathrm{GS}^{3}$ enables modelers to create and manage model versions and associate the input and output data with a particular model version.

\section{Semantic Markup: $\mathrm{GS}^{3}$} contains a data ingest pipeline that can be used to markup text files in various formats with terms that are of interest to modelers. Once marked up, these documents can be easily searched using the $\mathrm{GS}^{3}$ user interface.

\section{Metadata Extraction: $\mathrm{GS}^{3}$} ingest pipelines also read structured data formats such as well logs and extract metadata from these, for example, to show the geographical location of a well on a Google Maps interface in $\mathrm{GS}^{3}$.

During FY 2010, we presented a paper on $\mathrm{GS}^{3}$ in the Knowledge Management Track at the Hawaii International Conference on System Sciences.

For FY 2011, we will focus on the $\mathrm{GS}^{3}$ provenance interface will enable users to 1) explore the data that has been used to create a given set of simulation results for quality assurance purpose, and 2) modelers in $\mathrm{GS}^{3}$ require the ability to secure various data sets and model outputs due to the sensitivity of the information and projects they work on. 


\section{Information Integration for Forecasting Dynamic Organizational Behaviors}

Paul D. Whitney, Kenneth D. Jarman, Amanda M. White, Stephen J. Walsh, Alan J. Brothers, Seán J. Kreyling, Angela C. Dalton

The objective of this project is to develop a mathematical and computational methodology to forecast organization behavior using models, empirical data, and expert opinions.

$\mathbf{T}$ he ability to estimate the likelihood of future events based on current and historical data is paramount to the decision-making process of many government agencies. If successfully accomplished, numerous predictive tasks have significant value to these agencies: notably, predictive tasks related to deterrence of weapons of mass destruction events, countering terror events, characterizing the risks associated with terror events, and understanding the effects of options for resource deployment. There is significant experience that supports the integration of diverse information sources for challenging prediction problems. A successful approach to addressing problems in this category involves expertise and multiple options. Currently, the approach to solving these problems do not involve significant computational modeling for integration, and model and information re-use can be limited.

This project addresses this challenging integration problem by developing formal mathematical and computational methodologies to combine multiple models, multiple experts' opinions, and data. The technical approaches in this project for integrating predictive information have several unique and significant aspects: the approaches to validation, formal modeling of experts' assessments, and support for model integration. Each of these is an essential step in making progress in forecasting organizational behaviors. We are taking an "engineering" approach to validating the integrated forecasting model and executing a detailed quantitative comparison between observations and dynamic behavior models. With this approach, we can arrive at calibrated forecasting models, reject forecasting models as insufficient or useless, and leverage existing modeling technology to drive more accurate and complete models. We will incorporate experts' assessments by developing a mathematical structure that links the experts' input with the computational model. Our approach is analogous to using experts as "sensors" and building a sensor model. This is in contrast with existing approaches that treat experts' opinions as raw data. The standard approach places empirical observations and opinions as entities of the same type, but they are not. Accomplishing this part of the research can significantly change the nature of expert assessment and modeling in behavioral modeling and analysis.

Finally, support for model integration is driven by a diverse set of concepts and expertise required to address the significant challenges outlined above. Each of these involves significant, traditional science and technology expertise combined with the detailed knowledge of extant social systems. Enabling and supporting integration of models (data and experts' assessments) are critical steps in addressing these significant national challenges.

While often couched in terms of physical sciences and engineered technologies, the fundamental problems facing the United States in energy, security, transportation, and others are driven by people and organizations. We will develop the science, technology, and capability to enable predictive analysis of organizational behaviors

and address the human and organizational aspects of these problems. To combat these issues, our project has three specific aims. Because human behavior modeling is still in its earliest development, these aims are focused on enabling model construction, calibration, and validation.

Develop supporting mathematics and algorithms for model combinations. Complex human enterprises such as energy consumption, illicit trafficking, improvised explosive device deployments, and transportation are comprised of diverse human activities, technical expertise, technologies, and motivating human drivers. These activities or drivers are often separately modeled and understood by distinct individuals within distinct technical communities. This separation results in concepts and models abstractly and computationally 
challenging to link. Currently, linkages are either ad hoc or extremely high level. We will develop mathematical and computational methodologies to link disparate model components. Accomplishing this aim is a critical step toward integrative and collaborative modeling across diverse communities and expertise.

\section{Develop systematic methodologies for combining} predictive information from human experts' assessments with formal computational models. There is abundant expertise available to assess aspects of techno-social predictions, and there are numerous elicitation methodologies for taking advantage of this information. These expert assessments are executed in isolation from formal and computational models; accordingly, the interaction between assessments and models are ad hoc at best. A primary aim is to remedy this deficiency and more closely link the expert assessments with models. This combination of expert and model information will result in the ability to assess and improve the reliability, accuracy, explanatory, and diagnostic value of information provided directly by experts.

Develop clear and
understandable
explanatory
diagnostics for the
models, evidence and
experts' input
associated with the
models in an analysis
Predictive models
(especially those that
are mathematically
complex) can be
challenging to

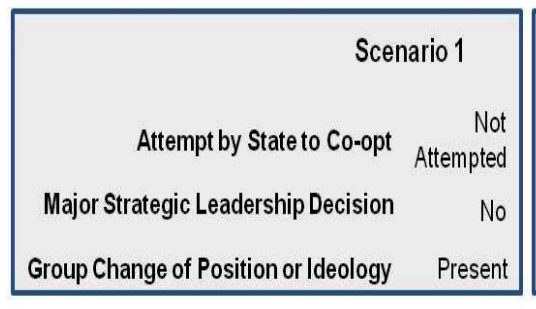

\section{Scenario 2}

Not Attempted

NNo Major Strategic Leadership Decision

Absent Group Change of Position or Ideology

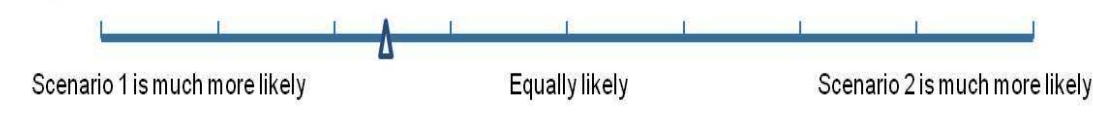

Scenarios are constructed by sampling from a Bayesian network model. The expert assessment is the relative likelihood of the two scenarios indicated by the location of the slider. These assessments are mapped back into the underlying model parameters. allowing those models to communicate. During FY 2010, we derived a new, maximum entropy based formalism for integrating Bayes net components that describes different behavioral constructs, constraining the integrated model according to the component distributions and general dependencies imposed between them. By reducing the human effort in building large Bayes nets to component-wise model building, this approach may alleviate the exponential growth

Conjoint analysis elicitation-based model calibration. In the absence of data, models may be calibrated using domain experts. A simple, efficient user interface has been developed to elicit opinions from experts regarding the relative likelihood of scenarios that are described by a Bayes net model. Computational methodologies developed and published as part of this research project then allow for model calibration by translating the expert opinions into model parameters. We have applied these methods to problems in nuclear non-proliferation.

\section{Agent-based} modeling. We developed mathematics and methods to calibrate agent-based models with observed data. We then demonstrated the mathematical, computational, and organizational expertise to use games as an experimental apparatus for human challenging to

understand. As a result, user and community acceptance of the models can be hard to gain. We address this challenge by developing explanatory diagnostics to communicate critical aspects among the models, forecasts, experts' opinion, and the associated driving evidence. This project will develop explanatory diagnostics that 1) assess consistency of the models with the evidence, 2) examine the importance of each piece of evidence to a prediction in the context of the models, 3 ) determine whether there are particular pieces of evidence not consistent with the model set, 4) perform uncertainty assessments for particular outcomes and predictions of interest, and 5) evaluate the historical performance of the prediction models.

In FY 2010, we made good progress toward achieving the above research objectives.

Model integration. Model integration enables the holistic approach by combining disparate modeling frameworks and behavior modeling. This research will generate the capacity to answer research questions at a more micro-individual actor level.

Graphical diagnostics for consistency of Bayes net models and data. We developed the first ever graphical diagnostics for Bayes nets that visualize the consistency of model assumptions with data. These diagnostics are widely applicable to Bayes nets in any domain, not simply human behavior research. This scientific breakthrough is a key step toward achieving our objective of creating transparent and understandable diagnostics for models given the data, expert assessments, and offers.

To date, our project makes a significant scientific contribution through developing the mathematical and computational capabilities to develop, calibrate, validate, and combine computational models of human behavior. Our outcomes can help decision-makers better address the critical problems challenging our nation. 


\section{Integrating Power and Performance Modeling for Exascale Systems}

Kevin J. Barker

This project will develop a power modeling methodology analogous to existing and proven

performance modeling techniques. The methodology will then be integrated with performance modeling in order to allow for the rapid study of architecture and workload designs.

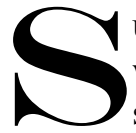
upercomputer systems have been designed primarily with performance as the main criterion that must be satisfied. However, as we move into the exascale era, power consumption will increasingly provide the pressure that will drive system design. Simply scaling up current technology will not provide an acceptable solution; extrapolating from today's largest machines indicates that on the order of $10 \mathrm{GW}$ of power will be required for an exascale system of similar design. New solutions will be required at all levels of the system architecture from hardware through middleware tools and to algorithms and applications with an eye towards minimizing power consumption as well as performance. And, new approaches and technology will be required to achieve such performance within an acceptable power budget.

Under this project, we will develop a methodology integrating both performance modeling and power modeling and use it to explore potential future exascale architectures and architectural components. We will develop new analytical methods to model and explore both the performance of large-scale computer installations as well as their power consumption before the construction and installation of such systems. Due to the cost of such large-scale supercomputing platforms, and the fact that they typically are unavailable for benchmarking and measurement prior to installation, analytic methods such as these that allow for quantitative evaluation of these systems contribute directly to the responsible use of procurement funds.

This project involves two primary components: 1) developing methodologies to model quantitatively and analytically the power consumption of large-scale parallel computing systems, and 2) integrating these techniques with existing proven performance modeling capabilities in order to explore the trade-offs between power consumption and performance and full system scale. Up until this point, procurements of large systems have been concerned with delivered performance on workloads of interest. However, as we move into the future and towards exascale capability, power will become a primary constraint on system design. It can easily be envisioned that insufficient power will be available to "light up" the entire machine simultaneously. In such a regime, novel methods will be required to deliver power in such a way as to maximize performance.

The outputs of this project will include methods to model power consumption of parallel systems as well as techniques to integrate power and performance modeling. This work will leverage previous work in the area of performance modeling; the methods for modeling power will in some ways parallel our performance modeling techniques. Both include empirical measurements at small scales combined with analytical techniques to derive predictions at full machine scales.

This project began at midyear, during FY 2010. We procured a measurement harness consisting of a power analyzer that will be used to gather measurements of power consumed by relevant workloads running on current and nearterm future hardware. We performed and initial examination of the energy consumption of single-sided communication primitives, where we used an interrupt signaling mechanism and dynamic frequency scaling to down-clock processors. We found that in both cases, as message sizes increase, these methods produce improvements, showing reductions in energy consumption. Additionally, we are leveraging existing relationships with major hardware designers (e.g., AMD and Intel) to gain access to the next-generation hardware and gathering data to characterize applications with different requirements (e.g., compute, memory, and network). We have commenced developing a successful performance modeling methodology to be applied to a large number of systems and workloads.

This learning process will apply directly to our modeling methodology development. We were invited to speak at Scalable Approaches to High Performance and High Productivity Computing (ScalPerf 2010), where this project was featured in the context of a tool for addressing exascale computing challenges.

For FY 2011, we will develop benchmarks to gather system power characteristics and obtain access to hardware platforms with appropriate power measurement sensors. We will develop and enhance the power modeling methodology, which is envisioned to be analogous with successful performance modeling methods and will contain static representations of workload characteristics parameterized in terms of system power consumption metrics. In addition, we will work to validate power consumption predictions provided by the created model against measurements taken on hardware platforms, where access to hardware resources with appropriate power sensors is critical. 


\title{
Knowledge Encapsulation Framework
}

\author{
Andrew J. Cowell, A. Keith Fligg, Peter C. Ellis, Liam R. McGrath, Kelly A. O'Hara, Michelle L. Gregory
}

We aim to develop a framework of components that can be used to develop collaborative information analysis and discovery environments. These components can be then be arranged to build a domain-specific, highly customizable environments for a range of domains.

A knowledge encapsulation framework (KEF) is a collection of components aligned to produce novel information analysis environments. The core project work is based around a natural language pipeline, a semantic wiki environment, and numerous software components that can customize the wiki for particular uses. In previous years, we have focused on supporting modelers and their information requirements working on climate and security in the Indian subcontinent, security of the U.S. energy grid, and the use of improvised explosive devices in theaters of war. In FY 2010, our focus was on improving those components built in previous years, create new components, and support of an integrated demonstration of illicit nuclear trafficking.

A suitable metaphor for the capabilities provided by these components is of an automated research assistant. Consider a piece of software that can take information important for a task (for modelers, typically journal articles, conference papers, technical reports, etc.) and place the material into a collaborative environment for the team members to collaborate over. Components allow users to comment on individual documents (either through a page-wide note taking facility or through annotations to parts of the page), engage in threaded dialogue or even synchronous chat. The environment is able to "understand" what colleagues find interesting about the shared material and can query traditional media sites (e.g., academic portals, news sites) and social media (e.g., blog search engines), bringing relevant hits into the environment for review. This process enables a constant stream of new, potentially relevant material for the collective user base to evaluate and utilize. All documents coming into the environment pass through a pipeline that extracts text from multiple file formats (e.g., .doc, PDF) and performs entity extraction (identifies people, places, things, events, and groups), and evaluates topics, sentiment (how people feel about a topic), and rhetoric (what people are saying, to whom, and for what reason).

On review, if the newly found material is deemed useful, it becomes part of the growing collection of information within the environment. This material is made available for all users through multiple methods. The environment supports typical wiki browsing metaphors as well as a graphical faceted browser. The user may perform regular or semantic searches, the latter of which allows for disambiguation (e.g., users pose queries such as "show me all documents that include Paris [as a location] and climate change [as an event] discussed negatively"). Such an environment provides data selection beyond what traditional keyword search systems can provide increased accuracy.

Information within the environment is then used as evidence to satisfy the domain model. After using the discussion board features to engage modelers, subject matter experts and analysts in the development of the model, an internal representation of the model is encoded within the environment. Collectively, users can review all relevant content in the environment and align documents, paragraphs, or even simple tabular statements to elements of the model. Simple parameterization of the model can occur within the environment, but more typically, modelers can export all the aligned evidence in a format that can be simply imported into their modeling engine.

A number of challenges exist with identifying information accurately through a named entity recognizer process. In FY 2008, we explored the best methods for co-reference resolution within and across documents and aliases. As model accuracy requires that information is correctly associated with time expressions, we explored a solution to these areas, which led to integrated technologies in FY 2009. At that time, our goal was to develop a tight linkage to models. The wider workflow from information identification to parameterizing resulting model nodes provided an effective environment. We placed considerable effort on the user experience to make the environment usable and compatible with other tools. Finally, we made several changes to the natural language pipeline, enabling the attachment of new annotators for different missions.

For FY 2010, we used machine-learning approaches to classify text being aligned with model nodes. Understanding the terms aligned with a model node, we can review information already within the environment, bring it to the modelers' attention, and prioritize those documents coming into the environment through discovery. We also built a formal build process that enables us quickly and efficiently to create new environments for different missions and domains. During FY 2010, a number of new domains were introduced using the build system, including marine hydro-kinetic modeling, cataloging of CRNE models, and the study of the science of science and innovation policy. 


\title{
Machine Learning String Tools for Operational and Network Security
}

\author{
Christopher S. Oebmen, Bobbie-Jo M. Webb-Robertson, Patrick J. Nichols, William K. Nickless
}

The goal of this project is to move toward proactive exploit detection by applying the mathematics and theory of bioinformatics. This approach is based on the concept that text strings describing biomolecules such as genes and proteins are analogous to text strings that describe many aspects of the cyber universe. We address the need to predict potential vulnerabilities in information technology or energy infrastructures, detect and remediate malware, and enable resilient, self-defending networks.

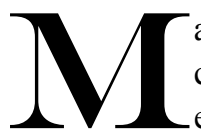

any cyber security challenges result from a combination of scale and complexity. For example, understanding the intent of network transactions in the context of anomaly detection within the DOE complex requires analysis of an enormous, ever-growing body of digital information to identify increasingly sophisticated attempts at infiltration and exploitation in an environment where the vast majority of transactions are benign. One limitation of rule-based detection schemes is that we must first know what to look for, which is a challenge in the face of constantly evolving exploit methodologies.

This project is directed at developing capabilities to enable the construction of a collection of solutions to challenges in cyber and network security. The primary approach is to train computational systems to recognize patterns of interest in string-based information (such as network transactions or legacy

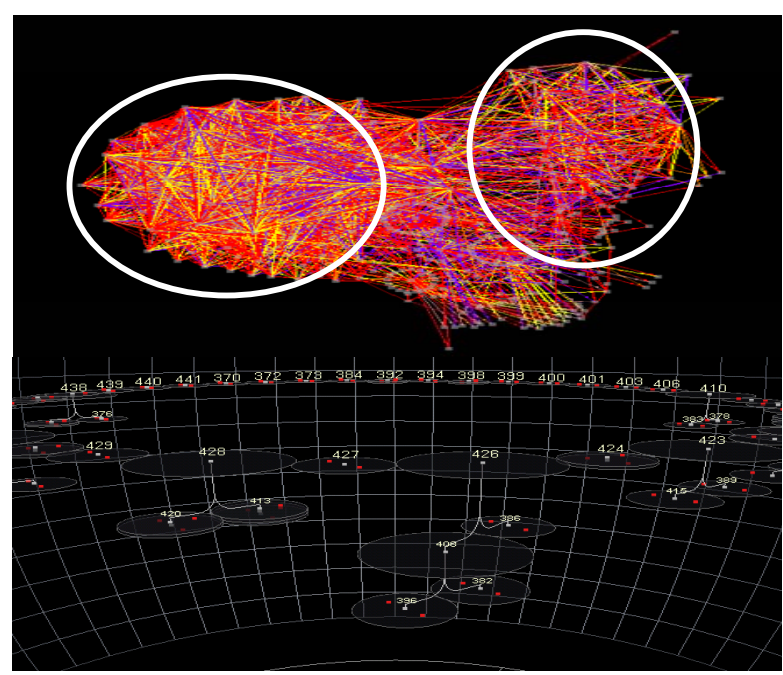

Top: Network view of similar servers illustrates commonly behaving servers on the PNNL network. Densely connected regions (circles) are indicative of many servers with similar behavior. Bottom: Hierarchical cluster of servers indicates high-confidence server families.
In FY 2008, we developed and implemented vectorization strategies for network transactions. FY 2009 brought a feasibility study for classifying cyber events, improving the vectorization strategy from the previous fiscal year, and creating a strategy for HPC binaries. Additionally, we worked on algorithm development to optimize the training phase. In FY 2010, we accomplished the following milestones with this project.

Completion of the ingestion/translation pipeline. The MLSTONES ingestion engine takes raw cyber data as input. Using user-specified mapping and translation tables, it accumulates cyber events by individual entities and creates a string representation of that entity's behaviors or events. The ingestion engine can be used to create large library files in a format suitable for bioinformatics analysis. Once in this format, an arbitrary list of individual event sequences can be compared against libraries for strings with a high degree of similarity. This metric can also be used to detect similarities with a high degree of sensitivity using the support vector machine technique classification developed in a prior LDRD project.

Demonstration of analytical capabilities on cyber data. Using the ingestion and translation pipeline described above, we demonstrated that families of network-attached servers at PNNL can be identified quantitatively using their sequence of warnings posted to the Microsoft Active Directory logfile. Using the nearness metric derived for all pairs of servers, a network representation of mutually source code) by using biological

theory to capture the inexact and evolving relationships between text strings associated with digital information and use machine-learning principles to extract patterns without a priori knowledge of those patterns. Success would have significant operational impact by enabling pattern-based, data-driven identification schemes grounded in a theoretical framework to augment the current rule-based approaches to cyber security. The intent is to develop the algorithmic and computational infrastructure needed to deploy this concept rapidly into new problem spaces of cyber security. similar behaviors was generated. To improve on this initial representation, we used hierarchical clustering to generate a full "family tree" of all servers. The collection of smaller tree structures illustrates high-confidence collections of servers having very similar behavior measured by string similarity in their translated warning streams. Several of these trees map to functional families such as web servers, database servers, email servers, and unused servers.

To complete our project in FY 2011, we will demonstrate and publish our research results illustrating the MLSTONES method, displaying its cross-disciplinary value. 


\title{
Managing Complexity of High-Volume Predictive and Adaptive Network Operations
}

\author{
Zhenyu (Henry) Huang, Yousu Chen, Pak Chung Wong
}

The goal of this project is to develop real-time decision support to network operators to ensure safe and reliable critical infrastructures.

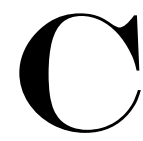
ritical infrastructures play an important role in modern societies. Failures such as power grid blackouts can lead to significant disruption of lives, industry, commercial activities, and result in massive economic losses. Operation of these networks is challenging, as they all have complex structures, wide geographical coverage, complex data/information technology systems, and highly dynamic and nonlinear behaviors with numerous internal configurations and external factors including physical attacks, cyber threats, human errors, and natural disasters. None of the complex network operations is fully automated; human-in-the-loop operation is critical. Given the complexity involved, there may be thousands of possible configurations at any given time, and operators need to examine them in near real time during an emergency to choose the best option and operate the network effectively. In today's practice, network operations are largely based on operators' experience with very limited real-time decision support, resulting in inadequate management of complex predictions and inability to anticipate, recognize, and respond to situations caused by human errors, natural disasters, and cyber attacks.

A systematic approach is needed to manage the numerous predictions and choose the best option within the near-real-time constraints. This project aims to establish a decision support system to manage complex predictions and help operators predict potential network failures and adapt the networks to adverse situations. The resultant decision support system will enable the continuous monitoring of network performance, turn large amounts of data into actionable information, and provide significant decision support for operating infrastructures.

During FYs 2008 and 2009, we successfully developed methods for network risk analysis and visualization, graph trending analysis, configuration clustering, data integrity assessment, multiple predictions for future network operations. In FY 2010, we further enhanced the features of our visual analytics method called the Graphical Contingency Analysis (GCA) Tool, which has graphical representations, ranking of contingencies, and an assessment of possible actions. This tool has three distinct advantages: 1) its intuitive graphical representation enables faster, more accurate identification of potential operational problems, 2) its ranking of contingencies helps focus the operator/planner's attention on the most severe problems and prioritize preventative actions, adding meaning to the results of contingency analysis for an operator or analyst, and 3) its interactive assessment of alterative actions provides more informed decision making.

The GCA tool was evaluated by power grid operational personnel in a WECC training course held in the Electricity Infrastructure Operations Center (EIOC). A user study was carefully designed and embedded into the training course. Preliminary analysis of results of experimental studies conducted within the workshop indicate that the GCA tool is effective in addressing the complexity of the contingency analysis problem by facilitating proactive rather than reactive strategies and reducing the number of "steps" that operators must examine in arriving at effective solutions, compared to the tabular representation in today's tool. If this advantage translates into time-savings, one may project that the elapsed time to address an "alarm condition" will be significantly reduced using the GCA tool.

In practice, the time-savings can yield a significant amount, as much as $\$ 1$ million per sanction event that is prevented. Other findings from the WECC study were that several operators who participated in the workshop expressed strong interest in obtaining the GCA tool for their control centers. Because of the unique features of the GCA tool, WECC is planning to use the GCA tool in its future training workshops.

Our goal of establishing a decision support system that effectively manages complex predictions, helps operators predict potential network failures, and takes effective actions in response to adverse situations - has been successfully achieved. The research products are in transition to possible commercial use. The effort strongly supports PNNL in advancing science and technology for information and infrastructure integrity, security, and reliability.

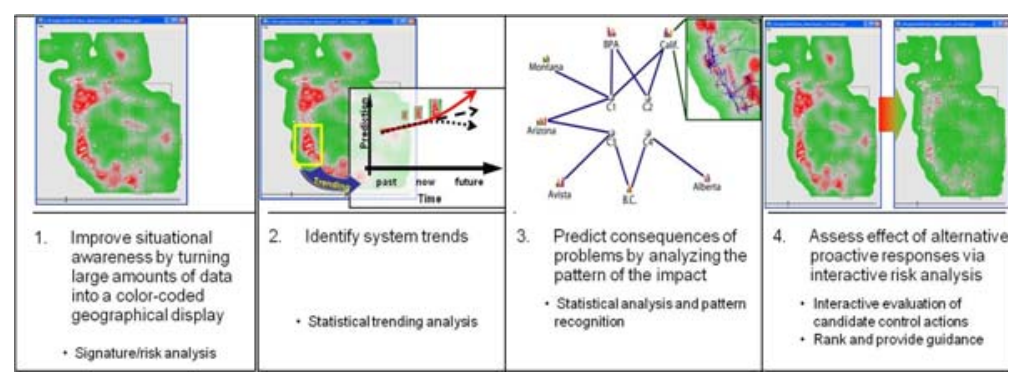

The decision support system consists of four basic elements: visually represent large amount of data, recognize imminent problems, assess consequences of problems, and evaluate remedial actions. 


\title{
Multiscale Models for Microbial Communities
}

\author{
Haluk Resat, Allan E. Konopka, Vanessa L. Bailey, Jay W. Grate
}

This project will develop mathematical models that incorporate the possible effects of heterogeneous three-dimensional structure, functional biodiversity of the microbial population, and geochemical processes into a unified model to improve our understanding of the role of interactions between co-existing organisms in a microbial community.

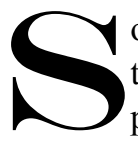
oil is a highly complex heterogeneous system in terms of inhabitant microorganisms and spatial properties. Soil structure can be viewed as a dynamic hierarchy of sizes that contain microbial cells with different metabolic properties. Such variations in local environmental conditions are expected to lead to a widely varying distribution in microbial cell dynamics. For mathematical modeling, this translates to having a heterogeneous system in which participating objects have different classes of dynamic properties: some would show strong individual characteristics that require explicit treatment, some would be part of multi-cellular assemblies that mimic large well mixed systems, and some would be endure in-between conditions.

The kinetic properties of microbial organisms are dependent on the physical, chemical, and biological composition of the systems in which they are a part. Therefore, investigation at the whole community level is required for a more comprehensive understanding of microbial systems. The main aim of this project is to develop novel mathematical models that incorporate the possible effects of heterogeneous three-dimensional structure, functional biodiversity of the microbial population, and geochemical processes into a unified model. This will improve our understanding of the role of interaction of bacterial and fungal metabolism in the transformation and sequestration of soil carbon, and contribute to predicting the impact of microbial community based management practices in controlling and regulating carbon flow in soils.

Variations in local conditions can impose different environmental cues on microbial cells, leading to widely distributed physiological responses in a population of cells. This is particularly the case for microbial communities in heterogeneous systems, where differences in local environmental conditions can have great effect on cellular dynamics and physiology. Building on recent advances in modeling bacterial ecosystems, we are constructing a hybrid, multiscale mathematical model for resource generation and utilization in environments representative of microbial

communities in natural soil aggregates. As sequestration of terrestrial carbon via microbial and chemical reactions in soil can offset some fossil fuel carbon emissions to lower greenhouse gas release, a better understanding of the transformation of carbon in soil systems and the flux and residence times of different carbon forms will enable greater understanding of terrestrial carbon sequestration processes.

In FY 2009, our modeling and simulation approach allowed for the dynamic coupling of the cells to their internal kinetics in a single, unified model while retaining the strengths of earlier efforts. We investigated the role of heterogeneity on the dynamics of microbial communities, which can arise both in terms of organism composition and spatial distributions. Instead of constructing accurate network models for a particular organism, our initial model-building efforts emphasized interdependent relationships between observed species in terms of their shared energy and metabolite utilization dynamics.

Using the metabolic network model developed in FY 2009, we performed a series of simulations in FY 2010 to predict whether steady-state oxygen gradients could form along channels of microfluidics devices. This is important because such oxygen gradients may lead to oxygen-poor (i.e., anaerobic) regions that co-exist with the oxygen-rich aerobic regions within the devices. It was found that oxygen gradients can indeed form if the inoculated biomass is high and fluid flow rate through the device is slow. Such predictions aid with the design of experimental studies pursued in complementary projects.

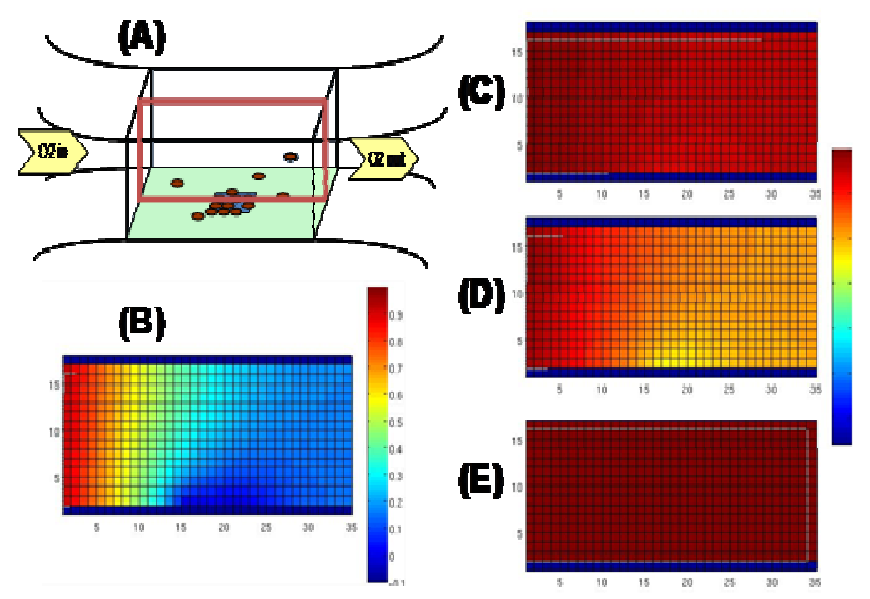

(A) Section of a channel in microfluidics devices where the role of oxygen input, biomass, and fluid flow rates on oxygen gradient formation was investigated; (B-E) Oxygen levels across the cross section indicated with the rectangle in (A). Shading bars show oxygen in $\mathrm{mg} / \mathrm{L}$ units. 
We also investigated which type of cellulose degradation mechanism might give a survival/growth advantage to bacterial organisms in heterogeneous environments such as the soil system. We discovered that the shedding of the expressed hydrolases as soluble enzymes to the surrounding media provides the greatest advantage in locating and degrading the cellulose used for growth. Additionally, our study highlighted the importance of substrate losses through diffusion in determining the ability of microbes to survive and grow in natural settings. As

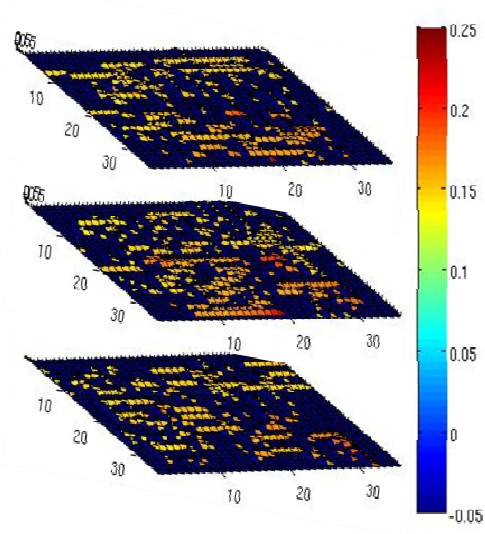

Soil aggregate is modeled as a heterogeneous porous structure mapped on a layered $35 \times 35 \times 35$ grid structure. For simplicity, only three of the 35 layers are shown. Numbers reveal the grid unit number, and spacing between units is $3 \mu \mathrm{m}$ (i.e., the aggregate is a cube with a $105 \mu \mathrm{m}$ sidelength). This is a typical distribution for the soluble substrate within aggregate pores at a particular point in time. As seen, the distribution is non-uniform. active microbial growth requires production and uptake of a substantial amount of soluble carbon substrates, cells need to work cooperatively to increase the concentration of degrading hydrolase enzymes near the cellulose source. Cooperation between cells makes it possible to overcome the diffusional losses.

Our FY 2011 studies will continue investigating the dynamics of model microbial communities in microfluidic devices and natural systems to determine which physical and chemical environmental characteristics have an impact on the microbial community dynamics. 


\title{
Precision Information Fusion Environment
}

\author{
Andrew J. Cowell, Alan R. Chappell, Tara D. Gibson, Aaron S. Harper, Jared M. Chase
}

- This research will design and implement technologies to support the next-generation precision fusion centers that tackle issues relating to timely information sharing, access and alignment, and arrangement of information. Our created prototype environment has the potential to become an integral part of threat analysis, emergency planning, training, response, recovery, and decision-making across multiple domains

I nformation fusion brings together information from distributed sources and models for the purpose of integration, analysis, decision-making, and dissemination. While information fusion is a powerful concept in theory, information access policies and data incompatibilities reduce the efficiency of such processes. This project aims to design and implement technologies to support next generation precision fusion centers that tackle issues relating to timely information sharing, access and alignment, and arrangement of information.

Responding to emergency situations is a challenging task. Data are either overwhelming or in short supply, and defined protocols useful in training become vulnerable in the stark reality of a real emergency. People start to rely on historical situations, personal experience, and "best guesses" to get them through, unsure if their actions will help or hinder unfolding event. While technology could help, inserting such solutions in these high stress environments is challenging. This effort proposes to use integrated modeling and simulation to provide a tactical capability.

Our focus in FY 2009 was to develop a prototype interface to compare and contrast the utility and importance of the different aspects of precision information fusion environments. We designed the architecture for the environment, explored a suitable set of models, and identified an appropriate middleware architecture in MeDICi. In addition to architecture design, we executed a literature review to understand the technological landscape in emergency management situations. We then performed a series of contextual inquiries (e.g., ethnographic studies), where we viewed emergency management staff in their regular work setting to understand how to introduce tactical modeling into workflow.

In FY 2010, we implemented the architecture designed in the previous year and focused specifically on social media content to sidestep the issues of using real data. Leveraging the MeDICi framework, this architecture enabled us to distribute the potentially large load on incoming data across a number of nodes in a cluster. As this was not a model-building endeavor, we looked to use prebuilt models that could be dropped into the environment for testing. Because system complexity can be an issue in stressful decision-making, the user interface for the environment was designed to be simple and efficient, offering from a small number of dedicated options. We focused on three domain areas:xxsocial media for situational awareness, contaminated building safety, and marine contaminate detection.

The system user is prompted with a simple menu where a domain of interest is selected (e.g., social media for situational awareness) with information related to this domain. A set of keywords and a geographical limiter (enabled via a map display) produce results of direct interest to the user. Search terms are submitted to the system, which in turn send them to one of four architecture media components (blogs, micro-blogs, photographs, and video). Each component sends the terms to search aggregators for that media type. For example, the blog component sends the request to 10 different blog search engines, each which covers a small part of the blogosphere. As they return, the component de-duplicates the results and passes them on to another component that pulls the content (e.g., downloads the picture or video, or the page containing the blog or micro-blog posting). For video, photographs and micro-blog, this is fairly simple, although the blog workflow is much more complex due to the issue of isolating the blog post content from the rest of the content on the webpage. For this instance, we developed a Firefox plug-in that enables the user to help define content within the page of interest, leading to a universal parsing agent template that can be used for other instances of the same blog engine.

As material is gathered, the environment is populated, and the user can visualize this information in different ways, such as on a timeline, on a map, or in a simple table. If results are not as promising as expected, we integrated Wordnet capability so users can expand keywords to cover other terms that people may have used to describe their posted content. Wordnet provides words that would disambiguate the term. As there were no models found to utilize this dataset, we provided two potential use cases. The first is an operations center, where the user enters a predefined list of terms of interest, and the environment running on a large display on the way could run continuously, alerting users when a report is made in the social media space (which can often preempt an official report through typical emergency management channels). The second use case was to enable users to make selections from the resulting information and push them into a semantic wiki environment based on the Knowledge Encapsulation Framework for further collaboration. 


\title{
Scalable Performance Diagnostics and Feedback for Massively Parallel Computers
}

\author{
Sriram Krishnamoorthy
}

The effective utilization of current and future supercomputers for science requires an application performance at scale. This project aims to develop capabilities to identify performance bottlenecks quickly and inhibitors of scalability, focusing on inter-process communication.

W hile characterizing communication behavior has been widely studied, a fundamental limitation of existing tools and approaches has been the limited usability in parallel programs running on large numbers of processors and the inflexibility in the information collected. Among the issues encountered are overhead to the profiled running program, managing the sheer size of the profiles generated, and providing insightful feedback to users rather than flooding them with raw data. Improvements in single processor performance through an increase in clock speed have been severely limited by the problems of power consumption and dissipation. High-end computers used for large-scale scientific calculations employ increasingly larger processor counts to achieve greater performance. The future exascale computers can be expected to contain up to millions of processors, with their complex interaction further complicating application scalability.

We are addressing these issues in the context of the global arrays $(\mathrm{GA})$

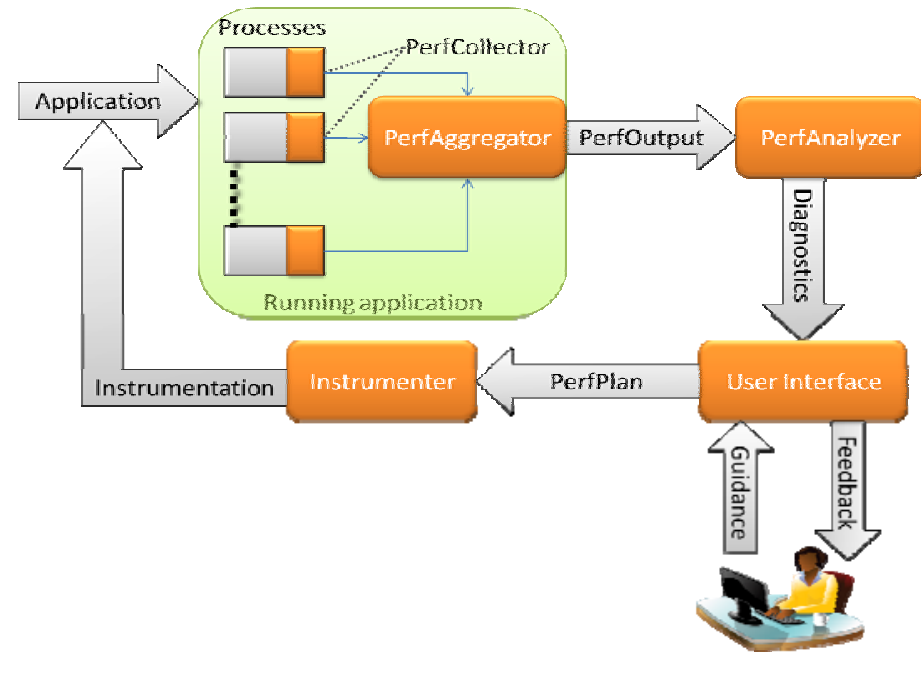

The envisioned iterative optimization process. We will develop and provide support for global arrays at each of these stages to enable effective application performance tuning. characteristics of their application, enabling a more accurate estimate of the impact of their design choices on overall application performance before implementation is complete and a large enough system is available to evaluate the implementation. Applications can thus be developed that will be ready to exploit the next generation of parallel systems when they become available.

The performance and analysis tuning has a specific process that we envision. The application developer can provide useful domain specific information to simplify performance collection and analysis. Additionally, the user can decide the level of detail and the components measured to tailor the data collected to meet the needs at hand. Such information would be used to decide the appropriate instrumentation in the program, which is then executed. The performance information from the different processes is collected and aggregated to derive a composite performance output. This output can be directly viewed by the user or run through various analyses for common performance bottlenecks. These steps can be repeated by the user to further tune the application.

During FY 2009, we developed a scalable communication trace compression technique. This unique approach extended a popular text compression algorithm to locate and compress identical patterns of communication across processes participating in programming suite, the

model used to develop key applications at PNNL. This project targets the development of suite capabilities to identify performance bottlenecks and inhibitors of scalability. We will increase understanding of the application characteristics through profiling to provide insights into their performance on future parallel systems. We will also develop capabilities to identify the inhibitors of scalability in parallel applications. Our approaches aim to allow better tuning of application performance, which will reduce time spent in tuning, allowing users to focus on newer functionality. In turn, scientists will obtain a better understanding of the performance the parallel execution. The evaluation using a standard benchmark suite demonstrated improved compression achieved over existing techniques. We also developed algorithms to identify similar (rather than just identical) patterns that would further improve the compression achieved. The results obtained from the combined algorithm were submitted for publication.

A preliminary version of a profile collection framework has been developed. The profile consists of an aggregate remote memory copy interface (ARMCI) communication information 
stored in a relational database format. The framework will allow development of a performance data repository that can be queried for performance analysis and feedback. A demonstration of the framework and associated tools was given to some members of the subsurface modeling project. We are involved in providing an improved version that incorporates their feedback to the scientists for their usage.

In FY 2010, we developed multiple tools and extensions, which are described below, to support some of the capabilities required in the tuning process.

Instrumenter - wrapper generators for ARMCI/GA. We have a python-based wrapper generator for ARMCI, which was refactored to provide a PARMCI interface enabling the wrapping of the public functions. The python-based generator was then used to develop a variety of wrappers for ARMCI, including for thread safety, tracing, profiling, and integration with other tools.

PerfCollector and PerfAggregator - communication trace compression. Communication traces retains temporal order of communication operations. However, lossless communication traces can get prohibitively large, causing programmers to resort to variety of other techniques. We developed a novel approach to lossless communication trace compression. The evaluation shows improved compression and reduced overhead over other approaches.
PerfCollector and PerfAggregator - profile collection. A preliminary version of a profile collection framework has been developed. Wrappers to ARMCI routines (written using the wrapper generator described above) were used to collect the communication profiles. In addition, the instrumenter enables collection of performance information for additional user-specified functions.

Tool integration. We have developed support for ARMCI profiling in TAU and SCALASCA, two widely distributed tools employed to profile MPI programs. Given the inter-operability between message passing interface and ARMCI/GA, this support enables users to obtain a better snapshot of the impact of the various components of their applications on the overall performance.

Application evaluation. The artifacts of this work were used to characterize a key module in NWChem using the TAU performance system. This analysis explained how subtle changes in the execution environment impact NWChem performance. We are already working with computation chemists towards tackling the algorithmic bottlenecks identified. Work on characterizing STOMP is underway.

The plans for FY 2011 include making the above capabilities developed available to application scientists. To this end, we shall incorporate feedback from a demonstration provided to the scientists and complete the tool development. 


\title{
The Analysis and Discovery of Influencing Factors in Social Media
}

\author{
Michelle L. Gregory, Eric J. Marshall, Andrew J. Cowell, Liam R. McGrath, Eric B. Bell
}

Through this research, we are developing algorithms and methods to glean information from social media sources. In particular, we are developing algorithms and methods to determine how information flows through social media so we can identify influential media channels. This project has impact both in scientific breakthroughs and research integration.

$\mathbf{T}$ here is a rich history of text analytics tools and methods that enable the analysis and understanding of large collections of text. Since the 1950s but exponentially growing in the last 25 years, algorithms were not developed to be used with the unique characteristics of today's social media data. The aspects of social media that make it difficult to handle by traditional text analytics are that it is usually messy and sparse data, not content rich like single source news articles. Often, lexical items are introduced and unconventional shorthand is commonplace (for example, IMHO for "in my humble opinion" in texting). In addition, the conversational nature of social media makes it very difficult to track topics and entities. However, the major shift to the use of this data makes it the new place to get information for political news, health services, scientific fields, and intelligence gathering. As such, new text analytics algorithms need to be developed to handle these data types.

In particular, data types have facets of information not found in traditional text types; they carry information about the relationship between users. New algorithms need to be developed to isolate these sorts of information from social media as well. We illustrate that the process of social media analytics is very similar to text analytics in general, but that new algorithms need to be developed to make use of the information provided in these data types. The scientific innovation of this work lies in addressing this research gap.

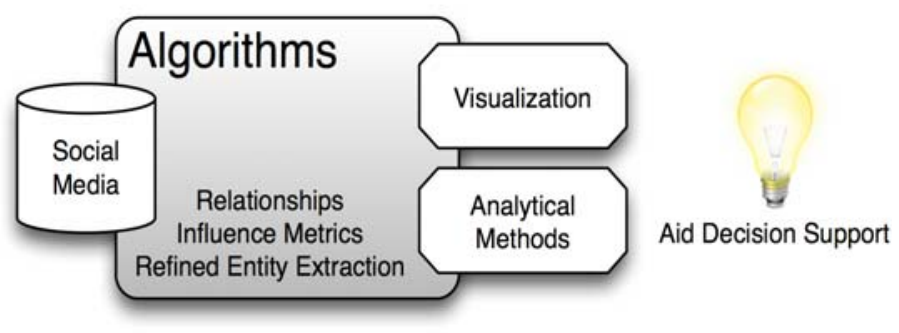

Text analytics for social media.
The specific focus of the proposed work is to build algorithm and methods that can aid in identifying opinion leaders within a small social media community. The ability to identify influential people or opinion leaders is paramount to the success of any mission that attempts to understand or influence the public response to an event.

Opinion leaders are not necessarily the decision-makers or those in charge. Often, opinion leaders are those who represent the voice and feelings of their audience and it is their natural skills in communication and leadership that enable them to influence opinion. With the emergence of new media types such as blogs, Twitter, and other social media sites, reaction to news or events is instantaneous, often well before traditional media sites can digest and produce their own stories. As such, data from these social media can be extremely useful in identifying the opinion drivers within a community. While we have begun building some basic capabilities in the area of social media analytics, this work has highlighted major open research problems in this domain. While our focus is on identifying metrics of influence, to accomplish this, we will also need to make scientific headway in the area of harvesting blogs.

In FY 2010, we developed a mathematical proof that combines our metrics of influence into a single metric. This has been extremely useful and has received good reviews (KDD Social Media Analytics Workshop). While we have begun to implement the algorithm, we need to understand the features necessary to determine influence, we need a meaningful way to combine these features into a predictive model, and this algorithm is at the center of that effort. In addition, we were able to build an early prototype for blog searches and harvesting. While the concept and outline is developed, and the methods defined, we need to incorporate the machine learning methods. This will require an annotating task to annotate a large number of blog templates so that we can train the system.

In FY 2011, we will be working on a case study that demonstrates the new capabilities developed through this researchicular client. 


\title{
Ultrascalable Solvers for Subsurface Simulation
}

\author{
Glenn E. Hammond, Barry Lee
}

We are researching the development of enhanced solver algorithms for parallel subsurface groundwater flow and contaminant transport codes for DOE use for investigating environmental remedial action alternatives and performance assessments. These improved solver algorithms will maximize using the costly high performance computing resources by subsurface simulators.

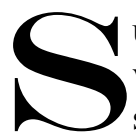
ubsurface flow and reactive transport codes play a vital role in accomplishing specific environmental stewardship objectives. These simulators enable scientists to predict the migration of contaminants in the earth's subsurface and evaluate the efficacy of proposed long-term waste repositories and site remediation alternatives, long before the technologies are deployed in the field. Built on the mathematical equations that govern groundwater flow and contaminant transport in the subsurface, these codes utilize solvers to resolve these equations much like an automobile uses an engine for propulsion. For years, solver algorithms have been effective at resolving these mathematical equations on desktop computers and small parallel computers. However, as the size of supercomputers approaches the exascale, it is unclear how these solvers will perform on these advanced machines.

The purpose of this research is to investigate the performance of conventional solver algorithms on today's largest supercomputers and improve their performance. Good performance is exemplified when a solver algorithm effectively utilizes computational power. For example, if the scientist employs 10 times more processor cores to run a given simulation, an ideal solver algorithm would solve the problem 10 times faster, a concept termed ideal efficiency. Because existing solver algorithms are known to perform with less than ideal efficiency on small supercomputers, we anticipate that their performance will degrade on larger, exascale computers. Therefore, we are developing improved solver algorithms that exhibit better efficiency than current techniques. The end goal is to incorporate these increasingly efficient solver algorithms within PNNL's eSTOMP simulator and employ them to resolve real-world flow and transport problems.

Early in FY 2010, we built upon scalability analyses conducted toward the end of FY 2009 to demonstrate that communication overhead associated with solving problems at the extreme scale (e.g., tens of thousands of processor cores) using state of the art multilevel solvers becomes excessive and dominates the time spent on computations. Essentially, the ratio of communication to computation (measured through a work unit based on a matrix-vector product) grows to excessive proportions as the problem size per processor core becomes too small (a well understood phenomenon) or the entire problem becomes too large necessitating global communication across the entire supercomputer (not so well understood). With further inspection of the communication algorithms employed within these multilevel solvers, hypotheses were developed regarding the complex communication patterns within the algorithms and potential bottlenecks impeding performance.

During FY 2010, we initiated the investigation of multilevel solver algorithms for the coupled systems of equations associated with carbon sequestration (water, $\mathrm{CO}_{2}$, temperature, and salt transport). This work reinforced the need for increased mathematical understanding of the coupling of equations governing carbon sequestration within multilevel preconditioners. Nodal relaxation was determined to be effective for smoothing these coupled equations. Our collaborators at the University Colorado at Boulder demonstrated that their novel multilevel algorithms can accommodate basis swapping, a capability need for simulating the dynamic state of the $\mathrm{CO}_{2}$ degree of freedom in the carbon sequestration problem. Through fine-grid nodal relaxation coupled with independent hierarchal processing of the variables associated with carbon sequestration processes, excellent convergence rate was achieved within their multilevel preconditioner.

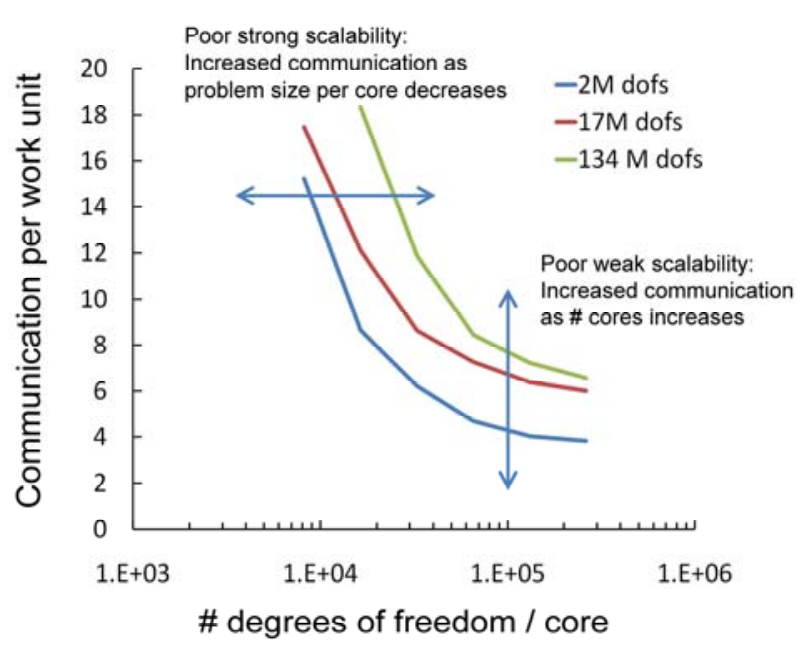

Comparison of communication per work unit ratio for three problem sizes (\# dofs) solved with various numbers of processor cores. As problem size grows (larger \# dofs) and problem size per processor core decreases, the ratio grows exponentially indicative of increased communication overhead and degrading solver scalability. 


\title{
Visualizing Uncertainty in Conceptual and Numerical Models for Geological Sequestration
}

\author{
Luke J. Gosink, Mark D. White, Diana H. Bacon, Bruce J. Palmer, Landon H. Sego
}

This research will help scientists identify and characterize uncertainty in the numerical simulations that are commonly used to assess the viability and long-term impacts of deploying sequestration at select geological regions.

D espite being actively researched for several decades, the field of uncertainty quantification, despite being is still rapidly evolving and developing. Existing research in this field attempts to quantify the uncertainty in a given system based on the system's reliance on instrumentation (used to obtain ground truth), conceptual modeling structures (the mathematical models describing a system for a real-life event), and numerical approximations (the methods used to approximate the operation of the system). A fundamental challenge to assessing uncertainty and accuracy in sequestration models arises from the fact that ground truth data - essential for most uncertainty quantification work - is largely unavailable; reliable, sufficient subsurface ground-truth is typically too costly or simply infeasible to obtain.

This project's objective, therefore, is to address this challenge by developing novel methods for characterizing uncertainty that can be used in the absence of ground truth. To achieve this goal, we combine the analytical strengths of statistics and signature science, with the interactive power of scientific visualization and visual analytic methods. Our underlying approach addresses uncertainty through the comparative analysis of varying scenarios. In this "ensemble analysis," we consider all likely events predicted by numerous systems for a fixed sequestration site. In characterizing the ensemble's "predictive uncertainty," we can identify regions for obtaining ground-truth that will (once ground truth has been acquired) provide the greatest benefit for model calibration and validation. Our integrated approach is novel in the geological sequestration, visualization, and uncertainty quantification communities.

Carbon sequestration is the process of capturing and storing greenhouse gasses deep in underground reservoirs; sequestration efforts are designed to help reduce the contributions that fossil fuels make (e.g., through their refinement and use in power generation) toward accelerating global climate change. This project, which is an integrated component of the Geological Sequestration Software Suite $\left(\mathrm{GS}^{3}\right)$, supports risk analysis, management, and mitigation tasks for sequestration activities through a unique combination of techniques derived from the fields of signature science, scientific visualization, and visual analytics. Our work will ultimately allow scientists to advance the understanding of sequestration modeling and more accurately assess the performance of and long-term impacts to sequestration activities at potential reservoirs.

Our work during FY 2010 focused on three stages: identify aspects of uncertainty to address within $\mathrm{GS}^{3}$, identify viable strategies to address these uncertainties, and acquire data for testing and validating our strategies. First, we worked to identify the varying sources of uncertainty present in each of the existing projects supporting $\mathrm{GS}^{3}$. Toward this goal, we are closely collaborating and consulting with other projects and have identified both elements of uncertainty that need to be addressed, and potential strategies for detecting and characterizing these uncertainties. Importantly, we have identified uncertainty to address in two stages of $\mathrm{GS}^{3}$ 's modeling framework: its conceptual and numerical model development stages.

To address these separate elements of uncertainty, we are developing a suite of analytical tools that combine signature characterization strategies with fuzzy clustering methods, time-series analysis, and regression analysis. We are extending this analysis with visual analytic and scientific visualization methods to help visualize and explore uncertainty characterized within sequestration data. We are working with two universities to help co-develop our visualization tool-suite: the University of North Caroline at Charlotte and the University of California at Davis. Both of these institutions have demonstrated expertise in developing novel information and scientific visualization strategies. Results from efforts made in these sub-contracts will benefit our project and directly integrate into $\mathrm{GS}^{3}$. Finally, we have acquired the data necessary to develop and validate our methods. This data consist of eight separate conceptual models, each of which is realized numerically over a time span of approximately one year.

In FY 2011, we will focus on developing and validating our methods, as well as integrating these methods into $\mathrm{GS}^{3}$. We anticipate demonstrating our first approach during the fiscal year, and anticipate full integration of our methods into $\mathrm{GS}^{3}$, including the integration of priority modeling data formats with user-downloadable application capabilities. 


\title{
Vulcan: Unexpressed Communication
}

\author{
Joseph R. Bruce, Glenn A. Fink
}

This project will create a secure, privacy enhanced collaborative environment for computer security professionals whose data sensitivities make sharing via normal means infeasible. Our work will enable rapid, global coordination of cyber defenders without interrupting analyst workflows.

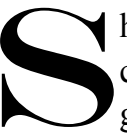

haring information is necessary for effective collaboration. In the post-September 11 world, government agencies have changed their attitudes from a "need-to-know" mindset to a "need-to-share" requirement. Cyber security problems are often far-reaching, global affairs that cannot easily be understood from a single vantage point. However, sharing introduces potential security and privacy problems, and sharing information takes work, both to share and to use the shared information. Simply automating the sharing process can exacerbate the security issues. Cyber defenders need a way to share information with others outside their organizations, governments, and countries without divulging sensitive or proprietary information and without revealing their own identities.

Our aim is to produce a system

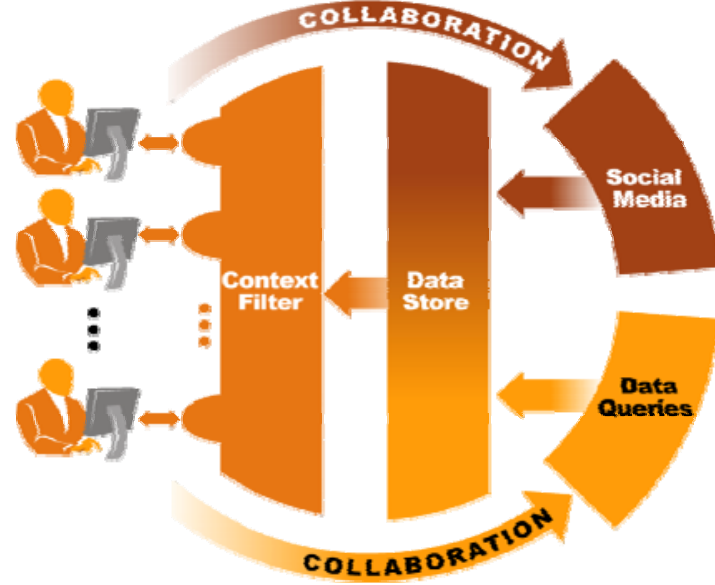

$V$ ulcan provides automatic collaboration using two sources of data: queries into internal data and accesses of Internet information such as social media. the code base of the eventual system. We also conducted an experiment in conjunction with the Pacific-Rim Regional Collegiate Cyber Defense Competition (PRCCDC).

The Vulcan collaboration system tracks activities of cyber analysts as they go about their work. As long as they stay within the tracked window or windows, queries to internal data and the external information sources are recorded and passed on to other Vulcan users. Users are never identified, but the aggregate information they generate can help cyber analysts more efficiently decide on avenues of further investigation based on the actions of others. Additionally, the Vulcan prototype will compare internal data queries of its user base for similarity and use this information as a recommendation system to propose related query terms. The prototype will gather Internet information sources being consulted by its constituency and track actual usage of this information to determine trends and suggest additional sources to users. We anticipate that Vulcan will in some ways resemble a web 2.0 social media and collaboration tool for cyber analysts.

Accomplishments during FY 2010 included publishing a journal article on cyber analyst processes. We wrote another paper (yet to be published) on our experiment at the PRCCDC that was designed to determine what metrics would help us measure the effectiveness of a collaboration treatment such as Vulcan. We used in situ situational awareness queries as a measure of team awareness and found that there is a relationship between the time and accuracy of a team's answers and its ultimate score. We plan to use situational awareness as an indicator of effectiveness of collaborative technologies in situations where there is no objective score, simulating real life situations.

For FY 2011, we will create a system that incorporates selected parts of the cyber analytic collaboration system and design studies for evaluation of the demonstration system, including an initial user interface to facilitate conduct of the studies. We will also define requirements for both the future contextualization of results and for future security of an implemented Vulcan system. 
Nuclear Science and Engineering 


\title{
Adaptation of Existing Probabilistic Risk Assessments to Support Reactor Aging Management
}

\author{
Stephen D. Unwin, Peter P. Lowry
}

This project is intended to make sure that regulatory decision-making on reactor life extension is properly risk-informed such that reactor aging management and research resources are most effectively expended. We will establish methods to identify those component aging issues that have the greatest influence on accident and operational risk and determine the most effective maintenance and monitoring strategies as well as materials research priorities for reactor aging management.

$\mathbf{P}$ robabilistic risk assessment (PRA) methodology has provided the principal basis for risk-informed decision-making in the nuclear power industry, yet limitations on conventional PRA methods constrain their value as effective tools to address aging effects and quantify risk and reliability impacts of component aging management strategies. PRA and associated decision technologies will provide the framework in which the significance of materials reliability issues can be assessed and important areas for improvement in structural materials reliability and monitoring methods identified. Although PRA models have proven to be a critical information resource in risk-informed regulatory decision-making, limitations in current methods and models constrain their prospective value in reactor aging management.

The objective of our work is to establish the methodological basis for adapting existing probabilistic risk and reliability studies to support integrated reactor aging management programs for passive nuclear power plant components. We will expand the scope and underlying models of an existing PRA study to allow reactor aging issues to be addressed and alternative aging management strategies to be evaluated and compared. The principal challenge in this expansion of methods is to model agedependent equipment failure rates and include in the PRA model certain classes of components (principally passive) that have not traditionally been captured in PRA studies. This is critical because it is passive components generally not amenable to refurbishment or replacement that are expected to govern the accident and operational risks associated with an aging fleet of nuclear power plants. There are three pillars to the methodology base being developed: 1) a means of screening passive components and associated aging degradation mechanisms to determine which could significantly influence operating risk in an aging reactor, 2) a process to systematically incorporate new failure events into a PRA, taking account of the potential dependences introduced, and 3) a method for quantifying the importance of components and associated aging stressors to operating risk.

A preliminary activity for FY 2008 was obtaining models and associated plant documentation to initiate methods development. We reconstructed a limited set of models and obtained relevant plant system engineering drawings and isometrics to facilitate an interpretation of the reconstructed models. An extensive literature review was conducted (which continued into FY 2009), with a compendium of documents describing nuclear power plant system and component aging studies, degradation management methods, and risk-based methodologies for degradation modeling. In FY 2009, a component/aging stressor screening process was developed and applied to limited scope PRA models.

In FY 2010, the methodology set previously created was applied to a full scope Level 1 PRA. This process drew on results generated under the U.S. Nuclear Regulatory Commission Proactive Management of Materials Degradation (PMMD) program in which an international expert panel assessed 3,500 nuclear power plant component classes against 16 aging degradation mechanisms. Our integration of these insights into an expanded failure modes and effects analysis produced a strong basis for component screening. Also, a new class of risk importance measure was developed that integrates conventional PRA importance measures with aging susceptibility metrics produced under the PMMD program.

Also in FY 2010, substantial progress was made in defining the means of inserting passives failure events into a PRA. We are drawing on a class of materials degradation models being developed under companion projects that apply a combination of physics and parametric analyses to model component degradation from flaw precursors to functional failure. These Markov models also provide the risk context in which the efficacies of various prognostic monitoring technologies can be assessed. The means of interfacing such models with an existing PRA structure was developed in FY 2010, and a demonstration of the methodological principles was conducted.

In FY 2011, we will refine the basis for quantification of component unavailabilities associated with specific aging degradation mechanisms and evaluate insights from application of the methodology to issues such as the risk benefits of improved system monitoring technologies, risk associated with extended operation, and priorities in materials science research. 


\title{
Advanced Nondestructive Assay for Safeguards
}

\author{
Glen A. Warren, L. Eric Smith, Sonya M. Bowyer, Luke W. Campbell, \\ Christopher J. Gesh, Derek. A. Haas, Alex C. Misner
}

This project is aimed at expanding the science base of direct measurement of uranium and plutonium isotopes in spent nuclear fuel and fresh mixed-oxide fuel. Lead slowing-down spectroscopy (LSDS) and high-energy delayed gamma spectroscopy will be studied as a means to improve material accountancy for international safeguards.

A

ccurate nondestructive measurements of the plutonium content in spent fuel assemblies is a well documented but still an unmet challenge in international safeguards. As nuclear fuel cycles propagate around the globe, the need for improved fuel accounting techniques for spent light-water reactor fuel and fresh mixed-oxide fuel will only increase (e.g., for verification at interim or permanent storage or at the head end of a reprocessing plant). Current methods for estimating plutonium mass are based on the detection of signatures from non-plutonium isotopes, which are then coupled to computational predictions to infer plutonium mass in the assembly. A nondestructive assay technology that could provide timely (tens of minutes), independent (no operator-declared information), and direct measurement of plutonium mass with high accuracy would be a significant step toward real-time accountancy and the prevention of materials diversion.

This project is aimed at expanding the science base of direct measurement of uranium and plutonium isotopes in spent nuclear fuel and fresh mixed-oxide (MOX) fuel. The foundational assay method will be LSDS, but high-energy delayed gamma spectroscopy and combinations thereof will also be studied. Both techniques have shown promise in proof-of-principle theoretical and simulation studies but require additional theoretical study coupled to empirical validation. This project will: 1) design, build, and characterize an LSDS instrument, 2) develop and use the capabilities to design, fabricate, and characterize the specialized fission chambers required by LSDS, 3) perform benchmarking measurements, and 4) develop and test analysis methods that address the complexities of neutron and gamma-ray self-shielding. This system will be used to study critical physics issues and to test instrumentation and data acquisition methods to develop non-destructive assay of spent fuel.

This project will significantly extend and validate the science base relevant to the direct measurement of plutonium in nuclear fuel. Additional outcomes of the research include the following:

Cross-sectional view of the LSDS showing the room, the lead cube, and the table (structure under the lead cube).

- Development of techniques required for the design, fabrication, and characterization of specialized fission chambers required for and LSDS instrument, and for other fuel cycle research applications (e.g., burn-up credit reactivity measurements, absolute fission cross-sections)

- Precise characterization of the time-dependent neutron transport and reactions, and the development of simulation methods to predict that behavior for viability studies will be benchmarked using a unique detection instrumentation suite

- The ability to quantify the strengths and limitations of lead slowing-down spectroscopy and high-energy delayed gamma spectroscopy for spent and MOX fuel assay using empirically validated modeling.

The combination of novel sensor development, modeling, experiment, analysis and evaluation in this project will provide needed capabilities in nuclear safeguards.

The primary focus of the project for FY 2010 was the design and construction of the LSDS. The principle components of the instrument are a large cube of lead $1.5 \mathrm{~m}$ on a side, with a neutron generator, a measurement sample, and detector instrumentation. The lead cube has a vertical hole and horizontal holes for placing the neutron generator, samples and instrumentation packages. The size of the lead cube was optimized for cost and measurement efficiency. The lead is naturally occurring and of high purity, which is important for maximizing the energy resolution of the system. An existing neutron generator will be used for system.

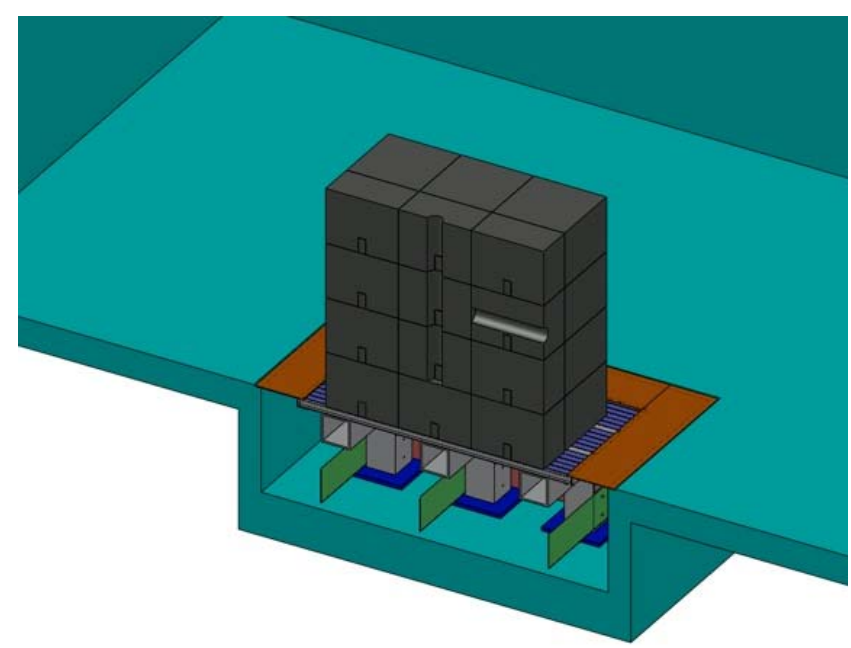


The initial measurements of the LSDS will be to characterize the energy-time relationship of the neutrons. This will be accomplished measuring the time difference between the entrance of the neutrons into the lead and the detection of gamma rays from specific foils. Those gamma rays will be generated at specific neutron energies, which depend on the material, so that a relationship between neutron energy and time can be determined. We ordered the detector for these measurements, while the foils and data acquisition system have been received. We completed a test of data acquisition system to demonstrate that the time resolution was adequate for these measurements.

Fast neutron detectors are a critical component of the LSDS as a means of monitoring fission rates. This project is developing actinide-loaded glass scintillators for this purpose. These glass scintillators may provide significantly more active material than the traditional fission chamber, and hence potentially higher sensitivity as well as the ability to differentiate fission events from alpha and gamma-ray radiation. Various silicate glass buttons containing 10 percent Th-232 loading by weight with four different rare earth activators were formulated and produced. While the samples demonstrated a response when exposed to gamma rays, preliminary measurements using a neutron source showed no obvious fast neutron response.

For FY 2011, the project will conduct measurements to characterize the LSDS as well as conduct initial measurements on simple nuclear materials. These measurements will enable us to begin the empirical validation of theoretical/numerical models that indicate the promise of these techniques for the direct measurement of plutonium in spent fuel. 


\section{Advanced Radiation Transport Methods}

Erin A. Miller, Jacob M. Benz, Aaron M. Bevill, Christopher J. Gesh, Kenneth D. Jarman, Sean M. Robinson, L. Eric Smith, Richard S. Wittman

Simulating radiation transport is important for finding or identifying radioactive materials. It provides a tool for designing detectors, evaluating detectors, and interpreting measurements. This project investigates ways of using, combining, and developing radiation transport methods in order to improve these often-lengthy calculations.

$\mathbf{R}$ adiation measurements are an important component of many security systems, either for detecting radioactive materials or to investigate objects (such as with radiography). Most radiation transport simulations are based on a Monte Carlo method, where random individual particles are simulated as they travel through a system and interact. This method works well in many cases, but can become prohibitively slow if many particles are involved, as is the case for heavily shielded problems or active interrogation. An alternative approach, deterministic radiation transport, is based on solving a continuity equation for a steady-state particle distribution. Often used for nuclear reactor design, this approach is well suited to describing large numbers of particles. Prior work at PNNL has developed a successful hybrid method using both deterministic and Monte Carlo methods to simulate gamma-spectroscopy problems.

This project develops advanced transport methods and approaches based on deterministic or hybrid approaches. Further methods development offers the possibility of faster simulations, greater solution confidence, and applications of deterministic or hybrid simulations to new problem types. Three main tasks are included: imaging, hybrid methods, and inverse problems. For imaging applications, such as radiography, Monte Carlo calculations can be extremely slow due to the large number of particles. Deterministic methods are investigated as an alternative. The hybrid methods investigated here use a deterministic adjoint calculation (which reveals the importance of any region of the problem to a detector, as opposed to the count rate due to a source) to improve the efficiency of a Monte Carlo calculation. Adjoint calculations may also be used directly to attempt to infer source characteristics and location.

For imaging, work completed in FY 2009 found that deterministic transport has potential to accelerate cone-beam radiography simulations by up to two orders of magnitude over Monte Carlo. However, it is often not feasible to use a finite element mesh with mesh size comparable to the imaging resolution. The more coarse computational mesh leads to mesh effects in the solution. Ray tracing allows the primary (unscattered) beam to be calculated without mesh artifacts; an alternative calculation method involving ray tracing in addition to the finite element calculation was used to eliminate mesh effects in the single-scatter component. This allows for a good, spatially smooth estimate of the majority of the signal. This calculation method may provide a useful tool for cone-beam radiography calculations.

Hybrid methods for detector response calculations and for transport biasing were identified and investigated in FY 2009, with modest gains seen for simple test problems. Work in FY 2010 focused on more challenging and more realistic test problems, in particular the assay of $\mathrm{UF}_{6}$ cylinders. For more challenging and strongly scattering problems, hybrid methods show considerable value, offering tens to hundreds of times improvements in efficiency. An effort was made to identify potential errors or difficulties in implementing hybrid methods, including the effects of insufficiently precise deterministic calculations, the ability to capture important problem features, and certain types of sampling which may require care in implementation with a transport biasing scheme. These results will aid in the implementation of hybrid methods to difficult simulation problems.

Work completed throughout the two years of this project has also identified and developed a method for inferring source position and strength based on adjoint calculations and count rates at multiple detectors. This information was used to produce a probability map of likely source location and strength, and successfully tested with a simple analytic test problem. The method was extended during FY 2010 to a more realistic test problem with moderate scattering, which was solved numerically. The test problem generally consists of 4 meter-scale boxes, surrounded by 8 detectors (or detector locations). Given a small source plus background radiation (resulting in a signal-to-noise ranging from 0.1 to at most 10 , but more typically around 1 ), the source location was correctly identified. A more simple method that did not take into account scattered radiation failed to locate the source in all three cases. Most prior source localization work has involved scatter-free approximations (or even free space approximations), and most produce only an estimate of location, not a complete probability map.

The general mathematical framework developed here may provide the basis of an improved localization capability, particularly for highly shielded, low-count sources. 


\title{
Application of Nitrogen Trifluoride $\left(\mathrm{NF}_{3}\right)$ to the Nuclear Fuel Cycle
}

\author{
Randall D. Scheele, Bruce K. McNamara, Andrew M. Casella
}

Using nitrogen trifluoride as a fluorinating agent offers the promise of a less hazardous process with the potential for using temperature as another tool to effect separations of fission products and transuranics from uranium, thus providing a new and more attractive fuels reprocessing approach

A ctinide and fission product fluoride volatility have long been considered as a potential compact and efficient approach for recovering valuable fuel constituents from spent nuclear fuels. As we investigated approaches for decontaminating the Portsmouth Gaseous Diffusion Uranium Enrichment Plant in Piketon, Ohio, we found that nitrogen trifluoride was an effective thermal fluorinating agent for the uranium and technetium deposit materials within the enrichment cascade. Nitrogen trifluoride converted the uranyl fluoride and various technetium compounds to volatile fluorides. Recognizing that nitrogen trifluoride had the potential to be an effective fluorinating agent for producing volatile fluorides from nuclear materials such as uranium, other actinide, and fission product compounds, we began this project to determine if nitrogen trifluoride had application in the nuclear fuel cycle as a fluorinating and oxidizing agent with the ability to enhance separations through thermal selectivity. Uranium fluoride volatility is key to uranium isotopic enrichment.

The objective of this project is to determine whether nitrogen trifluoride is an effective fluorinating agent for nuclear materials and fission products and whether it could be used as the fluorinating agent in the fluoride volatility fuels reprocessing flowsheet or other portions of the nuclear fuel cycle to produce volatile actinide and fission product fluorides. To investigate, we are using thermoanalytical methods to study nitrogen trifluoride thermal fluorination of uranium, other actinide, and fission product metal, oxides, and fluorides. We will develop the thermal chemistry needed to demonstrate the application of a recently identified actinide fluorinating and oxidizing agent into the nuclear fuel cycle. The new agent is attractive because it would provide a non-hazardous alternative to highly toxic and reactive tandem fluorinating agents that are currently used for nuclear fuel cycle applications. It has been demonstrated to convert uranium metal, common oxides, and fluorides effectively to the volatile uranium hexafluoride with different thermal selectivity. Thermoanalytical, calorimetric, and physiochemical solids characterization techniques will be used to investigate the reaction of the new fluorinating agent with increasingly complex chemical oxide systems representative of used nuclear fuel and its constituents.

During FY 2009, we performed screening studies using simultaneous thermogravimetric and differential thermal analysis to determine the thermal reaction sensitivity and mechanisms of the fluorination of uranium, uranium dioxide, triuranium sequioxide, uranium trioxide, molybdenum metal and trioxide, ruthenium oxide, and rhodium oxide. Nitrogen trifluoride effectively converted all of the uranium compounds, the molybdenum compounds, and ruthenium oxide to volatile fluorides. These experimental studies indicate that nitrogen trifluoride can potentially be used as a fluorinating agent for materials found in the nuclear fuel cycle, specifically including reprocessing.

In FY 2010, we completed preliminary studies on fluorination and/or oxidation of fission product oxides that form volatile fluorides, lanthanide oxides, and plutonium and neptunium oxides, which demonstrated that nitrogen trifluoride is an effective but temperature-sensitive fluorinating agent depending on the material. Next, we demonstrated the separation of uranium from plutonium in a mixed uranium and plutonium oxide, and molybdenum from uranium in a mixed molybdenum and uranium oxide. We also demonstrated fluorination of TRISO fuel components carbon, silicon carbide. Finally, we investigated low-temperature (approximately $100^{\circ} \mathrm{C}$ ) fluorination/oxidation of uranium and plutonium metals to elucidate the mechanism and kinetics. The latter studies indicate that the fluorination/ oxidation of the metal ultimately to uranium hexafluoride is a step-wise mechanism proceeding through heretofore unknown uranium compounds.

Our studies show nitrogen trifluoride's promise as a fluorinating agent for nuclear and nuclear-bred materials found in the nuclear fuel cycle including nuclear fuel reprocessing. A journal article on the fluorination of uranium compounds was finalized and published, and we presented papers at an American Nuclear Society Annual Conference and the Actinides Separations Conference.

For FY 2011, we will continue to refine our knowledge on the thermal sensitivities and reaction mechanisms for fission product and actinide oxides, develop reaction kinetics for the fluorination and/or oxidation of metallic uranium and plutonium and uranium dioxide, and investigate the separation of selected fission products and/or actinides from uranium. 


\section{Damage Maps for Waste Forms: Stage 1-Charge Imbalance Damage Sources}

Rick E. Williford, Joseph V. Ryan

This work considers transport of the microdamage across the waste form radius under competing thermal, charge, stress, and concentration gradients that cause localized damage accumulation, determine whether the waste form is inherently stable or unstable, and define the initial conditions for subsequent waste form corrosion.

$\mathbf{E}$ ngineered nuclear waste forms such as glasses and ceramics undergo microscale damage from radioactive decay and self-heating long after disposal. Low-level waste forms immobilize non-fissile radionuclides such as ${ }^{137} \mathrm{Cs}^{+1}$ and ${ }^{90} \mathrm{Sr}^{+2}$ that undergo beta decay to produce ${ }^{137} \mathrm{Ba}^{+2}$ and ${ }^{90} \mathrm{Zr}^{+4}$. Ionic radii decrease by about 20 and 29 percent, respectively, and in turn introduce localized charge and stress imbalances (damage) into the host glass matrix. The decay heat causes a roughly parabolic radial temperature distribution across the cylindrical waste form and introduces stress gradients. Chemical diffusion can disperse the defects, while temperature, stress, and charge gradients cause the radionuclides to migrate into defect clusters. The formation of such clusters at the waste form surface would provide corrosion sensitive regions such as highly soluble $\mathrm{Ba}$ or microcracks that would limit the functional lifetime of the waste form.

This work addresses the interactive competition between all four of the above thermodynamic migration forces in a unified analysis. It defines initial conditions for subsequent corrosion mechanisms after the 300 -year cool down period. Such long times are experimentally inaccessible, so sophisticated modeling techniques must be employed. We have developed modeling techniques that can predict the migration of damage sites due to competitive thermal, stress, charge, and chemical gradients that will determine whether $\mathrm{Cs}$ or Ba defect sites accumulate at the waste form surface (leading to corrosion-sensitive regions) and thus whether glass waste form is inherently stable or unstable.

In FY 2010, borosilicate glass was selected as the model waste form. Data for simulations were assembled for the Cs and $\mathrm{Ba}$ defect thermo-physical properties; the dimensions, mechanical and thermal properties of the glass, along with the decay heat generation rate and surface convective cooling conditions. A transient, multi-scale finite difference computer model was written to perform the simulations, and includes charge effects on diffusivities.

Simulations show that $\mathrm{Cs}$ and $\mathrm{Ba}$ migrate down the waste form radial temperature gradient (toward the surface), via the Soret effect. The compressive $\mathrm{Cs}_{\mathrm{Na}}$ defect (initial doping) migrates towards the far field tensile region at the waste form surface, while the tensile $\mathrm{Ba}_{\mathrm{Cs}}$ defect (after decay) migrates back towards the compressive region at the waste form center, both to balance local strain energies. Self-generated near field misfit stresses between the defect centers $\left(\mathrm{Cs}_{\mathrm{Na}}\right.$ or $\mathrm{Ba}_{\mathrm{Cs}}$ substitution sites) can also cause defect agglomeration that leads to void formation or microcracking sites that are corrosion sensitive. Near field effects are dominated by initial doping concentrations that determine competition between defect spacing (agglomeration forces) and decay heat generation (allowable temperatures).

Predicted thermal and stress distribution histories for initial Cs loadings of $2.33 \times 10^{-4}$ moles $/ \mathrm{cm}^{3}$ were quite reasonable compared to previously published studies, thus establishing a benchmark for further comparisons. Simulations up to 95 years (about 3 Cs half-lives) have been completed, with only small accumulation of $\mathrm{Cs}$ or $\mathrm{Ba}$ at the waste form surface (the site of initial corrosion). Moderate charge effects due to the small self-generated electrical fields appear to retard Cs migration. Extensions to 300 years are underway. To date, thermal diffusion dominates $\mathrm{Cs}$ and $\mathrm{Ba}$ defect localization effects, with far-field stress effects contributing about 10 percent. Near-field stress effects have so far been negligible for the defect radii simulated (the lower limit: atomic radii). Larger defect radii are presently under investigation, consistent with atomistic simulations.

At long times when the Cs has mostly decayed, Ba accumulated at the surface may reverse migration direction in the far field stress distribution if temperatures remain high enough for enhanced mobility. Preliminary results for longer simulations indicate that this reversal time is between 100 and 300 years. This is an important factor for initial surface conditions in waste form corrosion studies. Future work will address other, more mobile species (e.g., $\mathrm{Na}$ ), because they were found to be important for corrosion sensitivity in the literature. The ${ }^{90} \mathrm{Sr}^{+2} \rightarrow{ }^{90} \mathrm{Zr}^{+4}$ decay will also be treated to further assess charge effects.

Additional simulations are underway to assess the effects of uncertainties in initial loadings, diffusivities, defect radii, and heat of thermal transport as well as a radially oriented "channel" of high Cs concentration (type of "manufacturing defect") that can lead to defect localization. The results will help define manufacturing and corrosion rate tolerances for glass waste forms. The value is that the work predicts waste form stability where experiments are not possible from the long time periods. It is the first known unified treatment of interacting charge-diffusion-thermal-stress effects for waste forms and defines initial conditions for corrosion studies, while the latter defines boundary conditions for this work: the two are inter-connected parts of the same problem. 


\title{
Dissolution of Actinides Under Oxidizing Alkaline Conditions for Nuclear Energy Applications
}

\author{
Matthew Douglas, Shane M. Peper, Bruce K. McNamara, Matthew J. O'Hara
}

With more than 20 new nuclear power plants proposed in the United States in the next few years and over 160 proposed worldwide, it is of paramount importance that efficient and preferably environmentally benign ("green") processes be available to reprocess the current and future inventories of commercial spent nuclear fuel (SNF).

$\mathbf{T}$ o date, the Plutonium Uranium Redox EXtraction process (or so-called PUREX) process is the only technology that has been globally employed at a significant scale to reprocess SNF. This liquid-liquid extraction process uses highly concentrated $\mathrm{HNO}_{3}$ solutions $(6-11 \mathrm{M})$ at elevated temperatures as the head-end process for dissolving SNF. Following dissolution, organic extractants are dissolved in volatile organic solvents and used to facilitate the separation of actinides from each other and from other fission products. This process has several inherent shortcomings based on its design, such as the use of combustible organic solvents and corrosive acids, radiation-induced solvent degradation, the loss of minor actinides among fission product waste, and the generation of highly radiotoxic mixed waste. Conversely, we are attempting to recycle uranium from SNF by selectively leaching it from the irradiated fuel using optimized process conditions, which will dramatically reduce the amount of radioactive waste requiring long-term storage because uranium comprises approximately 94 percent of the total SNF generated by commercial power reactors in the United States.

The work being done in this effort focuses on optimizing dissolution behavior of U(IV) and other actinide oxides under oxidizing alkaline conditions and understanding the solution and solid-state coordination chemistry in this environment to design a "green" process for SNF dissolution. Recently, members of our research team discovered that the principal component of SNF, namely U(IV) oxide, can rapidly be dissolved at room temperature using a combination of common household reagents. This opens up the possibility for the first time to create an environmentally benign SNF recycle process that is also economically favorable and that has the potential to be operated as a "closed" system. This environmentally friendly approach results in the generation of benign yet recyclable byproducts, namely oxygen, water, and carbon dioxide. The key is to optimize the dissolution of U(IV) oxide under mild conditions at room temperature with the goal of designing an environmentally benign approach suitable for head-end SNF reprocessing. It is anticipated that this technology could potentially be integrated into existing reprocessing flowsheets currently under consideration by DOE (such as UREX-1a) or it could be used as the basis for developing an entirely green alkaline SNF recycling process. From a technical perspective, due to the paucity of data on the behavior of actinides in peroxide-containing alkaline media it is expected that many seminal scientific contributions will be made in several key areas, including actinide separations and actinide coordination chemistry.

During FY 2009, research was focused in uranium oxide $\left(\mathrm{UO}_{2}, \mathrm{U}_{3} \mathrm{O}_{8}\right.$, and $\left.\mathrm{UO}_{3}\right)$ dissolution studies, fabrication and testing of an automated system for monitoring actinide oxide dissolutions, solid-state f-element chemistry, and development of a new capability to analyze single crystals containing f-elements spectroscopically. For $\mathrm{UO}_{2}$, we continued our studies from FY 2008 and examined the effect of several key experimental parameters on the rate of uranium dissolution. The purpose of these experiments was to study the optimal reaction conditions to perform a dissolution demonstration using spent nuclear fuel containing approximately 95 percent $\mathrm{UO}_{2}$. It was found that the ammonium cation yields the most optimal initial uranium dissolution rates.

In FY 2010, research focused on three tasks: dissolution of uranium oxides in aqueous peroxide-containing carbonate solutions using an atmosphere-controlled digestion vessel, solution and solid-state speciation of actinides and lanthanides in aqueous peroxide-containing carbonate solutions, and use of microscope-based platforms for characterizing f-elements in solid and solution state by Raman, ultraviolet-visible-near infrared (UV-vis-NIR) absorption and classical fluorescence emission spectroscopies. The digestion vessel developed under the first task was modified so that either aqueous or gaseous reactants could be evaluated for their efficacy in increasing the dissolution rate of various uranium oxides. Specific parameters investigated included carbonate concentration, carbonate countercation, stir rate, and the use of gaseous reactants. A key phenomenon observed was the effect of oxygen on the kinetic rate of uranium dissolution. It was observed that oxygen significantly increased the rate of uranium dissolution by acting as a co-oxidant (together with peroxide). This result was welcomed due to the fact that oxygen is one of the key decomposition products of hydrogen peroxide, which is an important reactant.

Under the second task, various preparatory methods were used to synthesize single crystals of uranium, neptunium, plutonium, cerium, neodymium, and holmium. Most of the chemical structures observed in the peroxide-carbonate 
system were previously undiscovered and exhibited

unprecedented f-element coordination motifs. These single crystals were also analyzed using a new capability developed under this work, namely a high-resolution microscope-based Raman spectrometer. The data acquired from solutions of these key elements was used as a complementary technique for supported nuclear magnetic resonance data. The most exciting discovery was the determination of new series of trimeric actinide species, whereby one of the three uranium atoms could be replaced by either neptunium or plutonium. This finding allows us to understand better some of the fundamental coordination mechanisms that occur in aqueous solutions containing multiple actinide elements.

Finally, under the third task, a new laboratory technique was developed to collect high-resolution UV-vis-NIR absorbance and fluorescence spectra using a microscope-based platform. This technology will be invaluable for characterizing the solutions and solids previously analyzed using the aforementioned Raman spectroscopy technique. 


\title{
Dual-Mode Imaging for Dismantlement Transparency
}

\author{
Allen Seifert, Kenneth D. Jarman, Benjamin S. McDonald, Erin A. Miller, Alex C. Misner, \\ Mitchell J. Myjak, W. Karl Pitts, Sean M. Robinson, Mitchell L. Woodring
}

The detail discernible in imaging techniques has generally excluded them from consideration as verification tools in inspection regimes, even though this technology would help detect the improper diversion of special nuclear material. This project develops a number of image analysis algorithms that neither store nor disclose sensitive information, enabling their use in an arms control context.

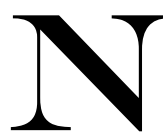
uclear nonproliferation and nuclear arms reduction are central objectives of many international treaties and other agreements. The signatory parties agree on a process by which to secure transparency on all sides. Inspectors might confirm that a small number of dismantled warheads are in a set of storage containers by making key measurements after these containers have been sealed. These measurements form the basis of comparison for later inspections of equivalent sealed containers. Preventing disclosure of sensitive information is critical for multiparty agreements. Hence, information barriers are typically established using physical obstructions, instrument firewalls, volatile data storage, rigorous procedural control, and other techniques. An information barrier is a collection of hardware, software, and procedures designed to prevent disclosure of sensitive information acquired during measurement. The inspections must achieve a balance between protecting sensitive data and disclosing sufficient information to establish that the container holds the declared item(s).

Radiation imaging is not normally incorporated into the verification process. However, imaging gives the spatial relationship between subassemblies in the object to be verified, which provides one of the strongest diagnostics in assessing the intended function of that item. As the focus on arms control shifts to greater discrimination of items after the reduction in nuclear weapons from the Cold War era, the simple attributes defined for material control may not have sufficient fidelity to distinguish treaty-limited items (such as weapons) from non-limited items (such as components or stored material). At the same time, a dismantlement regime might utilize imaging to assess whether a subassembly is a weapon component such as a pit or just a lump of special nuclear material having a similar radiation signature. Despite these advantages, imaging has been routinely dismissed as a verification technique, due in large part to assumptions that it is too difficult to implement behind an information barrier. While imaging is invasive, its capability to assess form and function could be key to enabling future arms control agreements. Operating the analysis algorithm reliably and autonomously behind an information barrier presents a technical challenge; our work has attempted to gauge the magnitude of that challenge. Whether imaging is accepted or rejected is ultimately a policy, not a technical, decision.

In FY 2009, we established a general computational framework for comparing any potential image comparison metric against a set of image data to establish the value of comparison metrics we devise. We vetted this framework against real data produced from active imaging of test objects with varying density using initial metrics based on comparison of one-dimensional histograms of two-dimensional images. This process provided a level of information barrier through data reduction. We then demonstrated the power of metrics to resolve true differences in terms of object densities on several similar objects with varying densities. From there, we explored a direct active image analysis method to estimate the overall density of spherical objects within an image as a means to verify declared materials.

For FY 2010, we developed three image analysis algorithms that reduce full image information to non-sensitive feature information.

Histogram comparison. This algorithm computes a histogram of pixel intensities from an image taken from an active imaging (radiography) system. The histogram from the interrogated object is compared to a previously generated "template" histogram to determine a degree of similarity with the expected object. This template would be stored on the system. The histogram should be a sufficient one-way transform to protect sensitive information from the original image, but further work remains to prove this assertion. Initial tests indicate that the histogram comparison algorithm distinguishes very well between phantom objects of the same shape but containing different densities.

Material recognition. This algorithm examines the pixels near the edges of an object in the radiography image. The intensity gradient corresponds to the density of the material: the higher the density, the sharper the edge transition. We developed algorithms for spherical objects as a relatively simple example. Like the histogram comparison algorithm, the material recognition technique is designed not to disclose sensitive information. Although the radius of the object is computed as part of the process, only the material attenuation factor needs to be reported. We obtained good results with both real and simulated images of spheres of different sizes and materials. 
Active/passive pixel correlation. This algorithm studies the correlation between pixel intensities in two images of the same container: one taken with an active (radiography) system, and one taken with a passive (autoradiography) system. The key idea behind this algorithm is that the dense items of interest will also be the most radioactive, whereas the surrounding filler material will be less dense and not emissive. If the dense items in the active image line up with the bright items in the passive image, the method yields a strong correlation between intensity values. A weaker correlation may denote an attempt to replace the true items with surrogate materials. The key advantage of the pixel correlation algorithm is the lack of stored parameters for image analysis. Hence, there are no information barrier restrictions to the technique. Simulated results indicate that the algorithm performs as expected for phantom objects.

Together with knowledge of the imaging systems, the use of these analysis algorithms either alone or in combination can potentially improve verification capability and increase the likelihood of detecting material diversion. 


\section{Isotopic Ratio Fluence Monitors for Canadian Deuterium Uranium (CANDU) and Pebble Bed Modular Reactor (PBMR) Plutonium Production Verification}

David C. Gerlach, Christopher J. Gesh, Scott C. Szechenyi

We are developing a concept to create monitoring devices that can be attached to reactor vessels and/or in-core structures, enabling the quantification of plutonium produced with sufficient accuracy for safeguards purposes, without direct analyses of fuel. The monitoring devices will provide a method where adherence to declared reactor operation can be objectively verified.

$\mathbf{T}$ he design of CANDU reactors presents unique nonproliferation and safeguards challenges. With respect to material accountancy, the relatively small physical size of CANDU fuel bundles, their short in-core duty cycle, and the ability of the CANDU reactor to be reloaded while in operation complicate item accounting considerably when compared to light water reactor (LWR). PBMR reactors represent less of a proliferation threat but pose additional and novel safeguards and accounting challenges due to the reactor design. The PBMR uses fuel pebbles rather than traditional pellets housed in fuel rods such as an LWR would use. The sheer number of fuel elements can present an accountability challenge.

We are developing and demonstrating techniques for measuring ratios of relative abundance of certain impurity elements for determining the fluence to which ex-reactor materials have been exposed, providing for the ability to calculate the amount of any other isotope produced, including plutonium. While our team has previously demonstrated these measurements using naturally occurring impurity elements, this can be time-consuming and impractical for large numbers of samples. Consequently, we are developing purpose-built indicators for CANDU and PBMR reactors and fuel assemblies that should make such measurements more practical.

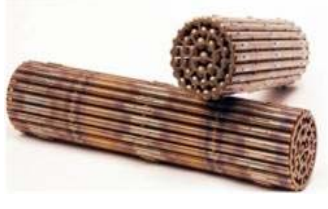

CANDU fuel assemblies
Our approach to developing and calibrating these monitoring devices is to conduct irradiation of graphite (for PBMR) and metals (for CANDU) in the Missouri University Research Reactor (MURR) and the
Advanced Test Reactor (ATR), followed by analysis at PNNL using a secondary ionization mass spectrometer (SIMS) to establish calibration curves of isotope ratio changes versus fluence. The resulting data and calibration curves should provide a means to quantify plutonium production in both reactor types and should give an indication of which of the four
candidate
metals would
make the
most practical
monitor for
CANDU.

In

FY 2009,

research

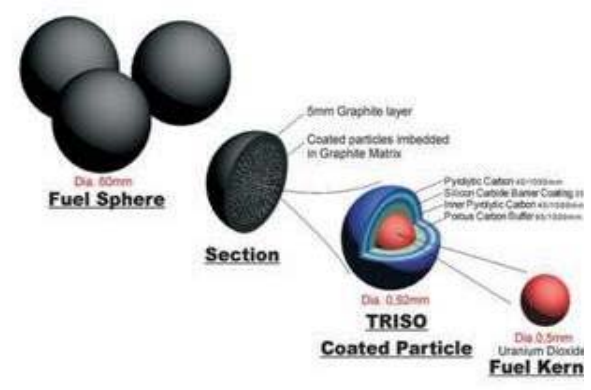

Construction of PBMR fuel elements focused on

potential designs for CANDU monitors and preparing for the irradiation of samples at the MURR reactor. In FY 2010, our primary tasks included packaging the graphite samples for irradiation at MURR, irradiation of samples, shipment of the first samples from MURR to PNNL, and presentations of the work. The first MURR sample was shipped to PNNL in mid-September, and the aluminum capsule was opened in a glove box after a delay due to high activity. The graphite samples from this first exposure package were then shipped to the SIMS laboratory for analysis. The removal sequence of three sample capsules at MURR had to be reversed due to unanticipated swelling of the lowest fluence capsule. While the two higher fluence samples were apparently operating above self-annealing temperatures, the lowest fluence capsule was below the critical temperature and Wigner energy with its associated swelling unexpectedly occurred. We had originally planned for this lowest fluence capsule to remain in the reactor for 18 months, but by reversing the order, we removed this capsule before enough swelling occurred to threaten integrity, and obtained a greater variation between the three levels of irradiation.

The SIMS analysis of the graphite samples will take place in FY 2011. Original plans were to irradiate metals with graphite at MURR. Because MURR did not have safety analyses on file for these metals, we applied to irradiate platinum, rhenium, hafnium, and tungsten samples at ATR during FY 2010. Due to higher fluence available at ATR, the exposure time can be shorter and still simulate reasonable exposures for our calibrations, and SIMS analyses of these metal samples can easily be accomplished in FY 2011.

In FY 2011, the remaining samples irradiated at MURR and ATR will be removed at two intervals for analyses. The samples will be used as reference standards and to construct calibration curves for CANDU monitors and to develop approaches for PBMR reactor accountability. Preliminary designs for CANDU monitors will be refined. 


\section{On-Line Flaw Detection in Reactor Piping using Acoustic Emission and Guided Wave Ultrasonic Techniques}

Stephen E. Cumblidge, Ryan M. Meyer, Steven R. Doctor

A shift from periodic local inspections of welds to global continuous monitoring would greatly improve crack detection, especially for the fast-growing flaws. An effective, online, continuous monitoring of the pressure vessel and important Class 1 piping systems using acoustic emission and guided wave ultrasound may be able to detect the fast-growing cracks and notify reactor operators before cracks have the opportunity to cause a leak in reactor components.

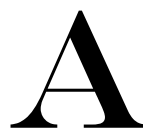

combination of acoustic emission and guided wave ultrasound can be used to monitor nuclear reactor cracking in two ways. Acoustic emission sensors can passively listen for characteristic signs of cracking during reactor operation. With an array of sensors mounted in strategic locations, one can determine the approximate location of the cracking. Guided wave ultrasound uses a series of transducers to project low-frequency ultrasound down the length of a pipe. The ultrasonic wave interacts with discontinuities and returns a series of echoes based on pipe characteristics. The pipe geometry and welds contribute to a constant ultrasonic signature for the guided-wave system. These guided waves would be emitted at regular intervals, and specific changes in the reflected and transmitted guided waves would show signs of degradation. While acoustic emission and guided wave ultrasound have been well developed, they have yet to be used together for continuous, on-line monitoring of nuclear components. The aim of this project is to experiment with these techniques to allow for continuous on-line monitoring of reactor components for cracking.

In FY 2009, a digital 8-channel acoustic emission system was purchased to capture and analyze acoustic emission signals real time. This system was tested on a large diameter pipe specimen from a cancelled nuclear power plant. The tests consisted of pencil-lead breaks and fatigue cracking of surrogate samples attached to the pipe. The system was also tested on fatigue cracks grown in a 4-inch carbon steel pipe using a four-point bending test. A notch was introduced to the pipe before undergoing cyclic loadings. With these tests, the acoustic emission system could discriminate between noise signals and those originating from fatigue flaw growth.

Activities for FY 2010 included developing and fabricating acoustic emission waveguides that allow acoustic monitoring of components subject to harsh environments by coupling the acoustic emission signal through a long metal rod. Of general concern is the effect of waveguides on the signal-to-noise ratio of the acoustic emission monitoring system. The aim of the acoustic emission waveguide studies is to determine the effect that relevant waveguide parameters have on the acoustic emission signal.

A software package was purchased to simulate and model guided wave propagation in test specimens. A guided wave ultrasound system was developed and tested on a 4-inch diameter carbon steel pipe. A saw cut in the pipe verified that flaws have a noticeable influence on the wave signal. An experiment was designed to fatigue stress a 4-inch diameter stainless steel pipe to failure while monitoring the pipe with guided wave ultrasonic transducers and acoustic emission transducers to correlate acoustic emissions and guided wave signals to fatigue crack growth. Baseline data (pre-loading) for this experiment has been collected.
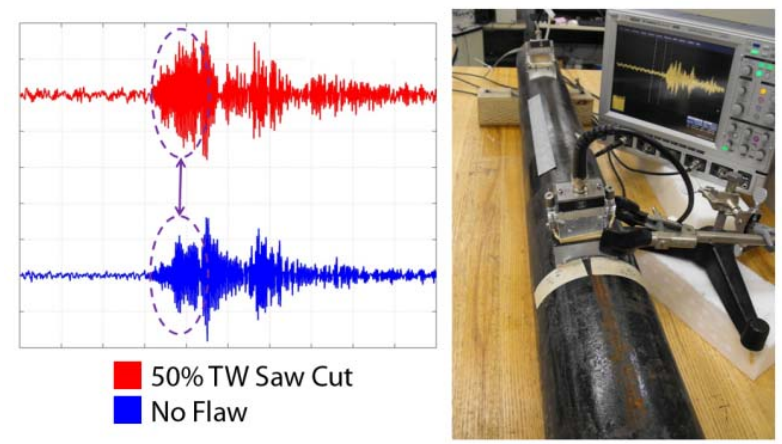

The affect of a saw cut flaw on a guided wave signal.

The results of FY 2009 activities were presented at the 2009 ANS winter meeting in Washington D.C. An abstract based on 2010 activities has also been submitted to the 2011 SPIE Smart Structures/NDE meeting in San Diego.

For FY 2011, we will continue our experiments with waveguides to understand their impact on signal-to-noise ratio. Our investigations of acoustic emission and guided wave techniques will be expanded to explore hybrid guided wave and acoustic emission monitoring techniques in an effort to develop methods for on-line crack detection that are less susceptible to typical sources of noise in nuclear power plant. In addition, we will explore improved methods of injecting guided ultrasonic waves into pipes that allow for more control over mode propagation and simplify analysis of measurement data. 


\title{
Theoretical Modeling and Ex-Reactor Testing of Fuel Properties to Accelerate Fuel Qualification
}

\author{
Carl E. Beyer, Brady D. Hanson, Andrew M. Casella, Rick E. Williford, Kenneth J. Geelhood
}

This project is intended to shorten fuel development and qualification time by providing better data early in the program as opposed to waiting for completion of lengthy irradiation test campaigns.

$\mathbf{T}$ here is renewed U.S. interest in expanding the use of the country's clean, safe nuclear power for domestic needs. Likewise, the rest of the world is considering the expansion of nuclear power as part of an effort to meet growing energy needs while minimizing carbon dioxide emissions. The Global Nuclear Energy Partnership (GNEP) program bears witness to these renewed interests in nuclear power. One aspect of GNEP is the development of advanced burner reactors (ABRs) to recycle spent fuel from the existing worldwide fleet of light water reactors. The primary fuel types identified for the ABR is either oxide or metal fuel with minor actinides. Additionally, DOE is pursuing the Generation IV Nuclear Energy Systems Initiative, which is aimed at developing and demonstrating advanced nuclear energy systems that can meet future needs for safe, sustainable, environmentally responsible, economical, proliferation resistant and physically secure energy. Under the GenIV initiative, the very high temperature and supercritical water cooled reactors were considered, both of which offer greater overall efficiencies and enable the production of hydrogen for use in the transportation industry as well as the gas, lead, and sodium cooled fast reactors.

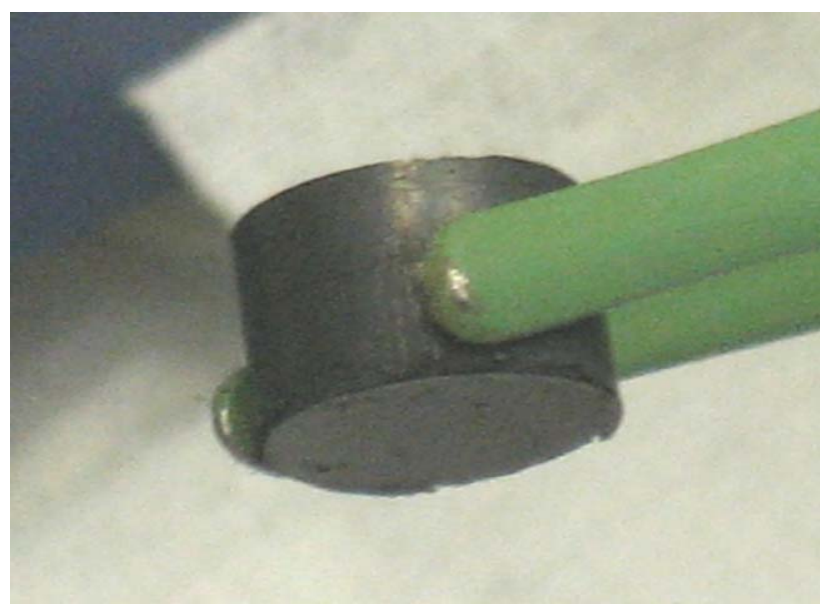

Image of a fabricated fuel pellet.

Our research is focused on two goals to assist in accelerating the qualification of new fuel types for new reactors: advancing the science of nuclear fuel performance modeling by developing first principle (theoretical) models and developing out of reactor testing capabilities to verify theoretical models and screen new fuel types utilizing a unique irradiated fuel (RADFUEL) concept for early modeling and simulation activities in support of in-reactor testing campaigns. The latter goal will assist with demonstrating that the theoretical modeling is valid. Overall, this project intends to demonstrate PNNL's ability to accelerate fuel development and qualification for transuranic fuel to be burned in the proposed advanced recycle reactor.

The first goal of the project resulted in a theoretical model for fuel thermal conductivity for mixed oxide (MOX) fuel containing minor actinides. The second goal was to develop and refine the capability to fabricate (on a small scale) and characterize fuels, including advanced fuels for any of the proposed reactor types prior to irradiation and RADFUELs to simulate advanced fuels at various fuel burn up conditions to support fuel performance modeling needs in advance of in reactor testing. While the initial focus has been on oxide fuels, the methodology and processes developed may be expanded for other fuel types. These data and model development are intended to advance the modeling efforts for new fuel types.

Modeling activities performed in FY 2009 included developing atomic potentials for fission products to be used in atomistic calculations to determine thermal diffusivity and thermal conductivity of irradiated oxide fuels. A model was developed for irradiated fuel based on these calculations. Our activities concentrated on pellet fabrication and installing the Netzsch laser flash analyzer in a radiologic fume hood for measuring the thermal diffusivity of RADFUELs. Additionally, pellets of uranium dioxide were successfully fabricated.

A thermal conductivity model was developed for irradiated MOX with actinides based on molecular dynamics simulations during FY 2010. This model included the 11 most abundant fission products of ruthenium, zirconium, cerium, palladium, neodymium, xenon, molybdenum, cesium, americium and curium, and neptunium. A model was developed in FY 2009 for unirradiated $\mathrm{UO}_{2}$ and $\mathrm{MOX}$ with actinides that agrees well with data. Our experimental work throughout the last fiscal year consisted of fabricating pellets with simulated fission products $(\mathrm{U}, \mathrm{Ru}) \mathrm{O}_{2}$ and $(\mathrm{U}, \mathrm{Mo}) \mathrm{O}_{2}$, also $\mathrm{UO}_{2}-\mathrm{Gd}_{2} \mathrm{O}_{3}$ and $\mathrm{UO}_{2}$ pellets. Thermal diffusivity measurements were made on the $\mathrm{UO}_{2}-\mathrm{Gd}_{2} \mathrm{O}_{3}$ and $\mathrm{UO}_{2}$ pellets as well as $\mathrm{UO}_{2}-\mathrm{Gd}_{2} \mathrm{O}_{3}$ pellets fabricated by a fuel vendor in the temperature range of 200 to $800^{\circ} \mathrm{C}$. 


\section{Underground Counting Capability Development and Potential Impacts}

Rosara F. Payne

The intended outcome of this project is a multi-faceted capability capitalizing on the expertise at PNNL to detect ultra-low levels of radioactivity in both gas and solid samples. The developed technologies and analysis protocols will contribute to ground-breaking applications, including materials assay, nuclear forensics, age dating, and large-scale physics experiments.

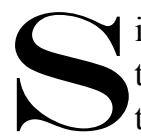
ignificant advances in ultra-low background technology of the past decade have yet to be applied to neutron activation analysis assay methods. The aim of neutron activation analysis and ultra-low background counting is to demonstrate improved neutron activation analysis and ultra-low background measurement science that is now possible in light of recent detector development and determine how the synergy between these technologies can be applied to enhance the speed and cost of analyses for current non-proliferation needs. Increased sensitivity offered by neutron activation analysis and ultra-low background can be shown, along with better throughput, speed, and cost having a potentially dramatic impact on the scenarios targeted with these kinds of samples.

This project was initiated to measure ultra-low background (ULB) radiation. The scope involves three tasks, each of which builds one important yet unique aspect of this capability. The three tasks all require a highly specialized set of skills and considerations needed when counting very low levels of radioactive materials: neutron activation analysis (NAA) and ULB counting, ultra-low radon emanation measurements, and precision gas measurements. The culmination of the tasks will result in a broad-based, world-class suite of capabilities at PNNL related to underground ultra-low level radiation detection. The research is broad, with the aim of developing a strong and diverse capability related to underground ULB radiation detection measurements.

Previous work allows this project to make significant technological advances. Dramatic improvements in ULB measurement science positions NAA and ULB counting to surpass mass spectrometry in analytical elemental analysis. Radon emanation systems are used to quantify the amount of radon in permeable, semi-porous materials. As ULB detectors have become increasingly sensitive, the actual materials they are constructed from contribute to their background. State-ofthe-art ULB detection facilities have radon emanation methods to assay materials used for detector construction, but no U.S. laboratory has this capability. Work done previously at PNNL has been on precise gas processing and ultra-low level detectors, making PNNL uniquely suited to build a radon emanation system along with the precision gas counting task. While gas counting employs length compensated proportional tubes to measure the absolute specific activity of a radioactive gas without the need of a reference standard (a method used by the National Institute of Standards and Technology [NIST] and others), this counting technique has not been applied to measure ultra-low specific activity gas samples.

For FY 2010, the NAA task has completed irradiations on one specific element of high interest, which lead to the realization that the relationship between NAA and ULB detection is complicated. The primary implication of using ULB detection systems is that the matrix of other elements that are present with the element of interest can cause interfering background for the low levels of radioactivity targeted. In turn, this has also led to an investigation into the materials that are appropriately clean and pure. The precision gas counting task completed the first phase of building a test apparatus for conducting specific activity measurements of gas samples. This assembly was tested and provided preliminary data that will be vital in the design and fabrication of the ULB proportional counters which will be built in year two of the project. PNNL is also trying to form a collaboration with the gas counting group at NIST.

Near the end of FY 2010, the radon emanation system was designed and built. It was determined that the detection efficiency for radon is expected to be about 83 percent. With this in mind, FY 2011 will focus on optimizing the gas purification/processing chemistry and samples will be counted in ULB proportional counters. Additionally, a suite of samples will be assayed to identify their radon levels. The goal will also be to submit a scientific article describing PNNL's ultra-low background absolute activity measurement capability.

The outcome of this project will leverage PNNL expertise and form the basis for measurement proficiency in various applications as demonstrated by measurements in a specific subset of applications. The NAA and ULB counting task will demonstrate the improvement that is now possible in light of recent ULB detector development. The Radon emanation apparatus will allow the identification of ULB suitable materials, the precision gas counting task will give us the capability to measure the specific activity of low-level gas samples raising the possibility of a number of novel measurements. Overall, the unique capabilities at PNNL enable a wide range of ULB measurements in the underground counting facility that will lead to worldwide recognition in different science communities and lend itself to continued business development. 


\section{Physics}




\title{
Advanced Computing Architectures for Smart Sensors and Sensor Analytics
}

\author{
Harold E. Trease, Brett G. Amidan, W. Karl Pitts
}

This project will demonstrate the utility of building smart sensors that integrate modern low-cost, low-power computational processing capability directly with sensors, where computational resources are integrated with these components for generating intelligently compressed signatures and performing data fusion at data acquisition and/or at the centralized computing facilities.

$\mathbf{T}$ he amount of image data generated by airborne and remote sensor platforms is growing exponentially. For example, a single unmanned air vehicle platform may consume the entire bandwidth capacity of one satellite. Data are increasing at such a rate that communication, storage, and processing capabilities are overwhelmed so that sensor data is collected but never analyzed or in some cases not stored. This problem will become exponentially worse with future unmanned air vehicle platforms that provide wide area surveillance capability through arrays of sensors, swarms of networked sensors, and multisensor platforms. Even if all the raw data could be communicated to a terrestrial analysis center, there is insufficient time to analyze it using manual procedures. New software algorithms and advanced processing hardware must be explored to extract useful information from this explosion of sensor-based information to provide advanced data processing at the point of acquisition, where analysts would get a summary without needing to access volumes of original raw data.
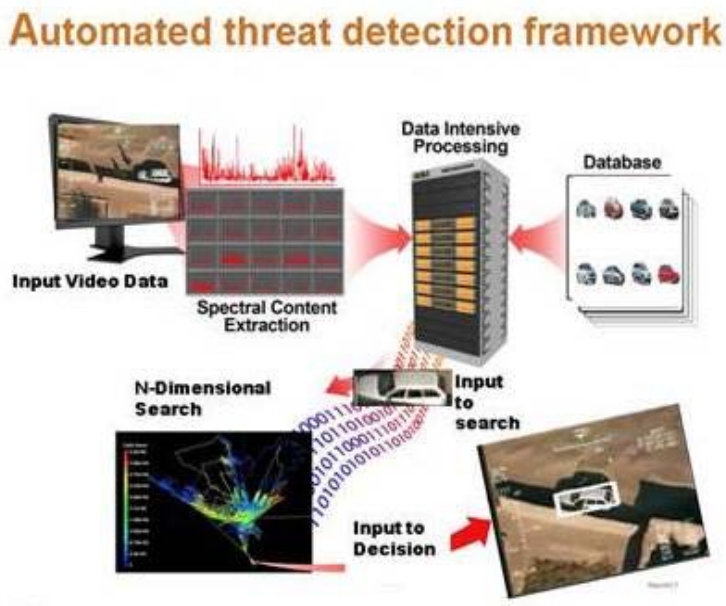

The new data processing and computer hardware system, based on compressive sensing and advanced micro-computer architectures.
We explored a new integrated platform consisting of three major components: sensor hardware, processing algorithms, and advanced micro-computing architectures. Our platform takes advantage of a new sensor/algorithm processing paradigm called compressive sensing. We added modern low-power, high performance parallel processing hardware to address the throughput issue. This activity enabled us accelerate the throughput of the sensor by using Moore's Law for semiconductors, based on computing capability growing exponentially, in conjunction with using the sensor to form a better sensing platform.

Compressive sensing uses a concept called sparse representations to adaptively construct a map of the sparse patterns contained in the sensor output, where the sparseness pattern identifies components of the sensor data that contains valuable sensor information. Using compressive sensing and sparse representations, we can process information on remote platforms and transmit only the data summarization to analysts, where it is a fraction of the original raw data but contains the basic information content.

During FY 2009, we commenced construction of a processing system that would demonstrate the concepts of integrating compressive sensing, sparse representation, and micro-computer architectures using video data as senor data. We defined the specific sensor data for collection and acquired sample data. After configuring the centralized computing capabilities, we gathered, processed, and analyzed data from the sensors and the centralized platforms.

In FY 2010, we continued construction of the processing system. We prepared codes, interfaces, and the computing device for testing in the real sensor environment. The system uses a portable computer as the remote computing platform and an Android cell phone or an iPad as the client device that communicates with the sensor platform and receives results. Our research improved the data signature and clustering methods, which also increases the accuracy of searches. Major results included the successful processing of the unmanned air vehicle platform and surveillance video, improving the data signature and clustering methods, and presenting the results to several interested clients, leading to additional work and opportunities. 


\section{Develop Ar-37 Measurement Capability for Treaty Verification Applications}

Jobn L. Orrell, Craig E. Aalseth, Derek A. Haas, Martin E. Keillor, Emily K. Mace, Allen Seifert, Vincent T. Woods

A capability to measure the radioactive noble gas argon (Ar)-37 will enhance the nation's ability to detect underground nuclear weapon testing. Development of specialized radiation detector equipment to measure Ar-37 in trace quantities supports this objective. The science and technological basis that this work will develop supports a broader objective of monitoring and deterring international nuclear weapon proliferation.

I $\mathrm{n}$ the Comprehensive Nuclear-Test-Ban Treaty that provides the basis for the onsite inspection of suspected nuclear facilities, Ar-37 has been identified as a relevant isotope for measurement. It is not produced in quantity at nuclear reactors or in the atmosphere but does appear in subsurface nuclear explosions due to the interaction of neutrons with calcium. We will develop a basis for a future laboratory capability for Ar-37 measurement, a prerequisite for advancing the state-of-the-art in technology applied to onsite inspection in treaty verification regimes. This project advances these goals by combining prior work on low background proportional counter development and energy threshold reduction. The outcome of this work will be a demonstrated detection apparatus focused on laboratory measurement of Ar-37. Included in this outcome is the specified measurement sensitivity quantified as the minimum detectable concentration of Ar-37 in the detection instrument. Estimations for improvement to the sensitivity of the apparatus as well as an assessment of the apparatus as a field instrument will be reported.

Measuring Ar-37 is challenging because it emits only difficult-to-detect low energy x-rays. The method of measurement relies on high sensitivity gas proportional counters. At present, only Switzerland and China possess dedicated gas proportional counter equipment capable of measuring Ar-37. Recent development of an ultra-low radioactive background gas proportional counter at PNNL has now shown that the United States has a potentially competitive and sensitive measurement capability. The primary development objective of this project is to prepare a laboratory-based Ar-37 measurement capability relevant to international treaty monitoring.

In FY 2009, research focused on production handling of the Ar-37 gas. Ar-37 is not naturally available in sufficient quantities for measurement due to its short, 35-day half-life. For this reason, Ar-37 is created using neutron irradiation at a nuclear reactor. There are two methods for generating the radioactive gas in quantities sufficient for measurement: neutron irradiation of Ca-40 and neutron irradiation of natural argon gas. The University of Texas worked with PNNL to develop the ability to irradiate natural argon gas and provide a sample for Ar-37 for measurement in a gas proportional counter.

During FY 2010, the first measurement of Ar-37 was made using the ultra-low-background proportional counter, result shown in the figure. A series of studies were made to optimize the detector performance (e.g., energy resolution and lifetime), determine the background sources (primarily cosmic-ray induced), and increase the signal to noise ratio by operation at higher pressure (10 atm instead $2 \mathrm{~atm}$ of gas). From these results, an Ar-37 sensitivity is inferred equivalent to 45 milli-Becquerel per standard cubic meter $(45 \mathrm{mBq} / \mathrm{SCM})$ of whole air. This result is relevant because naturally occurring backgrounds of Ar-37 are expected at the $1-10 \mathrm{mBq} / \mathrm{SCM}$. This recent measurement of Ar-37 was a demonstration. Refinement of the shield or measurements made at a moderate underground depth to exclude many cosmic-ray induced particles should lead to a system sensitive at the natural background level.

Work completed in the last few months of FY 2010 focused on the international treaty monitoring perspective of the work and field deployable measurement systems. Specifically, the counter gas used (P-10) is flammable, creating an operational challenge. Recent work has looked at measurements made using a non-flammable $\mathrm{Ar}-\mathrm{CO}_{2}$ counter gas mixture. 


\title{
First Operation of a Novel, High Mass Detector as a Weakly Interacting Massive Particle (WIMP) Dark Matter Detector
}

\author{
Martin E. Keillor, Jobn L. Orrell
}

This project will collect and analyze data covering a low energy region not previously explored with large high purity germanium spectrometers. We will assess this new detector technology for future large-scale WIMP dark matter searches.

$\mathbf{P}$ ublic and scientific interest in dark matter stems from the our knowledge and understanding of approximately 5 percent of the universe's composition. Direct detection of dark matter particles is perhaps the single most pressing astroparticle physics measurement sought by cosmologists and theoretical particle physicists alike. Theoreticians postulate the existence of a WIMP whose gravitational influence is ubiquitous but otherwise does not participate in the familiar interactions of the standard model of particle physics. Dark matter is better understoodperhaps better constrained —of major unknown categories. The search for WIMP dark matter has more than a decade of history, dove-tailing on efforts of cosmologists to account for the evolution of the mass distribution of the universe.

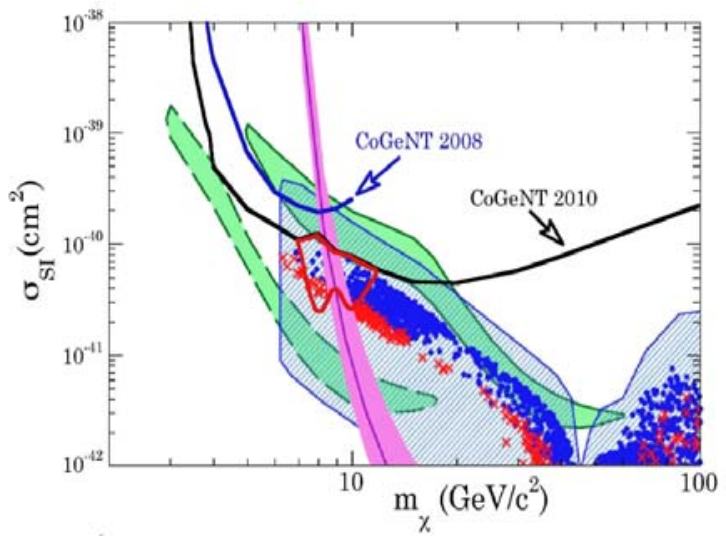

Dark matter exclusion limits by the CoGeNT Collaboration from a low-threshold detector located at the Soundan Underground Laboratory.
Measurements are expected to cover the region of the disputed positive result reported by the DAMA Collaboration.

The low background detector shield, designed in FY 2008, was physically installed at the Soudan Underground Laboratory in early FY 2009. That year, PNNL and researchers from the University of Washington's Center for Experimental Nuclear Physics and Astrophysics investigated the suitability to deploy an improved low-energy-threshold germanium detector for dark matter research. In FY 2010, a low-energy-threshold germanium detector was installed. Scientific data collection began in late 2009 and is continuing. We prepared our first experimental exclusions to light-mass

dark matter parameters (shown in the figure as CoGeNT 2010). Theoretical expectations for possible dark matter candidate parameter values are shown as "x." Limited areas are the DAMA experiment's favored dark matter signal regions, which are contentious within the dark matter community. A band approximately in the middle is a region generated by third-party theorists showing how a December 2009 CDMS paper could be consistent with DAMA's
Where these observations have

led to the proposal of an astroparticle solution, a confrontation with the standard model of particle physics is inevitable. No known particle has properties that account for astronomical observations, yet a clear prediction emerges that WIMP dark matter should interact with the matter of our everyday experience via elastic scattering.

Experimental searches for WIMP dark matter span a decade, dove-tailing on cosmologists' efforts to account for the discrepancy between the amounts of visible mass in the universe with observed gravitational effects, which indicate that 95 percent of the mass of the universe is unseen. This project is based on recognition of an advance in low energy threshold (about $0.3 \mathrm{keV}$ ) germanium detector technology, which could exploit the exponential increase in WIMP signal by measuring decreasingly energetic nuclear scattering recoils. We will identify backgrounds to the WIMP signature and investigate a region of WIMP mass and interaction cross-section not excluded by current experiments. results. The $2010 \mathrm{CoGeNT}$ work submitted to Physical Review Letters for publication is clearly highly relevant to disentangling the purported DAMA dark matter measurement, CDMS results, and theoretical expectations for light-mass WIMPs.

One of the advantages of the CoGeNT low-energy threshold germanium detector technology is cost. In contrast to large-scale liquid cryogen-based experiments or bolometer arrays, the CoGeNT germanium spectrometers are easy and inexpensive to operate. If light-mass dark matter of order approximately $10 \mathrm{GeV} / \mathrm{c}^{2}$ are indeed nature's choice, the CoGeNT technology is an ideal candidate for a future dark matter observatory that will help map the local dark matter density in our region of the galaxy. Such future measurements provide empirical information on questions of galaxy formation, the matter composition of the universe, and extensions beyond the $20^{\text {th }}$ century's standard model of particle physics. 


\title{
Multimodal X-ray Imaging with a Grating-Based Interferometer
}

\author{
Erin A. Miller, Timotby A. White, Benjamin S. McDonald, Allen Seifert
}

This project will use a PNNL-based system to image samples with relevance to a variety of applications, investigate the mechanisms of multimodal imaging, and explore qualitative and quantitative data processing.

$\mathbf{M}$ ultimodal or phase contrast $\mathrm{x}$-ray imaging is similar to conventional $x$-ray imaging, but image contrast is produced by local variations in the object's x-ray index of refraction rather than in its absorption. The technique has been used for several years with synchrotron $\mathrm{x}$-ray sources, where it has been of great use for imaging low-density and low- $Z$ samples, such as biological specimens or polymers. Operational applications that are incompatible with synchrotron sources have been limited due to stringent source coherence requirements. Recent work has demonstrated a new technique that allows the use of a conventional $x$-ray source. A series of gratings is used to improve the $\mathrm{x}$-ray source characteristics, set up an x-ray interference pattern that is sensitive to any distortions in the $\mathrm{x}$-rays due to the object, and detect those fine distortions. This raises for the first time the possibility of using phase contrast imaging in an operational setting.

Using a gratings-based interferometer, multimodal imaging can produce images based on three distinct contrast mechanisms: $\mathrm{x}$-ray absorption, $\mathrm{x}$-ray refractive index variations, and small angle scattering due to sample texture. This project constructed such a system and imaged a variety of materials. We see distinct, quantitative differences between different modes that will enable better material discrimination and, in some cases, lowered detection limits. Our project seeks to investigate the application of gratings-based multimodal x-ray imaging to explosives detection. Work on previous projects resulted in a test bed for phase contrast $\mathrm{x}$-ray imaging constructed off site using a decommissioned mammography machine for a source. Several different techniques were investigated for gratings fabrication, culminating in a working apparatus.

We constructed a multimodal imaging system on site at PNNL. The $\mathrm{x}$-ray source is a tube commonly used for non-destructive evaluation, with energies up to $160 \mathrm{kVp}$ (although for this measurement, the source was run at $40 \mathrm{kVp}$ ). The previous test system used an air-cooled source that had to be cooled between exposures. The new source is water cooled and can be run continuously, speeding data acquisition by almost 50 times. We made improvements in grating positioning, allowing for faster setup relative to the off-site test bed. Additional enhancements were made to data processing to remove phase wrapping artifacts and correct the datasets properly, which enabled more quantitative measurements and cleaner images. Finally, automation was incorporated into the measurement process, allowing for longer measurements and producing better statistics.

A number of samples were imaged, including explosives stimulants and composite and biological materials, and there were clear differences between the various contrast mechanisms. For example, absorption images emphasize a higher atomic number of materials such as metals. Phase images can indicate lower atomic number materials more strongly than absorption, and the phase images also highlight edges and boundaries. Finally, scatter images are sensitive to small-scale texture, materials such as powders or paper may show strongly in scatter even when nearly invisible to other contrast mechanisms. For powdered explosives simulants, the scatter signal-to-noise was approximately 50 percent higher than absorption, indicating that for this particular material, scatter can lower detection limits.

Additional work was performed to understand the physical basis of the contrast mechanisms. Phase shift is known to depend on the local derivative of the electron density, and absorption has a strong dependence on the material atomic number. Scatter, however, is less well understood. We observed that the scatter signal is sensitive to anisotropy in the sample. For a fabric with fibers parallel and perpendicular to grating lines, both fiber directions are visible in absorption, but only parallel fibers are strongly visible in scatter, which also depends on the length scale. Looking at glass beads ranging from $850 \mu$ to as small as $7 \mu$, the scatter signal clearly increased with decreasing size.

The combination of these distinct contrast modes holds promise for better material discrimination and lower detection limits in a wide variety of fields, including security screening, medical imaging, and materials science. 


\section{Spectroscopic X-ray Computed Tomography for Improved Explosives Detection}

Timothy A. White, Erin A. Miller; Frédéric Noo (University of Utab)

We are investigating a novel approach to computed tomography that will enhance the ability to identify materials. If successful, this technique has applications for homeland security (e.g., detection of explosives in baggage) as well as medical imaging (e.g., calcification scoring for coronaryartery disease).

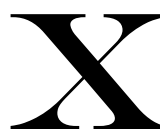

-ray computed tomography is a non-destructive evaluation tool that generates a three-dimensional image of the structure of an object and is used extensively in security screening for the examination of checked bags at the airport and as a medical diagnosis tool, commonly known as a CAT scan. In general, the information obtained in a computed tomography scan is proportional to a parameter of materials known as the linear attenuation coefficient, which is proportional to density and elemental composition - specifically, the effective atomic number, $Z_{\text {eff. }}$ Some tomography systems use a geometry known as dual-energy scanning in which data are collected with information in two x-ray energy ranges or windows; this geometry allows better discrimination between density and $Z_{\text {eff }}$ of a material, enabling better material identification. Our hypothesis is that using more energy windows will further enhance material identification and thus discrimination between benign and threat or disease materials; for instance, explosives in the case of security screening or plaque in the case of cardiac imaging.

However, full computed tomography scanning with an energy discriminating detector is not efficient with currently available electronics and detector materials. The principle constraint is that the required dynamic range, the range of $\mathrm{x}$-ray intensities that must be detected, is too great when considering attenuation paths through both the center and edge of the object. If, however, we relax the dynamic range constraint by considering only the central (and highly attenuating) region of the object, then we can develop a geometry and reconstruction algorithm to use the information from a detector with multiple energy windows. The geometry we propose includes a detector of sufficient size but no energy discrimination for full computed tomography acquisition and another, smaller spectroscopic detector that is spatially limited to viewing only the central region of the object. The goals of the project are to determine if such a geometry can indeed provide better material discrimination and to develop reconstruction algorithms to realize this advantage.

FY 2009 efforts identified a range of approaches. We considered whether the interior region of support could be increased in size with additional information from a current integration detector. If we could map an attenuation coefficient accurately with a single pixel detector with higher spectral resolution, then we might be able to determine the presence of threat material without requiring imaging. Even if this is not a practical approach, the evaluation should provide an upper limit on the utility of spectroscopic capability. Also, we considered a primary/secondary interrogation scheme in which a very fast scan with a non-spectroscopic detector defines a region of interest that could be interrogated with the detector.

We have shown that judicious choice of basis functions allows material discrimination and, perhaps more importantly, that the choice of discriminant - method or algorithm for discriminating between threat and benign material - critically affects the choice of basis function. In simulation experiments during FY 2010, we created a three-dimensional object with common benign materials encountered in security screening (e.g., apparel, electronics, printed material, alcohol) and an explosive material (DetaSheet) and simulated a fan-beam computed tomography imaging geometry using a photoncounting detector. A multiple-basis function decomposition process was invoked to transform the multiple-energy window projection data (sinograms) into projection data representing line integrals through coefficients. These data were reconstructed into images corresponding to materials that most closely matched the basis functions. For instance, using the mass attenuation coefficients of the explosive and wearing apparel as the basis functions results in an image of "things that have similar attenuation properties to the explosive material" and an image of "things that have similar attenuation properties to the wearing apparel." The addition of a third basis function would result in a third image in which the pixel intensities are proportional to how similar a region of the image is to that basis function.

We were to use the image data to discriminate explosive from benign material. The naïve approach would be to examine only the image corresponding to the explosive material basis function. This approach, however, ignored important information incorporated in the other basis function images. We showed that discriminants operating on different transformations of the original data could achieve different discriminabilities. This led us to hypothesize about other potential discriminants and began discussions about methods for exploring the multidimensional feature space. While we could not demonstrate a definitive explosive detection capability using photon-counting detectors, we feel there are advantages to this technique and higher fidelity observation of material signatures available through transmission $\mathrm{x}$-ray imaging techniques. 


\section{Appendix A}

\section{Fiscal Year 2010 Refereed Publications}




\section{Appendix A}

\section{Fiscal Year 2010 Refereed Publications}

During FY 2010, PNNL's LDRD Program resulted in 161 refereed publications. These journals articles, book chapters, and select conference papers are listed below in alphabetical order by the author's last name.

Aalseth CE, AR Day, EW Hoppe, TW Hossbach, BJ Hyronimus, ME Keillor, KE Litke, EE Mintzer, A Seifert, and GA Warren. 2009. "Design and Construction of a Low-Background, Internal-Source Proportional Counter." Journal of Radioanalytical and Nuclear Chemistry 282(1):233-237.

Arntzen EV, DR Geist, and PE Dresel. 2006. "Effects of Fluctuating River Flow on Groundwater/Surface Water Mixing in the Hyporheic Zone of a Regulated, Large Cobble Bed River." River Research and Applications 22(8):937-946.

Arntzen EV, DR Geist, KJ Murray, J Vavrinec III, EM Dawley, and DE Schwartz. 2009. "Influence of the Hyporheic Zone on Supersaturated Gas Exposure to Incubating Chum Salmon." North American Journal of Fisheries Management 29(6):1714-1727.

Baird CL, CJ Fischer, NB Pefaur, KD Miller, J Kagen, S Srivastava, and KD Rodland. 2010. "Developing Recombinant Antibodies for Biomarker Detection." Cancer Biomarkers 6(5-6):271-279.

Baker ES, EA Livesay, DJ Orton, RJ Moore, WF Danielson, DC Prior, YM Ibrahim, BL Lamarche, AM Mayampurath, AA Schepmoes, DF Hopkins, K Tang, RD Smith, and ME Belov. 2010. "An LC-IMS-MS Platform Providing Increased Dynamic Range for High-Throughput Proteomic Studies.” Journal of Proteome Research 9(2):997-1006.

Beagley N, KG Stratton, and BJM Webb-Robertson. 2010. "VIBE 2.0: Visual Integration for Bayesian Evaluation." Bioinformatics 26(2):280-282.

Best DM, SJ Bohn, DV Love, AS Wynne, and WA Pike. 2010. "Real-Time Visualization of Network Behaviors for Situational Awareness." In Proceedings of the Seventh International Symposium on Visualization for Cyber Security, pp. 79-90. ACM, New York, NY.

Bhattacharjee PS, YC Sud, X Liu, GK Walker, R Yang, and J Wang. 2010. "Importance of Including Ammonium Sulfate $\left(\left(\mathrm{NH}_{4}\right)_{2} \mathrm{SO}_{4}\right)$ Aerosols for Ice Cloud Parameterization in GCMs." Annales Geophysicae 28(2):621-631.

Bohner SA, B Hartman, MY Eltoweissy, and D Gracanin. 2010. "Agents in Service-Oriented Wireless Sensor Networks." International Journal of Wireless and Mobile Computing 4(3):218-227.

Bond-Lamberty B and AM Thomson. 2010. "Temperature-Associated Increases in the Global Soil Respiration Record." Nature 464(7288):579-582.

Bonneville AHR, GD Black, I Gorton, PSY Hui, EM Murphy, ML Rockhold, KL Schuchardt, C Sivaramakrishnan, MD White, MD Williams, and SK Wurstner. 2010. "Geologic Sequestration Software Suite: A Collaborative Approach to the Management of Geological GHG Storage Projects." In Energy Procedia: 10th Greenhouse Gas Control Technologies Conference (GHGT10), Amsterdam, NLD.

Buchko GW. 2009. "Pentapeptide Repeat Proteins and Cyanobacteria." In Handbook on Cyanobacteria: Biochemistry, Biotechnology and Applications, pp. 233-257. Nova Science, Hauppauge, NY. 
Buchko GW, G Niemann, ES Baker, ME Belov, RD Smith, F Heffron, JN Adkins, and JE McDermott. 2010. "A Multi-Pronged Search for a Common Structural Motif in the Secretion Signal of Salmonella enterica serovar Typhimurium Type III Effector Proteins." Molecular Biosystems 6(12):2448-2458.

Buchko GW, BJ Tarasevich, J Roberts, ML Snead, and WJ Shaw. 2010. "A Solution NMR Investigation into the Murine Amelogenin Splice-Variant LRAP (Leucine-Rich Amelogenin Protein)." Biochimica et Biophysica Acta-Proteins and Proteomics 1804(9):1768-1774.

Carroll TE and D Grosu. 2010. "Incentive Compatible Online Scheduling of Malleable Parallel Jobs with Individual Deadlines." In 39th International Conference on Parallel Processing (ICPP-2010), pp. 516-524. IEEE Computer Society, Los Alamitos, CA.

Chen L, O Villa, S Krishnamoorthy, and GR Gao. 2010. "Dynamic Load Balancing on Single- and Multi-GPU Systems." In Proceedings of the 24th IEEE International Symposium on Parallel \& Distributed Processing (IPDPS 2010), pp. 1-12. IEEE, Piscataway, NJ.

Chen Y, Z Huang, and D Chavarría-Miranda. 2010. "Performance Evaluation of Counter-Based Dynamic Load Balancing Schemes for Massive Contingency Analysis with Different Computing Environments." In Proceedings of the 2010 IEEE Power and Energy Society General Meeting. IEEE, Piscataway, NJ.

Chen Y, Z Huang, PC Wong, PS Mackey, CH Allwardt, J Ma, and FL Greitzer. 2010. "An Advanced Decision Support Tool for Electricity Infrastructure Operations." In Critical Infrastructure Protection IV, IFIP Advances in Information and Communication Technology 342(2010), eds T Moore and S Shenoi, pp. 245-260. Springer, New York, NY.

Curtis MM. 2010. "Supplemental Systems for Unattended UF6 Cylinder Monitoring." In Proceedings of the 51st Annual Meeting of the Institute of Nuclear Materials Management. INMM, Deerfield, IL.

DeMott PJ, AJ Prenni, X Liu, SM Kreidenweis, MD Petters, CH Twohy, MS Richardson, T Eidhammer, and DC Rogers. 2010. "Predicting Global Atmospheric Ice Nuclei Distributions and Their Impacts on Climate." Proceedings of the National Academy of Sciences of the United States of America 107(25):11217-11222.

Du D, Z Zou, Y Shin, J Wang, H Wu, MH Engelhard, J Liu, IA Aksay, and Y Lin. 2010. "Sensitive Immunosensor for Cancer Biomarker Based on Dual Signal Amplification Strategy of Graphene Sheets and Multi-Enzyme Functionalized Carbon Nanospheres." Analytical Chemistry 82(7):2989-2995.

Du P, Z Huang, R Diao, B Lee, and KK Anderson. 2010. “Application of Kalman Filter to Improve Model Integrity for Securing Electricity Delivery." In Fifth International CRIS Conference on Critical Infrastructures (CRIS 2010). Beijing, China.

Du P, Z Huang, R Diao, B Lee, and KK Anderson. 2010. "PMU Placement for Enhancing Dynamic Observability of a Power Grid." In 2010 IEEE Conference on Innovative Technologies for an Efficient and Reliable Electricity Supply, pp. 15-21. IEEE, New York.

Eltoweissy MY, DHC Du, M Gerla, S Giordano, M Gouda, H Schulzrinne, and M Youssef. 2010. "Mission Critical Networking." IEEE Journal on Selected Areas in Communications 28(5):625-629.

Erbacher R, DA Frincke, PC Wong, S Moody, and GA Fink. 2010. "A Multi-Phase Network Situational Awareness Cognitive Task Analysis." Information Visualization 9(3):204-219.

Ewing RG and MJ Waltman. 2010. "Production and Utilization of $\mathrm{CO}_{3}$-Produced by a Corona Discharge in Air for Atmospheric Pressure Chemical Ionization." International Journal of Mass Spectrometry 296(1-3):53-58. 
Fan J, T Yuan, JM Comstock, SJ Ghan, A Khain, LYR Leung, Z Li, VJ Martins, and M Ovchinnikov. 2009. "Dominant Role by Vertical Wind Shear in Regulating Aerosol Effects on Deep Convective Clouds." Journal of Geophysical Research D (Atmospheres) 114, Art D22206.

Fernandez CA, PK Thallapally, J Liu, and CHF Peden. 2010. "Effect of Produced HCl During the Catalysis on Micro- and Mesoporous MOFs." Crystal Growth \& Design 10(9):4118-4122.

Fifield LS and JW Grate. 2010. "Hydrogen-Bond Acidic Functionalized Carbon Nanotubes (CNTs) with Covalently-Bound Hexafluoroisopropanol Groups.” Carbon 48(7):2085-2088.

Ford R and DA Frincke. 2010. “Building a Better Boot Camp.” IEEE Security \& Privacy 8(1):68-71.

Frank DP, DC Gerlach, CJ Gesh, DE Hurley, GH Meriwether, MR Mitchell, and BD Reid. 2010. "Application of the Isotope Ratio Method to a Boiling Water Reactor." In Proceedings of the 51st Annual Meeting of the Institute of Nuclear Materials Management. INMM, Deerfield, IL.

Gettelman A, X Liu, SJ Ghan, H Morrison, S Park, AJ Conley, SA Klein, J Boyle, DL Mitchell, and JLF Li. 2010. "Global Simulations of Ice Nucleation and Ice Supersaturation with an Improved Cloud Scheme in the Community Atmosphere Model.” Journal of Geophysical Research D (Atmospheres) 115, Art D18216.

Glaesemann KR, N Govind, S Krishnamoorthy, and K Kowalski. 2010. "EOMCC, MRPT, and TDDFT Studies of Charge Transfer Processes in Mixed-Valence Compounds: Application to the Spiro Molecule." The Journal of Physical Chemistry A 114(33):8764-8771.

Goodman EL, DJ Haglin, C Scherrer, D Chavarría-Miranda, JA Mogill, and JT Feo. 2010. "Hashing Strategies for the Cray XMT.” In IEEE International Symposium on Parallel \& Distributed Processing, Workshops and Phd Forum (IPDPSW 2010), pp. 1-8. IEEE, Piscataway, NJ.

Govind N, PV Sushko, WP Hess, M Valiev, and K Kowalski. 2009. "Excitons in Potassium Bromide: A Study Using Embedded Time-dependent Density Functional Theory and Equation-of-Motion Coupled Cluster Methods." Chemical Physics Letters 470(4-6):353-357.

Grate JW, C Zhang, TW Wietsma, MG Warner, NC Anheier Jr, BE Bernacki, G Orr, and M Oostrom. 2010. "A Note on the Visualization of Wetting Film Structures and a Nonwetting Immiscible Fluid in a Pore Network Micromodel Using a Solvatochromic Dye." Water Resources Research 46:W11602.

Gray S, KM Weigel, KD Miller, J Ndung'u, P Buscher, TN Tran, CL Baird, and GA Cangelosi. 2010. "Flow Cytometry-Based Methods for Assessing Soluble scFv Activities and Detecting Pathogen Antigens in Solution." Biotechnology and Bioengineering 105(5):973-981.

Greitzer FL. 2010. "Wide-Area Situation Awareness in Electric Power Grid." In Proceedings of the SPIE: Cyber Security, Situation Management, and Impact Assessment II; and Visual Analytics for Homeland Defense and Security II, eds JF Buford, G Jakobson, J Erickson, WJ Tolone and W Ribarsky, Vol 7709, Art 77090F. Bellingham, WA.

Greitzer FL and DH Andrews. 2009. "Training Strategies to Mitigate Expectancy-Induced Response Bias in Combat Identification: A Research Agenda." In Human Factors Issues in Combat Identification, eds DH Andrews, RP Herz and MB Wolf, Ch 11, pp. 173-190. Ashgate, Burlington, VT.

Heldebrant DJ, PK Koech, MTC Ang, C Liang, JE Rainbolt, CR Yonker, and PG Jessop. 2010. "Reversible Zwitterionic Liquids, the Reaction of Alkanol Guanidines, Alkanol Amidines, and Diamines with $\mathrm{CO}_{2}$." Green Chemistry 12(4):713-721.

Henne KL, CN Nakatsu, DK Thompson, and A Konopka. 2009. "High-Level Chromate Resistance in Arthrobacter sp. Strain FB24 Requires Previously Uncharacterized Accessory Genes.” BMC Microbiology 9:199-213. 
Hinderliter PM, KR Minard, G Orr, WB Chrisler, BD Thrall, JG Pounds, and JG Teeguarden. 2010. "ISDD: A Computational Model of Particle Sedimentation, Diffusion and Target Cell Dosimetry for In Vitro Toxicity Studies." Particle and Fibre Toxicology 7(11), Art 36.

Hoppe EW, CE Aalseth, RL Brodzinski, AR Day, OT Farmer III, TW Hossbach, JI McIntyre, HS Miley, EE Mintzer, A Seifert, JE Smart, and GA Warren. 2008. "Use of Electrodeposition for Sample Preparation and Rejection Rate Prediction for Assay of Electroformed Ultra High Purity Copper for ${ }^{232} \mathrm{Th}$ and ${ }^{238} \mathrm{U}$ Prior to Inductively Coupled Plasma Mass Spectrometry (ICP/MS)." Journal of Radioanalytical and Nuclear Chemistry 277(1):103-110.

Hoppe EW, A Seifert, CE Aalseth, AR Day, OT Farmer III, TW Hossbach, JI McIntyre, HS Miley, JE Smart, and GA Warren. 2008. "A Method for Removing Surface Contamination on Ultra-Pure Copper Spectrometer Components." Journal of Radioanalytical and Nuclear Chemistry 276(3):645-650.

Hsu EW, LJ Healy, DR Einstein, and AP Kuprat. 2010. "Imaging-Based Assessment and Modeling of the Structures of the Myocardium." In Computational Cardiovascular Mechanics: Modeling and Applications in Heart Failure, Ch 2, pp. 23-29. Springer, New York, NY.

Hu Q, P Wang, and J Laskin. 2010. "Effect of the Surface on the Secondary Structure of Soft Landed Peptide Ions." Physical Chemistry Chemical Physics 12(39):12802-12810.

Huang Z, Y Chen, S Jin, D Chavarría-Miranda, BK Kalahar, DJ Baxter, and I Gorton. 2009. “A High-Performance Hybrid Computing Approach to Massive Contingency Analysis in the Power Grid." In 5th IEEE International Conference on e-Science. IEEE, Piscataway, NJ.

Huang Z, B Yang, and D Kosterev. 2008. "Benchmarking of Planning Models Using Recorded Dynamics." In Proceedings of IEEE Power and Energy Society Power Systems Conference and Exposition 2009. IEEE, Piscataway, NJ.

Huang Z, N Zhou, FK Tuffner, Y Chen, DJ Trudnowski, W Mittelstadt, JF Hauer, and JE Dagle. 2010. "Improving Small Signal Stability through Operating Point Adjustment." In Proceedings of the 2010 IEEE Power and Energy Society General Meeting. IEEE, Piscataway, NJ.

Hui PSY, JR Bruce, GA Fink, ML Gregory, DM Best, LR McGrath, and A Endert. 2010. "Towards Efficient Collaboration in Cyber Security." In International Symposium on Collaboration and Security (CTS '10), pp. 489-498. IEEE, Piscataway, NJ.

Jaffe JE, TC Kaspar, T Droubay, T Varga, ME Bowden, and GJ Exarhos. 2010. "Electronic and Defect Structure of CuSCN." The Journal of Physical Chemistry C 114(19):9111-9117.

Jin S, Z Huang, Y Chen, D Chavarría-Miranda, JT Feo, and PC Wong. 2010. "A Novel Application of Parallel Betweenness Centrality to Power Grid Contingency Analysis." In IEEE International Symposium on Parallel \& Distributed Processing (IPDPS 2010), pp. 1-7. IEEE, Piscataway, NJ.

Johnson GE, M Lysonski, and J Laskin. 2010. "In Situ Reactivity and TOF SIMS Analysis of Surfaces Prepared by Soft and Reactive Landing of Mass Selected Ions." Analytical Chemistry 82(13):5718-5727.

Kang X, J Wang, H Wu, J Liu, IA Aksay, and Y Lin. 2010. “A Graphene-Based Electrochemical Sensor for Sensitive Detection of Paracetamol." Talanta 81(3):754-759.

Keillor ME, CE Aalseth, AR Day, JE Fast, EW Hoppe, BJ Hyronimus, TW Hossbach, HS Miley, A Seifert, and GA Warren. 2009. "Design and Construction of an Ultra-Low-Background 14 Crystal Germanium Array for High Efficiency and Coincidence Measurements." Journal of Radioanalytical and Nuclear Chemistry 282(3):703-708.

Khurana H, MD Hadley, N Lu, and DA Frincke. 2010. "Smart-Grid Security Issues." IEEE Security \& Privacy $8(1): 81-85$. 
King SM, T Rosenoern, JE Shilling, Q Chen, Z Wang, G Biskos, KA McKinney, U Poschl, and ST Martin. 2010. "Cloud Droplet Activation of Mixed Organic-Sulfate Particles Produced by the Photooxidation of Isoprene." Atmospheric Chemistry and Physics 10(8):3953-3964.

Konopka A. 2009. “What is Microbial Community Ecology?” The ISME Journal 3(11):1223-1230.

Kourtev PS, CH Nakatsu, and A Konopka. 2009. "Inhibition of Nitrate Reduction by Chromium (VI) in Anaerobic Soil Microcosms.” Applied and Environmental Microbiology 75(19):6249-6257.

Kowalski K, S Krishnamoorthy, O Villa, JR Hammond, and N Govind. 2010. “Active-Space Completely-Renormalized Equation-of-Motion Coupled-Cluster Formalism: Excited-State Studies of Green Fluorescent Protein, Free-Base Porphyrin, and Oligoporphyrin Dimer." Journal of Chemical Physics 132(15), Art 154103.

Krishnamoorthy S and K Agarwal. 2010. "Scalable Communication Trace Compression." In The 10th IEEE/ACM International Conference on Cluster, Cloud and Grid Computing, pp. 408-417. IEEE, Piscataway, NJ.

Kulkarni GR and S Dobbie. 2010. "Ice Nucleation Properties of Mineral Dust Particles: Determination of Onset $\mathrm{RH}_{\mathrm{i}}$, IN Active Fraction, Nucleation Time-Lag, and the Effect of Active Sites on Contact Angles." Atmospheric Chemistry and Physics 10(1):95-105.

Kulkarni GR, MS Pekour, A Afchine, DM Murphy, and DJ Cziczo. 2011. "Comparison of Experimental and Numerical Studies of the Performance Characteristics of a Pumped Counterflow Virtual Impactor." Aerosol Science and Technology 45(3):382-392.

Kwak JH, JZ Hu, DW Hoyt, JA Sears Jr, CM Wang, KM Rosso, and AR Felmy. 2010. "Metal Carbonation of Forsterite in Supercritical $\mathrm{CO}_{2}$ and $\mathrm{H}_{2} \mathrm{O}$ Using Solid State ${ }^{29} \mathrm{Si},{ }^{13} \mathrm{C}$ NMR Spectroscopy." The Journal of Physical Chemistry C 114(9):4126-4134.

Lawrence P, W Kittichotirat, RE Bumgarner, JE McDermott, D Herndon, DP Knowles, and S Srikumaran. 2010. "Genome Sequences of Mannheimia haemolytica Serotype A2: Ovine and Bovine Isolates." Journal of Bacteriology 192(4):1167-1168.

Lawrence P, W Kittichotirat, JE McDermott, and RE Bumgarner. 2010. “A Three-Way Comparative Genomic Analysis of Mannheimia haemolytica Isolates.” BMC Genomics 11:535.

Levitskaia TG, JA Creim, TL Curry, T Luders, JE Morris, AD Woodstock, B Levinson, and KD Thrall. 2010. "Evaluation of Cuprimine ${ }^{\circledR}$ and Syprine ${ }^{\circledR}$ for Decorporation of 60 Cobalt and 210 Polonium." Health Physics 98(3):471-479.

Liao H, H Wu, J Wang, J Liu, Y Jiang, S Sun, and Y Lin. 2010. "Direct Electrochemistry and Electrocatalysis of Myoglobin Immobilized on Graphene-CTAB-Ionic Liquid Nanocomposite Film.” Electroanalysis 22(19):2297-2302.

Lin G, AM Tartakovsky, and DM Tartakovsky. 2010. "Uncertainty Quantification via Random Domain Decomposition and Probabilistic Collocation on Sparse Grids.” Journal of Computational Physics 229(19-20):6995-7012.

Lin G and AM Tartakovsky. 2010. "Numerical Studies of Three-Dimensional Stochastic Darcy's Equation and Stochastic Advection-Diffusion-Dispersion Equation.” Journal of Scientific Computing 43(1):92-117.

Lu X, GW Coffey, KD Meinhardt, VL Sprenkle, Z Yang, and JP Lemmon. 2010. "High Power Planar Sodium-Nickel Chloride Battery.” ECS Transactions 28(22):7-13.

Lu X, JP Lemmon, VL Sprenkle, and Z Yang. 2010. "Sodium-Beta Alumina Batteries: Status and Challenges.” JOM: The Journal of the Minerals, Metals and Materials Society 62(9):31-36. 
Lu X, G Xia, JP Lemmon, and Z Yang. 2010. “Advanced Materials for Sodium-Beta Alumina Batteries: Status, Challenges and Perspectives.” Journal of Power Sources 195(9):2431-2442.

Ma J, Y Chen, Z Huang, and PC Wong. 2010. "Using State Estimation Residuals to Detect Abnormal SCADA Data." In 2010 IEEE PES Transmission and Distribution Conference and Exposition. IEEE, Piscataway, NJ.

Ma W, S Krishnamoorthy, O Villa, and K Kowalski. 2010. "Acceleration of Streamed Tensor Contraction Expressions on GPGPU-Based Clusters." In Proceedings of the IEEE International Conference on Cluster Computing (CLUSTER 2010), pp. 207-216. IEEE, Piscataway, NJ.

Manz DO, TW Edgar, and GA Fink. 2010. "A Hybrid Authentication and Authorization Process for Control System Networks." In 2010 Sixth International Conference on Information Assurance and Security (IAS), August 23-25, 2010, Atlanta, GA, p. 36. IEEE, Piscataway, NJ.

Matson DW, GL Graff, JL Male, BR Johnson, Z Nie, AG Joly, and LC Olsen. 2010. "Synthesis and Screening of Thin Films in the $\mathrm{CeCl}_{3}-\mathrm{CeBr}_{3}$ System for Scintillator Applications." Thin Solid Films 518(12):3194-3198.

McDermott JE, MN Archuleta, BD Thrall, JN Adkins, and KM Waters. 2011. "Controlling the Response: Predictive Modeling of a Highly Central, Pathogen-Targeted Core Response Module in Macrophage Activation." PLoS One 6(2):e14673.

McDermott JE, AL Corrigan, ES Peterson, CS Oehmen, G Niemann, E Cambronne, D Sharp, JN Adkins, R Samudrala, and F Heffron. 2011. "Computational Prediction of Type III and IV Secreted Effectors in Gram-negative Bacteria." Infection and Immunity 79(1):23-32.

McDermott JE, MN Costa, DB Janszen, M Singhal, and SC Tilton. 2010. "Separating the Drivers from the Driven: Integrative Network and Pathway Approaches Aid Identification of Disease Biomarkers from High-Throughput Data." Disease Markers 28(4):253-266.

McDermott JE, CS Oehmen, LA McCue, EA Hill, DM Choi, HB Pakrasi, and LA Sherman. 2011. “A Model of Cyclic Transcriptomic Behavior in Cyanothece species ATCC 51142.” PNNL-SA-74409, Richland, WA. [Submitted]

McFarquhar G, SJ Ghan, J Verlinde, A Korolev, W Strapp, B Schmid, JM Tomlinson, M Wolde, SD Brooks, DR Collins, DJ Cziczo, MK Dubey, J Fan, CJ Flynn, I Gultepe, JM Hubbe, MK Gilles, A Laskin, P Lawson, WR Leaitch, P Liu, X Liu, D Lubin, C Mazzoleni, AM Macdonald, RC Moffet, H Morrison, M Ovchinnikov, DM Ronfeld, MD Shupe, DD Turner, S Xie, A Zelenyuk, K Bae, M Freer, and A Glen. 2010. "Indirect and Semi-Direct Aerosol Campaign (ISDAC): The Impact of Arctic Aerosols on Clouds." Bulletin of the American Meteorological Society online, September 3, 2010.

Mei D, JH Kwak, J Szanyi, Q Ge, and CHF Peden. 2010. "Catalyst Size and Morphological Effects on the Interaction of $\mathrm{NO}_{2}$ with $\mathrm{BaO} / \gamma-\mathrm{Al}_{2} \mathrm{O}_{3}$ Materials." Catalysis Today 151(3-4):304-313.

Mei D, RJ Rousseau, SM Kathmann, VA Glezakou, MH Engelhard, W Jiang, CM Wang, MA Gerber, JF White, and DJ Stevens. 2010. "Ethanol Synthesis from Syngas over Rh-Based $/ \mathrm{SiO}_{2}$ Catalysts: A Combined Experimental and Theoretical Modeling Study." Journal of Catalysis 271(2):325-342.

Miracle AL, C Evans, E Ferguson, B Greenberg, P Kille, A Schaeffner, M Sprenger, R van Aerle, and D Versteeg. 2008. "Applications of Genomic Technologies to Ecological Risk Assessments at Remediation/Restoration Sites." In Genomics in Regulatory Ecotoxicology: Applications and Challenges, ed. EJ Perkins, GP Daston, GT Ankley, AL Miracle, pp. 123-150. CRC Press, Boca Raton, FL.

Niemann G, RN Brown, JK Gustin, A Stufkens, AS Shaikh-Kidwai, J Li, JE McDermott, HM Brewer, AA Schepmoes, RD Smith, JN Adkins, and F Heffron. 2011. "Discovery of Novel Secreted Virulence Factors from Salmonella enterica serovar typhimurium by Proteomic Analysis of Culture Supernatants." Infection and Immunity 79(1):33-43. 
Oehmen CS and WR Cannon. 2008. "Bringing High Performance Computing to the Biologist's Workbench: Approaches, Applications and Challenges." In SciDAC 2008: Journal of Physics: Conference Series 125, p. 012052. IOP Publishing Ltd., Bristol, UK.

Oehmen CS, ES Peterson, and ST Dowson. 2010. “An Organic Model for Detecting Cyber Events.” In CSIIRW '10: Proceedings of the Sixth Annual Workshop on Cyber Security and Information Intelligence Research. ACM, New York, NY.

Oehmen CS and BJM Webb-Robertson. 2010. "Evaluating the Computational Requirements of Using SVM Software to Train Data-Intensive Problems." In Machine Learning Research Progress. Nova Science, Hauppauge, NY.

Ovchinnikov M and RC Easter Jr. 2010. "Modeling Aerosol Growth by Aqueous Chemistry in Nonprecipitating Stratiform Cloud." Journal of Geophysical Research D (Atmospheres) 115, Art D14210.

Pan A, J Liu, J Zhang, GH Cao, W Xu, Z Nie, J Xiao, D Choi, BW Arey, CM Wang, and S Liang. 2010. "Template Free Synthesis of LiV3O8 Nanorods as a Cathode Material for High-Rate Secondary Lithium Batteries." Journal of Materials Chemistry 21(4):1153-1161.

Pan A, J Liu, J Zhang, W Xu, GH Cao, Z Nie, BW Arey, and S Liang. 2010. "Nano-structured $\mathrm{Li}_{3} \mathrm{~V}_{2}\left(\mathrm{PO}_{4}\right)_{3} /$ Carbon Composite for High Rate Lithium Ion Batteries.” Electrochemistry Communications 12(12):1674-1677.

Pan A, J Zhang, Z Nie, GH Cao, BW Arey, G Li, S Liang, and J Liu. 2010. "Facile Synthesized Nanorod Structured Vanadium Pentoxide for High-Rate Lithium Batteries.” Journal of Materials Chemistry 20(41):9193-9199.

Pan Y, C Liu, D Mei, and Q Ge. 2010. "Effects of Hydration and Oxygen Vacancy on $\mathrm{CO}_{2}$ Adsorption and Activation on $\mathrm{B}_{-} \mathrm{Ga}_{2} \mathrm{O}_{3}(100)$." Langmuir 26(8):5551-5558.

Prince RN, ER Schreiter, P Zou, HS Wiley, AY Ting, RT Lee, and DA Lauffenburger. 2010. "The Heparin-Binding Domain of HB-EGF Mediates Localization to Sites of Cell-Cell Contact and Prevents HB-EGF Proteolytic Release." Journal of Cell Science 123(13):2308-2318.

Rodland KD. 2010. “Systems Biology and Biomarker Discovery.” Disease Markers 28(4):195-197.

Runde W, LF Brodnax, GS Goff, SM Peper, FL Taw, and BL Scott. 2007. "Synthesis and Structural Characterization of a Molecular Plutonium(IV) Compound Constructed from Dimeric Building Blocks." Chemical Communications Issue 17:1728-1729.

Rutledge RD, CL Warner, JW Pittman, RS Addleman, MH Engelhard, W Chouyyok, and MG Warner. 2010. "Thiol-Ene Induced Diphosphonic Acid Functionalization of Superparamagnetic Iron Oxide Nanoparticles." Langmuir 26(14):12285-12292.

Shah AR, JL Davidson, ME Monroe, AM Mayampurath, WF Danielson III, Y Shi, AC Robinson, BH Clowers, ME Belov, GA Anderson, and RD Smith. 2010. "An Efficient Data Format for Mass Spectrometry Based Proteomics." Journal of the American Society for Mass Spectrometry 21(10):1784-1788.

Shah AR, K Agarwal, ES Baker, M Singhal, AM Mayampurath, YM Ibrahim, LJ Kangas, ME Monroe, R Zhao, ME Belov, GA Anderson, and RD Smith. 2010. "Machine Learning Based Prediction for Peptide Drift Rimes in Ion Mobility Spectrometry.” Bioinformatics 26(13):1601-1607.

Shankaran H and HS Wiley. 2010. "Oscillatory Dynamics of the Extracellular Signal-regulated Kinase Pathway." Current Opinion in Genetics \& Development 20(6):650-655.

Shao Y, J Wang, H Wu, J Liu, IA Aksay, and Y Lin. 2010. "Graphene Based Electrochemical Sensors and Biosensors: A Review." Electroanalysis 22(10):1027-1036. 
Shao Y, S Zhang, MH Engelhard, G Li, G Shao, Y Wang, J Liu, IA Aksay, and Y Lin. 2010. "Nitrogen-Doped Graphene and its Electrochemical Applications." Journal of Materials Chemistry 20(35):7491-7496.

Sheaff CN, D Eastwood, CM Wai, and RS Addleman. 2008. "Fluorescence Detection and Identification of Tagging Agents and Impurities Found in Explosives." Applied Spectroscopy 62(7):739-746.

Sheen DM, TE Hall, RH Severtsen, DL McMakin, BK Hatchell, and PLJ Valdez. 2010. "Standoff Concealed Weapon Detection Using a $350 \mathrm{GHz}$ Radar Imaging System.” In Passive Millimeter Wave Imaging Technology XIII, eds DA Wikner and AR Luukanen, Vol 7670, Paper 767008. SPIE, Bellingham, WA.

Sheen DM, DL McMakin, and TE Hall. 2010. "Near-Field Three-Dimensional Radar Imaging Techniques and Applications." Applied Optics 49(19):E83-E93.

Shin Y, A Dohnalkova, and Y Lin. 2010. "Preparation of Homogeneous Gold-Silver Alloy Nanoparticles Using the Apoferritin Cavity as a Nanoreactor." The Journal of Physical Chemistry C 114(13):5985-5989.

Siegel J, O Villa, S Krishnamoorthy, A Tumeo, and X Li. 2010. "Efficient Sparse Matrix-Matrix Multiplication on Heterogeneous High Performance Systems." In Proceedings of the IEEE International Conference on Cluster Computing Workshops, pp. 1-8. IEEE, Piscataway, NJ.

Soderquist CZ, AM Johnsen, BK McNamara, BD Hanson, JW Chenault, KJ Carson, and SM Peper. 2011. "Dissolution of Irradiated Commercial $\mathrm{UO}_{2}$ Fuels in Ammonium Carbonate and Hydrogen Peroxide." Industrial and Engineering Chemistry Research 50(4):1813-1818.

Su Y, HM Brown, G Li, XD Zhou, JE Amonette, JL Fulton, DM Camaioni, and ZC Zhang. 2011. “Accelerated Cellulose Depolymerization Catalyzed by Paired Metal Chlorides in Ionic Liquid Solvent." Applied Catalysis A, General 391(1-2):436-442.

Sud YC, E Wilcox, WK Lau, GK Walker, X Liu, A Nenes, D Lee, KM Kim, Y Zhou, and PS Bhattacharjee. 2009. "Sensitivity of Boreal-Summer Circulation and Precipitation to Atmospheric Aerosols in Selected Regions: Part I Africa and India." Annales Geophysicae 27(10):3989-4007.

Sun K, N Stander, CS Jhun, Z Zhang, T Suzuki, GY Wang, M Saeed, AW Wallace, E Tseng, AJ Baker, D Saloner, DR Einstein, MB Ratcliffe, and JM Guccione. 2009. "A Computationally Efficient Formal Optimization of Regional Myocardial Contractility in a Sheep with Left Ventricular Aneurysm." Journal of Biomechanical Engineering 131(11), Art 111001.

Sun K, Z Zhang, T Suzuki, JF Wenk, N Stander, DR Einstein, D Saloner, AW Wallace, JM Guccione, and MB Ratcliffe. 2010. "Dor Procedure for Dyskinetic Anteroapical Myocardial Infarction Fails to Improve Contractility in the Borderzone." Journal of Thoracic and Cardiovascular Surgery 140(1):233-239.

Sushko ML and J Liu. 2010. "Structural Rearrangements in Self-Assembled Surfactant Layers at Surfaces." The Journal of Physical Chemistry B 114(11):3847-3854.

Sushko ML, KM Rosso, and J Liu. 2010. "Mechanism of Li+/Electron Conductivity in Rutile and Anatase $\mathrm{TiO}_{2}$ Nanoparticles." Journal of Physical Chemistry C 114(47):20277-20283.

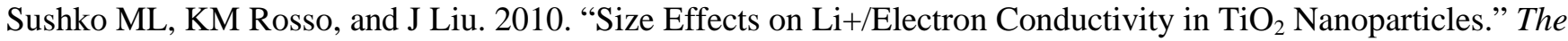
Journal of Physical Chemistry Letters 1(13):1967-1972.

Sushko ML, PV Sushko, IV Abarenkov, and AL Shluger. 2010. "QM/MM Method for Metal - Organic Interfaces." Journal of Computational Chemistry 31(16):2955-2966.

Talbot E, DA Frincke, and M Bishop. 2010. "Demythifying Cybersecurity.” IEEE Security \& Privacy 8(3):56-59. 
Teeguarden JG, BJM Webb-Robertson, KM Waters, A Murray, E Kisin, SM Varnum, JM Jacobs, JG Pounds, RC Zangar, and A Shvedova. 2011. "Comparative Proteomics and Pulmonary Toxicity of Instilled Single Walled Carbon Nanotubes, Crocidolite Asbestos and Ultrafine Carbon Black in Mice.” Toxicological Sciences 120(1):123-135.

Teeguarden JG, MS Bogdanffy, TR Covington, C Tan, and AM Jarabek. 2008. "A PBPK Model for Evaluating the Impact of Aldehyde Dehydrogenase Polymorphisms on Comparative Rat and Human Nasal Tissue Acetaldehyde Dosimetry." Inhalation Toxicology 20(4):375-390.

Tumeo A, and O Villa. 2010. "Accelerating DNA Analysis Applications on GPU Clusters.” In 8th IEEE Symposium on Application Specific Processors (SASP). IEEE, Piscataway, NJ.

Unwin SD, PP Lowry, and MY Toyooka. 2010. "A New Class of Risk-Importance Measures to Support Reactor Aging Management and the Prioritization of Materials Degradation Research." In 10th International Probabilistic Safety Assessment \& Management Conference - PSAM10. IPSAM, Seattle, WA.

Unwin SD, PP Lowry, and MY Toyooka. 2010. "Component Degradation Susceptibilities as the Bases for Modeling Reactor Aging Risk.” In 2010 Pressure Vessels \& Piping Conference (PVP 2010): Pressure Vessel Technology for Energy Challenge, Paper PVP2010-25750. ASME, New York, NY.

Valiev M, EJ Bylaska, N Govind, K Kowalski, TP Straatsma, HJJ van Dam, D Wang, J Nieplocha, E Apra, TL Windus, and WA De Jong. 2010. "NWChem: A Comprehensive and Scalable Open-Source Solution for Large Scale Molecular Simulations." Computer Physics Communications 181(9):1477-1489.

Vijayakumar M, SD Burton, C Huang, L Li, Z Yang, GL Graff, J Liu, JZ Hu, and M Skyllas-Kazacos. 2010. "Nuclear Magnetic Resonance Studies on Vanadium(IV) Electrolyte Solutions for Vanadium Redox Flow Battery." Journal of Power Sources 195(22 SP ISS):7709-7717.

Vijayakumar M, SN Kerisit, KM Rosso, SD Burton, JA Sears Jr, Z Yang, GL Graff, J Liu, and JZ Hu. 2011. "Lithium Diffusion in Li4Ti5O12 at High Temperatures." Journal of Power Sources

196(4):2211-2220.

Vijayakumar M, BM Sivakumar, P Nachimuthu, B Schwenzer, S Kim, Z Yang, J Liu, GL Graff, S Thevuthasan, and JZ Hu. 2011. "Spectroscopic Investigations of the Fouling Process on Nafion Membranes in Vanadium Redox Flow Batteries." Journal of Membrane Science 366(1-2):325-334.

Villa O, D Chavarría-Miranda, V Gurumoorthi, A Marquez, and S Krishnamoorthy. 2009. "Effects of Floating-Point Non-Associativity on Numerical Computations on Massively Multithreaded Systems."

In Cray User Group Meeting (CUG 2009). Cray User Group, Corvallis, OR.

Villa O, D Chavarría-Miranda, and KJ Maschhoff. 2009. "Input-Independent, Scalable and Fast String Matching on the Cray XMT.” In IEEE International Symposium on Parallel \& Distributed Processing. IEEE, Piscataway, NJ.

Villa O, L Chen, and S Krishnamoorthy. 2010. "High Performance Molecular Dynamic Simulation on Single and Multi-GPU Systems." In IEEE International Symposium on Circuits and Systems (ISCAS 2010), pp. 3805-3808. IEEE, Piscataway, NJ.

Villa O, A Tumeo, and D Sciuto. 2010. "Efficient Pattern Matching on GPUs for Intrusion Detection Systems." In Proceedings of the 7th ACM International Conference on Computing Frontiers, pp. 87-88. Association for Computing Machinery, New York, NY.

Viswanathan VV, D Choi, D Wang, W Xu, SA Towne, RE Williford, J Zhang, J Liu, and Z Yang. 2010. "Effect of Entropy of Lithium Intercalation in Cathodes and Anodes on Li-ion Battery Thermal Management." Journal of Power Sources 195(11):3720-3729. 
Wang CM, W Xu, J Liu, D Choi, BW Arey, LV Saraf, J Zhang, Z Yang, S Thevuthasan, DR Baer, and N Salmon. 2010. "In-Situ Transmission Electron Microscopy and Spectroscopy Studies of Interfaces in Li-ion Batteries: Challenges and Opportunities." Journal of Materials Research 25(8):1541-1547.

Wang D, R Kou, D Choi, Z Yang, Z Nie, J Li, LV Saraf, D Hu, J Zhang, GL Graff, J Liu, MA Pope, and IA Aksay. 2010. "Ternary Self-Assembly of Ordered Metal Oxide-Graphene Nanocomposites for Electrochemical Energy Storage.” ACS Nano 4(3):1587-1595.

Warner CL, S Addleman, AD Cinson, T Droubay, MH Engelhard, MA Nash, W Yantasee, and MG Warner. 2010. "High-Performance, Superparamagnetic, Nanoparticle-Based Heavy Metal Sorbents for Removal of Contaminants from Natural Waters." ChemSusChem 3(6):749-757.

Warner MG, CL Warner, RS Addleman, and W Yantasee. 2009. "Magnetic Nanomaterials for Environmental Applications." In Magnetic Nanomaterials (Nanomaterials for the Life Sciences), ed CSSR Kumar, Vol 4: Magnetic Nanomaterials, pp. 311-344. Wiley-VCH.

Webb-Robertson BJM, AL Bunn, and VL Bailey. 2011. "Phospholipid Fatty Acid Biomarkers in a Freshwater Periphyton Community Exposed to Uranium: Discovery by Non-linear Statistical Learning." Journal of Environmental Radioactivity 102(1):67-71.

Webb-Robertson BJM, LA McCue, KM Waters, MM Matzke, JM Jacobs, TO Metz, SM Varnum, and JG Pounds. 2010. "Combined Statistical Analyses of Peptide Intensities and Peptide Occurrences Improves Identification of Significant Peptides from MS-based Proteomics Data.” Journal of Proteome Research 9(11):5748-5756.

Webb-Robertson BJM, K Ratuiste, and CS Oehmen. 2010. "Physicochemical Property Distributions for Accurate and Rapid Pairwise Protein Homology Detection.” BMC Bioinformatics 11, Art 145.

Weber TJ, H Shankaran, HS Wiley, LK Opresko, WB Chrisler, and RD Quesenberry. 2010. "Basic Fibroblast Growth Factor Regulates Persistent ERK Osciliations in Premaligant but Not Malignant JB6 Cells." Journal of Investigative Dermatology 130(5):144-156.

Wegener KM, AK Singh, JM Jacobs, TR Elvitigala, EA Welsh, NS Keren, MA Gritsenko, BK Ghosh, DG Camp II, RD Smith, and HB Pakrasi. 2010. "Global Proteomics Reveal An Atypical Strategy for Carbon/Nitrogen Assimilation by a Cyanobacterium Under Diverse Environmental Perturbations." Molecular \& Cellular Proteomics. MCP 9(12):2678-89.

Welsh EA, ML Liberton, J Stockel, T Loh, TR Elvitigala, C Wang, A Wollam, RS Fulton, SW Clifton, JM Jacobs, R Aurora, BK Ghosh, LA Sherman, RD Smith, RK Wilson, and HB Pakrasi. 2008. "The Genome of Cyanothece 51142, A Unicellular Diazotrophic Cyanobacterium Important in the Marine Nitrogen Cycle." Proceedings of the National Academy of Sciences of the United States of America 105(39):15094-15099.

Wenk JF, CS Jhun, Z Zhang, K Sun, M Burger, DR Einstein, MB Ratcliffe, and JM Guccione. 2010.

"In Vivo Left Ventricular Geometry and Boundary Conditions." In Computational Cardiovascular Mechanics: Modeling and Applications in Heart Failure, eds JM Guccione, GS Kassak and MB Ratcliffe, Ch 1, pp. 3-21. Springer, New York, NY.

Werth CJ, C Zhang, ML Brusseau, M Oostrom, and T Baumann. 2010. "A Review of Non-Invasive Imaging Methods and Applications in Contaminant Hydrogeology Research.” Journal of Contaminant Hydrology 113(1-4):1-24.

Whitney PD and SJ Walsh. 2010. "Calibrating Bayesian Network Representations of Social-Behavioral Models." Lecture Notes in Computer Science 6007(2010):338-345.

Wick CD, TM Chang, and LX Dang. 2010. "Molecular Mechanism of $\mathrm{CO}_{2}$ and $\mathrm{SO}_{2}$ Molecules Binding to the Air/Liquid Interface of 1-Butyl-3-Methylimidazolium Tetrafluoroborate Ionic Liquid: A Molecular Dynamics Study with Polarizable Potential Models." Journal of Physical Chemistry B 114(46):14965-14971. 
Wischgoll T, DR Einstein, AP Kuprat, X Jiao, and GS Kassab. 2010. "Vascular Geometry Reconstruction and Grid Generation." In Computational Cardiovascular Mechanics: Modeling and Applications in Heart Failure, Ch 7 , pp. 103-119. Springer, New York, NY.

Wunschel DS, BJM Webb-Robertson, CW Frevert, SJ Skerret, N Beagley, AR Willse, HA Colburn, and KC Antolick. 2009. "Differentiation of Gram-Negative Bacterial Aerosol Exposure Using Detected Markers in Bronchial-Alveolar Lavage Fluid.” PLoS One 4(9):e7047.

Xiao J, D Choi, L Cosimbescu, PK Koech, J Liu, and JP Lemmon. 2010. "Exfoliated $\mathrm{MoS}_{2}$ Nanocomposite as an Anode Material for Lithium Ion Batteries." Chemistry of Materials 22(16):4522-4524.

Xiao J, W Xu, D Wang, and J Zhang. 2010. "Hybrid Air-Electrode for Li/Air Batteries." Journal of the Electrochemical Society 157(3):A294-A297.

Xiao J, W Xu, D Wang, D Choi, W Wang, X Li, GL Graff, J Liu, and J Zhang. 2010. "Stabilization of Silicon Anode for Li-Ion Batteries.” Journal of the Electrochemical Society 157(10):A1047-A1051.

Xu W, NL Canfield, D Wang, J Xiao, Z Nie, XS Li, WD Bennett, CC Bonham, and J Zhang. 2010. "Approach to Make Macroporous Metal Sheets as Current Collectors for Lithium-Ion Batteries." Journal of the Electrochemical Society 157(7):A765-A769.

$\mathrm{Xu}$ W, NL Canfield, D Wang, J Xiao, Z Nie, and J Zhang. 2010. "A Three-Dimensional Macroporous Cu/SnO ${ }_{2}$ Composite Anode Sheet Prepared via a Novel Method.” Journal of Power Sources 195(21):7403-7408.

Xu W, J Xiao, D Wang, J Zhang, and J Zhang. 2010. "Crown Ethers in Nonaqueous Electrolytes for Lithium/Air Batteries.” Electrochemical and Solid-State Letters 13(4):A48-A51.

Yang Y, CA Mims, RS Disselkamp, JH Kwak, CHF Peden, and CT Campbell. 2010. “(Non) Formation of Methanol by Direct Hydrogenation of Formate on Copper Catalysts." Journal of Physical Chemistry C 114(40):17205-17211.

Yantasee W, RD Rutledge, W Chouyyok, V Sukwarotwat, G Orr, CL Warner, MG Warner, GE Fryxell, RJ Wiacek, C Timchalk, and RS Addleman. 2010. "Functionalized Nanoporous Silica for Removal of Heavy Metals from Biological Systems; Adsorption and Application.” ACS Applied Materials \& Interfaces 2(10):2749-2758.

Yu J, KM Rosso, and J Liu. 2010. "Charge Localization and Transport in Lithiated Olivine Phosphate Materials." PNNL-SA-72778, Richland, WA. [Submitted]

Zaveri RA, JC Barnard, RC Easter Jr, N Riemer, and M West. 2010. "Particle-resolved Simulation of Aerosol Size, Composition, Mixing State, and the Associated Optical and Cloud Condensation Nuclei Activation Properties in an Evolving Urban Plume.” Journal of Geophysical Research D (Atmospheres) 115, Art D17210.

Zentner MD, I Therios, RA Bari, L Cheng, M Yue, R Wigeland, J Hassberger, B Boyer, and J Pilat. 2010. “An Expert Elicitation Based Study of the Proliferation Resistance of a Suite of Nuclear Power Plants." In Proceedings of the 51st Annual Meeting of the Institute of Nuclear Materials Management. INMM, Deerfield, IL.

Zhang C, KJ Dehoff, NJ Hess, M Oostrom, TW Wietsma, AJ Valocchi, BW Fouke, and CJ Werth. 2010. "Pore-Scale Study of Transverse Mixing Induced $\mathrm{CaCO}_{3}$ Precipitation and Permeability Reduction in a Model Subsurface Sedimentary System.” Environmental Science \& Technology 44(20):7833-7838.

Zhong L, JE Szecsody, ZF Zhang, and SV Mattigod. 2010. "Foam Delivery of Amendments for Vadose Zone Remediation - Propagation Performance in Unsaturated Sediments." Vadose Zone Journal 9(3):757-767. 


\section{Appendix B}

\section{Fiscal Year 2010 Non-Refereed Publications}




\section{Appendix B}

\section{Fiscal Year 2010 Non-Refereed Publications}

In this appendix, 221 non-refereed publications or presentations resulting from PNNL's LDRD Program during FY 2010 are arranged in alphabetical order by the first author's last name.

2010. PNNL-SA-73987 [Limited Distribution]

2010. PNNL-SA-73988 [Limited Distribution]

2010. PNNL-SA-74007 [Limited Distribution]

2010. PNNL-SA-74023 [Limited Distribution]

2010. PNNL-SA-74367 [Limited Distribution]

Aalseth CE, AR Day, DA Haas, EW Hoppe, BJ Hyronimus, ME Keillor, EK Mace, JL Orrell, A Seifert, and VT Woods. 2010. "Measurement of Ar-37 in a Low-background Proportional Counter for On-site Inspection under the Comprehensive Nuclear-Test-Ban Treaty." Presented by Allen Seifert at the 2010 Symposium on Radiation Measurements and Applications, Ann Arbor, MI, May 26, 2010.

Aalseth CE, AR Day, DA Haas, EW Hoppe, BJ Hyronimus, ME Keillor, EK Mace, JL Orrell, A Seifert, and VT Woods. 2010. "Experimental Optimization of Low-Background Proportional Counter Measurements of Ar-37 for On-Site Inspection under the Comprehensive Nuclear-Test-Ban Treaty." Presented by John Orrell (invited speaker) at IEEE - 2010 Nuclear Science Symposium and Medical Imaging Conference, Knoxville, TN, November 1, 2010.

Aalseth CE, AR Day, JC Hayes, EW Hoppe, TW Hossbach, BJ Hyronimus, ME Keillor, KE Litke, JI McIntyre, EE Mintzer, JL Orrell, A Seifert, and GA Warren. 2009. “Argon-37: Research Supporting On-Site Inspection Background Measurements." Presented by Craig E Aalseth (invited speaker) at INGE 2009 Workshop, Daejaeon South Korea, November 10, 2009.

Aalseth CE, AR Day, EW Hoppe, TW Hossbach, BJ Hyronimus, ME Keillor, KE Litke, EE Mintzer, A Seifert, and GA Warren. 2009. "Design and Construction of a Low-Background, Internal Source Proportional Counter from Electroformed Copper.” Presented by Craig E Aalseth at MARC VIII, Kona, HI, April 7, 2009.

Aalseth CE, ME Keillor, JC Hayes, JI McIntyre, JL Orrell, and A Seifert. 2009. "37Ar Background Measurements Supporting On-Site Inspection.” Presented by Craig E Aalseth (invited speaker) at International Scientific Studies Conference, Vienna, Austria, June 10, 2009.

Amidan BG and HE Trease. 2010. "Cluster Analysis Investigation of Video Streams and Archives." Presented by Brett G Amidan at Joint Statistical Meetings, Vancouver, BC Canada, August 3, 2010.

Anheier Jr NC and BA Bushaw. 2009. "Isotope Enrichment Detection by Laser Ablation - Laser Absorption Spectrometry." Presented by Norm Anheier (invited speaker) and Bruce Bushaw at the 2nd JAPAN-IAEA Workshop on Advanced Safeguards Technology for the Future Nuclear Fuel Cycle, Tokai-mura, Ibaraki, Japan, November 10, 2009. 
Anheier Jr NC and BA Bushaw. 2010. "Isotope Enrichment Detection by Laser Ablation - Laser Absorption Spectrometry: Automated Environmental Sampling and Laser-Based Analysis for HEU Detection." In Proceedings of the 2nd JAPAN-IAEA Workshop on Advanced Safeguards Technology for the Future Nuclear Fuel Cycle, vol INIS-XA1073. IAEA, Vienna, Austria.

Anheier Jr NC and BA Bushaw. 2010. "Unattended HEU Detection in Enrichment Plants using Automated Aerosol Collection and Laser-based Enrichment Assay." Presented by Norm Anheier at the Pacific Northwest International Conference on Global Nuclear Security: The Decade Ahead, Portland, OR, April 13, 2010.

Anheier Jr NC and BA Bushaw. 2010. "Unattended Monitoring of HEU Production in Gaseous Centrifuge Enrichment Plants using Automated Aerosol Collection and Laser-based Enrichment Assay." Presented by Norm Anheier at the Institute of Nuclear Materials Management Conference, Baltimore, MD, July 12, 2010.

Anheier Jr NC and BA Bushaw. 2010. "Unattended Monitoring of HEU Production in Gaseous Centrifuge Enrichment Plants using Automated Aerosol Collection and Laser-based Enrichment Assay." In Proceedings of the 51st Annual Meeting of the Institute of Nuclear Materials Management. INMM, Deerfield, IL.

Arrigo LM, HM Cho, and SC Smith. 2010. "Low Level Fluoride Analysis.” Presented by Leah Arrigo at the Joint 65th Northwest/22nd Rocky Mountain Regional Meeting of the American Chemical Society (NORM/RMRM 2010), Pullman, WA, June 22, 2010.

Atkinson DA, RG Ewing, and MJ Waltman. 2010. "Detection of TATP by Ion Mobility Spectrometry." Presented by David A Atkinson (invited speaker) at the 2nd Annual Trace Explosives Detection Workshop, Baltimore, MD, April 26, 2010.

Atkinson DA. 2010. "Explosives Detection: Exploitation of the Chemical Signatures." Presented by David Atkinson (invited speaker) at the Joint 65th Northwest/22nd Rocky Mountain Regional Meeting of the American Chemical Society (NORM/RMRM 2010), Pullman, WA, June 21, 2010.

Barker EI, X Sun, D Li, and R Devanathan. 2010. "Performance Prediction Using Mesoscale Model Informed Statistical 3D Polycrystalline Models." Presented by Erin Barker (invited speaker) at the 2nd Bienniel Symposium of Predictive Science and Technology of Mechanics and Materials, Mississippi State University, Starkville, MS, June 24, 2010.

Bearden MD and C Valkenburg. 2010. "Revisiting Condensation Gas Cleaning." Presented by Corinne Valkenburg at the Ninth Annual Conference on Carbon Capture \& Sequestration, Pittsburgh, PA, May 12, 2010.

Beliaev AS. 2009. "Novel Cultivation Techniques for Optimization of Phototrophic Metabolism." Presented by Alex Beliaev (invited speaker) at the 2009 Pacific Rim Summit on Industrial Biotechnology and Bioenergy, Honolulu, HI, November 10, 2009.

Beliaev AS. 2010. "Systems-level Studies of Photosynthetic Metabolism for Biofuel Applications." Presented by Alex Beliaev (invited speaker) at PNNL/LANL Meeting, Los Alamos, NM, May 11, 2010.

Bernacki BE and MC Phillips. 2010. "Standoff Hyperspectral Imaging of Explosives Residues Using Broadly Tunable External Cavity Quantum Cascade Laser Illumination.” In Chemical, Biological, Radiological, Nuclear, and Explosives (CBRNE) Sensing XI, vol 7665, Art 76650I, eds AW Fountain III and PJ Gardner. SPIE, Bellingham, WA.

Best DM, SJ Bohn, DV Love, AS Wynne, and WA Pike. 2010. "Real-Time Visualization of Network Behaviors for Situational Awareness." Presented by Daniel Best at Symposium on Visualization for Cyber Security, Ottawa, ON Canada, September 14, 2010.Best DM, DV Love, SJ Bohn, and WA Pike. 2010. "High-Throughput Real-Time Network Flow Visualization.” Presented Daniel M Best at FloCon 2010, New Orleans, LA, January 13, 2010. 
Bond LJ and P Ramuhalli. 2010. "Status Report TAG \#3: Prognostics and Structural Material Integrity." Presented by Leonard Bond (invited speaker) at IAEA Coordinated Research Project on Advanced Surveillance, Diagnostics, and Prognostics Techniques, Richland, WA, June 7, 2010.

Bond-Lamberty B and AM Thomson. 2009. "Can We Detect Changes in High-latitude Soil Respiration over Decadal Time Scales?" Presented by Ben Bond-Lamberty at the American Geophysical Union 2009 Fall Meeting, San Francisco, CA, December 17, 2009.

Bonneville AHR, GD Black, DH Bacon, I Gorton, LJ Gosink, ML Rockhold, AM Tartakovsky, MD White, MD Williams, and EM Murphy. 2010. "Geologic Sequestration Software Suite (GS3): An Integrated Tool to Manage GHG Storage Projects." Presented by Ellyn Murphy at Carbon Capture and Sequestration Conference, Pittsburgh, PA, May 10, 2010.

Bonneville AHR, I Gorton, EM Murphy, ML Rockhold, C Sivaramakrishnan, and MD White. 2010. "Geologic Sequestration Software Suite: A Collaborative Approach to the Management of Geological GHG Storage Projects." Presented by Alain Bonneville at Green House Gas Technology 10 (GHGT10), Amsterdam, NLD, September 20, 2010.

Breithaupt SA and TP Khangaonkar. 2009. "Water Quality of the Nearshore Environment - Modeling Dissolved Oxygen in Port Susan and the Lower Stillaguamish River." Presented by Stephen A Breithaupt at Puget Sound-Georgia Basin, Seattle, WA, February 17, 2009.

Buchko GW and H Robinson. 2010. "Structural Characterization of a Protein Associated with Nitrogen Fixation in the DUF269 Family - Crystal Structure of cce_0566 from the Cyanobacterium Cyanothece 51142." Presented by Garry W Buchko at the Northwest Crystallography Workshop 2010, Vancouver, BC Canada, June 18, 2010.

Bylaska EJ and JH Weare. 2010. "Next Generation Parameter-free Petascale Simuation Technology for Solution Species, Nanoparticles, and Geochemical Interfaces." Presented by Eric Bylaska (invited speaker) at the American Chemical Society Spring Meeting 2010, San Francisco, CA, March 23, 2010.

Carroll TE and D Grosu. 2010. "Incentive Compatible Online Scheduling of Malleable Parallel Jobs with Individual Deadlines." Presented by Thomas E Carroll at the 39th International Conference on Parallel Processing, San Diego, CA, September 16, 2010.

Carroll TE, PR Paulson, FL Greitzer, RE Hohimer, and L Franklin. 2010. "Reasoning About the Insider Threat." Presented by Thomas E Carroll at the IEEE Sympoisum on Security and Privacy 2010, Oakland, CA, May 18, 2010.

Casella AJ, JM Peterson, and TG Levitskaia. 2009. " $\mathrm{CO}_{2}$ Separation and Capture by Chitosan.” Presented by Amanda Casella at the 16th Symposium on Separation Science and Technology for Energy Applications, Gatlinburg, TN, October 22, 2009.

Casella AJ, TG Levitskaia, and JM Peterson. 2010. “Chitosan Materials for $\mathrm{CO}_{2}$ Separation and Capture.” Presented by Tatiana Levitskaia (invited speaker) at the ACS Spring Meeting, San Francisco, CA, March 25, 2010.

Chen Y and FL Greitzer. 2010. "Contingency Analysis and Visualization.” Presented by Yousu Chen (invited speaker) at BPA visit, Richland, WA, June 4, 2010.

Chen Y and Z Huang. 2010. "An Advanced Framework for Enabling Electricity Infrastructure Real-Time Decision Support." Presented by Yousu Chen at the Second International Conference on Computational Sustainability, Cambridge, MA, June 30, 2010.

Chen Y, Z Huang, PS Mackey, CH Allwardt, J Ma, and FL Greitzer. 2010. “An Advanced Visual Analytic Decision Support Tool for Electricity Infrastructure Operations." Presented by Yousu Chen (invited speaker) at the Fourth Annual IFIP WG 11.10 International Conference on Critical Infrastructure Protection, Fort McNair, DC, March 16, 2010. 
Choi D, D Wang, IT Bae, J Xiao, W Xu, Z Nie, J Zhang, Z Yang, GL Graff, and J Liu. 2010. "Synthesis, Orientation and Electrochemical Properties of Nanostructured $\mathrm{LiMPO}_{4}(\mathrm{M}: \mathrm{Fe}, \mathrm{Mn}, \mathrm{Co})$ Cathode for Li-ion Battery." Presented by Daiwon Choi at TMS2010: 139th Annual Meeting \& Exhibition, Seattle, WA, February 18, 2010.

Collett JR, RW Heck, and A Zwoster. 2010. "Dissolved Carbonic Anhydrase for Enhancing Post-Combustion Carbon Dioxide Hydration in Aqueous Ammonia." Presented by James R Collett at the 10th International Conference on Greenhouse Gas Control Technologies, Amsterdam, NLD, September 19, 2010.

Costa MN and JE McDermott. 2010. "Comparative Network Analysis of Specific Host-pathogen Interactions." Presented by Michelle Costa (invited speaker) at AMIA Translational Bioinformatics, San Francisco, CA, March 10, 2010.

Costa MN, JE McDermott, H Shankaran, BD Thrall, and KM Waters. 2010. "Predictive Modeling of Macrophage Transcriptional Response to Nanoparticle Exposure." Presented by Michelle N Costa at ISMB 2010, Boston, MA, July 13, 2010.

Cumblidge SE, SR Doctor, LJ Bond, and BE Watson. 2009. "A New Look at Acoustic Emission for Aging Management." Presented by Stephen Cumblidge at American Nuclear Society 2009 Winter Meeting and Nuclear Technology Expo, Washington DC, November 19, 2009.

Curtis MM. 2010. "Supplemental Systems for Unattended UF 6 Cylinder Monitoring." Presented by Michael Curtis (invited speaker) at 51st INMM Annual Conference, Baltimore, MD, July 14, 2010.

Cziczo DJ, H Herich, B Friedman, SJ Gallavardin, GR Kulkarni, M Kohn, K Pratt, and U Lohmann. 2010. "Field Studies of the Effect of Particle Composition on Droplet and Ice Nucleation." Presented by Dan Cziczo at International Aerosol Conference, Helsinki, Finland, September 3, 2010.

Cziczo DJ, O Stetzer, A Worringen, M Ebert, M Kamphus, J Curtius, S Metes, O Moehler, U Lohmann, GR Kulkarni, SJ Gallavardin, KD Froyd, PJ DeMott, D Thomson, and DM Murphy. 2010. "Which Aerosol Particles End Up Inside Clouds?” Presented by Daniel J Cziczo (invited speaker) at Princeton Seminar, Princeton, NJ, March 9, 2010.

De Jong WA. 2010. "NW Chem: Pushing the Scientific Envelope on Large Computing Platforms." Presented by Bert A de Jong (invited speaker) at Scalable Software Workshop, Arlington, VA, June 2, 2010.

del Pin F, DR Einstein, SR Idelsohn, AP Kuprat, X Jiao, JM Guccione, and MB Ratcliffe. 2009. "Lagrangian Fluid-Structure Interaction of an Aortic Valve Including Contact." Presented by Facundo Del Pin (invited speaker) at 10th National Congress on Computational Mechanics, Columbus, OH, July 16, 2009.

Du P, Z Huang, R Diao, B Lee, and KK Anderson. 2010. "PMU Placement for Enhancing Dynamic Observability of a Power Grid." Presented by Pengwei Du (invited speaker) at the 2010 IEEE Conference on Innovative Technologies for an Efficient and Reliable Electricity Supply, Waltham, MA, September 27, 2010.

Duckworth DC, LM Arrigo, M Liezers, M Douglas, MA Green, OT Farmer, JM Schwantes, and SM Peper. 2010. "Electrochemically-Modulated Separations for Material Accountability Measurements." Presented by Doug Duckworth at MPACT Working Group Meeting, Berkeley, CA, January 20, 2010.

Dyedov V, DR Einstein, X Jiao, AP Kuprat, and D Wang. 2009. "Extensions and Comparisons of Generation of Hybrid Prism-Tetrahedron Meshes with Biomedical Applications." Presented by Volodymyr Dyedov at $10^{\text {th }}$ US National Congress on Computational Mechanics/MeshTrends VII, Columbus, OH, July 16, 2009.

Edgar TW, MD Hadley, DO Manz, and JD Winn. 2010. "Secure and Efficient Routable Control Systems." PNNL-19474, Richland, WA.

Edgar TW, SL Clements, MD Hadley, WM Maiden, DO Manz, and SJ Zabriskie. 2010. "Cryptographic Trust Management System Design Document." PNNL-18744, Richland, WA. 
Eltoweissy MY, R Eltarras, S Olariu, and IR Chen. 2010. "Towards Trustworthy Shared Sensor-Actuator Networks." Presented by Mohamed Eltoweissy (invited speaker) at CSIIR '10 (Cyber Security and Information Intelligence Research Workshop), Oakridge, TN, April 21, 2010.

Enke C, SJ Ray, AW Graham, GM Hieftje, DW Koppenaal, and CJ Barinaga. 2010. "Distance-of-Flight Mass Spectrometry - A Proof-of-Concept Instrument." Presented by Chris Enke (invited speaker) at Pittcon Conference, Orlando, FL, March 1, 2010.

Ewing RG, MJ Waltman, and DA Atkinson. 2010. "Atmospheric Pressure Ionization Chemistry of Explosives." Presented by Robert Ewing at the 19th International Conference on Ion Mobility Spectrometry, Albuquerque, NM, July 19, 2010.

Ewing RG, MJ Waltman, and DA Atkinson. 2010. "Selective Atmospheric Pressure Chemical Ionization of Explosive Compounds." Presented by Robert G Ewing (invited speaker) at the 2nd Annual Trace Explosives Detection Workshop, Baltimore, MD, April 26, 2010.

Fang Y, BJ Palmer, and SB Yabusaki. 2010. "Parallel Implementation of a Subsurface Simulator using a Novel Grid Component and Global Array Toolkit." Presented by Yilin Fang at SIAM Conference on Parallel Processing and Scientific Computing, Seattle, WA, February 25, 2010.

Fast JD. 2009. "How Do We Know that Aerosol Forecasts are Improving for the Right Reasons?" Presented by Jerome E Fast at International Workshop on Air Quality Forecasting Research, Boulder, CO, December 2, 2009.

Fast JD, JC Barnard, EG Chapman, RC Easter Jr, SJ Ghan, WI Gustafson Jr, RA Zaveri, G Grell, SE Peckham, and SA McKeen. 2010. "Aerosol-Radiation-Microphysics Interactions: Aerosol Direct and Indirect Forcing." Presented by Jerome Fast at WRF-Chem Tutorial, National Center for Atmospheric Research, Boulder, CO, August 2, 2010.

Fast JD, WI Gustafson Jr, EG Chapman, and RC Easter Jr. 2009. "Applying the Aerosol Modeling Testbed to Assess the Performance of Simulated Particle Properties and Radiative Forcing from Different Process Modules." Presented by Jerome Fast at ARM Aerosol Working Group Meeting, Boulder, CO, October 1, 2009.

Fast JD, WI Gustafson Jr, EG Chapman, RC Easter Jr, GA Grell, M Barth, SA Klein, and Y Liu. 2010. "Using testbeds to address modeling challenges." Presented by Jerome D Fast at DOE Office of Biological and Environmental Research, Germantown, MD, February 4, 2010.

Fast JD, WI Gustafson Jr, RC Easter Jr, EG Chapman, and JP Rishel. 2010. "The Aerosol Modeling Testbed: Tools Available to Evaluate WRF-Chem Predictions using Field Campaign Data." Presented by Jerome Fast at WRF-Chem Tutorial, National Center for Atmospheric Research, Boulder, CO, August 2, 2010.

Fast JE, EW Hoppe, EE Mintzer, CE Aalseth, AR Day, DC Gerlach, OT Farmer III, M Liezers, EA Lepel, HS Miley, and DJ Edwards. 2010. "Majorana DUSEL R\&D Final Report." PNNL-19719, Richland, WA.

Felmy AR, JH Kwak, JZ Hu, KM Rosso, CM Wang, DW Hoyt, ES Ilton, and DA Dixon. 2010. "The Role of Intermediates During Metal Carbonation of Forsterite in Wet Supercritical $\mathrm{CO}_{2}$." Presented by Kevin Rosso at Goldschmidt 2010, Knoxville, TN, June 18, 2010.

Feo JT, G Chin Jr, A Marquez, S Choudhury, LJ Kangas, C Scherrer, M Halappanavar, DJ Haglin, D ChavarríaMiranda, JA Mogill, Y Chen, Z Huang, PC Wong, PS Mackey, RD Adolf, S Jin, PSY Hui, JR Johnson III, JC Mount, J Yin, S Shrestha, JB Manzano Franco, S Bokhari, O Villa, J Siegel, A Tumeo, S Secchi, S Sarkar, RM Farber, CD Corley, W Reynolds, BL Chamberlain, L Prokowich, DA Bader, D Ediger, K Jiang, and EJ Riedy. 2010. "CASS-MT Quarterly Review Presentations." Presented by John Feo, George Chin, David Haglin, Chad Scherrer, Jace Mogill, Yousu Chen, et al. (invited speakers) at CASS-MT Quarterly Review, Baltimore, MD, August 30, 2010. 
Feo JT, A Marquez, S Choudhury, LJ Kangas, K Maschhoff, C Scherrer, DJ Haglin, JA Mogill, TR Shippert, D Chavarría-Miranda, H Trease, Z Huang, Y Chen, PC Wong, PS Mackey, S Jin, M Halappanavar, RD Adolf, S Shrestha, YJ Liu, S Bokhari, JB Manzano Franco, DA Bader, D Ediger, K Jiang, EJ Riedy, CA Joslyn, S al-Saffar, SH Kahan, DW Mizell, EL Goodman, GE Mackey, J Berry, L Prokowich, CD Corley, AJ Cowell, ML Gregory, W Reynolds, JR Johnson III, JC Mount, J Yin, and G Chin Jr. 2010. "CASS Quarterly Review.” Presented by John Feo, George Chin, Chad Scherrer, Henry Huang, Mahantesh Halappanavar, Andres Marquez, et al. at CASS Quarterly Review Meeting, Annapolis Junction, MD, May 13, 2010.

Frank DP, DC Gerlach, CJ Gesh, DE Hurley, GH Meriwether, MR Mitchell, and BD Reid. 2010. "Application of the Isotope Ratio Method to a Boiling Water Reactor." In Proceedings of the 51 st Annual Meeting of the Institute of Nuclear Materials Management, Baltimore, MD. INMM, Deerfield, IL.

Frincke DA and JM Anderson. 2010. "Meeting Face(book) to Face: CyberSecurity in Our Brave New World." Presented by Deborah Frincke (invited speaker) at INSR, University of Washington, Seattle, WA, January 21, 2010.

Frincke DA and JM Anderson. 2010. "Virtually Yours: CyberSecurity in Our Brave New World.” Presented by Deb Frincke (invited speaker) at 2010 Borah Symposium, University of Idaho, Moscow, ID, April 5, 2010.

Frincke DA. 2010. "You want to do what?????, Vitrually Yours: Cyber Security in Our Brave New World." Presented by Deborah Frincke (invited speaker), Borah Symposium, Moscow, ID, April 6, 2010.

Frincke DA. 2010. "PNNL Portfolio of Cyber Programs and Capabilities." Presented by Deb A Frincke (invited speaker) at I3P Workshop at the University of Idaho, Moscow, ID, June 18, 2010.

Gerlach DC, BD Reid, CJ Gesh, BK McNamara, and MR Mitchell. 2009. "Isotope Ratio Techniques to Determine Energy Production in Reactors." Presented by David Gerlach at Asia Pacific Society for Radiochemistry 2009 Conference, Napa, CA, December 3, 2009.

Gerlach DC, BD Reid, CJ Gesh, MR Mitchell, SC Szechenyi, M Douglas, BK McNamara, TA Ellis, and RM Ermi. 2010. "Secondary Ion Mass Spectrometry Analysis of Materials to Develop In-core Safeguards Reactor Monitoring Devices.” Presented by David C Gerlach, INMM Baltimore 2010, Baltimore, MD, July 12, 2010.

Gerlach DC, BD Reid, CJ Gesh, SC Szechenyi, MR Mitchell, M Douglas, BK McNamara, TA Ellis, and RM Ermi. 2010. "Secondary Ion Mass Spectrometry Analysis of Materials to Develop In-core Safeguards Reactor Monitoring Devices." In Proceedings of the 51st Annual Meeting of the Institute of Nuclear Materials Management. INMM, Deerfield, IL.

Gesh CJ, DP Frank, DC Gerlach, DE Hurley, MR Mitchell, GH Meriwether, and BD Reid. 2010. "Application of the Isotope Ratio Method to a Boiling Water Reactor.” Presented by Doug Frank at INMM, Baltimore, MD, July 12, 2010.

Gibson TD, KL Schuchardt, and EG Stephan. 2009. "Application of Named Graphs Towards Custom Provenance Views." Presented by Tara Gibson at 1st Workshop on the Theory and Practice of Provenance (TaPP '09),

San Francisco, CA, February 23, 2009.

Gibson TD and EG Stephan. 2009. "Provenance: From Arts to Systems Science." Presented by Tara Gibson at SLAC Presentation, Palo Alto, CA, September 11, 2009.

Gorton I, GD Black, KL Schuchardt, C Sivaramakrishnan, SK Wurstner, and PSY Hui. 2010. "GS3: A Knowledge Management Architecture for Collaborative Geologic Sequestration Modeling." In Proceedings of the 43rd Hawaii International Conference on System Sciences (HICSS 2010). IEEE Computer Society Press, New York, NY.

Gosney A, CS Oehmen, AS Wynne, and JP Almquist. 2010. "An Adaptive Middleware Framework for Scientific Computing at Extreme Scales." In The 2010 IEEE International Conference on Information Reuse and Integration (IRI 2010), pp. 232-238. IEEE, Piscataway, NJ. 
Green MA, LM Arrigo, M Liezers, CR Orton, M Douglas, SM Peper, JM Schwantes, SG Hazelton, and DC Duckworth. 2010. "Electrochemically-Modulated Separations for Safeguards Measurements." Presented by Sandra G Hazelton at the Institute of Nuclear Materials Management 51st Annual Meeting, Baltimore, MD, July 14, 2010.

Greitzer FL. 2010. "Insider Threats.” Presented by Frank L Greitzer (invited speaker) at University of Hawaii online course ICS425, Computer Security and Ethics online, July 15, 2010.

Greitzer FL. 2010. "Insider Threat Behavior.” Presented by Frank L Greitzer (invited speaker) at Workshop on Accelerated Learning to Mitigate Insider Threats, Washington DC, June 22, 2010.

Greitzer FL. 2010. "Wide Area Situation Awareness in Electric Power Grid.” Presented by Frank L Greitzer (invited speaker) at SPIE Defense, Security and Sensing 2010, Cyber Security, Situation Management, and Impact Assessment, Orlando, FL, April 9, 2010.

Greitzer FL, PM Dauenhauer, TG Wierks, R Podmore, and AC Dalton. 2009. "Experimental Evaluation of Electric Power Grid Visualization Tools in the EIOC.” PNNL-19103, Richland, WA.

Greitzer FL, LJ Kangas, CF Noonan, and AC Dalton. 2010. "Identifying At-Risk Employees: A Behavioral Model for Predicting Potential Insider Threats.” PNNL-19665, Richland, WA.

Griffin JW, P Ramuhalli, M Dixit, and LJ Bond. 2009. "Experimental Assessment of NDE Methods for Long-Term Monitoring of Materials Degradation in Nuclear Power Plant Components." Presented by Pradeep Ramuhalli at American Nuclear Society 2009 Winter Meeting and Nuclear Technology Expo, Washington DC, November 19, 2009.

Gustafson Jr WI, JD Fast, EG Chapman, and JP Rishel. 2009. "The Aerosol Modeling Testbed: A Tool for Efficient Development of Aerosol Process Modules." Presented by William Gustafson (invited speaker) at International Aerosol Modeling Algorithms Conference, Davis, CA, December 11, 2009.

Gustafson Jr WI, JD Fast, RC Easter Jr, and PJ Rasch. 2010. "Porting the CAM5 Physics Suite into WRF." Presented by William Gustafson at 11th Annual WRF Users' Workshop, Boulder, CO, June 24, 2010.

Haas DA, M Bliss, SM Bowyer, JD Kephart, and LE Smith. 2010. “Actinide Loaded Glass Scintillators for Fast Neutron Detection." Presented by Derek A Haas at Symposium on Radiation Measurements and Applications, Ann Arbor, MI, May 27, 2010.

Haas DA, JL Orrell, TW Bowyer, JI McIntyre, HS Miley, CE Aalseth, and JC Hayes. 2010. "The Science Case for 37Ar as a Monitor for Underground Nuclear Explosions." PNNL-19458, Richland, WA.

Hager GJ, CJ Barinaga, M Liezers, and DW Koppenaal. 2009. “Towards High-Resolution ICP-MS with Orbital Trapping Technology." Presented by David W Koppenaal at 8th International Sector Field ICP-MS Conference, Gent, Belgium, September 15, 2009.

Hager GJ, M Liezers, CJ Barinaga, and DW Koppenaal. 2010. "New Directions in ICPMS Performance - Attaining Simultaneity and Resolution with New Approaches and Technology." Presented by David W Koppenaal (invited speaker) at 2010 Winter Conference, Fort Myers, FL, January 4, 2010.

Halappanavar M and UV Catalyurek. 2010. "Parallel Graph Matching and Coloring for Petascale Scientific Computing." Presented by Mahantesh Halappanavar (invited speaker) at 14th SIAM Conference on Parallel Processing for Scientific Computing, Seattle, WA, February 25, 2010.

Heredia-Langner A, RD Lins, R Tanguay, S Harper, J Hutchison, JG Teeguarden, JG Pounds, BJM Webb-Robertson, and BD Thrall. 2009. "Quantitative Structure Activity Modeling of Gold Nanoparticle Toxicity in a Zebrafish Developmental System." Presented by Alejandro Heredia-Langner at SOT 48th Annual Meeting \& ToxExpo, Baltimore, MD, March 17, 2009. 
Higgins SR, M Xu, P Cubillas, AS Lea, KG Knauss, and KM Rosso. 2010. “Scanning Probe Microscopy and Mineral-Water Interfaces: Have We Reached a Limit?” Presented by Steven R Higgins at Goldschmidt 2010, Knoxville, TN, June 17, 2010.

Hoffman MG, MCW Kintner-Meyer, A Sadovsky, and JG DeSteese. 2010. "Analysis Tools for Sizing and Placement of Energy Storage for Grid Applications - A Literature Review.” PNNL-19703, Richland, WA.

Hoppe EW, CE Aalseth, OT Farmer III, JE Fast, TW Hossbach, M Liezers, HS Miley, EE Mintzer, and JH Reeves. 2010. "Reductions of Radioactive Backgrounds in Ultra-High Purity Electroformed Copper." Presented by Eric Hoppe (invited speaker) at DURA Annual Meeting and DUSEL PDR Rollout, Fermilab, Batavia, IL, September 2, 2010.

Hoppe EW, CE Aalseth, OT Farmer III, JE Fast, TW Hossbach, HS Miley, EE Mintzer, and JH Reeves. 2010. "Reductions of Radioactive Backgrounds in Ultra-High Purity Electroformed Copper." Presented by Eric Hoppe (invited speaker) at LRT2010, Sudbury, ON Canada, August 28, 2010.

Hu JZ, JH Kwak, DW Hoyt, JA Sears, KM Rosso, and AR Felmy. 2010. "Studies of Metal Carbonation of Forsterite in Supercritical $\mathrm{CO}_{2}$ and $\mathrm{H}_{2} \mathrm{O}$ Using Solid State NMR Spectroscopy." Presented by Jian Zhi Hu at 239th ACS National Meeting, San Francisco, CA, March 22, 2010.

Hu Q, P Wang, PL Gassman, and J Laskin. 2009. "In situ Characterization of Surfaces Following Soft and Reactive Landing of Complex Ions." Presented by Qichi Hu at AVS 56th International Symposium, San Jose, CA, November 10, 2009.

Huang Z. 2010. "Smart Grid Challenges." Presented by Zhenyu (Henry) Huang (Invited speaker) at Math for Power System Workshop, Richland, WA, August 17, 2010.

Huang Z, D Chavarría-Miranda, N Zhou, B Lee, P Du, ST Elbert, R Diao, G Welch, G Bishop, and J Zhang. 2010. "Advanced Kalman Filter Applications for Smart Grid Operation." Presented by Zhenyu Huang (invited speaker) at 2010 DOE Applied Mathematics Program Meeting, May 4, 2010.

Huang Z, P Du, and K Dmitry. 2009. "Model Parameter Calibration.” Presented by Henry Huang (invited speaker) at WECC Modeling and Validation Group Meeting, San Diego, CA, March 5, 2009.

Huang Z, P Du, D Kosterev, and B Yang. 2009. "Application of Extended Kalman Filter Techniques for Dynamic Model Parameter Calibration." Presented by Zhenyu (Henry) Huang (invited speaker) at EEE Power and Energy Society General Meeting 2009, July 29, 2009.

Huang Z, B Yang, and D Kosterev. 2008. "Model Parameter Calibration Using Recorded Dynamics.” Presented by Zhenyu Huang (invited speaker) at NASPI Working Group Meeting, Charlotte, NC, October 16, 2008.

Huang Z, B Yang, and D Kosterev. 2009. "Benchmarking of Planning Models Using Recorded Dynamics.” Presented by Henry Huang at IEEE PES Power System Conference, Seattle, WA, March 16, 2009.

Huhmann B, FA Tilton, and AL Miracle. 2010. "Microbial Characterization of Hanford Site Sediments." Presented by Brittany Huhmann at DOE 2010 Science and Energy Research Challenge, Argonne National Laboratory, IL, November 14, 2010.

Hui PSY and ML Gregory. 2010. "Quantifying Sentiment and Influence in Blogspaces.” Presented by Peter Hui at 2010 ACM Workshop on Social Media Analytics (SOMA 2010), Washington DC, July 25, 2010.

Izaurralde RC, AM Thomson, R Sands, and HM Pitcher. 2010. "Bringing Water into an Integrated Assessment Framework.” PNNL-19320, Richland, WA. 
Jin S, Z Huang, Y Chen, D Chavarría-Miranda, JT Feo, and PC Wong. 2010. “A Novel Application of Parallel Betweenness Centrality to Power Grid Contingency Analysis.” Presented by Shuangshuang Jin (invited speaker) at IEEE International Parallel \& Distributed Processing Symposium, Atlanta, GA, April 19, 2010.

Johnsen AM, JM Billing, AJ Casella, BD Hanson, GJ Lumetta, SA Bryan, and TG Levitskaia. 2009. "Counter-Current Solvent Extraction Behavior of Neptunium." Presented by Amanda M Johnsen at Sixteenth Symposium on Separation Science and Technology for Energy Applications, Gatlinburg, TN, October 21, 2009.

Johnsen AM, BK McNamara, SM Peper, and CZ Soderquist. 2010. "Separation of Actinides by Anion Exchange in Carbonate Solution." Presented by Chuck Soderqist at 56th Annual Radiobioassay and Radiochemistry Measurements Conference, Richland, WA, October 25, 2010.

Kaspar TC, T Droubay, JE Jaffe, V Shutthanandan, T Varga, SA Chambers, and GJ Exarhos. 2009. "XPS Band Offset Measurements of $\mathrm{ZnO}, \mathrm{CdTe}$, and CuSCN for Application in Nanostructured Photovoltaics." Presented by Tiffany Kaspar at AVS 56th International Symposium, San Jose, CA, November 10, 2009.

Kaspar TC, T Droubay, JE Jaffe, V Shutthanandan, T Varga, SA Chambers, and GJ Exarhos. 2009. "XPS Band Offset Measurements of ZnO, CdTe, and CuSCN for Application in Nanostructured Photovoltaics." Presented by Tiffany Kaspar at Pacific Northwest Chapter AVS Symposium, Troutdale, OR, September 9, 2009.

Keillor ME, CE Aalseth, AR Day, LE Erikson, JE Fast, BD Glasgow, EW Hoppe, TW Hossbach, BJ Hyronimus, HS Miley, AW Myers, and A Seifert. 2010. "Initial Results: An Ultra-Low-Background Germanium Crystal Array." Presented by Martin E Keillor (invited speaker) at Monitoring Research Review 2010, September 21, 2010.

Keillor ME, CE Aalseth, AR Day, JE Fast, EW Hoppe, TW Hossbach, BJ Hyronimus, HS Miley, and A Seifert. 2009. "Assembly and Initial Results: An Ultra-Low-Background Germanium Crystal Array for High Efficiency and Coincidence Measurements.” Presented by Martin E Keillor (invited speaker) at Monitoring Research Review 2009, Tucson, AZ, September 21, 2009.

Keillor ME, CE Aalseth, AR Day, JE Fast, EW Hoppe, BJ Hyronimus, TW Hossbach, HS Miley, A Seifert, and GA Warren. 2009. "Design and Construction of an Ultra-Low-Background 14 Germanium Crystal Array for High Efficiency and Coincidence Measurements." Presented by Marty Keillor (invited speaker) at MARC VIII, Kona, HI, April 8, 2009.

Keillor ME, CE Aalseth, AR Day, JE Fast, EW Hoppe, BJ Hyronimus, TW Hossbach, and A Seifert. 2009. "Initial Component Testing for a Germanium Array Cryostat.” PNNL-18515, Richland, WA

Kreyling SJ, AJ Brothers, SM Short, JR Phillips, and MR Weimar. 2010. "An Analytical Framework for Assessing Reliable Nuclear Fuel Service Approaches: Economic And Non-Proliferation Merits of Nuclear Fuel Leasing." In Proceedings of the 51st Annual Meeting of the Institute of Nuclear Materials Management. INMM, Deerfield, IL.

Kulkarni GR, MS Pekour, KA Pratt, BJ Friedman, G Haller, I McCubbin, and DJ Cziczo. 2010. "Understanding ice formation in the atmosphere." Presented by Gourihar Kulkarni at AAAR Conference, Portland, OR, October 27, 2010.

Lee B. 2010. "Algorithmic Scalability for the Boltzmann Transport Equation.” Presented by Barry Lee (invited speaker) at SIAM Conference on Parallel Processing for Scientific Computing (PP10), Seattle, WA, February 26, 2010.

Lee B and GE Hammond. 2010. "Parallel Newton-Krylov Methods for Ultrascale Subsurface Reactive Multiphase Flow." Presented by Glenn E Hammond and Barry Lee (invited speakers) at 11th Copper Mountain Conference on Iterative Methods, 2010, Copper Mountain, CO, April 4, 2010.

Lemmon JP, Z Yang, X Lu, G Xia, VL Sprenkle, and KD Meinhardt. 2010. "High Power Planar Sodium Metal Halide Battery." Presented by John P Lemmon (invited speaker) at 217 ECS Meeting, Vancouver, BC Canada, April 27, 2010. 
Levitskaia TG, AJ Casella, and JM Peterson. 2010. "Chitosan Materials for $\mathrm{CO}_{2}$ Separation and Capture." Presented by Tatiana Levitskaia at 9th Annual Conference on Carbon Capture \& Sequestration, Pittsburgh, PA, May 12, 2010.

Levitskaia TG, JM Peterson, KD Thrall, and JA Creim. 2010. "Evaluation of Cuprimine ${ }^{\circledR}$ and Syprine ${ }^{\circledR}$, FDA-approved Therapeutics for Wilson Disease, as Decorporation Agents for Multiple Radionuclides." Presented by Tatiana Levitskaia (invited speaker) at International Workshop on Emergency Radiobioassay, Ottawa, ON Canada, September 2, 2010.

Lin G. 2010. "Stochastic Nonlinear Data-reduction Methods with Detection \& Prediction of Critical Rare Events." Presented by Guang Lin (invited speaker) at 2010 DOE Applied Mathematics Program Meeting, Berkeley, CA, May 4, 2010.

Liu X, X Shi, A Nenes, D Barahona Orjuela, and E Jensen. 2010. "Ice Nucleation in NCAR CAM5: Uncertainties and Challenges.” Presented by Xiaohong Liu at EGU, Vienna, Austria, May 4, 2010.

Liu X, S Xie, SJ Ghan, J Boyle, SA Klein, PJ DeMott, and AJ Prenni. 2009. "Ice Nucleation in Mixed-Phase Clouds: Parameterization Evaluation and Climate Impacts." Presented by Xiaohong Liu at AGU, San Francisco, CA, December 16, 2009.

Loring JS, Z Wang, CJ Thompson, AG Joly, DS Sklarew, KM Rosso, and AR Felmy. 2010. "Carbonation of Forsterite Exposed to Water-Saturated Supercritical Carbon Dioxide." Presented by John S Loring at Goldschmidt 2010, Knoxville, TN, June 15, 2010.

Lowry PP, SD Unwin, and MY Toyooka. 2010. "Development of Age-Dependent Failure Models For Incorporation Into Existing NPP PRAs.” Presented by Peter Lowry at American Nuclear Society: 2010 Annual Meeting, San Diego, CA, June 16, 2010.

Lu N, P Du, PR Paulson, FL Greitzer, X Guo, and MD Hadley. 2010. “A Smart Grid Testbed for Multi-layer Information Management System Development.” Presented by Ning Lu at Grid-InterOp 2010, Chicago, IL, December 1, 2010.

Lu N, PR Paulson, FL Greitzer, MD Hadley, and Y Chen. 2010. "A Predictive Defense Model for the Smart Grid." Presented by Ning Lu at Grid-Interop 2009, Denver, CO, November 17, 2010.

Lu N, MR Weimar, YV Makarov, F Rudolph, S Murthy, J Arseneaux, and C Loutan. 2010. "An Evaluation of the Flywheel Potential for Providing Regulation Service in California." In 2010 IEEE Power and Energy Society General Meeting. IEEE, Piscataway, NJ.

Lu S, YV Makarov, Y Zhu, N Lu, N Prakash Kumar, and BB Chakrabarti. 2010. "Unit Commitment Considering Generation Flexibility and Environmental Constraints." In 2010 IEEE Power and Energy Society General Meeting. IEEE, Piscataway, NJ.

Lu X, G Xia, KD Meinhardt, JP Lemmon, VL Sprenkle, and Z Yang. 2010. "Development of Advanced Low-Temperature Sodium Beta-Alumina Batteries." Presented by Xiaochuan Lu at The Minerals, Metals \& Materials Society (TMS2010) 139th Annual Meeting \& Exhibition, Seattle, WA, February 18, 2010.

Lu X, JP Lemmon, G Xia, KD Meinhardt, VL Sprenkle, and Z Yang. 2010. "Development of Advanced Low-Temperature Sodium Beta-Alumina Batteries." Presented by Xiaochuan Lu at Materials Challenges in Alternative \& Renewable Energy, Cocoa Beach, FL, February 24, 2010.

Maiden WM, I Dionysiou, DA Frincke, GA Fink, and DE Bakken. 2010. "DualTrust: A Distributed Trust Model for Swarm-Based Autonomic Computing Systems." Presented by Harald Gjermundrod at Third International Workshop on Autonomous and Spontaneous Security, Athens, Greece, September 23, 2010. 
Manz DO. 2010. "PNNL/ISI Proposed Collaboration.” Presented by David Manz (invited speaker) at Usenix Workshop, Washington DC, August 9, 2010.

McCue LA, VL Bailey, SJ Fansler, and A Konopka. 2010. "Community Diversity in Individual Soil Aggregates." Presented by Lee Ann McCue at International Society for Microbial Ecology, Seattle, WA, August 26, 2010.

McDermott JE, JN Brown, BJM Webb-Robertson, PJ Sabourin, CL Sabourin, S Belisle, R Palermo, RD Smith, MG Katze, and KM Waters. 2010. "Network-based Proteomic and Transcriptomic Integration of Macaque Host-response to Influenza Infection.” Presented by Jason E. McDermott at Keystone Symposium on Biomolecular Interaction Networks, Quebec, QC Canada, March 8, 2010.

McNamara BK, SM Peper, SI Sinkov, and LE Sweet. 2010. "13C-NMR Studies of Uranium, Neptunium, and Plutonium, in a Novel Spent Fuel Dissolver Matrix.” Presented by Bruce K McNamara (invited speaker) at the $239^{\text {th }}$ Meeting of the American Chemical Society, San Francisco, CA, March 23, 2010.

McNamara BK, SM Peper, CZ Soderquist, and KL Ziegelgruber. 2009. "Observation of Cation Affects on Uranium Speciation and Stability in Peroxidic Carbonated Solution." Presented by Bruce McNamara at FCR\&D Annual Meeting, Albuquerque, NM, October 20, 2009.

McNamara BK, SM Peper, LA Snow, and CZ Soderquist. 2009. "Origen of Cation Affects on Uranium Speciation and Stability in Peroxidic Carbonated Solution." Presented by Bruce McNamara at FCR\&D Annual Meeting, Albuquerque, NM, October 21, 2009.

McNamara BK, SM Peper, LA Snow, KL Ziegelgruber, and CZ Soderquist. 2009. "Dramatic Affects of Cation Identity on Uranium Speciation and Stability in Peroxidic Carbonated Solution.” Presented by Bruce McNamara (invited speaker) at Fall 2009 ACS National Meeting, Washington DC, August 19, 2009.

McNamara BK, SI Sinkov, LE Sweet, LA Snow, HM Cho, and SM Peper. 2010. "Adjustment of the Paramagnetic Content of the Actinide Carbonate Trimers: Structural Characterization by 13C-NMR, Raman, and Visible Spectroscopy." Presented by Lanee A Snow at Plutonium Futures: The Science 2010, Keystone, CO, September 20, 2010.

Moran JJ, MK Newburn, ML Alexander, and HW Kreuzer. 2010. "Spatially Resolved Stable Isotope Analysis at the Microbial Community Level." Presented by James Moran (Invited speaker) at International Society for Microbial Ecology Conference, Seattle, WA, August 23, 2010.

Moss RH. 2010. "Scenarios and Uncertainties: Role of IAMs (and Other Methods)." Presented by Richard Moss (invited speaker) at Energy Modeling Forum Workshop on Climate Change Impacts and Integrated Assessment, Snowmass, CO, July 30, 2010.

Moss RH. 2010. "Scenarios for Climate Research and Assessment." Presented by Richard Moss (invited speaker) at International Food Policy Research Institute Scenario Planning, Washington DC, March 3, 2010.

Narvaez J, BE Endicott-Popovsky, C Seifert, CU Aval, and DA Frincke. 2010. "Drive-by-Downloads." In Proceedings of the 43rd Hawaii International Conference on System Sciences, pp. 1-10. IEEE Computer Society Press, Los Alamitos, CA.

Oehmen CS. 2009. "Minisymposium MS28 Mathematics of Cybersecurity: Advancing Applications with Proactive Capabilities." Presented by Christopher Oehmen at SIAM CSE 09, March 2, 2009.

Oehmen CS, ES Peterson, and ST Dowson. 2010. “An Organic Model for Detecting Cyber Events." In Proceedings of the Sixth Annual Workshop on Cyber Security and Information Intelligence Research, Art 66. Association for Computing Machinery, New York, NY. 
Ovchinnikov M, SJ Ghan, A Korolev, X Liu, G McFarquhar, H Morrison, and S Xie. 2009. "Modeling Aerosol Effects on Arctic Stratus: Preliminary Results from the ISDAC Case Study." Presented by Mikhail Ovchinnikov at ARM Science Team Meeting, Louisville, KY, April 1, 2009.

Peper SM. 2010. "Methyl Methacrylate-Decyl Methacrylate Copolymer - A Plasticizer-Free Alternative for Ionophore-Based Chemical Sensors." Presented by Shane Peper on July 14, 2010. MACRO2010: 43rd IUPAC (International Union of Pure and Applied Chemistry) World Polymer Congress, Glasgow, UK.

Peper SM, LE Sweet, KL Ziegelgruber, and LM Arrigo. 2010. "Speciation Studies of Lanthanides and Actinides in Carbonate-Peroxide Systems.” Presented by Shane Peper at Pittsburgh Conference, Orlando, FL, March 1, 2010.

Peterson ES, ST Dowson, and CS Oehmen. 2010. “An Organic Model for Detecting Cyber-Events.” Presented by Elena Peterson (invited speaker) at Cyber Security \& Information Intelligence Research Workshop (CSIIRW), Oak Ridge, TN, April 22, 2010.

Peterson JM, TG Levitskaia, and KD Thrall. 2009. "Evaluation of Chitosan for Uranium Decorporation in the Rat Model." Presented by James M Peterson at Health Effects of Incorporated Radionuclides, Santa Fe, NM, May 10, 2009.

Pike WA. 2009. "Visual Analytics for Data-Intensive Computing." Presented by William A Pike at Supercomputing 2009, Portland, OR, November 17, 2009.

Pike WA. 2009. "Visualization for Discovering, Predicting, and Explaining Cyber Threats." Presented by William A Pike (invited speaker) at Global Cyber Security Conference, Washington DC, August 5, 2009.

Pike WA. 2010. "Discover, Resolve, Share, Prepare: Visualization for Real-Time Behavior Analysis of Network Flows." Presented by William Pike (invited speaker) at GFIRST, San Antonio, TX, August 19, 2010.

Pike WA, J Gerth, and J Talbot. 2009. "Cyber Analytics for US-CERT: Transitioning Network Flow Visualization from the Laboratory to the Watch Floor." Presented by Bill Pike (invited speaker) at Third Annual DHS University Network Summit, Washington DC, March 19, 2009.

Pitts WK, KD Jarman, EA Miller, BS McDonald, AC Misner, MJ Myjak, SM Robinson, A Seifert, CE Seifert, and ML Woodring. 2010. "Advantages of Dual Mode Imaging for Managing Sensitive Imaging Information." In Proceedings of the 51st Annual Meeting of the Institute of Nuclear Materials Management, Baltimore, MD.

Pitts WK, KD Jarman, EA Miller, BS McDonald, AC Misner, MJ Myjak, SM Robinson, A Seifert, CE Seifert, and ML Woodring. 2010. “Advantages of Dual Mode Imaging for Managing Sensitive Imaging Information.” Presented by William Karl Pitts at INMM 2010 Meeting, Baltimore, MD, July 12, 2010.

Pool DH and DL DuBois. 2010. "Modifying Electrodes with Nickel Based Molecular Electrocatalysts for the Production of Hydrogen." Presented by Douglas Pool at 2010 ACS Spring Meeting, San Francisco, CA, March 22, 2010.

Ramuhalli P and SE Cumblidge. 2010. "Online Diagnostics and Prognostics Tools for Residual Life Estimation in Ageing LWR Components." Presented by Pradeep Ramuhalli (Invited speaker) at IAEA Coordinated Research Project on Advanced Surveillance, Diagnostics, and Prognostics Techniques, Richland, WA, June 7, 2010.

Ramuhalli P, CH Henager Jr, JW Griffin, LJ Bond, and M Dixit. 2010. "Diagnostic and Prognostic Tools for Residual Life Estimation in Ageing Nuclear Power Plant Components." Presented by Pradeep Ramuhalli at Review of Progress in Quantitative Nondestructive Evaluation, San Diego, CA, July 20, 2010.

Ramuhalli P, JW Griffin, M Dixit, LJ Bond, and CH Henager Jr. 2010. "Experimental Assessment of NDE Methods for Online Monitoring of Materials Degradation in Nuclear Power Plant Components." Presented by Pradeep Ramuhalli at 2010 American Nuclear Society Meeting, San Diego, CA, June 15, 2010. 
Ray SJ, AW Graham, GM Hieftje, CG Enke, DW Koppenaal, and CJ Barinaga. 2010. "WP02, A New Paradigm for Elemental Mass Spectrometry: The Distance-of-Flight Mass Spectrometer." Presented by David Koppenaal at 2010 Winter Conference on Plasma Spectrochemistry, Ft. Meyers, FL, January 4, 2010.

Reid DJ, MD Bearden, and JE Cabe. 2010. “Commissioning an Engineering Scale Coal Gasifier.” PNNL-19636, Richland, WA.

Reid DJ, JE Cabe, and MD Bearden. 2010. “PNNL Coal Gasification Research.” PNNL-19606, Richland, WA.

Rice JS. 2010. "Risk Management of Sea Level Rise Uncertainty--Insights from a Decision Analysis Approach." Presented by Jennie Rice at Storm Surges Congress 2010, Hamburg, DEU, September 15, 2010.

Robinson SM, KD Jarman, EA Miller, AC Misner, MJ Myjak, WK Pitts, A Seifert, CE Seifert, and ML Woodring. 2010. "Image Analysis Algorithms for Dual Mode Imaging Systems." In Proceedings of the INMM 51st Annual Meeting, Palm Desert, CA.

Robinson SM, KD Jarman, EA Miller, AC Misner, MJ Myjak, WK Pitts, A Seifert, CE Seifert, and ML Woodring. 2010. "Verification Algorithms for Dual Mode Imaging Systems." Presented by Sean Robinson at 2010 INMM Conference, Baltimore, MD, July 12, 2010.

Rosso KM, EM Murphy, AHR Bonneville, JZ Hu, DW Hoyt, Z Wang, AS Lea, M Oostrom, C Zhang, HT Schaef, and BP McGrail. 2010. "Fundamental Science for Geologic Carbon Storage: Laboratory Probes for Understanding Mineralization and Trapping Mechanisms at the Microscopic Scale." Presented by Ellyn M Murphy at $10^{\text {th }}$ International Conference on Greenhouse Gas Control Technologies, Amsterdam, NLD, September 20, 2010.

Rosso KM, MD White, EM Murphy, JZ Hu, DW Hoyt, Z Wang, AS Lea, HT Schaef, and BP McGrail. 2009. "Fundamental Science for Geologic Carbon Sequestration: Molecular Probes for Understanding Wet $\mathrm{CO}_{2}$ Interaction with Caprock Minerals." Presented by Kevin M Rosso (invited speaker) at 2009 AGU Fall Meeting, San Francisco, CA, December 17, 2009.

Shi L, D Richardson, TA Clarke, SM Belchik, DW Kennedy, A Dohnalkova, AE Plymale, Z Wang, MJ Marshall, JN Adkins, KM Rosso, JM Zachara, and JK Fredrickson. 2010. "Functional Characterization of Microbial Proteins Involved in Biogeochemical Electron Transfer Reactions." Presented by Liang Shi (invited speaker) at ERSP PI Meeting, Washington DC, March 29, 2010.

Snow LA, SI Sinkov, LE Sweet, SM Peper, and BK McNamara. 2010. "Adjustment of the Paramagnetic Content of the Actinide Carbonate Trimers: Structural Characterization by 13C-NMR, Raman, and Visible Spectroscopy." Presented by Lanee Snow at Plutonium Futures “The Science," Keystone, CO, September 20, 2010.

Soderquist CZ, AM Johnsen, BK McNamara, BD Hanson, SM Peper, KJ Carson, and JW Chenault. 2009. "A Simplified and More Environmentally Benign Spent Nuclear Fuel Dissolution Process." Presented by Chuck Soderquist (invited speaker) at Fuel Cycle Research and Development Annual Meeting, Albuquerque, NM, October 21, 2009.

Stephan EG, TD Halter, and BD Ermold. 2010. "Leveraging the Open Provenance Model as a Multi-Tier Model for Global Climate Research.” Presented by Eric Stephan at IPAW 2010, Troy, NY, June 14, 2010.

Stephan EG, TD Halter, TJ Critchlow, P Pinheiro Da Silva, and L Salayandia. 2010. "Using Domain Requirements to Achieve Science-Oriented Provenance." In Provenance and Annotation of Data and Processes - Third International Provenance and Annotation Workshop, IPAW 2010: Lecture Notes in Computer Science 6378:301-303, eds DL McGuinness, JR Michaelis, and L Moreau, Springer, NY.

Sushko PV and KM Rosso. 2010. "Interaction of Intercalated Li with Oxygen Vacancies in $\mathrm{TiO}_{2}$ and its Effect on the Mobility of Li+ and E-species." Presented by Peter V Sushko at 217th Electrochemical Society Meeting, Vancouver, BC Canada, April 26, 2010. 
Sweet LE, KL Ziegelgruber, LM Arrigo, and SM Peper. 2009. "Speciation Studies of Ce and Pu Peroxo-Carbonate Systems." Presented by Lucas E Sweet (invited speaker) at Fuel Cycle Research \& Development Annual Review Meeting, Albuquerque, NM, October 20, 2009.

Szecsody JE, MJ Truex, L Zhong, MD Williams, CT Resch, and JP McKinley. 2010. "Remediation of Uranium in the Hanford Vadose Zone Using Gas-Transported Reactants: Laboratory Scale Experiments in Support of the Deep Vadose Zone Treatability Test Plan for the Hanford Central Plateau." PNNL-18879, Richland, WA.

Teeguarden JG. 2010. "Biomonitoring and Variability of Bisphenol A in Human Subjects Linking Urine Biomarkers to Exposure and Effects Through Computational Pharmacokinetics and Pharmacodynamics." Presented by Justin Teeguarden (invited speaker) at Toxicology Forum, Aspen, CO, July 14, 2010.

Teeguarden JG. 2009. "Genomic and Proteomic Assessment of the Pulmonary Response to Asbestos and Single Walled Carbon Nanotubes in Mice." Presented by Justin Teeguarden (invited speaker) at Nanotechnology Health and Safety Forum, Seattle, WA, June 8, 2009.

Teeguarden JG. 2010. “In Vitro Nanotoxicology: The Risk Assessor's Little House of Horrors.” Presented by Justin Teeguarden (invited speaker) at Oregon Nanoscience and Nanotechnologies Institute Green Nano Meeting, Portland, OR, June 17, 2010.

Teeguarden JG. 2010. "Physics, Physiology and Kinetics: The Foundation for Predictive Modeling of Nanomaterial Dosimetry for EHS Assessments." Presented by Justin Teeguarden (invited speaker) at National Academy of Sciences Committee on Environmental, Health, and Safety Research on Nanomaterials, Richland, WA, May 3, 2010.

Teeguarden JG. 2010. "Solutions to Key Challenges in Dosimetry for Nanotoxicology." Presented by Justin Teeguarden (invited speaker) at Multiscale Toxicology Initiative, Jefferson, AR, April 8, 2010.

Teeguarden JG. 2010. "The Dosimetry Contribution to An Integrative Program for Multi-Scale Nanotoxicology." Presented by Justin Teeguarden (invited speaker) at Multiscale Toxicology Initiative External Review Board Meeting, Oak Ridge, TX, June 16, 2010.

Teeguarden JG, PM Hinderliter, BD Thrall, G Orr, KM Waters, RA Corley, and JG Pounds. 2010. "The Particokinetic and Physiological Basis for In Vitro-In Vivo Extrapolation of Nanomaterial Toxicity Studies." Presented by Joel Pounds (invited speaker) at ATS 2010 Conference, New Orleans, LA, May 15, 2010.

Thompson CJ, Z Wang, AG Joly, JS Loring, DS Sklarew, KM Rosso, and AR Felmy. 2010. "Investigation of Forsterite Transformation in Supercritical $\mathrm{CO}_{2}$ by In-situ Optical Spectroscopy." Presented by Christopher J Thompson at 239th ACS National Meeting, San Francisco, CA, March 22, 2010.

Vishnu A, HJJ van Dam, WA De Jong, P Balaji, and S Song. 2010. "Data Centric Fault Tolerance." Presented by Abhinav Vishnu at International Conference for High Performance Computing, Networking, Storage and Analysis, November 16, 2010.

Walsh SJ and PD Whitney. 2010. "Calibrating Bayesian Network Representations of Social-Behavioral Models." Presented by Paul Whitney (invited speaker) at Social Computing, Behavior Modeling and Prediction, March 1, 2010.

Walsh SJ, AC Dalton, PD Whitney, and AM White. 2010. "Parameterizing Bayesian Network Representations of Social-Behavioral Models by Expert Elicitation." In IEEE International Conference on Intelligence and Security Informatics (ISI 2010), pp. 227-232. IEEE, Piscataway, NJ.

Wang D, J Xiao, W Xu, and J Zhang. 2010. "Electrochemical Investigation of Novel 5V Cathode Material: $\mathrm{LiNiPO}_{4}$ and $\mathrm{Li}_{2} \mathrm{CoPO}_{4} \mathrm{~F}$." Presented by David Wang at The 15th International Meeting on Lithium Batteries, Montreal, PQ Canada, July 1, 2010. 
Wang D, J Xiao, W Xu, and J Zhang. 2010. "Electrochemical Investigation of Novel Cathode Material $\mathrm{Li}_{2} \mathrm{CoPO}_{4} \mathrm{~F}$ for Lithium Ion Batteries." Presented by David Wang (invited speaker) at ICMA Workshop of Physics of Novel Energy Materials, Beijing, China, June 2, 2010.

Wang D, J Xiao, W Xu, D Choi, and J Zhang. 2009. "Investigation of the Novel Cathode Material $\mathrm{Li}_{2} \mathrm{CoPO}_{4} \mathrm{~F}$ for Lithium Ion Batteries.” Presented by Deyu Wang at MRS 2009 Fall Meeting, Boston, MA, December 1, 2009.

Wang Z, CJ Thompson, AG Joly, DS Sklarew, LJ Poindexter, and KM Rosso. 2009. "In-situ Optical Spectroscopy Investigation of Water and Its influence on Forsterite Transformation in Supercritical $\mathrm{CO}_{2}$." Presented by Zheming Wang at 2009 AGU Fall Meeting, San Francisco, CA, December 15, 2009.

Waters KM. 2009. "Bioinformatic Integration of Omics Data for Network and Pathway Reconstruction." Presented by Katrina Waters (invited speaker) at WSU Tri-Cities - BioinformaticIntegration of 'OmicsData for Network and Pathway Reconstruction, WA, November 13, 2009.

Waters KM. 2010. "Dynamic Network Analysis of Nanosilica-induced Toxicity Pathways using Microarray and Proteomic Data." Presented by Katrina Waters (invited speaker) at Society of Toxicology 2010 Annual Meeting, Salt Lake City, UT, March 10, 2010.

Waters KM, BJM Webb-Robertson, NJ Karin, X Zhang, JM Jacobs, DJ Bigelow, and KM Lee. 2010. "Integration of Microarray and Proteomic Data Reveals Susceptibility Factors for Exacerbated Immune Response in Diet-Induced Obese Mice Exposed to Cigarette Smoke." Presented by Katrina Waters (invited speaker) at Society of Toxicology, Salt Lake City, UT, March 9, 2010.

Webb-Robertson BJM. 2009. “A Bayesian Integration Model of High-Throughput Proteomics Data for Improved Early Detection of Microbial Infections." Presented by Bobbie-Jo Webb-Robertson (invited speaker) at Pacific Symposium on Biocomputing, Kohala, HI, January 9, 2009.

Webb-Robertson BJM, KM Waters, JM Jacobs, X Zhang, SM Varnum, DJ Bigelow, JR Hoidal, MB Scholand, and JG Pounds. 2010. "Bayesian Feature Selection Identifies Human Plasma Proteomic Biosignatures of Systemic Chronic Inflammatory and Oxidative Stress." Presented by Bobbie-Jo Webb-Robertson (invited speaker) at Society of Toxicology, Salt Lake City, UT, March 9, 2010.

Weil KS, GJ Grant, Y Hovanski, and JT Darsell. 2010. "Joining Technologies for Coal Power Applications." Presented by Scott Weil, 24th Annual Conference on Fossil Energy Materials, Pittsburgh, PA, May 25, 2010.

White MD, BP McGrail, HT Schaef, JZ Hu, DW Hoyt, AR Felmy, KM Rosso, and SK Wurstner. 2010. "Multiphase Sequestration Geochemistry: Model for Mineral Carbonation.” In GHGT-10, Energy Procedia. PNNL, Richland, WA.

White MD, BP McGrail, HT Schaef, JZ Hu, DW Hoyt, KM Rosso, AR Felmy, and SK Wurstner. 2010. "Multiphase Sequestration Geochemistry: Model for Mineral Carbonation." Presented by Mark D White at GHGT-10, Amsterdam, NLD, September 22, 2010.

White TA. 2010. “AIT Technology Overview." Presented by Tim White (invited speaker) at Algorithm Development for Security Applications III: Advanced Imaging Technologies, Boston, MA, April 27, 2010.

Whitney PD. 2010. "Illicit Nuclear Trafficking." Presented by Paul Whitney at TPAI 2010 Review, Richland , WA, June 2, 2010.

Whitney PD. 2010. "Information Integration." Presented by Paul Whitney at TPAI Advisory Review, Richland, WA, June 2, 2010.

Xia G, Z Yang, VL Sprenkle, KD Meinhardt, X Lu, NL Canfield, and JP Lemmon. 2009. "Development of Planar Sodium b-Alumina Battery Modules for Utility Applications." Presented by Gordon Xia at MS\&T09, Pittsburgh, PA, October 28, 2009. 
Xiao J, W Xu, D Choi, and J Zhang. 2009. "Synthesis and Characterization of $\mathrm{LiMnPO}_{4}$ by a Novel Precipitation Method." Presented by Jie Xiao, Natasha Chernova, Wu Xu, Daiwon Choi, M Stanley Whittingham, and Ji-Guang Zhang at Materials Research Society, Boston, MA, December 2, 2009.

Xu W, J Xiao, D Wang, J Zhang, and J Zhang. 2010. "Effects of Nonaqueous Electrolytes on Primary Li-Air Batteries." In Proceedings of the 44th Power Sources Conference, pp. 43-46. U.S. Army Electronics Research and Development Laboratory, Fort Monmouth, NJ.

Zaveri RA, JC Barnard, RC Easter Jr, N Riemer, and M West. 2009. "Simulating the Influence of Aerosol Mixing State on Optical and Cloud Nucleating Properties with a Particle-Resolved Aerosol Model." Presented by Rahul Zaveri, American Association for Aerosol Research (AAAR) 28th Annual Conference, Minneapolis, MN, October 28, 2009.

Zaveri RA, RC Easter Jr, and JC Barnard. 2009. "Evaluating the Effects of Mixing State Assumptions in Aerosol Modules on the Predicted Optical and CCN Activation Properties." Presented by Rahul Zaveri, International Aerosol Modeling Algorithms Conference, Davis, CA, December 11, 2009.

Zhang J. 2010. "Nanostructured Materials for Energy Storage Applications.” Presented by Jason Zhang (invited speaker) at Workshop of Physics of Novel Energy Materials and Collaborating Universities, Beijing, China, May 31, 2010.

Zhang J, L Li, S Kim, W Wang, B Schwenzer, B Chen, Z Nie, M Vijayakumar, J Liu, Z Yang, MA Hickner, and M Skyllas-Kazacos. 2010. "Research on Flow Batteries at Pacific Northwest National Laboratory." Presented by Jianlu Zhang, IFBF 2010: 1st International Flow Battery Forum, Vienna, Austria, June 16, 2010.

Zhang J, D Wang, W Xu, J Xiao, RE Williford, and GL Graff. 2010. "High Capacity Li-Air Batteries." Presented by Jason Zhang at 15th International Meeting on Lithium Batteries, Montreal, PQ Canada, July 1, 2010.

Zhang J, J Xiao, J Liu, D Choi, CM Wang, Z Yang, W Xu, and GL Graff. 2009. "Silicon Based High Capacity Anode for Li-Ion Battery Applications." Presented by Jason Zhang at 2009 MRS Fall Meeting, Boston, MA, December 2, 2009.

Zhang J, W Xu, J Xiao, D Wang, D Wang, RE Williford, and J Liu. 2009. “Ambient Operation of Li/Air Batteries." Presented by Jason Zhang (invited speaker) at Lithium Mobil Power 2009, Boston, MA, November 12, 2009.

Zhou N, FK Tuffner, G Lin, TA Ferryman, and Z Huang. 2010. "A State Prediction Methodology for Electric Power Grid Operation.” Presented by Ning Zhou at 2nd International Conference on Computational Sustainability, MIT, Cambridge, MA, June 30, 2010. 


\section{Appendix C}

\section{Index of Investigators}




\section{Appendix C}

\section{Index of Investigators}

\section{A}

Aalseth, Craig E. - 232

Addleman, R. Shane - 148, 154

Alexander, M. Lizabeth - 3, 12, 30

Amidan, Brett G. - 231

Amonette, James E. 94

Anderson, David - 114

Anheier Jr., Norman C. - 3, 62, 71

Arey, Bruce W. - 172

Arrigo, Leah M. - 52

Atkinson, David A. -76

Autrey, S. Thomas -70

\section{B}

Bacon, Diana H. - 100, 210

Baer, Donald R. - 56

Bailey, Vanessa L. - 24, 34, 35, 203

Baird, Cheryl L. $-21,25$

Baker, Eddie G. - 122

Bandara, Uditha C. -67

Barinaga, Charles J. -8

Barker, Erin I. - 166

Barker, Kevin J. - 199

Barnard, James C. -87

Batdorf, Michael T. - 124

Baxter, Douglas J. - 194

Beagley, Nathaniel - 93, 180

Bearden, Mark D. - 64, 122, 127

Beliaev, Alex S. -40

Bell, Eric B. - 208

Benz, Jacob M. - 218

Bernacki, Bruce E. -15

Bevill, Aaron M. - 218

Beyer, Carl E. - 227

Black, Gary D. -84

Blake, Thomas A. - 14

Bond, Leonard J. - 137

Bond-Lamberty, Benjamin - 117

Bonheyo, George T. - 47

Boschek, Curt B. - 25

Bowyer, Sonya M. -216

Boyd, Brian K. - 127

Brambley, Michael R. - 133
Brockman, Fred J. -72

Brooks, Kriston P. -48

Brothers, Alan J. - 197

Bruce, Joseph R. - 211

Bruemmer, Stephen M. - 161, 172

Bryan, Samuel A. - 73

Bryant, Donald - 40

Buchko, Garry W. - 21, 25

Bunch, Kyle J. - 138

Burton, Sarah D. - 118

Busche, Brad J. - 154

Bushaw, Bruce A. -3

Bylaska, Eric J. - 188

C

Cabe, James E. - 127

Callister, Stephen J. - 39

Calvin, Katherine V. - 117

Camaioni, Donald M. - 70

Campbell, James A. - 47, 53

Campbell, Luke W. - 216

Cannon, Bret D. -52

Carado, Anthony J. -8

Carroll, Kenneth C. -83

Carroll, Thomas E. - 183, 185, 187

Casella, Amanda J. - 73

Casella, Andrew M. - 219, 227

Chambers, Scott A. -63

Chao, Yulain -74

Chappell Alan R. - 205

Chase, Jared M. - 205

Chassin, David P. - 124, 132

Chavarría-Miranda, Daniel G. - 184, 193

Chen, Feng - 163

Chen, Yousu - 202

Cho, Herman M. - 52

Choi, Daiwon - 152

Chouyyok, Wilaiwan - 144

Clarke, Leon E. - 91

Cloutier, Janet M. -61

Clowers, Brian H. - 12, 30, 57

Coffey, Gregory W. - 143 
Comstock, Jennifer M. -86

Cowell, Andrew J. - 200, 205, 208

Coyle, Christopher A. - 163

Cumblidge, Stephen E. - 226

Cziczo, Daniel J. - 16, 86

\section{D}

Dagle, Robert A. -58

Dalton, Angela C. -197

Dang, Liem X. -50

Danielson, Gary R. - 190

Darsell, Jens T. -155

Davies, Richard W. -150

de Jong, Wibe A. - 179

Devanathan, Ramaswami - 166

Diao, Ruisheng - 112

Dirks, James A. - 91, 114, 133

Dixit, Manish - 133

Dixit, Mukul - 137, 138

Doctor, Steven R. - 226

Dohnálek, Zdenek - 60

Douglas, Matthew - 221

Droubay, Timothy C. -162

Du, Pengwei - 124, 130

DuBois, Daniel L. - 54, 164

Duckworth, Douglas C. - 26, 61

Dupuis, Michel - 68

\section{$\mathbf{E}$}

Easter, Richard C. - 87, 101

Edmonds, James A. - 91

Edwards, Danny J. - 172

Eiden, Gregory C. - 61

Elizondo, Marcelo A. - 112

Elliott, Douglas C. - 121, 123

Elliott, Michael L. -122

Ellis, Peter C. -200

Enke, Christie G. -8

Ewing, Robert G. $-62,76$

$\mathbf{F}$

Fan, Jiwen -86

Fang, Yilin -81

Fast, Jerome D. - 101

Felmy, Andrew R. - 139, 157

Feo, John T. - 184

Fernandez, Nick - 133

Ferryman, Thomas A. - 113
Fink, Glenn A. - 211

Flahert, Julia E. -92

Fligg, A. Keith -200

Franklin, Lyndsey R. - 190

Freedman, Vicky L. - 119

Freeman, Charles J. - 64, 122

Fryxell, Glen E. - 144

G

Ganguly, Dilip - 93

Gassman, Paul L. - 52

Gastelum, Zoe N. - 108

Geelhood, Kenneth J. - 227

Gerlach, David C. - 3, 225

Gesh, Christopher J. - 216, 218, 225

Ghan, Steven J. - 86

Gibson, Tara D. -205

Gill, Gary A. - 61

Glass, Kevin A. - 194

Gorrissen, Will J. - 133

Gorton, Ian 88 - 195

Gosink, Luke J. - 210

Graff, Gordon L. - 74

Grant, Glenn J. - 155

Grate, Jay W. - 5, 34, 62, 71, 72, 77, 97, 148, 159, 203

Gregory, Michelle L. - 200, 208

Greitzer, Frank L. - 181

Griffin, Jeffrey W. - 137

Gustafson Jr., William I. - 101

\section{H}

Haas, Derek A. - 216, 232

Hadley, Mark D. - 177

Halappanavar, Mahantesh M. - 184

Hamilton, Erin L. - 114

Hammond, Glenn E. - 209

Hampton, Shawn D. - 181

Hanrahan, Timothy P. - 99

Hanson, Brady D. - 227

Harper, Aaron S. - 205

Hart, Garret L. -61

Harvey, Julia B. - 108

Harvey, Scott D. -77

Hess, Nancy J. - 35

Hess, Wayne P. -63

Hieftje, Gary M -8

Higgins, Steven R. - 94

Hiranuma, Naruki - 6

Hjerpe, Andrea L. -53 
Hoffman, Michael J. - 124

Hohimer, Ryan E. - 181

Hovanski, Yuri - 155

Hoyt, David W. - 157

$\mathrm{Hu}$, Dehong -72

Hu, Jianzhi - 116, 146, 157

Huang, Cheng - 129, 163

Huang, Zhenyu (Henry) - 130, 202

Huesemann, Michael H. - 123

Humble, Paul H. - 64

I

Izaurralde, Roberto C. - 117

$\mathbf{J}$

Jacob, Richard E. - 21, 33

Jarman, Kenneth D. - 180, 197, 218, 223

Jarman, Kristin H. - 180

Jin, Chunlian - 112, 114

Johnson, Grant E. - 55

Johnson, John R. - 184

Joly, Alan G. -45

$\mathbf{K}$

Karkamkar, Abhijeet J. - 70

Kaspar, Tiffany C. -162

Katipamula, Srinivas - 133

Kay, Bruce D. -60

Keillor, Martin E. - 232, 233

Kelly, James F. - 14, 30

Kim, Jin Yong - 169

Kim, Son H. -91

King, Dale A. -48

King, David L. $-58,125$

Kintner-Meyer, Michael C.W. - 114, 124

Kirkham, Harold - 112, 132

Kleese-Van Dam, Kerstin - 191

Koech, Phillip K. - 118

Konopka, Allan E. - 203

Koppenaal, David W. -8

Kowalski, Karol - 188

Kozelisky, Anne E. - 107

Kreuzer, Helen W. - 26, 30

Kreyling, Seán J. - 108, 197

Krishnamoorthy, Sriram - 206

Krishnan, Manoj Kumar - 186

Kulkarni, Gourihar R. - 16, 86

Kumar, Nirupama Prakash - 124
Kuprat, Andrew P. - 119

Kwak, Ja Hun - 157

Kwon, Jae W. - 107

$\mathbf{L}$

LaMarche, Brian L. - 39

Laskin, Julia - 55

Last, George V. -84

Lavender, Curt A. - 150, 155

Lea, A. Scott - 94

Lebarbier, Vanessa M. - 58

Lee, Barry - 209

Lei, Hanwu - 121

Lemmon, John P. - 143, 163, 173

Leung, Lai-Yung (Ruby) - 89, 95, 102

Levitskaia, Tatiana G. - 73

Li, Dongsheng - 166

Li, Guosheng - 143

Li, Liyu - 127

Li, Xiaolin (Shari) - 152, 173

Li, Zhaohui - 151

Lin, Guang - 68, 81, 113

Lin, Xiao -60

Lin, Yuehe -151

Linggi, Bryan E. - 21

Liu, Jun - 74, 164

Liu, Xiaohong - 86

Lizarazo-Adarme, Jair A. - 58

Loring, John S. -45

Lowry, Peter P. - 215

Lu, Ning -177

Lu, Shuai - 112

Lu, Xiaochuan - 143

M

Mace, Emily K. - 232

Magnuson, Jon K. - 30, 32

Marquez, Andres - 192

Marshall, Eric J. - 208

Martin, Paul F. -9

Martucci, Louis M. - 190

Mayhorn, Ebony T. - 112

McCue, Lee Ann - 24

McDonald, Benjamin S. - 223, 234

McFarlane, Sally A. - 92

McGrath, Liam R. - 200, 208

McKinley, James P. - 61

McNamara, Bruce K. - 219, 221

Mei, Donghai - 68 
Meier, David E. - 107

Meyer, Ryan M. - 226

Miller, Erin A. - 218, 223, 234, 235

Miller, Keith D. - 5, 72, 148

Minard, Kevin R. -33

Misner, Alex C. - 216, 223

Moran, James J. - 30

Morgan, William F. - 36

Moss, Richard H. - 102

Mueller, Karl T. - 56

Murray, Christopher J. - 84

Myjak, Mitchell J. - 223

\section{$\mathbf{N}$}

Naes, Benjamin E. - 61

Neorr, Peter A. - 185

Nichols, Patrick J. - 201

Nickless, William K. - 201

Noo, Frédéric - 235

\section{$\mathbf{O}$}

O’Hara, Kelly A. - 200

O'Hara, Matthew J. - 221

Oehmen, Christopher S. - 194, 201

Olszta, Matthew J. - 172

Oostrom, Martinus - 97

Orr, Galya - 34, 72

Orr, Heather M. -110

Orrell, John L. - 232, 233

Ovchinnikov, Mikhail - 86

Owen, A.T. -9

Ozanich, Richard M. - 148

\section{$\mathbf{P}$}

Palmer, Bruce J. - 67, 81, 186, 210

Palo, Daniel R. - 58

Pan, Anqiang - 152

Panisko, Ellen A. - 23, 32

Parab, Kshitij K. -70

Paulson, Patrick R. - 181, 185, 190

Payne, Rosara F. - 228

Pekour, Mikhail S. -16

Peper, Shane M. - 3, 221

Petersen, Catherine E. - 47, 53

Peterson, James M. - 73

Phillips, Mark C. -15

Pitman, Stan G. - 150
Pitts, W. Karl - 223, 231

Purohit, Sumit -83

Q

Qiao, Hong (Amy) - 138

Quadrel, Richard W. - 183

$\mathbf{R}$

Ramuhalli, Pradeep - 137

Rasch, Philip J. - 93, 101

Ray, Steven J. -8

Rector, David R. - 139

Reid, Douglas J. - 127

Resat, Haluk - 203, 34

Rice, Jennie S. - 102

Rice, Jennie S. -114

Richmond, Marshall C. - 99

Rieke, Peter C. -64

Riemer, Nicole -87

Riensche, Roderick M. - 190

Robertson, J. David - 107

Robinson, Sean M. - 218, 223

Rockhold, Mark L. -84

Rodland, Karin D. - 21

Roesijadi, Guritno - 123

Rohatgi, Aashish - 154

Rosso, Kevin M. - 157, 167

Rousseau, Roger J. - 68

Runci, Paul J. - 102

Ryan, Joseph V. - 220

S

Sadovsky, Artyom - 124

Samaan, Nader A. - 112

Sams, Robert L. -30

Sanquist, Thomas F. -110

Schaef, H. Todd - 9

Scheele, Randall D. - 107, 219

Scheibe, Timothy D. -81

Schwantes, Jon M. - 107, 148

Schwenzer, Birgit -, 129

Scott, Michael J. - 91, 102

Sears, Jesse A - 157

Sego, Landon H. - 210

Seifert, Allen - 223, 232, 234

Shilling, John E. -6

Shin, Yongsoon - 72, 144, 151, 169 
Short, Luke C. $-47,53$

Short, Steven M. - 108

Shui, Bin - 110

Singh, Baldwinder - 101

Siple, Timothy E. - 114

Sivaramakrishnan, Chandrinka - 83

Sivaraman, Chitra -185

Smith, L. Eric - 216, 218

Smith, Richard D. -27

Smith, Steven C. - 52, 110

Soares, Thereza A. -25

Song, Chen -6

Song, Shuaiwen - 179

Stephens, Elizabeth V. - 150

Stewart, Mark L. - 139

Stolyar, Sergey - 32

Straatsma, Tjerk P. -25

Sullivan, E. Charlotte - 84

Szechenyi, Scott C. -225

$\mathbf{T}$

Tagestad, Jerry D. - 114

Tardiff, Mark F. - 180

Tartakovsky, Alexandre M. - 67, 68

Thallapally, Praveen K. - 77, 148

Thevuthasan, Theva -63

Thom, Ronald M. - 123

Thompson, Christopher J. - 45

Thomson, Allison M. - 117

Thorne, Paul D. - 119

Thrall, Karla D. -21

Timchalk, Charles A. - 21, 53

Toloczko, Mychailo B. - 161, 172

Towne, Silas A. - 116

Trease, Harold E. - 231

Tuffner, Francis K. - 113

Turcu, Romulus V - 157

\section{$\mathbf{U}$}

Unwin, Stephen D. - 102, 215

V

Vishnu, Abhinav - 179

Viswanathan, Vilayanur V. - 116
W

Walsh, Stephen J. - 197

Waltman, Melanie J. - 76

Wang, Deyu (David) - 116, 118, 152

Wang, Hailong - 93

Wang, Hongfei -66

Wang, Jun - 151

Wang, Liang (Frank) - 11

Wang, Zheming -45

Ward, Anderson L. -81

Warner, Marvin G. - 5, 71, 72, 148, 159

Warren, Glen A. - 216

Washton, Nancy M. - 56

Webb-Robertson, Bobbie-Jo M. - 201

Weil, K. Scott - 155

Weimar, Mark R. - 108

West, Matt -87

West, Tristram O. -117

White, Amanda M. - 197

White, Mark D. - 83, 100, 210

White, Timothy A. - 234, 235

Whitney, Paul D. - 197

Whyatt, Greg A. - 64

Wiedner, Susan D. - 23

Wigmosta, Mark S. -99

Wilkins, Michael J. - 34, 39

Williams, Mark D. - 119

Williford, Rick E. - 220, 227

Wise, Marshall A. - 91, 117

Wittman, Richard S. -218

Wong, Pak Chung - 202

Woodring, Mitchell L. - 223

Woods, Vincent T. -232

Wright, Aaron T. - 23, 32, 39

Wunschel, David S. - 26

Wurstner, Signe K. - 119

$\mathbf{X}$

Xia, Guan-Guang (Gordon) - 129

Xiao, Jie - 116, 118, 152, 173

$\mathrm{Xu}, \mathrm{Wu}-116,118,152$

$\mathbf{Y}$

Yabusaki, Steven B. - 81, 100

Yang, Feng - 21

Yang, Jenny Y. - 54

Yang, Zhenguo (Gary) - 74, 143

Yantasee, Wassana - 144

Young, Jonathan - 185

Yu, Jianguo - 152 


\section{$\mathbf{Z}$}

Zangar, Richard C. -21

Zaveri, Rahul A. - 87

Zhang, Changyong - 34, 97

Zhang, Jiguang (Jason) - 116, 118, 152, 173

Zhang, Yanfeng - 112
Zhang, Yanwen - 159

Zheng, Feng (Richard) - 48, 64

Zhou, Ning -113

Zhou, Xiao-Dong - 171

Zuljevic, Nino - 114, 190 


\section{Pacific Northwest}

NATIONAL LABORATORY

Proudly Operated by Battelle Since 1965

\section{ANNUAL REPORT 2010}

\section{Laboratory Directed}

\section{Research \& Development}

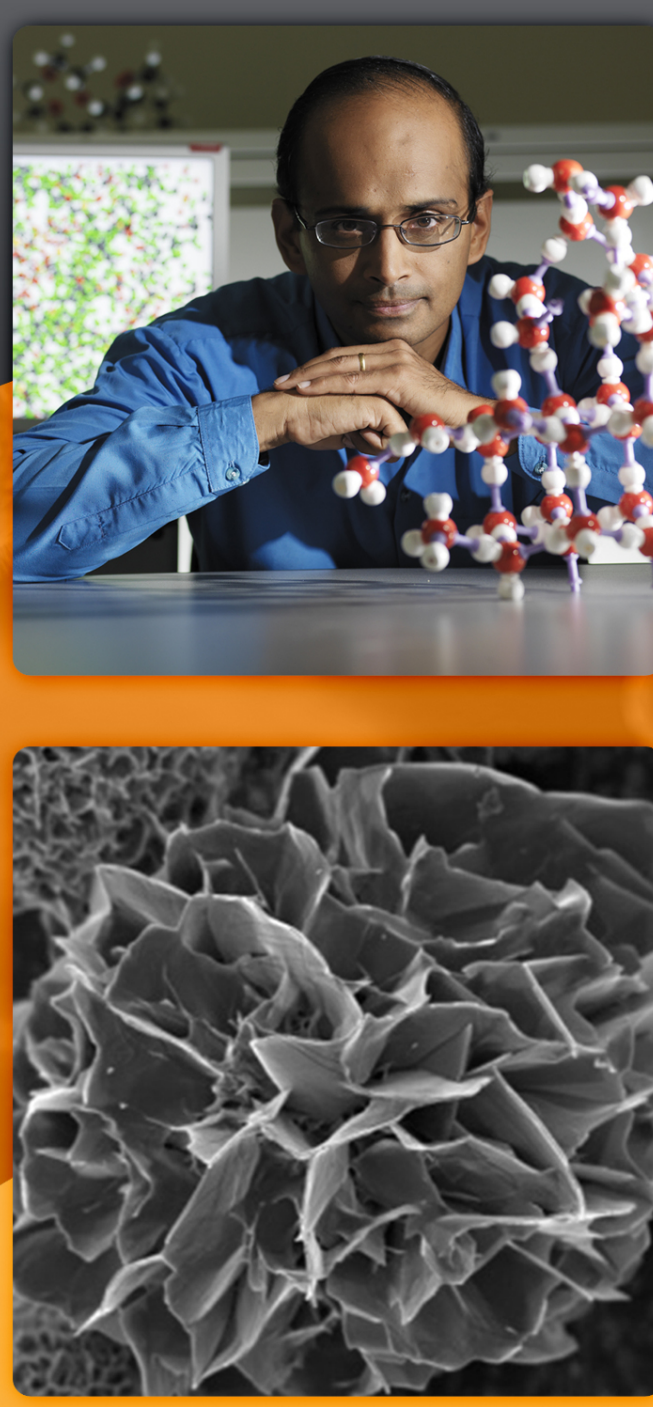

\section{www.ldrd.pnl.gov}

Pacific Northwest National Laboratory has an enduring mission to bring molecular and environmental sciences and engineering strengths to bear on DOE missions and national needs. Our vision is to be recognized worldwide and valued nationally for leadership in accelerating the rate of discovery and deployment of solutions to challenges in energy, national security, and the environment. 